\title{
Novel Methodologies for the Synthesis of Multivalent Glycoconjugates
}

By

\author{
Stefan Munneke
}

\author{
A thesis \\ submitted to the Victoria University of Wellington \\ in fulfilment of the requirements for the degree of \\ Doctor of Philosophy
}


"A man can be as great as he wants to be. If you believe in yourself and have the courage, the determination, the dedication, the competitive drive, and if you are willing to sacrifice the little things in life and pay the price for the things that are worthwhile, it can be done."

Vince Lombardi 


\section{Abstract}

Glycoconjugates, such as glycolipids and glycoproteins, play an important role in health and disease. The synthesis and biological evaluation of these glycoconjugates allows for the development of novel carbohydrate-based therapeutics and analytical tools. Traditionally, the conjugation of glycans to other substrates required the installment of an anomeric linker during the total synthesis of the glycan, however, this strategy does not allow for the conjugation of naturally isolated glycans. To address this concern, glycan conjugation methodologies without the need for protecting groups have been developed, including the use of oxyamine conjugation methodologies. In particular, the synthesis of a variety of novel bi-functional oxyamine linkers enabled the rapid assembly of various types of glycoconjugates, including fluorescent- and biotinylated-glycans, glycoproteins and multivalent glycodendrons.

The multivalent presentation of glycans to cell surface lectins is often required to induce a measurable biological response. This multivalent binding can be achieved by the presentation of glycans on dendrons as these 'glycodendrons' have increased affinity for their corresponding lectin compared to monovalent glycans. Moreover, these glycodendrons have several advantages, including high synthetic control, low cytotoxicity and in addition can be derivatised with molecular probes of choice, which can aid in the biological evaluation of these glycoconjugates. Accordingly, novel biotinylated and fluorescent dendrons were synthesised from a highly convergent second generation dendron core scaffold. These functionalised dendrons then allow for the nonavalent conjugation of carbohydrates, such as Lewis antigens, for their biological evaluation in the selective targeting of lectins.

Lewis antigens play an important role in host cell recognition, but these glycans are also involved in disease, such as in cancer metastasis and HIV-infection. The synthesis of Lewis antigens allows for the biological evaluation of these glycans, and moreover, could be employed in the development of novel glycan-based therapeutics and analytical tools. Accordingly, a novel high-yielding and efficient synthesis of a crystalline trisaccharide building block is presented, which can then be utilised in the synthesis of most Type-2 Lewis antigens. In particular, the global deprotection of the crystalline material gave the Lewis ${ }^{\mathrm{X}}$ glycan antigen, a natural ligand for the C-Type lectin DC-SIGN on dendritic cells and macrophages.

Finally, the rapid assembling of complex multivalent glycodendrons is discussed by conjugating the glycan antigens to the functionalised multivalent dendrons through the use of the bi-functional oxyamine linker methodology. In particular, the synthesis of a fluorescent Lewis ${ }^{\mathrm{X}}$ glycodendron is presented, and to demonstrate a potential biological application of this methodology, the fluorescent Lewis ${ }^{\mathrm{X}}$ glycodendron is evaluated as a flow cytometry marker for the C-type lectin DC-SIGN on human macrophages. 


\section{Acknowledgements}

After years of hard work, my journey has come to an end, and what a journey it has been. This incredible adventure started four years ago, when I moved from the Netherlands to the 'Land of the Long White Cloud', to 'the Middle of Middle Earth', to a place I have come to love: Wellington, New Zealand. Here I had the privilege to study at Victoria University of Wellington in the Immunoglycomics Reseach group, in collaboration with the Ferrier Research Institute (former IRL).

First and foremost, I would like to thank my supervisors Mattie Timmer, Bridget Stocker and Gavin Painter, for their guidance, encouragement, support, and belief in me, as well as giving me the opportunity to study in Wellington. I have really enjoyed the conversations and discussions on both chemistry and biology, and I admire you for your curiosity and excitement, your dedication and commitment in teaching me the trade of chemistry and immunology.

Special thanks to those with whom I got to work with more closely during the last four years. Thanks to Kristiana for your cheerful collaboration, encouragement and for accompanying me during late night hours. Thanks to Julien and Jaimé for your help on the oxyamine conjugation project, and Kristel for testing the glycoconjugates, Janice for bouncing ideas, and in addition, Alex, Gert, Hilary, Ben, Billy, Ashna, Emma, Anna, Janelle, Jessie, Amy, Charlotte, Shamal, Rhia, Chriselle and other SCPS students for great times in the lab.

I would like to acknowledge the academic and supporting staff of Victoria University of Wellington, and in particular Ian Vorster for all your help, instant availability and positive attitude. I would also like to thank Colin Hayman from the Ferrier Research Institute for your help and guidance with chromatography and Graeme Gainsford (Callaghan Innovation) for your help with crystallography. Thanks to Industrial Research Limited (Ferrier Research Institute), Victoria University of Wellington, New Zealand Institute of Chemistry who have financially supported me throughout my Ph.D. studies.

A big thumbs up for my friends and family, for riding this journey with me, for your company, laughter and support. In particular, my lovely mum, awesome dad and amazing brother for your belief in me, your interest in my research, and the privilege to Skype almost every week.

Finally, I especially would like to thank my fiancée, Marji. Without you I could not have done this. I would like to thank you for all our adventures, your encouragement, caring and love. It has been a long road and I truly believe that my accomplishments are yours to share. 


\section{Preface}

This doctoral thesis consists of six indivual chapters. Each chapter contains individual compound numbering and contains separate citation and reference lists. To further clarify; the compound numbering and citations are chapter bound, and the identical compounds and citations numbers may differ between the chapters.

The findings in this doctoral thesis has been/will also be presented in the following manuscripts:

- $\quad$ Munneke, S.; Prevost, J. R. C.; Painter, G. F.; Stocker, B. L.; Timmer, M. S. M. 'The Rapid and Facile Synthesis of Oxyamine Linkers for the Preparation of Hydrolytically Stable Glycoconjugates' Org. Lett. 2015, 17, 624-627.

- $\quad$ Munneke, S.; Painter, G. F.; Gainsford, G. J.; Stocker, B. L.; Timmer, M. S. M. 'Total Synthesis of Lewis ${ }^{\mathrm{X}}$ Using a Late-Stage Crystalline Intermediate' Carbohydr. Res. 2015, $414,1-7$.

- $\quad$ Munneke, S.; Stocker, B. L.; Timmer, M. S. M.; Gainsford, G. J. 'N-(2-Acetamido-2deoxy- $\beta$-D-glucopyranosyl)- $N$-(3-azidopropyl)-O-methyl-hydroxylamine' Submitted to Acta Cryst. E

- $\quad$ Munneke, S.; Hill, J. C.; Timmer, M. S. M.; Stocker, B. L. 'Synthesis and Hydrolytic Stability of $N$ - and $O$-methyloxyamine linker Glycoconjugates' Manuscipt in preparation

- Munneke, S.; Stocker, B. L.; Timmer, M. S. M. 'The versatility of $N$-alkyl-methoxyamine bi-functional linkers for the preparation of various glycoconjugates' Manuscipt in preparation

- $\quad$ Munneke, S.; Kodar, K.; Painter, G. F.; Stocker, B. L.; Timmer, M. S. M. 'Synthesis of fluorescent multivalent glycodendrons for detection of DC-SIGN on macrophages' Manuscipt in preparation 


\section{Table of Contents}

CHAPTER 1: Introduction

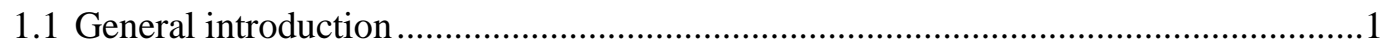

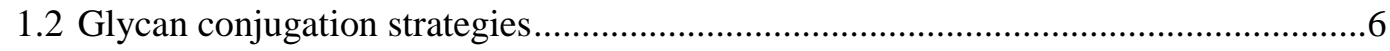

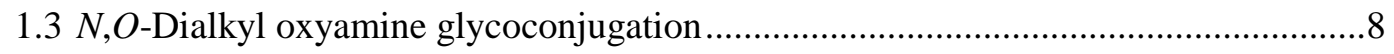

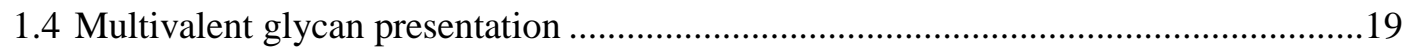

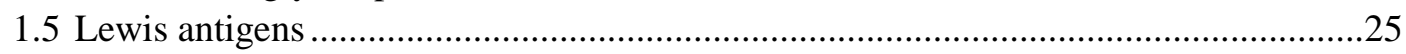

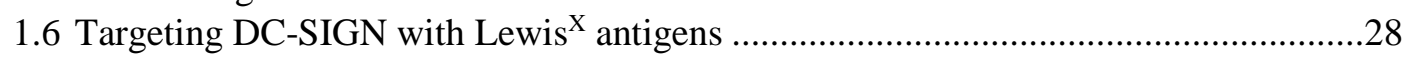

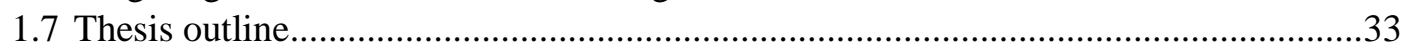

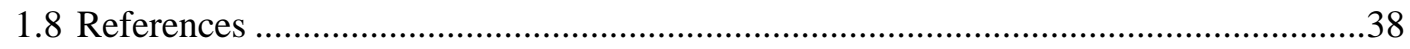

CHAPTER 2: The rapid and facile synthesis of oxyamine linkers for the preparation of novel glycoconjugates

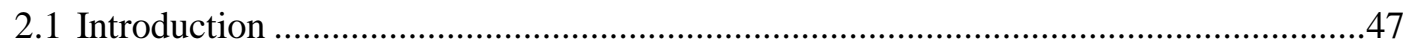

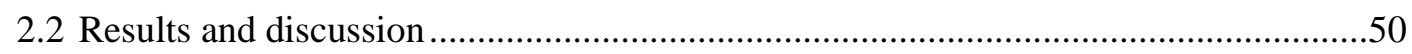

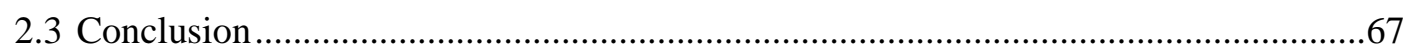

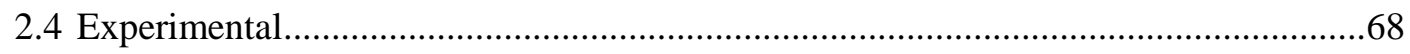

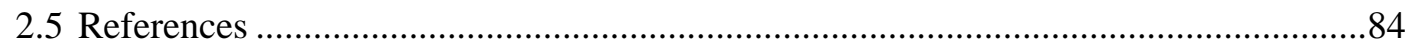

CHAPTER 3: Design and synthesis of novel fluorescent and biotinylated multivalent glycodendrons

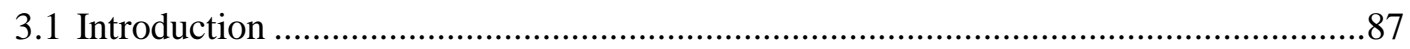

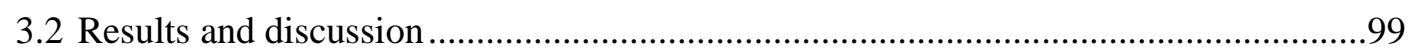

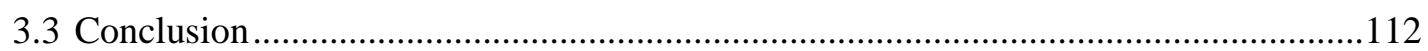

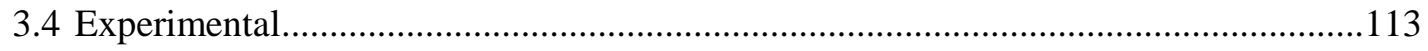

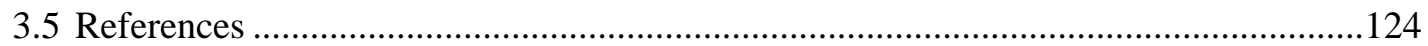

CHAPTER 4: Highly efficient synthesis of Lewis ${ }^{\mathrm{X}}$ via a versatile late-stage crystalline trisaccharide intermediate.

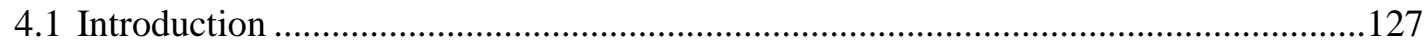

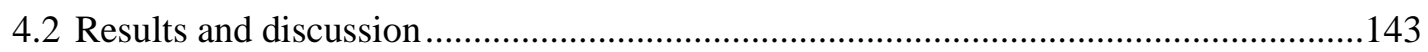

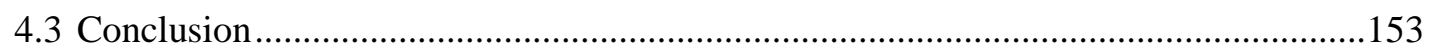

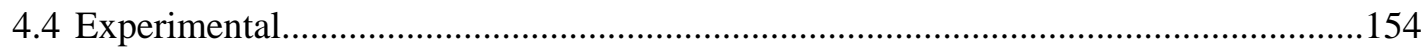

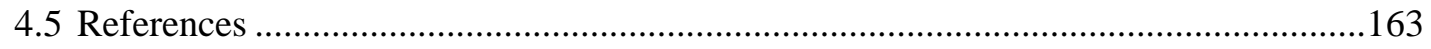


CHAPTER 5: The synthesis and biological evaluation of fluorescent Lewis ${ }^{\mathrm{X}}$ glycodendrons to target DC-SIGN lectins on macrophages.

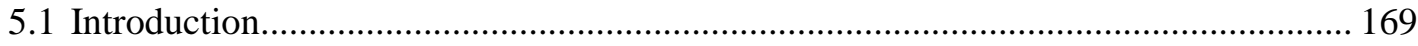

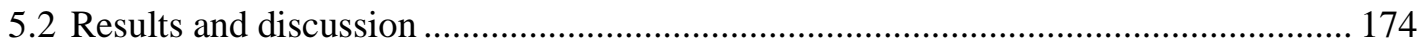

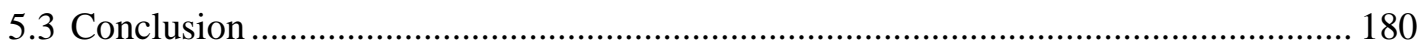

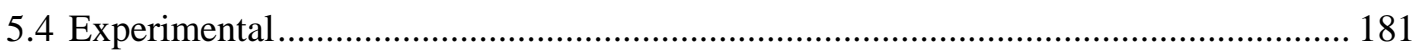

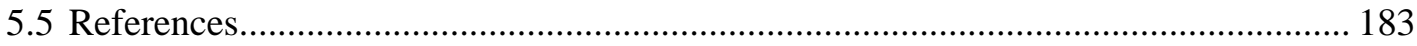

CHAPTER 6: Conclusion and future prospects

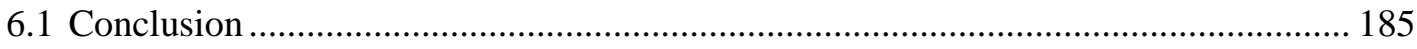

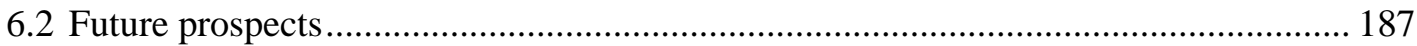

\section{APPENDIX}

\begin{tabular}{|c|c|}
\hline \multicolumn{2}{|l|}{ MR Spectra } \\
\hline Chapter $2 .$. & . Appendix - 1 \\
\hline Chapter 3 .. & Appendix - 61 \\
\hline Chapter 4 .. & Appendix - 83 \\
\hline Chapter $5 .$. & Appendix - 101 \\
\hline
\end{tabular}




\section{List of abbreviations}

\begin{tabular}{|c|c|c|c|}
\hline $1 \mathrm{G}$ & First generation & DMTST & Dimethyl(methylthio)sulfonium \\
\hline $2 \mathrm{G}$ & Second generation & & triflate \\
\hline $3 \mathrm{G}$ & Third generation & DNA & Deoxyribonucleic acid \\
\hline Ac & Acetyl & EBP & Egg-binding protein \\
\hline $\mathrm{AcOH}$ & Acetic acid & EGCase & Recombinant endoglycoceramidase \\
\hline $\mathrm{AcSH}$ & Thioacetic acid & ELISA & Enzyme-linked immunosorbent assay \\
\hline $\mathrm{AgOTf}$ & Silver triflate & EPO & Erythropoietin \\
\hline AIBN & Azobisisobutyronitrile & ER & Endoplasmic reticulum \\
\hline Alk & Alkyl & EtOAc & Ethyl acetate \\
\hline All & Allyl & FCS & Fetal calf serum \\
\hline APC & Antigen presenting cell & FI & Fluorescent intensity \\
\hline ATPase & Adenosinetriphosphatase & FITC & Fluorescein isothiocyanate isomer I \\
\hline $\mathrm{Bn}$ & Benzyl & Fmoc & Fluorenylmethyloxycarbonyl \\
\hline Boc & tert-Butyloxycarbonyl & Fuc & Fucose \\
\hline BODIPY & Boron-dipyrromethene & FUT & Fucosyltransferase \\
\hline BSA & Bovine serum albumin & Gal & Galactose \\
\hline $\mathrm{Bu}$ & Butyl & GalNAc & $N$-Acetylgalactosamine \\
\hline $\mathrm{Bz}$ & Benzoyl & G-CSF & Granulocyte-colony stimulating \\
\hline $\mathrm{BzCl}$ & Benzoyl chloride & & factor \\
\hline CAD & Charged aerosol detector & GDP & Guanosine diphosphate \\
\hline Calcd & Calculated & Glc & Glucose \\
\hline CD44 & Cluster of differentiation & GlcN & Glucosamine \\
\hline $\mathrm{ClAc}$ & Chloroacetyl & GlcNAc & $N$-Acetylglucosamine \\
\hline $\mathrm{ClZ}$ & 2-Chlorobenzyloxycarbonyl & GM-CSF & Granulocyte macrophage-colony \\
\hline COSY & $\begin{array}{l}\text { Homonuclear correlation } \\
\text { spectroscopy }\end{array}$ & GMP & Good manufacturing practices \\
\hline Cys & Cysteine & gp120 & Envelope glycoprotein-120 \\
\hline$d$ & doublet & HBTU & $N, N, N^{\prime}, N^{\prime}$-Tetramethyl-O- $(1 H-$ \\
\hline DC & Dendritic cell & & $\begin{array}{l}\text { benzotriazol-1-yl)uronium } \\
\text { hexafluorophosphate }\end{array}$ \\
\hline DCC & $N, N^{\prime}$-Dicyclohexylcarbodiimide & HIV & Human immunodeficiency virus \\
\hline DCE & Dichloroethane & HMBC & Heteronuclear multiple bond \\
\hline DC-SIGN & Dendritic cell-specific intercellular & & correlation \\
\hline & adhesion molecule-3-grabbing non- & HRMS & High resolution mass spectometry \\
\hline$d d$ & $\begin{array}{l}\text { integrin } \\
\text { Doublet of doublets }\end{array}$ & HSQC & $\begin{array}{l}\text { Heteronuclear single quantum } \\
\text { coherence }\end{array}$ \\
\hline DDQ & $\begin{array}{l}\text { 2,3-Dichloro-5,6-dicyano-1,4- } \\
\text { benzoquinone }\end{array}$ & $\mathrm{IC}_{50}$ & $\begin{array}{l}\text { Half maximal inhibitory } \\
\text { concentration }\end{array}$ \\
\hline $\mathrm{dfBz}$ & 2,5-Difluorobenzoyl & IL & Interleukin \\
\hline DIC & $N, N^{\prime}$-Diisopropylcarbodiimide & IFN & Interferon \\
\hline DiPEA & $N, N$-Diisopropylethylamine & IR & Infrared \\
\hline DMAP & 4-Dimethylaminopyridine & Lac & Lactosyl \\
\hline DME & Dimethyl ether & LacCer & Lactosyl ceramide \\
\hline DMF & Dimethylformamide & LCMS & Liquic chromatography mass \\
\hline DMSO & Dimethylsulfoxide & & spectrometer \\
\hline
\end{tabular}




\begin{tabular}{|c|c|c|c|}
\hline Lev & Levulinoyl & Siglec & Sialic-acid-binding immunoglobulin- \\
\hline $\operatorname{Le}^{\mathrm{X}}$ & Lewis $^{\mathrm{X}}$ & & like lectin \\
\hline LPS & Lipopolysaccharide & SMCC & Succinimidyl-4-[ $N$-maleimido- \\
\hline$m / z$ & mass/charge & & methyl]cyclohexane-1-carboxylate \\
\hline MALDI & Matrix-assisted laser desorption & SPPS & $\begin{array}{l}\text { Solid phase peptide synthesis } \\
\text { Triplet }\end{array}$ \\
\hline Man & $\begin{array}{l}\text { ionization } \\
\text { Mannose }\end{array}$ & $\mathrm{TBABr}$ & Tetrabutylammonium bromide \\
\hline $\mathrm{Me}$ & Methyl & TBAI & Tetrabutylammonium iodide \\
\hline $\mathrm{MeOH}$ & Methanol & TBDPS & Tert-butyl-diphenylsilyl \\
\hline MFI & Median fluorescent intensity & $t \mathrm{Bu}$ & Tert-butyl \\
\hline MGL & Macrophage galactose-type lectin & TCA & Trichloroacetyl \\
\hline $\mathrm{MHC}$ & Major histocompatibility complex & TCEP & Tris(2-carboxyethyl)phosphine \\
\hline MRI & Magnetic resonance imaging & TDS & Tert-butyldimethylsilyl \\
\hline mRNA & Messenger ribonucleic acid & TES & Triethylsilyl \\
\hline $\mathrm{MsCl}$ & Mesyl chloride & TFA & Trifluoroacetic acid \\
\hline $\mathrm{NaOAc}$ & Sodium acetate & $\mathrm{TfOH}$ & Trifluorosulfonic acid \\
\hline NHS & $N$-Hydroxysuccinimide & TGF & Transforming growth factor \\
\hline NIS & $N$-Iodosuccinimide & THF & Tetrahydrofuran \\
\hline NMR & Nuclear magnetic resonance & THP-1 & Leukemic human monocytic cell line \\
\hline NO & Nitric oxide & TIC & Total ion count \\
\hline NP & Nanoparticle & TLC & Thin layer chromatography \\
\hline NZD & New Zealand dollar & TLR & Toll-like receptor \\
\hline Obsd & Observed & TNF & Tumour necrosis factor \\
\hline $\mathrm{OMe}$ & Methoxy & TOCSY & Total correlation spectroscopy \\
\hline OVA & Ovalbumin & TOF & Time-of-flight \\
\hline PAMAM & Poly(amido amine)amine & Tol & Toluene \\
\hline PBS & Phosphate buffered saline & TRIS & Tris(hydroxymethyl)amino-methane \\
\hline $\mathrm{PE}$ & Petroleum ether $50-60$ & Troc & 2,2,2-Trichloroethoxycarbonyl \\
\hline$p$ & Pentet & $\mathrm{TsOH}$ & para-Toluenesulfonic acid \\
\hline Pept & Peptide & UDP & Uridine diphosphate \\
\hline PG & Protecting group & UV & Ultraviolet \\
\hline $\mathrm{Ph}$ & Phenyl & WGA & Wheat germ agglutinin \\
\hline Phth & Phthaloyl & $\mathrm{Z}$ & Benzyloxycarbonyl \\
\hline Piv & Pivaloyl & & \\
\hline PMA & Phorbol-12-myristate-13-acetate & & \\
\hline PMB & para-Methoxybenzyl & & \\
\hline ppm & Parts per million & & \\
\hline PRR & Pathogen recognising receptor & & \\
\hline PSGL-1 & P-Selectin glycoprotein ligand-1 & & \\
\hline Pyr & Pyridine & & \\
\hline$q$ & Quartet & & \\
\hline RaNi & Raney-nickel & & \\
\hline $\mathrm{rt}$ & Room temperature & & \\
\hline$s$ & Singlet & & \\
\hline sat & Saturated & & \\
\hline
\end{tabular}




\section{Chapter 1.}

\section{Introduction.}

\subsection{General Introduction}

The beginning of the $21^{\text {st }}$ century has seen significant advances within the fields of chemistry and biology related to human health and disease. Nowadays, genomic- and proteomic-based treatments are readily available, and in addition carbohydrate-based treatments are emerging. ${ }^{1}$ Glycoconjugates, such as glycolipids and glycoproteins, are essential to all forms of life. Cells are coated in glycans and these fundamental constituents are involved in a variety of biological processes such as host cell recognition, inflammation, cell signalling, proliferation and protein folding. ${ }^{2,3}$ A better understanding of the role of glycans, and the successful use of glycan based treatments has pushed the field of glycobiology to the front of academic and pharmaceutical research.

The growing interest in glycobiology has led to the design of numerous novel agents such as glycan-based therapeutics, ${ }^{4}$ immunotherapies, ${ }^{5}$ small molecule drugs, ${ }^{6}$ and analytical tools such glycan-arrays. ${ }^{7,8}$ These agents often target lectins (carbohydrate binding proteins) which are expressed on the cell surface. Lectins generally have a weak affinity for their carbohydrate ligands and require multivalent binding in order to induce a measurable biological response. Accordingly, for therapeutic application the glycan epitope is often conjugated to a multivalent substrate such as proteins, liposomes or dendrimers. This conjugation, however, is not straightforward and often does not allow for the use of naturally isolated glycans and/or requires complicated multi-step synthetic protocols.

In this thesis the development of novel chemical tools for the synthesis of multivalent glycoconjugates is described. Several aspects of carbohydrate chemistry are discussed including the rapid assembly of glycoconjugates through the use of bi-functional oxyamine linkers, the 
efficient total synthesis of the Lewis ${ }^{\mathrm{X}}$ antigen and the multivalent presentation of these glycans through the use of functionalised dendrons. In addition, the synthesis and biological evaluation of a fluorescent glycodendron for the C-type lectin DC-SIGN will be presented.

\subsubsection{Natural roles of glycans}

Carbohydrates are essential for life in all organisms and are found either in, or on the surface of all cells. They are fundamental constituents of glycoproteins and glycolipids and are involved in a variety of biological processes. ${ }^{2,3}$ Glycans, however, also play important roles in disease where defects in glycosylation processes or the overexpression of glycan antigens can lead to a wide variety of disorders, including cancers. ${ }^{9}$ Moreover, pathogens, such as the human immunodeficiency virus (HIV), can evade and infect the host immune system by using a nonpathogenic glycan display. ${ }^{10,11}$ Nevertheless, the immune system is quite efficient in protecting us against pathogens by identifying foreign glycan displays through the use of pathogen recognising receptors (PRRs), despite the extreme diversity of glycans found in nature.

\subsubsection{Carbohydrate diversity}

The structural diversity of glycoconjugates in nature is enormous due to the complexity of the carbohydrate constituents. ${ }^{3,12}$ A hexose such as glucose contains five chiral centres, and can adopt four different configurations: $\alpha$-pyranose, $\beta$-pyranose, $\alpha$-furanose and $\beta$-furanose. Besides the stereochemistry of the individual carbohydrate moieties and the anomeric configuration of the hexose, the regiospecificity (i.e. $1^{\prime} \rightarrow 1,1^{\prime} \rightarrow 2,1^{\prime} \rightarrow 3$, etc.) of the glycosidic linkages in oligosaccharides gives rise to the vast number of glycans found in nature. ${ }^{3}$ In addition, each glycan has specific functions, which may vary depending on the type of conjugate (i.e. glycolipid or glycoprotein). In contrast to proteins, the glycan structure is not encoded within the genome of an organism but it is post-translationally modified by glycosyltransferases and glycosidases. ${ }^{13,14}$

\subsubsection{Glycoprotein synthesis and post-translational modification}

Proteins are biosynthesised by ribosomes which translate messenger ribonucleic acid (mRNA) into polypeptide chains. The peptides and/or proteins then undergo post-translational modifications in order to change the phenotype of the protein, for example by folding, glycosylation, phosphorylation, methylation, lipidation, $N$-acetylation or proteolysis. This process can occur at any time during the life cycle of a protein. Despite the limited human genome the diversity of the post-translational modifications gives rise to the wide variety of proteins. Indeed, the glycosylation of proteins is thought to be one of the most important post-translational 
modification processes due to the variety of functional modifications that the carbohydrates can impart on the protein. ${ }^{12}$

\subsubsection{N-glycan and O-glycan biosynthesis}

The biosynthesis of glycoproteins starts in either the endoplasmic reticulum (ER) or the Golgi apparatus depending on the type of glycan. $\mathrm{N}$-glycans often terminate in $\mathrm{N}$-acetylglucosamine (GlcNAc) and are bound through asparagine to proteins, whereas O-glycans are often initiated by $\mathrm{N}$-acetylgalactosamine (GalNAc) and can either be linked to the hydroxyl of threonine, serine or tyrosine (Figure 1).

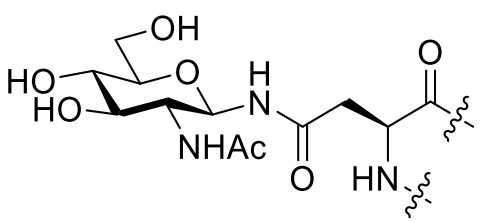

N-Glycan

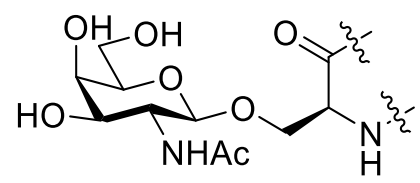

O-Glycan

Figure 1. $\mathrm{N}$-glycans are often terminated in $\mathrm{N}$-acetylglucosamine which is bound to the amide functionality of the amino acid asparagine, whereas $O$-glycans are often initiated by $\mathrm{N}$-acetylgalactosamine and can either be bound to the hydroxyl of threonine, serine or tyrosine.

The synthesis of N-glycans starts on the cytoplasmic surface of the endoplasmic reticulum (ER), where phosphorylated dolichol is glycosylated by a GlcNAc-1-phophotransferase, followed by other glycosylations to generate $\mathrm{Glc}_{3} \mathrm{Man}_{9} \mathrm{GlcNAc}_{2}$ on the phosphorylated lipid carrier (Figure 2). ${ }^{15,16}$ The glycosyltransferases require nucleotide-sugar donors, which are synthesised in the cytoplasm or nucleus. After dolichol glycosylation on the ER surface, the glycolipid flips to the inside of the ER, where glucosyl-transferases glycosylate the core saccharide. Next, the glycan is transferred to the asparagine of new proteins, which are biosynthesised by the ribosome, by an oligosaccharyltransferase to form the N-linkage, and the lipid carrier is recycled to the surface of the ER for subsequent glycosylation. The new glycoprotein is checked by calnexin, which is a chaperone that determines the protein folding and the quality of proteins. The correctly folded glycoprotein is then transferred to the Golgi apparatus where more than 200 glycosidases and glycosyltransferases further diversity the $\mathrm{N}$-glycan structures. The newly formed glycoproteins can either be transported to the cell surface or excreted from the cell. 


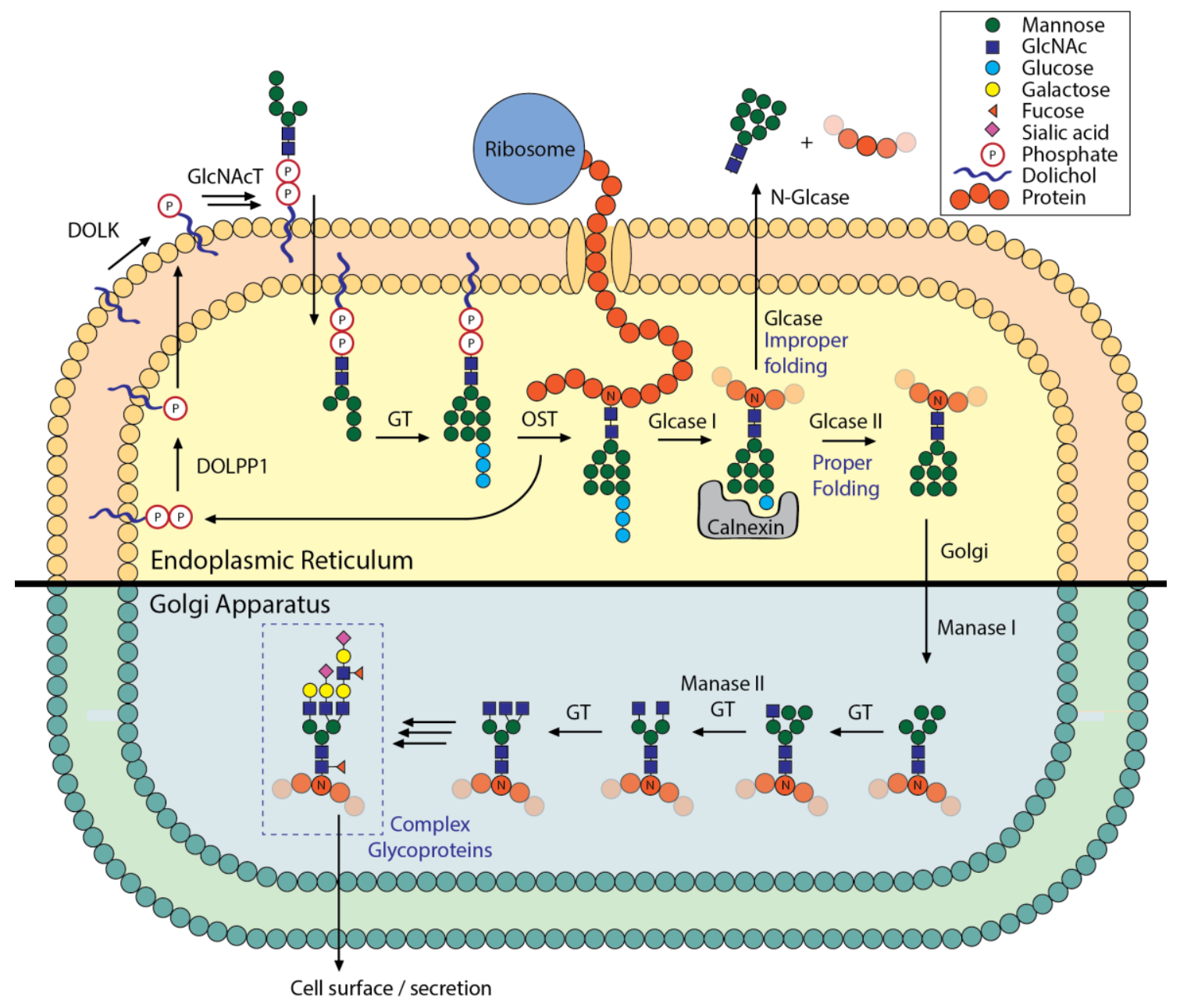

Figure 2. Biosynthesis of protein bound N-glycans. Figure based on publications of Wolfert \& Boons as well as Cantagrel \& Lefeber. ${ }^{15,16}$

The O-glycans are biosynthesised in the Golgi apparatus where the biosynthesis commences with the transfer of a GalNAc moiety onto the hydroxyl group of either serine or threonine by an $\mathrm{N}$-acetylgalactosaminyltransferase. ${ }^{17}$ From here, the core structure can be converted into complex O-glycoproteins in a similar fashion to N-glycans.

As mentioned above, post-translational modifications occur intracellularly, however, once glycoconjugates are expressed extracellularly, glycosidase and glycosyltransferase activity can still alter the glycan moieties as required, depending on the cellular environment. ${ }^{13}$ This glycan alteration can tag proteins to target specific lectins or immune cells, which, for example, can affect cellular uptake, intracellular processing and antigen presentation.

It is important to note that glycosylation pathways differ between organisms, but also within classes, families and individuals. The presence or absence of glycosyltransferases and glycosidases, which are encoded within the DNA, greatly influences the glycan structure and diversity displayed on cells. The immune system can utilise these glycan displays for host cell 
and pathogen recognition. Detection of foreign glycans expressed by pathogens, however, can result in pathogen digestion and antigen presentation, which ultimately can lead to immune activation against a particular pathogen. ${ }^{15}$

Unfortunately, many viruses can use (host) glycosylation mechanisms to not only avoid detection by immune cells but also aid infection of the host. ${ }^{18}$ For example, the HIV envelope protein gp120 is highly glycosylated (with an average of $25 \mathrm{~N}$-glycans per protein) in order to block antibody binding and proteolysis of the protein antigen, which is necessary for pathogen recognition by the immune system. In addition, these glycan antigens can bind to specific C-type lectins expressed on immune cells, which results in cellular internalisation and infection of the host cell. ${ }^{19}$

\subsubsection{Carbohydrate-based therapeutics}

While carbohydrates play an important role in both health and disease, it has taken a considerable amount of time for scientists to appreciate the role of carbohydrates in nature. ${ }^{1}$ The structural elucidation and chemical conjugation of carbohydrates was pioneered in the 1890's when Emil Hermann Fischer, who later received a Nobel Prize for this work, reacted carbohydrates under acidic conditions with alcoholic solvents to form Fischer glycosides. ${ }^{20}$ During the next 100 years, many different types of glycoconjugates were discovered and synthesised. ${ }^{21}$ The field of glycomics, however, did not start to expand until the 1980s with significant breakthroughs such as the development of small molecule glycosidase and glycosyl transferase inhibitors, ${ }^{6}$ and carbohydrate-based vaccines. ${ }^{1,5}$

Most of these small molecules target glycosyltransferases and glycosidases, whereas other glycan therapies often target lectins. For example, many iminosugars can be used as potent inhibitors for glycosidase and glycosyltransferases by mimicking the transition state of the natural substrate. ${ }^{22,23}$ In contrast, glycans can be utilised in a therapeutic setting to target specific lectins to induce a biological response or for cell-selective targeting for immune activation or cell-depletion. This, however, requires the conjugation of the glycan antigen to a multivalent substrate, such as proteins or dendrimers, in order to obtain a significant biological response upon lectin binding. 


\subsection{Glycan Conjugation strategies}

As described above, a number of biological functions have been attributed to glycoconjugates and, accordingly, there has been much interest in the construction of glycoconjugate mimetics, ${ }^{21,24,25}$ glycopeptides,${ }^{26}$ and carbohydrate arrays, ${ }^{7,27,28}$ and in the synthesis of fluorescent or biotinylated glycoconjugate probes. ${ }^{29,30}$ The conjugation of carbohydrates to the substrate of choice often requires the use of a chemical linker at the non-reducing end of the glycan. Traditionally, the installation of such linkers requires the total synthesis of the glycan antigen, in which the linker is installed in an early stage of the synthesis. Such a strategy, however, is timeconsuming and does not allow for the conjugation of carbohydrates from natural sources. Accordingly, much effort has been expended in the development of linker strategies that can be performed at the reducing end of free sugars without the need for protecting groups. These approaches include the use of Kochetkov amination, ${ }^{31}$ reductive amination, ${ }^{32}$ oximes/hydrazides ${ }^{33-35}$ and the use of oxyamines (Scheme 1). ${ }^{36}$

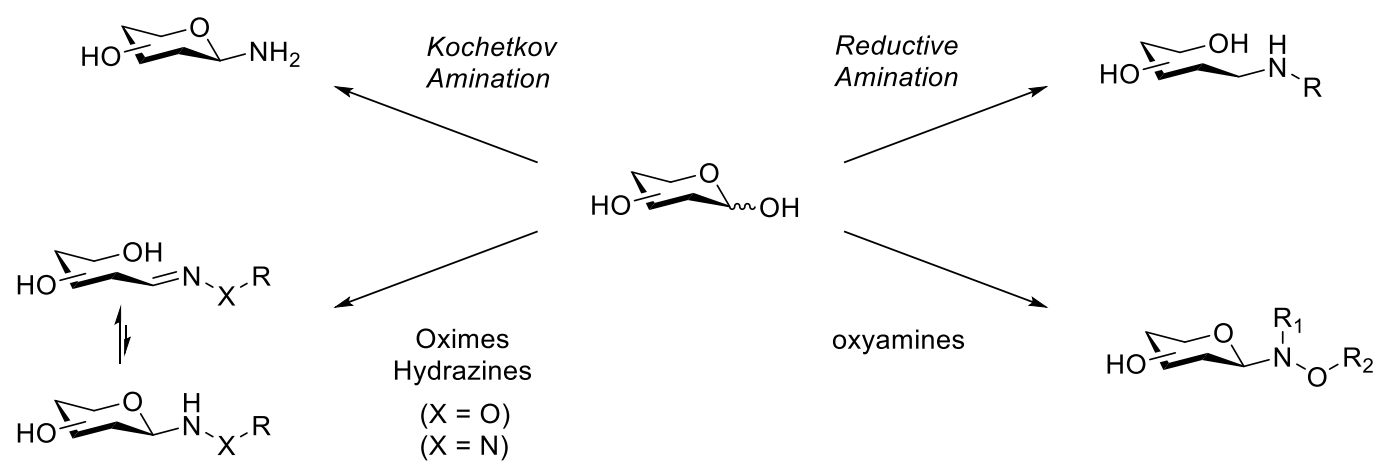

Scheme 1. Chemoselective chemical ligation methods for free sugars.

\subsubsection{Kochetkov Amination}

The Kochetkov amination allows for the formation of a $\beta$-D-glycosylamine by incubating a carbohydrate with a free reducing end in an ammonium carbonate solution at $30-40{ }^{\circ} \mathrm{C}$ for more than 24 hours. ${ }^{31}$ Although the scope and application of the Kochetkov amination is limited to amine conjugation chemistry, acylation reactions with the glycosylamine can then result in complex glycopeptide conjugates. This was nicely demonstrated by Danishefsky and co-workers who used the Kochetkov amination to conjugate complex sialylated tri-branched N-glycans to native peptide sequences so as to synthesise various glycoforms of erythropoietin (EPO) and study their role in prostate cancer. ${ }^{37}$ Although the Kochetkov amination results in the native N-glycan linkage, often incomplete amination is observed. ${ }^{38}$ Moreover, given the instability of the glycosylamine, the crude product is often used in amide ligation reactions. Here, removal of the large excess of ammonium formate is critical to prevent the reaction with activated peptides to form asparagine derivatives. The methodology was later adapted by Davies and co-workers, 
whereby the glycoside in the presence of Lawesson's reagent was converted into a glycosylthiol, which then allowed for sulfur conjugation chemistry. ${ }^{39}$

\subsubsection{Reductive Amination}

Reductive amination is widely used for the preparation of glycoconjugates. Here, the aldehyde functionality of the sugar is converted into the imine, which in turn is reduced by a reducing agent to give the 'ring-opened' glycoconjugate. There are many examples of the use of reductive amination for the synthesis of glycoconjugates, with a representative example being that of Van Kooyk and co-workers who synthesised Lewis ${ }^{\mathrm{X}}$ glycodendrons to target DC-SIGN on dendritic cells. ${ }^{40}$ It can also be used to conjugate glycans directly to lysine moieties on proteins in order to obtain glycoproteins. ${ }^{41}$ This technique, however, affects the structural integrity of the reducing end carbohydrate, which in turn could affect lectin binding.

\subsubsection{Oxime/Hydrazine Ligation}

Another widely studied conjugation method is the oxime and hydrazine glycan ligation. In 1895, Wolff discovered that hydrazines can react with carbohydrates to form stable glycoconjugates, ${ }^{42}$ and since then, it has been determined that both oximes and hydrazines can be used in a condensation reaction under aqueous conditions to create glycoconjugates without the need for protecting groups. ${ }^{43}$ Accordingly, both hydrazine and oximes have been used in various applications, which include the characterisation and isolation of glycan antigens, ${ }^{44-46}$ and glycoconjugate synthesis. ${ }^{47-50}$ Moreover, oximes and hydrazines can be used in analytical applications. For example, fluorescent hydrazines have been synthesised for use in HPLC analysis ${ }^{46}$ whereas biotinylated analogues were used to immobilise glycan antigens on plates for ELISA assays. ${ }^{51}$ Although the use of oxime and hydrazine ligations is a robust technique for the conjugation of glycan antigens, in solution the obtained glycoconjugate excists in the ring-opened hydrazine and the closed pyranose and furanose forms, ${ }^{52}$ which could affect lectin affinity and thus the biological activity of the conjugate. ${ }^{48}$ In 2014, Unverzagt and co-workers reported the reaction of hydroxylamine glycosides with $\alpha$-ketoacids, such as pyruvic acid, to form the native $\mathrm{N}$-glycan acetamide linkage in various yields $(23-80 \%)$. When these $\alpha$-ketoacids were incorporated into more complex peptides, low yields were obtained. ${ }^{53}$ This novel method, however, has great potential in the preparation of glycoconjugates. Nevertheless, in 1998 it was determined by Peri et al. that the use of $N, O$-dialkylated oxyamines in the condensation reaction resulted in the exclusive formation of the ring-closed glycoconjugate. ${ }^{54}$ Thus, this latter strategy has particular merit as it does not affect the structural integrity of the reducing end sugar. 


\section{$1.3 \mathrm{~N}, \mathrm{O}$-Dialkyl oxyamine glycoconjugation}

The efficacy of oxyamines as a means to synthesise glycoconjugates was significantly enhanced with the introduction of $\mathrm{N}, \mathrm{O}$-dialkyl oxyamines. To this end, Peri et al. synthesised two types of oxyamine substrates for conjugation with carbohydrates (Scheme 2). These oxyamines are divided in two sub-types, the "Type A" $O$-alkyl- $N$-methyl oxyamines 1 and the "Type B" $N$-alkyl$O$-methyl oxyamines 2 . Both types of oxyamines have been successfully conjugated to carbohydrates under mildly acidic aqueous conditions to form the ring-closed glycoconjugates 3 and $\mathbf{4}$, respectively. The scope and application of the "Type A"oxyamine linker, however, has been well studied, whereas the "Type B" has been utilised less extensively.

\section{Type $A$}

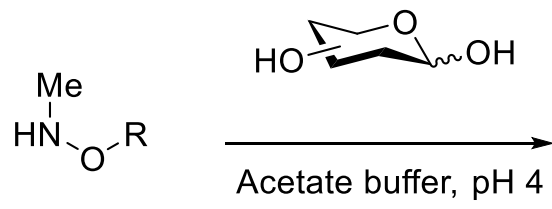

1

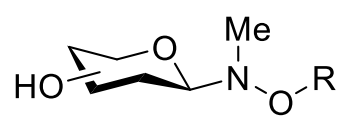

3

\section{Type B}<smiles>[R]NOC</smiles>

2

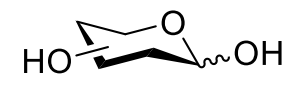

Acetate buffer, $\mathrm{pH} 4$

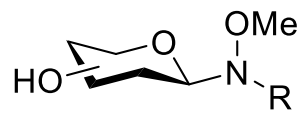

4

Scheme 2. Oxyamine linkers for the conjugation of carbohydrates.

\subsubsection{Oxyamine glycan conjugation Scope and Limitations}

The condensation reaction between reducing sugars and both "Type A" and "Type B" oxyamine derivatives can be performed under various conditions. ${ }^{54,55}$ For example, solvent mixtures such as DMF:AcOH, Pyr:AcOH, MeOH: $\mathrm{CHCl}_{3}$ or an aqueous acetate buffer at $\mathrm{pH} 4.5$ can be used to give glycosides in good yields. It should be noted that these conjugation reactions are equilibrium reactions and accordingly require high concentrations of either (or both) the glycan or the oxyamine in order to obtain high yields. ${ }^{43}$ Moreover, depending on the stereochemistry of the carbohydrate, it is possible to form mixtures of $\alpha-, \beta-$, pyranosyl- and furanosyl-glycosides (Table 1)..$^{55}$ For example, upon reaction of D-glucose with either $N$-methoxy-4methylbenzylamine (entry 1) or $N$-benzyloxy-4-methylbenzylamine (entry 2), exclusive formation of $\beta$-pyranose glycoconjugates was observed, as the formation of $\alpha$-pyranosides is disfavoured due to 1,3-diaxial interactions (Figure 3). Morover, the reaction of D-GlcNAc (entry 3 ) with oxyamines also resulted in the $\beta$-pyranose glycoconjugates. On the other hand, 
reaction of the methoxyamine with $N$-acetyl-D-galactosamine (entry 4) and D-galactose (entry 5) gave a 41:59 and 94:6 mixture of $\beta$-pyranose and $\beta$-furanose, respectively. Here, it was suggested that the $\beta$-galactofuranosides do not experience the unfavourable steric interactions between the O-3 and C-5 groups which are experienced by glucofuranoside derivatives. Isolation of the individual structural isomers proved ineffective as the single isomers rapidly equilibrate to the pyranose/furanose mixtures. Moreover, it was found that the reaction with the more "Type A"like linkers ( $R=$ Allyl, Bn; entry 6 and 7 ) results in different pyranose/furanose ratios, indicating that oxyamine substituents influence the outcome of these reactions. In the case of mannose (entry 8 ), the $\beta$-pyranose, $\alpha$-pyranose and $\alpha$-furanose isomers are formed, whereas the $\beta$-furanoside was not observed due to the unfavourable 1,2-cis and 4,5-cis configuration of this adduct. The formation of the $\alpha$-pyranoside can be explained by the favourable 1,2-trans configuration of the hydroxyl substituents and the C-1/C-2 dipole effect. ${ }^{55}$ It is also interesting to note that the $\alpha$ furanose is formed as a minor product, despite the undesired O-4 and C-5-cis configuration of the hydroxyls. This indicates that the electronic effects of the 1,2-trans configuration may play a role in the formation of the $\alpha$-pyranoside and $\alpha$-furanoside of the mannose conjugates.

Table 1. The relative stereochemistry of the obtained glycosides.

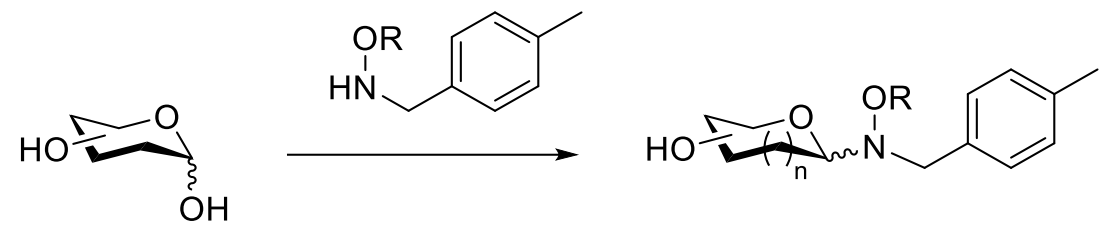

\begin{tabular}{c|l|c|c|cccc}
\hline Entry & Carbohydrate & R & Yield & $\boldsymbol{\beta}$-Pyr & $\boldsymbol{\alpha}$-Pyr & $\boldsymbol{\beta}$-Fur & $\boldsymbol{\alpha}$-Fur \\
\hline 1 & Glc & Me & 71 & 100 & - & - & - \\
2 & Glc & Bn & 67 & 100 & - & - & - \\
3 & GlcNAc & Me & 47 & 100 & - & - & - \\
4 & GalNAc & Me & 78 & 41 & - & 59 & - \\
5 & Gal & Me & 82 & 96 & - & 4 & - \\
6 & Gal & Allyl & 47 & 89 & - & 11 & - \\
7 & Gal & Bn & 56 & 59 & - & 41 & - \\
8 & Man & Me & 91 & 37 & 41 & - & 21 \\
\hline
\end{tabular}



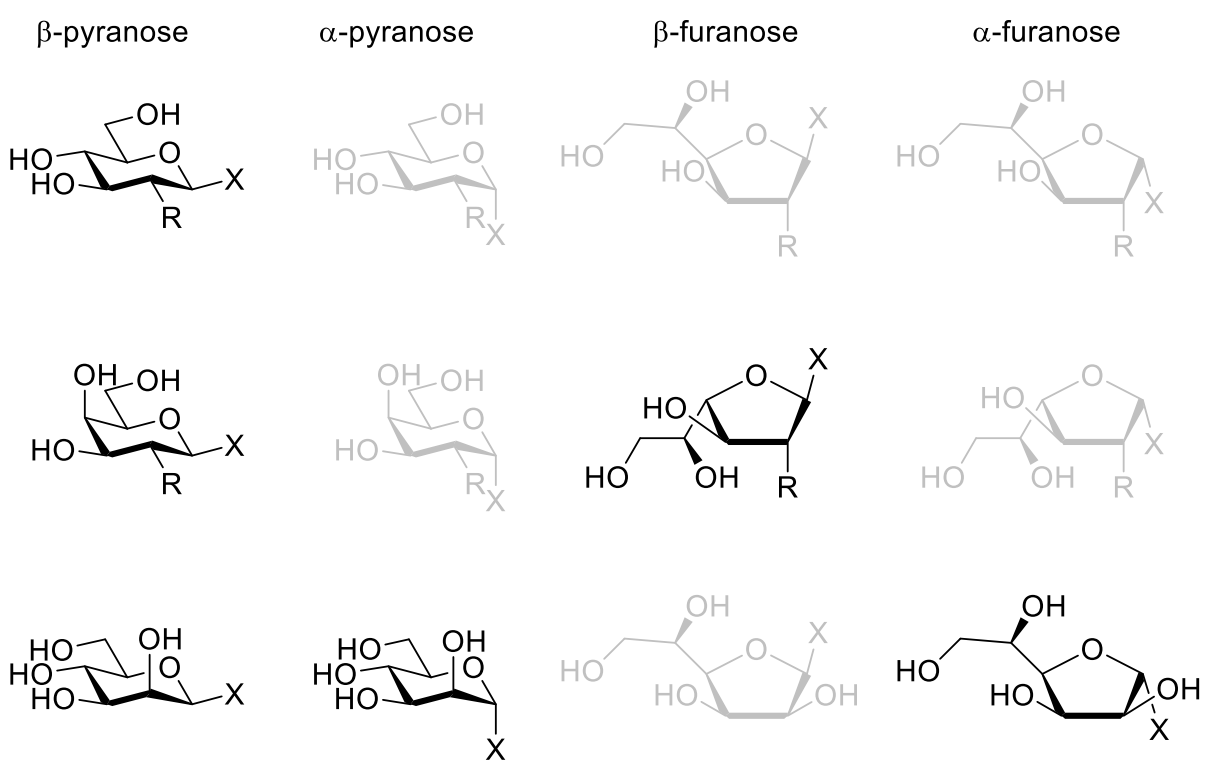

Figure 3. Possible isomer formation in the glycosylation reaction of both "Type A" and "Type B" oxyamines with glucose (top row, $\mathrm{R}=\mathrm{OH}$ ), GlcNAc (top row $\mathrm{R}=\mathrm{NHAc}$ ), galactose (middle row, $R=O H$ ), $N$-acetylgalactosamine (middle row, $R=N H A c$ ) and mannose (bottom row). The isolated isomers are depicted in black whereas the grey isomers were not observed. ${ }^{55}$

Although many carbohydrates are obtained as isomeric mixtures when using oxyamines, glucose and $\mathrm{N}$-acetylglucosamine reducing end glycans result in the selective formation of the $\beta$-pyranoside. Given the importance of reducing end $\mathrm{N}$-acetylglucosamine in $\mathrm{N}$-glycans, these oxyamine glycoside conjugations nevertheless have much potential in the synthesis of biologically relevant glycoconjugates.

\subsubsection{Oxyamine Stability}

Since oxyamine glycosides are formed under acidic equilibrium conditions, the readily formed glycoconjugates can be hydrolysed to the hemiacetal glycan when subjected to aqueous conditions below pH 7. The hydrolytic stability of both "Type A" and "Type B" glycoconjugates has been investigated. ${ }^{55,56}$ For both types, it was observed that the hydrolysis reaction is pseudo-first order, highly $\mathrm{pH}$ dependent, and that the half-life depends on the substituents on the nitrogen, the oxygen and the carbohydrate. When more electron withdrawing substituents are present, the half-life is increased. For example, benzyloxyamine glucosides $\left(t_{1 / 2}=7 \mathrm{~d}\right)$ are more stable than methoxyamine glucosides $\left(t_{1 / 2}=1.4 \mathrm{~d}\right)$ when submitted to an aqueous buffer of $\mathrm{pH} 4 .{ }^{56}$ Moreover, the hydrolysis rate of $\mathrm{N}$-acetylglucosamine is much slower than glucose, which in turn hydrolyses slower than xylose oxyamines. Furthermore, $\mathrm{N}$-acetylglucosamine derivatives show excellent hydrolytic stability at physiological $\mathrm{pH}$. 


\subsubsection{Glycoprotein synthesis}

Peri et al. synthesised novel glycopeptides employing the "Type A" oxyamine glycan conjugation (Scheme 3) ${ }^{54}$ Here, the oxyamine functionalised peptide 5 was methylated via a two-step reductive amination with formaldehyde and $\mathrm{NaCNBH}_{3}$. Next, the ligation with various glycans, including glucose, lactose and maltotriose, was performed under mildly acidic aqueous conditions to give the desired glycopeptides 6-9 in $35-70 \%$ yield.

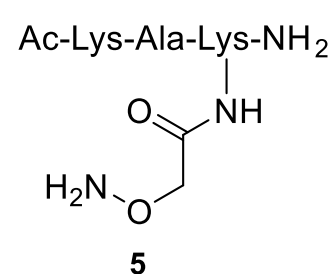

5
1) $\mathrm{CH}_{2} \mathrm{O}, \mathrm{NaOAc}, \mathrm{AcOH}$ $\mathrm{H}_{2} \mathrm{O}, \mathrm{pH} 4$

2) $\mathrm{NaCNBH}_{4}, \mathrm{AcOH}$

3) Sugar, $60^{\circ} \mathrm{C}$, Acetate buffer, $\mathrm{pH} 4$

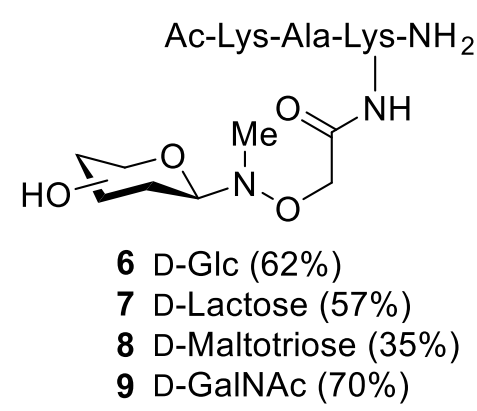

Scheme 3. Synthesis of oxyamine functionalised glycopeptides 6-9. ${ }^{54}$

Following the initial work of Peri et al., Carrasco et al. synthesised a novel series of both "Type A" and "Type B" oxyamine functionalised amino acids $\mathbf{1 0}-12$ (Scheme 4). ${ }^{57,58}$ These amino acids were then utilised in solid phase peptide synthesis (SPPS) to form oxyamine peptides 13 - 15, which in turn could be used in the conjugation with D-glucose to obtain glycosylated peptides 16 and 17 in good (75-80\%) yields. Here, it should be noted, however, that the secondary oxyamine 15 could not be conjugated to D-glucose due to the steric hindrance of the methyl substituent. In a similar synthetic approach, oxyamine derived aspartate amino acids were synthesised by Filira et al. for the synthesis of small glycopeptides. ${ }^{59}$ 
Type $A$
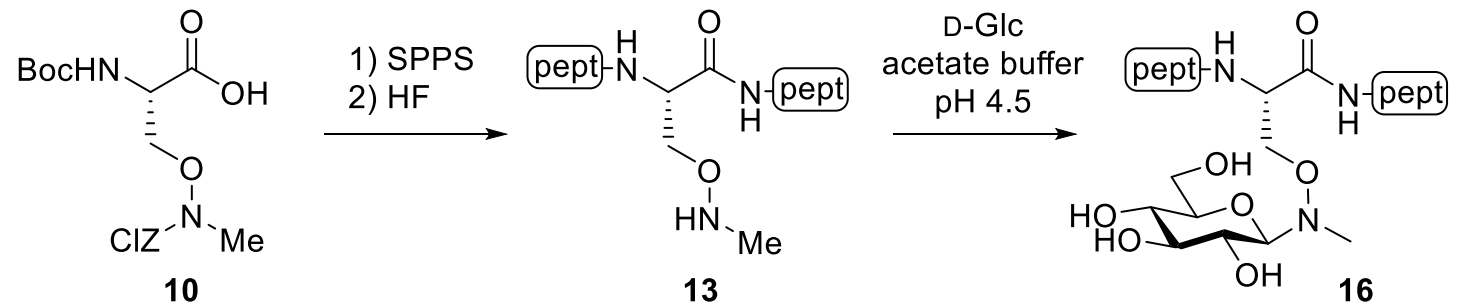

\section{Type B}
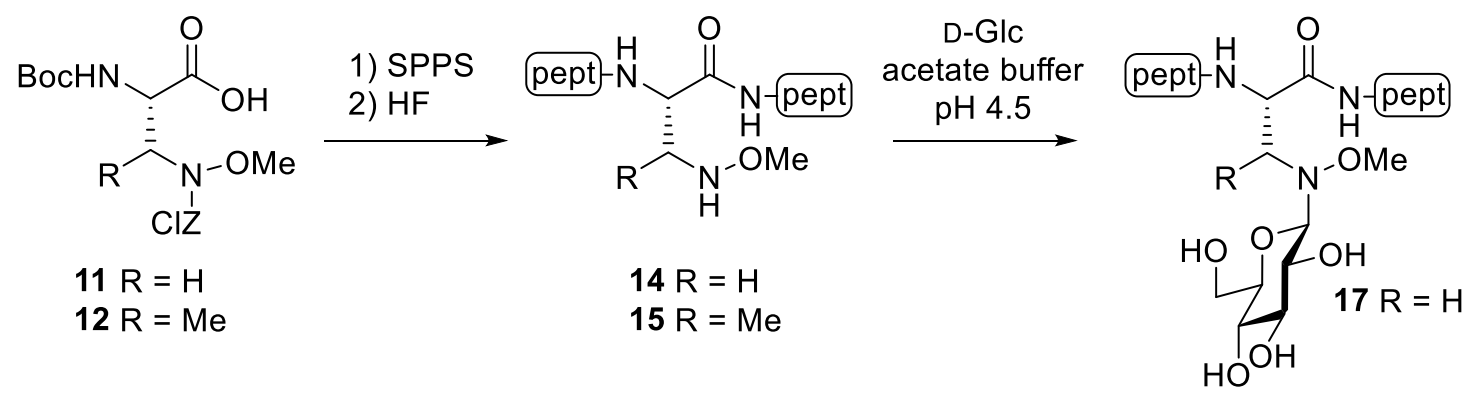

Scheme 4. Oxyamine functionalised amino acids were used to synthesise glycopeptides. ${ }^{57}$

\subsubsection{Glycan-mimetics}

Besides glycopeptide synthesis, Peri et al. also synthesised novel glycan mimetics by using the methoxyamine as an inter-glycosidic linkage to form oligosaccharides (A. Scheme 5). ${ }^{60,61}$ These “Type B" oxyamine glycans could be obtained via a Dess-Martin oxidation of the 6-hydroxyl of methyl glucoside 18, followed by imine formation with methoxyamine and subsequent deacetylation and imine reduction to give methoxyamine functionalised glucoside 19. Next, the conjugation with a variety of reducing sugars, including Glc, Man, Gal and GlcNAc was performed to give disaccharide glycan mimetics 20, however, as described above, the use of galactose and mannose resulted in a mixture of $\alpha / \beta$ - and furanose/pyranose-adducts.

This strategy was then further exploited towards the synthesis of Lipid A derivatives, however, in an attempt to conjugate glycolipid $\mathbf{2 1}$ and $\mathbf{2 2}$ via this methodology, disaccharide $\mathbf{2 3}$ was not observed despite varying the experimental conditions such as solvents and temperature (B. Scheme 5). Thus, a traditional glycosylation between donor $\mathbf{2 4}$ and acceptor $\mathbf{2 5}$ was performed to obtain the glycoside 26. In addition, the use of the hydroxyl acceptor $\mathbf{2 7}$ gave the $O$-glycoside 28. Both the oxyamine 26 and $O$-glycoside were deprotected to give glycolipids $\mathbf{2 3}$ and 29 and were tested for their ability to induce macrophages to release nitric oxide (NO), however, no response was observed. Instead, the glycolipids competitively bind Toll-like receptor-4 (TLR-4) compared to its natural ligand lipopolysaccharide (LPS), and thus can be used as antagonists for TLR-4. Both glycolipids $\mathbf{2 3}$ and $\mathbf{2 9}$ show very similar results, which indicates that the "Type B" methoxyamine glycosidic linkage does not interfere binding with the TLR-4 receptor. 
A.

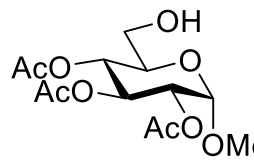

18
1) Dess-Martin periodinane 2) $\mathrm{MeONH}_{2}$

3) $\mathrm{NaOMe}$

4) $\mathrm{NaCNBH}_{3}, \mathrm{AcOH}$ $69 \%$ (over 4 steps)

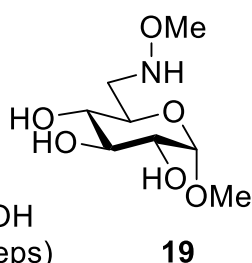

19

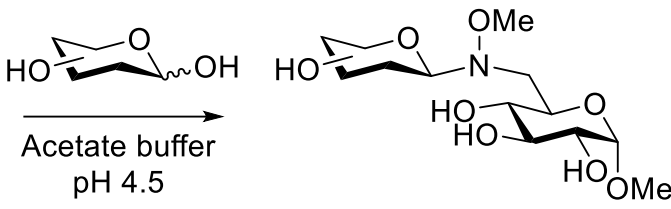

20

B.

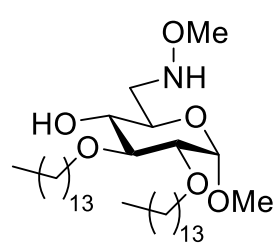

21

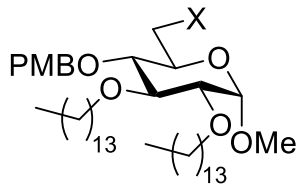

$25 \mathrm{X}=\mathrm{NHOMe}$

$27 \mathrm{X}=\mathrm{OH}$

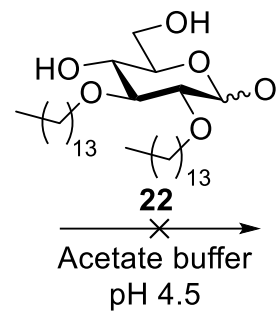

$\mathrm{pH} 4.5$

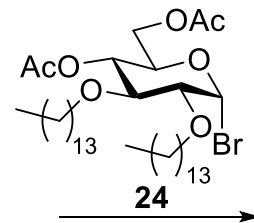

$\mathrm{Ag}_{2} \mathrm{CO}_{3}, \mathrm{AgOTf}$ $\mathrm{CH}_{2} \mathrm{Cl}_{2}, 99 \%$

1) $\mathrm{DDQ}$
2) $\mathrm{NaOMe}$

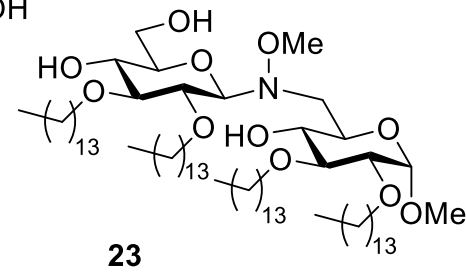

23

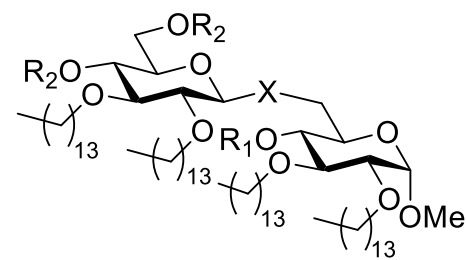

$26 X=$ NOMe $R_{1}=P M B, R_{2}=A c$

$28 X=O \quad R_{1}=P M B, R_{2}=A C$

$23 X=$ NOMe $R_{1}=R_{2}=H$

Scheme 5. The use of oxyamines towards glycan mimetics. ${ }^{61}$

This mimetic concept was recently further explored by Ishida et al. who synthesised $N$-LacCer glycolipid 30 (Figure 4) to inhibit the hydrolytic activity of recombinant endoglycoceramidase-II (EGCase-II). ${ }^{62}$ In this work, a "Type B" oxyamine derived ceramide lipid was synthesised and used in the conjugation with lactose to form $N$-LacCer 30, which can inhibit EGCase II-mediated hydrolysis of $O$-LacCer 31. The advantage of this route is that it is relatively easy to vary the carbohydrate moiety in order to alter the activity of the glycolipid, as the carbohydrate is introduced in the last step. In addition, the oxyamine glycosidic linkage shows potential for other glycosidase and glycosyl transferase inhibitors.

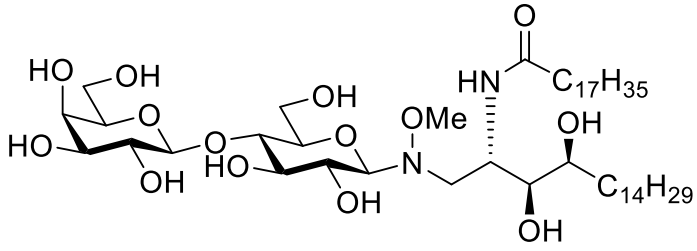

$30 \mathrm{~N}$-LacCer

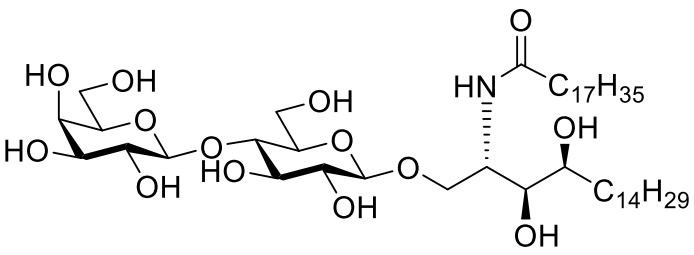

31 O-LacCer

Figure 4. $\mathrm{N}$-LacCer 30 can inhibit the EGCase II-mediated hydrolysis of O-LacCer $31 .{ }^{62}$ 


\subsubsection{Neoglycorandomisation}

In an effort to further explore the use of oxyamine glycan conjugation, the research group of Thorson and co-workers conjugated glycan libraries of both natural and un-natural glycans to aglycon natural products via "Type B" oxyamine conjugation. (Scheme 6). ${ }^{63}$ This process, also known as neoglycorandomisation, started with the conjugation of 78 glycans to oxyamine derivatised digitoxin. ${ }^{64}$ Digitoxin is a glycosylated cardenolide (steroid) with potential anti-cancer activity that is attributed to its ability to mediate the inhibition of the plasma membrane $\mathrm{Na}^{+} / \mathrm{K}^{+}-$ adenosinetriphosphatase (ATPase). By changing the glycan antigen, it was envisioned that the cancer cell specificity could be enhanced, and thus the library of oxyamine-glycan conjugates were tested in a high-throughput screening against nine human cancer cell lines. Although most glycoconjugates were obtained as furanose/pyranose mixtures, it was observed that some conjugates had a 20 fold increase in $\mathrm{IC}_{50}$ compared to the natural digitoxin. This nicely illustrated that the glycan moiety can alter the cell specificity of the drug and since then the concept of neoglycorandomisation has been employed for other drugs, such as colchicine, ${ }^{65}$ vancomycin, ${ }^{66}$ betulinic acid, ${ }^{67}$ chlorambucil, ${ }^{48}$ warfarin ${ }^{68}$ cyclopamine, ${ }^{69}$ amphimedosides. ${ }^{70}$ and perillyl glycosides. ${ }^{71}$ This work has been recently summarised in an excellent review. ${ }^{36}$
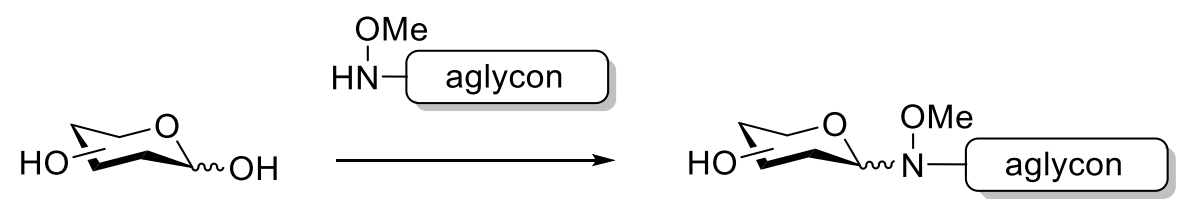

Glycan Library

Scheme 6. Neoglycorandomisation: reaction aglycons through oxyamines to glycan libraries allows for rapid screening of biological active glycoconjugates.

Despite the potential of the neoglycorandomisation strategy and the fact that the glycoconjugates can be obtained in good yields, a large excess of the carbohydrate is typically required for the glycan conjugation step. This is a serious limitation of the methodolgy when complex and less readily available $\mathrm{N}$-glycans are needed for conjugation. Accordingly, a high yielding glycan conversion was investigated through the use of bi-functional linkers. 


\subsubsection{Bi-functional linkers}

Bi-functional oxyamine linkers of "Type A" were first reported by Bohorov et al. for use in glycan arrays. ${ }^{72}$ In this 5 -step linker synthesis, hydroxylamine 32 was protected with an $N$-Boc protecting group. The alcohol was then mesylated and subjected to bromide substitution to give bromide $\mathbf{3 3}$ (Scheme 7). Next, $N$-methylhydroxylamine was $N$-Boc protected to give oxyamine $\mathbf{3 4}$, which was used in the alkylation reaction with bromide 33. After alkylation, the linker was treated with TFA to give the bi-functional linker 35 in $8 \%$ over 5 -steps. With the "Type A" linker in hand, various glycans 36 were conjugated, including complex oligosaccharides, and the resulting conjugates $\mathbf{3 7}$ were printed on an NHS-ester activated glycan-array chip. This linker was later employed by Boons and co-workers to print complex asymmetrical $N$-glycans on micro-array slides. ${ }^{73}$ Moreover, these linkers would allow for amide ligation reactions with the substrate of choice to form various glycoconjugates.
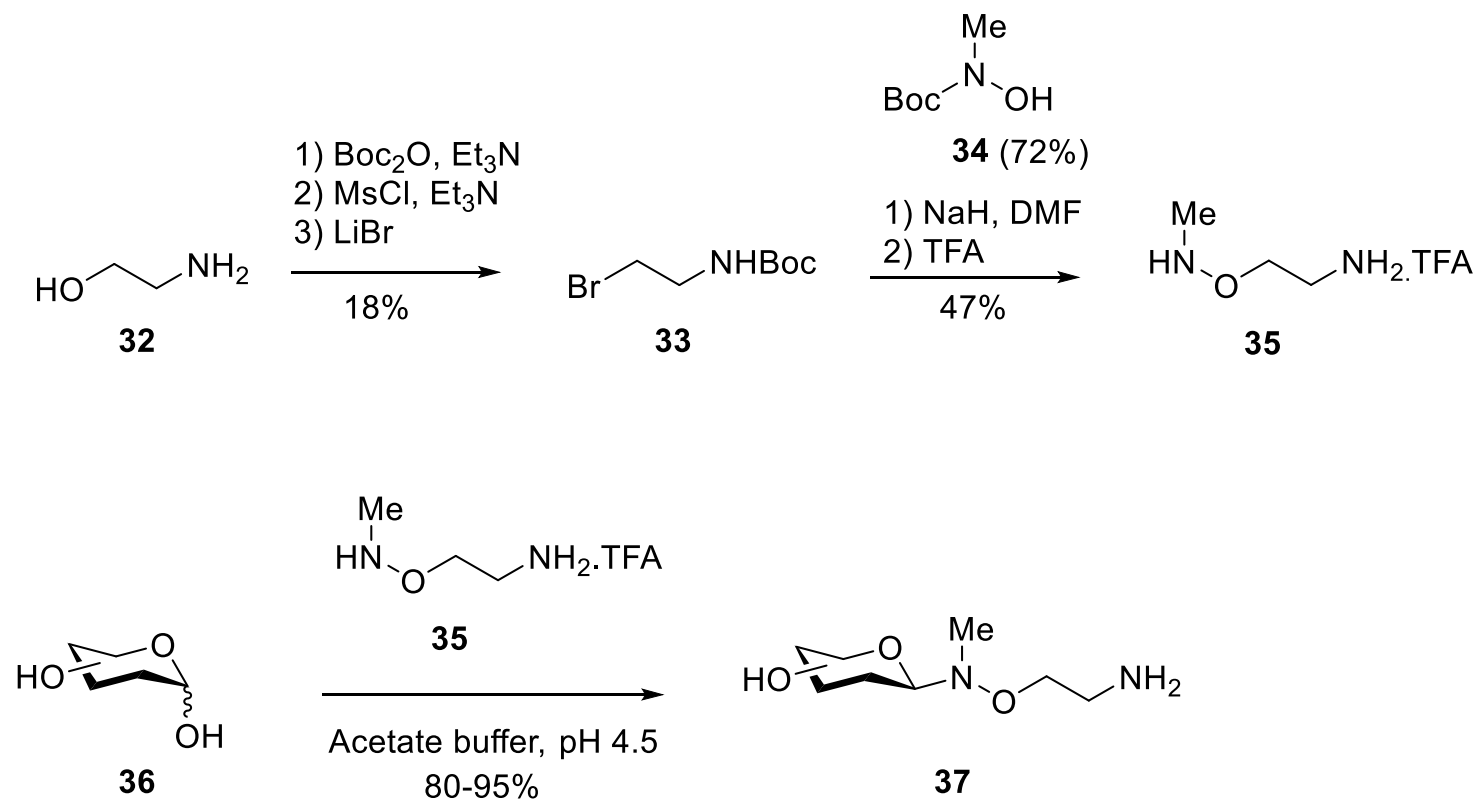

Scheme 7. "Type A" bi-functional linker synthesis and subsequent glycan conjugation by Bohorov et al. ${ }^{72}$

Recently, the group of Boons and co-workers reported the use of a $N$-Fmoc protected bi-functional "Type A" oxyamine linker in the chemoenzymatic synthesis of complex oligosaccharides (Scheme 8). ${ }^{74}$ Instead of conjugating these linkers to readily synthesised glycoconjugates, linker 38 was condensed with lactose (39) to give disaccharide oxyamine 40, which in turn was used throughout the chemoenzymatic synthesis of complex glycan ligands such as $\mathbf{4 1}$. In this way, other complex glycans were synthesised, such as dodecasaccharide $\mathbf{4 2}$. 


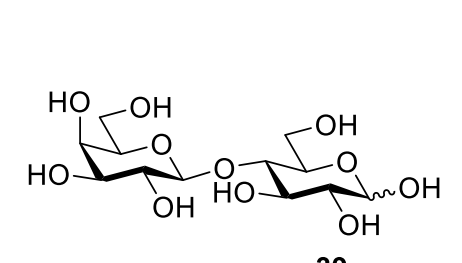

39

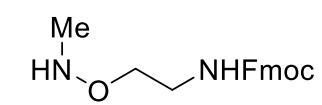

38

Acetate buffer, $\mathrm{pH} 4.5$

$80-95 \%$

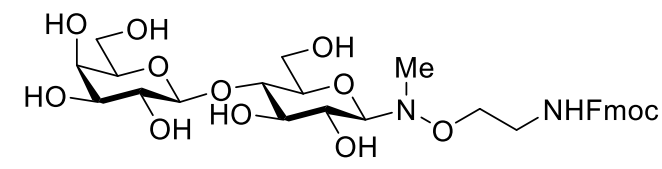

40
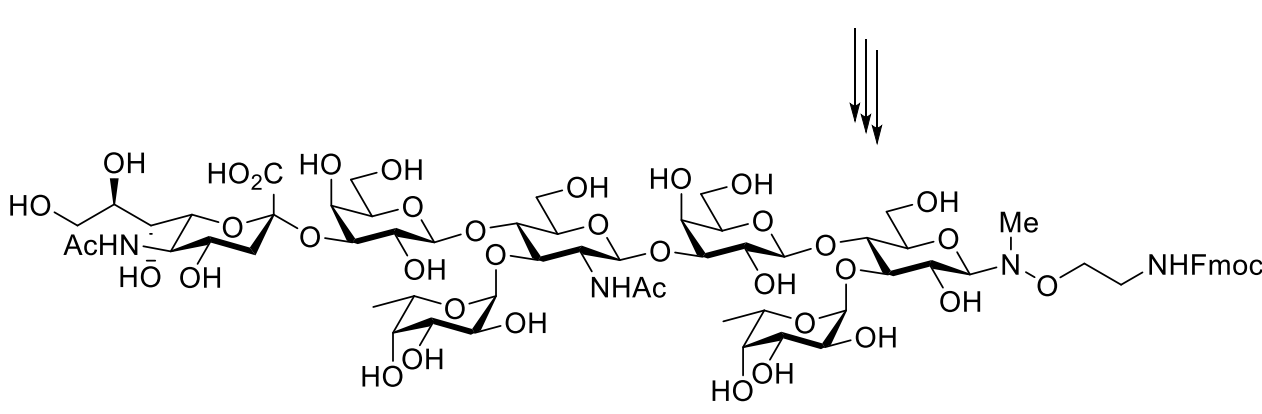

41

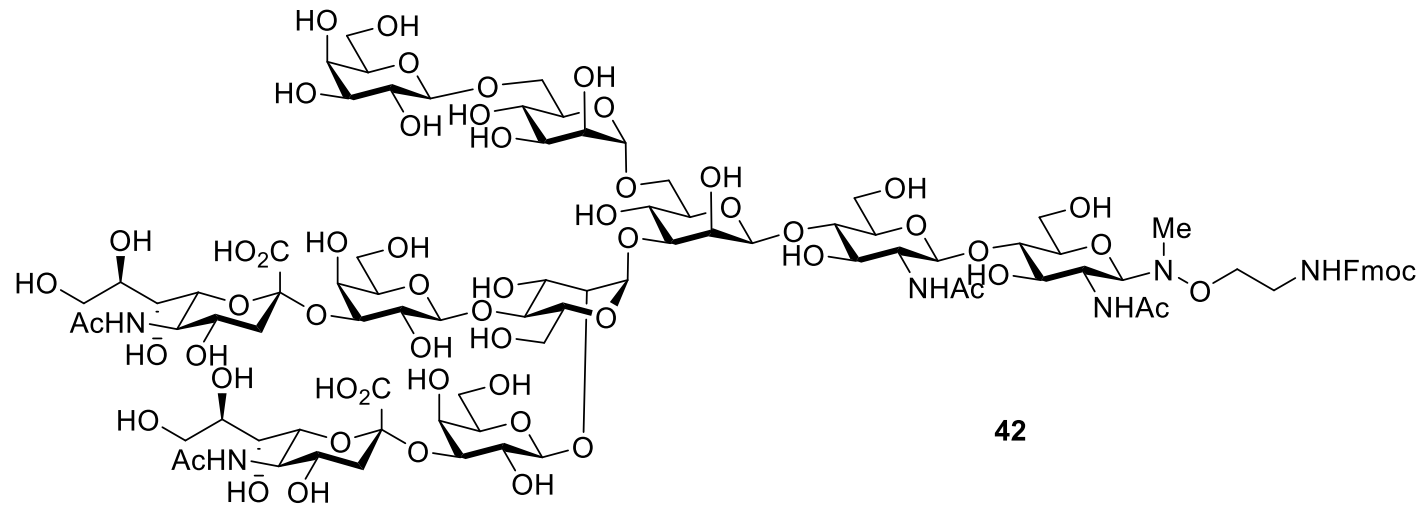

Scheme 8. Chemoenzymatic synthesis of oxyamine functionalised complex glycans. ${ }^{74}$

In addition to the work of Bohorov and Boons, other "Type A" bi-functional linkers $\mathbf{4 3}$ - $\mathbf{4 6}$ have also been synthesised (Figure 5), as well as linker 35. These linkers were conjugated to glucose so as to evaluate the relative stability of the constructs $(\mathbf{4 7}-\mathbf{5 1})$ as well as their lectin binding. Here, the latter was determined by immobilising $N$-acetyllactosamine oxyamine glycoconjugates to an array slide and employing a fluorescent Wheat Germ Agglutinin (WGA) binding assay. From these studies it was determined that the benzyl derivatives 49 and 51 were more stable glycoconjugates when compared to methyl derivatives $\mathbf{4 8}$ and $\mathbf{5 0}$, however, the size of the benzyl group affects lectin binding. Moreover, oxime 47 is more stable than the methylated glycoconjugates 48 and $\mathbf{5 0}$, but when the lectin binding of $\mathbf{4 7}$ is compared to the methyl- and benzyl-oxyamine conjugates $\mathbf{4 8}$ - 51, oxime $\mathbf{4 7}$ showed significantly lower affinity for the lectin. This could be explained by the fact that oxime $\mathbf{4 7}$ exists in the ring-opened form in solution, which affects the structural integrity of the glycan. 
<smiles>[R]NOCc1ccc(C(=O)NCCN)cc1</smiles><smiles>[R]NOCCN</smiles>

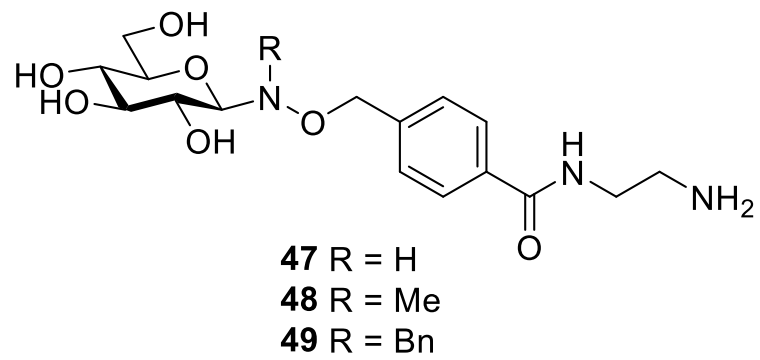<smiles>[R]OC(O)C1(O)C(O)C(O)C(O)C1ON([R])OCCN</smiles>

Figure 5. "Type A" bi-functional oxyamine linkers and glycoconjugates were tested on hydrolytic stability and lectin binding. ${ }^{56}$

In a similar fashion, a thiol bi-functional oxyamine linker of "Type A" was synthesised for the conjugation of glycans to bromoacetyl functionalised proteins (Scheme 9) ${ }^{75}$ Here, 3 -chloropropyl 4-methylbenzenesulfonate (52) was alkylated with $N$-Boc- $N$-methyl-hydroxylamine $\mathbf{5 3}$ to give chloride 54. A thiol substitution of the chloride with KSCN, followed by thiocyanate hydrolysis and subsequent $N$-Boc cleavage gave bi-functional linker 55. With the "Type A" linker in hand, $\mathrm{N}$-acetylglucosamine oligosaccharide $\mathbf{5 6}$ was then reacted with bromoacetyl functionalised bovine serum albumin (BSA) 57 to give glycoprotein $\mathbf{5 8}$, bearing four to eight oligosaccharides. ${ }^{75}$
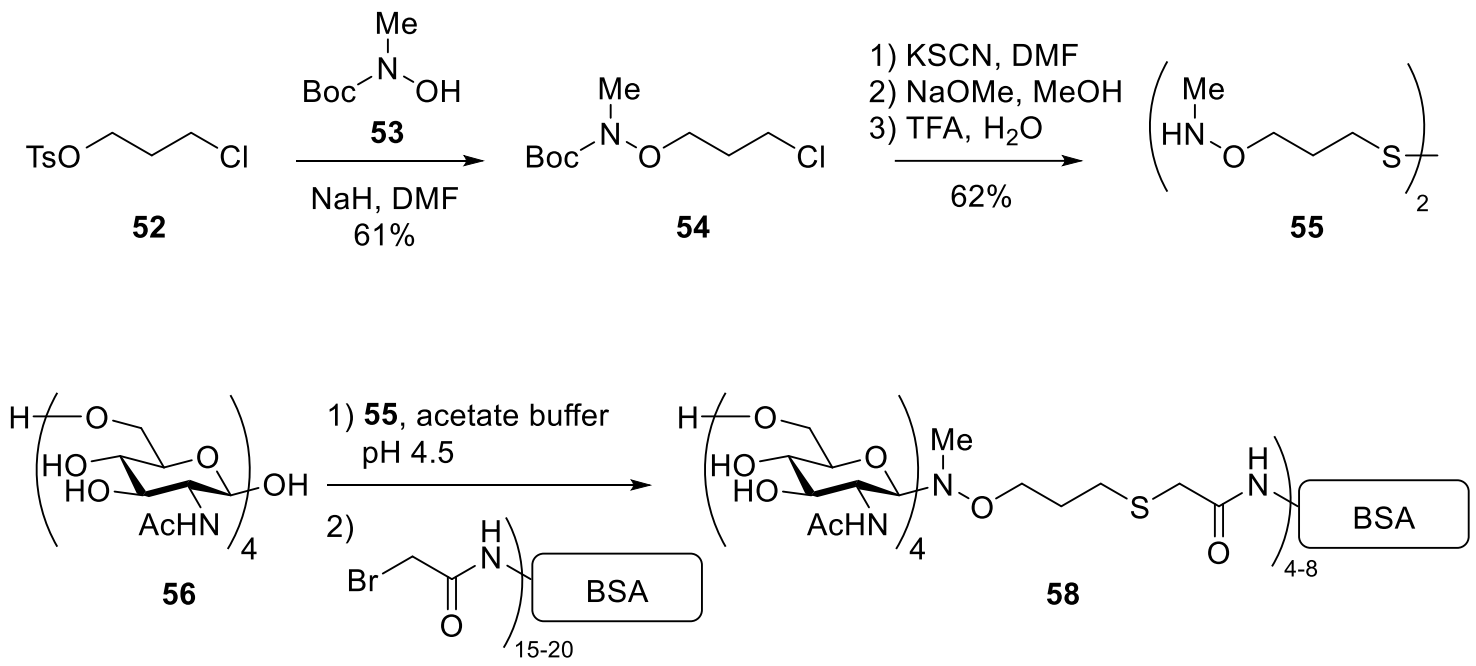

57

Scheme 9. Synthesis of "Type A" bi-functional linker 55 and conjugation of glucosamine antigens to $B S A .{ }^{75}$ 
Beside the synthesis of oxyamine functionalised amino-acids, only one "Type B" bi-functional linker has been synthesised. El-Noubbou et al. demonstrated the use of oxyamines in the assembly of nanoparticles via the utilisation of the azide-alkyne Huisgen cycloaddition (Scheme 10). In this work, methoxyamine (59) was benzoylated, alkylated with bromopropyne, and de-benzoylated to give the "Type B" alkyne bi-functional linker $\mathbf{6 0}$ in three steps. Next, condensation of $N$-acetylglucosamine (61) and oxyamine 60 was performed, followed by the Huisgen cycloaddition with azide-functionalised iron oxide nanoparticle $\mathbf{6 2}$, to form glycosylated nanoparticle 63.
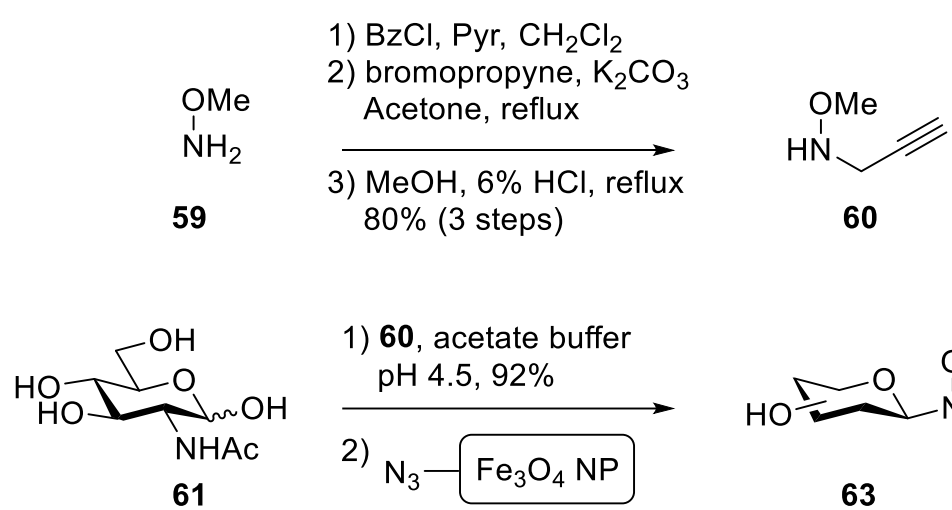

1) 60 , acetate buffer $\mathrm{pH} 4.5,92 \%$

2) $\mathrm{N}_{3}-\underbrace{\mathrm{Fe}_{3} \mathrm{O}_{4} \mathrm{NP}}_{62}$

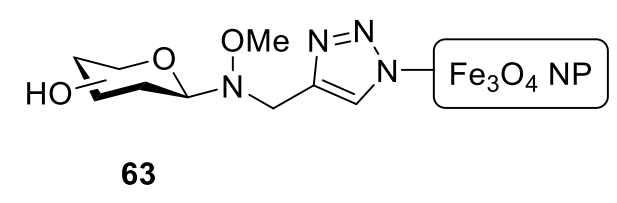

Scheme 10. Synthesis of "Type B" alkyne oxyamine 60, which allows for the synthesis of carbohydrate functionalised iron oxide nanoparticle $\mathbf{6 3}$ through the use of the copper-mediated Huisgen cycloaddition. ${ }^{76}$

Given the ease of formation, and their potential use in the conjugation of naturally isolated reducing carbohydrate antigens without the need for protecting groups, oxyamine methodology has proven to be a useful strategy in the synthesis of glycoconjugates. In comparison to the Kochetkov amination, oxyamines have the advantage that they are more stable than the $\beta$-glycosylamine, and, in addition, are not restricted to amide ligations with other substrates. When compared to reductive amination and oxime/hydrazide conjugation, oxyamines have the advantage in that the ring-closed glycoconjugate is obtained which better mimics the native glycan.

As discussed above, both "Type A" $O$-linked oxyamine and "Type B" $N$-linked oxyamines have been utilised in the synthesis of various glycoconjugates, including glycopeptides, glycan mimetics and neoglycorandomised therapeutics. Moreover, the use of "Type A" bi-functional linkers has been extensively investigated for the conjugation of glycan antigens to the substrate of choice, whereas research towards "Type B" bi-functional linkers has been explored less. Although the use of oxyamine linkers allows for the synthesis of biologically relevant glycoconjugates, to induce a meaningful biological response, the multivalent presentation of glycan antigens is required, thus the synthesis of multivalent glycoconjugates will be discussed. 


\subsection{Multivalent Glycan presentation}

\subsubsection{Dendritic structures}

In biological systems polyvalent interactions are collectively much stronger than their corresponding monovalent interactions. ${ }^{77-79}$ Interactions between monomeric carbohydrate ligands and protein receptors are often of low affinity and, therefore, give weak or non-detectable biological response. Multivalent presentation of ligands, however, can enhance binding and downstream signalling. The cell wall is covered in carbohydrates and, as a result, interactions between cells are enhanced by multivalent presentation of glycan antigens to corresponding lectins. For example, the deadly chicken influenza virus H5N1 targets specific cells through multivalent binding to sialyl Lewis ${ }^{\mathrm{X}}$ ligands. ${ }^{80}$

There are many structures which can be used for multivalent interactions with cell surfaces (Figure 6). For example, (branched) polymers decorated with glycan antigens have been used to enhance the biological response upon lectin binding. ${ }^{81}$ More recently, glycan decorated liposomes have been used to transport toxins efficiently into B-cells. ${ }^{82}$ Liposomes, however, have limited shelf lives compared to dendritic molecules. Unlike polymeric chains and liposomes, the synthesis of dendritic structures more readily allows for the control of the size and functionality of the target structures. ${ }^{83}$ Moreover, it has been demonstrated that the multivalent presentation of glycan antigens on dendritic molecules significantly enhances lectin binding and results in a stronger biological response when compared to polymer-presented glycan antigens..$^{40}$

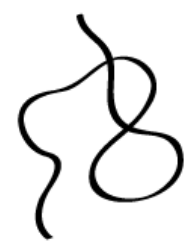

Linear Polymer

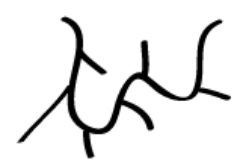

Branched Polymer

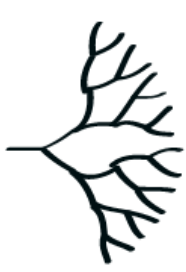

Dendron

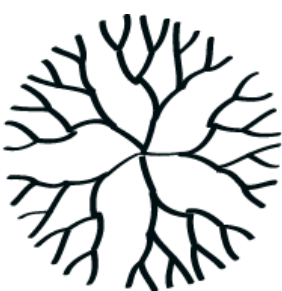

Dendrimer

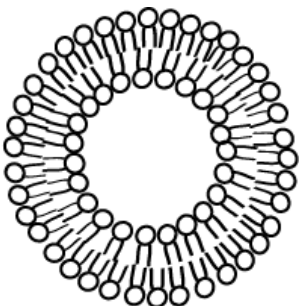

Liposome

Figure 6. Examples of multivalent structures.

\subsubsection{Dendritic features}

The names dendrimer and dendron originate from the Greek word 'dendros', meaning branch. An obvious example of a branched structure in nature is a tree, which uses a dendritic structure above the ground to enhance its exposure to sunlight, whereas an underground dendritic root system allows efficient nutrient uptake. Unlike trees, synthetic dendrons are often symmetrical and are structurally well-defined branched molecules of a specific size and functionalised terminal surface area. ${ }^{84}$ In contrast to other multivalent structures dendrons and dendrimers can be synthesised as single molecular species. Thus, dendron/dendrimer synthesis allows for the control 
of physical and chemical properties of biomolecules that are presented in a multivalent fashion, which in turn allows for the close monitoring of their biological behaviour. ${ }^{85}$ Accordingly, dendritic macromolecules have been used in various applications, including drug/gene delivery, cellular targeting, catalysis, and solubilisation. ${ }^{86}$

Dendrimers contain a core moiety and a layer-by-layer system that controls the size, branching and surface area of the dendritic structure. The central core of a dendrimer comprises either a single atom or a molecular moiety, such as a carbohydrate or amino acid. ${ }^{87}$ The size of the dendritic structure can be regulated by the number of generations, which is determined by the number of focal points between the core and the periphery of the dendrimer. For example, a dendrimer with two focal points is a second generation $(2 \mathrm{G})$-dendrimer. The outer shell can be decorated with the functional group of choice, for example glycan-decorated dendrimers are known as glycodendrimers. Dendrimer derivatisation with lectin binding specific carbohydrates can be used for dendrimer trafficking to specific cell types. Accordingly, this feature can be used to study biological processes or can be utilized in the development of therapeutic agents. ${ }^{88}$

Glycodendrons have similar properties to glycodendrimers, however, the former are bi-functional molecules. Glycodendrons also contain carbohydrates at the periphery of the dendron, however, instead of a symmetrical core, dendrons are synthesised from a bi-functional core. This allows for the additional functionalisation of dendrons with the substrate of choice, which can range from fluorescent molecules ${ }^{89}$ to small molecule drug carriers, ${ }^{90}$ as well as larger constructs such as peptide epitopes and proteins. ${ }^{91}$

\subsubsection{Dendron functionalisation}

\subsubsection{Fluorescent dendrons}

Fluorescent markers for the analysis of cellular processes have become a widely used tool in the field of biochemistry. ${ }^{92,93}$ To this end, fluorescent molecules have been conjugated to a variety of molecules including glycans, proteins, lipids, nucleic acids and dendrons, and have found application in a number of fluorescent-based readout assays, including microscopy, flow cytometry and ELISA. For example, BODIPY-labelled glycodendrons have been synthesised for the detection of the DC-SIGN receptor on dendritic cells, and subsequently used to study the lysosomal uptake of DC-SIGN antigens. ${ }^{89}$

\subsubsection{Biotinylated dendrons}


The interaction between biotin and avidin is the strongest non-covalent binding known to date $\left(K_{\mathrm{d}}=1.7 \times 10^{-15} \mathrm{M}\right)$, and accordingly, biotinylated dendrons have been conjugated to avidin or streptavidin proteins. ${ }^{94}$ The rapid conjugation of biotinylated substrates with streptavidin probes containing, for example, fluorescent groups, enzyme reporters or antigens, has many applications including use in microscopy, flow cytometry as well as vaccine development. ${ }^{95,96}$ Moreover, biotinylated constructs can be used in ELISA assays where the ELISA plate is coated with streptavidin, followed by the biotinylated dendron, which then results in the presentation of the glycodendrons on the surface of the plate. ${ }^{97}$

Biotinylated dendrimers have been used in various applications, including in the detection of influenza viruses, ${ }^{98}$ radioimmunotherapy, ${ }^{99}$ and cellular imaging. ${ }^{100}$ For example, in studies by Xu et al. a biotinylated dendrimer-based MRI contrast agent was synthesised and subsequently conjugated to fluorescent avidin. ${ }^{101}$ In this work, it was demonstrated that the supramolecular construct effectively targeted ovarian cancer tumours in mice, with efficient delivery of both the MRI contrast agent as well as the fluorophores to the cancer cells.

\subsubsection{Dendron drug delivery vehicles}

Dendrimers have been widely studied for their ability to deliver small molecule drugs to target cells. ${ }^{102}$ These small molecule pharmaceuticals can either be covalently attached to the dendron or encapsulated in the void spaces within the dendrimer. The encapsulation of drugs within dendrimers requires void spaces of a particular size and moreover, it requires various degrees of either hydrogen bonding, ionic interaction or hydrophobic void space. ${ }^{103}$ This encapsulation process has been used for many clinical drugs, such as diclofenac, doxorubicin and 10-hydroxycamptothecin. For the covalent attachment of drugs to dendrimers, key factors to consider are the stability of the conjugate in circulation and the cleavage of the drug within the target cell. ${ }^{102}$ To this end, most strategies involve the acid-dependent release of the drug, which allows for drug release when the dendrimer gets internalised in endosomal vesicles. Covalently attached drugs are often used in vaccination strategies, whereby antigenic peptides are delivered to immune cells to induce an immune response. Here, dendrimers and functional dendrons can be used instead of carrier proteins. The advantage of dendrons over proteins is that it is often easier to specifically functionalise dendrimers, while the conjugation of proteins can lead to a mixture of products. 


\subsubsection{Size and functionalisation of dendrons}

\subsubsection{The effect of dendron size on the efficiency of lectin binding}

The multivalent presentation of the glycan antigen is the most important property of glycodendrons when used for lectin binding studies. Although the synthesis of large dendrons seems desirable for the mimicry of biological events, several groups have reported on both the binding efficiency as well as the toxicity of large dendrons and have demonstrated that more highly branched dendrons are not necessarily better than their lesser branched counterparts. For example, Wang et al. showed that when comparing the half inhibition concentration $\left(\mathrm{IC}_{50}\right)$ of the 2G12 lectin between the mono-valent glycan and the tri-, nona- and 27-valent glycodendrons, all three dendrons showed significantly higher affinity for the lectin. ${ }^{104}$ When comparing the $\mathrm{IC}_{50}$ per dendron (290, 4.6 and $4.1 \mathrm{nM}$, respectively), the second generation dendron is just as effective as the third generation dendron. However, when the efficiency per glycan is compared, the second generation has a lower $\mathrm{IC}_{50}$ than the third generation (10 and $41 \mathrm{nM}$, respectively). Moreover, it should be noted that the synthesis of second generation dendrons is more efficient and requires fewer glycan antigens.

The size of the dendron (without increasing the number of antigens), however, is important for lectin binding. This was nicely demonstrated by van Kooyk and co-workers, who used the reductive amination between the antigen Lewis ${ }^{\mathrm{x}}$ and different size (3G, 4G and 5G) poly(amido amine)amine (PAMAM) dendrimers to obtain Lewis ${ }^{\mathrm{X}}$-glycodendrons of various sizes. ${ }^{40}$ Due to the coupling inefficiency and structural defects of the larger dendrimers, all dendrimers contained $16 \pm 3$ glycans, and thus the size of the dendrimers $(23 \pm 2,27 \pm 2$ and $45 \pm 3 \mathrm{kDa})$ could be compared in lectin binding studies. It was observed that larger dendrons resulted in more efficient binding of the lectin. This supports the theory that the size of the dendron, and thus the spacing between glycans, might be more important for binding than the number of antigens when enough antigens are present.

\subsubsection{Dendrimer cytotoxicity}

Another important aspect of dendrimer chemistry is the biocompatibility and cytotoxicity of dendrimer constructs, particularly when these macromolecules are used in a pharmaceutical setting. Although each dendrimer/dendrons has a specific cytotoxicity, depending on the dendron functionalisation, several studies have considered dendron toxicity. ${ }^{105}$ For example, it was found that the smaller generations $(<G 5)$ PAMAM-based dendrons have little or no harmful effect at concentration desired for biological applications, whereas larger $(>5 \mathrm{G})$ dendrimers are more cytotoxic. ${ }^{106}$ In addition, no evidence of immunogenicity was observed for the smaller generations $(<5 \mathrm{G})$, although the functionalisation of dendrons and dendrimers will affect the biological response too, and thus the potential cytotoxicity of these macromolecules. Notwithstanding, preliminary research indicates that small dendritic structures have few in vivo side effects. ${ }^{107}$ 


\subsubsection{Dendrimer design and synthetic approaches}

\subsubsection{Divergent vs convergent dendrimer synthesis}

Traditionally, dendrimers and dendrons have been synthesised either via divergent or convergent approaches (Scheme 11). ${ }^{108}$ In the divergent approach, the dendrimer is synthesised from the inside-out. Here a multifunctional core is activated and functionalised with a new generation. Repetition of these two steps increases the number of generations and thus the size of the dendrimer. It should be noted that structural defects developed during divergent dendrimer synthesis can be difficult to separate from the desired dendron. However, as divergent dendrimer synthesis allows for the use of cheap and readily available reagents, many commercially available dendrimers are synthesised via this approach.

The convergent synthetic route allows for the synthesis of dendron 'wedges' that are subsequently coupled to a dendron core structure to obtain a dendrimer. This allows for a more controlled synthesis, as structural defects are more easily removed. A drawback of the convergent synthesis is the requirement for excess dendron during the coupling with the core structure in order to obtain the dendrimer in sufficient yield.

More recently, double exponential growth methodology has been developed for the more efficient syntheses of dendrons ${ }^{83}$ Here, a protected first generation dendron is synthesised, which is then converted to a second generation dendron in a parallel synthesis. This route has particular merit since it minimises the number of steps to assemble larger dendrons, and in addition, it allows for the easy purification of the first generation dendron and accordingly, rapid assembly of second, third and fourth generation dendrons.

Using these methodologies, an overwhelming number of dendrons and dendrimers have been synthesised in the last three decades, each of them with unique structural features. ${ }^{83,84,109}$ However, each of these dendrons and dendrimers have three things in common: a core structure, branching units and a functionalised periphery.

As mentioned above, the core structures can differentiate between dendrimers and dendrons. However, both dendrons and dendrimers can be synthesised from a large variety of core molecules, such as ammonia, diamine-alkanes, benzenes, amino acids, lipids, hydrocarbons and heteroatoms. In particular, the use of tris(hydroxymethyl)aminomethane (TRIS) has been useful in dendrimer chemistry as it allows for three fold symmetry. A more comprehensive review on the use of TRIS in dendron synthesis will be discussed in Chapter 3. 

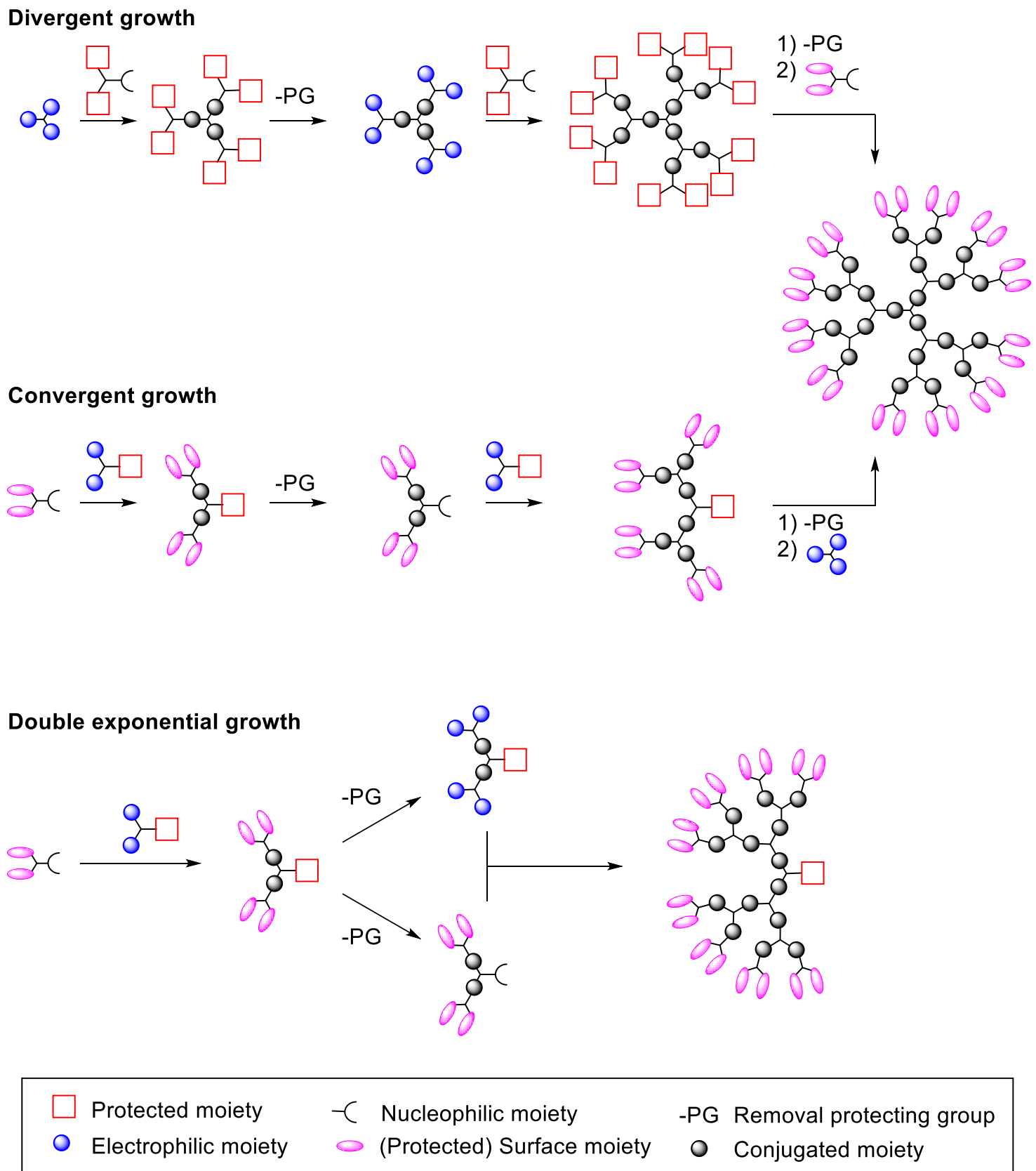

Scheme 11. Synthetic approaches towards dendrons and dendrimers. 


\subsection{Lewis Antigens}

The Lewis antigens are a related set of glycans found in nature. They are divided in two subgroups, the Type- 1 and Type-2 Lewis antigens, which are characterised by the presence of the $\alpha$-Fuc-(1 $\rightarrow 4)$-GlcNAc or $\alpha$-Fuc-( $1 \rightarrow 3$ )-GlcNAc residues, respectively (Figure 7$).{ }^{110}$ The structurally most basic Type-1 and Type-2 Lewis antigens are known as Lewis ${ }^{\mathrm{A}}$ and Lewis ${ }^{\mathrm{X}}$. In the presence of a fucosyltransferase (FUT), these structures are converted into Lewis ${ }^{\mathrm{B}}$ and Lewis ${ }^{\mathrm{Y}}$, whereas sialylation of the galactose moiety results in Sialyl Lewis ${ }^{\mathrm{A}}$ and Sialyl Lewis ${ }^{\mathrm{X}}$ derivatives. Moreover, sulfation of the 6-, 6'- or 3'-hydroxyl gives rise to sulfated derivatives.

\section{Type-1 Antigens}

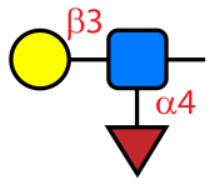

Lewis $^{A}$

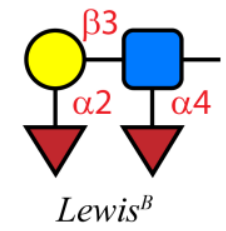

Lewis $^{B}$

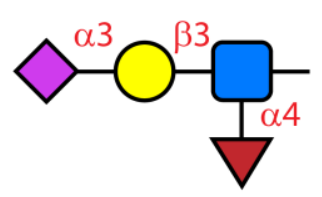

Sialyl Lewis ${ }^{4}$

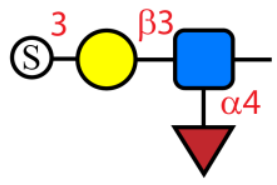

3'-Sulfo-Lewis ${ }^{A}$

Type-2 Antigens

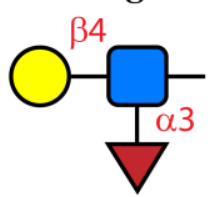

Lewis $^{X}$

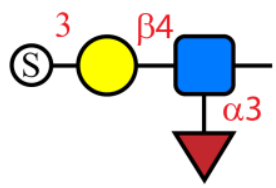

3'-Sulfo Lewis'
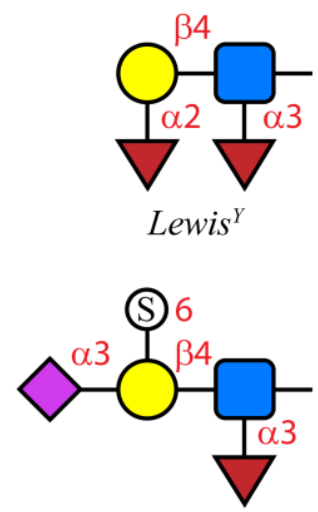

6'-Sulfo Sialyl Lewis ${ }^{X}$

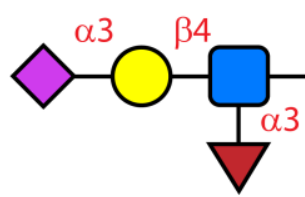

Sialyl Lewis ${ }^{X}$

Figure 7. Schematical presentation of Type-1 and Type-2 Lewis antigens; $\bigcirc=$ Galactose; $\square=N$-Acetylglucosamine; $\nabla=$ Fucose $; \diamond=$ Sialic acid; (S)= sulfate.

The biosynthesis of the Lewis antigens depends on the presence or absence of fucosyltransferases. The Type-1 Lewis antigens are encoded by genes on chromosomes FUT3 and FUT2. ${ }^{110}$ These genes have dominant alleles ( $\mathrm{Le}$ and $\mathrm{Se}$ ) which code for the fucosyltransferases, as well as non-functional recessive alleles (le and $s e$ ). Individuals with the Le allele (FUT3) have the $\alpha-(1 \rightarrow 3 / 4)$-fucosyltransferase which produce Lewis $^{\mathrm{A}}$ and small amounts of Lewis ${ }^{\mathrm{X}}$ antigens from the Type-1 and Type-2 precursor, respectively. The Se allele (FUT2) encodes for the $\alpha-(1 \rightarrow 2)$-fucosyltransferase, which in turn produces Lewis ${ }^{\mathrm{B}}$ and Lewis ${ }^{\mathrm{Y}}$ antigens. Individuals lacking both $\mathrm{Se}$ and $\mathrm{Le}$ genes are Lewis negative and do not produce Type-1 blood group antigens. The Type- 2 Lewis antigens, however, can also be synthesised by $\alpha-(1 \rightarrow 3)$-fucosyltransferases 
that are encoded within the FUT6 and FUT7 genes, of which the FUT6 gene is expressed by $96 \%$ of the population. ${ }^{111}$ In contrast to other erythrocyte antigens, Lewis glycans are synthesised in the tissue and distributed throughout the body via the excretion of glycolipids into the plasma. ${ }^{12}$ Accordingly, Lewis antigens are widely distributed in body fluids and tissues and are mainly expressed on epithelia of glandular tissues.

\subsubsection{Lewis Blood group antigens}

Unlike Type-2 antigens, the Type-1 Lewis antigens (e.g. Lewis ${ }^{\mathrm{A}}$ and Lewis $\left.{ }^{\mathrm{B}}\right)$, are incorporated by erythrocytes with variable expression levels between individuals. ${ }^{112}$ Moreover, the concentration of these Lewis blood group antigens may alter in case of disease, or during pregnancy. The incorporation of these antigens by immune cells might be involved with self-antigen detection, as anti-Lewis ${ }^{\mathrm{A}}$ and anti-Lewis ${ }^{\mathrm{B}}$ antibodies are found in Le(a-/b-) deficient individuals as well as pregnant women. ${ }^{113}$ Although the function of Lewis ${ }^{\mathrm{A}}$ and Lewis ${ }^{\mathrm{B}}$ antigens remains somewhat uncertain, the sialylated blood group antigen, Sialyl Lewis ${ }^{\mathrm{A}}$, is involved in binding with E-selectins.

\subsubsection{E-, L- and P-Selectin binding}

One of the major features of Lewis antigens is the reversible binding of E-, L- and P-selectins expressed on the endothelium as well as on individual leukocytes. ${ }^{114,115}$ The endothelium upregulates these cell adhesion selectins upon local inflammation or bacterial infection which, in turn, recruits leukocytes through the interaction of the glycan antigens expressed on immune cells. This causes the leukocyte to slow down through "rolling and tethering", and allows for the migration of the leukocyte into the tissue towards the site of inflammation. ${ }^{115}$ Sialyl Lewis ${ }^{\mathrm{X}}$ is found on the glycoprotein P-Selectin Glycoprotein Ligand-1 (PSGL-1) expressed on immune cells, which mediates the interaction with the selectins. ${ }^{116}$ Moreover, Lewis ${ }^{\mathrm{Y}}$ antigens are expressed on Cluster of Differentiation-44 (CD44), a transmembrane glycoprotein found on a large number of mammalian cell types. ${ }^{117}$ The primary function of CD44 is binding of hyaluronan, but it can also mediate cell-cell interactions, cell adhesion, and tissue migration through the interaction between the Lewis antigen and the selectins. ${ }^{114}$ The binding of glycan antigens to selectins, however, has also been linked to various disease, including viral infection, arthritis, thrombosis and tumour metastasis. ${ }^{118-120}$

\subsubsection{Lewis antigens in disease}

As mentioned above, the selectin mediated binding of leukocytes allows for the migration of these leukocytes into the tissue. Tumours, however, often upregulate Lewis antigens for migration of 
cancer cells into new organs (metastasis). Sialyl Lewis ${ }^{\mathrm{X}}$ overexpression is often found on cancer cells and is associated with cancer metastasis, and the overexpression of this biomarker can be used to detect these types of cancers through high-density antibody arrays. ${ }^{120} \mathrm{In}$ a similar fashion, Lewis ${ }^{\mathrm{Y}}$ has been found to be expressed at various levels in human malignancies, and can be used as a biomarker for cancer-progenitors. ${ }^{121,122}$ Besides tumour metastasis, Lewis antigens can be involved with other migratory complications. Sialyl Lewis ${ }^{\mathrm{A}}$, which is expressed on CD44v6 on human intestinal epithelial cells, mediates the transepithelial migration of granulocytes into the lumen. Patients suffering from inflammatory bowel disease showed highly upregulated Sialyl Lewis ${ }^{\mathrm{A}}$ antigen levels, which could explain the excess of granulocytes present in the lumen of patients suffering from Crohn's disease and ulcerative colitis. ${ }^{123}$ Moreover, sialylated glycoproteins are found on viruses such as the Herpes Simplex Virus Type-1 (HSV-1) and HIV, which allows for infection of the host through selectin-mediated binding. ${ }^{118,124}$

\subsubsection{Lewis antigens and human fertilisation}

Lewis $^{\mathrm{X}}$, Lewis ${ }^{\mathrm{Y}}$ and, in particular, Sialyl Lewis ${ }^{\mathrm{X}}$ are involved in human fertilisation. For fertilisation to occur, the sperm cells initially need to bind to the highly specialised extracellular translucent matrix coating the egg known as the zona pellucida. This first interaction is mediated between the egg-binding protein (EBP) on sperm cells, and Lewis antigens on the zona pellucida. ${ }^{125,126}$ Upon sperm cell binding to the zona pellucida, the acrosomal exocytosis is induced. Next, the acrosome-reacted sperm adheres to the plasma membrane of the egg cell and the fusion of the membranes results in the fusion of both nuclei.

\subsubsection{Sulfated Lewis Antigens}

Sulfated Lewis antigens have been found to have many important biological roles. For example, $3^{\prime}-O$-sulfo-Lewis ${ }^{\mathrm{X}}$ is synthesised by Gal-3-O-sulfotransferase ${ }^{127}$ and plays an important role in the regulation of integrin-associated cell adhesion, ${ }^{128}$ selectin binding, ${ }^{129}$ and is also overexpressed in particular tumour cells. ${ }^{130}$ Moreover, sulfated sialyl Lewis ${ }^{\mathrm{X}}$ ligands play an important role in the modulation of immune responses through the interaction with sialic-acid-binding immunoglobulin-like lectins (siglecs), ${ }^{131}$ as well as the selectins. ${ }^{132}$ The siglecs are a family of transmembrane cell-surface proteins which are selectively expressed on immune cells. Expression of the different siglecs ranges from single cell-types to a variety of immune cells. ${ }^{131,133}$ Currently, fourteen different human siglecs and nine mouse variants are known. Each siglec can bind to specific carbohydrate ligands, and in particular, Siglec-8 and Siglec-9 are of interest as they bind to $6^{\prime}-O$-sulfo and $6-O$-sulfo sialyl Lewis ${ }^{\mathrm{X}}$, respectively. ${ }^{131}$ Engagement of siglecs can result in various immunological functions, such as cellular activation, adhesion, endocytosis, proliferation and apoptosis. ${ }^{134,135}$ 


\subsubsection{Lectin binding Lewis antigens}

Besides the binding of selectins, Lewis antigens have been shown to bind other lectins, including macrophage galactose-type lectin (MGL), Langerin, and Dendritic Cell-Specific Intercellular adhesion molecule-3-Grabbing Non-integrin (DC-SIGN). The mouse MGL has been found to bind Lewis ${ }^{\mathrm{X}}$ antigens, however, the human MGL binds to $\alpha / \beta$-GalNAc Tn-antigens (O-glycans) instead of Lewis antigens, and thus cannot be used to target human DCs through the use of Lewis antigens. ${ }^{136}$ Langerin is expressed on Langerhans cells, which make up the outer cell layer of epithelial and mucosal tissue, and form the first line of defense against pathogens such as bacteria, fungi and viruses. Langerin is an extracellular C-type lectin with specific affinity for mannose and fucosylated glycans, including Lewis ${ }^{\mathrm{B}}$ and Lewis ${ }^{\mathrm{Y}}$. Upon binding of these pathogens, the pathogen-Langerin complex rapidly internalises into Birbeck granules for degradation of the pathogen, which results in antigen presentation and immune activation against the pathogens. This receptor has been the major focus of research against HIV. The binding of Lewis ${ }^{\mathrm{X}}$ to the C-type lectin DC-SIGN, however, results in internalisation of the complex in a similar fashion to Langerin, but instead of the formation of Birbank granules, internalisation results in lysosomal compartments for antigen degradation. This makes DC-SIGN an interesting target for vaccine development.

\subsection{Targeting DC-SIGN with Lewis ${ }^{\mathrm{X}}$ antigens}

\subsubsection{C-type lectin DC-SIGN}

Antigen-presenting cells (APCs), such as dendritic cells (DCs) and macrophages, are mostly located where pathogen invasion is most common, (i.e. on the skin, in the respiratory system as well as the digestive system). Both macrophages and DCs are capable of pathogen recognition and processing and play a crucial role in the early stages of pathogen elimination. ${ }^{137}$ By phagocytosis and intracellular processing of pathogens, APCs are capable of presenting specific peptide antigens to effector T-cells in order to produce and stimulate specific immune responses against these pathogens. ${ }^{138}$ They are efficient at detecting pathogens due to their display of pattern-recognition receptors (PRR), such as Toll-like receptors, Nod-like receptors, RIG-I-like receptors and C-type lectins, with each PRR recognising a different class of molecular patterns. ${ }^{138}$ The molecular recognition of these PRRs is extremely diverse, ranging from nucleic acids to lipids and from carbohydrates to proteins, making these cells extremely efficient at pathogen recognition.

DC-SIGN is a C-type lectin and is expressed on the extracellular cell wall of myeloid DCs, human alveolar and lymph node macrophages, and endothelial cells. ${ }^{19,139-143}$ Although DC-SIGN is highly expressed on most DCs, the expression on macrophages is dependent on the phenotype of 
the macrophage. ${ }^{141,144}$ DC-SIGN, also known as Cluster of Differentiation 209 (CD209), is encoded by the $C D 209$ gene and has a high affinity for the transmembrane glycoprotein ICAM3 on T-cells. ${ }^{143}$ The C-type lectin comprises a $\mathrm{Ca}^{2+}$ mediated binding site to which a variety of carbohydrate ligands have been found to adhere, including high-mannose glycans and fucosylated oligosaccharides such as Lewis ${ }^{\mathrm{A}}$ and Lewis ${ }^{\mathrm{X}} \cdot{ }^{145-147}$ Moreover, glycan array screening of the DC-SIGN receptor revealed that at least 20 different glycans have a significant interaction with DC-SIGN. ${ }^{148}$

DC-SIGN is organised in tetramers, ${ }^{146}$ and upon multivalent binding of the lectin, the complex is internalised by phagocytosis. This internalisation is independent of receptor cross-linking and the DC differentiation status. ${ }^{149}$ After internalisation the natural ligand is released from the DC-SIGN receptor and the receptor is recycled to the cell surface. While inside the lysosome, the antigens get processed into smaller peptide antigen fragments. These antigens are then presented on MHC-II to activate CD4+ T-cells. When using DC-SIGN antibodies, however, the recycling of the lectin is prevented which in turn inhibits the antigen uptake by DC-SIGN ${ }^{+}$cells. Moreover, the use of anti-DC-SIGN antibodies can result in an aberrant immune response with undesired immune activation. Accordingly, it could be desirable to utilise the DC-SIGN native glycan antigen to target these receptors in a therapeutic setting.

\subsubsection{DC-SIGN mediated treatment}

Given the importance of DC-SIGN in pathogen recognition and antigen presentation for immune activation, DC-SIGN has been studied for its potential use in therapeutic settings. In 2012, Van Kooyk and co-workers synthesised mannose and fucose functionalised fluorescent glycodendrons to target DC-SIGN on DCs (Figure 8). ${ }^{89}$ These BODIPY labelled glycodendrons 64 were synthesised via the copper-mediated Huisgen cycloaddition and it was found that these glycodendrimers bind DC-SIGN and internalise without activation or maturation of the DC. Using a mannose glycodendron, Davies and co-workers synthesised a Virus-like glycodendrinanoparticle displaying up to 1620 glycan ligands to potentially block viral infection by Ebola via DC-SIGN. ${ }^{91}$ Here, the second generation mannose dendrimer was conjugated to the Qß-Hpg16 180 nanoparticle through a copper-mediated Huisgen cycloaddition to give glycodendrinanoparticle $\mathbf{6 5}$. It was observed that 1620 glycan ligands were required to block viral infection, whereas the nanoparticle containing 540 antigens insufficiently blocked infection. Furthermore, it was found that a $25 \mathrm{nM}$ concentration of glycodendrinanoparticles was required to prevent HIV infection of DCs by more than $90 \%$ which was a similar result to that observed when using the anti-DC-SIGN antibody. ${ }^{91}$ 

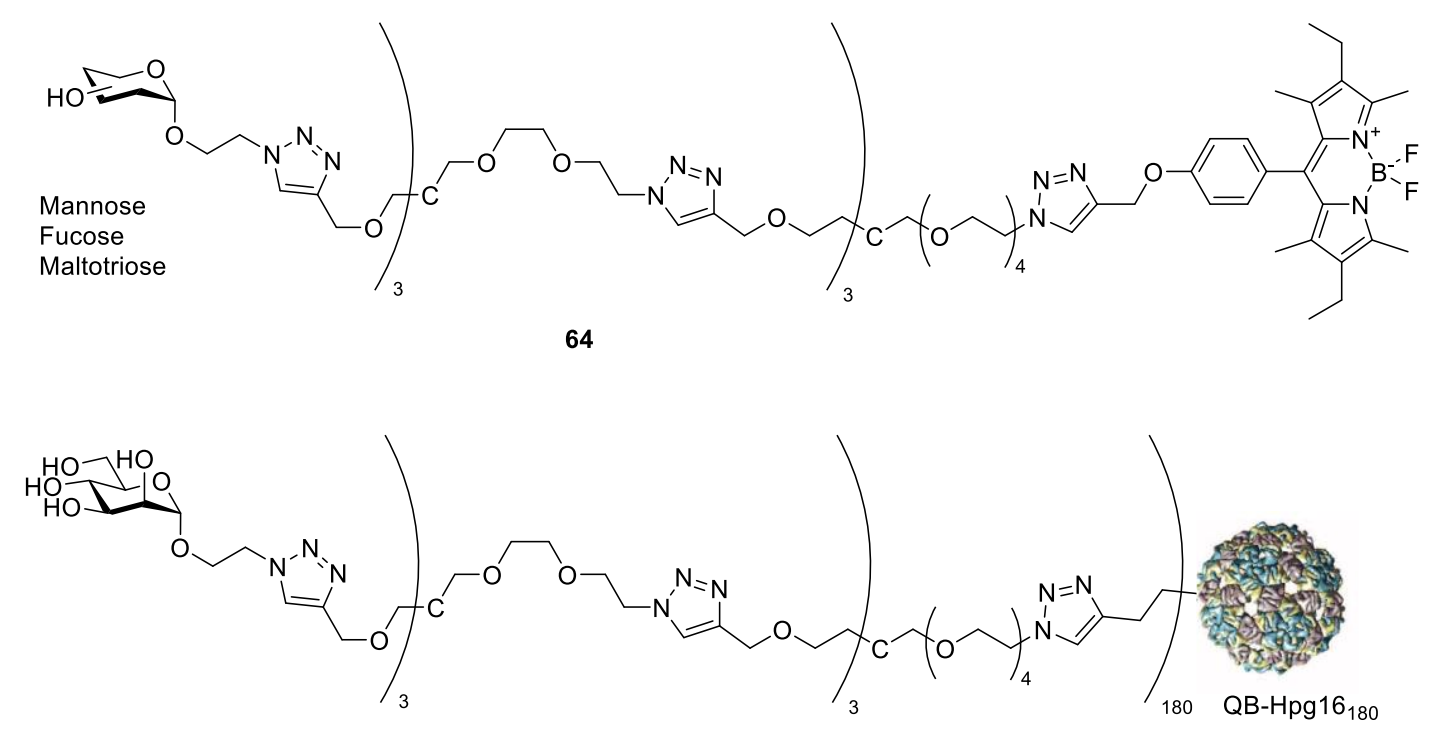

65

Figure 8. BODIPY-labelled mannose- and fucose-dendrimers 64 were used to target DC-SIGN on DCs. ${ }^{89}$ The mannose-glycodendrinanoparticle 65 can prevent DC-SIGN-mediated HIV-infection of DCs. ${ }^{91}$

Although mannose (micromolar affinity) and fucose (high micromolar affinity) glycans can be used for DC-SIGN mediated treatments, it was shown that the mannose derivatives also bind to the receptor Langerin which leads to an undesired side effect in the treatment of HIV. Langerin also binds HIV, however, instead of infection of the host, Langerin binding results in HIV capturing and destruction through rapid Birbeck granule degradation. Thus, blocking the Langerin receptor in HIV treatment is undesirable. To prevent Langerin binding, Lewis ${ }^{\mathrm{X}}$ glycan antigens can be used to target DC-SIGN. This approach was taken by Van Kooyk and co-workers who reported the synthesis of Lewis ${ }^{\mathrm{X}}$ glycodendrimers and it was postulated that these glycodendrimers can prevent HIV-transmission through DC-SIGN on dendritic cells. ${ }^{40}$ Using the reductive amination methodology, they conjugated the Lewis ${ }^{\mathrm{X}}$ antigen to the commercially available PAMAM dendrimers 66 of various size to yield the 3G-, 4G- and 5G-dendrimers 67 each bearing $16 \pm 2$ Lewis $^{\mathrm{X}}$ antigens (Scheme 12). It was observed that the spacing between glycan ligands is important for biological activity, where the larger dendrons more efficiently bound to DC-SIGN. Moreover, they showed that these glycodendrimers had higher binding affinity for DC-SIGN binding when compared to Lewis ${ }^{\mathrm{X}}$ containing polyacrylamide polymers. It was shown that over $95 \%$ of the DCs internalise these glycodendrons, and internalisation did not induce DC-activation. Notwithstanding, it was found that these glycodendrimers were able to completely inhibit HIV infection, even after two days in the presence of HIV. 

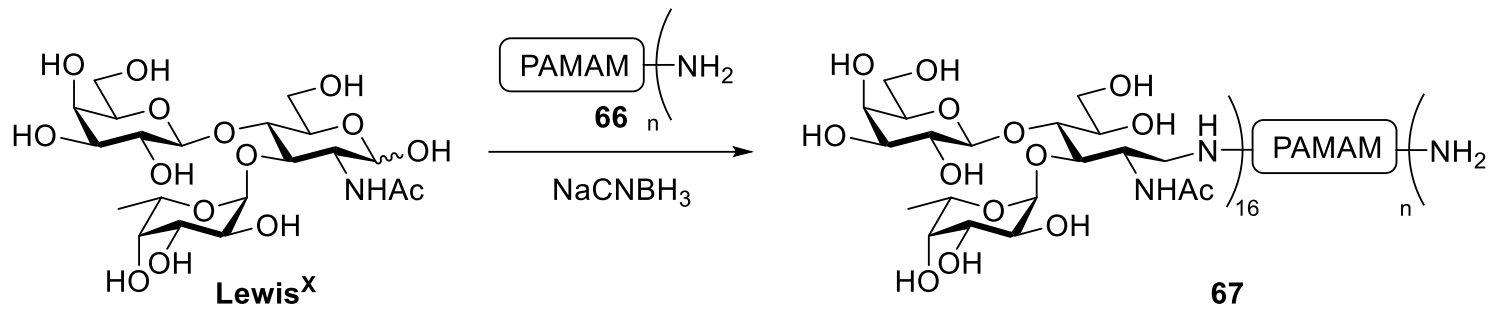

Scheme 12. Reductive amination between Lewis ${ }^{X}$ and various sizes PAMAM-NH 66 gave $3 G-, 4 G$ - and $5 G$-glycodendrons 67 bearing $16 \pm 2$ Lewis antigens. ${ }^{40}$

The ability of these Lewis antigens to bind DC-SIGN and internalise into DCs allows for cell-selective antigen delivery, and 'controlled' activation of the immune system against various diseases, and accordingly would allow for the development of novel vaccines. This requires the DC-SIGN mediated internalisation of glycodendrons containing antigenic peptides. Accordingly Garcia-Vallejo et al. prepared Lewis ${ }^{\mathrm{B}}$ containing glycopeptide-PAMAM conjugates 68 (Figure 9). ${ }^{150}$ Again, these glycodendrons showed sufficient binding to DC-SIGN, resulting in antigen delivery by internalization as was evidenced by flow cytometry. Most importantly, these glycodendrons were able to present these antigens to ovalbumin (OVA)-specific CD4 ${ }^{+} \mathrm{T}$-cells, which resulted in T-cell proliferation. Here it should be noted that the second generation was sufficient to induce a T-cell response, whereas larger dendrons did not induce stronger biological responses. Interestingly, these glycodendrons showed superior activity when compared to DCs activated with Lewis ${ }^{\mathrm{B}}$-OVA conjugates, which indicates that these glycodendrons are effective cell-targeting delivery vehicles.

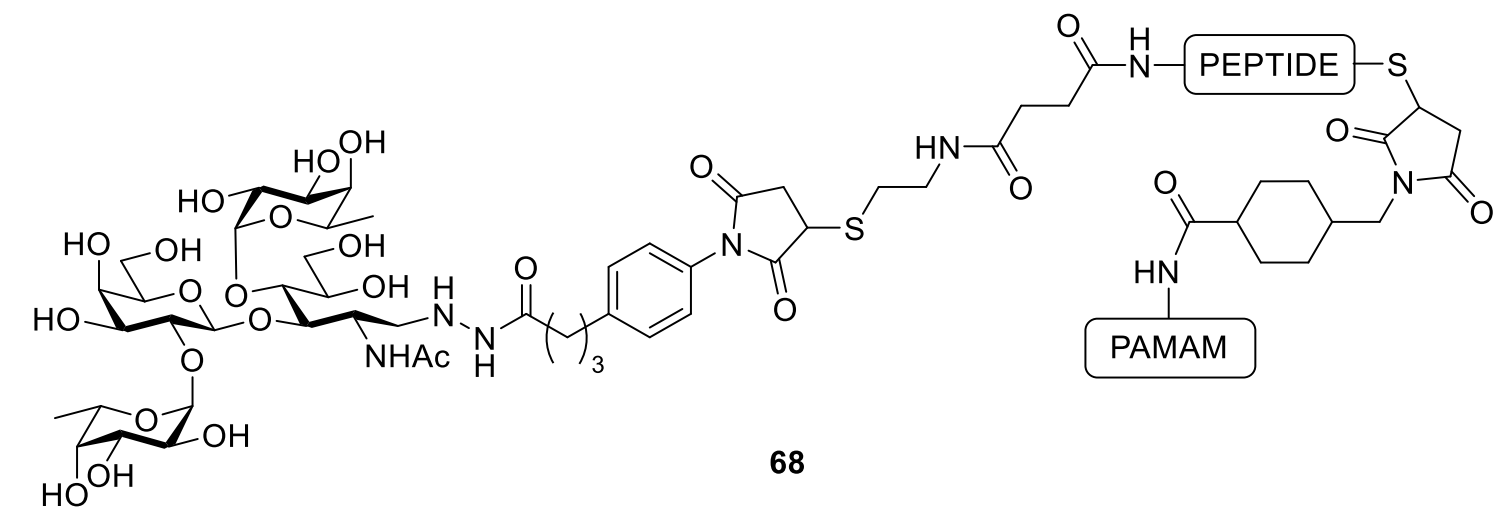

Figure 9. Synthesis of PAMAM based glycodendrons bearing antigenic peptides such as SIINFEKL and ISQAVHAAHAEINEAGR. ${ }^{150}$ 
Recently, targeting of DC-SIGN has also been achieved with the use of fucosylated gold nanoparticle 69 (Figure 10). ${ }^{151}$ It was observed that the $N$ - $\alpha$-fucosyl- $\beta$-alanylamide bound with similar if not higher affinity for DC-SIGN compared to the Lewis ${ }^{\mathrm{X}}$ antigen. ${ }^{152,153}$ It was demonstrated that these nanoparticles were internalised by DCs through binding with DC-SIGN without triggering inflammatory cytokine responses of the DCs. Thus the synthesis of fucosylated gold nanoparticles allows for a less expensive and effective alternative to Lewis ${ }^{\mathrm{X}}$ derived glycodendrons. However, many C-type lectins can bind fucosylated glycan antigens, and thus the selectivity for DC-SIGN C-type lectins remains questionable.

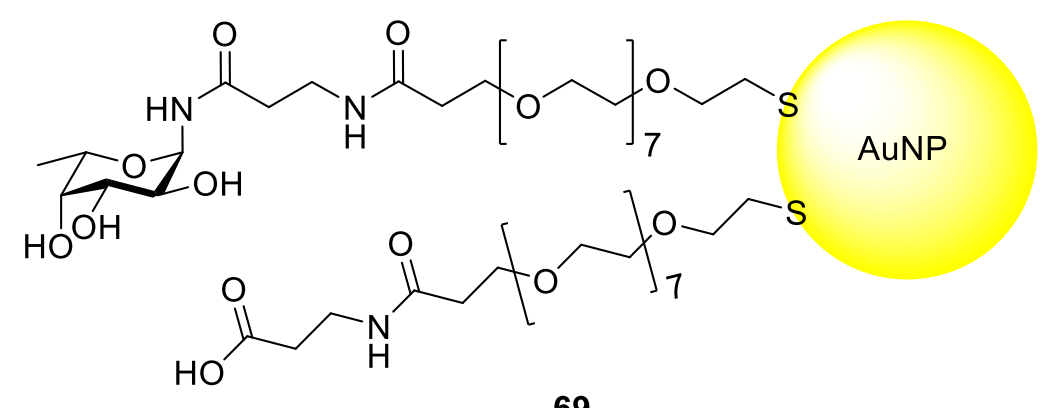

69

Figure 10. Fucosylated gold nanoparticle 77 for targeting of DC-SIGN.

Besides Lewis antigens, high mannose glycodendrons were synthesised to target DC-SIGN on DCs. Wong and co-workers synthesised $1 \mathrm{G}-3 \mathrm{G}$ glycodendrons bearing the $\mathrm{Man}_{9} \mathrm{GlcNAc}_{2}$ high mannose glycan antigen. ${ }^{104}$ These glycodendrons were tested for DC-SIGN binding and it was observed that second generation glycodendrons were the most efficient for lectin binding. The third generation only showed a slight increase in activity, however, with lower activity per glycan antigen. Moreover, it was demonstrated that these glycodendrons are potential candidates for carbohydrate vaccines as well as antiviral agents related to HIV. 


\subsection{Thesis outline}

The overall objective of the research described in this dissertation is to develop new and efficient strategies for the synthesis of multivalent glycodendrons. To achieve this, a novel bi-functional oxyamine linker strategy that allows for the conjugation of glycans to other complex biomolecules will first be discussed in Chapter 2. To enhance the binding affinity of these glycoconjugates, biotinylated and fluorescently labelled glycodendrons were then synthesised, as described in Chapter 3, while Chapter 4 concerns the synthesis of a representative carbohydrate antigen, Lewis ${ }^{\mathrm{X}}$. Finally, the combination of the bi-functional oxyamine linkers, the multivalent dendrons and the glycan antigen allows for the target goal of assembling a complex glycoconjugate, which is the focus of Chapter 5. To demonstrate a potential biological application of this methodology, the fluorescent Lewis ${ }^{\mathrm{x}}$ glycodendron is evaluated as a flow cytometry marker for the C-type lectin DC-SIGN on human DCs and macrophages. A brief overview of the content of each chapter is provided below.

\subsubsection{Proposed strategy for the rapid assembly of glycoconjugates through the use of bi-functional oxyamine linkers}

The use of oxyamine glycoconjugation strategies has made a significant impact in the field of carbohydrate chemistry, as it allows for the conjugation of both naturally sourced and synthetic reducing-end carbohydrate antigens without the use of protecting groups. In particular, bi-functional linkers have been used to conjugate complex glycans to microarray slides, to test for their lectin binding ability. Accordingly, it is proposed to synthesise various glycoconjugates through the use of oxyamine bi-functional linkers (Scheme 13), which allow for the conjugation of glycan antigens to substrates of choice using two orthogonal reactions. As described in sections 1.2 and 1.3 above, the use of "Type A" bi-functional linkers has been explored by various groups, however, these strategies often involve the use of long and/or low-yielding synthetic routes. Herein, it was proposed that the "Type B" oxyamines could be more readily synthesised via reductive amination methodology and this work forms the focus of Chapter 2.

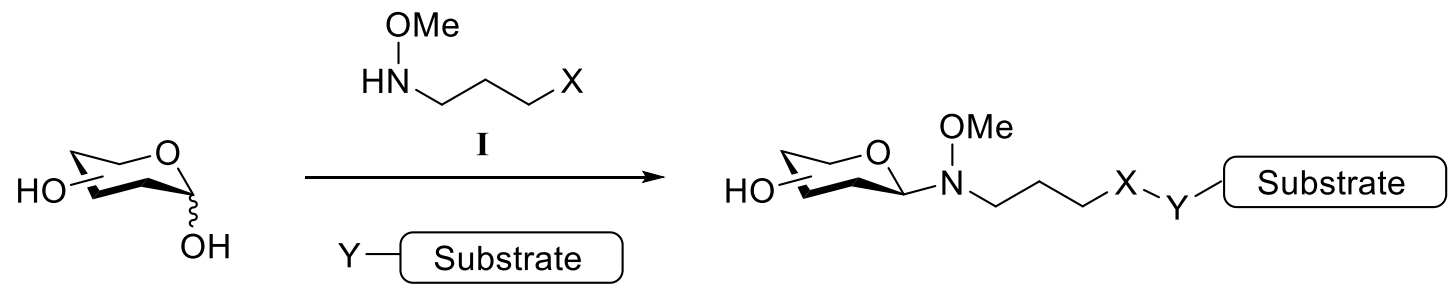

Scheme 13. Rapid assembly of glycoconjugates through the use of bi-functional linker I. 
Retrosynthetically, it was proposed that the bi-functional linker I could be obtained via the reduction of the corresponding methoxyimine $\mathbf{I I}$, which in turn can be obtained from the reaction of aldehyde III with methoxyamine (Scheme 14). The second functional group that gives rise to the bi-functionality of the oxyamine linker can be introduced via a Michael addition of various nucleophiles with acrolein IV. For example, sodium azide can be used to synthesise the azide functionalised bi-functional linker, whereas thiols can be used to synthesise thiol-derived linkers. This strategy allows for the synthesis of various bi-functional linkers which all contain the oxyamine moiety for glycan conjugation, but which also include a second functional group for the required chemical ligation.

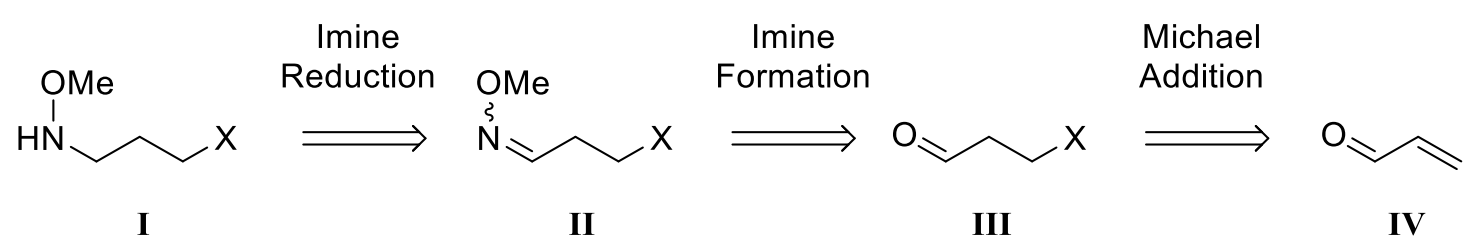

$X=N_{3}, S H, S R$

Scheme 14. Retrosynthesis of bi-functional linkers.

\subsubsection{Proposed synthesis of novel glycodendrons}

Glycosylated dendritic structures, such as glycodendrons, have been found to effectively bind cells with increased binding affinity for their corresponding lectin. Moreover, when compared to polymers and liposomes, dendritic structures can be prepared with high synthetic control, thereby allowing for more controlled biological studies to be undertaken. Accordingly, the synthesis of a multivalent second generation glycodendron was proposed in order to enhance glycan antigen activity (Scheme 15). The glycodendron $\mathbf{V}$ would contain various functionalities, including biotin and fluorescent groups, which would allow for the biological evaluation of these glycodendrons using various analytical techniques such as microscopy, ELISA, and flow cytometry. Thus, the synthesis of the target glycodendron $\mathbf{V}$ could be achieved using a highly convergent second generation dendron scaffold VI. Here, the functionalisation of dendron scaffold VI with the molecular probe VII, followed by glycosylation with oxyamine functionalised carbohydrate VIII gives the target glycoconjugate. This approach allows for the rapid modification of the dendron according to the required functionality. Specifically, in this thesis an oxyamine-functionalised Lewis $^{\mathrm{X}}$ glycan will be conjugated to a fluorescently labelled $2 \mathrm{G}$ dendron as a proof of concept. 

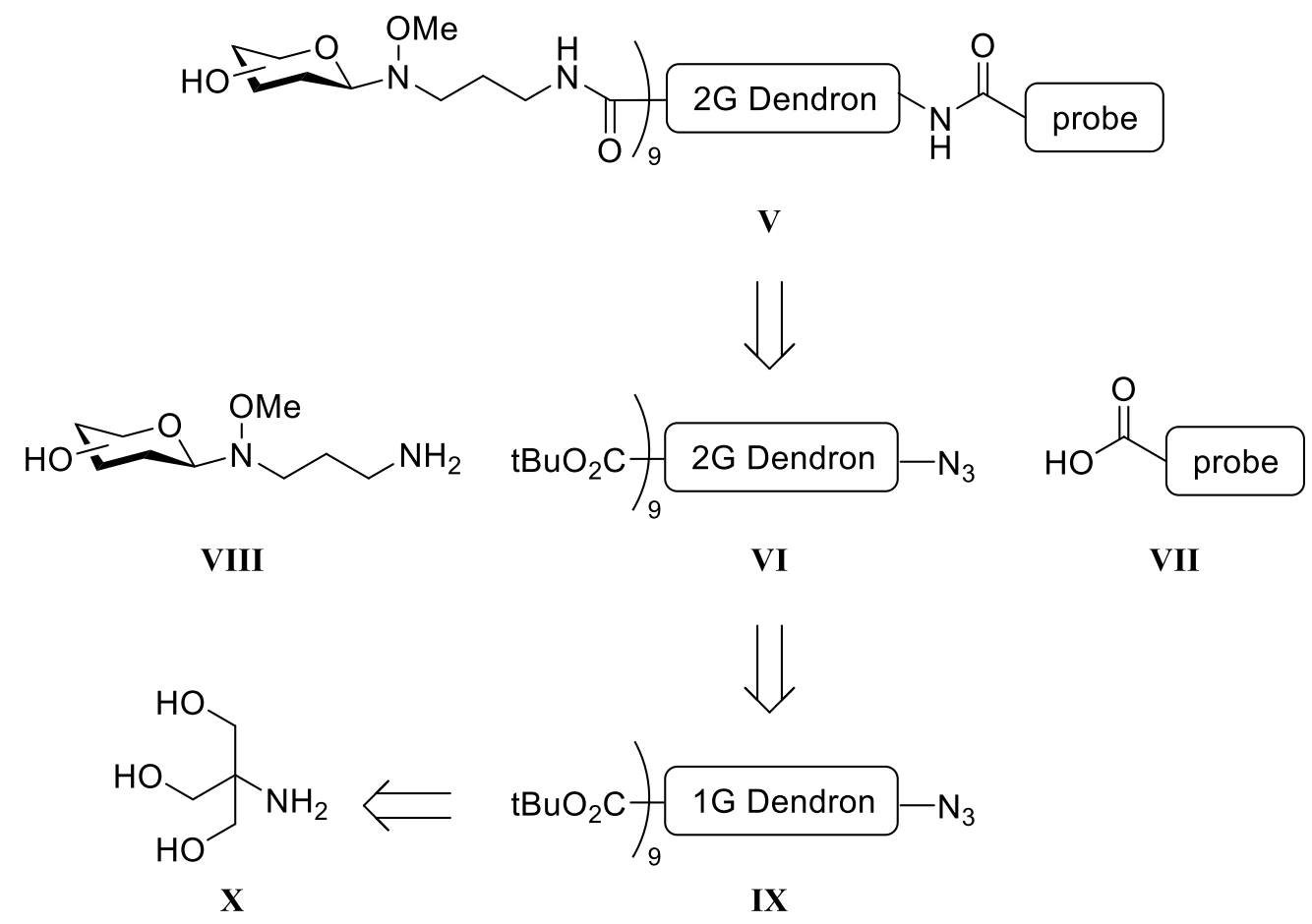

Scheme 15. Retrosynthesis of TRIS-based second generation glycodendrons.

There are several methodologies for dendron synthesis, including divergent, convergent and double exponential growth synthesis, of which the latter is the most efficient methodology. It was envisioned to use the double exponential growth synthetic route towards the highly convergent synthesis of the bi-functional second generation dendron scaffold VI. Accordingly, the second generation dendron core VI could be synthesised from the first generation dendron IX in three steps. Here, the synthesis of the first generation dendron IX can be achieved using tris(hydroxymethyl)aminomethane (TRIS) $\mathbf{X}$ as a core moiety. A more comprehensive review on the use of TRIS in dendron synthesis will be provided in the introduction of Chapter 3.

\subsubsection{Synthesis of Lewis ${ }^{\mathrm{X}}$ antigens}

As described in section 1.5, Lewis antigens possess a variety of biological functions including selectin-mediated cell-adhesion process and Lectin-mediated immune activation against pathogens. Moreover, the over-expression of these Lewis antigens often is associated with various tumours and the targeting of highly glycosylated cells or the synthesis of these tumour-associated carbohydrate antigens for use in vaccines could allow for the development of better anti-cancer therapies. In addition, glycotherapies could be further developed whereby the glycan-lectin binding process is used to target specific cell types and to study lectin-mediated biological events. 
Given the breath of biological activities attributed to the Lewis antigens in general, it was proposed that an efficient and rapid synthesis of the Lewis ${ }^{\mathrm{x}}$ antigens was required via a route that would allow for the synthesis of other Type 2 Lewis antigens (e.g. Sialyl Lewis ${ }^{\mathrm{X}}$, and sulfated Lewis ${ }^{\mathrm{X}}$ derivatives). Accordingly, it was proposed that these Lewis antigens, such as Lewis ${ }^{\mathrm{X}} \mathbf{X I}$, could be prepared from an orthogonal protected "Type 2" Lewis antigen intermediate XII, which in turn could be synthesised from the corresponding monosaccharide building blocks XIII-XV via the regioselective glycosylation between galactose donor XIII and GlcNAc acceptor XIV followed by the $[2+1]$ glycosylation with donor XV (Scheme 16). In this way, the synthetic strategy is not only highly efficient but also convergent. A thorough analysis of the previously reported syntheses of Lewis ${ }^{\mathrm{X}}$ derivatives, as well as a novel synthesis of the Lewis ${ }^{\mathrm{x}}$ antigen are presented in Chapter 4.

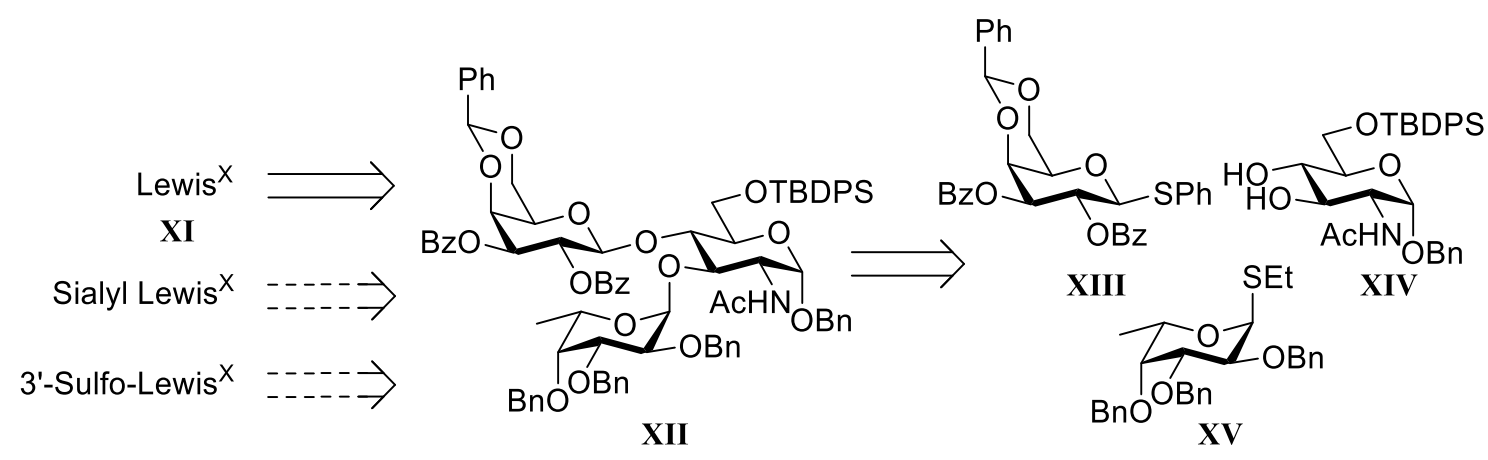

Scheme 16. Retrosynthesis of "Type 2" Lewis antigens through the use of an orthogonally protected trisaccharide scaffold XII.

\subsubsection{Biological evaluation of Lewis ${ }^{\mathrm{X}}$-glycodendrons}

The C-type lectin DC-SIGN is expressed on DCs and specific subsets of macrophages and binds to various Lewis antigens, including Lewis ${ }^{\mathrm{X}}$. The interaction of DC-SIGN with its ligands results in lysosomal uptake and degradation, followed by antigen presentation by the MHC-II complex to $\mathrm{CD}^{+}{ }^{+} \mathrm{T}$-cells, which leads to immune activation. Here, it is important to note that the simultaneous multivalent binding of these ligands to the receptor is required to induce a biological response, and accordingly various multivalent scaffolds have been developed to target lectins efficiently. Chapter 5 concerns the combination of the bi-functional oxyamine linker strategy with the multivalent dendrons and the Lewis ${ }^{\mathrm{x}}$ glycan antigen to prepare fluorescent glycodendrons XVI (Scheme 17). Here, the glycodendron will be prepared via the conjugation of Lewis ${ }^{\mathrm{X}} \mathbf{X I}$ to the bi-functional linker I, followed by the conjugation with the fluorescent dendron scaffold XVII. The glycodendron will then be used for the detection of DC-SIGN on human DCs as well as 'M1-like' and 'M2-like' macrophages via flow cytometry. The targeting of DC-SIGN by this glycodendron will provide proof-of-concept that the general targeting strategy works, and it is 
envisioned that future work could include the specific delivery of antigenic peptides to these APCs. For example, peptide antigen functionalised dendrons could be used to selectively deliver peptide antigens to ${\mathrm{DC}-\mathrm{SIGN}^{+}}^{+}$cells, which could potentially activate the immune system, mediated by MHC-II antigen presentation to $\mathrm{CD}^{+}{ }^{+} \mathrm{T}$-cells.
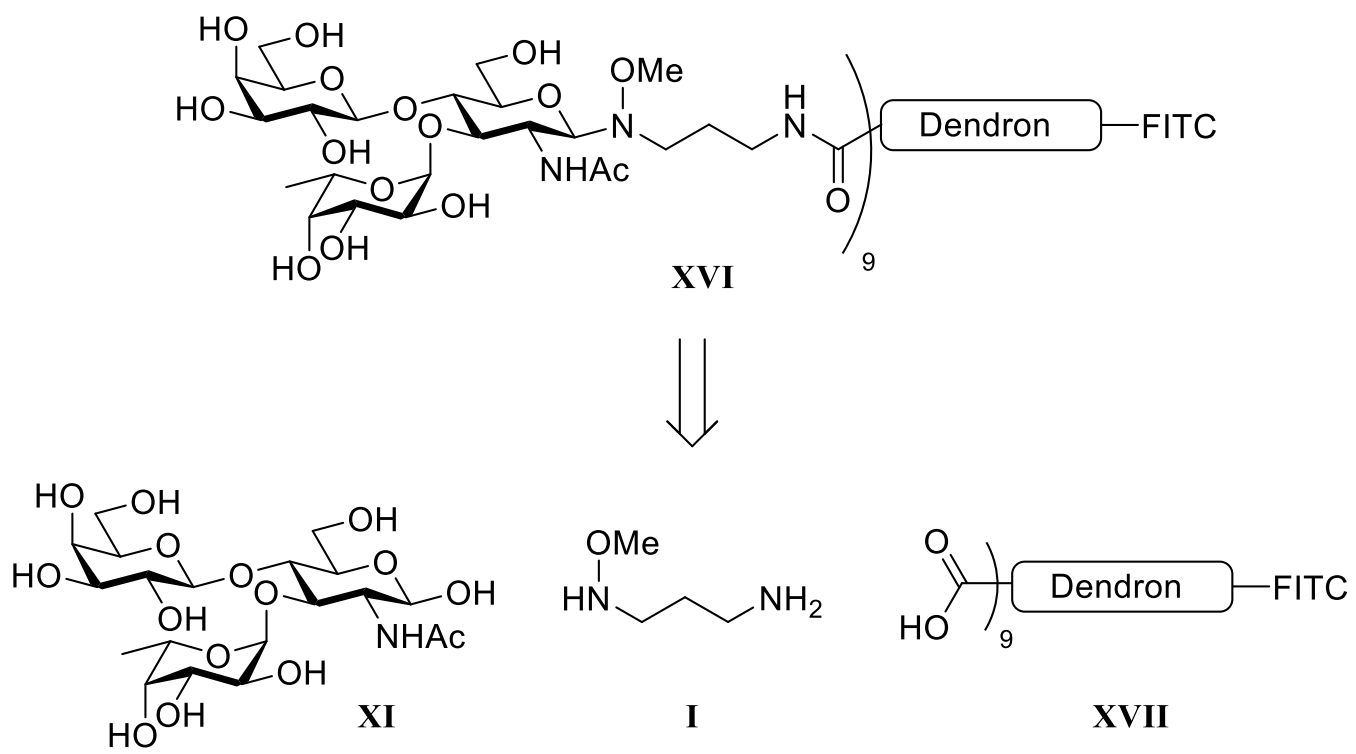

Scheme 17. Retrosynthesis of fluorescent multivalent Lewis ${ }^{X}$ glycodendron $\mathbf{X V}$. 


\subsection{References}

(1) Hudak, J. E.; Bertozzi, C. R. 'Glycotherapy: New Advances Inspire a Reemergence of Glycans in Medicine'. Chem. Biol., 2014, 21, 16-37.

(2) Weinbaum, S.; Tarbell, J. M.; Damiano, E. R. 'The Structure and Function of the Endothelial Glycocalyx Layer.' Annu. Rev. Biomed. Eng. 2007, 9, 121-167.

(3) Cummings, R. D. 'The Repertoire of Glycan Determinants in the Human Glycome.' Mol. Biosyst. 2009, 5, 1087-1104.

(4) Seeberger, P. H.; Werz, D. B. 'Synthesis and Medical Applications of Oligosaccharides.' Nature 2007, 446, 1046-1051.

(5) Morelli, L.; Poletti, L.; Lay, L. 'Carbohydrates and Immunology: Synthetic Oligosaccharide Antigens for Vaccine Formulation' Eur. J. Org. Chem. 2011, 2011, 5723 5777.

(6) Ernst, B.; Magnani, J. L. 'From Carbohydrate Leads to Glycomimetic Drugs.' Nat. Rev. Drug Discov. 2009, 8, 661-677.

(7) Oyelaran, O.; Gildersleeve, J. C. 'Glycan Arrays: Recent Advances and Future Challenges.' Curr. Opin. Chem. Biol. 2009, 13, 406-413.

(8) Liang, P.-H.; Wu, C.-Y.; Greenberg, W. A.; Wong, C.-H. 'Glycan Arrays: Biological and Medical Applications'. Curr. Opin. Chem. Biol., 2008, 12, 86-92.

(9) Freeze, H. H. 'Understanding Human Glycosylation Disorders: Biochemistry Leads the Charge.' J. Biol. Chem. 2013, 288, 6936-6945.

(10) Van Kooyk, Y.; Geijtenbeek, T. B. H. 'DC-SIGN: Escape Mechanism for Pathogens.' Nat. Rev. Immunol. 2003, 3, 697-709.

(11) Coleman, C. M.; St Gelais, C.; Wu, L. 'Adv. Exp. Med. Biol.'; Wu, L.; Schwartz, O., Eds.; Advances in Experimental Medicine and Biology; Springer: New York, 2013; Vol. 762.

(12) Mariño, K.; Bones, J.; Kattla, J. J.; Rudd, P. M. 'A Systematic Approach to Protein Glycosylation Analysis: A Path through the Maze.' Nat. Chem. Biol. 2010, 6, 713-723.

(13) Parker, R. B.; Kohler, J. J. 'Regulation of Intracellular Signaling by Extracellular Glycan Remodeling'. ACS Chem. Biol., 2010, 5, 35-46.

(14) Yarema, K. J.; Bertozzi, C. R. 'Characterizing Glycosylation Pathways.' Genome Biol. 2001, 2, 1-10.

(15) Wolfert, M. A.; Boons, G.-J. 'Adaptive Immune Activation: Glycosylation Does Matter.' Nat. Chem. Biol. 2013, 9, 776-784.

(16) Cantagrel, V.; Lefeber, D. J. 'From Glycosylation Disorders to Dolichol Biosynthesis Defects: A New Class of Metabolic Diseases.' J. Inherit. Metab. Dis. 2011, 34, 859-867.

(17) Brockhausen, I.; Schachter, H.; Stanley, P. O-GalNAc Glycans. In Essentials of Glycobiology; Varki, A.; Cummings, R. D.; Esko, J. D.; Freeze, H. H.; Stanley, P.; Bertozzi, C. R.; Hart, G. W.; Etzler, M. E., Eds.; Cold Spring Harbor : Cold Spring Harbor Laboratory Press., 2009; pp. 115-127.

(18) Vigerust, D. J.; Shepherd, V. L. 'Virus Glycosylation: Role in Virulence and Immune Interactions'. Trends Microbiol., 2007, 15, 211-218.

(19) Geijtenbeek, T. B.; Kwon, D. S.; Torensma, R.; van Vliet, S. J.; van Duijnhoven, G. C.; Middel, J.; Cornelissen, I. L.; Nottet, H. S.; KewalRamani, V. N.; Littman, D. R.; Figdor, C. G.; van Kooyk, Y. 'DC-SIGN, a Dendritic Cell-Specific HIV-1-Binding Protein That Enhances Trans-Infection of T Cells.' Cell 2000, 100, 587-597.

(20) Fischer, E. 'Ueber Die Glucoside Der Alkohole' Ber. Dtsch. Chem. Ges. 1893, 26, 24002412.

(21) Davis, B. G. 'Recent Developments in Glycoconjugates' J. Chem. Soc. Perk. T. 1 1999, 3215.

(22) Wolfenden, R. 'Conformational Aspects of Inhibitor Design: Enzyme-Substrate Interactions in the Transition State.' Bioorg. Med. Chem. 1999, 7, 647-652.

(23) Schramm, V. L. 'Transition States, Analogues, and Drug Development'. ACS Chem. Biol., 2013, 8, 71-81.

(24) Peri, F.; Cipolla, L.; La Ferla, B.; Nicotra, F. 'Glycoconjugate and Oligosaccharide Mimetics by Chemoselective Ligation' C.R. Chim. 2003, 6, 635-644. 
(25) Boltje, T. J.; Buskas, T.; Boons, G.-J. 'Opportunities and Challenges in Synthetic Oligosaccharide and Glycoconjugate Research.' Nat. Chem. 2009, 1, 611-622.

(26) Specker, D.; Wittmann, V. Synthesis and Application of Glycopeptide and Glycoprotein Mimetics. In Glycopeptides and Glycoproteins; Springer Berlin Heidelberg: Berlin, Heidelberg, 2007; pp. 65-107.

(27) Horlacher, T.; Seeberger, P. H. 'Carbohydrate Arrays as Tools for Research and Diagnostics.' Chem. Soc. Rev. 2008, 37, 1414-1422.

(28) Laurent, N.; Voglmeir, J.; Flitsch, S. L. 'Glycoarrays--Tools for Determining ProteinCarbohydrate Interactions and Glycoenzyme Specificity.' Chem. Comm. 2008, 44004412.

(29) Timmer, M. S. M.; Stocker, B. L.; Seeberger, P. H. 'Probing Glycomics.' Curr. Opin. Chem. Biol. 2007, 11, 59-65.

(30) Stocker, B. L.; Timmer, M. S. M. 'Chemical Tools for Studying the Biological Function of Glycolipids.' Chembiochem 2013, 14, 1164-1184.

(31) Likhosherstov, L. M.; Novikova, O. S.; Derevitskaja, V. A.; Kochetkov, N. K. 'A New Simple Synthesis of Amino Sugar B-D-Glycosylamines'. Carbohydr. Res., 1986, 146, C1C5.

(32) Song, X.; Xia, B.; Stowell, S. R.; Lasanajak, Y.; Smith, D. F.; Cummings, R. D. 'Novel Fluorescent Glycan Microarray Strategy Reveals Ligands for Galectins.' Chem. Biol. 2009, 16, 36-47.

(33) Jencks, W. P. 'Studies on the Mechanism of Oxime and Semicarbazone Formation 1' J. Am. Chem. Soc. 1959, 81, 475-481.

(34) Sayer, J. M.; Peskin, M.; Jencks, W. P. 'Imine-Forming Elimination Reactions. I. General Base Acid Catalysis and Influence of the Nitrogen Substituent on Rates and Equilibria for Carbinolamine Dehydration' J. Am. Chem. Soc. 1973, 95, 4277-4287.

(35) Helferich, B.; Schirp, H. 'Über N-Acyl-Hydrazone Einfacher Zucker, II. Mitteilung' Chem. Ber. 1953, 86, 547-556.

(36) Goff, R. D.; Thorson, J. S. 'Neoglycosylation and Neoglycorandomization: Enabling Tools for the Discovery of Novel Glycosylated Bioactive Probes and Early Stage Leads.' Medchemcomm 2014, 5, 1036-1047.

(37) Walczak, M. A.; Hayashida, J.; Danishefsky, S. J. 'Building Biologics by Chemical Synthesis: Practical Preparation of Di- and Triantennary N-Linked Glycoconjugates' $J$. Am. Chem. Soc. 2013, 135, 4700-4703.

(38) Urge, L.; Otvos, L.; Lang, E.; Wroblewski, K.; Laczko, I.; Hollosi, M. 'Fmoc-Protected, Glycosylated Asparagines Potentially Useful as Reagents in the Solid-Phase Synthesis of N-Glycopeptides' Carbohydr. Res. 1992, 235, 83-93.

(39) Bernardes, G. J. L.; Gamblin, D. P.; Davis, B. G. 'The Direct Formation of Glycosyl Thiols from Reducing Sugars Allows One-Pot Protein Glycoconjugation' Angew. Chem. Int. Ed. Engl. 2006, 45, 4007-4011.

(40) Garcia-Vallejo, J. J.; Koning, N.; Ambrosini, M.; Kalay, H.; Vuist, I.; Sarrami-Forooshani, R.; Geijtenbeek, T. B. H.; van Kooyk, Y. 'Glycodendrimers Prevent HIV Transmission via DC-SIGN on Dendritic Cells.' Int. Immunol. 2013, 25, 221-233.

(41) Gildersleeve, J. C.; Oyelaran, O.; Simpson, J. T.; Allred, B. 'Improved Procedure for Direct Coupling of Carbohydrates to Proteins via Reductive Amination' Bioconjug. Chem. 2008, 19, 1485-1490.

(42) Wolff, H. 'Ueber Dextrosebenzhydrazid' Ber. Dtsch. Chem. Ges. 1895, 28, 160-163.

(43) Kwase, Y. A.; Cochran, M.; Nitz, M. Protecting-Group-Free Glycoconjugate Synthesis : Hydrazide and Oxyamine Derivatives in N -Glycoside Formation. In Modern Synthetic Methods in Carbohydrate Chemistry: From Monosaccharides to Complex Glycoconjugates; Werz, D. B.; Vidal., S., Eds.; Wiley-VCH Verlag GmbH \& Co. KGaA, 2014; pp. 3-5.

(44) Niikura, K.; Kamitani, R.; Kurogochi, M.; Uematsu, R.; Shinohara, Y.; Nakagawa, H.; Deguchi, K.; Monde, K.; Kondo, H.; Nishimura, S. I. 'Versatile Glycoblotting Nanoparticles for High-Throughput Protein Glycomics' Chem. Eur. J. 2005, 11, 38253834.

(45) Furukawa, J. I.; Shinohara, Y.; Kuramoto, H.; Miura, Y.; Shimaoka, H.; Kurogochi, M.; Nakano, M.; Nishimura, S. I. 'Comprehensive Approach to Structural and Functional 
Glycomics Based on Chemoselective Glycoblotting and Sequential Tag Conversion' Anal. Chem. 2008, 80, 1094-1101.

(46) Kariya, Y.; Herrmann, J.; Suzuki, K.; Isomura, T.; Ishihara, M. 'Disaccharide Analysis of Heparin and Heparan Sulfate Using Deaminative Cleavage with Nitrous Acid and Subsequent Labeling with Paranitrophenyl Hydrazine.' J. Biochem. 1998, 123, 240-246.

(47) Godula, K.; Bertozzi, C. R. 'Synthesis of Glycopolymers for Microarray Applications via Ligation of Reducing Sugars to a Poly(acryloyl Hydrazide) Scaffold' J. Am. Chem. Soc. 2010, 132, 9963-9965.

(48) Goff, R. D.; Thorson, J. S. 'Assessment of Chemoselective Neoglycosylation Methods Using Chlorambucil as a Model.' J. Med. Chem. 2010, 53, 8129-8139.

(49) Peluso, S.; Imperiali, B. 'Asparagine Surrogates for the Assembly of N-Linked Glycopeptide Mimetics by Chemoselective Ligation' Tetrahedron Lett. 2001, 42, 20852087.

(50) Ng, P.-S.; Laing, B. M.; Balasundarum, G.; Pingle, M.; Friedman, A.; Bergstrom, D. E. 'Synthesis and Evaluation of New Spacers for Use as dsDNA End-Caps.' Bioconjug. Chem. 2010, 21, 1545-1553.

(51) Leteux, C.; Childs, R. a.; Chai, W.; Stoll, M. S.; Kogelberg, H.; Feizi, T. 'Biotinyl-L-3-(2Naphthyl)-Alanine Hydrazide Derivatives of N-Glyeans: Versatile Solid-Phase Probes for Carbohydrate-Recognition Studies' Glycobiology 1998, 8, 227-236.

(52) Plenkiewicz, J.; Szarek, W. A.; SIPOS, P. A.; PHIBBS, M. K. 'O -Benzyl Oximes of Carbohydrates' Synthesis (Stuttg). 1974, 1974, 56-58.

(53) Pöhner, C.; Ullmann, V.; Hilpert, R.; Samain, E.; Unverzagt, C. 'Chemoselective Coupling of Sugar Oximes and A-Ketoacids to Glycosyl Amides and N-Glycopeptides' Tetrahedron Lett. 2014, 55, 2197-2200.

(54) Peri, F.; Dumy, P.; Mutter, M. 'Chemo- and Stereoselective Glycosylation of Hydroxylamino Derivatives: A Versatile Approach to Glycoconjugates' Tetrahedron 1998, 54, 12269-12278.

(55) Langenhan, J. M.; Endo, M. M.; Engle, J. M.; Fukumoto, L. L.; Rogalsky, D. R.; Slevin, L. K.; Fay, L. R.; Lucker, R. W.; Rohlfing, J. R.; Smith, K. R.; Tjaden, A. E.; Werner, H. M. 'Synthesis and Biological Evaluation of RON-Neoglycosides as Tumor Cytotoxins' Carbohydr. Res. 2011, 346, 2663-2676.

(56) Cló, E.; Blixt, O.; Jensen, K. J. 'Chemoselective Reagents for Covalent Capture and Display of Glycans in Microarrays' Eur. J. Org. Chem. 2010, 540-554.

(57) Carrasco, M. R.; Brown, R. T. 'A Versatile Set of Aminooxy Amino Acids for the Synthesis of Neoglycopeptides.' J. Org. Chem. 2003, 68, 8853-8858.

(58) Carrasco, M. R.; Nguyen, M. J.; Burnell, D. R.; MacLaren, M. D.; Hengel, S. M. 'Synthesis of Neoglycopeptides by Chemoselective Reaction of Carbohydrates with Peptides Containing a Novel N'-Methyl-Aminooxy Amino Acid' Tetrahedron Lett. 2002, 43, 57275729.

(59) Filira, F.; Biondi, B.; Biondi, L.; Giannini, E.; Gobbo, M.; Negri, L.; Rocchi, R. 'Opioid Peptides: Synthesis and Biological Properties of [(N-Gamma-glucosyl,N-GammaMethoxy)-Alpha, Gamma-Diamino-(S)-butanoyl]4-Deltorphin-1-Neoglycopeptide and Related Analogues.' Org. Biomol. Chem. 2003, 1, 3059-3063.

(60) Peri, F.; Deutman, A.; La Ferla, B.; Nicotra, F. 'Solution and Solid-Phase Chemoselective Synthesis of (1-6)-Amino(methoxy) Di- and Trisaccharide Analogues.' Chem. Comm. 2002, 1504-1505.

(61) Peri, F.; Jiménez-Barbero, J.; García-Aparicio, V.; Tvaroska, I.; Nicotra, F. 'Synthesis and Conformational Analysis of Novel N(OCH3)-Linked Disaccharide Analogues.' Chemistry 2004, 10, 1433-1444.

(62) Ishida, J.; Hinou, H.; Naruchi, K.; Nishimura, S.-I. 'Synthesis of Neoglycosphingolipid from Methoxyamino-Functionalized Ceramide.' Bioorg. Med. Chem. Lett. 2014, 24, $1197-1200$.

(63) Langenhan, J. M.; Griffith, B. R.; Thorson, J. S. 'Neoglycorandomization and Chemoenzymatic Glycorandomization: Two Complementary Tools for Natural Product Diversification.' J. Nat. Prod. 2005, 68, 1696-1711. 
(64) Langenhan, J. M.; Peters, N. R.; Guzei, I. a; Hoffmann, F. M.; Thorson, J. S. 'Enhancing the Anticancer Properties of Cardiac Glycosides by Neoglycorandomization.' Proc. Natl. Acad. Sci. U. S. A. 2005, 102, 12305-12310.

(65) Ahmed, A.; Peters, N. R.; Fitzgerald, M. K.; Watson, J. a; Hoffmann, F. M.; Thorson, J. S. 'Colchicine Glycorandomization Influences Cytotoxicity and Mechanism of Action.' $J$. Am. Chem. Soc. 2006, 128, 14224-14225.

(66) Griffith, B. R.; Krepel, C.; Fu, X.; Blanchard, S.; Ahmed, A.; Edmiston, C. E.; Thorson, J. S. 'Model for Antibiotic Optimization via Neoglycosylation: Synthesis of Liponeoglycopeptides Active against VRE.' J. Am. Chem. Soc. 2007, 129, 8150-8155.

(67) Goff, R. R. D.; Thorson, J. S. J. 'Enhancing the Divergent Activities of Betulinic Acid via Neoglycosylation' Org. Lett. 2009, 11, 461-464.

(68) Peltier-Pain, P.; Timmons, S. C.; Grandemange, A.; Benoit, E.; Thorson, J. S. 'Warfarin Glycosylation Invokes a Switch from Anticoagulant to Anticancer Activity.' ChemMedChem 2011, 6, 1347-1350.

(69) Goff, R. D.; Thorson, J. S. 'Enhancement of Cyclopamine via Conjugation with Nonmetabolic Sugars.' Org. Lett. 2012, 14, 2454-2457.

(70) Langenhan, J. M.; Mullarky, E.; Rogalsky, D. K.; Rohlfing, J. R.; Tjaden, A. E.; Werner, H. M.; Rozal, L. M.; Loskot, S. a. 'Amphimedosides A-C: Synthesis, Chemoselective Glycosylation, and Biological Evaluation.' J. Org. Chem. 2013, 78, 1670-1676.

(71) Nandurkar, N. S.; Zhang, J.; Ye, Q.; Ponomareva, L. V; She, Q.; Thorson, J. S. 'The Identification of Perillyl Alcohol Glycosides with Improved Antiproliferative Activity' $J$. Med. Chem. 2014, 57, 7478-7484.

(72) Bohorov, O.; Andersson-Sand, H.; Hoffmann, J.; Blixt, O. 'Arraying Glycomics: A Novel Bi-Functional Spacer for One-Step Microscale Derivatization of Free Reducing Glycans.' Glycobiology 2006, 16, 21C - 27C.

(73) Wang, Z.; Chinoy, Z. S.; Ambre, S. G.; Peng, W.; McBride, R.; de Vries, R. P.; Glushka, J.; Paulson, J. C.; Boons, G.-J. 'A General Strategy for the Chemoenzymatic Synthesis of Asymmetrically Branched N-Glycans.' Science 2013, 341, 379-383.

(74) Prudden, A. R.; Chinoy, Z. S.; Wolfert, M. A.; Boons, G.-J. 'A Multifunctional Anomeric Linker for the Chemoenzymatic Synthesis of Complex Oligosaccharides.' Chem. Comm. 2014, 50, 7132-7135.

(75) Leung, C.; Chibba, A.; Gómez-Biagi, R. F.; Nitz, M. 'Efficient Synthesis and Protein Conjugation of Beta-(1-->6)-D-N-Acetylglucosamine Oligosaccharides from the Polysaccharide Intercellular Adhesin.' Carbohydr. Res. 2009, 344, 570-575.

(76) El-Boubbou, K.; Zhu, D. C.; Vasileiou, C.; Borhan, B.; Prosperi, D.; Li, W.; Huang, X. 'Magnetic Glyco-Nanoparticles: A Tool to Detect, Differentiate, and Unlock the GlycoCodes of Cancer via Magnetic Resonance Imaging.' J. Am. Chem. Soc. 2010, 132, 44904499.

(77) Mammen, M.; Choi, S.-K.; Whitesides, G. M. 'Polyvalent Interactions in Biological Systems: Implications for Design and Use of Multivalent Ligands and Inhibitors' Angew. Chem. Int. Ed. Engl. 1998, 37, 2754-2794.

(78) Pieters, R. J. 'Maximising Multivalency Effects in Protein-Carbohydrate Interactions.' Org. Biomol. Chem. 2009, 7, 2013-2025.

(79) Lundquist, J. J.; Debenham, S. D.; Toone, E. J. 'Multivalency Effects in ProteinCarbohydrate Interaction: The Binding of the Shiga-like Toxin 1 Binding Subunit to Multivalent C-Linked Glycopeptides' J. Org. Chem. 2000, 65, 8245-8250.

(80) Gambaryan, A. S.; Tuzikov, A. B.; Pazynina, G. V; Webster, R. G.; Matrosovich, M. N.; Bovin, N. V. 'H5N1 Chicken Influenza Viruses Display a High Binding Affinity for Neu5Aca2-3Galb1-4(6- $\left.\mathrm{HSO}_{3}\right)$ GlcNAc-Containing Receptors' Virology 2004, 326, 310316.

(81) Bovin, N. V; Korchagina EYu; Zemlyanukhina, T. V; Byramova, N. E.; Galanina, O. E.; Zemlyakov, a E.; Ivanov, a E.; Zubov, V. P.; Mochalova, L. V. 'Synthesis of Polymeric Neoglycoconjugates Based on N-Substituted Polyacrylamides.' Glycoconj. J. 1993, 10, $142-151$.

(82) Chen, W. C.; Completo, G. C.; Sigal, D. S.; Crocker, P. R.; Saven, A.; Paulson, J. C. 'In Vivo Targeting of B-Cell Lymphoma with Glycan Ligands of CD22.' Blood 2010, 115, 4778-4786. 
(83) Walter, M. V; Malkoch, M. 'Simplifying the Synthesis of Dendrimers: Accelerated Approaches'. Chem. Soc. Rev., 2012, 41, 4593.

(84) Astruc, D.; Boisselier, E.; Ornelas, C. 'Dendrimers Designed for Functions: From Physical, Photophysical, and Supramolecular Properties to Applications in Sensing, Catalysis, Molecular Electronics, Photonics, and Nanomedicine' Chem. Rev. 2010, 110, 1857-1959.

(85) Boas, U.; Heegaard, P. M. H. 'Dendrimers in Drug Research.' Chem. Soc. Rev. 2004, 33 , 43-63.

(86) Gajbhiye, V.; Palanirajan, V. K.; Tekade, R. K.; Jain, N. K. 'Dendrimers as Therapeutic Agents: A Systematic Review.' J. Pharm. Pharmacol. 2009, 61, 989-1003.

(87) Cloninger, M. J. 'Biological Applications of Dendrimers' Curr. Opin. Chem. Biol. 2002, $6,742-748$.

(88) Nanjwade, B. K.; Bechra, H. M.; Derkar, G. K.; Manvi, F. V; Nanjwade, V. K. 'Dendrimers: Emerging Polymers for Drug-Delivery Systems.' Eur. J. Pharm. Sci. 2009, 38, 185-196.

(89) Ribeiro-Viana, R.; García-Vallejo, J. J.; Collado, D.; Pérez-Inestrosa, E.; Bloem, K.; van Kooyk, Y.; Rojo, J. 'BODIPY-Labeled DC-SIGN-Targeting Glycodendrons Efficiently Internalize and Route to Lysosomes in Human Dendritic Cells.' Biomacromolecules 2012, 13, 3209-3219.

(90) Bernardes, G. J. L.; Kikkeri, R.; Maglinao, M.; Laurino, P.; Collot, M.; Hong, S. Y.; Lepenies, B.; Seeberger, P. H. 'Design, Synthesis and Biological Evaluation of Carbohydrate-Functionalized Cyclodextrins and Liposomes for Hepatocyte-Specific Targeting.' Org. Biomol. Chem. 2010, 8, 4987-4996.

(91) Ribeiro-Viana, R.; Sánchez-Navarro, M.; Luczkowiak, J.; Koeppe, J. R.; Delgado, R.; Rojo, J.; Davis, B. G. 'Virus-like Glycodendrinanoparticles Displaying Quasi-Equivalent Nested Polyvalency upon Glycoprotein Platforms Potently Block Viral Infection.' Nat. Commun. 2012, 3, 1303.

(92) Beahm, B. J.; Bertozzi, C. R. Imaging Cell-Surface Glycans in Animals with Bioorthogonal Chemistry. In Glycoscience: Biology and Medicine; 2014; pp. 1-11.

(93) Chang, P. V.; Bertozzi, C. R. 'Imaging beyond the Proteome'. Chem. Comm., 2012, 48, 8864.

(94) Ennen, F.; Boye, S.; Lederer, A.; Cernescu, M.; Komber, H.; Brutschy, B.; Voit, B.; Appelhans, D. 'Biohybrid Structures Consisting of Biotinylated Glycodendrimers and Proteins: Influence of the Biotin Ligand's Number and Chemical Nature on the Biotinavidin Conjugation' Polym. Chem. 2014, 5, 1323.

(95) Dundas, C. M.; Demonte, D.; Park, S. 'Streptavidin-Biotin Technology: Improvements and Innovations in Chemical and Biological Applications' Appl. Microbiol. Biotechnol. 2013, 97, 9343-9353.

(96) Stevens, E. J.; Peakman, M. 'Enhanced T Cell Proliferation and Increased Responder Frequency Following Delivery of Antigen to the Antigen-Presenting Cell; B Cell Dependency and Use in Detection of Autoreactive T Cells' J. Immunol. Methods 1998, 215, 59-70.

(97) Baek, M. G.; Roy, R. 'Simultaneous Binding of Mouse Monoclonal Antibody and Streptavidin to Heterobifunctional Dendritic L-Lysine Core Bearing T-Antigen Tumor Marker and Biotin' Bioorgan. Med. Chem. 2001, 9, 3005-3011.

(98) Kale, R. R.; Mukundan, H.; Price, D. N.; Harris, J. F.; Lewallen, D. M.; Swanson, B. I.; Schmidt, J. G.; Iyer, S. S. 'Detection of Intact Influenza Viruses Using Biotinylated Biantennary S-Sialosides' J. Am. Chem. Soc. 2008, 130, 8169-8171.

(99) Wilbur, D. S.; Park, S. I.; Chyan, M. K.; Wan, F.; Hamlin, D. K.; Shenoi, J.; Lin, Y.; Wilbur, S. M.; Buchegger, F.; Pantelias, A.; Pagel, J. M.; Press, O. W. 'Design and Synthesis of Bis-Biotin-Containing Reagents for Applications Utilizing Monoclonal Antibody-Based Pretargeting Systems with Streptavidin Mutants' Bioconjug. Chem. 2010, $21,1225-1238$.

(100) Yoo, B.; Cheal, S. M.; Torchon, G.; Dilhas, A.; Yang, G.; Pu, J.; Punzalan, B.; Larson, S. M.; Ouerfelli, O. 'N-Acetylgalactosamino Dendrons as Clearing Agents to Enhance Liver Targeting of Model Antibody-Fusion Protein.' Bioconjug. Chem. 2013, 24, 2088-2103. 
(101) Xu, H.; Regino, C. A. S.; Koyama, Y.; Hama, Y.; Gunn, A. J.; Bernardo, M.; Kobayashi, H.; Choyke, P. L.; Brechbiel, M. W. 'Preparation and Preliminary Evaluation of a BiotinTargeted, Lectin-Targeted Dendrimer-Based Probe for Dual-Modality Magnetic Resonance and Fluorescence Imaging' Bioconjug. Chem. 2007, 18, 1474-1482.

(102) Svenson, S. 'Dendrimers as Versatile Platform in Drug Delivery Applications'. Eur. J. Pharm. Biopharm., 2009, 71, 445-462.

(103) Jansen, J. F.; de Brabander-van den Berg, E. M.; Meijer, E. W. 'Encapsulation of Guest Molecules into a Dendritic Box.' Science 1994, 266, 1226-1229.

(104) Wang, S.-K.; Liang, P.-H.; Astronomo, R. D.; Hsu, T.-L.; Hsieh, S.-L.; Burton, D. R.; Wong, C.-H. 'Targeting the Carbohydrates on HIV-1: Interaction of Oligomannose Dendrons with Human Monoclonal Antibody 2G12 and DC-SIGN.' Proc. Natl. Acad. Sci. U. S. A. 2008, 105, 3690-3695.

(105) Jain, K.; Kesharwani, P.; Gupta, U.; Jain, N. K. 'Dendrimer Toxicity: Let's Meet the Challenge'. Int. J. Pharm., 2010, 394, 122-142.

(106) Roberts, J. C.; Bhalgat, M. K.; Zera, R. T. 'Preliminary Biological Evaluation of Polyamidoamine (PAMAM) Starburst Dendrimers.' J. Biomed. Mater. Res. 1996, 30, 5365.

(107) Duncan, R.; Izzo, L. 'Dendrimer Biocompatibility and Toxicity'. Adv. Drug. Deliver. Rev., 2005, 57, 2215-2237.

(108) Turnbull, W. B.; Stoddart, J. F. 'Design and Synthesis of Glycodendrimers.' Rev. Mol. Biotechnol. 2002, 90, 231-255.

(109) Gingras, M.; Chabre, Y. M.; Roy, M.; Roy, R. 'How Do Multivalent Glycodendrimers Benefit from Sulfur Chemistry?' Chem. Soc. Rev. 2013, 42, 4823-4841.

(110) Soejima, M.; Koda, Y. 'Molecular Mechanisms of Lewis Antigen Expression'. Legal Medicine, 2005, 7, 266-269.

(111) McCurley, R. S.; Recinos, A.; Olsen, a S.; Gingrich, J. C.; Szczepaniak, D.; Cameron, H. S.; Krauss, R.; Weston, B. W. 'Physical Maps of Human Alpha $(1,3)$ fucosyltransferase Genes FUT3-FUT6 on Chromosomes 19p13.3 and 11q21.' Genomics 1995, 26, 142-146.

(112) Pamela Stanley, R. D. C. Structures Common to Different Glycans. In Essentials of Glycobiology. 2nd edition.; 2009.

(113) Höglund, P.; Rosengren-Lindquist, R.; Wikman, A. T. 'A Severe Haemolytic Transfusion Reaction Caused by Anti-Lea Active at $37^{\circ} \mathrm{C}$ ' Blood Transfus. 2013, 11, 456-459.

(114) Zöller, M. 'CD44: Can a Cancer-Initiating Cell Profit from an Abundantly Expressed Molecule?' Nat. Rev. Cancer 2011, 11, 254-267.

(115) Ley, K. 'The Role of Selectins in Inflammation and Disease'. Trends Mol. Med., 2003, 9, 263-268.

(116) Carlow, D. A.; Gossens, K.; Naus, S.; Veerman, K. M.; Seo, W.; Ziltener, H. J. 'PSGL-1 Function in Immunity and Steady State Homeostasis'. Immunol. Rev., 2009, 230, 75-96.

(117) Gao, L.; Yan, L.; Lin, B.; Gao, J.; Liang, X.; Wang, Y.; Liu, J.; Zhang, S.; Iwamori, M. 'Enhancive Effects of Lewis Y Antigen on CD44-Mediated Adhesion and Spreading of Human Ovarian Cancer Cell Line RMG-I.' J. Exp. Clin. Cancer Res. 2011, 30, 15.

(118) Chung, T.-W.; Kim, S.-J.; Choi, H.-J.; Song, K.-H.; Jin, U.-H.; Yu, D.-Y.; Seong, J.-K.; Kim, J.-G.; Kim, K.-J.; Ko, J.-H.; Ha, K.-T.; Lee, Y.-C.; Kim, C.-H. 'Hepatitis B Virus X Protein Specially Regulates the Sialyl Lewis a Synthesis among Glycosylation Events for Metastasis' Mol. Cancer 2014, 13, 1-12.

(119) Remmers, N.; Anderson, J. M.; Linde, E. M.; DiMaio, D. J.; Lazenby, A. J.; Wandall, H. H.; Mandel, U.; Clausen, H.; Yu, F.; Hollingsworth, M. a. 'Aberrant Expression of Mucin Core Proteins and O-Linked Glycans Associated with Progression of Pancreatic Cancer' Clin. Cancer Res. 2013, 19, 1981-1993.

(120) Rho, J. H.; Mead, J. R.; Wright, W. S.; Brenner, D. E.; Stave, J. W.; Gildersleeve, J. C.; Lampe, P. D. 'Discovery of Sialyl Lewis A and Lewis X Modified Protein Cancer Biomarkers Using High Density Antibody Arrays' J. Proteomics 2014, 96, 291-299.

(121) Lin, W. M.; Karsten, U.; Goletz, S.; Cheng, R. C.; Cao, Y. 'Co-Expression of CD173 (H2) and CD174 (Lewis Y) with CD44 Suggests That Fucosylated Histo-Blood Group Antigens Are Markers of Breast Cancer-Initiating Cells' Virchows Arch. 2010, 456, 403-409.

(122) Liu, J.-J.; Lin, B.; Hao, Y.-Y.; Li, F.-F.; Liu, D.-W.; Qi, Y.; Zhu, L.-C.; Zhang, S.-L.; Iwamori, M. 'Lewis(y) Antigen Stimulates the Growth of Ovarian Cancer Cells via 
Regulation of the Epidermal Growth Factor Receptor Pathway.' Oncol. Rep. 2010, 23, 833-841.

(123) Brazil, J. C.; Liu, R.; Sumagin, R.; Kolegraff, K. N.; Nusrat, A.; Cummings, R. D.; Parkos, C. a; Louis, N. a. ' $\alpha 3 / 4$ Fucosyltransferase 3-Dependent Synthesis of Sialyl Lewis A on CD44 Variant Containing Exon 6 Mediates Polymorphonuclear Leukocyte Detachment from Intestinal Epithelium during Transepithelial Migration.' J. Immunol. 2013, 191, 4804-4817.

(124) Nordén, R.; Nyström, K.; Adamiak, B.; Halim, A.; Nilsson, J.; Larson, G.; Trybala, E.; Olofsson, S. 'Involvement of Viral Glycoprotein gC-1 in Expression of the Selectin Ligand Sialyl-Lewis X Induced after Infection with Herpes Simplex Virus Type 1' Apmis 2013, 121, 280-289.

(125) Pang, P.-C.; Chiu, P. C. N.; Lee, C.-L.; Chang, L.-Y.; Panico, M.; Morris, H. R.; Haslam, S. M.; Khoo, K.-H.; Clark, G. F.; Yeung, W. S. B.; Dell, A. 'Human Sperm Binding Is Mediated by the Sialyl-Lewis ${ }^{\mathrm{x}}$ Oligosaccharide on the Zona Pellucida' Science 2011, 333, 1761-1764.

(126) Pang, P. C.; Tissot, B.; Drobnis, E. Z.; Sutovsky, P.; Morris, H. R.; Clark, G. F.; Dell, A. 'Expression of Bisecting Type and Lewis ${ }^{\mathrm{x}} /$ Lewis $^{\mathrm{y}}$ Terminated N-Glycans on Human Sperm' J. Biol. Chem. 2007, 282, 36593-36602.

(127) Degroote, S.; Ducourouble, M. P.; Roussel, P.; Lamblin, G. 'Sequential Biosynthesis of Sulfated And/or Sialylated Lewis X Determinants by Transferases of the Human Bronchial Mucosa.' Glycobiology 1999, 9, 1199-1211.

(128) Zhang, C. Y.; Hu, P.; Fu, D.; Wu, W.; Jia, C. Y.; Zhu, X. C.; Wu, X. Z. '3'-Sulfo-Lex Is Important for Regulation of Integrin Subunit Av' Biochemistry 2010, 49, 7811-7820.

(129) Yuen, C. T.; Lawson, a M.; Chai, W.; Larkin, M.; Stoll, M. S.; Stuart, a C.; Sullivan, F. X.; Ahern, T. J.; Feizi, T. 'Novel Sulfated Ligands for the Cell Adhesion Molecule ESelectin Revealed by the Neoglycolipid Technology among O-Linked Oligosaccharides on an Ovarian Cystadenoma Glycoprotein.' Biochemistry 1992, 31, 9126-9131.

(130) Capon, C.; Wieruszeski, J. M.; Lemoine, J.; Byrd, J. C.; Leffler, H.; Kim, Y. S. 'Sulfated Lewis X Determinants as a Major Structural Motif in Glycans from LS174T-HM7 Human Colon Carcinoma Mucin' J. Biol. Chem. 1997, 272, 31957-31968.

(131) O'Reilly, M. K.; Paulson, J. C. 'Siglecs as Targets for Therapy in Immune-Cell-Mediated Disease.' Trends Pharmacol. Sci. 2009, 30, 240-248.

(132) Ohmori, K.; Kanda, K.; Mitsuoka, C.; Kanamori, A.; Kurata-Miura, K.; Sasaki, K.; Nishi, T.; Tamatani, T.; Kannagi, R. 'P- and E-Selectins Recognize Sialyl 6-Sulfo Lewis X, the Recently Identified L-Selectin Ligand.' Biochem. Biophys. Res. Commun. 2000, 278, 90 96.

(133) Paulson, J. C.; Macauley, M. S.; Kawasaki, N. 'Siglecs as Sensors of Self in Innate and Adaptive Immune Responses.' Ann. N. Y. Acad. Sci. 2012, 1253, 37-48.

(134) Macauley, M. S.; Crocker, P. R.; Paulson, J. C. 'Siglec-Mediated Regulation of Immune Cell Function in Disease' Nat. Rev. Immunol. 2014.

(135) Pillai, S.; Netravali, I. A.; Cariappa, A.; Mattoo, H. 'Siglecs and Immune Regulation'. Annu. Rev. Immunol., 2012, 30, 357-392.

(136) Van Kooyk, Y.; Ilarregui, J. M.; van Vliet, S. J. 'Novel Insights into the Immunomodulatory Role of the Dendritic Cell and Macrophage-Expressed C-Type Lectin MGL' Immunobiology 2015, 220, 185-192.

(137) Medzhitov, R. 'Recognition of Microorganisms and Activation of the Immune Response.' Nature 2007, 449, 819-826.

(138) Gordon, S. 'Pattern Recognition Receptors: Doubling up for the Innate Immune Response'. Cell, 2002, 111, 927-930.

(139) Krutzik, S. R.; Tan, B.; Li, H.; Ochoa, M. T.; Liu, P. T.; Sharfstein, S. E.; Graeber, T. G.; Sieling, P. a; Liu, Y.-J.; Rea, T. H.; Bloom, B. R.; Modlin, R. L. 'TLR Activation Triggers the Rapid Differentiation of Monocytes into Macrophages and Dendritic Cells.' Nat. Med. 2005, 11, 653-660.

(140) Lai, W. K.; Sun, P. J.; Zhang, J.; Jennings, A.; Lalor, P. F.; Hubscher, S.; McKeating, J. a; Adams, D. H. 'Expression of DC-SIGN and DC-SIGNR on Human Sinusoidal Endothelium: A Role for Capturing Hepatitis C Virus Particles.' Am. J. Pathol. 2006, 169, 200-208. 
(141) Soilleux, E. J.; Morris, L. S.; Leslie, G.; Chehimi, J.; Luo, Q.; Levroney, E.; Trowsdale, J.; Montaner, L. J.; Doms, R. W.; Weissman, D.; Coleman, N.; Lee, B. 'Constitutive and Induced Expression of DC-SIGN on Dendritic Cell and Macrophage Subpopulations in Situ and in Vitro.' J. Leukoc. Biol. 2002, 71, 445-457.

(142) Tailleux, L.; Pham-Thi, N.; Bergeron-Lafaurie, A.; Herrmann, J.-L.; Charles, P.; Schwartz, O.; Scheinmann, P.; Lagrange, P. H.; de Blic, J.; Tazi, A.; Gicquel, B.; Neyrolles, O. 'DC-SIGN Induction in Alveolar Macrophages Defines Privileged Target Host Cells for Mycobacteria in Patients with Tuberculosis.' PLoS Med. 2005, 2, e381.

(143) Geijtenbeek, T. B.; Torensma, R.; van Vliet, S. J.; van Duijnhoven, G. C.; Adema, G. J.; van Kooyk, Y.; Figdor, C. G. 'Identification of DC-SIGN, a Novel Dendritic Cell-Specific ICAM-3 Receptor That Supports Primary Immune Responses.' Cell 2000, 100, 575-585.

(144) Brown, M. B.; von Chamier, M.; Allam, A. B.; Reyes, L. 'M1/M2 Macrophage Polarity in Normal and Complicated Pregnancy' Front. Immunol. 2014, 5, 1-10.

(145) Appelmelk, B. J.; van Die, I.; van Vliet, S. J.; Vandenbroucke-Grauls, C. M. J. E.; Geijtenbeek, T. B. H.; van Kooyk, Y. 'Cutting Edge: Carbohydrate Profiling Identifies New Pathogens That Interact with Dendritic Cell-Specific ICAM-3-Grabbing Nonintegrin on Dendritic Cells' J. Immunol. 2003, 170, 1635-1639.

(146) Mitchell, D. A.; Fadden, A. J.; Drickamer, K. 'A Novel Mechanism of Carbohydrate Recognition by the C-Type Lectins DC-SIGN and DC-SIGNR. Subunit Organization and Binding to Multivalent Ligands' J. Biol. Chem. 2001, 276, 28939-28945.

(147) Granelli-Piperno, A.; Pritsker, A.; Pack, M.; Shimeliovich, I.; Arrighi, J.-F.; Park, C. G.; Trumpfheller, C.; Piguet, V.; Moran, T. M.; Steinman, R. M. 'Dendritic Cell-Specific Intercellular Adhesion Molecule 3-Grabbing Nonintegrin/CD209 Is Abundant on Macrophages in the Normal Human Lymph Node and Is Not Required for Dendritic Cell Stimulation of the Mixed Leukocyte Reaction' J. Immunol. 2005, 175, 4265-4273.

(148) Guo, Y.; Feinberg, H.; Conroy, E.; Mitchell, D. a; Alvarez, R.; Blixt, O.; Taylor, M. E.; Weis, W. I.; Drickamer, K. 'Structural Basis for Distinct Ligand-Binding and Targeting Properties of the Receptors DC-SIGN and DC-SIGNR.' Nat. Struct. Mol. Biol. 2004, 11, 591-598.

(149) Tacken, P. J.; Ter Huurne, M.; Torensma, R.; Figdor, C. G. 'Antibodies and Carbohydrate Ligands Binding to DC-SIGN Differentially Modulate Receptor Trafficking.' Eur. J. Immunol. 2012, 42, 1989-1998.

(150) García-Vallejo, J. J.; Ambrosini, M.; Overbeek, A.; van Riel, W. E.; Bloem, K.; Unger, W. W. J.; Chiodo, F.; Bolscher, J. G.; Nazmi, K.; Kalay, H.; van Kooyk, Y. 'Multivalent Glycopeptide Dendrimers for the Targeted Delivery of Antigens to Dendritic Cells' Mol. Immunol. 2013, 53, 387-397.

(151) Arosio, D.; Chiodo, F.; Reina, J. J.; Marelli, M.; Penadés, S.; van Kooyk, Y.; GarciaVallejo, J. J.; Bernardi, A. 'Effective Targeting of DC-SIGN by A-Fucosylamide Functionalized Gold Nanoparticles.' Bioconjug. Chem. 2014, 25, 2244-2251.

(152) Andreini, M.; Doknic, D.; Sutkeviciute, I.; Reina, J. J.; Duan, J.; Chabrol, E.; Thepaut, M.; Moroni, E.; Doro, F.; Belvisi, L.; Weiser, J.; Rojo, J.; Fieschi, F.; Bernardi, A. 'Second Generation of Fucose-Based DC-SIGN Ligands: Affinity Improvement and Specificity versus Langerin.' Org. Biomol. Chem. 2011, 9, 5778-5786.

(153) Doknic, D.; Abramo, M.; Sutkeviciute, I.; Reinhardt, A.; Guzzi, C.; Schlegel, M. K.; Potenza, D.; Nieto, P. M.; Fieschi, F.; Seeberger, P. H.; Bernardi, A. 'Synthesis and Characterization of Linker-Armed Fucose-Based Glycomimetics' Eur. J. Org. Chem. 2013, 5303-5314. 


\section{Chapter 2.}

\section{The rapid and facile synthesis of oxyamine linkers for the preparation of novel glycoconjugates.}

\subsection{Introduction}

\subsubsection{Conjugation of carbohydrates}

Glycoconjugates are ubiquitous in nature and can be present in many different forms ranging from mono- to polysaccharides, and from glycolipids to glycoproteins. ${ }^{1}$ A number of biological functions have been attributed to these glycoconjugates and, accordingly, there has been much interest in the construction of glycoconjugate mimetics, ${ }^{2-4}$ glycopeptides, ${ }^{5}$ and carbohydrate arrays, ${ }^{6-8}$ and in the synthesis of fluorescent or biotinylated glycoconjugate probes. ${ }^{9,10}$ To this end, synthetic strategies have been developed which allow for the conjugation of the carbohydrate of interest to a bifunctional linker that can then be further functionalised as required. These linkers can be introduced during the total synthesis of oligosaccharides, however, this is time-consuming and does not allow for the conjugation of carbohydrates from natural sources. Accordingly, much effort has been expended in the development of linker strategies at the reducing end of unprotected carbohydrates. These approaches include the use of Kochetkov amination, ${ }^{11}$ reductive amination, ${ }^{12}$ oximes/hydrazides ${ }^{13-15}$ and oxyamines, ${ }^{16}$ which were previously discussed in the introduction of this thesis.

\subsubsection{Oxyamine Linkers}

The focus of this thesis chapter concerns the synthesis of new oxyamine linkers that can be used for the construction of glycoconjugates. Here, it is important to note that the conjugation of carbohydrates with an oxyamine results in the formation of ring-closed glycoconjugates. ${ }^{17}$ This has particular merit as it allows for the facile introduction of a variety of functionalised linkers, 
without affecting the structural integrity of the reducing end sugar. $N$-Glycosyl oxyamines were first synthesised in the late 1970 's to study $N$-aryl- $N$-hydroxy-glucuronylamine metabolites of anticancer drugs. ${ }^{17-19}$ However, twenty years passed before oxyamines were realised in glycoconjugation when Peri et al. prepared neoglycopeptides and neoglycolipids. ${ }^{17}$ In brief, other elegant applications of oxyamines include their use in the synthesis of glycan and glycolipid analogues, ${ }^{20-22}$ in the neoglycorandomisation of methoxyamine-appended drug targets, ${ }^{16}$ and to conjugate carbohydrates to amino acids. ${ }^{23-29}$ Seminal examples include work by the groups of Blixt, ${ }^{30}$ Carraso, ${ }^{28} \mathrm{Nitz},{ }^{31}$ and Jensen, ${ }^{32}$ and more recently, Boons and co-workers demonstrated the power of oxyamine-linkers by attaching complex $\mathrm{N}$-glycans to microarrays ${ }^{33}$ through the use of a 2-[(methylamino)oxy]ethylamine linker. ${ }^{30}$ In all previously described studies, the oxyamine linkers were of 'Type A' (Scheme 1), and while the linkers clearly showed much potential in the synthesis of glycoconjugates, the linker synthesis was low yielding (10-25\%) and required 5-6 synthetic steps. The strategies employed also required different approaches for different terminal functional groups. At the time these studies were initiated, there was an unmet need for the efficient, scalable, and preferably one-pot synthesis of a series of differentially functionalised oxyamine linkers.

A.
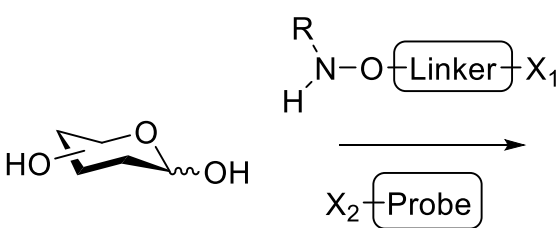

B.

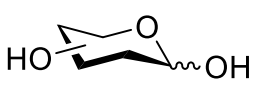

RO

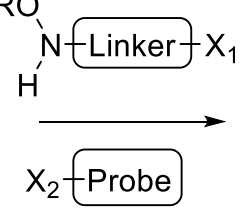

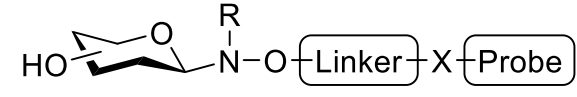

$\mathrm{R}=\mathrm{Me}, \mathrm{Bn}$, etc.

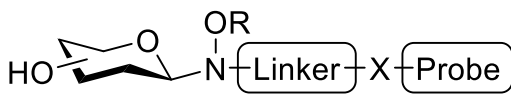

Scheme 1. The two general strategies for the conjugation of carbohydrates to oxyamine linkers and further functionalisation with the probe of interest.

\subsubsection{Bifunctional Linker Design}

\subsubsection{Retrosynthesis of linkers}

It was postulated that a variety of bifunctional linkers could be synthesised, including the azido-, amino-, thiol- and aminoacid linkers, by using a 3-step bifunctional linker synthesis (Scheme 2). Retrosynthetically, it was envisioned that the methoxyamine functionality in the linker could be obtained via a reductive amination of the corresponding aldehyde. Accordingly, the target oxyamine I, which can be equipped with different functional handles, was proposed to be prepared from the corresponding imine II via the reduction of the imine with $\mathrm{NaCNBH}_{3}$. Imine 
II could be synthesised from the condensation reaction of aldehyde III with methoxyamine. This reductive amination of aldehydes was first explored in 2002 by Peri et al. towards the synthesis of dimeric neoglycosides. ${ }^{20}$ The aldehyde in turn could be obtained via the Michael addition of different nucleophiles with acrolein IV. Herein it should be noted that sodium azide, as well as thiols (e.g., thiolacetic acid, cysteamine, $N$-Boc-cysteine and propane-1,3-dithiol), are known to undergo 1,4-addition to acrolein. ${ }^{34}$ Accordingly, it was envisioned that these nucleophiles give rise to azide and thiol functionalised linkers, whereas the amine linker could be derived via the Staudinger reduction of the azide bifunctional linker.

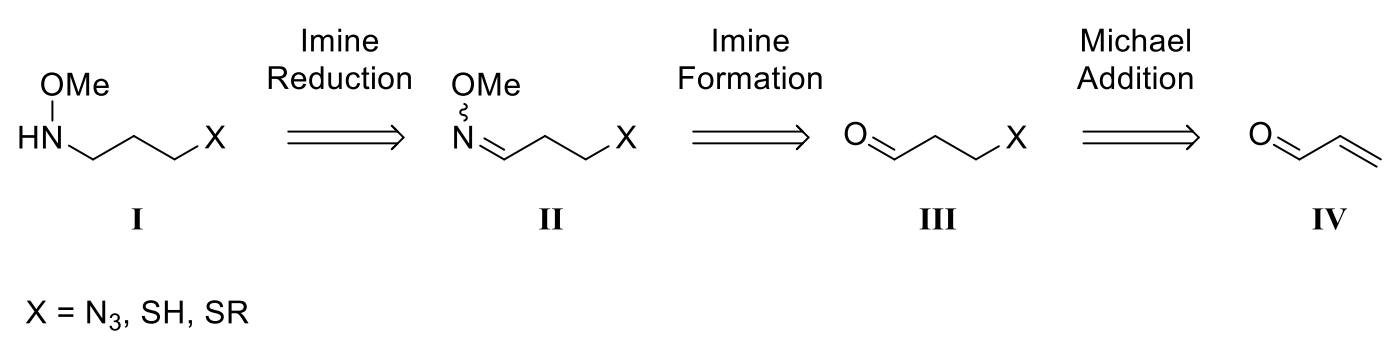

Scheme 2. Retrosynthesis of bifunctional methoxyamine linkers.

\section{Chemical ligation methods}

Upon the successful synthesis of a variety of oxyamine linkers, it was proposed that the chemical ligation with the carbohydrate would be performed first, followed by the conjugation to the probe of choice (Scheme 3). For example, the azide derived bifunctional linker can be used in the conjugation with a glycan, followed by the copper mediated azide-alkyne Huisgen cycloaddition with alkynes, ${ }^{35}$ or a copper-free strain-promoted alkyne-azide cycloaddition, ${ }^{36}$ to obtain triazole containing glycoconjugates, while amine functionalised bifunctional linkers are suitable for classical peptide chemistry. ${ }^{30}$ Thiol linkers are extremely useful for maleimide conjugate additions which are often used in protein conjugation ${ }^{37,38}$ in thiol-ene addition reactions, ${ }^{39}$ or the displacement of $\alpha$-halo ketones. Accordingly, to illustrate the versatility of the oxyamine linker, it was proposed that a variety of glycoconjugates (e.g., containing biotin, a fluorophore, a protein, or presented in a multivalent fashion on a dendrimer scaffold) would be synthesised.

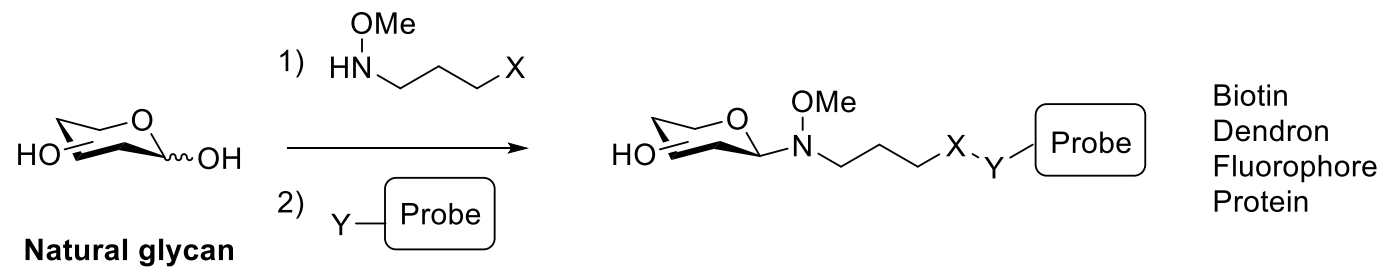

Scheme 3. Synthesis of glycoconjugates utilising the chemoselective bifunctional linkers. 


\subsection{Results and discussion}

\subsubsection{Linker synthesis}

To explore the proposed synthetic strategy, the azide-functionalised oxyamine linker $\mathbf{1}$ was first prepared (Scheme 4). Here, acrolein was subjected to a Michael addition using sodium azide to generate 3-azidopropanal (2) in good yield on a gram scale. ${ }^{34}$ Due to the instability and reactivity of the aldehyde, the reaction was performed at $-15^{\circ} \mathrm{C}$ and the mixture was directly used after basic aqueous work-up. Next, the condensation of propanal 2 with methoxyamine was performed under basic conditions to give imine 3. At first, pyridine/ethanol was used as a solvent system, ${ }^{20}$ however, the use of sodium acetate in $\mathrm{CH}_{2} \mathrm{Cl}_{2}$ was preferred due to practical advantages such as easier extraction. ${ }^{40}$ Imine $\mathbf{3}$ could then be purified by silica gel column chromatography before being reduced with $\mathrm{NaCNBH}_{3}$ in the presence of ethanolic $\mathrm{HCl}$ (freshly prepared from $\mathrm{AcCl}$ in ethanol) to give the desired bifunctional linker 1. Purification of the methoxyamine was achieved by aqueous workup followed by column chromatography. In addition, it should be noted that amine 1 can either be purified by Kugelrohr distillation (bp. of ca. $150{ }^{\circ} \mathrm{C}$ at $10 \mathrm{mbar}$ ) or by silica gel column chromatography. Using this approach, azido methoxyamine 1 was synthesised in $80 \%$ yield over three steps. Purification of the intermediate products, however, is not required and indeed, the methoxyamine $\mathbf{1}$ can be prepared on a gram scale and in a higher (96\%) yield if the intermediates are not isolated, but used directly after each extraction without concentration of the reaction mixture. Next, to prepare the amine functionalised oxyamine linker $\mathbf{4}$, the azide $\mathbf{1}$ can be reduced using a Staudinger reaction in excellent yield. Oxyamine $\mathbf{4}$ can readily be purified by a reverse phase plug to remove the phosphine by-products. Infrared spectroscopy confirmed the reduction of the azide to the amine by the lack of the distinct azide stretch at $2100 \mathrm{~cm}^{-1}$, which was previously observed for azide $\mathbf{1}$, and all other characterization data, including ${ }^{1} \mathrm{H}$ and ${ }^{13} \mathrm{C}$ NMR, were in full accordance with the anticipated structures.

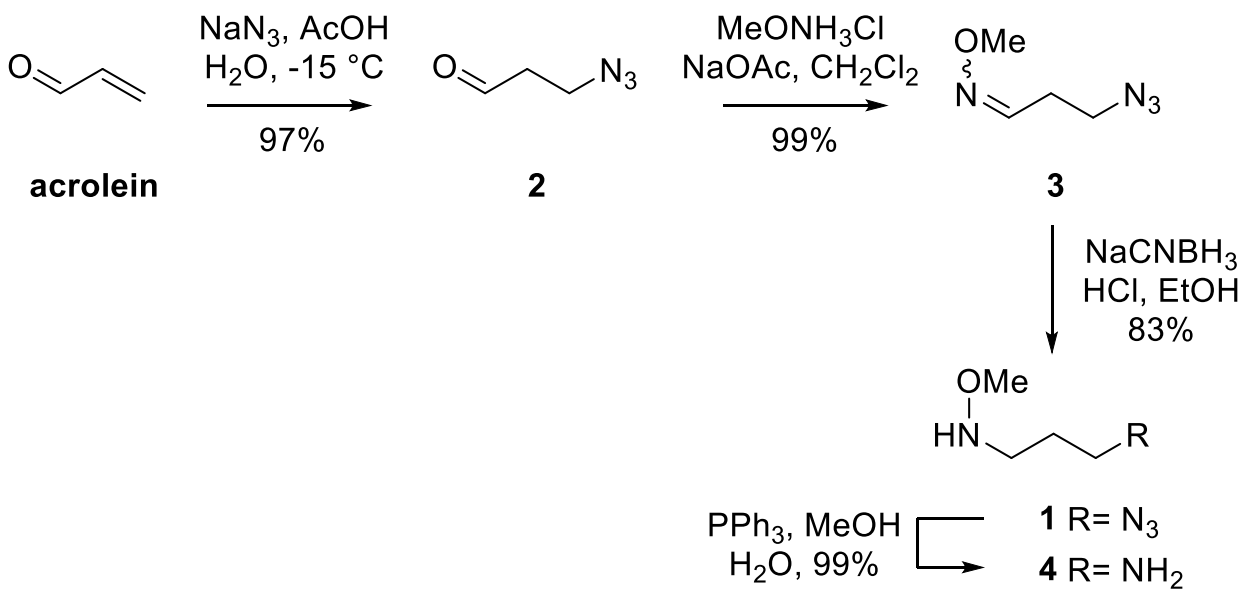

Scheme 4. Synthesis of azide and amine functionalised oxyamine linkers. 
Having established the potential of the methodology, the repertoire of oxyamine functionalised bifunctional linkers was then extended by exploring the construction of a thiol-functionalised linker (Scheme 5). To this end, thioacetic acid was reacted with acrolein to provide the 1,4-adduct $\mathbf{5}$, which was again condensed with methoxyamine to give the corresponding oxime $\mathbf{6}$. While initial attempts to reduce the oxime in a one-pot approach led to the formation of the amine, S-to-N acyl migration from the thiol could not be prevented and the acetamide product was isolated in high yield. Therefore, the intermediate thioacetate was deacetylated under Zemplén conditions to give thiol 7, in excellent yield. Finally, the reduction of imine $\mathbf{7}$ under the agency of $\mathrm{NaCNBH}_{3}$ allowed for the successful synthesis of thiol-functionalised linker $\mathbf{8}$ in $79 \%$ overall yield (4 steps). Moreover, in 1965 Bauer and Ghosh prepared a similar thiol-functionalised methoxyamine, however, with an ethyl-, instead of propyl-linker length. ${ }^{41}$

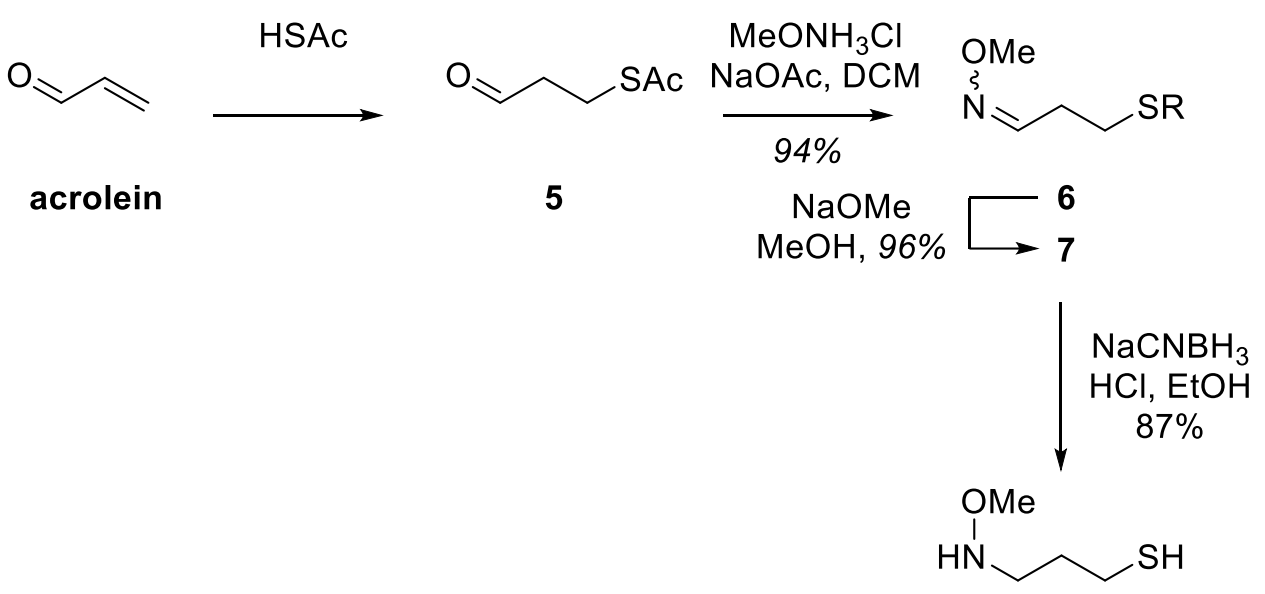

8

Scheme 5. Synthesis of the thiol functionalised oxyamine linker 8.

With the goal of developing a one-pot procedure, the use of functionalised thiols for the Michael addition was explored (Table 1). To this end, the reaction of cysteamine with acrolein, followed by the addition of methoxyamine, led to the in situ formation of the oxime, which was subsequently reduced by the addition of $\mathrm{NaCNBH}_{3}$, leading to the one-pot three-step synthesis of the amine-functionalised linker 9 (entry 1). The desired oxyamine 9 could be purified by silica gel column chromatography to yield the target linker in a good (66\%) yield. Given the success of this methodology, it was subsequently extended to the use of alternative thiol nucleophiles in order to synthesise an array of bifunctional linkers. 
Table 1. Three-step one-pot synthesis of oxyamine linkers.

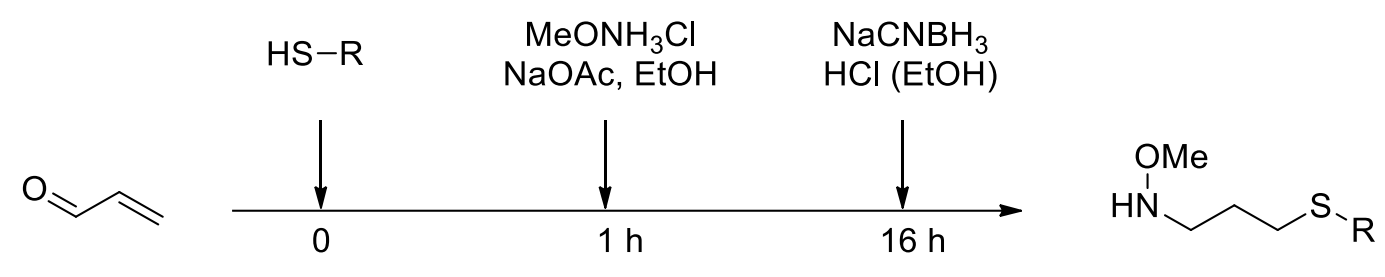

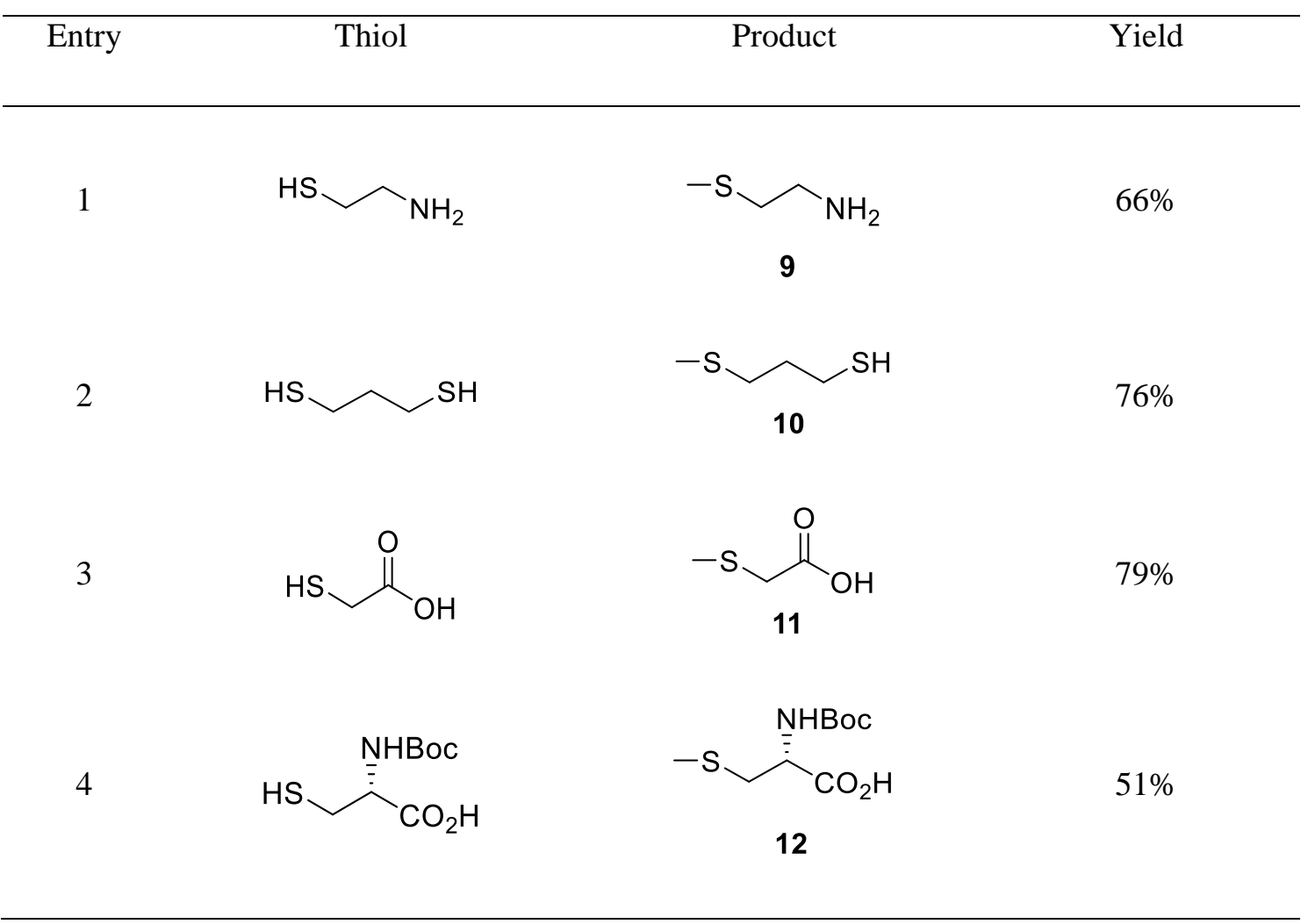

For the synthesis of a thiol-functionalised linker, neat propane-1,3-dithiol was then used to allow for the rapid synthesis of linker $\mathbf{1 0}$ in $\mathbf{7 6 \%}$ yield (Table 1, entry 2). Here, 1,3-propanedithiol simultaneously acts as a reducing agent, which allows for the isolation of the sulfhydryl instead of the disulfide oxyamine after silica gel chromatography. In order to produce a carboxy-modified linker, 2-mercaptoacetic acid was used (entry 3) Again, the required linker 11 was readily prepared via the three-step one pot strategy and in $79 \%$ overall yield. It should be noted that concentration of the crude reaction mixture results in the formation of the ethyl ester derivative $\left({ }^{1} \mathrm{H}\right.$ NMR $\delta 4.22\left(\mathrm{q}, 2 \mathrm{H}, J_{\mathrm{CH} 2, \mathrm{CH} 3}=7.1 \mathrm{~Hz}, \mathrm{CH}_{2} \mathrm{OEt}\right), 3.53\left(\mathrm{~s}, 3 \mathrm{H}, \mathrm{OCH}_{3}\right), 2.97\left(\mathrm{t}, 2 \mathrm{H}, J_{l, 2}=7.3\right.$ $\left.\mathrm{Hz}, \mathrm{CH}_{2}-1\right), 2.68\left(\mathrm{t}, 2 \mathrm{H}, J_{2,3}=7.3 \mathrm{~Hz}, \mathrm{CH}_{2}-3\right), 1.81\left(\mathrm{p}, 2 \mathrm{H}, J_{1,2}=J_{2,3}=7.3 \mathrm{~Hz}, \mathrm{CH}_{2}-2\right), 1.27(\mathrm{t}$, $3 \mathrm{H}, J_{\mathrm{CH} 2 \mathrm{CH} 3}=7.1 \mathrm{~Hz}, \mathrm{CH}_{3} \mathrm{OEt}$ ); $\mathrm{m} / z$ for $\left[\mathrm{C}_{8} \mathrm{H}_{18} \mathrm{NO}_{3} \mathrm{~S}\right]^{+}$calc. 208.1002, obs. 208.1013). Due to the high water solubility of linker 11, an aqueous work-up procedure was not feasible and instead the reaction was quenched by the addition of $1 \mathrm{M} \mathrm{NaOH}$ (aq.) before concentration and purification by silica gel flash column chromatography to give the target carboxy-modified linker. Finally, to 
determine if the methodology was amenable to the use of more complex substrates, acrolein was subjected to a Michael addition with $N$-Boc-cysteine, followed by oxime formation and reduction (entry 4). Again, ethyl ester formation $\left(\mathrm{m} / z\right.$ for $\left[\mathrm{C}_{14} \mathrm{H}_{29} \mathrm{~N}_{2} \mathrm{O}_{5} \mathrm{~S}\right]^{+}$calc. 337.1792, obs. 337.1820) was observed as well as some $N$-Boc cleavage $\left(\mathrm{m} / z\right.$ for $\left[\mathrm{C}_{9} \mathrm{H}_{21} \mathrm{~N}_{2} \mathrm{O}_{3} \mathrm{~S}\right]^{+}$calc. 237.1267, obs. 237.1280), and thus the reaction was quenched with $1 \mathrm{M} \mathrm{NaOH}$ followed by concentration and silica gel flash column chromatography to afford amino-acid derivative 12. While the overall yield for the synthesis of cysteine linker 12 was modest (51\% yield over three steps), this route nonetheless provides a rapid entry to an alkoxyamine functionalised amino acid.

\subsubsection{Carbohydrate Ligation of methoxyamine linkers}

With a series of oxyamine linkers in hand, the efficiency of glycan conjugation was then investigated. Given the importance of GlcNAc as an N-linked reducing end terminal sugar, ${ }^{42}$ this substrate was initially used for linker conjugation. To this end, $N$-acetyl-glucosamine (13) and the appropriate linker (10 equiv.) were stirred in an aqueous $2 \mathrm{M} \mathrm{AcOH} / \mathrm{NH}_{4} \mathrm{OAc}$ buffer at $\mathrm{pH} 4.5$ (0.1 M glycan concentration) for $16 \mathrm{~h}$ at room temperature (Scheme 6). These solvent conditions were chosen as they should allow for the solubilisation of most glycans. The conjugation of azide linker 1 proceeded smoothly and in excellent yield to give $\mathrm{N}$-glycan $\mathbf{1 4}$ in 87\%. Here, it was found that direct concentration or lyophilisation of the reaction mixture resulted in neoglycoside hydrolysis, therefore reverse phase chromatography was used to purify glycoconjugate $\mathbf{1 4}$. Alternatively, the neoglycosides was purified using a water based size exclusion column (BioGel $\mathrm{P}-2,1200 \times 18 \mathrm{~mm}$, flow-rate $10 \mathrm{~mL} /$ hour), whereby the crude reaction mixture was directly loaded onto the size exclusion column, to give the neoglycoside in excellent yields. The conjugation of the sugar to the linker was confirmed by $\mathrm{HMBC}$ between the $\mathrm{CH}_{2}-1$ of the linker and the $\mathrm{CH}^{-1}{ }^{\prime}$ of the glycan (Figure 1). Moreover, the exclusive formation of the $\beta$-pyranoside was confirmed by the $J_{1^{\prime}, 2^{\prime}}=9.8 \mathrm{~Hz}$. In addition, the $\mathrm{N}$-glycan 14 was crystallised from $\mathrm{MeOH} / \mathrm{Et}_{2} \mathrm{O}$ and analysed by X-ray crystallography, which confirmed that the linker was in the $\beta$-configuration (Figure 2). 


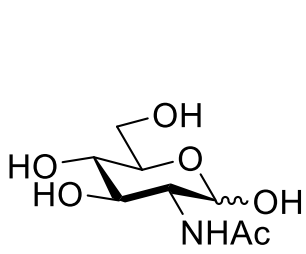

13
OMe
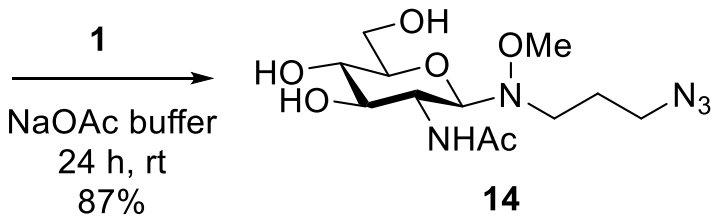
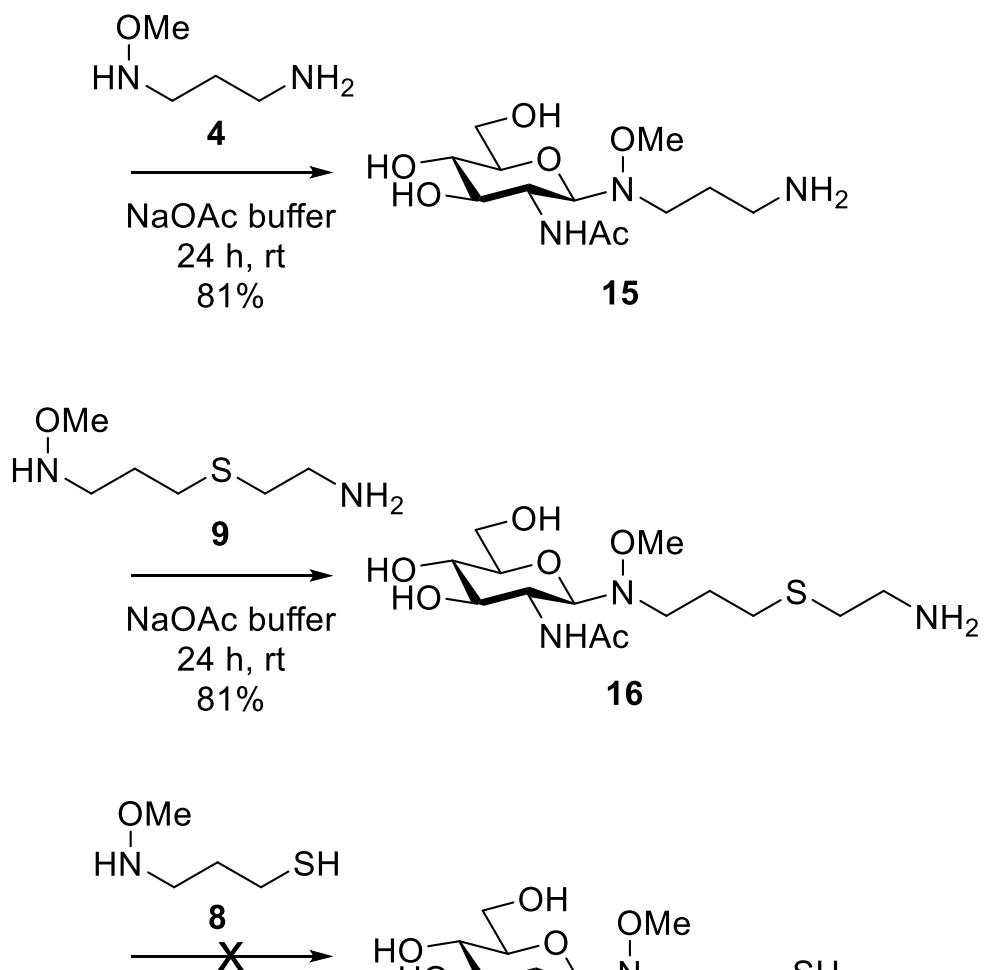

NaOAc buffer TCEP, $24 \mathrm{~h}, \mathrm{rt}$
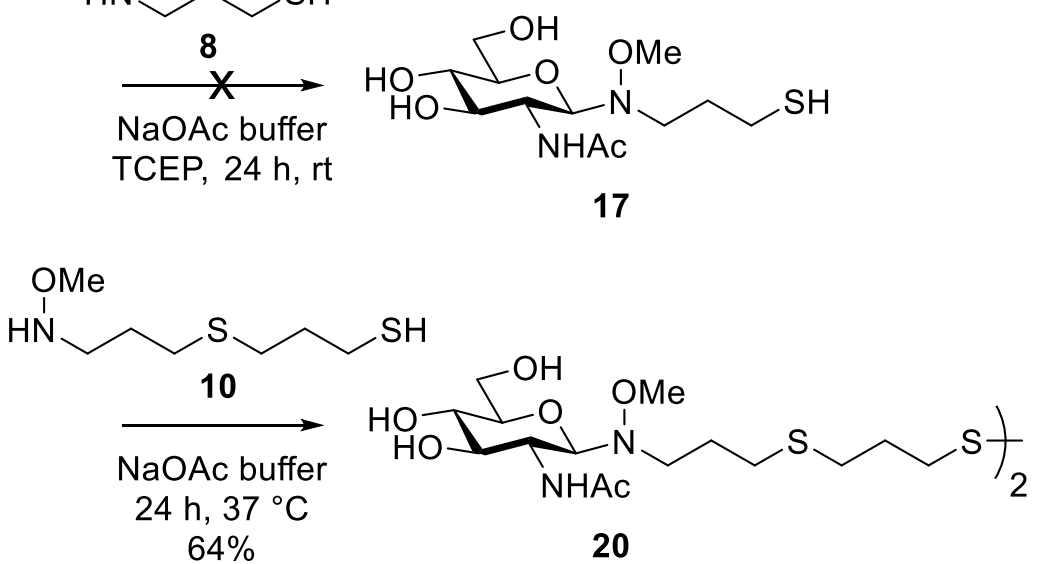

Scheme 6. Conjugation of oxyamine linkers to GlcNAc.

Next, $N$-acetylglucosamine (13) was successfully condensed with the amine functionalised linkers $\mathbf{4}$ and $\mathbf{9}$ to give both the corresponding N-glycans $\mathbf{1 5}$ and $\mathbf{1 6}$ in 81\% yield. Interestingly, amine 15 had no retention using reverse phase chromatography and thus required purification by size exclusion chromatography. Size exclusion chromatography with a $0.1 \mathrm{M}$ ammonium formate solution results in the formation of the formate salt of $\mathbf{1 5}$. This, however, can be converted to the free amine or hydrochloride salt by filtering over a ion exchange resin, Dowex $\mathrm{OH}^{-}$or Dowex $\mathrm{Cl}^{-}$ respectively (see SI for corresponding NMR spectra). The less polar glycoside 16, however, was obtained after purification with reverse phase and silica gel column chromatography. 


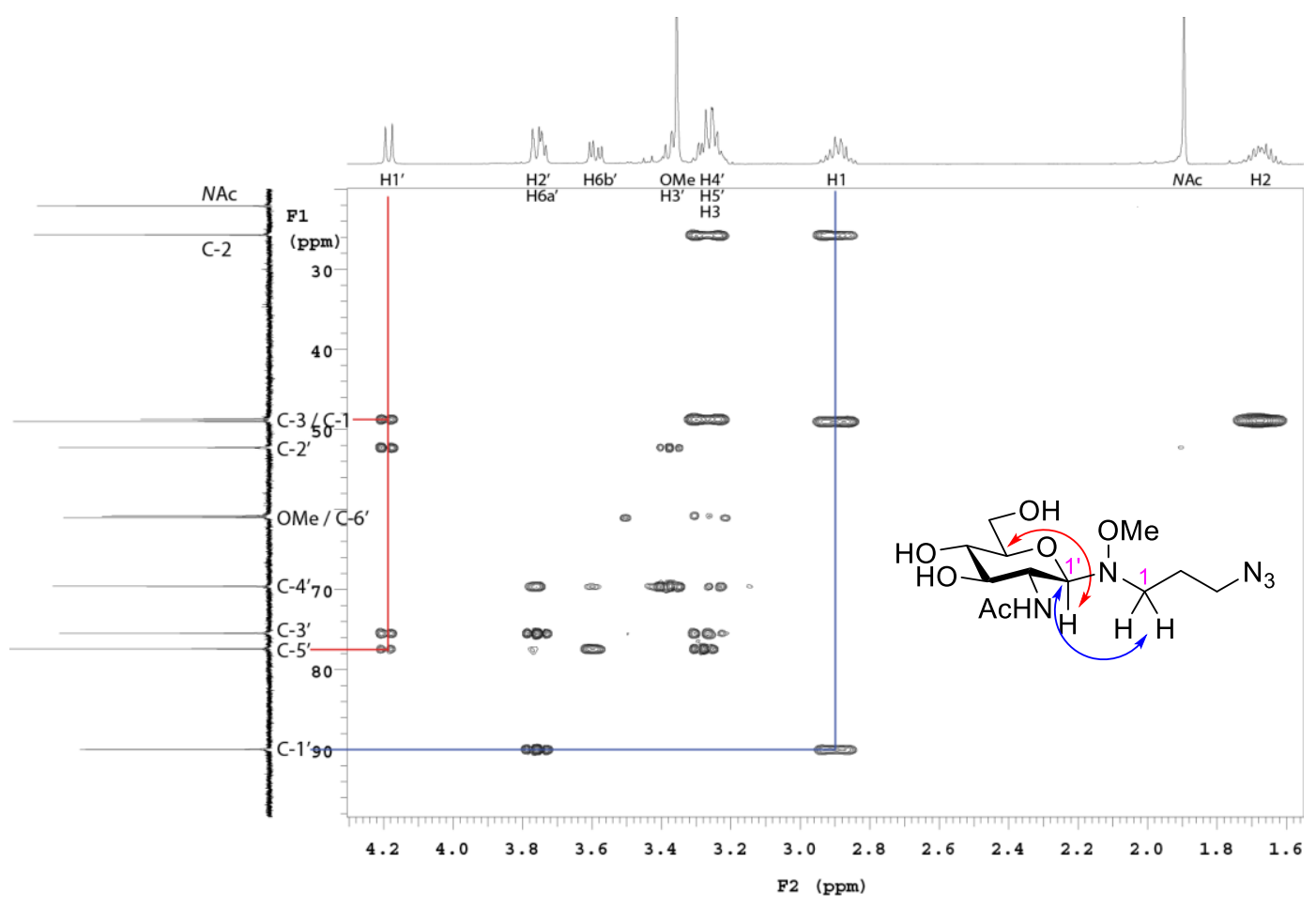

Figure 1. $\mathrm{HMBC}$ of neoglycoside $14\left(\mathrm{D}_{2} \mathrm{O}, 500 \mathrm{MHz}\right)$.
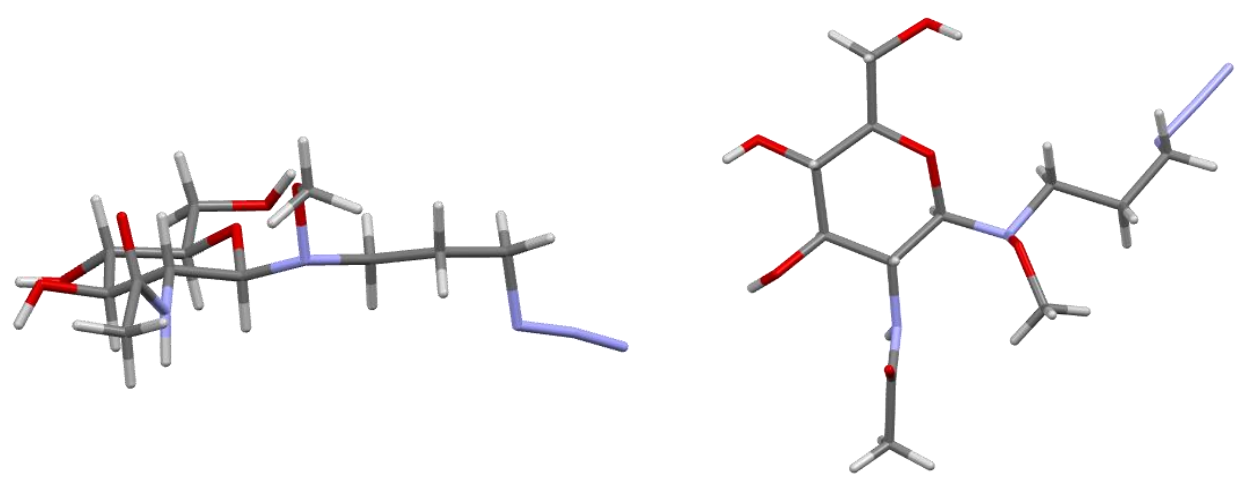

Figure 2. X-ray crystallography structure for glycoside 14. A) Sideways view B) Topview.

Having succesfully prepared the azide-functionalised glycan 14, $N$-acetylglucosamine (13) was then reacted with thiol functionalised linker 8 to obtain a condensation product in $81 \%$ yield, however, this did not turn out to be the desired glycosylamine 17, but instead a diastereomeric mixture of $\mathrm{N}$-methoxythiazinanes $\mathbf{1 8}$, which are formed via the intramolecular 6-endo-trig ringclosure of the thiol and the imine. (Scheme 7). Here, TLC analysis indicated the formation of this newly formed glycoconjugate, and although this was supported by mass spectrometry $(\mathrm{m} / \mathrm{z}$ for $\left[\mathrm{C}_{12} \mathrm{H}_{25} \mathrm{~N}_{2} \mathrm{O}_{6} \mathrm{~S}\right]^{+}$calc. 325.1428 , obs. 325.1445), the structures could not be conclusively confirmed using NMR analysis. Surprisingly, NMR spectroscopy showed peak broadening in both the ${ }^{1} \mathrm{H}$ and the ${ }^{13} \mathrm{C}$ experiments, even when using different solvents $\left[\mathrm{D}_{2} \mathrm{O}, \mathrm{CD}_{3} \mathrm{OD}, \operatorname{Pyr}\left(\mathrm{D}_{5}\right)\right.$ and $\left.\operatorname{DMSO}\left(\mathrm{D}_{6}\right)\right]$ and varying temperatures ( $\mathrm{rt}, 30,40,50$ and $60^{\circ} \mathrm{C}$ ). Nevertheless, the structure 
of the newly formed thiazinanes could be confirmed after acetylation of the crude condensation reaction products.

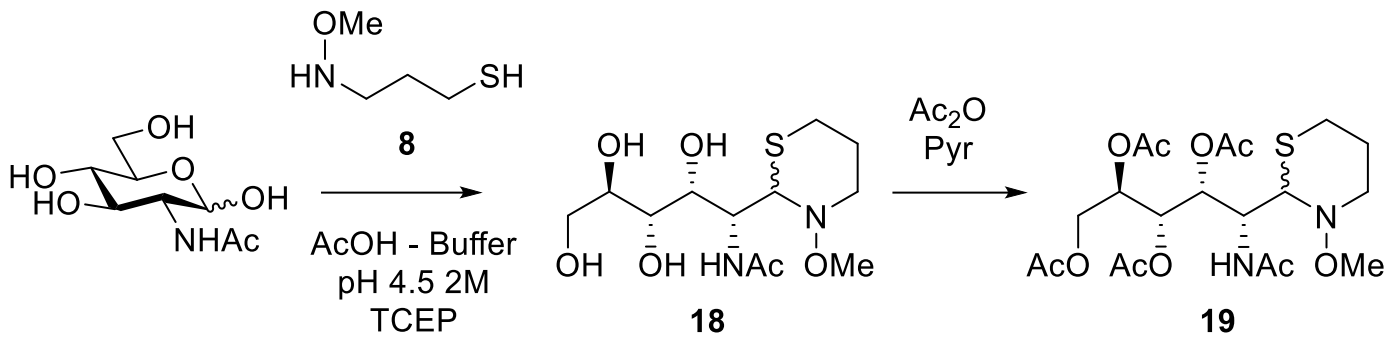

Scheme 7. The formation of N-methoxy thiazinanes 18, which after per-acetylation could be confirmed by NMR analysis.

After acetylation of tetraols 18, two products were observed by NMR-analysis, although peak broadening complicated the ${ }^{1} \mathrm{H}$ and ${ }^{13} \mathrm{C}$ NMR spectra. Fortunately NMR analysis at $50{ }^{\circ} \mathrm{C}$ proved sufficient for structural confirmation of the two diastereomeric thioaminals 19. Although the relative stereochemistry of the two diastereoisomers could not be determined, a combination of COSY, HSQC (coupled and decoupled) and HMBC NMR-experiments enabled all resonances of each diastereoisomer to be assigned. To this end, the presence of the thiazinane was confirmed for both isomers by the $\mathrm{HMBC}$ between $\mathrm{C}-1$ ' and $\mathrm{CH}_{\text {eq }} \mathrm{N}$ as well as $\mathrm{CH}_{\mathrm{eq}} \mathrm{S}$. In addition, a coupled HSQC experiment showed a ${ }^{1} J_{C I^{\prime}-H l^{\prime}}=146 \mathrm{~Hz}$ for isomer $a$ and ${ }^{1} J_{C 1^{\prime}-H l^{\prime}}=145 \mathrm{~Hz}$ for isomer $b$, indicating they both contain the thioaminal functional group. Moreover, 1D-TOCSY experiments were used to analyse the 6-membered ring (Figure 3). Here, excitation of the isomer a equatorial $\mathrm{CH}_{2} \mathrm{NO}\left(\mathrm{H}_{\mathrm{a}}\right)$ at $3.60 \mathrm{ppm}$ gave the TOCSY resonances, throughout the spin system, at the relaxation delay times of 20, 40, 80 and $120 \mathrm{~ms}$. Here, it was observed that for isomer a the coupling constants of the protons were indicative of the presence of a 6-membered ring. Unfortunately, selective excitation of $\mathrm{CH}_{2} \mathrm{NO}\left(\mathrm{H}_{\mathrm{a}}\right)$ for isomer $b$ could not be used for structure confirmation, due to overlapping TOCSY resonances. 


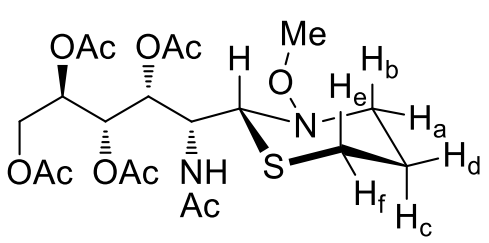

$19 a$

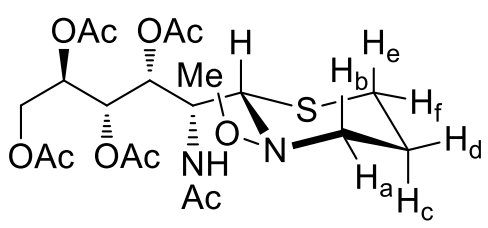

$19 b$

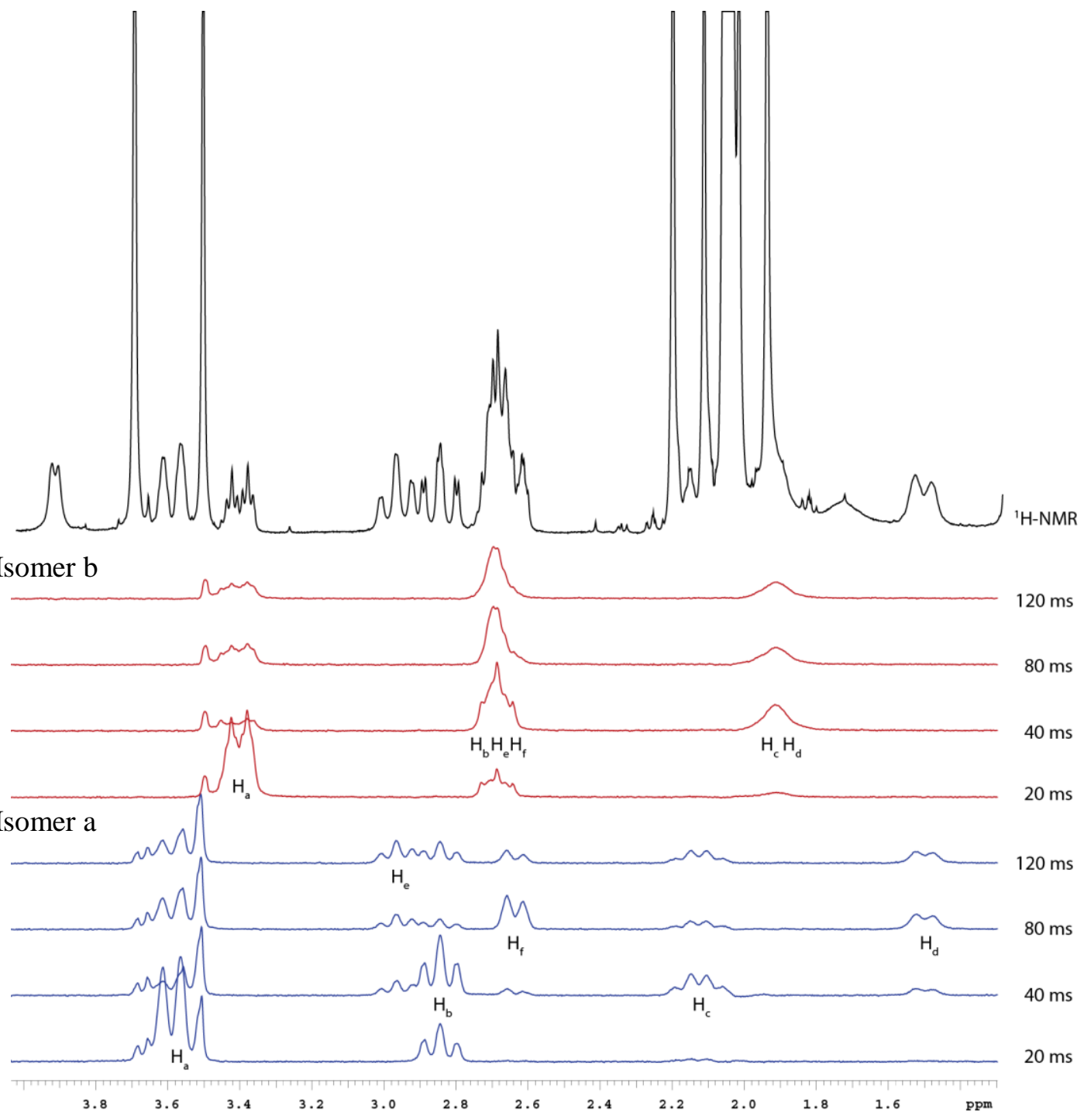

Figure 3. TOCSY-NMR experiment with relaxation delay times of 20, 40, 80 and $120 \mathrm{~ms}$. Selective excitation at $3.58 \mathrm{ppm}\left(H_{a}\right)$ for isomer a (blue), and at $3.40 \mathrm{ppm}\left(H_{a}\right)$ for isomer $b(\mathrm{red})$ and the corresponding ${ }^{1}$ H-NMR spectrum (black).

In an attempt to prevent the 6-endo-trig cyclisation and formation of the thioaminals, the extended thiol linker chain $\mathbf{1 0}$ was used (Scheme 6). Gratifyingly, this led to the successful formation of the disulfide glycosylamine $\mathbf{2 0}$ in $64 \%$ yield. Here, it should be noted that the disulfide was obtained after exposure to air, however, the use of a reducing agent, such as tris(2-carboxyethyl)phosphine (TCEP), prevented the formation or reduced the already formed disulfide to the corresponding sulfhydryl. 


\subsubsection{Kinetic Stability Studies}

As oxyamine-linkers are hydrolysed under acidic aqueous conditions, we investigated the rates of hydrolysis of glycoconjugate $\mathbf{1 4}$ at varying $\mathrm{pH}$. Previous work in this field has shown that the substitution pattern of the nitrogen and oxygen in oxyamines affects their hydrolytic stability, with the electron-donating Bn group on either the oxygen or nitrogen of the oxime decreasing the rate of hydrolysis. ${ }^{32}$ The disadvantage of the bulky benzyl group, however, is that conjugation to the glycan is not as efficient and lectin binding can be compromised. ${ }^{32}$ Thus, glycoconjugate $\mathbf{1 4}$ was subjected to aqueous conditions at $\mathrm{pH} 4.75,5,6,7$ and 9 to evaluate linker stability (Figure 4). Using a ${ }^{1} \mathrm{H}-\mathrm{NMR}$ experiment, the reactions were monitored over time and relative integration between $\mathrm{CH}_{2}-1$ and $\mathrm{H}-1$ ' gave the respective ratios between neoglycoside $\mathbf{1 4}$, the hydrolysed glycan 13 and the linker $\mathbf{1}$. As anticipated, the rates of hydrolysis were $\mathrm{pH}$ dependent, with the glycoconjugate 14 showing no appreciable cleavage at $\mathrm{pH} 7$ and 9 over a 100 day period. At $\mathrm{pH}$ 6 , only limited cleavage was observed with a $\mathrm{t}_{1 / 2}=2$ years, which indicates that the linker is suited for use under physiological conditions. At $\mathrm{pH} 5$ and 4.5, $\mathrm{t}_{1 / 2}=120$ and 22 days, respectively, which was 2 to 5 -fold better than the analogous benzylated oxyamine of 'Type A' (Figure 4). ${ }^{32}$ Accordingly, the $N$-linked methoxyamine linker of 'Type B' can not only be readily prepared but, in addition, shows excellent hydrolytic stability.
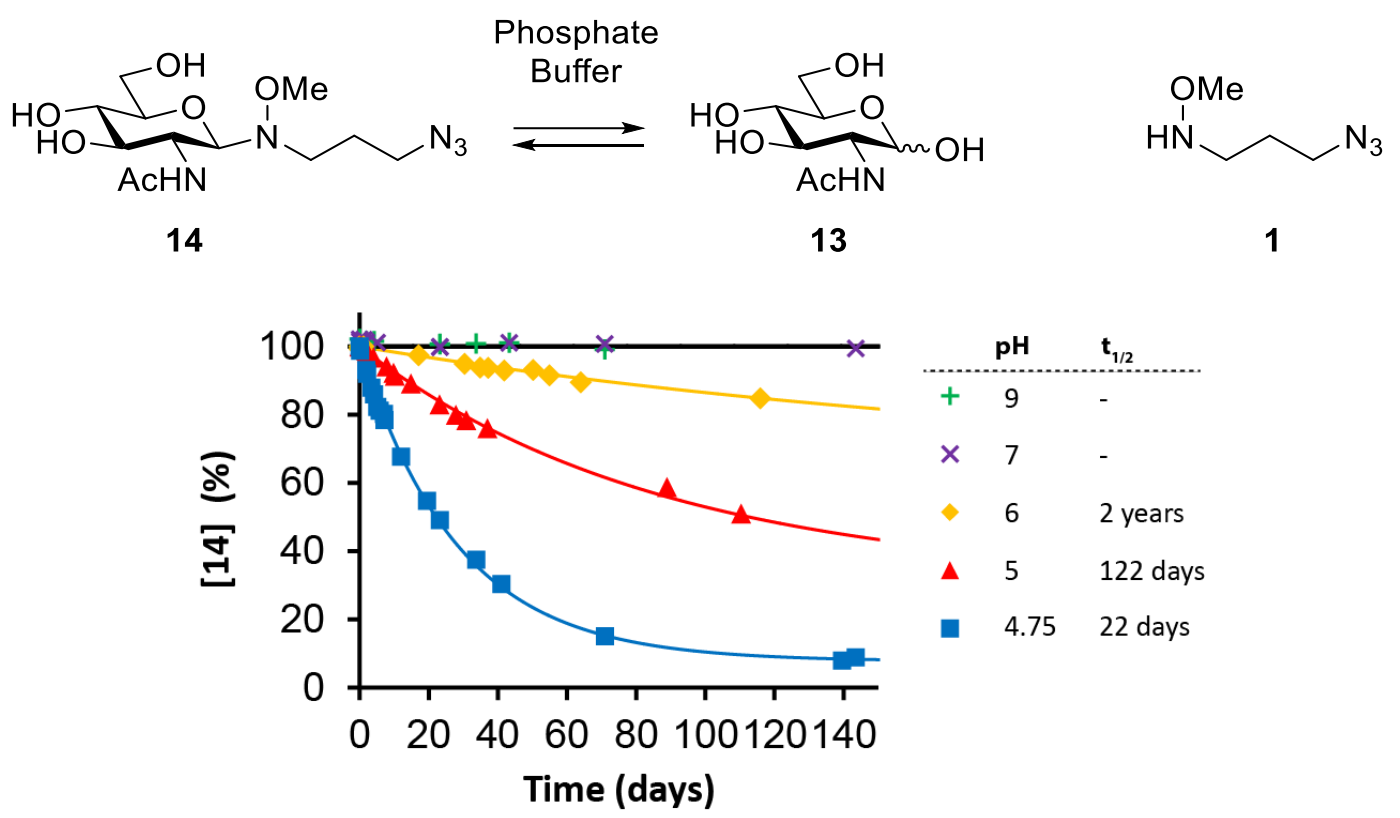

Figure 4. Rates of hydrolysis of oxyamine linked GlcNAc 14 at pH 4.75, 5, 6, 7 and 9. As determined by ${ }^{1} \mathrm{H}$-NMR analysis of $50 \mathrm{mM}$ solutions of glycoside 4 in sodium phosphate buffered $\mathrm{D}_{2} \mathrm{O}$. 
To calculate the half-life for each reaction, the rate equation for chemical decay of the glycoside is described with:

$$
N(t)=N_{0} e^{-\lambda t}
$$

Where $N(t)$ is the percentage of starting material left at time $(t), N_{0}$ is the amount of starting material at $t=0, \lambda$ is the probability of one molecule to decay per time unit (decay constant) and $t$ is the time. However, given the fact that the reaction reaches an equilibrium, the equation must be modified to describe that the reaction reaches the equilibrium $N_{e q}$ :

$$
N(t)=\left(N_{0}-N_{e q}\right) e^{-\lambda t}+N_{e q}
$$

To determine the half-life a theoretical line is fitted to the data according to the least squares method. By fitting the line to the data, both the rate constant $\lambda$ and the equilibrium $N_{e q}$ were determined for each reaction. Next, the half-life can be determined by solving the equation (2) above for $N(t)=50$. For example, if $N_{e q}=7$ and $\lambda=0.035$ then a half-life of 22 days is calculated:

$$
\begin{aligned}
& 50=(100-7) e^{-0.035 t}+7 \\
& t_{\left(\frac{1}{2}\right)}=\frac{\ln \left(\frac{1}{43 / 93}\right)}{0.035}=22 \text { days }
\end{aligned}
$$

Currently, further investigations towards oxyamine stability are underway. In these experiments the glycoconjugates of 'Type A' and 'Type B' are directly compared under the same reaction conditions. Here, the effect of the glycan concentration, the $\mathrm{pH}$ and the buffer strength are examined. These results will be reported in due course.

\subsubsection{Scope and limitation of carbohydrates ligation}

Having demonstrated that a variety of oxyamine linkers can be conjugated to $N$-acetyl glucosamine (13), the chemoselective ligation of methoxyamine linkers to other carbohydrates was investigated. As nicely demonstrated in the neoglycorandomisation research field, a variety of carbohydrates can be used for the chemoselective ligation with methoxyamine linkers. However, only a few neoglycosides are selectively obtained in the $\beta$-pyranose form, where most glycans are obtained as $\alpha / \beta$-mixtures as well as furanose/pyranose configurations. ${ }^{43}$ To determine whether comparable $\alpha / \beta$, pyranose/furanose configurations would be obtained when using methoxyamine linker $\mathbf{1}$, a variety of reducing end sugars were conjugated to this linker and subsequently analyzed by ${ }^{1} \mathrm{H}-\mathrm{NMR}$. Accordingly, it was found that both GlcNAc and glucose were selectively obtained as the $\beta$-pyranoside. Moreover, D-galactose was successfully converted into the corresponding neoglycoside, however, with a 90:10 $\beta$-pyranose: $\beta$-furanose ratio. 
Furthermore, mannose was obtained as an $\alpha$-furanose and $\alpha / \beta$-pyranose mixture, which is in line with Langenhans' study. ${ }^{43}$ Accordingly, the selectivity of the ligation with all forms of oxyamine linkers appears to be similar and thus no further investigation was performed towards the nature of the reducing end sugar.

To further illustrate the versatility of the linker strategy, conjugation to more sterically hindered carbohydrates were undertaken. To this end, the disaccharide maltose (21) was successfully converted into glycoside 22 in excellent yield and with complete $\beta$-selectivity. Conjugation of the biologically relevant trisaccharide Lewis $^{\mathrm{X}}(\mathbf{2 3})$ was then performed, whereby the amine linker 4 and Lewis ${ }^{\mathrm{X}} \mathbf{2 3}$ were stirred in a $2 \mathrm{M} \mathrm{NaOAc} / \mathrm{NH}_{4} \mathrm{OAc}$ buffer at room temperature (Scheme 4). The reaction proved sluggish and only $c a$. $25 \%$ conversion was observed after 24 h by TLCanalysis. Increasing the temperature to $40{ }^{\circ} \mathrm{C}$, however, led to a marked increase in reaction rate, with complete conversion to $\mathrm{N}$-glycan $\mathbf{2 4}$ being observed after $36 \mathrm{~h}$ at this temperature. Purification by direct loading of the reaction mixture onto a Bio-Gel P-2 column for size exclusion chromatography then allowed for the isolation of conjugate $\mathbf{2 4}$ in $88 \%$ yield and as only the $\beta$ pyranosyl glycoside, as evidenced by ${ }^{1} J_{\mathrm{CH}-1^{\prime}}=154 \mathrm{~Hz}$, and an HMBC between H-1'and C-5' (Figure 5).

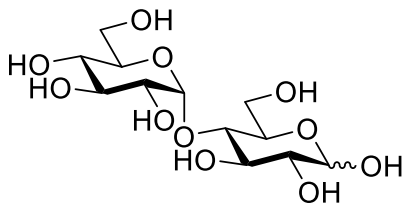

21

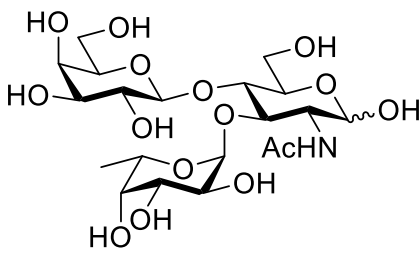

23

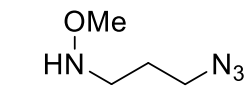

1

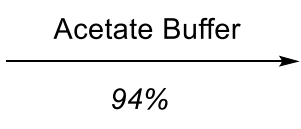

$\mathrm{OMe}$

HN $\mathrm{NH}_{2}$

4

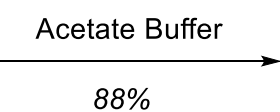

$88 \%$

24

Scheme 8. Neoglycosylation of the more bulky maltose 21 and the biological relevant Lewis ${ }^{X} 2$. 

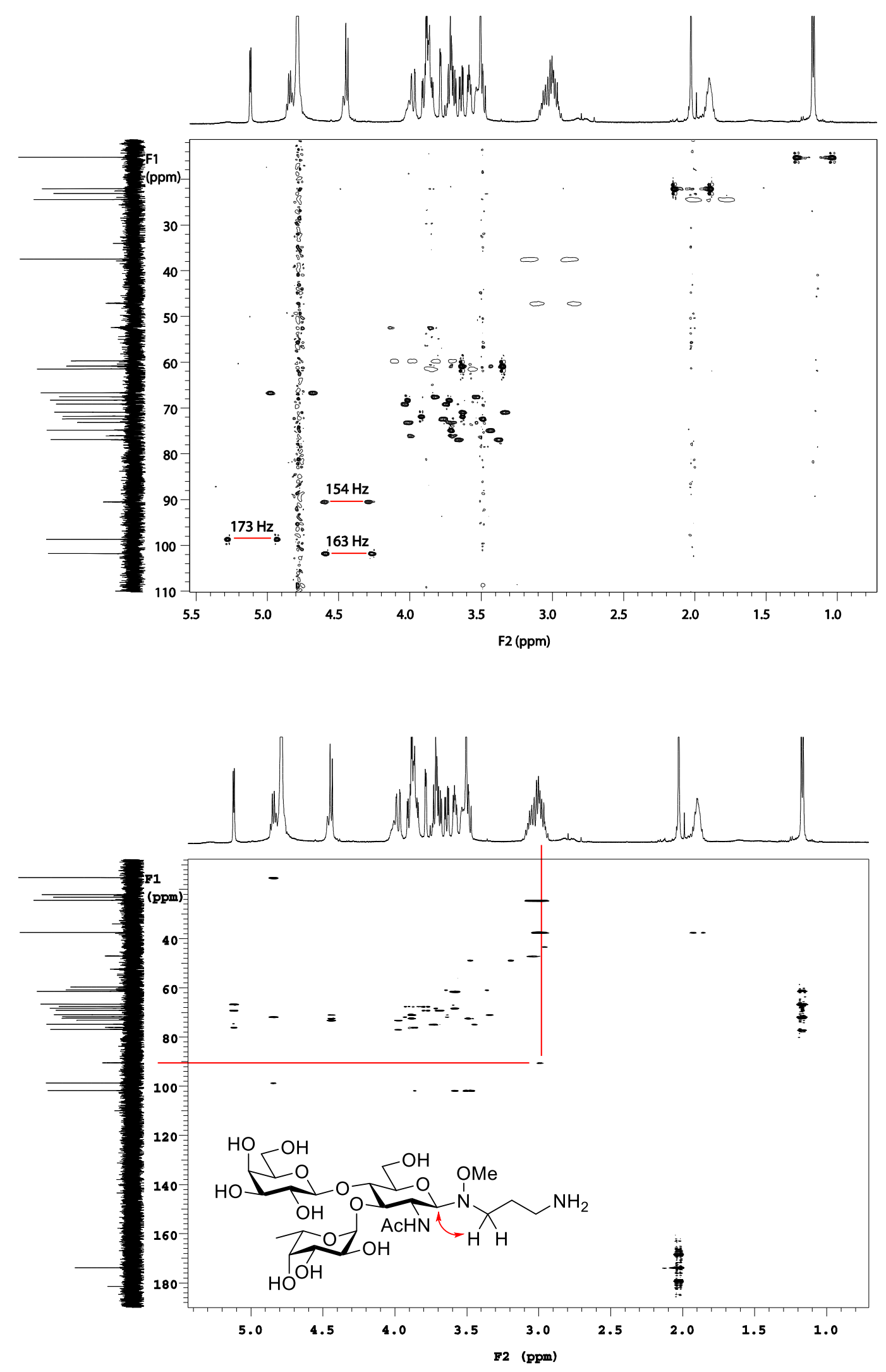

Figure 5. Coupled HSQC (top) and HMBC (bottom) NMR experiments of Lewis ${ }^{X}$-glycoconjugate 24. $\left(\mathrm{D}_{2} \mathrm{O}, 500 \mathrm{MHz} \mathrm{NMR}\right)$ 


\subsubsection{Conjugation of neoglycosides to probes}

In order to determine whether the azide derived neoglycoside $\mathbf{1 4}$ could be used in the copper mediated azide-alkyne Huisgen cycloaddition, the azidoglycoconjugate 14 was successfully reacted with propargyl alcohol (Scheme 9). This led to the formation of triazole $\mathbf{2 5}$ in good yield (79\%) and demonstrated that the azide-functionalised neoglycoside can indeed successfully be used in a cycloaddition reaction. The use of amine functionalised glycans was exemplified by the synthesis of a fluorescent glycoconjugate. Here, neoglycoside 16 was reacted with dansyl chloride under basic conditions to give sulfonamide $\mathbf{2 8}$ in $\mathbf{7 7 \%}$ yield. In this way the potential to generate fluorescent glycans for the study of biological processes has been demonstrated. ${ }^{10}$ Amine derivative 15 was also successfully conjugated with D-biotin using the $N, N, N^{\prime}, N^{\prime}$-tetramethyl-O(1H-benzotriazol-1-yl)uronium hexafluorophosphate (HBTU) mediated peptide coupling, to give glycoconjugate $\mathbf{2 6}$ in $71 \%$ yield. Here, the biotinylated glycan was purified by a combination of silica gel and size exclusion chromatography. The conjugation of neoglycoside $\mathbf{1 5}$ with a nonavalent bifunctional second generation dendron allows for the multivalent presentation of the glycan in $\mathbf{2 7}$ which forms the subject of Chapter 3.

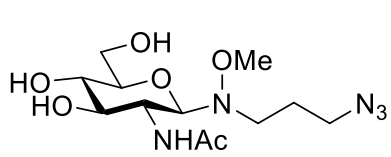

14

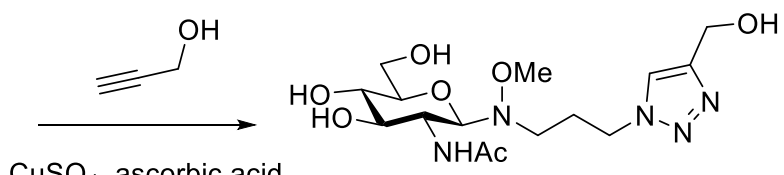

$\mathrm{CuSO}_{4}$, ascorbic acid

$\mathrm{EtOH}, 79 \%$

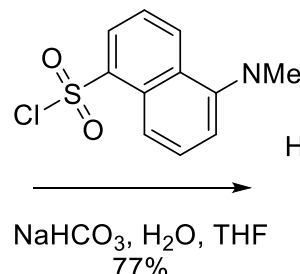

16

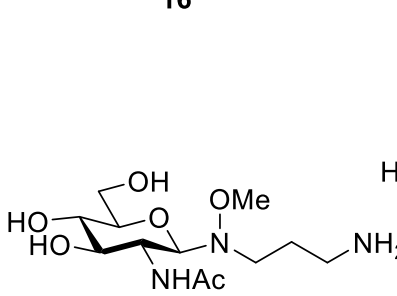

15

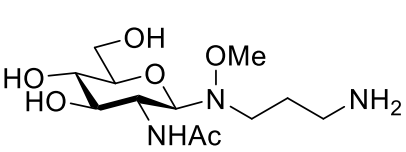

15

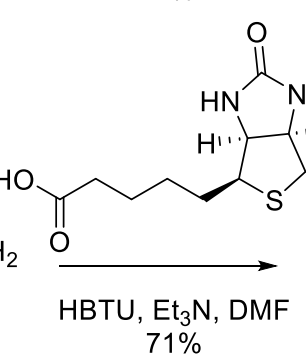

$71 \%$

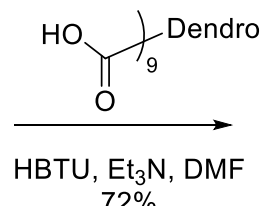
$72 \%$
25

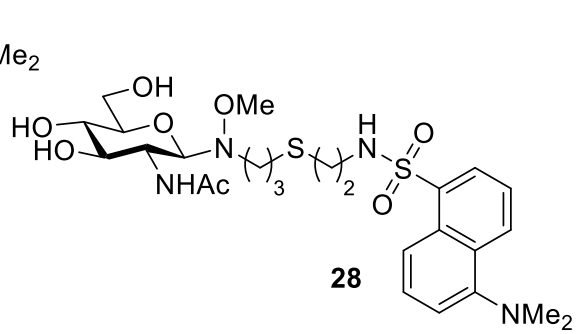

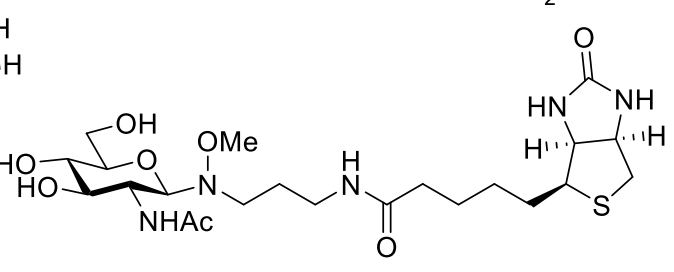

26

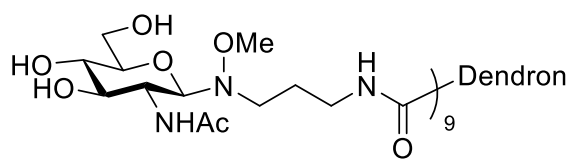

27

Scheme 9. Conjugation of neoglycosides with various probes. 
To further demonstrate the scope and efficiency of the developed oxyamine linker methodology, it was proposed to conjugate thiol functionalised glycosides to maleimide $\mathbf{2 9}$. This required the synthesis of biotinylated-maleimide $\mathbf{2 9}$ via the amide coupling of D-biotin with previously reported 2-aminoethyl-maleimide intermediate $\mathbf{3 0}$ (Scheme 10). ${ }^{44}$ This intermediate was prepared from ethylene diamine $(\mathbf{3 1})$ via mono $N$-Boc protection $(\rightarrow \mathbf{3 2})$, reaction with maleic anhydride and subsequent cyclisation to give maleimide 33, and TFA treatment to give amine $\mathbf{3 0}$. Subsequently, disulfide 20 and Michael acceptor 29 were successfully reacted in $\mathrm{H}_{2} \mathrm{O}$ to give crude biotinylated adduct $\mathbf{3 4}$, which was then purified by a combination of reverse phase $\left(\mathrm{C}_{18}\right)$ and size exclusion chromatography (Scheme 11). Both biotinylated adducts 26 and 34 can be used for the detection of carbohydrate binding antibodies in patients' blood following conjugation of the probes to streptavidin-coated plates, and subsequent incubation with patients' serum. ${ }^{45,46}$

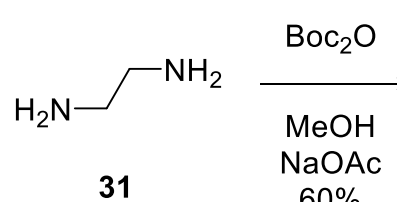

$60 \%$

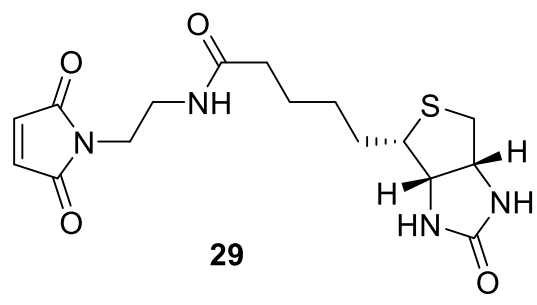

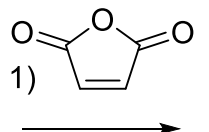

2) $\mathrm{Ac}_{2} \mathrm{O}, \mathrm{Pyr}$ $41 \%$ (2-steps)

32

D-Biotin HBTU, Et ${ }_{3} N$ DMF

$92 \%$<smiles>CC(C)(C)NCCN1C(=O)C=CC1=O</smiles>

33 TFA, $\mathrm{CH}_{2} \mathrm{Cl}_{2}$ $82 \%$<smiles>NCCN1C(=O)C=CC1=O</smiles>

Scheme 10. Synthesis of maleimide acceptor 29. 


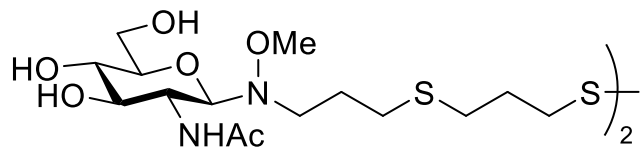

20<smiles>O=C(CCCC[C@@H]1SC[C@@H]2NC(=O)N[C@H]12)NCCN1C(=O)C=CC1=O</smiles>

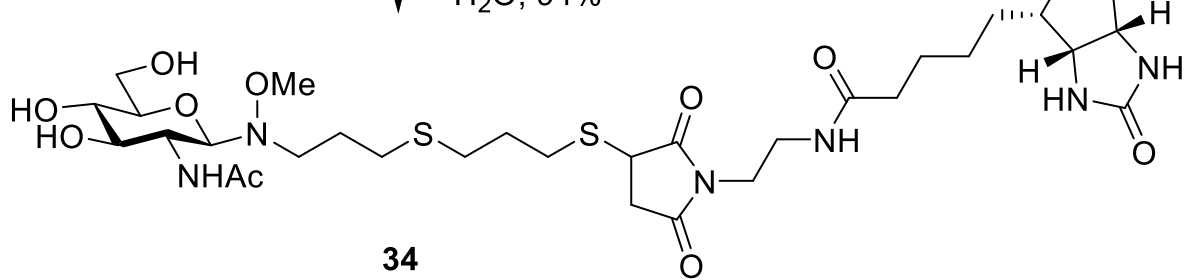

Scheme 11. Synthesis of biotinylated neoglycoside 34.

The use of maleimide chemistry can also be extended to the formation of highly glycosylated proteins. Here, the commercially available bifunctional cross-linker succinimidyl-4-[Nmaleimidomethyl]cyclohexane-1-carboxylate (SMCC) 35 was first used for the maleimide functionalisation of the serine residues in Bovine Serum Albumin (BSA) 36 (Scheme 12). The SMCC linker can then be used in the thiol-maleimide conjugation with the neoglycoside 20 to give glycoprotein 37 . The reaction progress was monitored using MALDI-TOF analysis, whereby the average molecular weight of the protein was measured after SMCC conjugation and subsequent glycosylation. Initially, a number of reaction conditions were explored to find the optimal conjugation conditions to obtain highly glycosylated proteins. 

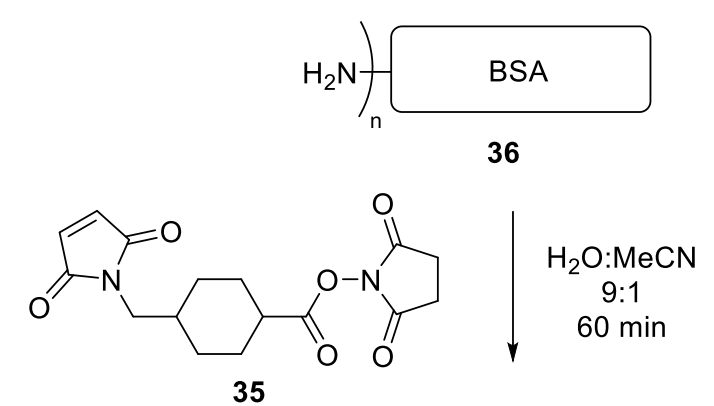

35
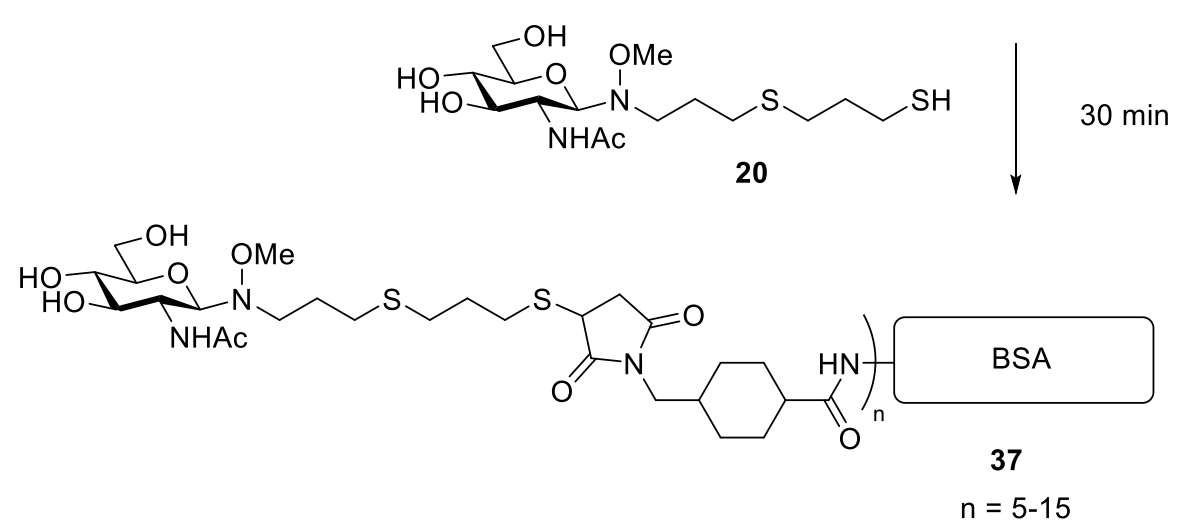

Scheme 12. Glycosylation of BSA 36 using SMCC cross-linker 35 and neoglycoside 20 to give glycoprotein 37.

Given that SMCC 35 is hydrophobic, the cross-linker conjugation was first examined using different concentrations of DMSO in water. To this end, the protein was dissolved in water ( $1 \mathrm{mg} / \mathrm{mL}$ ), followed by the addition of cross-linker (60 equiv.) in DMSO to give 10, 25, 50 and $75 \%$ DMSO in water. In addition, a duplicate experiment was performed using $\mathrm{MeCN}$ instead of DMSO. The reactions were incubated at $\mathrm{rt}$ for $30 \mathrm{~min}$. and a MALDI-TOF sample was prepared. Next, 120 molar equivalents of thiol functionalised glycoside $\mathbf{2 0}$ in water was added and the reaction was incubated for a further 30 minutes, before another MALDI-TOF sample was prepared. From these experiments it was observed that both $10 \%$ DMSO and $10 \% \mathrm{MeCN}$ were sufficient for conjugating the cross-linker to BSA, whereas higher concentrations of organic solvent did not result in much higher cross-linking. That said, low cross-linking $(<5$ linkers $)$ as well as GlcNAc binding was observed throughout all the experiments, which could be explained by low solubility of the protein. Accordingly, when the protein was dissolved in PBS an increased conjugation of both the cross-linker as well as the glycan was observed. Next, the amount of SMCC-linker was explored (60, 120, 180, 360, 720 equiv.) and it was observed that optimal conjugation was achieved using 180 equivalents of SMCC. Here it should be noted that a large excess of cross-linker requires an even larger excess of glycan. With these results in hand, the efficiency of the SMCC cross-linking step as well as the glycan conjugation was analysed over time (Figure 6). Again, BSA $36(66.2 \mathrm{kDa})$ was dissolved in PBS, followed by the addition of SMCC 35 in MeCN (10\% final concentration) and MALDI-TOF samples, as well as $10 \mu \mathrm{L}$ 
samples for glycoconjugation, were taken at $0,15,30,60,120,240$ and $480 \mathrm{~min}$. To each of the $10 \mu \mathrm{L}$ glycoconjugation samples, 10 equiv. of oxyamine $20(10 \mathrm{mg} / \mathrm{mL}$ in water) was added, and for each of these reactions MALDI-TOF samples were taken after 0, 30 and $60 \mathrm{~min}$. From these experiments, it was found that the SMCC conjugation reaction continued over time, however, the glycan conjugation was inhibited after 8-10 glycans were cross-linked. This decrease in glycan conjugation may be due to the SMCC-maleimide moiety cross-linking within the protein, or maleimide degradation without affecting the activated NHS-ester. Nevertheless, it was determined that optimal glycoconjugation can be achieved when the BSA protein $35(10 \mathrm{mg} / \mathrm{mL})$ was reacted with the SMCC cross linker 35 (180 equiv.) in 10\% MeCN in PBS for 60 min before adding an excess of 360 equiv. of the neoglycoside $\mathbf{2 0}$ and incubating the glycoprotein for $60 \mathrm{~min}$ at rt. Although, glycoprotein $\mathbf{3 7}$ was never purified, numerous literature procedures describe this process using dialysis or size exclusion chromatography purification methods, thereby demonstrating yet another application of the oxyamine ligation strategy. ${ }^{47}$

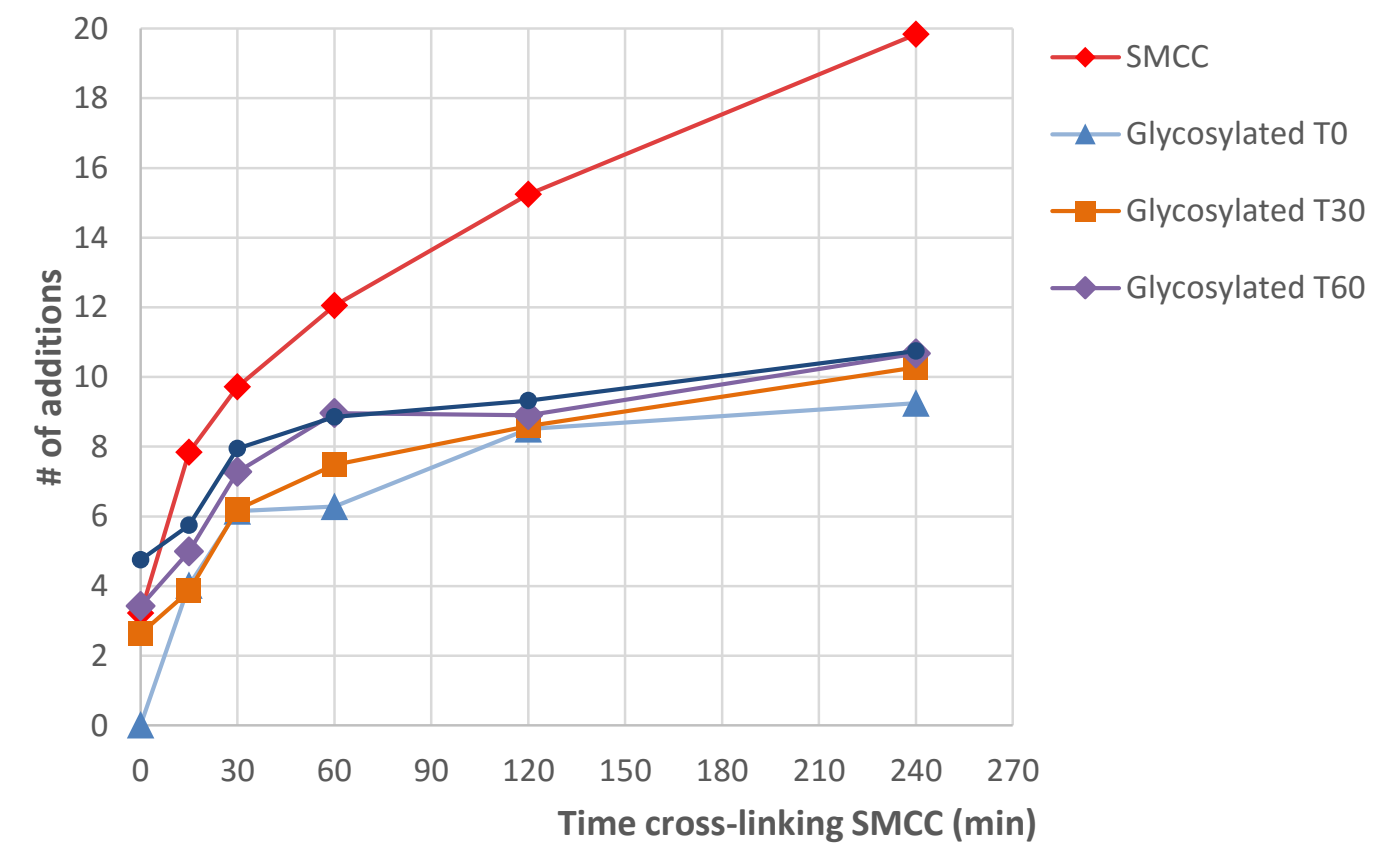

Figure 6. Synthesis of BSA glycoprotein 37. First conjugation of cross-linker SMCC 35 was monitored over time. In addition, glycoside 20 was added at each time point, and measured over a $4 \mathrm{~h}$. time frame. Here, the average addition of cross-linker is calculated by measuring the molecular weight difference between BSA and cross-linked protein. In a similar fashion the glycosylation state is calculated, by subtracting the final molecular weight over the molecular weight increase after conjugation at each time point. 


\subsection{Conclusion}

In conclusion, a variety of oxyamine linkers have been prepared in high yield and in few (3-4) steps. Key in the reaction sequence was the use of acrolein as a Michael acceptor which could then undergo reductive amination with methoxyamine. Many of the linkers can be synthesised using a one-pot protocol, and can be prepared on a gram scale. The linkers can be readily coupled to glycans in excellent yields, and hydrolytic stability studies revealed that the methoxyamine linker is stable over a large $\mathrm{pH}$ range, with exceptional stability at physiological $\mathrm{pH}$. The use of the linker for conjugation to more complex glycans has been demonstrated by the highly efficient coupling to Lewis ${ }^{\mathrm{x}}$. In addition, the versatility of the differently functionalised oxyamine linkers was demonstrated via the synthesis of biotinylated, fluorescently-labelled, and protein-conjugated probes, which were prepared using different reaction conditions, incluing peptide ligation, thiolmaleimide Michael addition and sulfonylation. The ability of the oxyamine linker to participate in the copper-mediated azide-alkyne Huisgen cyclisation reaction was also demonstrated using a model substrate.

Compared to the existing methodology for oxyamine glycan linker synthesis the route presented here has a number of advantages. In particular, the linkers in this thesis are prepared in higher yields (50-96\%) and fewer steps (3-4 steps) when compared to the the linkers of "Type A" (4-6 steps, 10-34\%). In addition, these linkers can be prepared with the functional handle of choice, and thus can be used in the assembly of the glycoconjugate of choice. 


\subsection{Experimental}

General Procedure. Unless otherwise stated all reactions were performed under atmospheric air. $\mathrm{H}_{2} \mathrm{O}$, methanol ( $\mathrm{MeOH}$, Pure Science), ethanol (EtOH, absolute, Pure Science), ethyl acetate (EtOAc, Pure Science), dichloromethane (LabServ) and petroleum ether (PE, Pure Science) were distilled prior to use. Acrolein (Merck), AcOH (Ajax Finechem), $\mathrm{NaN}_{3}$ (BDH), $30 \%$ aqueous $\mathrm{NH}_{3}$ (J. T. Baker Chemical Co.), AcCl (Sigma Aldrich), $\mathrm{PPh}_{3}$ (Acros), $\mathrm{NaCNBH}_{3}$ (Aldrich), $\mathrm{NaOH}$ (Pure Science), NaOAc (Riedel-de Haën), $\mathrm{NaHCO}_{3}$ (Pure Science), $\mathrm{MgSO}_{4}$ (Pure Science), $\mathrm{Na}_{2} \mathrm{CO}_{3}$ (Pure Science), $\mathrm{NH}_{4} \mathrm{OAc}$ (Sigma Aldrich), methoxyamine hydrochloride (Sigma Aldrich), thioacetic acid (Aldrich), sodium (Aldrich), 1,3-propanedithiol (Aldrich), cysteamine hydrochloride (Fluka), $N$-Boc-Cys-OH (Fluka), 2-mercaptoacetic acid (Aldrich) and $N$-Acetylglucosamine (Fluka) were used as received. All solvents were removed by evaporation under reduced pressure. Reactions were monitored by TLC-analysis on silica gel coated plastic sheets $(0.20 \mathrm{~mm}$, silicagel 60$)$ with detection by UV-absorption $(254 \mathrm{~nm})$, by dipping in $10 \%$ $\mathrm{H}_{2} \mathrm{SO}_{4}$ in EtOH followed by charring at $\sim 150{ }^{\circ} \mathrm{C}$, by dipping in $\mathrm{I}_{2}$ in silica, or by dipping in a solution of ninhydrin in $\mathrm{EtOH}$ followed by charring at $\sim 150{ }^{\circ} \mathrm{C}$. Column chromatography was performed on Pure Science silica gel (40-63 micron). AccuBOND II ODS-C18 (Agilent) was used for reverse phase chromatography. Bio-Gel P-2 $(600 \times 10 \mathrm{~mm})$ was used for size exclusion chromatography. Optical rotations were recorded using a Perkin-Elmer 241 polarimeter at the sodium D-line. Infrared spectra were recorded as thin films using a Bruker Tensor 27 FTIR spectrometer equipped with an Attenuated Total Reflectance (ATR) sampling accessory and are reported in wave numbers $\left(\mathrm{cm}^{-1}\right)$. High-resolution mass spectra were recorded on an Agilent Technology 6530 Accurate Mass Q-TOF/LCMS spectrometer using positive electro-spray ionisation. Nuclear magnetic resonance spectra were recorded at $20{ }^{\circ} \mathrm{C}$ in $\mathrm{D}_{2} \mathrm{O}, \mathrm{CDCl}_{3}$ or $\mathrm{CD}_{3} \mathrm{OD}$ using either a Varian Unity-INOVA operating at $300 \mathrm{MHz}$ or a Varian Unity operating at 500 MHz. Chemical shifts are given in ppm $(\delta)$ relative to tetramethylsilane. NMR peak assignments were made using COSY, HSQC and HMBC experiments. 


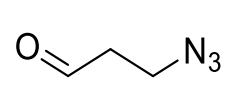

3-Azido-propanal (2). Acetic acid (2.0 mL) was cooled to $-20{ }^{\circ} \mathrm{C}$ and acrolein $(0.92 \mathrm{~mL}, 13.7 \mathrm{mmol})$ was added, followed by dropwise addition of a solution of $\mathrm{NaN}_{3}(1.34 \mathrm{~g}, 20.6 \mathrm{mmol})$ in water $(5.2 \mathrm{~mL})$ and the reaction mixture was stirred for $1 \mathrm{~h}$ at -20 ${ }^{\circ} \mathrm{C}$. The reaction mixture was extracted with $\mathrm{CH}_{2} \mathrm{Cl}_{2}(2 \times 10 \mathrm{~mL})$, and the combined organic extracts were washed with sat. aq. $\mathrm{Na}_{2} \mathrm{CO}_{3}(10 \mathrm{~mL})$ and water $(10 \mathrm{~mL})$, dried over $\mathrm{MgSO}_{4}$, filtered and concentrated in vacuo to afford 3-azido-propanal (1.32 g, $97 \%)$. Due to the instability of the aldehyde the crude material was directly used in the next step. $\mathrm{R}_{f}=0.52$ (PE/EtOAc, 80/20, v/v); IR (film) 2938, 2917, 2848, 2737, 2094, 1718, 1449, 1366, 1286, $1071 \mathrm{~cm}^{-1} ;{ }^{1} \mathrm{H}$ NMR (500 MHz, $\left.\mathrm{CDCl}_{3}\right) \delta 9.80(\mathrm{bs}, 1 \mathrm{H}, \mathrm{CH}-1), 3.61\left(\mathrm{t}, 2 \mathrm{H}, J_{2,3}=6.3 \mathrm{~Hz}, \mathrm{CH}_{2}-3\right), 2.72\left(\mathrm{dt}, 2 \mathrm{H}, J_{1,2}=0.9 \mathrm{~Hz}, J_{2,3}=\right.$ $\left.6.3 \mathrm{~Hz}, \mathrm{CH}_{2}-2\right) ;{ }^{13} \mathrm{C}$ NMR (125 MHz, $\left.\mathrm{CDCl}_{3}\right) \delta 199.4$ (C-1), 44.4 (C-3), 42.6 (C-2); MS (EI): m/z $99(\mathrm{M}+, 1 \%), 71(2 \%), 56(5 \%), 55(6 \%), 43(97 \%), 28(100 \%)$.

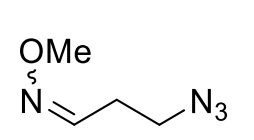

3-Azidopropanal $\boldsymbol{O}$-methyl oxime (3). To a solution of crude 3-azido-propanal ( $847 \mathrm{mg}, 8.55 \mathrm{mmol})$ in $\mathrm{CH}_{2} \mathrm{Cl}_{2}(10 \mathrm{~mL})$, methoxyamine hydrochloride ( $852 \mathrm{mg}$, $10.3 \mathrm{mmol})$ and sodium acetate $(1.40 \mathrm{~g}, 17.1 \mathrm{mmol})$ were added at $\mathrm{rt}$ and the reaction mixture was stirred for $16 \mathrm{~h}$. The reaction mixture was quenched by the addition of sat. aq. $\mathrm{Na}_{2} \mathrm{CO}_{3}(10 \mathrm{~mL})$ and extracted with $\mathrm{CH}_{2} \mathrm{Cl}_{2}(2 \times 10 \mathrm{~mL})$, and the combined organic extracts were washed with brine $(10 \mathrm{~mL})$, dried over $\mathrm{MgSO}_{4}$, filtered and concentrated in vacuo. The residue was purified by column chromatography (PE/EtOAc, 99/1 $\rightarrow$ 97/3, v/v) to give 3azidopropanal $O$-methyl oxime (1.08 g, $99 \%)$ as a colorless oil. $\mathrm{R}_{f}=0.61$ (PE/EtOAc, 90/10, v/v); IR (film) 2939, 2902, 2855, 2821, 2099, 1461, 1442, 1352, 1265, 1240, 1047, 910, $733 \mathrm{~cm}^{-}$ 1. ${ }^{1} \mathrm{H}$ NMR (500 MHz, $\left.\mathrm{CDCl}_{3}\right) \delta 7.39\left(\mathrm{t}, 1 \mathrm{H}, J_{l, 2}=5.5 \mathrm{~Hz}, \mathrm{H}-1\right.$ major $), 6.70\left(\mathrm{t}, 1 \mathrm{H}, J_{l, 2}=5.3 \mathrm{~Hz}\right.$, $\mathrm{H}-1$ minor), 3.89 (s, 3H, OCH 3 minor), 3.84 (s, 3H, OCH 3 major), $3.48\left(\mathrm{t}, 2 \mathrm{H}, J_{2,3}=6.8 \mathrm{~Hz}, \mathrm{CH}_{2}-\right.$ 3 major), 3.45 (t, 2H, $J_{2,3}=6.8 \mathrm{~Hz}, \mathrm{CH}_{2}-3$ minor), 2.60 (dt, $2 \mathrm{H}, J_{1,2}=5.8 \mathrm{~Hz}, J_{2,3}=6.6 \mathrm{~Hz}, \mathrm{CH}_{2}-$ 2 minor), 2.48 (dt, $2 \mathrm{H}, J_{1,2}=5.9 \mathrm{~Hz}, J_{2,3}=6.6 \mathrm{~Hz}, \mathrm{CH}_{2}-2$ major $) ;{ }^{13} \mathrm{C} \mathrm{NMR}\left(125 \mathrm{MHz}, \mathrm{CDCl}_{3}\right) \delta$ 147.3 (C-1 minor), 146.9 (C-1 major), $62.0\left(\mathrm{OCH}_{3}\right.$ minor $), 61.7\left(\mathrm{OCH}_{3}\right.$ major $), 48.5$ (C-3 major), 48.1 (C-3 minor), 29.6 (C-2 major), 25.6 (C-2 minor); HRMS(ESI) $m / z$ calcd. for $\left[\mathrm{C}_{4} \mathrm{H}_{9} \mathrm{~N}_{4} \mathrm{O}\right]^{+}$: 129.0771, obsd.: 129.0779 .<smiles>CONCCCN</smiles>

$\mathrm{N}$-(3-Azidopropyl)-O-methylhydroxylamine (1). Procedure A (Stepwise): To a solution of 3 -azidopropanal $O$-methyl oxime (934 mg, $7.29 \mathrm{mmol}$ ) in ethanol $(15 \mathrm{~mL}), \mathrm{NaCNBH}_{3}(550 \mathrm{mg}, 8.75 \mathrm{mmol})$ was added, followed by dropwise addition of $1 \mathrm{M}$ ethanolic $\mathrm{HCl}(8.75 \mathrm{~mL}$, freshly prepared from $\mathrm{AcCl}$ and $\mathrm{EtOH})$ and the reaction mixture was stirred at $\mathrm{rt}$ for $60 \mathrm{~min}$. The crude mixture was concentrated and the white solid was suspended in sat. aq. $\mathrm{Na}_{2} \mathrm{CO}_{3}(30 \mathrm{~mL})$ and extracted with $\mathrm{CH}_{2} \mathrm{Cl}_{2}(2 \times 30 \mathrm{~mL})$. The combined organic extracts were dried over $\mathrm{MgSO}_{4}$, filtered and concentrated in vacuo. 
Purification of the residue by silica gel column chromatography $\left(\mathrm{CH}_{2} \mathrm{Cl}_{2} / \mathrm{MeOH}, 99 / 1 \rightarrow 90 / 10\right.$, $\mathrm{v} / \mathrm{v}$ ) yielded oxyamine $\mathbf{1}$ as a colorless oil (787 mg, $83 \%)$.

Procedure B (Without isolation of intermediates): Acetic acid $(2.0 \mathrm{~mL})$ was cooled to $-20{ }^{\circ} \mathrm{C}$ and acrolein $(0.92 \mathrm{~mL}, 13.7 \mathrm{mmol})$ was added, followed by dropwise addition of a solution of $\mathrm{NaN}_{3}$ $(1.34 \mathrm{~g}, 20.6 \mathrm{mmol})$ in water $(5.2 \mathrm{~mL})$ and the reaction mixture was stirred for $1 \mathrm{~h}$ at $-20{ }^{\circ} \mathrm{C}$. The crude mixture was quenched by addition of sat. aq. $\mathrm{NaHCO}_{3}(10 \mathrm{~mL})$ and the reaction mixture was extracted with $\mathrm{CH}_{2} \mathrm{Cl}_{2}(2 \times 10 \mathrm{~mL})$, and the combined organic extracts were dried over $\mathrm{MgSO}_{4}$ and filtered. To the solution in $\mathrm{CH}_{2} \mathrm{Cl}_{2}$, methoxyamine hydrochloride (1.37 g, $16.4 \mathrm{mmol}$ ) and sodium acetate $(1.40 \mathrm{~g}, 17.1 \mathrm{mmol})$ were added at $\mathrm{rt}$ and the reaction mixture was stirred at $\mathrm{rt}$ for $16 \mathrm{~h}$. The reaction mixture was then quenched by the addition of sat. aq. $\mathrm{Na}_{2} \mathrm{CO}_{3}(10 \mathrm{~mL})$ and extracted with $\mathrm{CH}_{2} \mathrm{Cl}_{2}(2 \times 10 \mathrm{~mL})$. The combined organic extracts were dried over $\mathrm{MgSO}_{4}$ and filtered. To the solution of crude product in $\mathrm{CH}_{2} \mathrm{Cl}_{2}, \mathrm{NaCNBH}_{3}(1.03 \mathrm{~g}, 16.4 \mathrm{mmol})$ was added, followed by drop wise addition of $1 \mathrm{M}$ ethanolic $\mathrm{HCl}(18 \mathrm{~mL}$, freshly prepared from $\mathrm{AcCl}$ and $\mathrm{EtOH}$ ) and the reaction mixture was stirred at $\mathrm{rt}$ for $1 \mathrm{~h}$. The crude mixture was concentrated and the white solid was suspended in sat. aq. $\mathrm{Na}_{2} \mathrm{CO}_{3}(30 \mathrm{~mL})$ and extracted with $\mathrm{CH}_{2} \mathrm{Cl}_{2}(2 \times 30$ $\mathrm{mL}$ ). The combined organic extracts were dried over $\mathrm{MgSO}_{4}$, filtered and concentrated in vacuo. Purification of the residue by silica gel column chromatography $\left(\mathrm{CH}_{2} \mathrm{Cl}_{2} / \mathrm{MeOH}, 99 / 1 \rightarrow 90 / 10\right.$, v/v) yielded oxyamine 1 (1.71 g, $96 \%$ over 3 steps) as a colorless oil. $\mathrm{R}_{f}=0.15$ (in EtOAc); IR (film) 2937, 2897, 2876, 2811, 2093, 1677, 1464, 1352, 1262, 1125, $1044 \mathrm{~cm}^{-1}$; ${ }^{1} \mathrm{H}$ NMR (500 $\left.\mathrm{MHz}, \mathrm{D}_{2} \mathrm{O}\right) \delta 3.93\left(\mathrm{~s}, 3 \mathrm{H}, \mathrm{OCH}_{3}\right), 3.53\left(\mathrm{t}, 2 \mathrm{H}, J_{2,3}=6.6 \mathrm{~Hz}, \mathrm{CH}_{2}-3\right), 3.45\left(\mathrm{t}, 2 \mathrm{H}, J_{1,2}=7.4 \mathrm{~Hz}\right.$, $\left.\mathrm{CH}_{2}-1\right), 2.02\left(\mathrm{tt}, 2 \mathrm{H}, J_{1,2}=J_{2,3}=6.8 \mathrm{~Hz}, \mathrm{CH}_{2}-2\right) ;{ }^{13} \mathrm{C} \mathrm{NMR}\left(125 \mathrm{MHz}, \mathrm{CDCl}_{3}\right) \delta 61.6\left(\mathrm{OCH}_{3}\right)$, 48.2 (C-3), 46.6 (C-1), 22.8 (C-2); HRMS(ESI) $\mathrm{m} / z$ calcd. for $\left[\mathrm{C}_{4} \mathrm{H}_{11} \mathrm{~N}_{4} \mathrm{O}\right]^{+}$: 131.0927, obsd.: 131.0929.

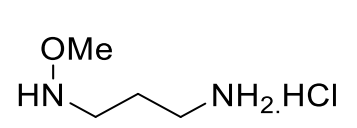

3-(Methoxyamino)propan-1-amine hydrochloride (4). To a solution of $\mathrm{N}$-(3-Azidopropyl)- $O$-methylhydroxylamine 1 (95.6 mg, $0.74 \mathrm{mmol}$ ) in $\mathrm{CH}_{2} \mathrm{Cl}_{2}: \mathrm{EtOH}: \mathrm{H}_{2} \mathrm{O}$ (1:3:1), triphenylphosphine (385 mg, $1.47 \mathrm{mmol}$ ) was added and the reaction mixture was stirred at $\mathrm{rt}$ for $16 \mathrm{~h}$. The crude mixture was concentrated in vacuo, $1 \mathrm{M} \mathrm{HCl}(5 \mathrm{~mL})$ was added and the mixture was filtered and the residue was purified by reverse phase chromatography $\left(\mathrm{C}_{18}, \mathrm{H}_{2} \mathrm{O}\right)$ to yield amine $\mathbf{4}$ as a white solid $(102 \mathrm{mg}, 0.73 \mathrm{mmol}$, $99 \%) . \mathrm{R}_{f}=0.24\left(\mathrm{CH}_{2} \mathrm{Cl}_{2}: \mathrm{EtOH}: \mathrm{MeOH}: \mathrm{NH}_{3}\right.$ (aq. 35\%), 10:2:2:1, v/v/v/v); IR (film) 3400, 2950, 2766, 2695, 2492, 1611, 1475, 1272, 1163, 1016, 954, $758 \mathrm{~cm}^{-1} ;{ }^{1} \mathrm{H}$ NMR (300 MHz, $\left.\mathrm{D}_{2} \mathrm{O}\right) \delta 3.85$ (s, $\left.3 \mathrm{H}, \mathrm{OCH}_{3}\right), 3.40\left(\mathrm{t}, 2 \mathrm{H}, J_{l, 2}=7.6 \mathrm{~Hz}, \mathrm{CH}_{2}-1\right), 3.07$ (t, $\left.2 \mathrm{H}, J_{2,3}=7.6 \mathrm{~Hz}, \mathrm{CH}_{2}-3\right), 2.08(\mathrm{tt}, 2 \mathrm{H}$, $\left.J_{1,2}=J_{2,3}=7.6 \mathrm{~Hz}, \mathrm{CH}_{2}-2\right) ;{ }^{13} \mathrm{C}$ NMR $\left(125 \mathrm{MHz}, \mathrm{D}_{2} \mathrm{O}\right) \delta 61.5\left(\mathrm{OCH}_{3}\right), 45.9(\mathrm{C}-1), 36.8(\mathrm{C}-3)$, 21.7 (C-2); HRMS(ESI) $m / z$ calcd. for $\left[\mathrm{C}_{4} \mathrm{H}_{13} \mathrm{~N}_{2} \mathrm{O}\right]^{+}:$105.1022, obsd.: 105.1023. 


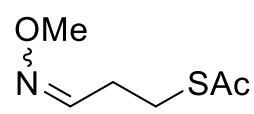

S-3-(Methoxyimino)propyl thioacetate (6). Acrolein $(0.90 \mathrm{~mL}, 13.5 \mathrm{mmol})$ was added dropwise to thioacetic acid $(1.04 \mathrm{~mL}, 14.8 \mathrm{mmol})$ and the reaction mixture was stirred at $\mathrm{rt}$ for $2 \mathrm{~h}$. The crude $S$-3-oxopropyl thioacetate 5 was used in the next step. To a solution of crude $S$-3-oxopropyl thioacetate 5 (13.5 mmol) in $\mathrm{CH}_{2} \mathrm{Cl}_{2}$ $(13.5 \mathrm{~mL})$, methoxyamine hydrochloride $(1.35 \mathrm{~g}, 16.2 \mathrm{mmol})$ and sodium acetate $(2.21 \mathrm{~g}, 26.9$ mmol) were added and the reaction mixture was stirred $16 \mathrm{~h}$ at $\mathrm{rt}$. The crude mixture was then quenched with sat. aq. $\mathrm{Na}_{2} \mathrm{CO}_{3}(25 \mathrm{~mL})$ and extracted with $\mathrm{CH}_{2} \mathrm{Cl}_{2}(2 \times 10 \mathrm{~mL})$, and the combined organic extracts were washed with brine $(10 \mathrm{~mL})$, dried over $\mathrm{MgSO}_{4}$, filtered and concentrated in vacuo. The residue was purified by silica gel column chromatography (PE/EtOAc, 95/5 $\rightarrow$ 80/20, $\mathrm{v} / \mathrm{v})$ to give a mixture of $S$-3-(methoxyimino)propyl thioacetate 6 (2.05 g, $94 \%$ over 2 steps) as a colorless oil. $\mathrm{R}_{f}=0.69(\mathrm{PE} / \mathrm{EtOAc}, 75 / 25$, v/v); IR (film) 2991, 2961, 2939, 2901, 2820, 1689, $1634,1466,1428,1355,1282,1132,1104,1085,1038,952,912,892,858,734,623 \mathrm{~cm}^{-1} ;{ }^{1} \mathrm{H}$ $\operatorname{NMR}\left(500 \mathrm{MHz}, \mathrm{CDCl}_{3}\right) \delta 7.29\left(\mathrm{t}, 1 \mathrm{H}, J_{l, 2}=5.7 \mathrm{~Hz}, \mathrm{H}-1\right.$ major $), 6.58\left(\mathrm{t}, 1 \mathrm{H}, J_{l, 2}=5.4 \mathrm{~Hz}, \mathrm{H}-1\right.$ minor), 3.80 (s, 3H, $\mathrm{OCH}_{3}$ minor), 3.75 (s, $3 \mathrm{H}, \mathrm{OCH}_{3}$ major), 2.97 (t, $2 \mathrm{H}, J_{2,3}=7.1 \mathrm{~Hz}, \mathrm{CH}_{2}-3$ major), $2.95\left(\mathrm{t}, 2 \mathrm{H}, J_{2,3}=7.2 \mathrm{~Hz}, \mathrm{CH}_{2}-3\right.$ minor), $2.54\left(\mathrm{dt}, 2 \mathrm{H}, J_{1,2}=5.4 \mathrm{~Hz}, J_{2,3}=7.2 \mathrm{~Hz}, \mathrm{CH}_{2}-2\right.$ minor), 2.41 (dt, $2 \mathrm{H}, J_{1,2}=5.7 \mathrm{~Hz}, J_{2,3}=7.1 \mathrm{~Hz}, \mathrm{CH}_{2}-2$ major), 2.28 (s, 3H, SAc minor), 2.27 (s, 3H, SAc major); ${ }^{13} \mathrm{C}$ NMR (125 MHz, $\left.\mathrm{CDCl}_{3}\right) \delta 195.22$ (C=O minor), 195.19 (C=O major), 148.6 (C-1, C=N minor), 148.1 (C-1, C=N major), $61.7\left(\mathrm{OCH}_{3}\right.$ minor), $61.4\left(\mathrm{OCH}_{3}\right.$ major), 30.6 ( $\mathrm{SAc}$ major + minor), 29.8 (C-2 major), 25.9 (C-3 major), 25.8 (C-2 minor), 25.6 (C-3 minor); HRMS(ESI) $m / z$ calcd. for $\left[\mathrm{C}_{6} \mathrm{H}_{12} \mathrm{NO}_{2} \mathrm{~S}\right]^{+}: 162.0583$, obsd.: 162.0585 .

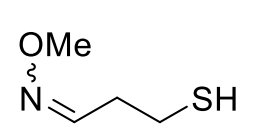

3-Mercaptopropanal $\boldsymbol{O}$-methyl oxime (7). To prevent disulfide formation all solvents used during the reaction and purification were pre-purged with argon gas. To imine 6 (151 mg, $0.93 \mathrm{mmol}$ ), 1M methanolic sodium methoxide ( $2 \mathrm{~mL}$, freshly prepared from sodium and methanol) was added at $\mathrm{rt}$ and the reaction mixture was stirred under argon for $30 \mathrm{~min}$. The reaction mixture was then washed with sat. aq. $\mathrm{Na}_{2} \mathrm{CO}_{3}(10 \mathrm{~mL})$ and extracted with $\mathrm{CH}_{2} \mathrm{Cl}_{2}(2 \times 10 \mathrm{~mL})$, and the combined $\mathrm{CH}_{2} \mathrm{Cl}_{2}$ solutions were dried over $\mathrm{MgSO}_{4}$, filtered and concentrated in vacuo to give crude 3-mercaptopropanal $O$-methyl oxime 7 (107 $\mathrm{mg}$, $96 \%$ ) as a colorless oil. $\mathrm{R}_{f}=0.63(\mathrm{PE} / \mathrm{EtOAc}, 75 / 25$, v/v); IR (film) 3002, 2990, 2937, 2923, 2819, 1465, 1437, 1279, 1080, 1036, 916, 857, $743 \mathrm{~cm}^{-1}$; ${ }^{1} \mathrm{H} \mathrm{NMR}\left(300 \mathrm{MHz}, \mathrm{CDCl}_{3}\right) \delta 7.39(\mathrm{t}$, $1 \mathrm{H}, J_{1-2}=5.6 \mathrm{~Hz}, \mathrm{H}-1$ major $), 6.71\left(\mathrm{t}, 1 \mathrm{H}, J_{1-2}=4.7 \mathrm{~Hz}, \mathrm{H}-1\right.$ minor $), 3.87\left(\mathrm{~s}, 3 \mathrm{H}, \mathrm{OCH}_{3}\right.$ minor $)$, 3.84 (s, 3H, $\mathrm{OCH}_{3}$ major), 2.88-2.46 (m, 4H, $\mathrm{CH}_{2}-2$ minor, $\mathrm{CH}_{2}-2$ major, $\mathrm{CH}_{2}-3$ minor, $\mathrm{CH}_{2}-3$ major), 1.52 (t, $1 \mathrm{H}, J_{3-S H}=8.0 \mathrm{~Hz}, \mathrm{SH}$ major), $1.44\left(\mathrm{t}, 1 \mathrm{H}, J_{3-S H}=7.8 \mathrm{~Hz}, \mathrm{SH}\right.$ minor $) ;{ }^{13} \mathrm{C} \mathrm{NMR}$ $\left(125 \mathrm{MHz}, \mathrm{CDCl}_{3}\right) \delta 149.0$ (C-1 minor) 148.4 (C-1 major), $61.9\left(\mathrm{OCH}_{3}\right.$ major $), 61.6\left(\mathrm{OCH}_{3}\right.$ minor), 33.9 (C-2 major), 30.0 (C-2 major), 21.8 (C-3 major), 21.4 (C-3 minor); HRMS(ESI) m/z calcd. for $\left[\mathrm{C}_{4} \mathrm{H}_{10} \mathrm{NOS}\right]^{+}: 120.0478$, obsd.: 120.0483 . 
SH

3-(Methoxyamino)propane-1-thiol (8). To prevent disulfide formation all solvents used during the reaction and purification were pre-purged with argon gas. To a solution of 3-mercaptopropanal $O$-methyl oxime ( $95 \mathrm{mg}, 0.80 \mathrm{mmol}$ ) and $\mathrm{NaCNBH}_{3}(60 \mathrm{mg}, 0.96 \mathrm{mmol})$ in ethanol, $1 \mathrm{M}$ ethanolic $\mathrm{HCl}(2 \mathrm{~mL}$, freshly prepared from $\mathrm{AcCl}$ and $\mathrm{EtOH}$ ) was added dropwise and the reaction mixture was stirred at $\mathrm{rt}$ for $1 \mathrm{~h}$. The crude mixture was concentrated in vacuo, suspended in sat. aq. $\mathrm{Na}_{2} \mathrm{CO}_{3}(30 \mathrm{~mL})$ and extracted with $\mathrm{CH}_{2} \mathrm{Cl}_{2}(2 \times 30 \mathrm{~mL})$. The combined organic extracts were dried over $\mathrm{MgSO}_{4}$, filtered and concentrated in vacuo. 1,3-propanedithiol was added to reduce disulfides. The residue was purified by silica gel flash column chromatography $\left(\mathrm{CH}_{2} \mathrm{Cl}_{2} / \mathrm{EtOH} / \mathrm{MeOH} / \mathrm{NH}_{3}\right.$ (aq. 33\%), $30 / 2 / 2 / 1 \rightarrow 20 / 2 / 2 / 1, \mathrm{v} / \mathrm{v} / \mathrm{v} / \mathrm{v}$ ) yielded 3-(methoxyamino)propane-1-thiol 8 (84 $\mathrm{mg}, 87 \%$ ) as a colorless oil. $\mathrm{R}_{f}=0.65\left(\mathrm{CH}_{2} \mathrm{Cl}_{2} / \mathrm{EtOH} / \mathrm{MeOH} / \mathrm{NH}_{3}\right.$ (aq. 33\%), 25/2/2/1, v/v/v/v); IR (film) 3246, 3237, 3064, 2939, 2842, 2411, 2366, 2360, 2343, 1739, 1676, 1440, 1365, 1217, 1121, $1023 \mathrm{~cm}^{-1}$; ${ }^{1} \mathrm{H}$ NMR (300 MHz, $\left.\mathrm{D}_{2} \mathrm{O}\right) \delta 4.04\left(\mathrm{~s}, 3 \mathrm{H}, \mathrm{OCH}_{3}\right), 3.59$ (t, 2H, $\left.J_{l-2}=7.5 \mathrm{~Hz}, \mathrm{CH}_{2}-1\right), 2.79(\mathrm{t}, 2 \mathrm{H}$, $\left.J_{2-3}=6.8 \mathrm{~Hz}, \mathrm{CH}_{2}-3\right), 2.16\left(\mathrm{p}, 2 \mathrm{H}, J_{l-2}=J_{2-3}=7.4 \mathrm{~Hz}, \mathrm{CH}_{2}-2\right) ;{ }^{13} \mathrm{C} \mathrm{NMR}\left(125 \mathrm{MHz}, \mathrm{D}_{2} \mathrm{O}\right) \delta 64.3$ $\left(\mathrm{OCH}_{3}\right), 50.4(\mathrm{C}-1), 30.2(\mathrm{C}-2), 23.7(\mathrm{C}-3)$; HRMS(ESI) $\mathrm{m} / \mathrm{z}$ calcd. for $\left[\mathrm{C}_{4} \mathrm{H}_{12} \mathrm{NOS}\right]^{+}: 122.0634$, obsd.: 122.0635 .

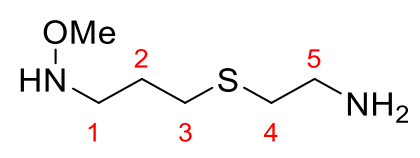

2-(3-(Methoxyamino)propylthio)ethanamine (9). To cysteamine hydrochloride $(186 \mathrm{mg}, 1.64 \mathrm{mmol})$ in ethanol $(2 \mathrm{~mL})$, acrolein $(0.10 \mathrm{~mL}, 1.50 \mathrm{mmol})$ was added dropwise and the reaction mixture was stirred at $\mathrm{rt}$ for $2 \mathrm{~h}$. The crude mixture was dissolved in EtOH $(5 \mathrm{~mL})$ and methoxyamine hydrochloride (150 mg, $1.80 \mathrm{mmol})$ and sodium acetate $(246 \mathrm{mg}, 3.00 \mathrm{mmol})$ were added and the reaction mixture was stirred at $\mathrm{rt}$ for $16 \mathrm{~h}$. To the mixture, $\mathrm{NaCNBH}_{3}(141 \mathrm{mg}, 2.24 \mathrm{mmol})$ was added, followed by dropwise addition of $1 \mathrm{M}$ ethanolic $\mathrm{HCl}(5 \mathrm{~mL}$, freshly prepared from $\mathrm{AcCl}$ and $\mathrm{EtOH}$ ), and the reaction mixture was stirred at $\mathrm{rt}$ for $1 \mathrm{~h}$. The crude mixture was concentrated and the white solid was suspended in sat. aq. $\mathrm{Na}_{2} \mathrm{CO}_{3}(30 \mathrm{~mL})$ and extracted with $\mathrm{CH}_{2} \mathrm{Cl}_{2}(2 \times 30$ $\mathrm{mL}$ ). The combined organic extracts were dried over $\mathrm{MgSO}_{4}$, filtered and concentrated. Purification of the residue by silica gel column chromatography $\left(\mathrm{CH}_{2} \mathrm{Cl}_{2} / \mathrm{EtOH} / \mathrm{MeOH} / \mathrm{NH}_{3}\right.$ (aq. $33 \%$ ), 20/2/2/1 $\rightarrow$ 10/2/2/1, v/v/v/v) yielded methoxyamine $9(163 \mathrm{mg}, 66 \%)$ as a colorless oil. $\mathrm{R}_{f}=0.56\left(\mathrm{CH}_{2} \mathrm{Cl}_{2} / \mathrm{EtOH} / \mathrm{MeOH} / \mathrm{NH}_{3}\right.$ (aq. 33\%), 25/2/2/1, v/v/v/v); IR (film) 3381, 3247, 3015, 2939, 2933, 1738, 1698, 1635, 1508, 1464, 1376, 1230, 1217, $1030 \mathrm{~cm}^{-1} ;{ }^{1} \mathrm{H}$ NMR (500 MHz, $\left.\mathrm{D}_{2} \mathrm{O}\right) \delta 3.55\left(\mathrm{~s}, 3 \mathrm{H}, \mathrm{OCH}_{3}\right), 3.21\left(\mathrm{t}, 2 \mathrm{H}, J_{4,5}=6.6 \mathrm{~Hz}, \mathrm{CH}_{2}-5\right), 3.00\left(\mathrm{t}, 2 \mathrm{H}, J_{1,2}=7.2 \mathrm{~Hz}, \mathrm{CH}_{2}-1\right)$, $2.86\left(\mathrm{t}, 2 \mathrm{H}, J_{4,5}=6.6 \mathrm{~Hz}, \mathrm{CH}_{2}-4\right), 2.65\left(\mathrm{t}, 2 \mathrm{H}, J_{2,3}=7.4 \mathrm{~Hz}, \mathrm{CH}_{2}-3\right), 1.86\left(\mathrm{p}, 2 \mathrm{H}, J_{1,2}=J_{2,3}=7.2\right.$ $\left.\mathrm{Hz}, \mathrm{CH}_{2}-2\right) ;{ }^{13} \mathrm{C}$ NMR $\left(125 \mathrm{MHz}, \mathrm{D}_{2} \mathrm{O}\right) \delta 60.5\left(\mathrm{OCH}_{3}\right), 49.1$ (C-1), $39.5(\mathrm{C}-5), 33.3(\mathrm{C}-3), 28.3(\mathrm{C}-$ 4), 26.0 (C-2); HRMS(ESI) $\mathrm{m} / z$ calcd. for $\left[\mathrm{C}_{6} \mathrm{H}_{12} \mathrm{NO}_{2} \mathrm{~S}\right]^{+}:$165.1056, obsd.: 165.1058. 


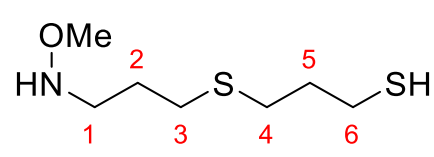

3-(3-(Methoxyamino)propylthio)propane-1-thiol (10). To neat 1,3-propanedithiol (1.50 mL, $15.0 \mathrm{mmol})$, acrolein $(0.20 \mathrm{~mL}, 2.99$ mmol) was added dropwise and the reaction mixture was stirred at $\mathrm{rt}$ for $2 \mathrm{~h}$. The crude reaction mixture was diluted with EtOH $(5 \mathrm{~mL})$ and methoxyamine hydrochloride ( $300 \mathrm{mg}, 3.59 \mathrm{mmol})$ and sodium acetate $(492 \mathrm{mg}, 6.00 \mathrm{mmol})$ were added and the reaction mixture was stirred at $\mathrm{rt}$ for $16 \mathrm{~h}$. To the crude mixture, $\mathrm{NaCNBH}_{3}(282 \mathrm{mg}, 4.49 \mathrm{mmol})$ was added, followed by dropwise addition of $1 \mathrm{M}$ ethanolic $\mathrm{HCl}(10 \mathrm{~mL}$, freshly prepared from $\mathrm{AcCl}$ and $\mathrm{EtOH}$ ), and the reaction mixture was stirred at $\mathrm{rt}$ for $1 \mathrm{~h}$. The crude mixture was concentrated in vacuo, suspended in sat. aq. $\mathrm{Na}_{2} \mathrm{CO}_{3}(30 \mathrm{~mL})$ and extracted with $\mathrm{CH}_{2} \mathrm{Cl}_{2}(2 \times 10$ $\mathrm{mL}$ ). The combined organic extracts were dried over $\mathrm{MgSO}_{4}$, filtered and concentrated in vacuo. Purification of the residue by silica gel column chromatography $\left(\mathrm{CH}_{2} \mathrm{Cl}_{2} / \mathrm{MeOH}, 100 / 0 \rightarrow 98 / 2\right.$, $\mathrm{v} / \mathrm{v}$ ) yielded methoxyamine 10 (442 mg, $76 \%$ ) as a colorless oil. $\mathrm{R}_{f}=0.60$ (PE/EtOAc, 50/50, v/v); IR (film) 3246, 3933, 2851, 2807, 1692, 1464, 1436, 1366, 1295, 1261, 1190, 1075, 1026, 958, 914, 834, 732, 647, $626 \mathrm{~cm}^{-1} ;{ }^{1} \mathrm{H}$ NMR (500 MHz, D $\left.{ }_{2} \mathrm{O}\right) \delta 5.59$ (bs, $\left.1 \mathrm{H}, \mathrm{NH}\right), 3.52$ (s, 3H, $\left.\mathrm{OCH}_{3}\right), 3.00\left(\mathrm{dt}, 2 \mathrm{H}, J_{l, N H}=1.0 \mathrm{~Hz}, J_{l, 2}=6.9 \mathrm{~Hz}, \mathrm{CH}_{2}-1\right), 2.66-2.61\left(\mathrm{~m}, 4 \mathrm{H}, \mathrm{CH}_{2}-4, \mathrm{CH}_{2}-6\right)$, $2.59\left(\mathrm{t}, 2 \mathrm{H}, J_{6, S H}=J_{5,6}=7.1 \mathrm{~Hz}, \mathrm{CH}_{2}-3\right), 1.88\left(\mathrm{p}, 2 \mathrm{H}, J_{4,5}=J_{5,5}=7.1 \mathrm{~Hz}, \mathrm{CH}_{2}-5\right), 1.81\left(\mathrm{p}, 2 \mathrm{H}, J_{1,2}\right.$ $\left.=J_{2,3}=7.0 \mathrm{~Hz}, \mathrm{CH}_{2}-2\right), 1.36\left(\mathrm{t}, 1 \mathrm{H}, J_{6, S H}=8.0 \mathrm{~Hz}, \mathrm{SH}\right) ;{ }^{13} \mathrm{C} \mathrm{NMR}\left(125 \mathrm{MHz}, \mathrm{D}_{2} \mathrm{O}\right) \delta 62.0\left(\mathrm{OCH}_{3}\right)$, 50.8 (C-1), 33.4 (C-5), 30.4 (C-4), 29.9 (C-3), 27.3 (C-2), 23.6 (C-6); HRMS(ESI) m/z calcd. for $\left[\mathrm{C}_{7} \mathrm{H}_{18} \mathrm{NOS}_{2}\right]^{+}: 196.0824$, obsd.: 196.0819 .

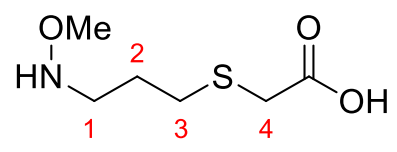

2-(3-(Methoxyamino)propylthio)acetic acid (11). To neat 2-mercaptoacetic acid $(0.16 \mathrm{~mL}, 2.25 \mathrm{mmol})$, acrolein $(0.10 \mathrm{~mL}, 1.50$ mmol) was added drop wise at $0{ }^{\circ} \mathrm{C}$ and the reaction mixture was stirred at $\mathrm{rt}$ for $30 \mathrm{~min}$. The crude was dissolved in ethanol $(1.0 \mathrm{~mL})$ and sodium acetate $(246 \mathrm{mg}$, $3.00 \mathrm{mmol})$ and methoxyamine hydrochloride $(250 \mathrm{mg}, 2.99 \mathrm{mmol})$ were added and the reaction mixture was stirred at $\mathrm{rt}$ for $16 \mathrm{~h}$. To the crude mixture, $\mathrm{NaCNBH}_{3}(141 \mathrm{mg}, 2.24 \mathrm{mmol})$ and $1 \mathrm{M}$ ethanolic $\mathrm{HCl}(7.5 \mathrm{~mL}$, freshly prepared from ethanol and $\mathrm{AcCl})$ were added and the reaction mixture was stirred for $1 \mathrm{~h}$. The crude reaction mixture was quenched by the addition of $1 \mathrm{M}$ $\mathrm{NaOH}$ to $\mathrm{pH} 9$ and the crude was concentrated in vacuo. Purification of the residue by silica gel column chromatography $\left(\mathrm{CH}_{2} \mathrm{Cl}_{2} / \mathrm{EtOH} / \mathrm{MeOH} / \mathrm{NH}_{3}\right.$ (aq. 33\%, 10/2/2/1 $\rightarrow 5 / 2 / 2 / 1$, v/v/v/v)) afforded amino acid 11 (214 mg, $79 \%) ; \mathrm{R}_{f}=0.31\left(\mathrm{CH}_{2} \mathrm{Cl}_{2} / \mathrm{EtOH} / \mathrm{MeOH} / \mathrm{NH}_{3}\right.$ (aq. 33\%), 10/2/2/1, v/v/v/v); IR (film) 3128, 3039, 2939, 2807, 1664, 1562, 1440, 1401, 1298, 1223, 1139 , 1043, 903, 785, 687, $587 \mathrm{~cm}^{-1}$; ${ }^{1} \mathrm{H}$ NMR $\left(500 \mathrm{MHz}, \mathrm{D}_{2} \mathrm{O}\right) \delta 4.04\left(\mathrm{~s}, 3 \mathrm{H}, \mathrm{OCH}_{3}\right), 3.59\left(\mathrm{t}, 2 \mathrm{H}, J_{1,2}\right.$ $\left.=7.6 \mathrm{~Hz}, \mathrm{CH}_{2}-1\right), 3.56\left(\mathrm{~s}, 2 \mathrm{H}, \mathrm{CH}_{2}-4\right), 2.88\left(\mathrm{t}, 2 \mathrm{H}, J_{2,3}=7.1 \mathrm{~Hz}, \mathrm{CH}_{2}-3\right), 2.17\left(\mathrm{t}, 2 \mathrm{H}, J_{l, 2}=J_{2,3}=\right.$ $\left.7.3 \mathrm{~Hz}, \mathrm{CH}_{2}-2\right) ;{ }^{13} \mathrm{C}$ NMR (125 MHz, $\left.\mathrm{D}_{2} \mathrm{O}\right) \delta 174.9(\mathrm{C}=\mathrm{O}), 61.9\left(\mathrm{OCH}_{3}\right), 47.7(\mathrm{C}-1), 33.4(\mathrm{C}-3)$, 28.9 (C-4), 22.7 (C-2); HRMS(ESI) $\mathrm{m} / z$ calcd. for $\left[\mathrm{C}_{6} \mathrm{H}_{12} \mathrm{NO}_{3} \mathrm{~S}\right]=:$ : 178.0543, obsd.: 178.0547. 


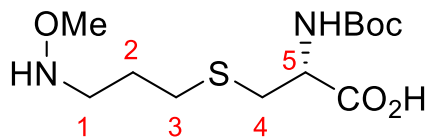

$S$-(3-(Methoxyamino)propyl)- $N$-Boc-L-cysteine (12). To neat $N$ Boc-Cys-OH (332 mg, $1.50 \mathrm{mmol})$, acrolein $(0.1 \mathrm{~mL}, 1.50 \mathrm{mmol})$ was added dropwise and the reaction mixture was stirred at $\mathrm{rt}$ for $2 \mathrm{~h}$. The crude reaction mixture was dissolved in a mixture of $\mathrm{CH}_{2} \mathrm{Cl}_{2}(1 \mathrm{~mL})$ and $\mathrm{EtOH}(1 \mathrm{~mL})$, methoxyamine hydrochloride $(250 \mathrm{mg}, 1.8 \mathrm{mmol})$ and sodium acetate $(246 \mathrm{mg}, 3.0 \mathrm{mmol})$ were added and the reaction mixture was stirred at $\mathrm{rt}$ for $16 \mathrm{~h}$. To the crude mixture, $\mathrm{NaCNBH}_{3}(141$ $\mathrm{mg}, 2.24 \mathrm{mmol})$ was added, followed by dropwise addition of $1 \mathrm{M}$ ethanolic $\mathrm{HCl}(7.5 \mathrm{~mL}$, freshly prepared from $\mathrm{AcCl}$ and $\mathrm{EtOH}$ ), and the reaction mixture was stirred at $\mathrm{rt}$ for $60 \mathrm{~min}$. The crude reaction mixture was quenched by the addition of $1 \mathrm{M} \mathrm{NaOH}$ to $\mathrm{pH} 9$ and the crude was concentrated in vacuo. Purification of the residue by silica gel column chromatography $\left(\mathrm{CH}_{2} \mathrm{Cl}_{2} / \mathrm{MeOH}, 95 / 5 \rightarrow 80 / 20\right.$, v/v) yielded methoxyamine 12 (236 mg, $\left.51 \%\right)$ as a colorless oil. $\mathrm{R}_{f}=0.15\left(\mathrm{CH}_{2} \mathrm{Cl}_{2} / \mathrm{EtOH} / \mathrm{MeOH} / \mathrm{NH}_{3}(\mathrm{aq} .33 \%), 10 / 2 / 2 / 1, \mathrm{v} / \mathrm{v} / \mathrm{v} / \mathrm{v}\right) ; \alpha_{\mathrm{D}}{ }^{20.1}=-7.5(\mathrm{c}=1, \mathrm{MeOH}) ;$ IR (film) 3335, 2976, 2934, 1687, 1584, 1509, 1416, 1394, 1367, 1249, 1167, 1051, 1025, 868, 777, 659, $578 \mathrm{~cm}^{-1} ;{ }^{1} \mathrm{H}$ NMR (500 MHz, CD $\left.{ }_{3} \mathrm{OD}\right) \delta 4.22-4.17(\mathrm{~m}, 1 \mathrm{H}, \mathrm{H}-5), 3.50\left(\mathrm{~s}, 3 \mathrm{H}, \mathrm{OCH}_{3}\right)$, $3.01\left(\mathrm{dd}, 1 \mathrm{H}, J_{4 a, 5}=4.4 \mathrm{~Hz}, J_{4 a, 4 b}=13.7 \mathrm{~Hz}, \mathrm{H}-4 \mathrm{a}\right), 2.95\left(\mathrm{t}, 2 \mathrm{H}, J_{1,2}=7.0 \mathrm{~Hz}, \mathrm{CH}_{2}-1\right), 2.84(\mathrm{dd}$, $\left.1 \mathrm{H}, J_{4 b, 5}=7.3 \mathrm{~Hz}, J_{4 a, 4 b}=13.7 \mathrm{~Hz}, \mathrm{H}-4 \mathrm{~b}\right), 2.64\left(\mathrm{t}, 2 \mathrm{H}, J_{2,3}=7.2 \mathrm{~Hz}, \mathrm{CH}_{2}-3\right), 1.79\left(\mathrm{p}, 2 \mathrm{H}, J_{1,2}=J_{2,3}\right.$ $\left.=7.1 \mathrm{~Hz}, \mathrm{CH}_{2}-2\right), 1.45\left(\mathrm{~s}, 9 \mathrm{H}, \mathrm{CH}_{3} t \mathrm{Bu}\right) ;{ }^{13} \mathrm{C} \mathrm{NMR}\left(125 \mathrm{MHz}, \mathrm{CD}_{3} \mathrm{OD}\right) \delta 177.7(\mathrm{COOH}), 157.7$ $(\mathrm{C}=\mathrm{ONH}), 80.5(\mathrm{Cq}), 61.6\left(\mathrm{OCH}_{3}\right), 56.1(\mathrm{C}-5 \alpha \mathrm{CH}), 50.8(\mathrm{C}-1), 35.5(\mathrm{C}-4), 30.9(\mathrm{C}-3), 28.8\left(\mathrm{CH}_{3}\right.$ $t \mathrm{Bu}), 27.7$ (C-2); HRMS(ESI) $\mathrm{m} / z$ calcd. for $\left[\mathrm{C}_{12} \mathrm{H}_{25} \mathrm{~N}_{2} \mathrm{O}_{5} \mathrm{~S}\right]^{+}:$309.1502, obsd.: 309.1479.
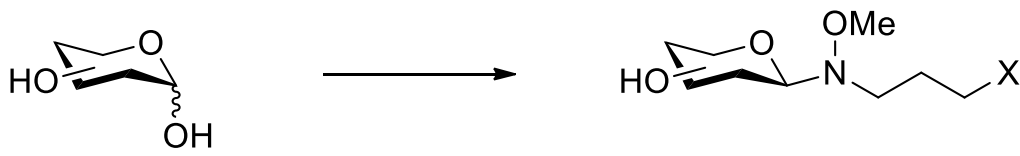

General procedure for the chemical ligation of methoxyamine linkers with carbohydrates. To a solution of carbohydrate $(0.15 \mathrm{mmol})$ in an $\mathrm{AcOH} / \mathrm{NH}_{4} \mathrm{OAc}$ buffer $(1.5 \mathrm{~mL}, 2 \mathrm{M}$, freshly prepared, $\mathrm{pH} 4.5)$, methoxyamine linker $(1.50 \mathrm{mmol})$ was added and the reaction mixture was stirred at $\mathrm{rt}$ for $24 \mathrm{~h}$ at the indicated temperature. The crude mixture was then directly loaded onto a size exclusion column $\left(\right.$ Bio-Gel P-2, $1200 \times 18 \mathrm{~mm}$ ) and eluted with a $0.1 \mathrm{M}$ aq. $\mathrm{NH}_{4} \mathrm{HCO}_{3}$ solution. Lyophilisation of the product fractions afforded the neoglycoside. Alternatively, the neoglycoside can be purified by directly loading the crude reaction mixture onto a reverse phase column $\left(\mathrm{C}_{18}\right)$ and/or by purification by silica gel column chromatography to afford the neoglycoside.

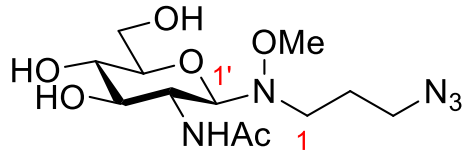

$N$-(2-Acetamido-2-deoxy- $\beta$-D-glucopyranosyl)- $N$-(3azidopropyl)- $\boldsymbol{O}$-methylhydroxylamine $\quad$ (14). $\quad N$-Acetyl-

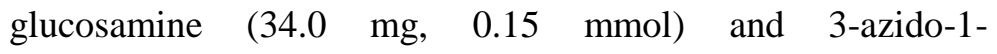


methoxyaminopropane $1(195 \mathrm{mg}, 1.50 \mathrm{mmol})$ were reacted in an $\mathrm{AcOH} / \mathrm{NH}_{4} \mathrm{OAc}$ buffer $(1.5$ $\mathrm{mL}, 2 \mathrm{M}$, freshly prepared, $\mathrm{pH} 4.5$ ) at $\mathrm{rt}$ for $24 \mathrm{~h}$. Purification by reverse phase chromatography $\left(\mathrm{C}_{18}, \mathrm{H}_{2} \mathrm{O} / \mathrm{MeOH}, 100 / 0 \rightarrow 60 / 40, \mathrm{v} / \mathrm{v}\right)$ afforded neoglycoside $14(44.6 \mathrm{mg}, 87 \%)$ as a white solid. $\mathrm{R}_{f}=0.64\left(\mathrm{CH}_{2} \mathrm{Cl}_{2} / \mathrm{EtOH} / \mathrm{MeOH} / \mathrm{NH}_{3}\right.$ (aq. 35\%), 10/2/2/1, v/v/v/v); $\alpha_{\mathrm{D}}{ }^{20.3}=-19(\mathrm{c}=0.1, \mathrm{MeOH}) ;$ IR (film) 3369, 3296, 3253, 3003, 2953, 2943, 2889, 2099, 2067, 1735, 1647, 1605, 1575, 1567, $1487,1446,1419,1377,1349,1308,1253,1079,1020,828 \mathrm{~cm}^{-1} ;{ }^{1} \mathrm{H}$ NMR $\left(500 \mathrm{MHz}, \mathrm{D}_{2} \mathrm{O}\right) \delta$ $4.33\left(\mathrm{~d}, 1 \mathrm{H}, J_{l^{\prime}, 2^{\prime}}=9.8 \mathrm{~Hz}, \mathrm{H}-1^{\prime}\right), 3.89\left(\mathrm{dd}, 1 \mathrm{H}, J_{I^{\prime}, 2^{\prime}}=J_{2^{\prime}, 3^{\prime}}=9.6 \mathrm{~Hz}, \mathrm{H}-2^{\prime}\right), 3.88\left(\mathrm{dd}, 1 \mathrm{H}, J_{5^{\prime}, 6^{\prime}}=1.5\right.$ $\left.\mathrm{Hz}, J_{6 a^{\prime}, 6 b^{\prime}}=12.3 \mathrm{~Hz}, \mathrm{H}-6 \mathrm{a}^{\prime}\right), 3.73\left(\mathrm{dd}, 1 \mathrm{H}, J_{5^{\prime}, 6^{\prime}}=5.3 \mathrm{~Hz}, J_{6 a^{\prime}, b^{\prime}}=12.3 \mathrm{~Hz}, \mathrm{H}-6 \mathrm{~b}^{\prime}\right), 3.51(\mathrm{dd}, 1 \mathrm{H}$, $\left.J_{2^{\prime}, 3^{\prime}}=J_{3^{\prime}, 4^{\prime}}=8.7 \mathrm{~Hz}, \mathrm{H}-3^{\prime}\right), 3.50\left(\mathrm{~s}, 3 \mathrm{H}, \mathrm{OCH}_{3}\right) 3.46-3.34\left(\mathrm{~m}, 4 \mathrm{H}, \mathrm{H}-4^{\prime}, \mathrm{H}-5^{\prime}, \mathrm{CH}_{2}-3\right), 3.09-$ 2.98 (m, 2H, $\left.\mathrm{CH}_{2}-1\right), 2.04$ (s, 3H, $\left.\mathrm{CH}_{3} \mathrm{Ac}\right), 1.88-1.74$ (m, 2H, $\left.\mathrm{CH}_{2}-2\right) ;{ }^{13} \mathrm{C}$ NMR (125 MHz, $\left.\mathrm{D}_{2} \mathrm{O}\right) 173.9(\mathrm{C}=\mathrm{O}), 90.0\left(\mathrm{C}-1^{\prime}\right), 77.4\left(\mathrm{C}-5^{\prime}\right), 75.5\left(\mathrm{C}-3^{\prime}\right), 69.6\left(\mathrm{C}-4^{\prime}\right), 61.0\left(\mathrm{OCH}_{3}\right), 60.8\left(\mathrm{C}^{\prime} 6^{\prime}\right)$, 52.3 (C-2'), 49.0 (C-3), 48.7 (C-1), 25.7 (C-2), $22.1\left(\mathrm{CH}_{3} \mathrm{Ac}\right)$; HRMS(ESI) $m / z$ for $\left[\mathrm{C}_{12} \mathrm{H}_{24} \mathrm{~N}_{5} \mathrm{O}_{6}\right]^{+}$ calcd.: 334.1721 , obsd.: 334.1719 .

Hydrolytic stability experiment. To a solution of neoglycoside $\mathbf{1 4}(8.0 \mathrm{mg})$ in $\mathrm{D}_{2} \mathrm{O}(0.5 \mathrm{~mL})$, $\mathrm{NaH}_{2} \mathrm{PO}_{4}$ and $\mathrm{Na}_{2} \mathrm{HPO}_{4}$ were added, resulting in a $100 \mathrm{mM}$ solution with the $\mathrm{pH}$ of $4.75,5,6,7$ or 9. The decay was measured over time, using ${ }^{1} \mathrm{H}-\mathrm{NMR}$ analysis, in which the intergral of $\mathrm{CH}_{2}-1$ was measured against the integral of $\mathrm{CH}-1^{\prime}$. As described in section 2.2.2.1, a line was fitted to the data according to the least-square method, and the half-lives were calculated based on the fitted line.

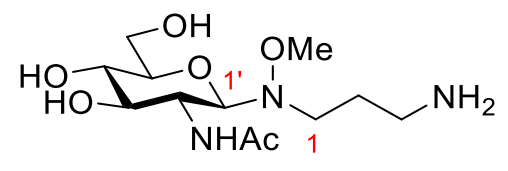

$N$-(2-Acetamido-2-deoxy- $\beta$-D-glucopyranosyl)- $N$-(3aminopropyl)- $O$-methylhydroxylamine (15). $\quad N$-Acetylglucosamine $\quad(34.0 \quad \mathrm{mg}, \quad 0.15 \quad \mathrm{mmol}) \quad$ and 3-(methoxyamino)propan-1-amine hydrochloride 4 (211 mg, $1.50 \mathrm{mmol})$ were reacted in an $\mathrm{AcOH} / \mathrm{NH}_{4} \mathrm{OAc}$ buffer $(1.5 \mathrm{~mL}, 2 \mathrm{M}$, freshly prepared, $\mathrm{pH} 4.5)$ at $\mathrm{rt}$ for $24 \mathrm{~h}$. Purification by size exclusion chromatography (Bio-Gel P-2) afforded neoglycoside 15 (28.3 mg, $0.092 \mathrm{mmol}, 81 \%$ ). $\mathrm{R}_{f}=0.16\left(\mathrm{CH}_{2} \mathrm{Cl}_{2} / \mathrm{EtOH} / \mathrm{MeOH} / \mathrm{NH}_{3}\right.$ (aq. 35\%), 5/2/2/1, v/v/v/v); $\alpha_{\mathrm{D}}^{20.3}=-21(\mathrm{c}=0.1, \mathrm{MeOH}) ;$ IR (film) 3401, 3375, 3322, 3311, 3291, 3278, 2938, 2885, 2727, 2058, 1651, 1567, 1490, 1459, 1437, 1376, 1315, 1257, 1108, 1023, $633 \mathrm{~cm}^{-1} ;{ }^{1} \mathrm{H}$ NMR $\left(500 \mathrm{MHz}, \mathrm{D}_{2} \mathrm{O}\right) \delta 4.41\left(\mathrm{~d}, 1 \mathrm{H}, J_{l^{\prime} 2^{\prime}}=\right.$ $\left.9.9 \mathrm{~Hz}, \mathrm{H}-1^{\prime}\right), 3.92\left(\mathrm{dd}, 1 \mathrm{H}, J_{5^{\prime}, 6^{\prime}}=0.9 \mathrm{~Hz}, J_{6 a^{\prime}, 6 b^{\prime}}=12.5 \mathrm{~Hz}, \mathrm{H}-6 \mathrm{a}^{\prime}\right), 3.85\left(\mathrm{dd}, 1 \mathrm{H}, J_{I^{\prime}, 2^{\prime}}=J_{2^{\prime}, 3^{\prime}}=9.8\right.$ $\left.\mathrm{Hz}, \mathrm{H}-2^{\prime}\right), 3.75\left(\mathrm{dd}, 1 \mathrm{H}, J_{5^{\prime}, 6^{\prime}}=4.9 \mathrm{~Hz}, J_{6 a^{\prime}, 6 b^{\prime}}=12.5 \mathrm{~Hz}, \mathrm{H}-6 \mathrm{~b}^{\prime}\right), 3.55\left(\mathrm{dd}, 1 \mathrm{H}, J_{2^{\prime}, 3^{\prime}}=J_{3^{\prime}, 4^{\prime}}=9.1\right.$ $\mathrm{Hz}, \mathrm{H}-3^{\prime}$ ), 3.52 (s, 3H, OCH 3 ), $3.45-3.40$ (m, 2H, H-4', H-5'), $3.12-3.01$ (m, 4H, $\mathrm{CH}_{2}-1, \mathrm{CH}_{2}-$ 3), 2.06 (s, 3H, CH $\left.\mathrm{CH}_{3} \mathrm{Ac}\right), 1.93\left(\mathrm{p}, 2 \mathrm{H}, J_{l, 2}=J_{2,3}=7.1 \mathrm{~Hz}, \mathrm{CH}_{2}-2\right) ;{ }^{13} \mathrm{C} \mathrm{NMR}\left(125 \mathrm{MHz}, \mathrm{D}_{2} \mathrm{O}\right) \delta$ 174.1 (C=O Ac), $90.5\left(\mathrm{C}-1^{\prime}\right), 77.4\left(\mathrm{C}-5^{\prime}\right), 75.2\left(\mathrm{C}-3^{\prime}\right), 69.6\left(\mathrm{C}-4^{\prime}\right), 61.1\left(\mathrm{OCH}_{3}\right), 60.8\left(\mathrm{C}-6^{\prime}\right), 52.3$ (C-2'), 47.9 (C-1), 37.6 (C-3), 24.5 (C-2), $22.1\left(\mathrm{CH}_{3}\right.$ Ac); HRMS(ESI) m/z calcd. for $\left[\mathrm{C}_{12} \mathrm{H}_{26} \mathrm{~N}_{3} \mathrm{O}_{6}\right]^{+}: 308.1816$, obsd.: 308.1820 . 


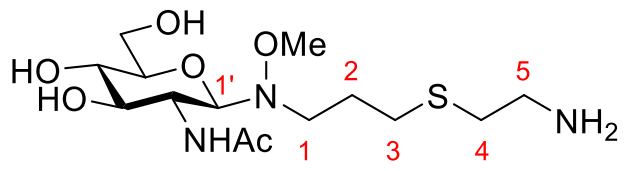

2-(3-[Methoxy(2-acetamido-2-deoxy- $\beta$-Dglucopyranosyl)amino] propylthio)ethan-1-amine (16). $N$-Acetylglucosamine $(12.0 \mathrm{mg}, 54.2 \mu \mathrm{mol})$ and oxyamine 8 ( $89.1 \mathrm{mg}, 0.54 \mathrm{mmol})$ were reacted in an $\mathrm{AcOH} / \mathrm{NH}_{4} \mathrm{OAc}$ buffer $(1.5 \mathrm{~mL}, 2 \mathrm{M}$, freshly prepared, $\mathrm{pH} 4.5)$ at $\mathrm{rt}$ for $72 \mathrm{~h}$. Purification with reverse phase chromatography $\left(\mathrm{C}_{18}, \mathrm{H}_{2} \mathrm{O} / \mathrm{MeOH}\right.$, $100 / 0 \rightarrow 90 / 10, \mathrm{v} / \mathrm{v}), \quad$ followed by silica gel column flash chromatography $\left(\mathrm{CH}_{2} \mathrm{Cl}_{2} / \mathrm{EtOH} / \mathrm{MeOH} / \mathrm{NH}_{3}\right.$ (aq. 33\%), 10/2/2/1 $\rightarrow$ 5/2/2/1, v/v/v/v) afforded neoglycoside 16 (16.1 mg, $81 \%) . \mathrm{R}_{f}=0.53\left(\mathrm{CH}_{2} \mathrm{Cl}_{2} / \mathrm{EtOH} / \mathrm{MeOH} / \mathrm{NH}_{3}\right.$ (aq. $33 \%$ ), 5/2/2/1, v/v/v/v); $\alpha_{D}^{20.3}=-25$ (c = 0.1, MeOH); IR (film) 3287, 2938, 2056, 1690, 1650, 1559, 1434, 1375, 1316, 1131, 1080, 1034, 947, $632 \mathrm{~cm}^{-1} ;{ }^{1} \mathrm{H}$ NMR $\left(500 \mathrm{MHz}, \mathrm{D}_{2} \mathrm{O}\right) \delta 4.34\left(\mathrm{~d}, 1 \mathrm{H}, J_{l^{\prime}, 2^{\prime}}=9.7 \mathrm{~Hz}, \mathrm{H}-1^{\prime}\right), 3.93-3.85$ $\left(\mathrm{m}, 2 \mathrm{H}, \mathrm{H}-2^{\prime}, \mathrm{H}-6 \mathrm{a}^{\prime}\right), 3.89\left(\mathrm{t}, 1 \mathrm{H}, J_{I^{\prime}, 2^{\prime}}=J_{2^{\prime}, 3^{\prime}}=9.7 \mathrm{~Hz}, \mathrm{H}-2^{\prime}\right), 3.73\left(\mathrm{dd}, 1 \mathrm{H}, J_{5^{\prime}, 6^{\prime}}=5.3 \mathrm{~Hz}, J_{6 a^{\prime}, 6 b^{\prime}}=\right.$ $\left.12.4 \mathrm{~Hz}, \mathrm{H}-6 \mathrm{~b}^{\prime}\right), 3.56$ - 3.47 (m, 4H, H-2', $\mathrm{OCH}_{3}$ ), 3.46 - 3.37 (m, 2H, H-4', H-5'), 3.07 - 3.00 (m, 2H, $\left.\mathrm{CH}_{2}-1\right), 2.85$ (t, 2H, J $\mathrm{J}_{4,5}=6.6 \mathrm{~Hz}, \mathrm{CH}_{2}-5$ ), $2.71-2.54\left(\mathrm{~m}, 4 \mathrm{H}, \mathrm{CH}_{2}-3, \mathrm{CH}_{2}-4\right), 2.04$ (s, $\left.3 \mathrm{H}, \mathrm{CH}_{3} \mathrm{Ac}\right), 1.81\left(\mathrm{~m}, 2 \mathrm{H}, \mathrm{CH}_{2}-2\right) .{ }^{13} \mathrm{C}$ NMR (125 MHz, $\left.\mathrm{D}_{2} \mathrm{O}\right) \delta 173.9(\mathrm{C}=\mathrm{O}), 90.1\left(\mathrm{C}-1^{\prime}\right), 77.5$ (C-5'), $75.5\left(\mathrm{C}-3^{\prime}\right), 69.7\left(\mathrm{C}-4^{\prime}\right), 61.1\left(\mathrm{OCH}_{3}\right), 60.9\left(\mathrm{C}-6^{\prime}\right), 52.3\left(\mathrm{C}-2^{\prime}\right), 50.3(\mathrm{C}-1), 39.4(\mathrm{C}-5), 32.9$ (C-3), $28.4(\mathrm{C}-4), 26.4(\mathrm{C}-2), 22.2\left(\mathrm{CH}_{3} \mathrm{Ac}\right)$; HRMS(ESI) $\mathrm{m} / \mathrm{z}$ calcd. for $\left[\mathrm{C}_{14} \mathrm{H}_{30} \mathrm{~N}_{3} \mathrm{O}_{6} \mathrm{~S}\right]^{+}$: 368.1850, obsd.: 368.1864 .

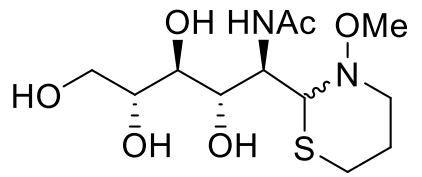

D-gluco-2-(1-Acetamido-2,3,4,5-tetrahydroxy-pentyl)-3methoxy-1,3-thiazinane (18). $N$-Acetylglucosamine (34 mg, 0.15 mmol) and 3-(methoxyamino)propane-1-thiol 8 (182 mg, 1.50 mmol) were reacted in an $\mathrm{AcOH} / \mathrm{NH}_{4} \mathrm{OAc}$ buffer $(1.5 \mathrm{~mL}, 2 \mathrm{M}$, freshly prepared, $\mathrm{pH} 4.5)$ containing 5\% TCEP at $\mathrm{rt}$ for $72 \mathrm{~h}$. Purification with reverse phase chromatography $\left(\mathrm{C}_{18}\right.$, $\mathrm{H}_{2} \mathrm{O} / \mathrm{MeOH}, 100 / 0 \rightarrow 60 / 40$, v/v) afforded thiazinane $18(44 \mathrm{mg}, 89 \%)$ as an colourless oil. $\mathrm{R}_{f}=$ $0.32\left(\mathrm{CH}_{2} \mathrm{Cl}_{2} / \mathrm{EtOH} / \mathrm{MeOH} / \mathrm{NH}_{3}\right.$ (aq. 33\%), 10/2/2/1, v/v/v/v); IR (film) 3298, 2940, 1646, 1545, 1428, 1374, 1318, 1284, 1190, 1079, 1037, 951, 867, 844, 694, $632 \mathrm{~cm}^{-1} ;{ }^{1} \mathrm{H}$ NMR (300 MHz, $\left.\mathrm{D}_{2} \mathrm{O}, 50{ }^{\circ} \mathrm{C}\right) \delta 4.85-4.62(\mathrm{~m}, 2 \mathrm{H}), 4.63-4.38(\mathrm{~m}, 2 \mathrm{H}), 4.12-3.72(\mathrm{~m}, 10 \mathrm{H}), 3.35-2.91(\mathrm{~m}$, $4 \mathrm{H}), 2.40-2.13(\mathrm{~m}, 5 \mathrm{H}), 2.11-1.77(\mathrm{~m}, 1 \mathrm{H}) ;{ }^{13} \mathrm{C}$ NMR $\left(150 \mathrm{MHz}, \mathrm{D}_{2} \mathrm{O}\right) \delta 174.1,173.8,72.2$, 71.3, 69.3, 68.4, 68.0, 62.6, 62.3, 58.6, 58.0 53.5, 50.6, 27.2, 22.1, 22.0, 17.8; HRMS(ESI) $\mathrm{m} / \mathrm{z}$ calcd. for $\left[\mathrm{C}_{12} \mathrm{H}_{25} \mathrm{~N}_{2} \mathrm{O}_{6} \mathrm{~S}\right]^{+}: 325.1428$, obsd.: 325.1426 .

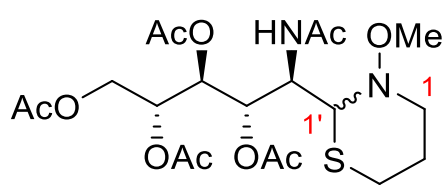

D-gluco-2-(1-Acetamido-2,3,4,5-tetra-acetoxy-pentyl)-3methoxy-1,3-thiazinane (19). The mixture of thiazinanes 18 (12.0 mg, $37.0 \mu \mathrm{mol})$ was co-evaporated with pyridine:acetic 
anhydride $(3 \mathrm{~mL}, 2 / 1, \mathrm{v} / \mathrm{v})$ and dissolved in acetic anhydride $(2 \mathrm{~mL})$ and pyridine $(1 \mathrm{~mL})$ and stirred overnight. The crude reaction mixture was concentrated and purified by silica gel column chromatography (PE/EtOAc, 95/5 $\rightarrow$ 80/20, v/v) to give a diastereomeric thioaminals 19 (17.8 $\mathrm{mg}, 98 \%$ ) as a colorless oil. $\mathrm{R}_{f}=0.38$ (EtOAc); IR (film) 2951, 2847, 2816, 1742, 1668, 1514, 1429, 1370, 1313, 1211, 1033, 953, 916, 859, 845, 795, 729, 646, $633 \mathrm{~cm}^{-1} ;{ }^{1} \mathrm{H}$ NMR $(300 \mathrm{MHz}$, $\left.\mathrm{D}_{2} \mathrm{O}, 50{ }^{\circ} \mathrm{C}\right) \delta 6.06\left(\mathrm{~d}, 1 \mathrm{H}, J_{N H, 2^{\prime}}=9.8 \mathrm{~Hz}, \mathrm{NH}\right.$ isomer $\left.A\right), 5.64\left(\mathrm{~d}, 1 \mathrm{H}, J_{N H, 2^{\prime}}=10.1 \mathrm{~Hz}, \mathrm{NH}\right.$ isomer $B), 5.50\left(\mathrm{dd}, 1 \mathrm{H}, J_{3^{\prime}, 4^{\prime}}=2.0 \mathrm{~Hz}, J_{2^{\prime}, 3^{\prime}}=9.4 \mathrm{~Hz}, \mathrm{H}-3^{\prime}\right.$ isomer $\left.A\right), 5.43\left(\mathrm{dd}, 1 \mathrm{H}, J_{3^{\prime}, 4^{\prime}}=2.0 \mathrm{~Hz}, J_{2^{\prime}, 3^{\prime}}=\right.$ $8.6 \mathrm{~Hz}, \mathrm{H}-3^{\prime}$ isomer $\left.B\right), 5.43-5.37\left(\mathrm{~m}, 2 \mathrm{H}, \mathrm{H}-4^{\prime}\right.$ isomer $\left.A+B\right), 5.23-5.13\left(\mathrm{~m}, 2 \mathrm{H}, \mathrm{H}-5^{\prime}\right.$ isomer $A+B), 4.84\left(\mathrm{dt}, 1 \mathrm{H}, J_{I^{\prime}, 2^{\prime}}=J_{2^{\prime}, 3^{\prime}}=5.5 \mathrm{~Hz}, J_{2^{\prime}, N H}=10.0 \mathrm{~Hz}, \mathrm{H}-2^{\prime}\right.$ isomer $\left.B\right), 4.53\left(\mathrm{bd}, 1 \mathrm{H}, J_{I^{\prime}, 2^{\prime}}=4.5\right.$ $\mathrm{Hz},{ }^{1} J_{C H}=147 \mathrm{~Hz}, \mathrm{H}-1^{\prime}$ isomer A), $4.40\left(\mathrm{dt}, 1 \mathrm{H}, J_{I^{\prime}, 2^{\prime}}=J_{2^{\prime}, 3^{\prime}}=4.9 \mathrm{~Hz}, J_{2^{\prime}, N H}=9.7 \mathrm{~Hz}, \mathrm{H}-2^{\prime}\right.$ isomer $7 A), 4.28\left(\mathrm{dd}, 1 \mathrm{H}, J_{5^{\prime}, 6 a^{\prime}}=3.2 \mathrm{~Hz}, J_{6 a^{\prime}, 6 b^{\prime}}=12.4 \mathrm{~Hz}, \mathrm{H}-6 \mathrm{a}^{\prime}\right.$ isomer $\left.B\right), 4.22\left(\mathrm{dd}, 1 \mathrm{H}, J_{5^{\prime}, 6 a^{\prime}}=2.9 \mathrm{~Hz}\right.$, $J_{6 a^{\prime}, 6 b^{\prime}}=12.5 \mathrm{~Hz}, \mathrm{H}-6 \mathrm{~b}^{\prime}$ isomer $\left.A\right), 4.15-4.07\left(\mathrm{~m}, 2 \mathrm{H}, \mathrm{H}-6 \mathrm{~b}^{\prime}\right.$ isomer $\left.A+B\right), 3.90\left(\mathrm{bd}, 1 \mathrm{H}, J_{1^{\prime}, 2^{\prime}}=\right.$ $5.2 \mathrm{~Hz},{ }^{1} J_{C H}=149 \mathrm{~Hz}, \mathrm{H}-1^{\prime}$ isomer $\left.B\right), 3.68\left(\mathrm{~s}, 3 \mathrm{H}, \mathrm{OCH}_{3}\right.$ isomer $\left.A\right), 3.63-3.52\left(\mathrm{~m}, 1 \mathrm{H}, \mathrm{H}-1_{\mathrm{eq}}\right.$ isomer A), $3.49\left(\mathrm{~s}, 3 \mathrm{H}, \mathrm{OCH}_{3}\right.$ isomer B), $3.39\left(\mathrm{dt}, J_{\text {leq,2eq }}=J_{\text {leq,2ax }}=4.3 \mathrm{~Hz}, J_{\text {leq, lax }}=13.3 \mathrm{~Hz}, \mathrm{H}\right.$ $1_{\mathrm{eq}}$ isomer B), $2.95\left(\mathrm{dt}, 1 \mathrm{H}, J_{2 e q, 3 a x}=1.7 \mathrm{~Hz}, J_{2 a x, 3 a x}=J_{3 e q, 3 a x}=13.0 \mathrm{~Hz}, \mathrm{H}-3_{\mathrm{ax}}\right.$ isomer B), $2.83(\mathrm{ddd}$, $1 \mathrm{H}, J_{\text {lax }, 2 e q}=2.9 \mathrm{~Hz}, J_{\text {lax, } 2 a x}=13.0 \mathrm{~Hz}, J_{\text {lax, }- \text { leq }}=15.5 \mathrm{~Hz}, \mathrm{H}-1_{\mathrm{ax}}$ isomer B), $2.75-2.57$ (m, 3H, H$3_{\mathrm{eq}}$ isomer B, H-1 ax isomer A, H-3 $3_{\mathrm{ax}}$ isomer $A, \mathrm{H}-3_{\mathrm{eq}}$ isomer $\left.A\right), 2.18$ (s, 3H, $\left.\mathrm{CH}_{3} \mathrm{Ac}\right), 2.10(\mathrm{~s}, 3 \mathrm{H}$, $\mathrm{CH}_{3} \mathrm{Ac}$ ), 2.04 (s, 3H, CH $\mathrm{Ac}$ ), 2.03 (s, 3H, $\mathrm{CH}_{3} \mathrm{Ac}$ ), 2.02 (s, 3H, $\mathrm{CH}_{3} \mathrm{Ac}$ ), 2.00 (s, 3H, $\mathrm{CH}_{3} \mathrm{Ac}$ ), $1.97-1.84$ (m, 5H, $\mathrm{CH}_{3}$ Ac, H-2 $2_{\mathrm{eq}}, \mathrm{H}-2_{\mathrm{ax}}$ isomer A), 1.49 (bd, $1 \mathrm{H}, \mathrm{H}-2_{\mathrm{eq}}$ isomer B); ${ }^{13} \mathrm{C} \mathrm{NMR}(150$ MHz, $\left.\mathrm{D}_{2} \mathrm{O}\right) \delta 171.2(\mathrm{C}=\mathrm{O}), 170.8(\mathrm{C}=\mathrm{O}), 170.53(\mathrm{C}=\mathrm{O}), 170.46(\mathrm{C}=\mathrm{O}), 170.3(\mathrm{C}=\mathrm{O}), 170.2$ $(\mathrm{C}=\mathrm{O}), 169.9(\mathrm{C}=\mathrm{O}), 169.8(2 \times \mathrm{C}=\mathrm{O}), 169.6(\mathrm{C}=\mathrm{O}), 70.9,70.5,69.9,69.4\left(\mathrm{C}-3^{\prime}, \mathrm{C}^{\prime} 4^{\prime}\right.$ isomer $A+B) 69.0\left(\mathrm{C}-5 '^{\prime}\right.$ isomer $\left.A+B\right), 68.6\left(\mathrm{C}-1^{\prime}\right.$ isomer $\left.B\right), 67.6\left(\mathrm{C}-1^{\prime}\right.$ isomer $\left.A\right), 62.3$ (C-6' isomer A/B), $62.1\left(\mathrm{C}-6{ }^{\prime}\right.$ isomer $\left.\mathrm{A} / \mathrm{B}\right), 59.8\left(\mathrm{OCH}_{3}\right.$ isomer $\left.B\right), 58.2\left(\mathrm{OCH}_{3}\right.$ isomer $\left.A\right), 52.9\left(\mathrm{CH}_{2} \mathrm{NO}\right.$ isomer $\left.B\right)$, $51.6\left(\mathrm{CH}_{2} \mathrm{NO}\right.$ isomer $\left.A\right), 51.5\left(\mathrm{C}-2^{\prime}\right.$ isomer $\left.A\right), 49.5\left(\mathrm{C}-2^{\prime}\right.$ isomer $\left.B\right), 28.1\left(\mathrm{CH}_{2} \mathrm{~S}\right.$ isomer $\left.B\right), 26.8$

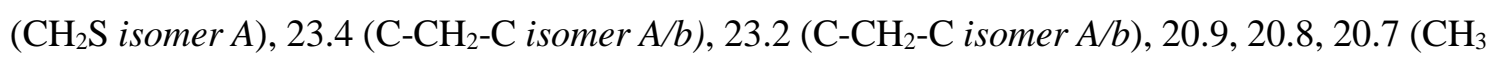
Ac); TOCSY NMR (300 MHz, $\mathrm{D}_{2} \mathrm{O}, 50{ }^{\circ} \mathrm{C}$ ) Isomer a $\delta 3.59$ (excited, app. bd, $J_{\text {lax, leq }}=14.6 \mathrm{~Hz}$, H-1eq), 2.84 (app. bt, $J_{\text {lax, Ieq }}=J_{\text {lax, } 2 a x}=13.1 \mathrm{~Hz}, \mathrm{H}-1 \mathrm{ax}$ ), 2.13 (app. bq, $J_{\operatorname{lax}, 2 a x}=J_{2 a x, 2 e q}=J_{2 a x-3 a x}=$ $13.0 \mathrm{~Hz}, \mathrm{H}-2 \mathrm{ax}$ ), 1.50 (app. bd, $J_{2 e q, 2 a x}=14.3 \mathrm{~Hz}, \mathrm{H}-2 \mathrm{eq}$ ), 2.97 (app. bt, $J_{2 a x, 3 a x}=J_{3 a x, 3 e q}=13.0$ $\mathrm{Hz}, \mathrm{H}-3 \mathrm{ax}$ ), 2.64 (app. bd, $J_{3 e q, 3 a x}=13.3 \mathrm{~Hz}, \mathrm{H}-3 \mathrm{eq}$ ); HRMS(ESI) $\mathrm{m} / z$ calcd. for $\left[\mathrm{C}_{20} \mathrm{H}_{33} \mathrm{~N}_{2} \mathrm{O}_{10} \mathrm{~S}\right]^{+}$: 493.1850, obsd.: 493.1856.

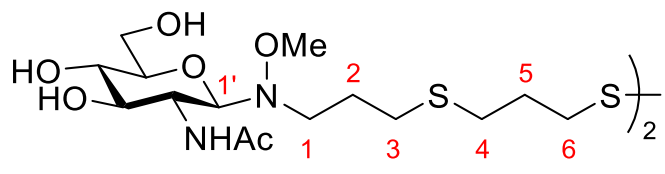

1,2-Bis(3-[3-(methoxy[2-acetamido-2-deoxy- $\beta$ D-glucopyranosyl]amino) propylthio]propyl)disulfane (20). $N$-Acetylglucosamine $(7.8 \mathrm{mg}$, $35.3 \mu \mathrm{mol})$ and 3-(3-(methoxyamino)propylthio)propane-1-thiol 10 (69.0 mg, $0.35 \mathrm{mmol}$ ) were reacted a mixture of $\mathrm{AcOH} / \mathrm{NH}_{4} \mathrm{OAc}$ buffer $(0.2 \mathrm{~mL}, 2 \mathrm{M}$, freshly prepared, $\mathrm{pH} 4.5)$, ethanol (0.2 $\mathrm{mL})$ and $\mathrm{CH}_{2} \mathrm{Cl}_{2}(0.1 \mathrm{~mL})$ at $40{ }^{\circ} \mathrm{C}$ for $72 \mathrm{~h}$. The crude reaction mixture was directly loaded on a 
size exclusion column (Bio-Gel P-2, $600 \times 10 \mathrm{~mm}$ ) and eluted with $0.1 \mathrm{M}$ aq. $\mathrm{NH}_{4} \mathrm{HCO}_{3}$. Lyophilisation of the product fractions afforded disulfide $\mathbf{2 0}(9.0 \mathrm{mg}, 64 \%)$ as a colorless oil. $\mathrm{R}_{f}$ $($ Sulfhydryl $)=0.34\left(10 / 2 / 2 / 10 \mathrm{CH}_{2} \mathrm{Cl}_{2} / \mathrm{EtOH} / \mathrm{MeOH} / \mathrm{NH}_{3}\right.$ (aq, $\left.\left.33 \%\right)\right), \mathrm{R}_{f}=($ disulfide $)=0.17$ $\left(10 / 2 / 2 / 1 \mathrm{CH}_{2} \mathrm{Cl}_{2} / \mathrm{EtOH} / \mathrm{MeOH} / \mathrm{NH}_{3}(\mathrm{aq}, 33 \%)\right) ; \alpha_{\mathrm{D}}{ }^{19.8}=+3.5$ (c = 0.1, MeOH); IR (film) 3313, 3273, 3094, 3070, 2656, 2909, 1632, 1557, 1487, 1439, 1355, 1215, 1195, 1111, 1038, 1000, 986, 903, 755, 721, 691, 620, $604 \mathrm{~cm}^{-1} ;{ }^{1} \mathrm{H}$ NMR (500 MHz, $\left.\mathrm{D}_{2} \mathrm{O}\right) \delta 4.34\left(\mathrm{~d}, 2 \mathrm{H}, J_{l^{\prime}, 2^{\prime}}=9.8 \mathrm{~Hz}, \mathrm{H}-1^{\prime}\right)$, $3.92-3.86$ (m, 4H, H-6a', H-2'), $3.74\left(\mathrm{dd}, 2 \mathrm{H}, J_{5^{\prime}, 6 b^{\prime}}=5.4 \mathrm{~Hz}, J_{6 a^{\prime}, 6 b^{\prime}}=12.4 \mathrm{~Hz}, \mathrm{H}-6 \mathrm{~b}^{\prime}\right), 3.55-3.46$ (m, 8H, $\left.\mathrm{OCH}_{3}, \mathrm{H}-3^{\prime}\right), 3.44-3.36$ (m, 4H, H-4', H-5'), $3.10-3.00$ (m, 4H, $\left.\mathrm{CH}_{2}-1\right), 2.85$ (t, 4H, $\left.J_{5,6}=7.1 \mathrm{~Hz}, \mathrm{CH}_{2}-6\right), 2.70\left(\mathrm{t}, 4 \mathrm{H}, J_{4,5}=7.1 \mathrm{~Hz}, \mathrm{CH}_{2}-4\right), 2.68-2.57\left(\mathrm{~m}, 4 \mathrm{H}, \mathrm{CH}_{2}-3\right), 2.04(\mathrm{~s}, 6 \mathrm{H}$, $\mathrm{CH}_{3} N \mathrm{Ac}$ ), $2.00\left(\mathrm{p}, 4 \mathrm{H}, J_{4,5}=J_{5,6}=7.0 \mathrm{~Hz}, \mathrm{CH}_{2}-5\right), 1.86-1.78\left(\mathrm{~m}, 4 \mathrm{H}, \mathrm{CH}_{2}-2\right) ;{ }^{13} \mathrm{C} \mathrm{NMR}(125$ $\left.\mathrm{MHz}, \mathrm{D}_{2} \mathrm{O}\right) \delta 173.8(\mathrm{C}=\mathrm{O}), 90.0\left(\mathrm{C}-1^{\prime}\right), 77.4\left(\mathrm{C}-5^{\prime}\right), 75.5\left(\mathrm{C}-3^{\prime}\right), 69.6\left(\mathrm{C}-4^{\prime}\right), 61.1\left(\mathrm{OCH}_{3}\right), 60.8$ (C-6'), 52.3 (C-2'), 50.3 (C-1), 36.5 (C-6), 29.4 (C-4), 28.6 (C-3), 27.9 (C-5), 26.3 (C-2), 22.1 $\left(\mathrm{CH}_{3} N A c\right)$; HRMS(ESI) $\mathrm{m} / z$ calcd. for $\left[\mathrm{C}_{30} \mathrm{H}_{59} \mathrm{~N}_{4} \mathrm{O}_{12} \mathrm{~S}_{4}\right]^{+}: 795.3007$, obsd:: 795.3018; HRMS(ESI) $m / z$ calcd. for $\left[\mathrm{C}_{15} \mathrm{H}_{31} \mathrm{~N}_{2} \mathrm{O}_{6} \mathrm{~S}_{2}\right]^{+}: 399.1618$, obsd.: 399.1609 .

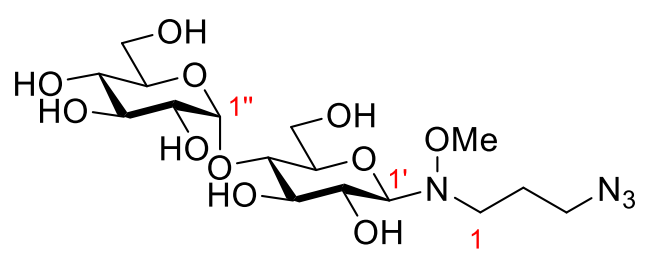

$N$-(4-O-( $\alpha$-D-glucopyranosyl)- $\beta$-Dglucopyranosyl)- $N$-(3-azidopropyl)- $O$ methylhydroxylamine (22). To a solution of maltose 21 ( $23 \mathrm{mg}, 67 \mu \mathrm{mol})$ in a $\mathrm{AcOH} / \mathrm{AcONH}_{4}$ buffer (0.5 mL, 2M, freshly prepared, pH 4.5), 3-azido-propan-1-methoxyamine 1 (88 mg, 0.067 mmol) was added and the reaction mixture was stirred at room temperature for $24 \mathrm{~h}$. The rude mixture was directly loaded on a size exclusion column (BioRad, P2) and eluted with $0.1 \mathrm{M}$ aq. $\mathrm{NH}_{4} \mathrm{HCO}_{3}$. Lyophilisation of the product fraction afforded neoglycoside $22(28.8 \mathrm{mg}, 94 \%)$ as a white foam. $\mathrm{R}_{f}=0.54\left(\mathrm{CH}_{2} \mathrm{Cl}_{2} / \mathrm{EtOH} / \mathrm{MeOH} / \mathrm{NH}_{3}\right.$ (aq. $\left.\left.35 \%\right), 10 / 2 / 2 / 1, \mathrm{v} / \mathrm{v} / \mathrm{v} / \mathrm{v}\right) ; \alpha_{\mathrm{D}}^{17.6}=+24(\mathrm{c}=$ 1, MeOH); IR (film) 3349, 2931, 2098, 1568, 1439, 1355, 1262, 1145, 1075, 1025, 906, 858, 783, $592,528 \mathrm{~cm}^{-1} ;{ }^{1} \mathrm{H}$ NMR $\left(500 \mathrm{MHz}, \mathrm{D}_{2} \mathrm{O}\right) \delta 5.41\left(\mathrm{~d}, 1 \mathrm{H}, J_{I^{\prime \prime}, 2^{\prime \prime}}=3.9 \mathrm{~Hz}, \mathrm{H}-1^{\prime \prime}\right), 4.19\left(\mathrm{~d}, 1 \mathrm{H}, J_{l^{\prime}, 2^{\prime}}=\right.$ $\left.9.2 \mathrm{~Hz}, \mathrm{H}-1^{\prime}\right), 3.91\left(\mathrm{dd}, 1 \mathrm{H}, J_{5^{\prime}, 6^{\prime}}=1.8 \mathrm{~Hz}, J_{6 a^{\prime}, 6 b^{\prime}}=12.1 \mathrm{~Hz}, \mathrm{H}-6 \mathrm{a}^{\prime}\right), 3.85\left(\mathrm{dd}, 1 \mathrm{H}, J_{5^{\prime \prime}, 6 a^{\prime \prime}}=1.9 \mathrm{~Hz}\right.$, $\left.J_{6 a^{\prime \prime}, 6 b^{\prime \prime}}=12.2 \mathrm{~Hz}, \mathrm{H}-6 \mathrm{a}^{\prime \prime}\right), 3.81$ - 3.64 (m, 5H, H-5', H-6b', H-6b", H-3", H-5"), 3.62 (s, 3H, $\mathrm{OCH}_{3}$ ), 3.61 - 3.38 (m, 7H, H-4', H-2', H-2", H-3', H-3a, H-3b, H-4"), 3.19 - 3.11 (m, 1H, H1a), $3.00-2.94$ (m, 1H, H-1b), $1.94-1.85$ (m, 2H, H-2); ${ }^{13} \mathrm{C}$ NMR (125 MHz, $\left.\mathrm{D}_{2} \mathrm{O}\right) \delta 99.5$ (C1"), $91.9\left(\mathrm{C}-1^{\prime}\right), 77.6\left(\mathrm{C}-5^{\prime}\right), 76.5\left(\mathrm{C}-4^{\prime}\right), 76.0\left(\mathrm{C}-3^{\prime}\right), 72.8,72.6$ (C-3", C-5"), $71.6\left(\mathrm{C}-2^{\prime \prime}\right), 69.6$ $\left(\mathrm{C}-2^{\prime}\right), 69.3\left(\mathrm{C}-4^{\prime \prime}\right), 62.0\left(\mathrm{OCH}_{3}\right), 60.8\left(\mathrm{C}-6^{\prime}\right), 60.4\left(\mathrm{C}-6^{\prime \prime}\right), 49.9$ (C-1), 49.1 (C-3), 25.9 (C-2); HRMS(ESI) $m / z$ calcd. for $\left[\mathrm{C}_{16} \mathrm{H}_{31} \mathrm{~N}_{4} \mathrm{O}_{11}\right]^{+}: 455.1984$, obsd.: 455.1986 . 


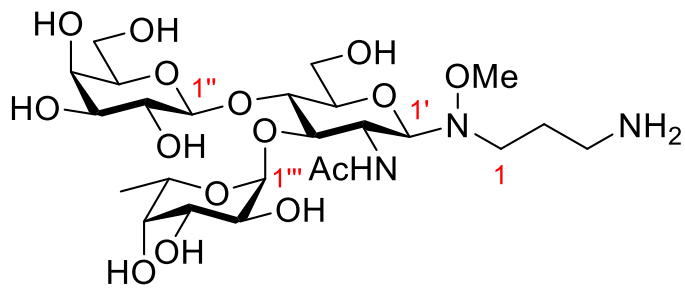

$N$-(2-Acetamido-2-deoxy-3-O-( $\alpha$-Lfucopyranosyl)-4- $O$-( $\beta$-D-galacto-pyranosyl)$\beta$-D-glucopyranosyl)- $\mathrm{N}$-(3-aminopropyl)- $\mathrm{O}$ methylhydroxylamine (24). To a solution of Lewis $^{\mathrm{X}} \quad \mathbf{2 3} \quad(5.6 \mathrm{mg}, \quad 10.6 \mu \mathrm{mol})$ in a

$\mathrm{AcOH} / \mathrm{NH}_{4} \mathrm{OAc}$ buffer $(0.5 \mathrm{~mL}, 2 \mathrm{M}$, freshly prepared, $\mathrm{pH} 4.5)$, 3-(methoxyamino)propan-1amine hydrochloride $4(15.9 \mathrm{mg}, 113.2 \mu \mathrm{mol})$ was added and the reaction mixture was stirred at $40{ }^{\circ} \mathrm{C}$ for $35 \mathrm{~h}$. The crude mixture was directly loaded on a size exclusion column (Bio-Gel P-2, $1200 \times 18 \mathrm{~mm}$ ) and eluted with $0.1 \mathrm{M}$ aq. $\mathrm{NH}_{4} \mathrm{HCO}_{3}$. Lyophilisation of the product fractions afforded neoglycoside 24 (5.7 mg, 88\%). $\mathrm{R}_{f}=0.10\left(\mathrm{CH}_{2} \mathrm{Cl}_{2} / \mathrm{EtOH} / \mathrm{MeOH} / \mathrm{NH}_{3}\right.$ (aq. 35\%), 5/2/2/1, $\mathrm{v} / \mathrm{v} / \mathrm{v} / \mathrm{v}) ; \alpha_{\mathrm{D}}^{19.5}=-9.3(\mathrm{c}=0.2, \mathrm{MeOH}) ;$ IR $($ film) $3341,2925,2852,17117,1647,1586,1466$, 1451, 1415, 1380, 1350, 1302, 1233, 1193, 1085, 1026, 968, $917 \mathrm{~cm}^{-1},{ }^{1} \mathrm{H}$ NMR (500 MHz, D $\left.\mathrm{O}\right)$ $\delta 5.12\left(\mathrm{~d}, 1 \mathrm{H}, J_{l^{\prime \prime}, 2^{\prime \prime \prime}}=3.9 \mathrm{~Hz}, \mathrm{H}-1^{\prime \prime}{ }^{\prime \prime \prime}\right), 4.84\left(\mathrm{q}, 1 \mathrm{H}, J_{5^{\prime \prime \prime}, 6^{\prime \prime \prime}}=6.7 \mathrm{~Hz}, \mathrm{H}-5^{\prime \prime \prime}\right), 4.49-4.42$ (m, 1H, H1', H-1"), 4.05 (m, 2H, H-2', H-6a'), 3.93 - 3.81 (m, 5H, H-3'", H-4", H-4', H-3', H-6b'), 3.80 (d, $\left.1 \mathrm{H}, J_{3^{\prime \prime \prime}, 4^{\prime \prime \prime}}=J_{4^{\prime \prime \prime}, 5^{\prime \prime \prime}}=2.9 \mathrm{~Hz}, \mathrm{H}-4^{\prime \prime \prime}\right), 3.76\left(\mathrm{~m}, 3 \mathrm{H}, \mathrm{H}-6 \mathrm{a}^{\prime \prime}, \mathrm{H}-6 \mathrm{~b}^{\prime \prime}, \mathrm{H}-2^{\prime \prime \prime}\right), 3.64\left(\mathrm{dd}, 1 \mathrm{H}, J_{3^{\prime \prime}, 4^{\prime \prime}}=3.2\right.$ $\left.\mathrm{Hz}, J_{2^{\prime \prime}, 3^{\prime \prime}}=9.9 \mathrm{~Hz}, \mathrm{H}-3^{\prime \prime}\right), 3.60-3.55$ (m, 1H, H-5"), $3.54-3.46$ (m, 1H, H-5"), $3.54-3.46$ (m, 5H, $\left.\mathrm{OCH}_{3}, \mathrm{H}-5^{\prime}, \mathrm{H}-2^{\prime \prime}\right), 3.11-2.92$ (m, 4H, $\left.\mathrm{CH}_{2}-1, \mathrm{CH}_{2}-3\right), 2.02$ (s, 3H, $\mathrm{CH}_{3} \mathrm{Ac}$ ), 1.95 (m, 2H, $\left.\mathrm{CH}_{2}-2\right), 1.17$ (d, 3H, $\left.J_{5^{\prime \prime \prime}, 6^{\prime \prime \prime}}=6.7 \mathrm{~Hz}, \mathrm{H}-6^{\prime \prime \prime}\right) ;{ }^{13} \mathrm{C} \mathrm{NMR}\left(125 \mathrm{MHz}, \mathrm{D}_{2} \mathrm{O}\right) 170.5(\mathrm{C}=\mathrm{O}), 101.8(\mathrm{C}-$ 1"), 98.7 (C-1'"'), 90.5 (C-1'), 76.9 (C-5'), 76.1 (C-3'), 74.8 (C-5"), 73.2 (C-4'), 72.4 (C-3"), 71.8 (C-4"'), 70.9 (C-2"), 69.1 (C-3"'), 68.3 (C-4"), 67.6 (C-2"'), 66.7 (C-5"'), 61.4 (C-6"), 60.9 $\left(\mathrm{OCH}_{3}\right), 59.7\left(\mathrm{C}-6^{\prime}\right), 52.5\left(\mathrm{C}-2^{\prime}\right), 47.2(\mathrm{C}-1), 37.5(\mathrm{C}-3), 24.5(\mathrm{C}-2), 22.1\left(\mathrm{CH}_{3} \mathrm{Ac}\right), 15.2\left(\mathrm{C}-6^{\prime \prime \prime}\right)$; HRMS(ESI) $m / z$ calcd. for $\left[\mathrm{C}_{24} \mathrm{H}_{46} \mathrm{~N}_{3} \mathrm{O}_{15}\right]^{+}:$616.2923, obsd.: 616.2938 .

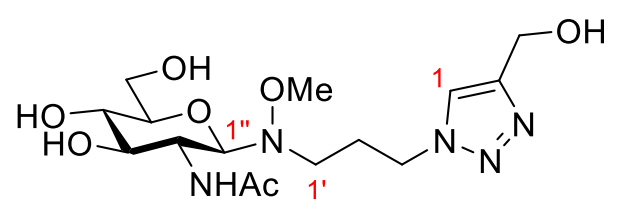

(1-(3-(Methoxy(2-acetamido-2-deoxy-b-Dglucosyl)amino)propyl)-1H-1,2,3-triazol-4yl)methanol (25). To a solution of azide $14(11.8 \mathrm{mg}$, $35 \mu \mathrm{mol})$ and propagyl alcohol $(0.1 \mathrm{~mL})$ in ethanol ( $1 \mathrm{~mL})$, a solution of L-ascorbic acid $(6.2 \mathrm{mg}, 35 \mu \mathrm{mol})$ and $\mathrm{CuSO}_{4}(1 \mathrm{mg})$ were added and the reaction mixture was stirred for $3 \mathrm{~d}$ at rt. The crude mixture was filtered and washed with $\mathrm{H}_{2} \mathrm{O}$ (2 $\mathrm{mL})$ and concentrated in vacuo. Purification with reverse phase chromatography $\left(\mathrm{C}_{18}\right.$, $\left.\mathrm{H}_{2} \mathrm{O} / \mathrm{MeOH}, 100 / 0 \rightarrow 90 / 10, \mathrm{v} / \mathrm{v}\right)$ yielded triazole $25(10.7 \mathrm{mg}, 79 \%)$ as a colourless oil. $\mathrm{R}_{f}=0.53$ $\left(\mathrm{CH}_{2} \mathrm{Cl}_{2} / \mathrm{EtOH} / \mathrm{MeOH} / \mathrm{NH}_{3}\right.$ (aq. $33 \%$ ), 5/2/2/1, v/v/v/v); $\alpha_{\mathrm{D}}{ }^{19}=-0.82(\mathrm{c}=0.1, \mathrm{MeOH}) ; \mathrm{IR}$ (film) 3350, 2934, 2874, 1643, 1555, 1442, 1378, 1319, 1227, 1104, 1054, 1033, $1021 \mathrm{~cm}^{-1}$; ${ }^{1} \mathrm{H}$ NMR $\left(500 \mathrm{MHz}, \mathrm{CD}_{3} \mathrm{OD}\right) \delta 7.99\left(\mathrm{~s}, 1 \mathrm{H}\right.$, triazole), $4.73\left(\mathrm{~s}, 2 \mathrm{H}, \mathrm{C}=\mathrm{C}-\mathrm{CH}_{2}\right), 4.58-4.45\left(\mathrm{~m}, 2 \mathrm{H}, \mathrm{CH}_{2} \mathrm{H}-\right.$ 3), $4.33\left(\mathrm{~d}, 1 \mathrm{H}, J_{I^{\prime}, 2^{\prime}}=9.8 \mathrm{~Hz}, \mathrm{H}-1^{\prime}\right), 3.91-3.85\left(\mathrm{~m}, 2 \mathrm{H}, \mathrm{H}-2^{\prime}, \mathrm{H}-6 \mathrm{a}^{\prime}\right), 3.73\left(\mathrm{dd}, 1 \mathrm{H}, J_{5^{\prime}, 6 b^{\prime}}=5.4\right.$ $\left.\mathrm{Hz}, J_{6 a^{\prime}, 6 b^{\prime}}=12.4 \mathrm{~Hz}, \mathrm{H}-6 \mathrm{~b}^{\prime}\right), 3.53\left(\mathrm{dd}, 1 \mathrm{H}, J_{2^{\prime}, 3^{\prime}}=8.7 \mathrm{~Hz}, J_{3^{\prime}, 4^{\prime}}=9.7 \mathrm{~Hz}, \mathrm{H}-3^{\prime}\right), 3.50\left(\mathrm{~s}, 3 \mathrm{H}, \mathrm{OCH}_{3}\right)$, $3.42\left(\mathrm{t}, 1 \mathrm{H}, J_{2^{\prime}, 3^{\prime}}=J_{4^{\prime}, 5^{\prime}}=8.7 \mathrm{~Hz}, \mathrm{H}-4^{\prime}\right), 3.40-3.35\left(\mathrm{~m}, 1 \mathrm{H}, \mathrm{H}-5^{\prime}\right), 3.03-2.89(\mathrm{~m}, 2 \mathrm{H}, \mathrm{H}-1), 2.24-$ 
2.10 (m, 2H, H-2), 2.06 (s, 3H, $\left.\mathrm{CH}_{3} \mathrm{Ac}\right) ;{ }^{13} \mathrm{C} \mathrm{NMR}\left(125 \mathrm{MHz}, \mathrm{CD}_{3} \mathrm{OD}\right) \delta 173.9(\mathrm{C}=\mathrm{O} \mathrm{Ac}), 146.7$ $(\mathrm{Cq} C=\mathrm{C}), 124.0(\mathrm{CH}=\mathrm{C}), 90.1\left(\mathrm{C}-1^{\prime \prime}\right), 77.4\left(\mathrm{C}-5^{\prime \prime}\right), 75.4\left(\mathrm{C}-3^{\prime \prime}\right), 69.6\left(\mathrm{C}-4^{\prime \prime}\right), 61.2\left(\mathrm{OCH}_{3}\right), 60.8$ (C-6"), $54.5\left(\mathrm{CH}_{2}\right.$ allylic), $52.3\left(\mathrm{C}-2^{\prime \prime}\right), 48.4\left(\mathrm{C}-1^{\prime}\right), 48.2\left(\mathrm{C}-3^{\prime}\right), 27.0\left(\mathrm{C}-2^{\prime}\right), 22.1\left(\mathrm{CH}_{3} \mathrm{Ac}\right)$; HRMS(ESI) $m / z$ calcd. for $\left[\mathrm{C}_{15} \mathrm{H}_{28} \mathrm{~N}_{5} \mathrm{O}_{7}\right]^{+}:$390.1983, obsd.: 390.1986 .

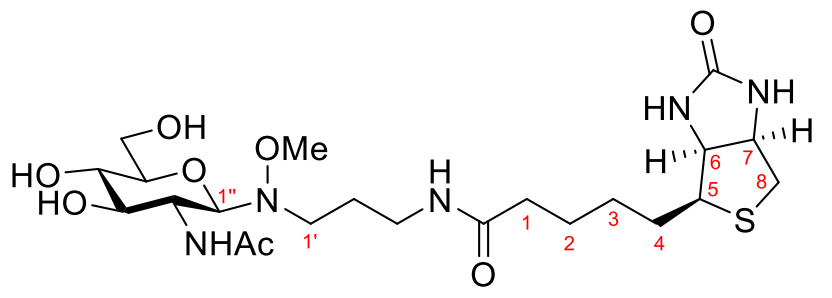

\section{$N$-(3-(methoxy[2-acetamido-2-deoxy-} $\beta$-D-glucopyranosyl]-amino)propyl)D-biotinamide (26). To a solution of amine 15 (12.0 mg, $39.1 \mu \mathrm{mol})$ in DMF

(0.2 mL), D-biotin $(13.0 \mathrm{mg}, 53.7 \mu \mathrm{mol}), \mathrm{Et}_{3} \mathrm{~N}(12 \mu \mathrm{L})$ and HBTU $(30.0 \mathrm{mg}, 79 \mu \mathrm{mol})$ were added and the reaction mixture was stirred for $2 \mathrm{~h}$. at $\mathrm{rt}$. To the reaction mixture, $\mathrm{H}_{2} \mathrm{O}(2 \mathrm{~mL})$ was added and the white participate was filtered and washed with water $(2 \times 1 \mathrm{~mL})$. The liquid residue was then purified by reverse phase chromatography (elute in $\mathrm{H}_{2} \mathrm{O}$ ), concentrated in vacuo, followed by silica gel flash chromatograpy $\left(\mathrm{CH}_{2} \mathrm{Cl}_{2} / \mathrm{EtOH} / \mathrm{MeOH} / \mathrm{NH}_{3}\right.$ (aq. 33\%), 15/2/2/1 $\rightarrow$ 10/2/2/1, v/v/v/v) to afford glyconjugate $26(17.6 \mathrm{mg}, 84 \%)$ as a colourless oil. $\mathrm{R}_{f}=0.58$ $\left(\mathrm{CH}_{2} \mathrm{Cl}_{2} / \mathrm{EtOH} / \mathrm{MeOH} / \mathrm{NH}_{3}\right.$ (aq. 33\%), 5/2/2/1, v/v/v/v); $\alpha_{\mathrm{D}}^{20.0}=+3.1(\mathrm{c}=0.2, \mathrm{MeOH}) ; \mathrm{IR}($ film) 3343, 2927, 2857, 1671, 1652, 1637, 1559, 1542, 1457, 1437, 1395, 1375, 1317, 1266, 1110, $1078,1053,1033 \mathrm{~cm}^{-1} ;{ }^{1} \mathrm{H}$ NMR $\left(500 \mathrm{MHz}, \mathrm{D}_{2} \mathrm{O}\right) \delta 4.58\left(\mathrm{dd}, 1 \mathrm{H}, J_{7,8}=4.7 \mathrm{~Hz}, J_{6,7}=7.9 \mathrm{~Hz}, \mathrm{H}-\right.$ 7), 4.39 (dd, $\left.1 \mathrm{H}, J_{5,6}=4.5 \mathrm{~Hz}, J_{6,7}=7.9 \mathrm{~Hz}, \mathrm{H}-7\right), 4.30$ (d, $\left.1 \mathrm{H}, J_{l^{\prime \prime}, 2^{\prime \prime}}=9.8 \mathrm{~Hz}, \mathrm{H}-1^{\prime \prime}\right), 3.90-3.84$ (m, 2H, H-6a", H-2"), $3.71\left(\mathrm{dd}, 1 \mathrm{H}, J_{5^{\prime \prime}, 6^{\prime \prime}}=5.4 \mathrm{~Hz}, J_{6 a^{\prime \prime}, 6 b^{\prime \prime}}=12.5 \mathrm{~Hz}, \mathrm{H}-6 \mathrm{~b}^{\prime \prime}\right), 3.49$ (t, 1H, $J_{3^{\prime \prime}, 4^{\prime \prime}}=$ $\left.J_{4^{\prime \prime}, 5^{\prime \prime}}=8.9 \mathrm{~Hz}, \mathrm{H}-3^{\prime \prime}\right), 3.47$ (s, 3H, OCH 3 ), $3.42-3.34$ (m, 2H, H-4", H-5"), $3.34-3.28$ (m, 1H, H-5), 3.28 - 3.22 (m, 1H, H-3a'), 3.22 - 3.15 (m, 1H, H-3b'), 3.03 (m, 3H, $\left.\mathrm{CH}_{2}-1^{\prime}, \mathrm{H}-8 \mathrm{a}\right), 2.75$ $\left(\mathrm{d}, 1 \mathrm{H}, J_{8 a, 8 b}=13.1 \mathrm{~Hz}, \mathrm{H}-8 \mathrm{~b}\right), 2.23$ (t, $\left.2 \mathrm{H}, J_{1,2}=7.2 \mathrm{~Hz}, \mathrm{CH}_{2}-1\right), 2.02\left(\mathrm{~s}, 3 \mathrm{H}, \mathrm{CH}_{3} N A c\right), 1.78-$ 1.50 (m, 6H, $\left.\mathrm{CH}_{2}-2^{\prime}, \mathrm{CH}_{2}-2, \mathrm{CH}_{2}-4\right), 1.44-1.34$ (m, 2H, $\left.\mathrm{CH}_{2}-3\right) ;{ }^{13} \mathrm{C} \mathrm{NMR}\left(125 \mathrm{MHz}, \mathrm{D}_{2} \mathrm{O}\right) \delta$ $176.6\left(\mathrm{C}=\mathrm{O}\right.$ amide), 174.0 ( $\mathrm{C}=\mathrm{O}$ acetamide), 165.3 ( $\mathrm{C}=\mathrm{O}$ carbamide), $90.0\left(\mathrm{C}-1^{\prime \prime}\right), 77.3\left(\mathrm{C}-5^{\prime \prime}\right)$, $75.5\left(\mathrm{C}-3^{\prime \prime}\right), 69.6\left(\mathrm{C}-4^{\prime \prime}\right), 62.0(\mathrm{C}-6), 61.0\left(\mathrm{OCH}_{3}\right), 60.8\left(\mathrm{C}-6{ }^{\prime \prime}\right), 60.1(\mathrm{C}-7), 55.3(\mathrm{C}-5), 52.2(\mathrm{C}-$ 2"), 48.7 (C-1'), 39.6 (C-8), 37.0 (C-3'), 35.4 (C-1), 27.7 (C-3), 27.6 (C-4), 25.9 (C-2'), 25.0 (C2), $22.1\left(\mathrm{CH}_{3} \mathrm{Ac}\right)$; HRMS(ESI) $\mathrm{m} / \mathrm{z}$ calcd. for $\left[\mathrm{C}_{22} \mathrm{H}_{39} \mathrm{~N}_{5} \mathrm{O}_{8} \mathrm{SNa}\right]^{+}: 556.2414$, obsd.: 556.2416.
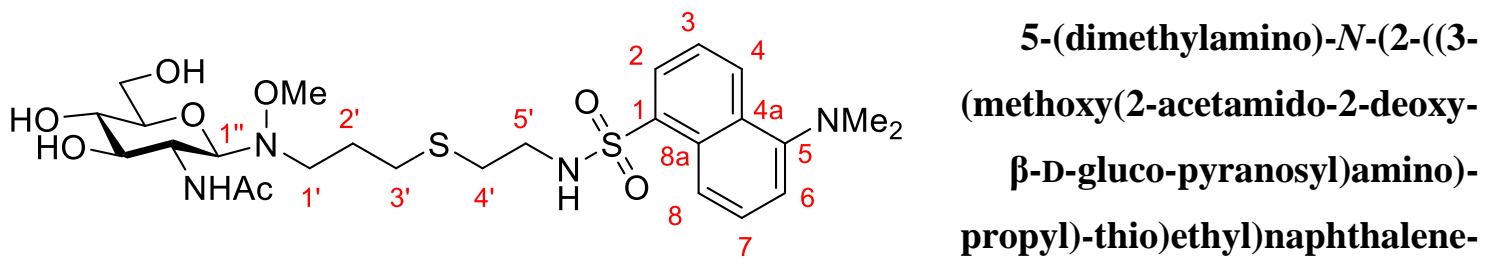

1-sulfonamide (28). To a solution of amine $\mathbf{1 6}(10.9 \mathrm{mg}, 29.7 \mu \mathrm{mol})$ in $\mathrm{H}_{2} \mathrm{O}(2 \mathrm{~mL}), \mathrm{NaHCO}_{3}$ (15 mg, $179 \mu \mathrm{mol})$ was added. Dansylchloride $(12.0 \mathrm{mg}, 44.5 \mu \mathrm{mol})$ was dissolved in distilled THF $(1 \mathrm{~mL})$ and added drop wise to the reaction mixture. After 2 hours the THF was concentrated 
in vacuo and the remaining aqueous reaction mixture was purified by reverse phase chromatography $\left(\mathrm{C}_{18}, \mathrm{H}_{2} \mathrm{O} / \mathrm{MeOH}, 100 / 0 \rightarrow 25 / 75, \mathrm{v} / \mathrm{v}\right)$ to give fluorescent glycoside 28 (13.8 $\mathrm{mg}, 77 \%) ; \mathrm{R}_{f}=0.50\left(\mathrm{CH}_{2} \mathrm{Cl}_{2} / \mathrm{MeOH}, 8 / 1, \mathrm{v} / \mathrm{v}\right) ; \alpha_{\mathrm{D}}{ }^{17.8}=-14.4(\mathrm{c}=0.5, \mathrm{MeOH}) ; \mathrm{IR}$ (film) 3304, 3283, 3088, 2939, 2795, 1650, 1572, 1503, 1456, 1409, 1374, 1356, 1316, 1232, 1201, 1142, $1076,1037,945,793,686,625,573,554,536 \mathrm{~cm}^{-1} ;{ }^{1} \mathrm{H}$ NMR $\left(500 \mathrm{MHz}, \mathrm{CD}_{3} \mathrm{OD}\right) \delta 8.57(\mathrm{~d}, 1 \mathrm{H}$, $\left.J_{2,3}=8.6 \mathrm{~Hz}, \mathrm{H}-2\right), 8.34\left(\mathrm{~d}, 1 \mathrm{H}, J_{3,4}=8.7 \mathrm{~Hz}, \mathrm{H}-4\right), 8.21\left(\mathrm{~d}, 1 \mathrm{H}, J_{7,8}=7.3 \mathrm{~Hz}, \mathrm{H}-8\right), 7.62-7.56$ (m, 2H, H-3, H-7), 7.28 (d, 1H, $\left.J_{6,7}=7.6 \mathrm{~Hz}, \mathrm{H}-6\right), 4.22$ (d, 1H, $\left.J_{l^{\prime \prime}, 2^{\prime \prime}}=9.8 \mathrm{~Hz}, \mathrm{H}-1^{\prime \prime}\right), 3.85$ (dd, $\left.1 \mathrm{H}, J_{5^{\prime \prime}, 6 a^{\prime \prime}}=1.8 \mathrm{~Hz}, J_{6 a^{\prime \prime}, 6 b^{\prime \prime}}=12.0 \mathrm{~Hz}, \mathrm{H}-6 \mathrm{a}^{\prime \prime}\right), 3.78\left(\mathrm{dd}, 1 \mathrm{H}, J_{l^{\prime \prime}, 2^{\prime \prime}}=J_{2^{\prime \prime}, 3^{\prime \prime}}=9.9 \mathrm{~Hz}, \mathrm{H}-2^{\prime \prime}\right), 3.68(\mathrm{dd}$, $\left.1 \mathrm{H}, J_{5^{\prime \prime}, 6 b^{\prime \prime}}=5.6 \mathrm{~Hz}, J_{6 a^{\prime \prime}, 6 b^{\prime \prime}}=12.0 \mathrm{~Hz}, \mathrm{H}-6 \mathrm{~b}^{\prime \prime}\right), 3.47\left(\mathrm{~s}, 3 \mathrm{H}, \mathrm{OCH}_{3}\right), 3.41\left(\mathrm{dd}, 1 \mathrm{H}, J_{2^{\prime \prime}, 3^{\prime \prime}}=J_{3^{\prime \prime}, 4^{\prime \prime}}=9.0\right.$ $\left.\mathrm{Hz}, \mathrm{H}-3^{\prime \prime}\right), 3.36-3.27$ (m, $\left.1 \mathrm{H}, \mathrm{H}-4^{\prime \prime}\right), 3.21\left(\mathrm{ddd}, 1 \mathrm{H}, J_{5^{\prime \prime}, 6 a^{\prime \prime}}=1.9 \mathrm{~Hz}, J_{5^{\prime \prime}, 6 b^{\prime \prime}}=5.4 \mathrm{~Hz}, J_{4^{\prime \prime}, 5^{\prime \prime}}=9.6\right.$ $\left.\mathrm{Hz}, \mathrm{H}-5^{\prime \prime}\right), 3.02$ (t, $\left.1 \mathrm{H}, J_{4^{\prime} 5^{\prime}}=7.2 \mathrm{~Hz}, \mathrm{H}-5^{\prime}\right), 2.98-2.85$ (m, 8H, H-1', $2 \times N-\mathrm{CH}_{3}$ ), $2.49-2.32$ (m, 4H, H-3', H-4') 1.95 (s, 3H, $\left.\mathrm{CH}_{3} \mathrm{Ac}\right), 1.69-1.59$ (m, 1H, H-2'); ${ }^{13} \mathrm{C}$ NMR (125 MHz, $\left.\mathrm{CD}_{3} \mathrm{OD}\right) \delta 173.3(\mathrm{C}=\mathrm{O}$ Ac), 153.2, 137.1, 131.32, 131.30 (C-1/4a/5/8a), 130.9 (C-2), 130.1 (C8), 129.2, 124.3 (C-3/C-7), 120.6 (C-4), 116.5 (C-6), 92.4 (C-1"), 79.8 (C-5"), 77.5 (C-3"), 71.8 $\left(\mathrm{C}-4^{\prime \prime}\right), 62.9\left(\mathrm{C}-6^{\prime \prime}\right), 62.2\left(\mathrm{OCH}_{3}\right), 54.1\left(\mathrm{C}-2^{\prime \prime}\right), 51.4\left(\mathrm{C}-1^{\prime}\right), 45.8\left(\mathrm{~N}\left(\mathrm{CH}_{3}\right)_{2}\right), 43.9\left(\mathrm{C}-5^{\prime}\right), 32.4(\mathrm{C}-$ $\left.4^{\prime}\right), 30.2\left(\mathrm{C}-3^{\prime}\right), 28.5\left(\mathrm{C}-2^{\prime}\right), 23.0\left(\mathrm{CH}_{3} \mathrm{Ac}\right)$; HRMS(ESI) $\mathrm{m} / \mathrm{z}$ calcd. for $\left[\mathrm{C}_{26} \mathrm{H}_{41} \mathrm{~N}_{4} \mathrm{O}_{8} \mathrm{~S}_{2}\right]^{+}$: 601.2360, obsd.: 601.2362 .

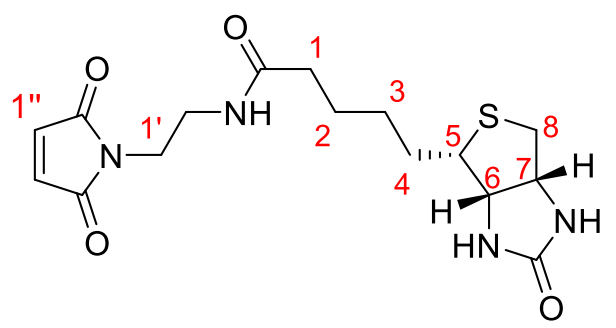

1-(2-D-biotinamidoethyl)-1H-pyrrole-2,5-dione (29). To a solution of 2-aminoethyl maleimide ${ }^{44}$ (159 $\mathrm{mg}, 1.13 \mathrm{mmol})$ and D-biotin $(363.4 \mathrm{mg}, 1.31 \mathrm{mmol})$ in DMF (10 mL), HBTU (516 mg, $1.36 \mathrm{mmol})$ and triethylamine $(0.1 \mathrm{~mL})$ was added and the reaction mixture was stirred at $\mathrm{rt}$ for $2 \mathrm{~h}$. The crude reaction mixture was concentrated and purified by reverse phase column chromatography $\left(\mathrm{C}_{18}, \mathrm{H}_{2} \mathrm{O} / \mathrm{MeOH}, 100 / 0 \rightarrow 70 / 30\right.$, v/v) and size exclusion chromatography (Sephadex LH-20, $\mathrm{CH}_{2} \mathrm{Cl}_{2} / \mathrm{MeOH}, 50 / 50$, v/v) to give biotin functionalised maleimide 29 as a white solid (380 mg, $1.04 \mathrm{mmol}, 92 \%)$. $\mathrm{R}_{f}=0.59\left(\mathrm{CH}_{2} \mathrm{Cl}_{2} / \mathrm{MeOH}, 80 / 20\right.$, v/v); $\alpha_{\mathrm{D}}^{20.6}=+26.5(\mathrm{c}=0.2, \mathrm{MeOH}) ;$ IR (film) $3283,3087,2936,2864,1701,1552,1461,1439,1408$, 1360, 1332, 1266, 1169, 1101, 1055, 1033, 828, 763, $696 \mathrm{~cm}^{-1} ;{ }^{1} \mathrm{H}$ NMR (500 MHz, CD $\left.{ }_{3} \mathrm{OD}\right) \delta$ $6.82(\mathrm{~s}, 2 \mathrm{H}, \mathrm{HC}=\mathrm{CH}), 4.60(\mathrm{~s}, 1 \mathrm{H}, \mathrm{NH}), 4.49\left(\mathrm{dd}, J_{6,7}=4.9 \mathrm{~Hz}, J_{7,8}=7.7 \mathrm{~Hz}, 1 \mathrm{H}, \mathrm{H}-7\right), 4.67$ (dd, $\left.1 \mathrm{H}, J_{6,7}=4.9 \mathrm{~Hz}, J_{5,6}=7.8, \mathrm{~Hz} \mathrm{H}-6\right), 3.61\left(\mathrm{dd}, 2 \mathrm{H}, J_{I^{\prime}, 2^{\prime}}=5.5 \mathrm{~Hz}, \mathrm{CH}_{2}-1^{\prime}\right), 3.36\left(\mathrm{dd}, 2 \mathrm{H}, J_{l^{\prime}, 2^{\prime}}=\right.$ $\left.5.5 \mathrm{~Hz}, \mathrm{CH}_{2}-2^{\prime}\right), 3.24-3.17(\mathrm{~m}, 1 \mathrm{H}, \mathrm{H}-5), 2.93\left(\mathrm{dd}, 1 \mathrm{H}, J_{7-8 a}=5.0 \mathrm{~Hz}, J_{8 a-8 b}=12.8 \mathrm{~Hz}, \mathrm{H}-8 \mathrm{a}\right)$, $2.70\left(\mathrm{~d}, 1 \mathrm{H}, J_{8 a-8 b}=12.8 \mathrm{~Hz}, \mathrm{H}-8 \mathrm{~b}\right), 2.12\left(\mathrm{dt}, 2 \mathrm{H}, J_{l a, 2}=3.4 \mathrm{~Hz}, J_{l b, 2}=7.4 \mathrm{~Hz}, \mathrm{CH}_{2}-1\right), 1.79-1.66$ (m, 1H, $\mathrm{CH}_{2}-4 \mathrm{a}$ ), 1.66 - 1.52 (m, 3H, $\left.\mathrm{CH}_{2}-2, \mathrm{CH}_{2}-4 \mathrm{~b}\right), 1.50-1.35$ (m, 2H, $\left.\mathrm{CH}_{2}-3\right)$; ${ }^{13} \mathrm{C}$ NMR $\left(125 \mathrm{MHz}, \mathrm{CD}_{3} \mathrm{OD}\right) \delta 176.3(\mathrm{C}=\mathrm{O}$ amide $), 172.6(2 \times \mathrm{C}=\mathrm{O}$ maleimide $), 166.1(\mathrm{C}=\mathrm{O}$ carbamate $)$, $135.5(\mathrm{C}=\mathrm{C}), 63.3$ (C-6), $61.6(\mathrm{C}-7), 56.9(\mathrm{C}-5), 41.0(\mathrm{C}-8), 38.8\left(\mathrm{C}-2^{\prime}\right), 38.4\left(\mathrm{C}-1^{\prime}\right), 36.7(\mathrm{C}-1)$, 
29.7 (C-3), 29.4 (C-4), 26.6 (C-2); HRMS(ESI) $m / z$ calcd. for $\left[\mathrm{C}_{16} \mathrm{H}_{23} \mathrm{~N}_{4} \mathrm{O}_{4} \mathrm{~S}\right]^{+}: 367.1435$, obsd.: 367.1429 .

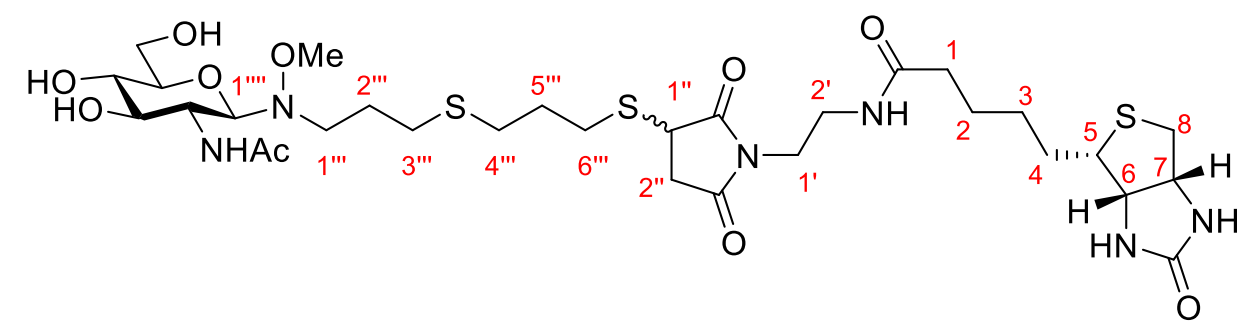

1-(2-D-biotinamidoethyl)-3-(3-(3-(methoxy[2-acetamido-2-deoxy- $\beta$-D-glucopyranosyl]amino)propylthio)propylthio)pyrrolidine-2,5-dione (34). To a solution of neoglycoside 20 (7.0 $\mathrm{mg}, 17.6 \mu \mathrm{mol})$ in $\mathrm{H}_{2} \mathrm{O}(1 \mathrm{~mL})$, maleimide $29(9.0 \mathrm{mg}, 24.6 \mathrm{mmol})$ was added, and the reaction mixture was stirred at $\mathrm{rt}$ for $1 \mathrm{~h}$. The crude reaction mixture was purified using size exclusion chromatography (Bio Gel P-2) to yield glycoconjugate 34 (12.6 mg, $16.5 \mu \mathrm{mol}, 94 \%$ ) as colourless oil. $\mathrm{R}_{f}=0.54\left(\mathrm{CH}_{2} \mathrm{Cl}_{2} / \mathrm{EtOH} / \mathrm{MeOH} / \mathrm{NH}_{3}\right.$ (aq. 33\%), 5/2/2/1, v/v/v/v); IR (film) 3338, 3286, 2933, 2871, 2859, 1700, 1648, 1559, 1452, 1439, 1332, 1265, 1188, 1107, 1080, 1026, 969 , 901, 847, $760699 \mathrm{~cm}^{-1} ;{ }^{1} \mathrm{H}$ NMR $\left(500 \mathrm{MHz}, \mathrm{D}_{2} \mathrm{O}\right) \delta 4.60\left(\mathrm{dd}, 1 \mathrm{H}, J_{7,8}=5.0 \mathrm{~Hz}, J_{6,7}=7.8 \mathrm{~Hz}, \mathrm{H}-\right.$ 7), 4.42 (dd, $\left.1 \mathrm{H}, J_{5-6}=4.5 \mathrm{~Hz}, J_{6-7}=7.8 \mathrm{~Hz}, \mathrm{H}-6\right), 4.32$ (d, $\left.1 \mathrm{H}, J_{I^{\prime \prime \prime}, 2^{\prime \prime \prime \prime}}=9.8 \mathrm{~Hz}, \mathrm{H}-1^{\prime \prime \prime \prime}\right), 4.02-$ $3.98\left(\mathrm{~m}, 1 \mathrm{H}, \mathrm{H}-1^{\prime \prime}\right), 3.91-3.85$ (m, 2H, H-2'"', $\left.\mathrm{H}-6 \mathrm{a}^{\prime \prime \prime \prime}\right), 3.72\left(\mathrm{dd}, 1 \mathrm{H}, J_{5^{\prime \prime \prime \prime}, 6^{\prime \prime \prime}}=5.2 \mathrm{~Hz}, J_{6 a^{\prime \prime \prime}, 6 b^{\prime \prime \prime \prime}}=\right.$ $\left.12.3 \mathrm{~Hz}, \mathrm{H}-6 \mathrm{~b}^{\prime \prime \prime \prime}\right), 3.71$ - 3.58 (m, 2H, $\left.\mathrm{CH}_{2}-1^{\prime}\right), 3.53$ - 3.46 (m, 4H, H-3'"'", $\mathrm{OCH}_{3}$ ), 3.45 - 3.35 (m, 4H, CH $\mathrm{CH}_{2} \mathrm{2}^{\prime}, \mathrm{H}-5^{\prime \prime \prime \prime}, \mathrm{H}-4^{\prime \prime \prime \prime}$ ), 3.35 - 3.24 (m, 2H, $\left.\mathrm{CH}_{2}-5, \mathrm{H}-2 \mathrm{a}^{\prime \prime}\right), 3.06-2.95$ (m, 3H, $\mathrm{CH}_{2}-1^{\prime \prime \prime}$, $\mathrm{H}-8 \mathrm{a}), 2.93-2.79$ (m, 2H, $\left.\mathrm{CH}_{2}-6^{\prime \prime \prime}\right), 2.77$ (d, 1H, $\left.J_{8 a-8 b}=13.0 \mathrm{~Hz}, \mathrm{H}-8 \mathrm{~b}\right), 2.72$ (m, 5H, H-2b", $\left.\mathrm{CH}_{2}-4^{\prime \prime \prime}, \mathrm{CH}_{2}-3^{\prime \prime \prime}\right), 2.19$ (t, $1 \mathrm{H}, J_{l, 2}=7.3 \mathrm{~Hz}, \mathrm{CH}_{2}-1$ ), 2.03 (s, 3H, $\mathrm{CH}_{3} \mathrm{Ac}$ ), $1.96-1.87$ (m, 2H, $\left.\mathrm{CH}_{2}-5^{\prime \prime \prime}\right), 1.84-1.76$ (m, 2H, $\left.\mathrm{CH}_{2}-2^{\prime \prime \prime}\right), 1.76-1.66$ (m, 1H, H-4a), 1.62 - 1.50 (m, 3H, H-4b, $\left.\mathrm{CH}_{2}-2\right), 1.42-1.33\left(\mathrm{~m}, 2 \mathrm{H}, \mathrm{CH}_{2}-3\right) ;{ }^{13} \mathrm{C}$ NMR $\left(125 \mathrm{MHz}, \mathrm{D}_{2} \mathrm{O}\right) \delta 182.0,180.8(2 \times \mathrm{C}=\mathrm{O}$ pyrrolidine $), 179.5$ ( $\mathrm{C}=\mathrm{O}$ biotin), 176.3 ( $\mathrm{C}=\mathrm{O}$ NAc), 167.8 (C=O carbamate-biotin), 92.6 (C-1"'"'), 80.0 (C-4"'''), 78.1 (C-3"'"'), 72.2 (C-5"'"'), 64.6 (C-6), $63.7\left(\mathrm{OCH}_{3}\right), 63.4$ (C-6"'"'), 62.8 (C-7), 57.8 (C-5), 54.9 (C-2'"'), 52.9 (C-1'"'), 42.5 (C-1"), 42.3 (C-8), 41.2 (C-1'), 39.1 (C-2'), 38.5 (C-2"), 38.0 (C-1), 32.4 (C-6"'), 32.3 (C-4"'), 31.2 (C-3"'), 30.6, 30.5 (C-5"', C-3), 30.2 (C-4), 28.9 (C$\left.2^{\prime \prime \prime}\right), 27.5$ (C-2), $24.8\left(\mathrm{CH}_{3}\right.$ Ac); HRMS(ESI) $\mathrm{m} / z$ calcd. for $\left[\mathrm{C}_{31} \mathrm{H}_{53} \mathrm{~N}_{6} \mathrm{O}_{10} \mathrm{~S}_{3}\right]^{+}:$765.2980, obsd.: 765.2975 . 


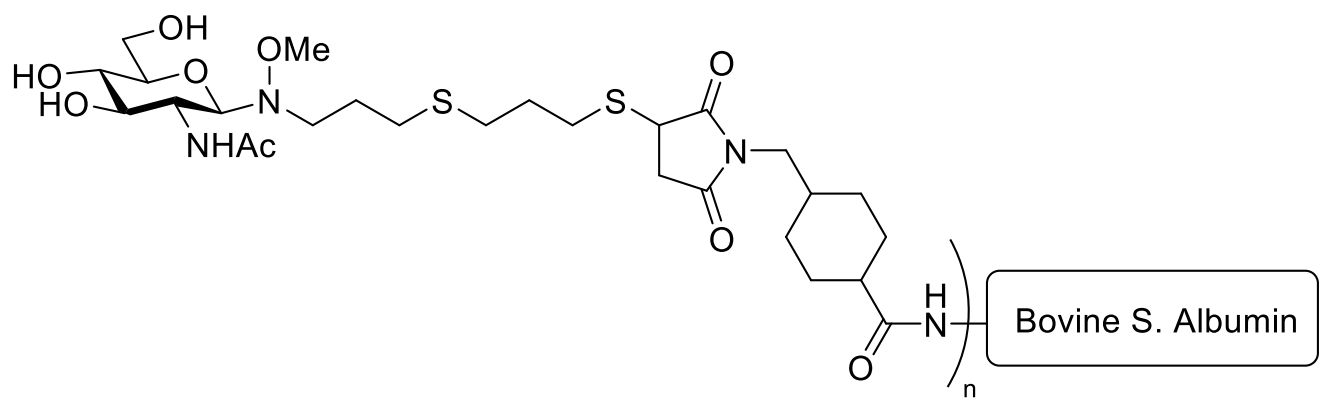

Glycoprotein 37. To a solution of Bovine Serum Albumin 36 (1 mg, $0.015 \mu \mathrm{mol})$ in PBS (1 mL), SMCC cross-linker $35(0.9 \mathrm{mg}, 2.73 \mu \mathrm{mol})$ in $\mathrm{MeCN}(0.1 \mathrm{~mL})$ was added and the reaction mixture was incubated at rt. To $10 \mu \mathrm{L}$ of the crude reaction mixture, neoglycoside $\mathbf{2 0}(2.2 \mathrm{mg}, 5.45 \mu \mathrm{mol})$ in water $(10 \mu \mathrm{L})$ was added and the reaction mixture was allowed to stir at $\mathrm{rt}$ to obtain crude glycoprotein 37.

\section{Preparation of MALDI-TOF Matrix solution.}

Matrix solution. To a solution of cinnapinic acid (15 mg) in $\mathrm{MeCN}: \mathrm{H}_{2} \mathrm{O}(1 \mathrm{~mL}, 1 / 1, \mathrm{v} / \mathrm{v})$, TFA (1 $\mu \mathrm{L}$ ) was added and the mixture was vortexed for $3 \mathrm{~min}$, and centrifuged for 10 minutes, to obtain the matrix solution.

Sample preparation. $1-2 \mathrm{uL}$ of the desired sample $(\sim 1.0 \mathrm{mg} / \mathrm{mL})$ was added to $20 \mu \mathrm{L}$ of the matrix solution and a pipette was used to mix the sample. NOTE, do not use vortex since this will precipitate the protein. Next, $2 \mu \mathrm{L}$ of the sample/matrix was spotted on the MALDI-plate and allowed to dry for $1 \mathrm{~h}$. before measuring the MALDI-TOF. 


\subsection{References}

(1) Allen, H. J., Kisailus, E. C., E. . M. D. Inc. In Glycoconjugates: Composition: Structure, and Function; New York, NY, 1992.

(2) Davis, B. G. 'Recent Developments in Glycoconjugates' J. Chem. Soc. Perk. T. 1 1999, 3215.

(3) Peri, F.; Cipolla, L.; La Ferla, B.; Nicotra, F. 'Glycoconjugate and Oligosaccharide Mimetics by Chemoselective Ligation' C.R. Chim. 2003, 6, 635-644.

(4) Boltje, T. J.; Buskas, T.; Boons, G.-J. 'Opportunities and Challenges in Synthetic Oligosaccharide and Glycoconjugate Research.' Nat. Chem. 2009, 1, 611-622.

(5) Specker, D.; Wittmann, V. Synthesis and Application of Glycopeptide and Glycoprotein Mimetics. In Glycopeptides and Glycoproteins; Springer Berlin Heidelberg: Berlin, Heidelberg, 2007; pp. 65-107.

(6) Horlacher, T.; Seeberger, P. H. 'Carbohydrate Arrays as Tools for Research and Diagnostics.' Chem. Soc. Rev. 2008, 37, 1414-1422.

(7) Laurent, N.; Voglmeir, J.; Flitsch, S. L. 'Glycoarrays--Tools for Determining ProteinCarbohydrate Interactions and Glycoenzyme Specificity.' Chem. Comm. 2008, 44004412.

(8) Oyelaran, O.; Gildersleeve, J. C. 'Glycan Arrays: Recent Advances and Future Challenges.' Curr. Opin. Chem. Biol. 2009, 13, 406-413.

(9) Timmer, M. S. M.; Stocker, B. L.; Seeberger, P. H. 'Probing Glycomics.' Curr. Opin. Chem. Biol. 2007, 11, 59-65.

(10) Stocker, B. L.; Timmer, M. S. M. 'Chemical Tools for Studying the Biological Function of Glycolipids.' Chembiochem 2013, 14, 1164-1184.

(11) Likhosherstov, L. M.; Novikova, O. S.; Derevitskaja, V. A.; Kochetkov, N. K. 'A New Simple Synthesis of Amino Sugar B-D-Glycosylamines'. Carbohydr. Res., 1986, 146, C1C5.

(12) Song, X.; Xia, B.; Stowell, S. R.; Lasanajak, Y.; Smith, D. F.; Cummings, R. D. 'Novel Fluorescent Glycan Microarray Strategy Reveals Ligands for Galectins.' Chem. Biol. 2009, 16, 36-47.

(13) Jencks, W. P. 'Studies on the Mechanism of Oxime and Semicarbazone Formation 1' J. Am. Chem. Soc. 1959, 81, 475-481.

(14) Sayer, J. M.; Peskin, M.; Jencks, W. P. 'Imine-Forming Elimination Reactions. I. General Base Acid Catalysis and Influence of the Nitrogen Substituent on Rates and Equilibria for Carbinolamine Dehydration' J. Am. Chem. Soc. 1973, 95, 4277-4287.

(15) Helferich, B.; Schirp, H. 'Über N-Acyl-Hydrazone Einfacher Zucker, II. Mitteilung' Chem. Ber. 1953, 86, 547-556.

(16) Goff, R. D.; Thorson, J. S. 'Neoglycosylation and Neoglycorandomization: Enabling Tools for the Discovery of Novel Glycosylated Bioactive Probes and Early Stage Leads.' Medchemcomm 2014, 5, 1036-1047.

(17) Peri, F.; Dumy, P.; Mutter, M. 'Chemo- and Stereoselective Glycosylation of Hydroxylamino Derivatives: A Versatile Approach to Glycoconjugates' Tetrahedron 1998, 54, 12269-12278.

(18) Moreno, H. R.; Radomski, J. L. 'Synthesis of the Urinary Glucuronic Acid Conjugate of N-Hydroxy-4-Aminobiphenyl.' Cancer Lett. 1978, 4, 85-88.

(19) Poupko, J. M.; Hearn, W. L.; Radomski, J. L. 'N-Glucuronidation of N-Hydroxy Aromatic Amines: A Mechanism for Their Transport and Bladder-Specific Carcinogenicity.' Toxicol. Appl. Pharmacol. 1979, 50, 479-484.

(20) Peri, F.; Deutman, A.; La Ferla, B. La; Nicotra, F. 'Solution and Solid-Phase Chemoselective Synthesis of (1-6)-Amino(methoxy) Di- and Trisaccharide Analogues.' Chem. Comm. 2002, 1504-1505.

(21) Peri, F.; Jiménez-Barbero, J.; García-Aparicio, V.; Tvaroska, I.; Nicotra, F. 'Synthesis and Conformational Analysis of Novel $\mathrm{N}(\mathrm{OCH} 3)$-Linked Disaccharide Analogues.' Chem. Eur. J. 2004, 10, 1433-1444. 
(22) Ishida, J.; Hinou, H.; Naruchi, K.; Nishimura, S.-I. 'Synthesis of Neoglycosphingolipid from Methoxyamino-Functionalized Ceramide.' Bioorg. Med. Chem. Lett. 2014, 24, 1197-1200.

(23) Carrasco, M. R.; Nguyen, M. J.; Burnell, D. R.; MacLaren, M. D.; Hengel, S. M. 'Synthesis of Neoglycopeptides by Chemoselective Reaction of Carbohydrates with Peptides Containing a Novel N'-Methyl-Aminooxy Amino Acid' Tetrahedron Lett. 2002, 43, 5727 5729.

(24) Filira, F.; Biondi, B.; Biondi, L.; Giannini, E.; Gobbo, M.; Negri, L.; Rocchi, R. 'Opioid Peptides: Synthesis and Biological Properties of [(N-Gamma-glucosyl,N-GammaMethoxy)-Alpha, Gamma-Diamino-(S)-butanoyl]4-Deltorphin-1-Neoglycopeptide and Related Analogues.' Org. Biomol. Chem. 2003, 1, 3059-3063.

(25) Carrasco, M. R.; Brown, R. T. 'A Versatile Set of Aminooxy Amino Acids for the Synthesis of Neoglycopeptides.' J. Org. Chem. 2003, 68, 8853-8858.

(26) Carrasco, M. R.; Brown, R. T.; Serafimova, I. M.; Silva, O. 'Synthesis of N-Fmoc-O-(N'Boc-N'-Methyl)-Aminohomoserine, an Amino Acid for the Facile Preparation of Neoglycopeptides.' J. Org. Chem. 2003, 68, 195-197.

(27) Jiménez-Castells, C.; de la Torre, B. G.; Gutiérrez Gallego, R.; Andreu, D. 'Optimized Synthesis of Aminooxy-Peptides as Glycoprobe Precursors for Surface-Based SugarProtein Interaction Studies.' Bioorg. Med. Chem. Lett. 2007, 17, 5155-5158.

(28) Seo, J.; Michaelian, N.; Owens, S. C.; Dashner, S. T.; Wong, A. J.; Barron, A. E.; Carrasco, M. R. 'Chemoselective and Microwave-Assisted Synthesis of Glycopeptoids.' Org. Lett. 2009, 11, 5210-5213.

(29) Zevgiti, S.; Zabala, J. G.; Darji, A.; Dietrich, U.; Panou-Pomonis, E.; SakarellosDaitsiotis, M. 'Sialic Acid and Sialyl-Lactose Glyco-Conjugates: Design, Synthesis and Binding Assays to Lectins and Swine Influenza H1N1 Virus.' J. Pept. Sci. 2012, 18, 52 58.

(30) Bohorov, O.; Andersson-Sand, H.; Hoffmann, J.; Blixt, O. 'Arraying Glycomics: A Novel Bi-Functional Spacer for One-Step Microscale Derivatization of Free Reducing Glycans.' Glycobiology 2006, 16, 21C - 27C.

(31) Leung, C.; Chibba, A.; Gómez-Biagi, R. F.; Nitz, M. 'Efficient Synthesis and Protein Conjugation of Beta-(1-->6)-D-N-Acetylglucosamine Oligosaccharides from the Polysaccharide Intercellular Adhesin.' Carbohydr. Res. 2009, 344, 570-575.

(32) Cló, E.; Blixt, O.; Jensen, K. J. 'Chemoselective Reagents for Covalent Capture and Display of Glycans in Microarrays' Eur. J. Org. Chem. 2010, 540-554.

(33) Wang, Z.; Chinoy, Z. S.; Ambre, S. G.; Peng, W.; McBride, R.; de Vries, R. P.; Glushka, J.; Paulson, J. C.; Boons, G.-J. 'A General Strategy for the Chemoenzymatic Synthesis of Asymmetrically Branched N-Glycans.' Science 2013, 341, 379-383.

(34) Davies, A. J.; Donald, A. S. R.; Marks, R. E. 'The Acid-Catalysed Decomposition of Some B-Azido-Carbonyl Compounds' J. Chem. Soc. C Org. 1967, 2109.

(35) Rostovtsev, V. V.; Green, L. G.; Fokin, V. V.; Sharpless, K. B. 'A Stepwise Huisgen Cycloaddition Process: Copper(I)-Catalyzed Regioselective "Ligation" of Azides and Terminal Alkynes' Angew. Chem. Int. Ed. Engl. 2002, 41, 2596-2599.

(36) Baskin, J. M.; Prescher, J. A.; Laughlin, S. T.; Agard, N. J.; Chang, P. V; Miller, I. A.; Lo, A.; Codelli, J. A.; Bertozzi, C. R. 'Copper-Free Click Chemistry for Dynamic in Vivo Imaging.' Proc. Natl. Acad. Sci. U. S. A. 2007, 104, 16793-16797.

(37) Yoshitake, S.; Imagawa, M.; Ishikawa, E.; Niitsu, Y.; Urushizaki, I.; Nishiura, M.; Kanazawa, R.; Kurosaki, H.; Tachibana, S.; Nakazawa, N.; Ogawa, H. 'Mild and Efficient Conjugation of Rabbit Fab' and Horseradish Peroxidase Using a Maleimide Compound and Its Use for Enzyme Immunoassay.' J. Biochem. 1982, 92, 1413-1424.

(38) Dickgreber, N.; Stoitzner, P.; Bai, Y.; Price, K. M.; Farrand, K. J.; Manning, K.; Angel, C. E.; Dunbar, P. R.; Ronchese, F.; Fraser, J. D.; Bäckström, B. T.; Hermans, I. F. 'Targeting Antigen to MHC Class II Molecules Promotes Efficient Cross-Presentation and Enhances Immunotherapy.' J. Immunol. 2009, 182, 1260-1269.

(39) Lowe, A. B. 'Thiol-Ene "click" Reactions and Recent Applications in Polymer and Materials Synthesis' Polym. Chem. 2010, 1, 17.

(40) Miyata, O.; Muroya, K.; Kobayashi, T.; Yamanaka, R.; Kajisa, S.; Koide, J.; Naito, T. 'Radical Cyclization in Heterocycle Synthesis. Part 13: Sulfanyl Radical Addition- 
cyclization of Oxime Ethers and Hydrazones Connected with Alkenes for Synthesis of Cyclic B-Amino Acids' Tetrahedron 2002, 58, 4459-4479.

(41) Bauer, L.; Ghosh, B. K. 'The Synthesis of (Hydroxylamino)alkyl Mercaptans' J. Org. Chem. 1965, 30, 4298-4303.

(42) Werz, D. B.; Ranzinger, R.; Herget, S.; Adibekian, A.; von der Lieth, C.-W.; Seeberger, P. H. 'Exploring the Structural Diversity of Mammalian Carbohydrates ("glycospace") by Statistical Databank Analysis.' ACS Chem. Biol. 2007, 2, 685-691.

(43) Langenhan, J. M.; Endo, M. M.; Engle, J. M.; Fukumoto, L. L.; Rogalsky, D. R.; Slevin, L. K.; Fay, L. R.; Lucker, R. W.; Rohlfing, J. R.; Smith, K. R.; Tjaden, A. E.; Werner, H. M. 'Synthesis and Biological Evaluation of RON-Neoglycosides as Tumor Cytotoxins' Carbohydr. Res. 2011, 346, 2663-2676.

(44) Richter, M.; Chakrabarti, A.; Ruttekolk, I. R.; Wiesner, B.; Beyermann, M.; Brock, R.; Rademann, J. 'Multivalent Design of Apoptosis-Inducing Bid-BH3 PeptideOligosaccharides Boosts the Intracellular Activity at Identical Overall Peptide Concentrations.' Chemistry 2012, 18, 16708-16715.

(45) Santoso, K. "The Synthesis of Carbohydrate Probes for the Diagnosis of Multiple Sclerosis," Victoria University Wellington, 2015.

(46) Huflejt, M. E.; Vuskovic, M.; Vasiliu, D.; Xu, H.; Obukhova, P.; Shilova, N.; Tuzikov, A.; Galanina, O.; Arun, B.; Lu, K.; Bovin, N. 'Anti-Carbohydrate Antibodies of Normal Sera: Findings, Surprises and Challenges.' Mol. Immunol. 2009, 46, 3037-3049.

(47) Bae, S. M.; Kim, J.-H.; Chung, S. W.; Byun, Y.; Kim, S. Y.; Lee, B.-H.; Kim, I.-S.; Park, R.-W. 'An Apoptosis-Homing Peptide-Conjugated Low Molecular Weight HeparinTaurocholate Conjugate with Antitumor Properties.' Biomaterials 2013, 34, 2077-2086. 


\section{Chapter 3.}

\section{Design and synthesis of novel fluorescent and biotinylated multivalent glycodendrons.}

\subsection{Introduction}

Dendrimers and dendrons are commonly used to enhance or decrease a specific activity, and accordingly have been investigated for their use in a large variety of applications ${ }^{1}$ including light harvesting and fluorescent sensors, ${ }^{2,3}$ catalysis, ${ }^{4}$ as well as for biomedical purposes. ${ }^{5}$ In particular, the use of glycodendrimers in biomedical applications has significantly increased over the last few decades and glycodendrons are now being used as magnetic resonance imaging contrast agents, 6,7 antigen carriers and adjuvants for vaccines, ${ }^{8,9}$ cell-specific delivery vehicles for the treatment and diagnosis of disease, ${ }^{10-12}$ or to simply solubilise active pharmaceuticals. ${ }^{13}$

On a molecular level, inter-cellular interactions are often mediated by multivalent interactions between carbohydrate antigens and their corresponding lectins. In fact, the low binding affinity between carbohydrate antigens and lectins requires multivalent interactions in order to induce a biological response. ${ }^{14}$ The key feature of glycodendrons is their multivalent display of carbohydrate antigens and accordingly such glycodendrons are able to mimic cellular interactions. This allows glycodendrons to be used in biomedical applications, as described above. ${ }^{9}$ As discussed in Chapter 1 , several synthetic methods have been developed for the assembly of multivalent dendrons, ${ }^{15}$ whereas this chapter provides an overview of the TRIS-based dendrimer/dendron syntheses. Moreover, the synthesis of a novel second generation dendron scaffold is discussed, which allows for the rapid 
functionalisation and oxyamine glycan conjugation towards the assembly of complex glycoconjugates for the biological purpose of choice.

\subsubsection{TRIS-based dendrons}

Tris(hydroxymethyl)aminomethane (TRIS) (1) as a branching unit in dendrons and dendrimers has been utilised in various synthetic methodologies. ${ }^{16-19}$ Key in the synthesis of TRIS-based dendrons is the tri- $O$-alkylation reaction, and accordingly, several alkylation reactions have been reported (Scheme 1). For example, reaction of TRIS (1) with acrylonitrile gives rise to trinitrile $\mathbf{3}$, whereas the conjugate addition of the $N$-protected TRIS 2 with either propargyl bromide, allyl bromide or tertbutyl bromoacetate gives rise to dendrons 4, 5 and 6, respectively. Alternatively, the $N$-protected TRIS 2 can be used as an acceptor in glycosylation reactions to give the trivalent glycodendron 7 .

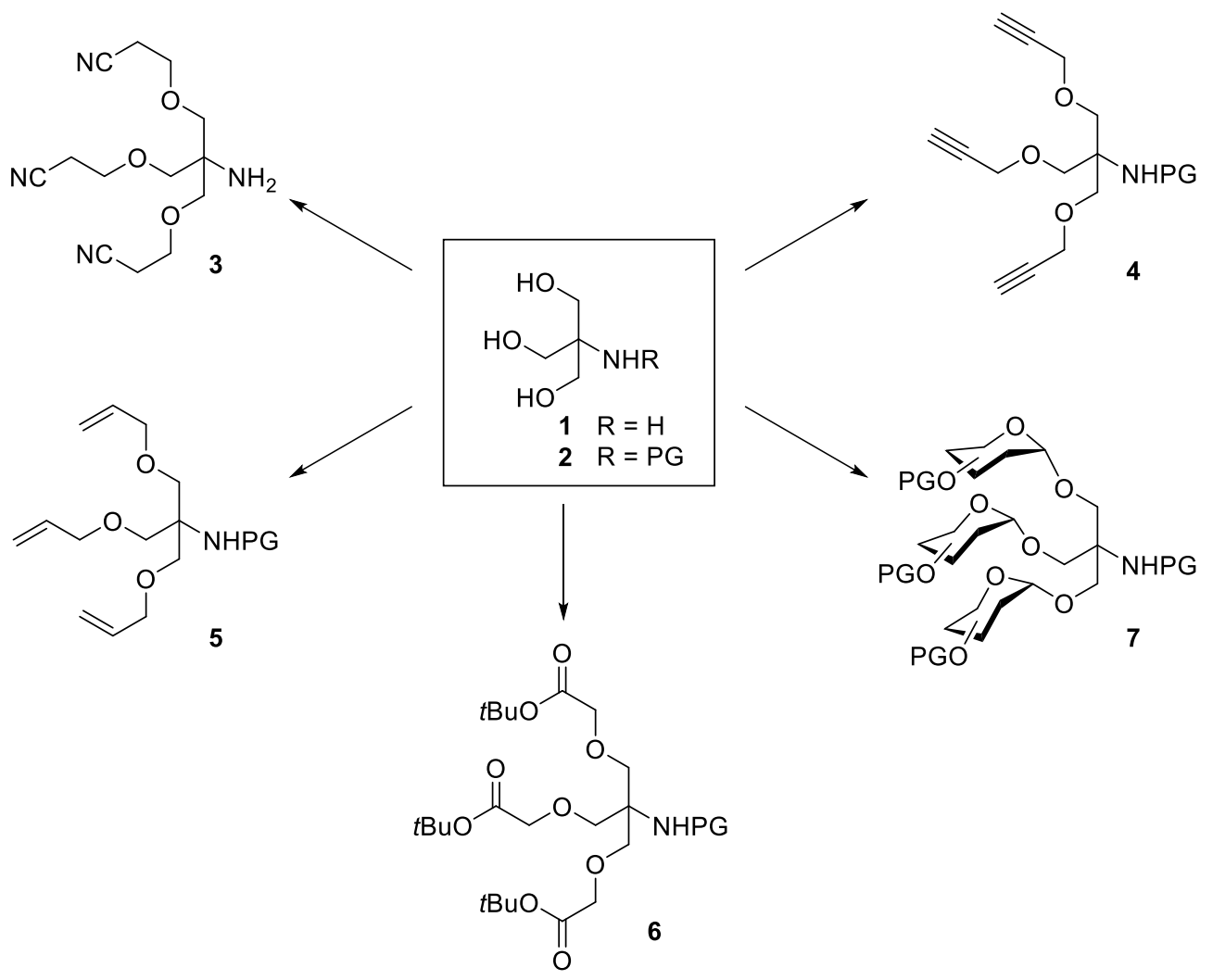

Scheme 1. Different approaches for the tri-O-alkylation of TRIS. 


\subsubsection{TRIS alkylation with acrylonitrile}

In 1991, Newkome et al. first reported the use of TRIS (1) as a core scaffold for dendrimer synthesis. ${ }^{16}$ Their synthesis commenced with a Michael addition of TRIS to acrylonitrile to give 1,4-adduct $\mathbf{8}$ in $81 \%$ yield (Scheme 2). Next, the nitrile functional groups were converted to the ethyl esters under acidic conditions to give first generation dendron 9. The amine was then reacted with 1,3,5benzenetricarbonyl trichloride (10) to obtain nonaester 11 which after ester hydrolysis gave the nonavalent acid $\mathbf{1 2}$.

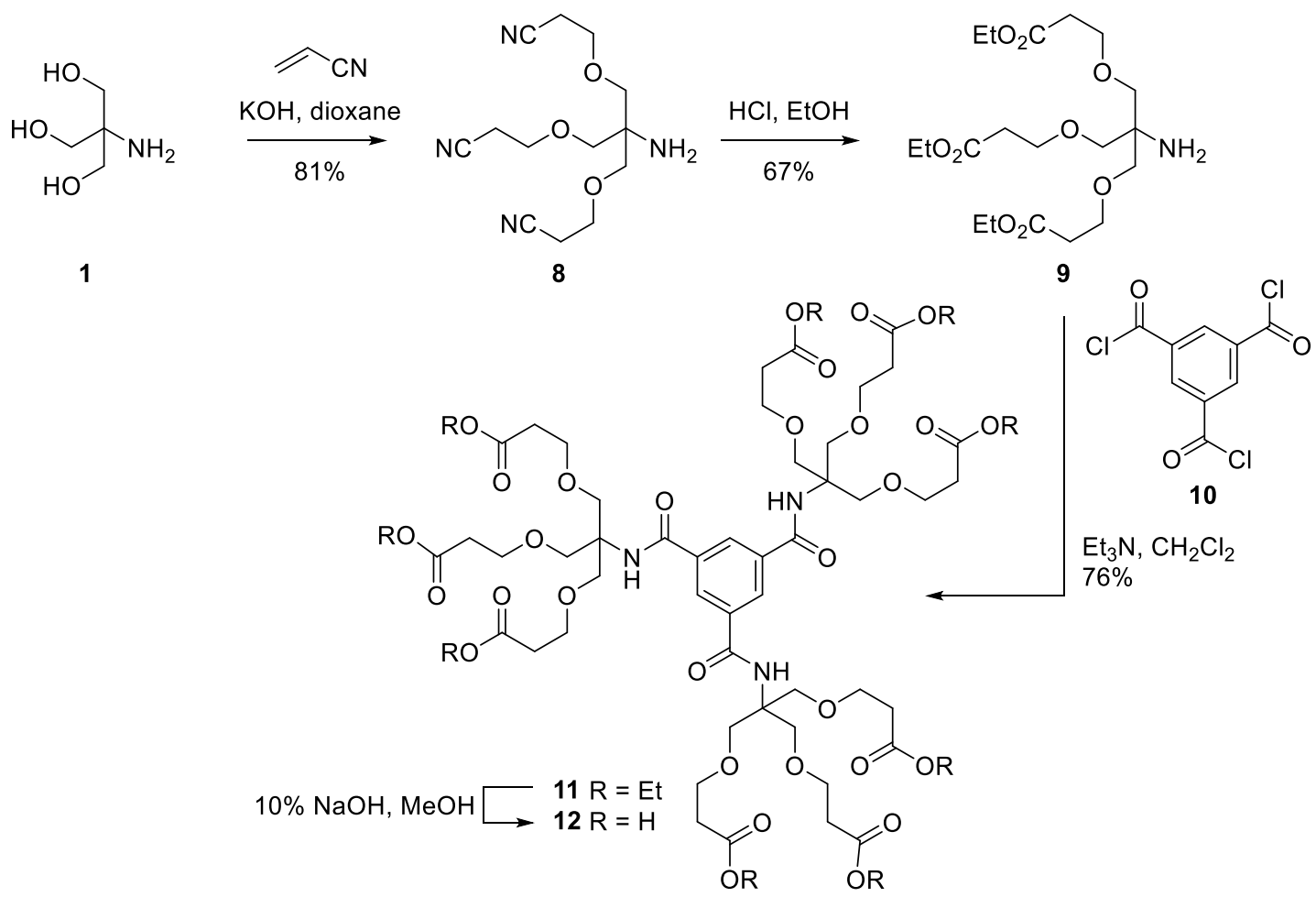

Scheme 2. First dendrimer synthesis featuring the TRIS moiety. ${ }^{16,20}$

Following the initial work by Newkome et al., many TRIS-based dendrons have been synthesised utilising the initial alkylation with acrylonitrile. For example, in 1999 Sliedregt et al. synthesised glycodendron glycolipid $\mathbf{1 3}$ in 9 steps from the afore mentioned common intermediate $\mathbf{9}$ in order to associate glycosides with liposomes via the incorporation of the acylated cholesterol derivative (Figure 1). ${ }^{21,22}$ The liposomes were then used to target liver cells via the selective binding of asialoglycoprotein receptors to the galactose residues of the glycodendron. 


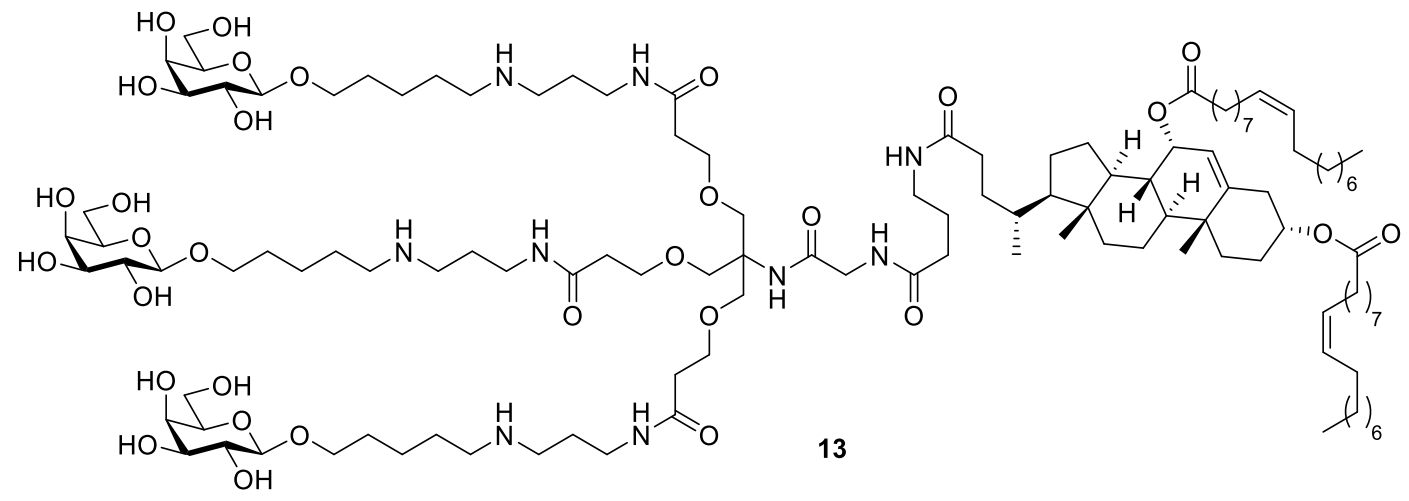

Figure 1. Galactose glycodendron lipids allow for liposome association to target liver cells. ${ }^{21}$

More recently, this synthetic route has been used by Seeberger and co-workers for the synthesis of second generation glycodendron $\mathbf{1 4}$ functionalised with a cyclodextrin for the selective delivery of doxorubicin to liver cells (Figure 2). ${ }^{11}$ Here, the doxorubicin was trapped within the cyclodextrin moiety, which allowed the release of the active drug in the target cells. It was found that doxorubicincontaining glycodendrons were able to selectively induce apoptosis in liver cells, whereas the empty cyclodextrin glycodendrons did not result in liver cell apoptosis. This nicely demonstrates the ability of multivalent glycodendrons to target cell-specific receptors and deliver cytotoxic drugs in order to deplete these cell types, and, therefore, validates the potential use of dendrimers as drug-delivery vehicles.

Although acrylonitrile has been successfully employed in glycodendrimer synthesis, an extra step is required for the conversion to the ester derivative, which ultimately lowers the overall efficiency of the synthesis. 


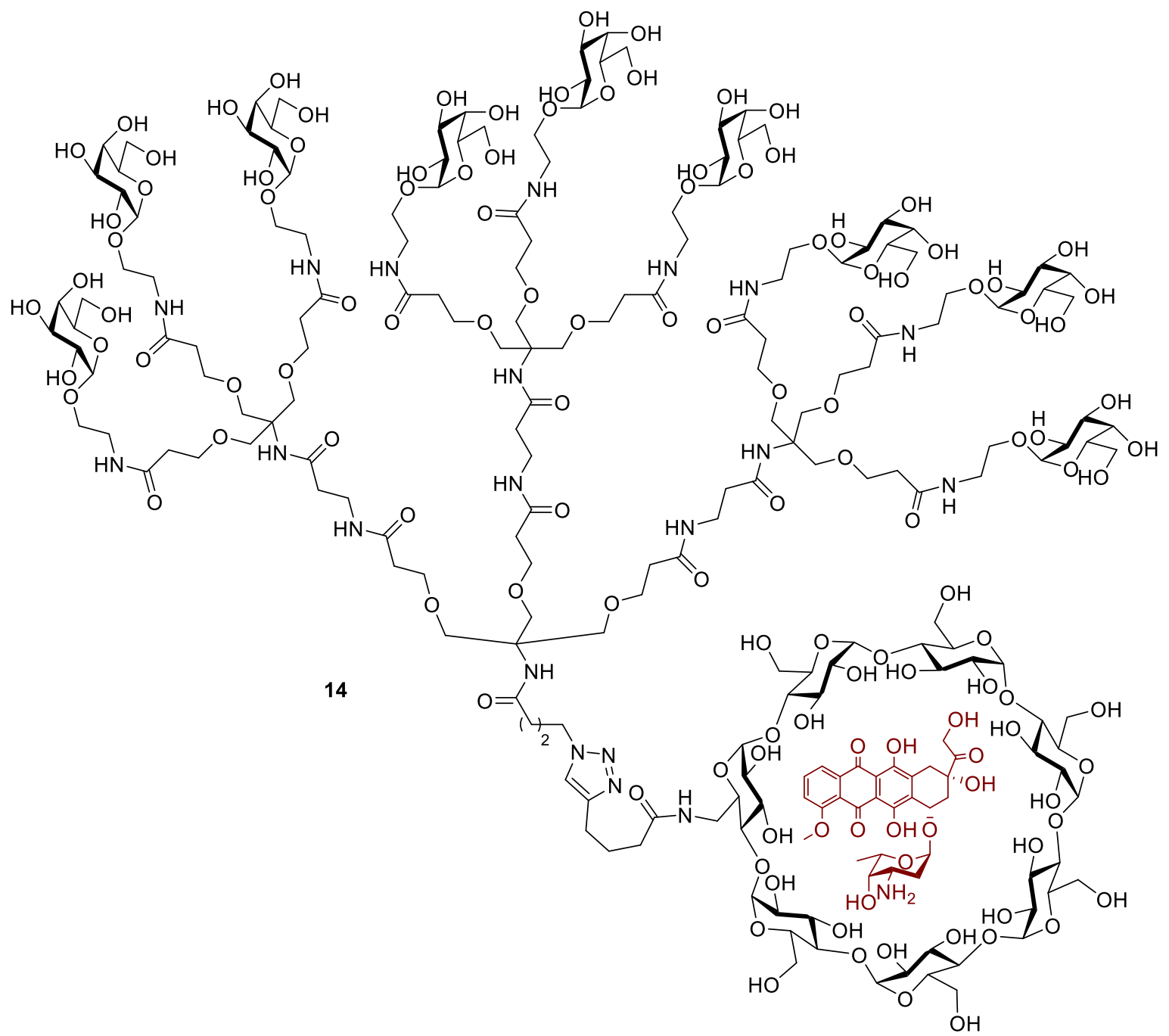

Figure 2. A galactose-glycodendron functionalised $\beta$-cyclodextrin for the liver cell-specific delivery of doxorubicin.

\subsubsection{Propargylation of TRIS}

The tri- $O$-propargylation of $N$-protected TRIS with propargyl bromide can be performed to give the first generation dendron, which in turn is suitable for the Huisgen cycloaddition reaction with azide derivatives. ${ }^{17,23-25}$ Although the use of copper in late stage dendrimer chemistry is not ideal due to the cytotoxicity of copper and the ability of dendrons to host metals in its void spaces, ${ }^{26,27}$ the Huisgen cycloaddition has been used extensively throughout the field of dendrimer chemistry. ${ }^{28,29}$ For example, in 2008 Chabre et al. presented the synthesis of triazole based dendrons and dendrimers, whereby TRIS (1) was reacted with acryloyl chloride to give triol $\mathbf{1 5}$, which subsequently was reacted with propargyl bromide to give dendron 16 (Scheme 3). ${ }^{17}$ Next, the dendron was reacted with thiol $\mathbf{1 7}$ to give the octadecapropargylated dendritic scaffold, which, was then used in the copper-mediated 
azide-alkyne Huisgen cycloaddition with azido-glycan $\mathbf{1 8}$ to give octadeca-valent glycodendrimer 19 after deacetylation.

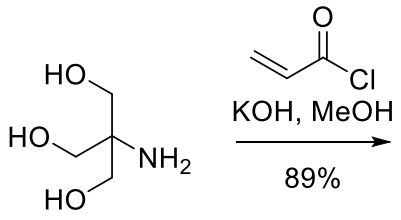<smiles>C#CCBr</smiles>

15<smiles>C#CCOCC(COCC#C)(COCC#C)NC(=O)C=C</smiles>

16 $\mathrm{NaBH}_{4}, \mathrm{EtOH}$ $88 \%$

2) $18, \mathrm{CuSO}_{4}$ $\mathrm{Na}$ ascorbate $\mathrm{THF} / \mathrm{H}_{2} \mathrm{O}$ $55^{\circ} \mathrm{C}, 6 \mathrm{~h}$ $\mathrm{rt}, 18 \mathrm{~h}(78 \%)$

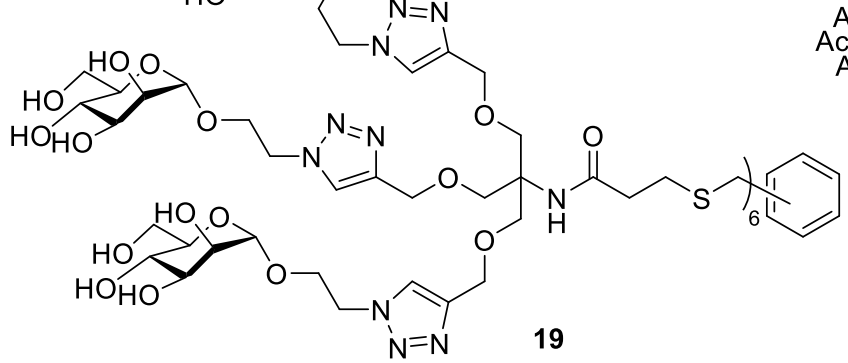

3) $\mathrm{NaOMe}, \mathrm{MeOH}$ $90 \%$

Scheme 3. Glycodendrimer 19 synthesis utilising an azide-alkyne Huisgen cycloaddition reaction. ${ }^{17}$

In a similar fashion, Rojo and co-workers synthesised fluorescently labelled glycodendrons $\mathbf{2 0 - 2 2}$ utilising the copper catalysed azide-alkyne cycloaddition reaction (Figure 3). ${ }^{25}$ Although, instead of TRIS, pentaerythritol was used as the branching moiety towards the synthesis of several second generation glycodendrons, which were then used to analyse the DC-SIGN-mediated internalization by dendritic cells. A synthetic intermediate was then used in the synthesis of virus-like glycodendrinanoparticles bearing up to 1620 glycans, to study its ability to block DC-SIGN and prevent infection by the Ebola virus. ${ }^{30}$ 


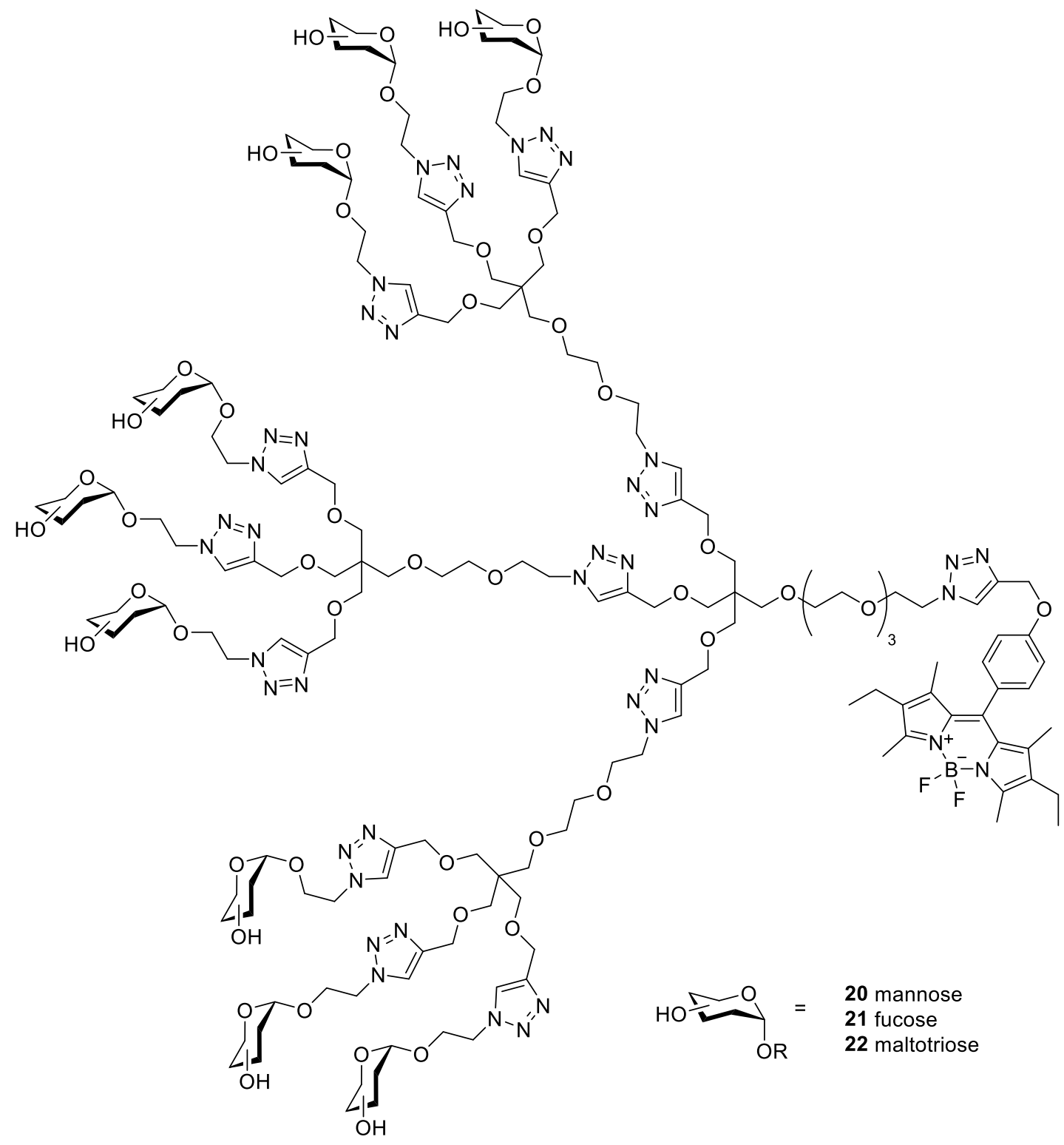

Figure 3. BODIPY-labelled glycodendrons were synthesised to study DC-SIGN-mediated internalization by human dendritic cells. ${ }^{25}$

\subsubsection{Allylation of TRIS}

Kaplánek et al. demonstrated the use of allyl bromide in the tri- $O$-alkylation of TRIS derivatives, where TRIS (1) was $N$-Boc protected $(\rightarrow \mathbf{2 3})$ and subsequently allylated with allyl bromide using $\mathrm{KOH}$ in DMSO to give trialkene $\mathbf{2 4}$ in good yields (Scheme 4). ${ }^{18}$ Although these dendrons were not used for conjugation of biomolecules, the tri- $O$-allylation would allow for the synthesis of sulfur derived dendrimers, through the use of thiol-ene conjugation reactions. ${ }^{31}$ The use of sulfur chemistry in the synthesis of glycodendrons has been discussed in an excellent review. ${ }^{32}$ 


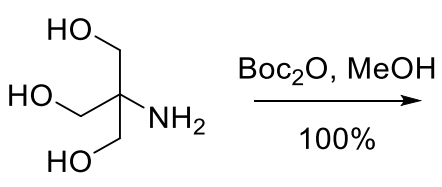

1

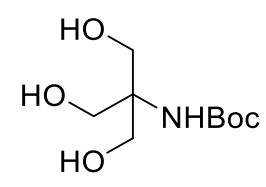

23
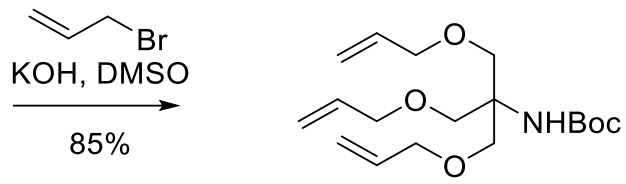

24

Scheme 4. Synthesis of triallyl dendron $24 .^{18}$

Similar trialkenyl dendrons such as $\mathbf{2 5}$, were used in thiol-ene conjugations, as was demonstrated by Gómes-Garcia et al. who used thioglycosides such as lactose derivative $\mathbf{2 6}$ to form glycodendron $\mathbf{2 7}$ using catalytic azobisisobutyronitrile (AIBN) (Scheme 5). ${ }^{33}$ On the other hand, more complex, asymmetrical glycodendrons could be obtained when the lactose glycan was coupled first followed by the thiol-ene conjugation with mannoside 28. This, however, resulted in the formation of a 3:1 mixture of $\mathbf{2 9}$ and $\mathbf{3 0}$ respectively. These glycodendron were then used in the convergent synthesis of a $\beta$-cyclodextrin glycodendron.

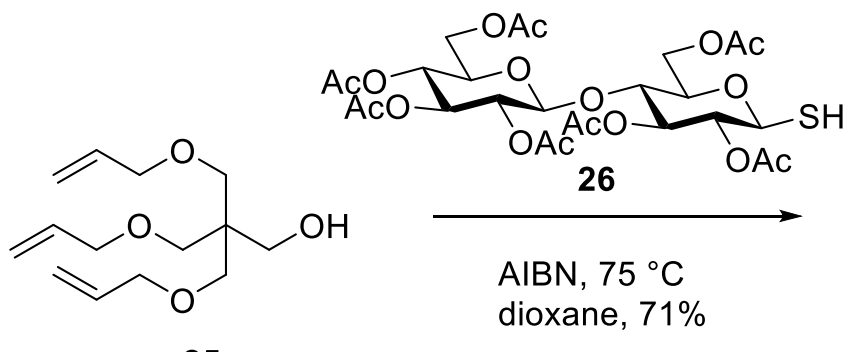

25

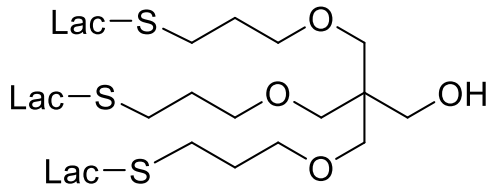

27

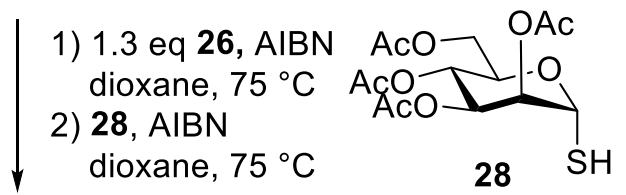

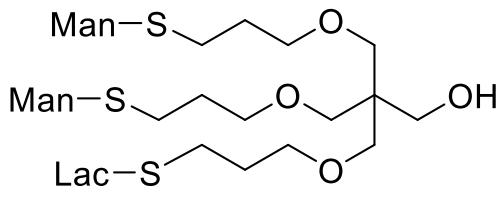

29

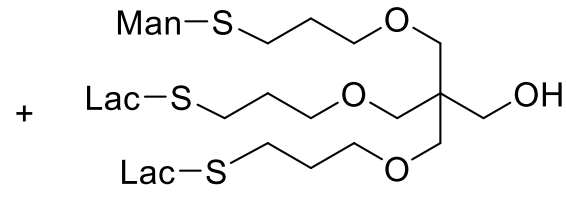

30

Scheme 5. Thiol-ene conjugation in the synthesis of glycodendrons. ${ }^{33}$

These thiol-ene conjugations, however, require radical initiation and conjugation at high temperatures which can complicate the conjugation of oligosaccharides. In addition, protected glycoconjugates were used which requires a late stage deprotection step on large glycoconjugates complicating the assembly of these macromolecules. 


\subsubsection{TRIS alkylation with tert-butyl bromoacetate}

In 2001, Dupuy et al. reported the tri- $O$-alkylation of $N, N$-diphenyl TRIS 31 with tert-butyl bromoacetate. ${ }^{34}$ The alkylation was performed in a two-phase system $\left(\mathrm{CH}_{2} \mathrm{Cl}_{2}: 50 \%\right.$ aq. $\left.\mathrm{NaOH}\right)$ with a phase transfer catalyst to give dendron 32 in $53 \%$ (Scheme 6). The resulting dendron, however, was synthesised for phase transfer methodology purposes and was not further utilised. In contrast to the use of acrylonitrile for the tri- $O$-alkylation of TRIS derivatives, the advantage of using tert-butyl bromoacetate as an alkylation agent is that it directly results in ester formation and thus shortens the synthetic route by one step. In 1999, a similar alkylation was included in patented methodology by Martinez, whereby a similar triol was reacted with tert-butyl bromoacetate and $\mathrm{KOtBu}$ in $t \mathrm{BuOH}$. The yield of this reaction, however, was not reported. ${ }^{35}$

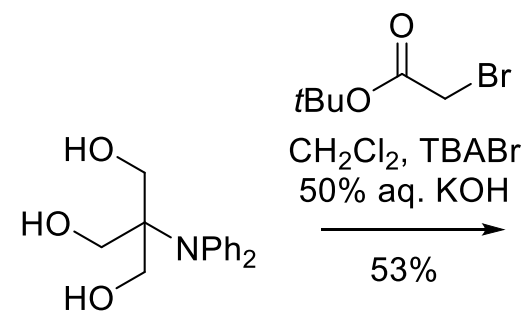

31

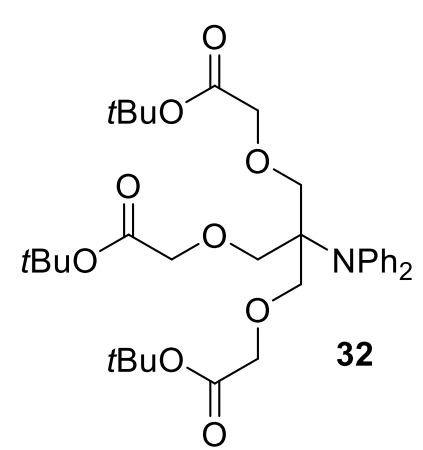

Scheme 6. Tri-O-alkylation of TRIS derivative 31 with tert-butyl bromoacetate. ${ }^{34}$

\subsubsection{Direct tri-O-glycosylation of TRIS}

As mentioned above, the use of TRIS derivatives as acceptors in glycosylation reactions gives rise to trivalent glycodendrons, which in turn can be used in a convergent route to make larger generation glycodendrons. In 1996, Stoddart and co-workers reported the synthesis of an octadeca-valent glycodendron via this convergent synthetic route (Scheme 7). ${ }^{19,36}$ Here, TRIS (1) was benzyloxycarboxyl (Z)-protected and the resulting carbamate $\mathbf{3 3}$ was glycosylated with glycosyl bromide $\mathbf{3 4}$ to give glycoside $\mathbf{3 5}$. With the dendron in hand, further derivatisation gave octadecavalent glycodendron 36. 


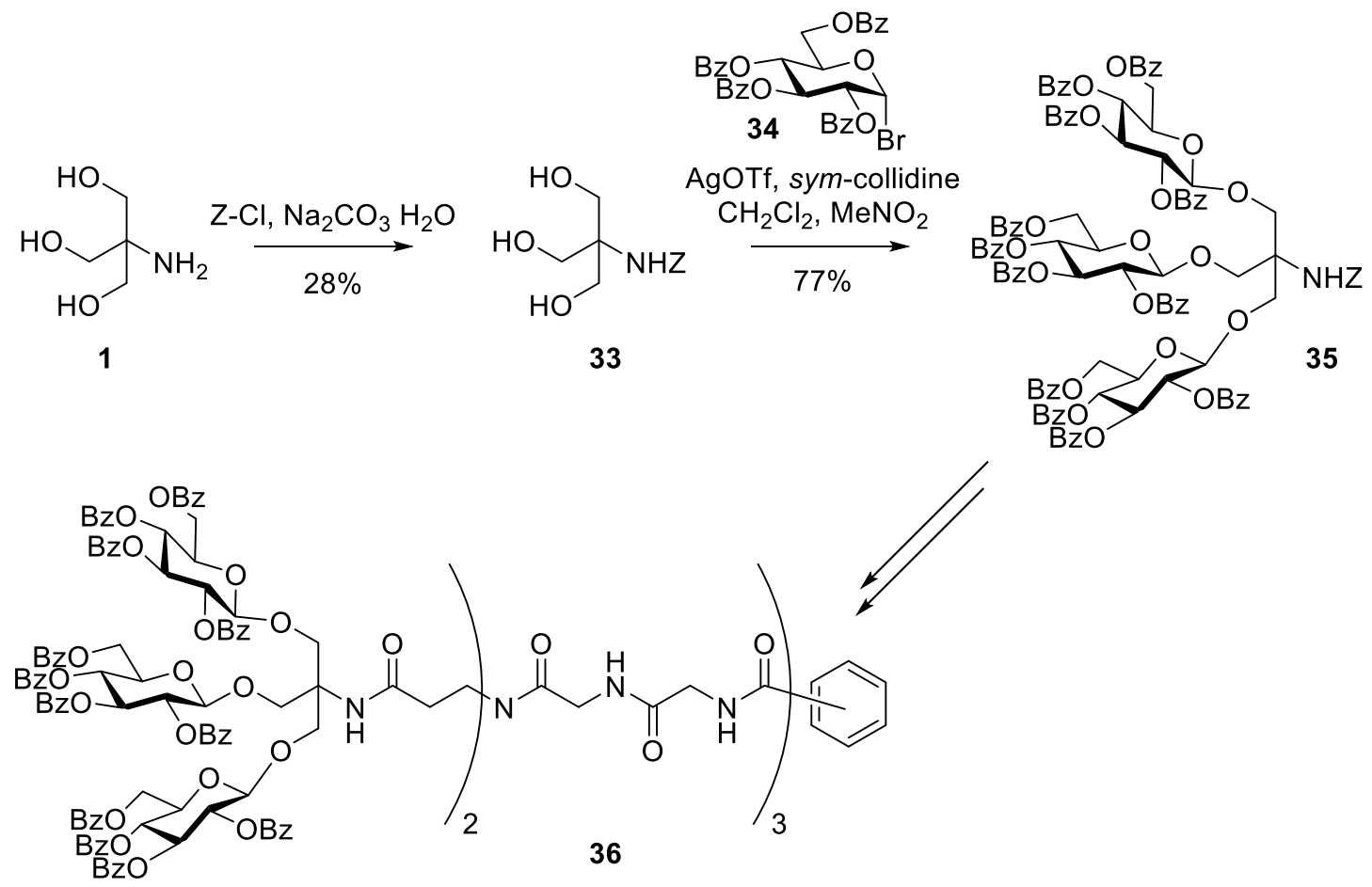

Scheme 7. Convergent synthesis of TRIS-glycodendrons by Stoddart and co-workers. ${ }^{19}$

Using this glycosylation method, Furneaux et al. synthesised trivalent $\alpha$-mannose-dendrons for the liposome-mediated antigen delivery to dendritic cells. ${ }^{37}$ In 2006, this type of glycodendron was functionalised with paramagnetic gadolinium $(\mathrm{Gd})$ metal for MRI purpose. ${ }^{6}$ More recently, glycodendrons were conjugated to photosensitisers to exclusively target cancer cell lines, but these glycodendrons did not show cell specificity in vitro, when compared between healthy cells and two tumour cell lines.

This method, however, requires the use of protected glycosyl donors, which then requires global deprotection of macromolecules, complicating the synthesis of large glycodendrons. Moreover, the short distance between the three antigens makes these dendrimers extremely compact. This might affect the lectin binding as these glycodendrons are relatively bulky when compared to the other TRIS-based glycodendrons. 


\subsubsection{Project aim and Retrosynthesis}

The aim of the research described in this chapter is to synthesise novel glycodendrons for use in biomedical applications. To this end, it was envisioned that an orthogonal dendron core, which allows for the rapid functionalisation with the antigen and probes of choice, should be prepared (Scheme 8). The carbohydrate antigen of choice can then be coupled to the periphery of the dendron. Here, it should be noted that the use of bifunctional methoxyamine linkers, as described in Chapter 2, will be utilised to conjugate the desired carbohydrate to the multivalent dendron scaffold. Thus, to obtain glycodendrons I, a peptide conjugation protocol was envisioned for the glycan conjugation, and accordingly a carboxylic acid functionalised periphery of the dendron was desired, which in turn could be readily obtained from ester derivatives II. Orthogonality at the core of the dendron allows for functionalisation with the probe (e.g. biotin and fluorophores), and again it was envisioned to use a peptide conjugation protocol to functionalise the amine core with carboxylic acid derivatised probes to obtain the dendrons II. Accordingly, the amine core dendron could be obtained from azide III via the Staudinger reaction. As described above, sufficient lectin binding can be achieved with nonavalent second generation glycodendrons, and accordingly, the synthesis of a bifunctional second generation dendron III was proposed. Here, it was anticipated that the efficient double exponential growth methodology could be used, thereby allowing for the synthesis of the second generation dendron III from the first generation dendron IV. Finally, dendron IV can be synthesised in two steps from the branching unit TRIS (1), via amine conjugation and tri- $O$-alkylation. 

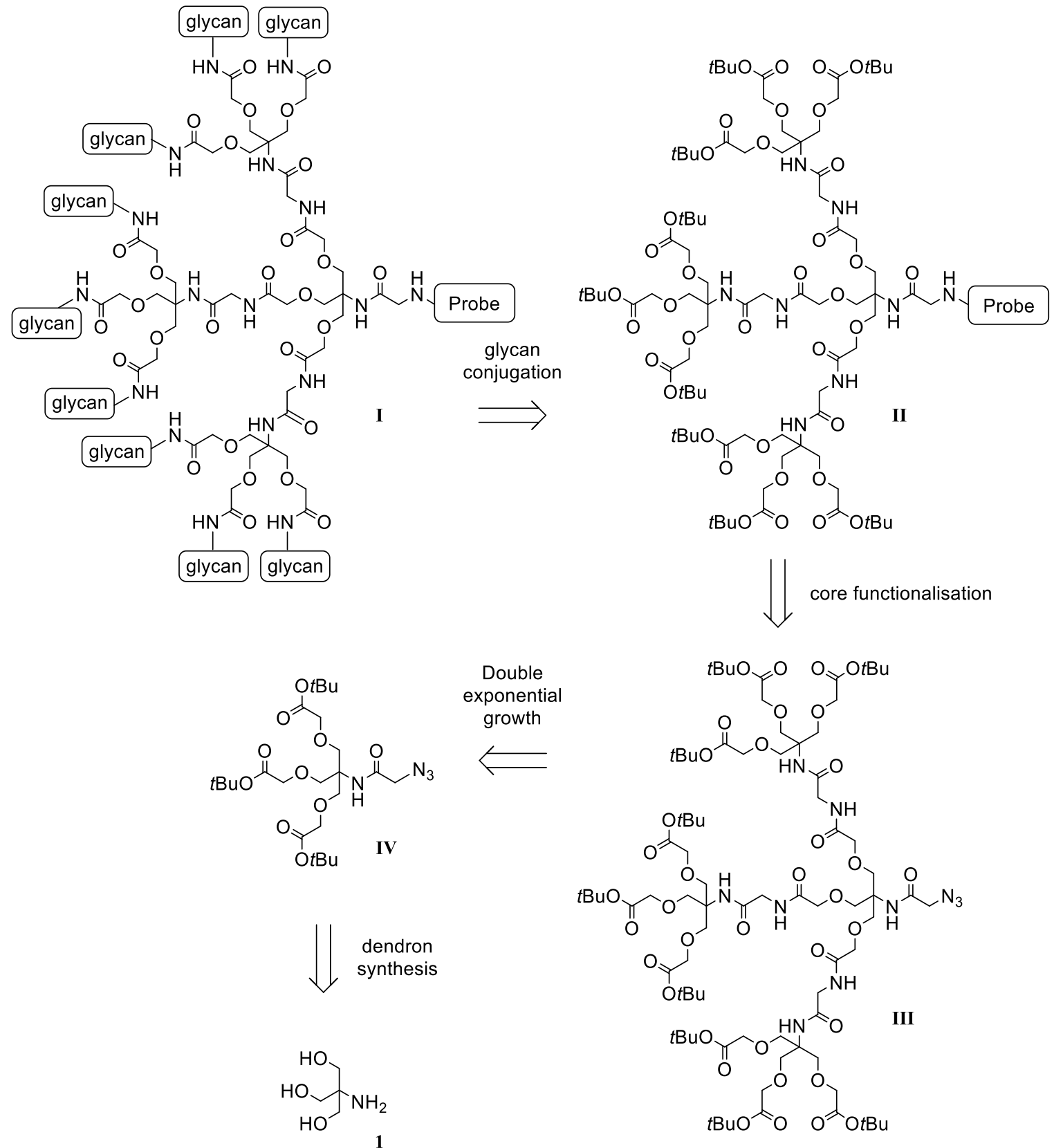

Scheme 8. Retrosynthesis of bi-functional glycodendrons. 


\subsection{Results and discussion}

\subsubsection{Initial strategy}

In an initial attempt to synthesise multivalent dendrons, it was anticipated that the synthesis of a first generation dendron could be achieved via the amine-protection and $O$-alkylation of the hydroxyls in TRIS (1). Thus the synthesis commenced with the smooth $N$-Boc protection of TRIS (1) to give triol 23 (Scheme 9). ${ }^{18}$ Next, the alkylation reaction was investigated and sodium hydride was slowly added to triol 23 in the presence of methyl bromoacetate in DMF. Here, a large number of products were observed using TLC-analysis. Although some of the desired tri-alkylated product $\mathbf{3 7}$ was observed using LC-MS analysis, a variety of byproducts were also observed in the LC-MS chromatogram (Figure 4). Here, intramolecular reaction of the alcohol with the carbamate of the $N$-Boc protecting group gave cyclic carbamate $\mathbf{3 8}$, which is a reaction that has previously been used in the synthesis of spiropiperidines. ${ }^{38,39}$ Moreover, the formation of $\alpha$-bromoacetate esters (i.e 39) indicates that esterification occurs instead of the desired alkylation reaction. Mono- and di-alkylated products were also observed in the LC-MS chromatogram.
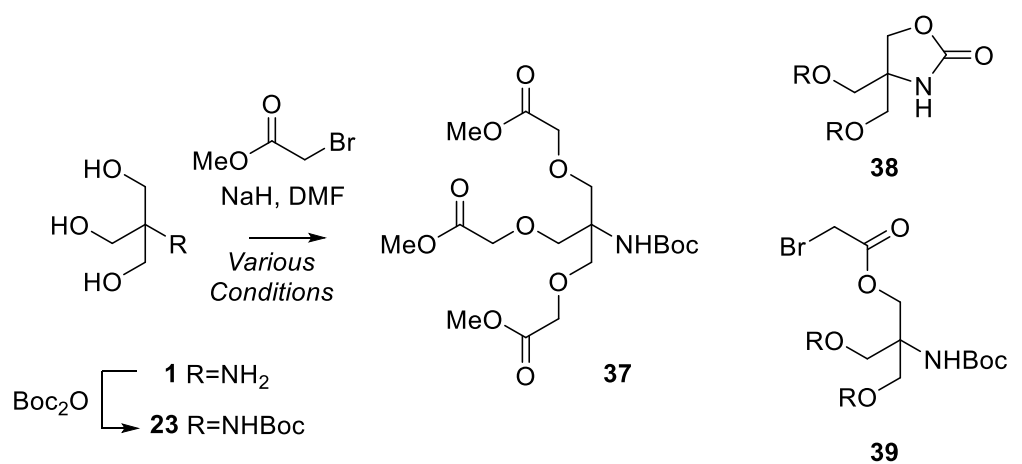

Scheme 9. Alkylation attempt of triol 23 to yield trimethyl ester 37. During the alkylation reaction numerous byproducts were formed, which could be separated and analysed using LCMS (Figure 4).

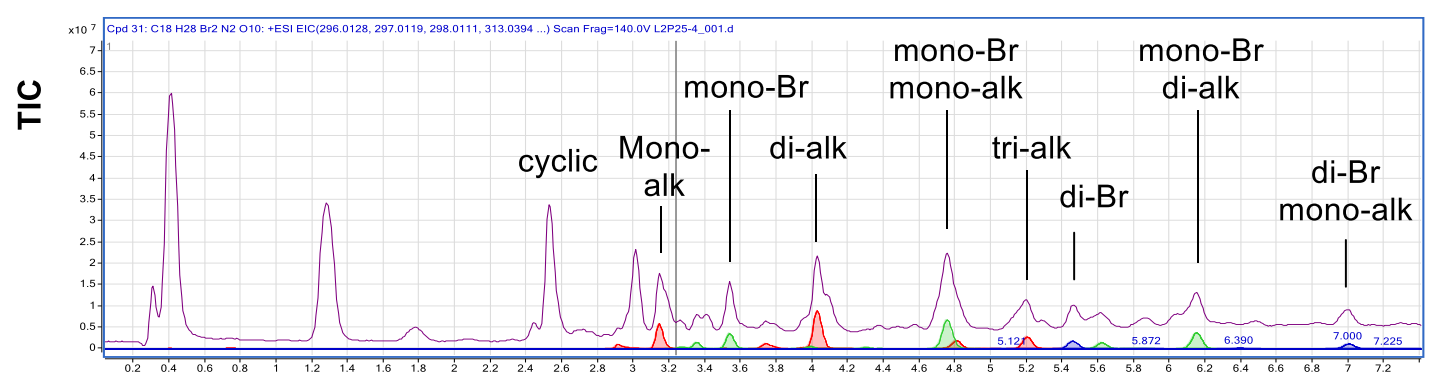

Retention time

Figure 4. LCMS (TIC) trace of alkylation reaction with some of the indicated byproducts. 
Accordingly, it was thought that conversion of the amine in TRIS to the glycinamide would prevent the cyclisation reaction and use of a bulky alkylating agent tert-butyl bromoacetate would prevent esterification byproducts. Thus, TRIS 1 was reacted with NHS-activated $N$-Z protected glycine $\mathbf{4 0}$ to give the desired amide 41 (Scheme 10). Next, alkylation of the triol with tert-butyl bromoacetate was explored. First, the tri- $O$-alkylation reaction was examined using the previously reported conditions. ${ }^{34}$ To this end, the triol was reacted with tert-butyl bromoacetate in a bi-phasic system comprising $50 \%$ aq. $\mathrm{KOH}, \mathrm{CH}_{2} \mathrm{Cl}_{2}$ and $\mathrm{TBABr}$ as a phase transfer catalyst. Using these conditions only small amounts of alkylation products were observed, therefore alternative alkylation conditions were explored. Initially, triol 41 was submitted to alkylation conditions utilizing tert-butyl bromoacetate, $\mathrm{NaH}$ and TBAI in DMF. While cyclisation with concomitant removal of the Z-group was not observed, overalkylation of the carbamate gave rise to tetraester 42, which was confirmed by HMBC NMR analysis. Even though the formation of the over-alkylated byproducts was undesired, the basis of successful dendron chemistry was unveiled: by changing the Z-protecting group to an azide derivative, formation of the over-alkylated product could be prevented.
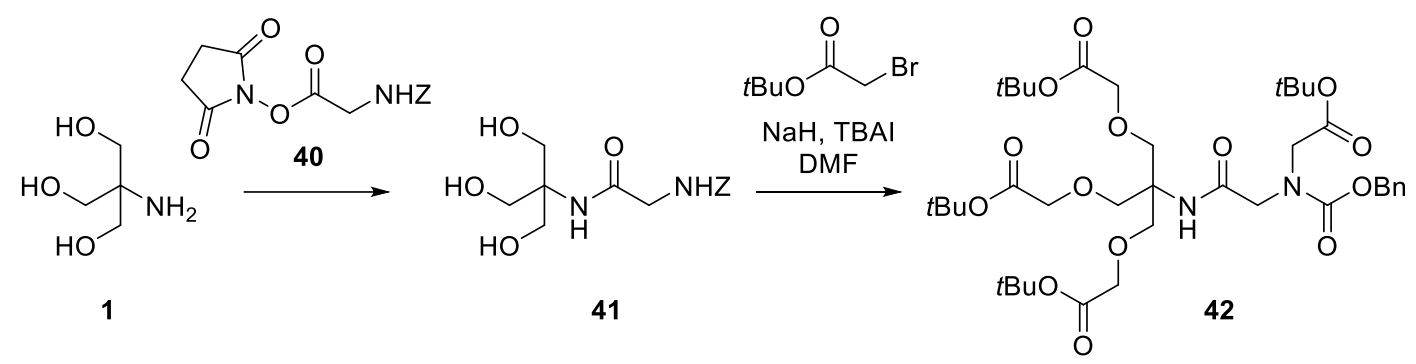

Scheme 10. Alterations in the dendron design led to the formation of the over-alkylated dendron 42.

\subsubsection{First generation dendron synthesis}

Thus, in the alternative approach, the synthesis of first generation dendron commenced with the quantitative substitution reaction of 2-bromoacetic acid (43) with sodium azide to give azidoacetic acid (44) (A, Scheme 11). Next, activation of the carboxylic acid by a DCC-mediated coupling with $N$-hydroxysuccinimide gave the activated ester $\mathbf{4 5}$, which was selectively conjugated with TRIS 1 to afford triol $\mathbf{4 6}$ in $80 \%$ yield over three steps. Here, it should be noted that an excess of TRIS prevents the formation of $O$-acylated byproducts. With the triol in hand, alkylation with $\mathrm{NaH}$ and tert-butyl bromoacetate in DMF yielded the first generation dendron in moderate yields (ca 30\%), however by LCMS there was no evidence of the previously found type of byproducts. Small-scale optimisation of the alkylation reaction (temperature, speed of addition and choice of solvent) revealed that a reasonably quick addition of $\mathrm{NaH}$ to the mixture of triol $\mathbf{4 6}$ and tert-butyl bromoacetate in a 1:1 
DMF:toluene mixture at room temperature gave dendron 47 in $47 \%$ yield. Similar yields could also be achieved on a gram scale, which allowed for the large scale synthesis of the novel dendron core structure. In order to prevent future purification problems of larger dendrons, the first generation dendron was purified using silica gel flash chromatography as well as reverse phase $\left(\mathrm{C}_{18}\right)$ chromatography to obtain the dendron in excellent (94\%) purity (B, Scheme 11).

A.

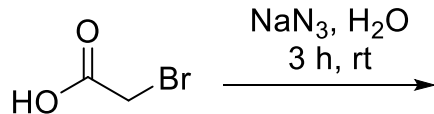

43

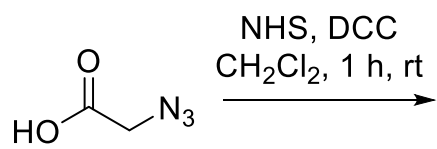

44<smiles>CCC(C)(C)OC(=O)COCC(COCC(=O)OC(C)(C)C)(COCC(=O)OC(C)(C)C)NC(=O)C[18O]</smiles>

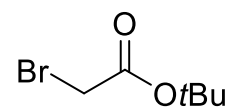

$\mathrm{NaH}, \mathrm{DMF}$ Toluene, $3 \mathrm{~h}, \mathrm{rt}$

$47 \%$<smiles>[13CH3]CC(=O)ON1C(=O)CCC1=O</smiles>

45

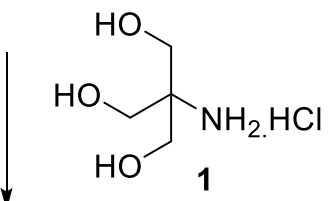

$\mathrm{Et}_{3} \mathrm{~N}, \mathrm{DMF}, 1 \mathrm{~h}, \mathrm{rt}$

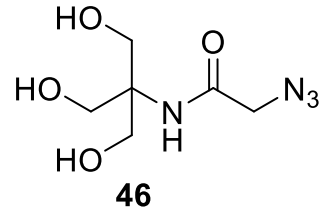

$80 \%$ (3-steps)

B.

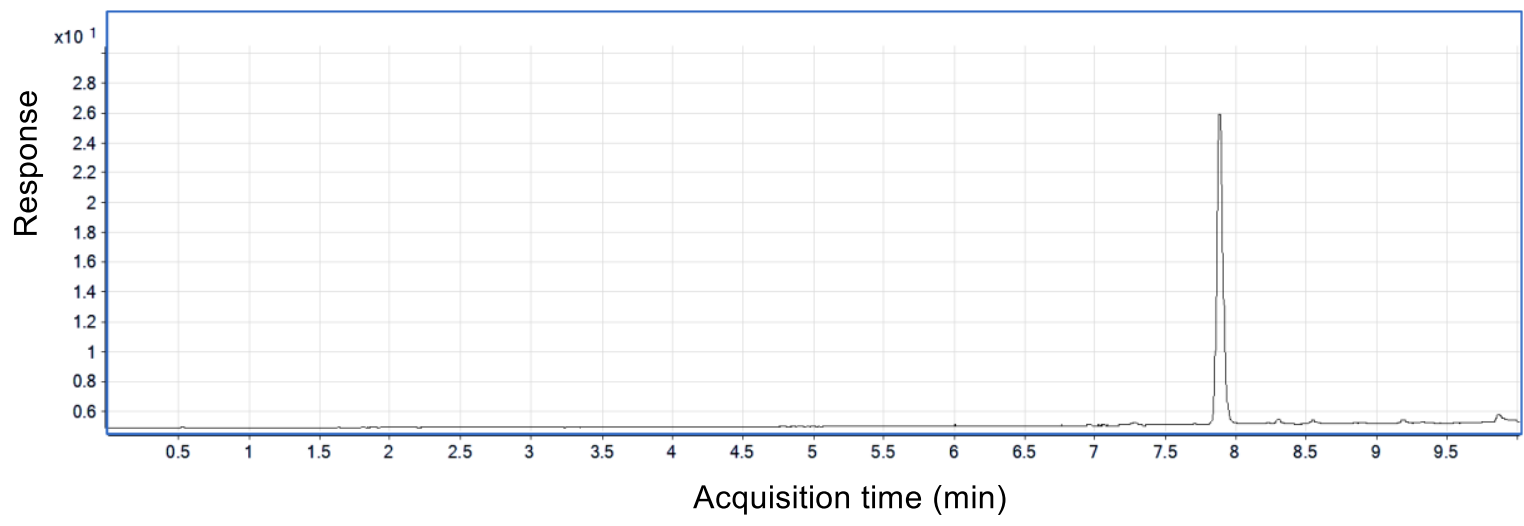

Scheme 11. Synthesis of first generation dendron 47. After purification, the LC-CAD trace shows a 94\% purity of the first generation dendron. C18 LC conditions: $50 / 50 \rightarrow 100 / 0 \mathrm{MeCN} / \mathrm{H}_{2} \mathrm{O}, \mathrm{v} / \mathrm{v}(10$ min gradient); Retention time (min): 7.9 (47).

Structural elucidation of the dendron with both ${ }^{1} \mathrm{H}$ and ${ }^{13} \mathrm{C}-\mathrm{NMR}$ spectra revealed the trilateral symmetry of the first-generation dendron (Figure 5). This symmetry simplifies the ${ }^{1} \mathrm{H}$ and ${ }^{13} \mathrm{C} \mathrm{NMR}$ spectra of these dendritic molecules. Incomplete coupling, however, results in less symmetrical products and almost certainly more complex NMR-spectra. 


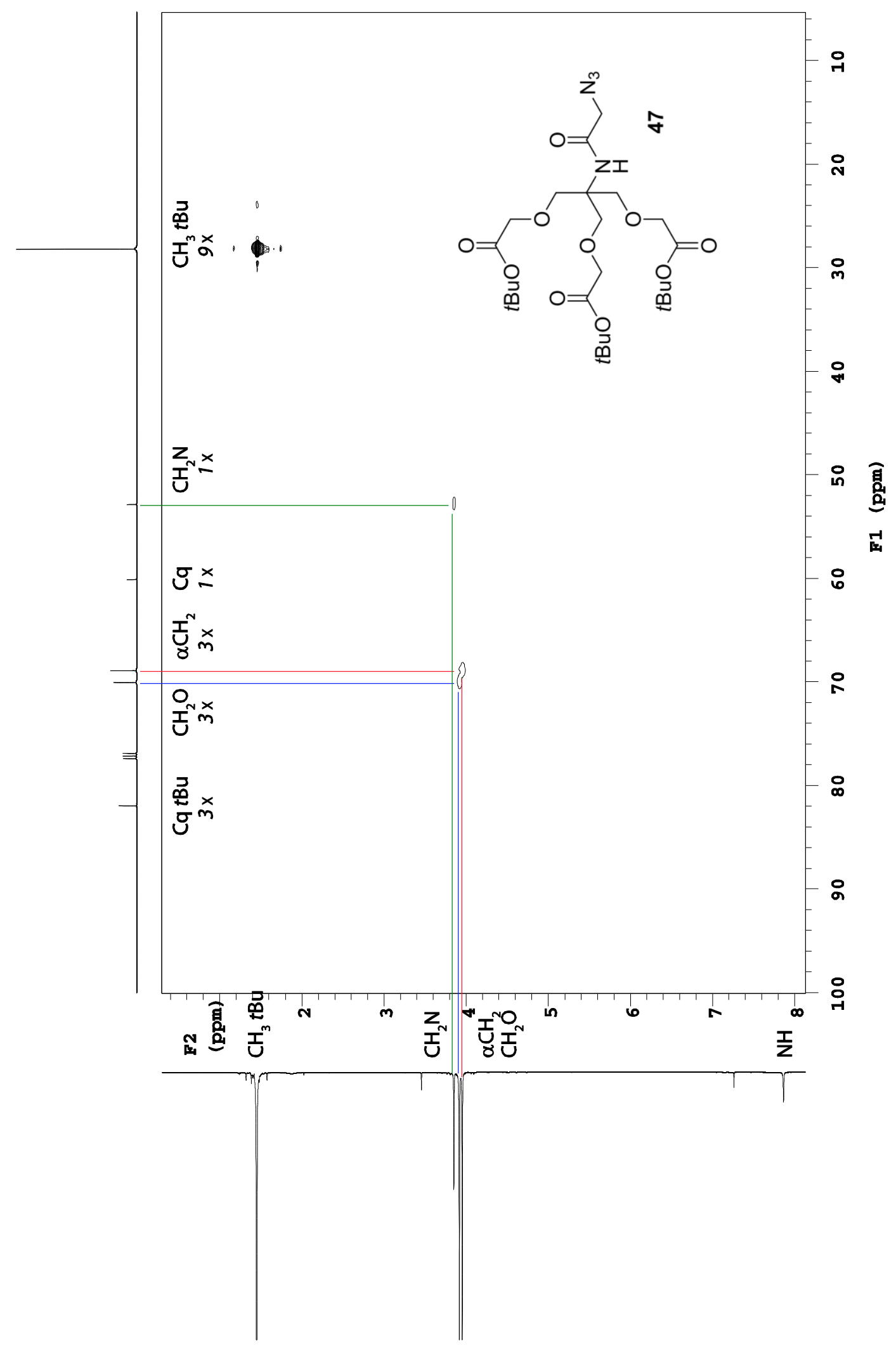

Figure 5. HSQC experiment of the first generation dendron 47 showing the $C_{3}$-symmetry in both the proton as well as the carbon NMR spectra. 


\subsubsection{Second generation dendron synthesis}

With the first generation in hand, the synthesis of the second generation dendron was explored using the double exponential growth approach. Here, the first generation dendron $\mathbf{4 7}$ was divided into two batches for the conversion into two different reactive substrates, followed by the conjugation of both compounds to afford the second generation dendron. Accordingly, for the tert-butyl deprotection of 47, aqueous $6 \mathrm{M} \mathrm{HCl}$ was initially used, but this reaction proved slow and sluggish due to the insolubility of the ester-protected dendron in water. When the hydrolysis was performed in a mixture of aqueous $6 \mathrm{M} \mathrm{HCl}$ and tert-butanol, the reaction showed full conversion of the starting material, however, cleavage of the glycinamide was also observed as evidenced by MS analysis. Accordingly, other deprotection strategies were explored and gratifyingly, the use of $\mathrm{TFA} / \mathrm{CH}_{2} \mathrm{Cl}_{2}(1 / 1$, v/v) at room temperature for 1 hour led to the formation of the tri-valent acid $\mathbf{4 8}$ in excellent yield. Here, it is important to note that the use of alcoholic solvents should be avoided, in order to prevent ester formation, until the TFA is eluted with water on the reverse phase chromatography column.

Next, azide reduction of the first generation dendron $\mathbf{4 7}$ was performed using a Staudinger reaction in $\mathrm{CH}_{2} \mathrm{Cl}_{2} / \mathrm{MeOH} / \mathrm{H}_{2} \mathrm{O}(2 / 9 / 1, \mathrm{v} / \mathrm{v} / \mathrm{v})$ to give primary amine 49 which was purified using reverse phase chromatography and isolated in a quantitative yield. IR-spectroscopy was used to confirm the absence of the azide peak $\left(\sim 2100 \mathrm{~cm}^{-1}\right)$. Alternatively, a Raney-Nickel hydrogenation reaction was used to give the primary amine $\mathbf{4 9}$ in quantitative yield, which has the advantage that it is readily purified via a silica gel plug. 


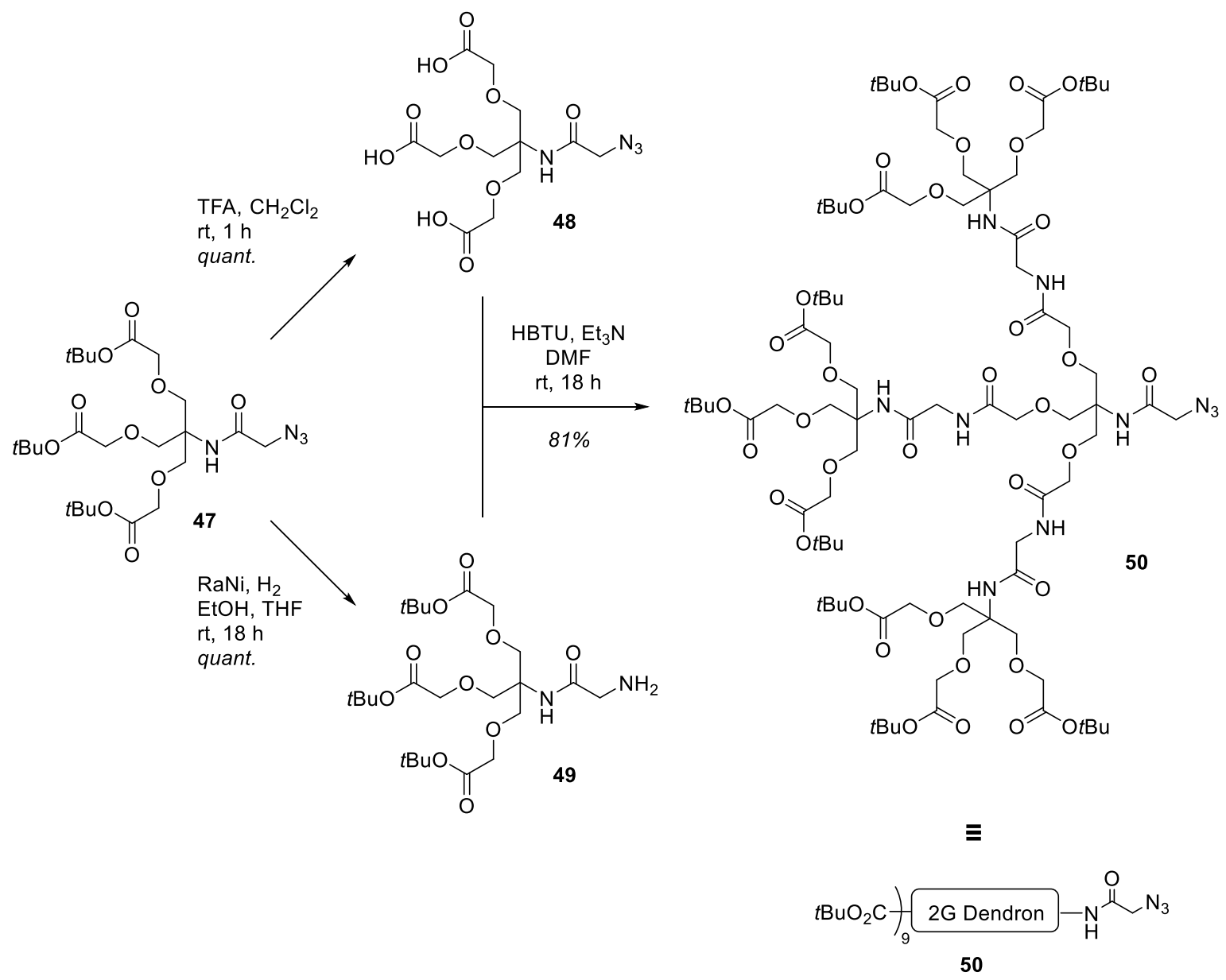

Scheme 12. Synthesis of second generation dendron 50 using a double exponential growth coupling strategy.

With both triacid 48 and amine 49 in hand, the coupling of triacid 48 and 4.5 equivalents of amine 49 using HBTU and $\mathrm{Et}_{3} \mathrm{~N}$ was then undertaken to give second generation dendron $\mathbf{5 0}$ in $81 \%$ yield and in $95 \%$ purity after chromatography on both silica gel and $\mathrm{C}_{18}$ beads, respectively (Figure 6). The combination of both purification techniques was required in order to obtain a sufficiently pure sample of the dendron. Again, these reactions could be performed on a gram scale in order to produce the second generation dendron $\mathbf{5 0}$ on a multi-gram scale.

In terms of characterisation, both ${ }^{1} \mathrm{H}$ and ${ }^{13} \mathrm{C}$-NMR spectra showed the $\mathrm{C}_{3}$-symmetry of the second generation dendron 50. In addition, two-dimensional data (HMBC and HSQC) were used to confirm the coupling between building blocks. With the desired bifunctional dendron core in hand, further functionalisation of the dendron, as well as the glycan conjugation were examined. 


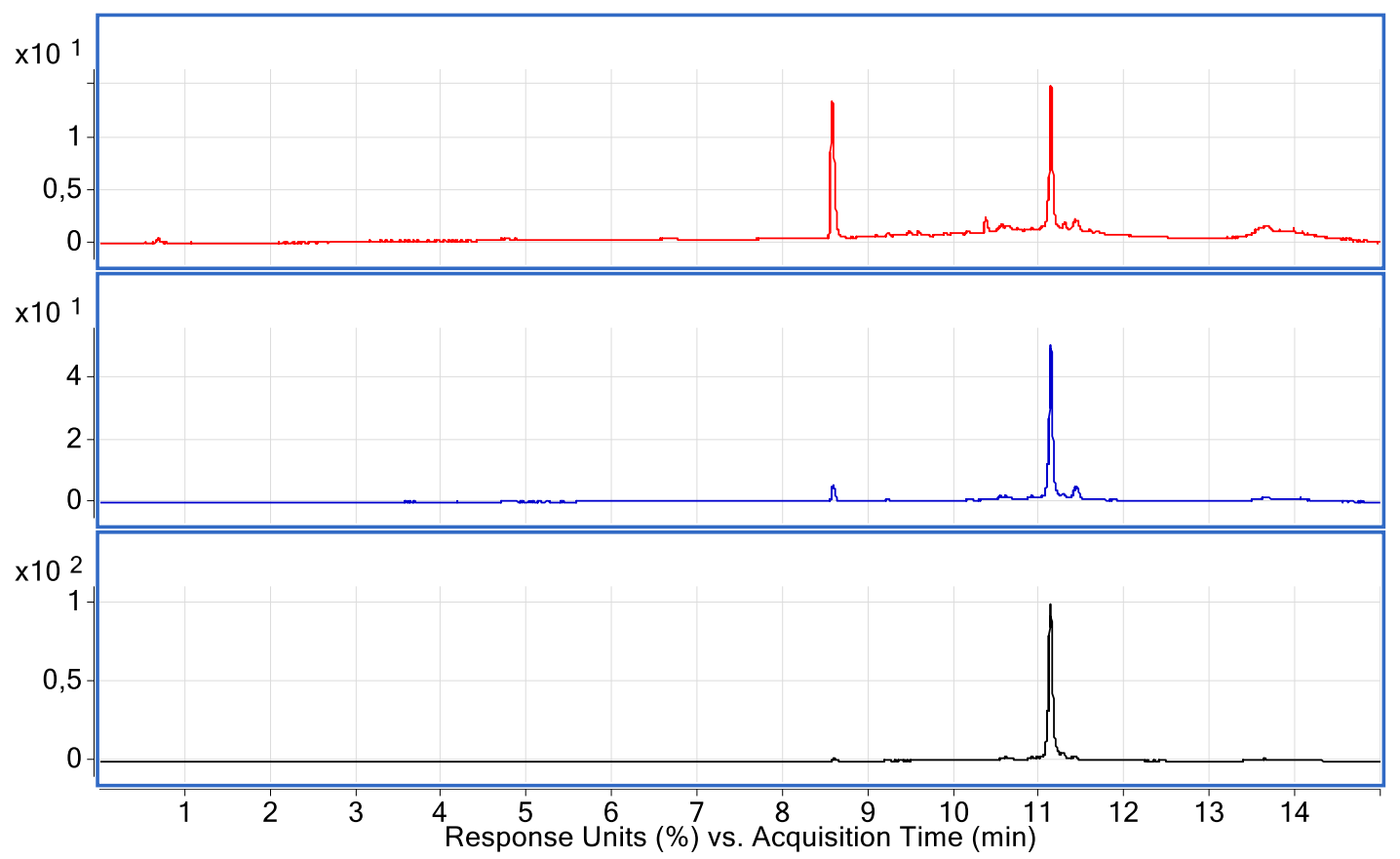

Figure 6. LC-CAD trace for dendron 50 after extraction (red, top), after silica gel column chromatography (blue, middle) and ODS-reverse phase chromatography (black, bottom). $C_{18} L C$ conditions: $5 / 95 \rightarrow 100 / 0 \mathrm{MeCN} / \mathrm{H}_{2} \mathrm{O}$, v/v (10 min gradient), 2 min 100/0 MeCN: $\mathrm{H}_{2} \mathrm{O}, \mathrm{v} / \mathrm{v}$; Retention times (min): $8.6(49), 11.1(50)$;

\subsubsection{Dendron functionalisation}

\subsubsection{Biotinylated glycodendrons}

After the successful preparation of the orthogonally protected dendron $\mathbf{5 0}$, the synthesis of the second generation biotin-functionalised glycodendron was undertaken. To this end, the azide was first converted to the corresponding amine via a Raney-Nickel hydrogenation reaction and the crude product was then coupled to D-biotin using an HBTU-mediated peptide ligation (Scheme 13). This gave the biotin functionalised dendron $\mathbf{5 1}$ in $96 \%$ yield over two steps. Again, glycodendron $\mathbf{5 1}$ was purified by both silica gel as well as reverse phase chromatography. 

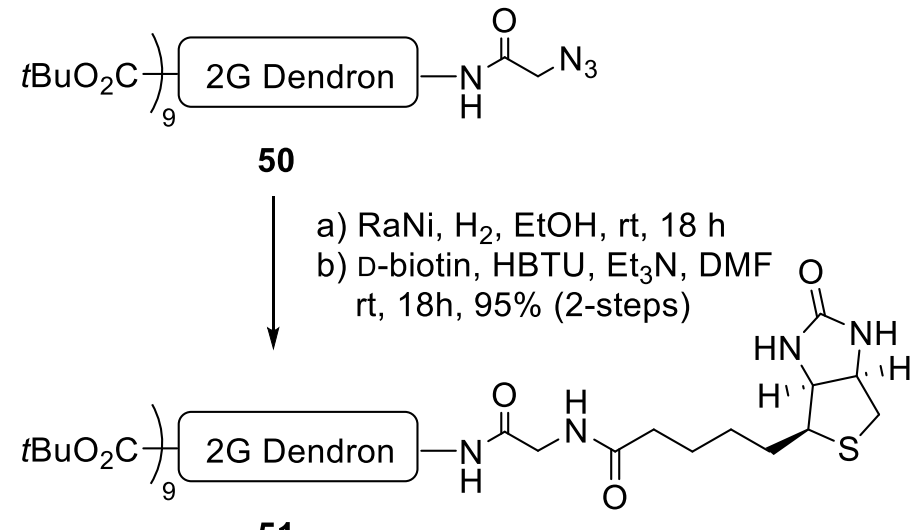

51

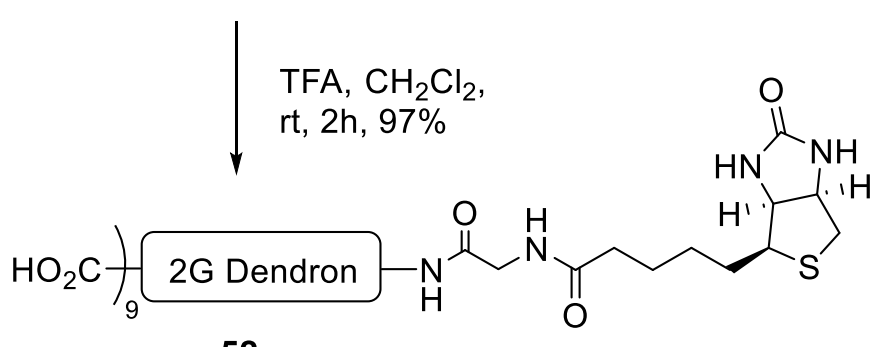

52

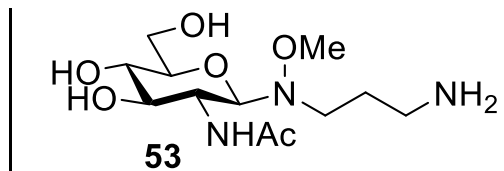
HBTU, $\mathrm{Et}_{3} \mathrm{~N}, \mathrm{DMF}$
rt, $18 \mathrm{~h}, 72 \%$

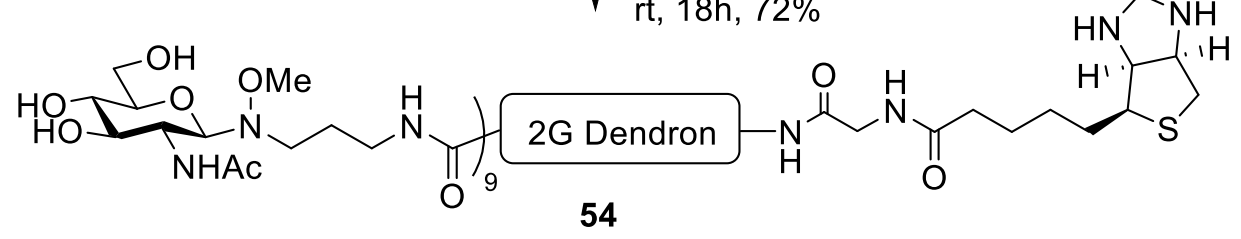

Scheme 13. Functionalisation of the second generation dendron 54.

Removal of the tert-butyl esters in dendron 51 using the previously optimised $\mathrm{TFA} / \mathrm{CH}_{2} \mathrm{Cl}_{2}$ methodology yielded $\mathbf{5 2}$ in 2 hours. The crude reaction mixture was then concentrated, co-evaporated with $\mathrm{H}_{2} \mathrm{O}$ and subsequently subjected to a coupling reaction with amine-functionalised carbohydrates. To establish that the bifunctionalised methoxyamine linker (see Chapter 2) was suitable for multivalent conjugation strategies, an amide coupling between dendron $\mathbf{5 2}$ and oxyamine glycoside 53 was attempted whereby an excess of 18 molar equivalents of amine $\mathbf{5 3}$ was used in order to facilitate the conjugation. Using HRMS-analysis of the crude reaction mixture, it was determined that the previous conjugation conditions ( $\mathrm{HBTU}$ and $\mathrm{Et}_{3} \mathrm{~N}$ in $\mathrm{DMF}$ ) gave rise to the desired glycodendron 
54 in about an hour, however, the reaction mixture was stirred overnight to ensure optimal conjugation. Purification of the glycodendron 54 was then achieved by membrane dialysis (cellulose ester dialysis membrane, molecular weight cut-off 500D). Here, the crude reaction mixture was diluted with water and dialysed for 48 hours with a $0.1 \mathrm{M} \mathrm{Na}_{2} \mathrm{HPO}_{4}$ buffer at $\mathrm{pH} 7.5$ to prevent oxyamine linker hydrolysis (see Chapter 2). After dialysis, the glycodendron was lyophilised and the product was purified by reverse phase chromatography $\left(\mathrm{C}_{8}\right)$ to give glycodendron $\mathbf{5 4}$ in a good (72\%) yield. The glycodendron 54 was analysed using ${ }^{1} \mathrm{H}$ NMR analysis, and in addition, HSQC analysis (Figure 7) showed the presence of all $\mathrm{CH}$ resonances, which could not be observed in the ${ }^{13} \mathrm{C}$ spectrum. Taken as a whole, the synthesis of the biotin functionalised glycodendron demonstrated the use of the dendron scaffold to present carbohydrates in a multivalent fashion and moreover, the effectiveness of the oxyamine linker for glycan conjugation. 


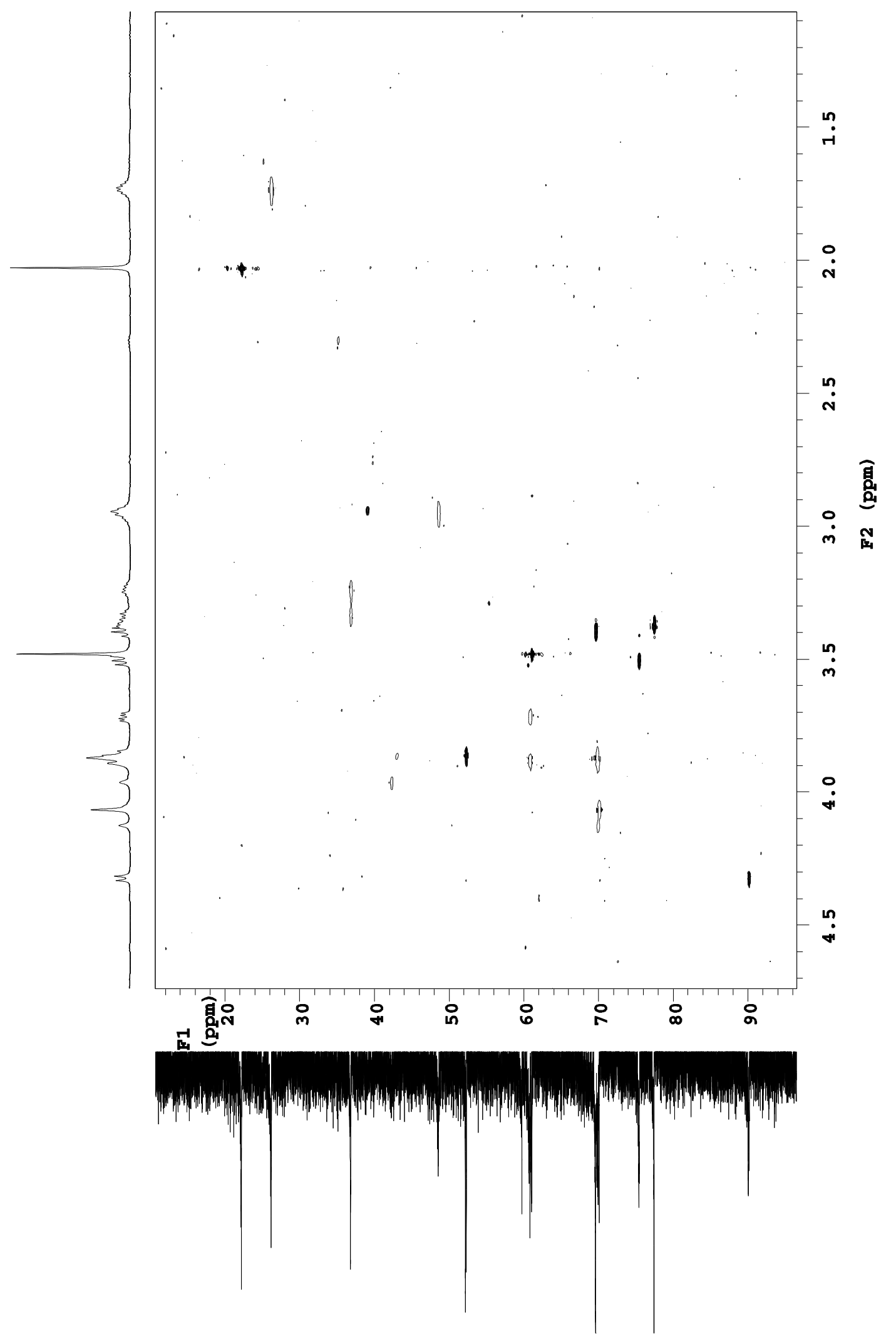

Figure 7. HSQC of glycodendron 54. $\left(\mathrm{D}_{2} \mathrm{O}, 500 \mathrm{MHz}\right)$ 


\subsubsection{Fluorescent glycodendrons}

In addition to biotin-functionalised glycodendrons, a strategy for the synthesis of fluorescent glycodendrons was explored as these latter constructs have wide application in microscopy and in flow cytometry. To this end, the azide moiety of the second generation dendron $\mathbf{5 0}$ was reduced to the corresponding amine, followed by the smooth ligation with fluorescein isothiocyanate isomer I (FITC) to give the fluorescent dendron 55 (Scheme 14). Next, TFA treatment of $\mathbf{5 5}$ in $\mathrm{CH}_{2} \mathrm{Cl}_{2}$ effectively removed the tert-butyl esters, however, in addition the Edman degradation reaction, which can be used to determine amino acid sequences, ${ }^{40}$ was observed. Here, the glycinamide moiety was eliminated to give the cyclic byproduct 56 [HRMS(ESI) $\mathrm{m} / z$ calcd. for $\left[\mathrm{C}_{23} \mathrm{H}_{15} \mathrm{~N}_{2} \mathrm{O}_{6} \mathrm{~S}\right]^{+}: 447.0645$, obsd.: 447.0660] ${ }^{41}$ and the corresponding dendron without glycine moiety 57 [ ${ }^{1} \mathrm{H}$ NMR (500MHz, $\left.\mathrm{D}_{2} \mathrm{O}\right) \delta 4.16\left(\mathrm{~s}, 18 \mathrm{H}, 9 \times \mathrm{CH}_{2} \mathrm{O}\right), 4.14\left(\mathrm{~s}, 6 \mathrm{H}, 3 \times \mathrm{CH}_{2} \mathrm{O}\right), 3.93\left(\mathrm{~s}, 6 \mathrm{H}, 3 \times \mathrm{CH}_{2} \mathrm{C}=\mathrm{O}, 3.84(\mathrm{~s}, 18 \mathrm{H}, 9\right.$ $\left.\times \mathrm{CH}_{2} \mathrm{C}=\mathrm{O}\right), 3.81\left(\mathrm{~s}, 6 \mathrm{H}, 3 \times \mathrm{CH}_{2} \mathrm{~N}\right) ; \mathrm{HRMS}(\mathrm{ESI}) \mathrm{m} / \mathrm{z}$ calcd. for $\left[\mathrm{C}_{46} \mathrm{H}_{73} \mathrm{~N}_{7} \mathrm{O}_{36}\right]^{2+}: 649.7043$, obsd.: 649.7051]. Optimisation of this reaction was thought to be difficult due to the fact that nine esters need to be cleaved without the undesired Edman degradation reaction, which occurs below pH 3 . Thus, an alternative method for the synthesis of the fluorescent glycodendrons was investigated.

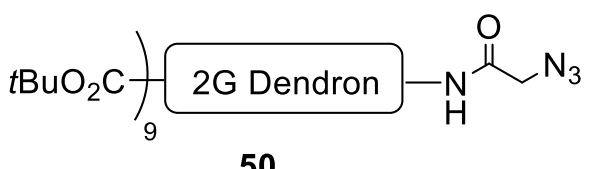

50

a) $\mathrm{PPh}_{3}, \mathrm{MeOH}, \mathrm{H}_{2} \mathrm{O}, \mathrm{rt}, 18 \mathrm{~h}$

b) FITC, $\mathrm{MeOH}, \mathrm{CH}_{2} \mathrm{Cl}_{2}$, rt, $18 \mathrm{~h}$
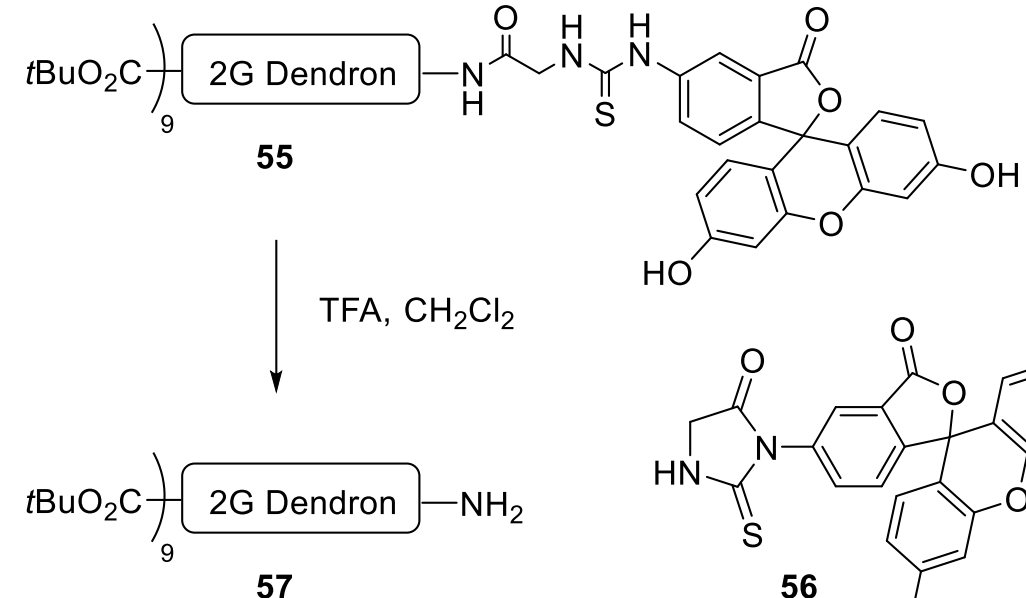

57

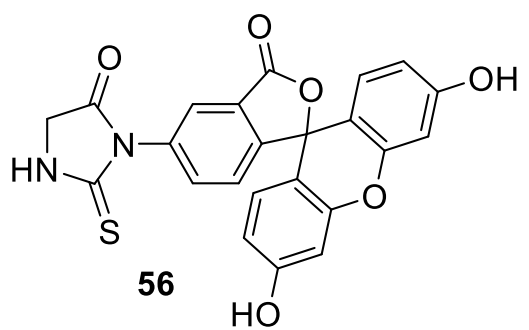

Scheme 14. Attempted synthesis of fluorescein-based dendrons. 
To prevent the Edman degradation byproduct, the azide of the second generation dendron $\mathbf{5 0}$ was converted to the corresponding amine and conjugated to Fmoc-6-aminocaproic acid $\mathbf{5 8}^{42}$ to give dendron 59 in an excellent yield (91\% over 2 -steps) (Scheme 15). Fmoc deprotection with piperidine then gave the hexanamide amine dendron, which was readily purified using size exclusion chromatography to remove the piperidine. Next, FITC conjugation in DMF with $\mathrm{Et}_{3} \mathrm{~N}$ as a base gave the fluorescent dendron 60 in good $(80 \%)$ yield. In an attempt to synthesise dendron $\mathbf{6 1}$, dendron 60 was treated with TFA, however, degradation of the fluorophore resulted in the isolation of an unknown dendron. Thus, another synthetic approach was required.

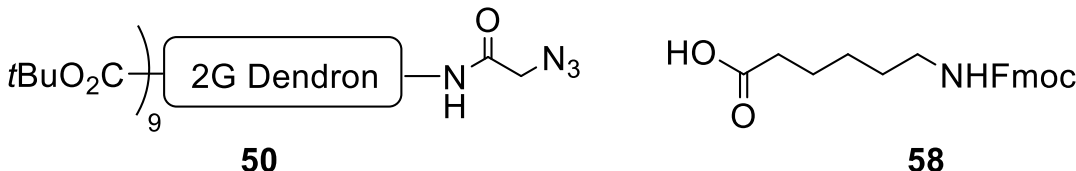

a) $\mathrm{RaNi}, \mathrm{H}_{2}$, EtOH, rt, $18 \mathrm{~h}$

b) 58, HBTU, Et ${ }_{3} \mathrm{~N}, \mathrm{DMF}$, rt, $2 \mathrm{~h}$, $91 \%$ (2 steps)

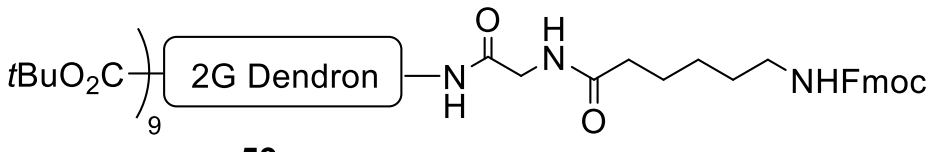

59

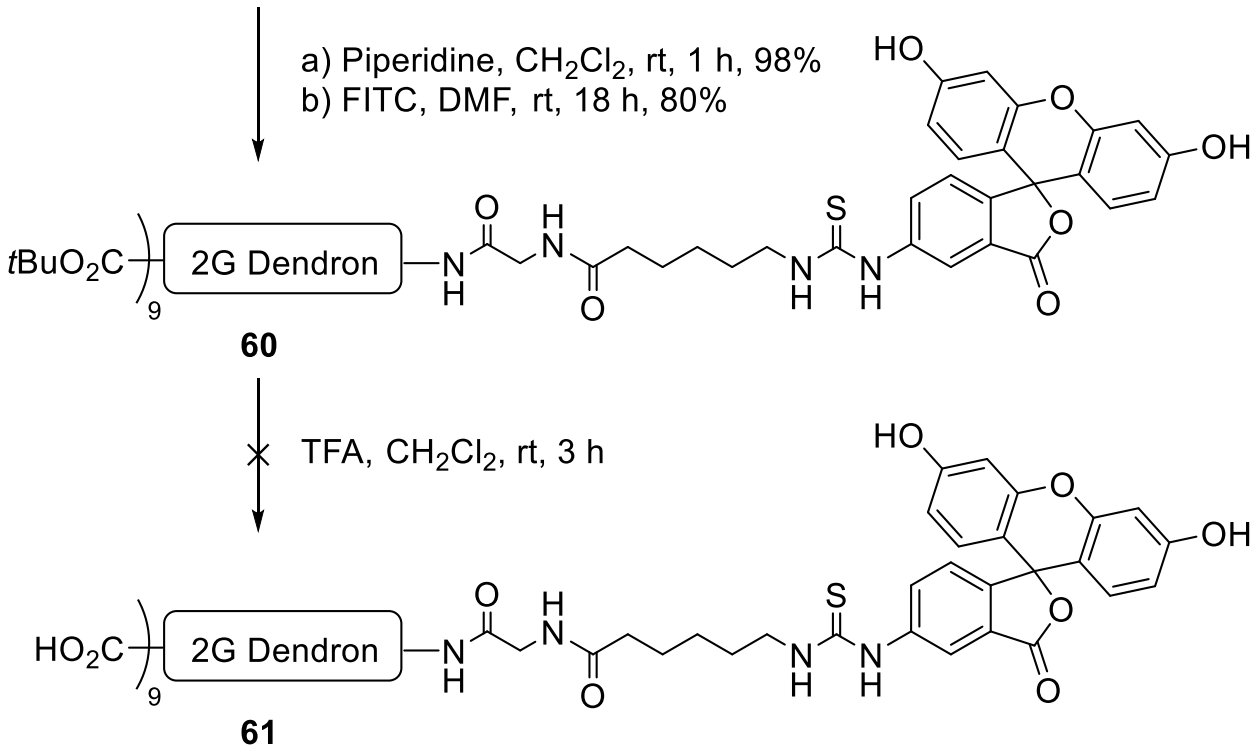

Scheme 15. An alternative attempt to synthesise a fluorescent dendron. 
In the next approach, dendron $\mathbf{5 0}$ was deprotected to the amino acid, followed by conjugation with FITC (Scheme 16). Here, the second generation dendron was deprotected using the Staudinger azide reduction and subsequent TFA treatment to give the amino-acid 62 in an excellent yield (99\%), which could be readily purified by filtration to remove the triphenylphosphine salts. Next, conjugation with FITC in DMF and $\mathrm{Et}_{3} \mathrm{~N}$ gave the desired fluorescent dendron 63 in an excellent yield (74\%). Here it should be noted that the dendron was obtained as the triethylamine salt after size exclusion chromatography, however, the dendron could be obtained as the nona-valent acid after an ion exchange plug $\left(\right.$ Dowex $\mathrm{H}^{+}$). With the fluorescent dendron in hand, chemical ligation with amine functionalised glycans gives rise to the desired fluorescent dendrons. In Chapter 5, the conjugation of the Lewis ${ }^{\mathrm{X}}$ glycan with dendron $\mathbf{6 3}$, as well as the biological evaluation of these glycodendrons is discussed.

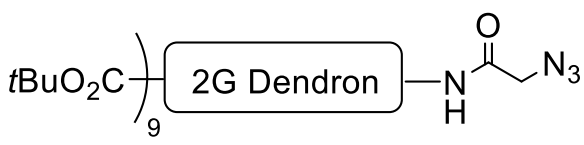

50

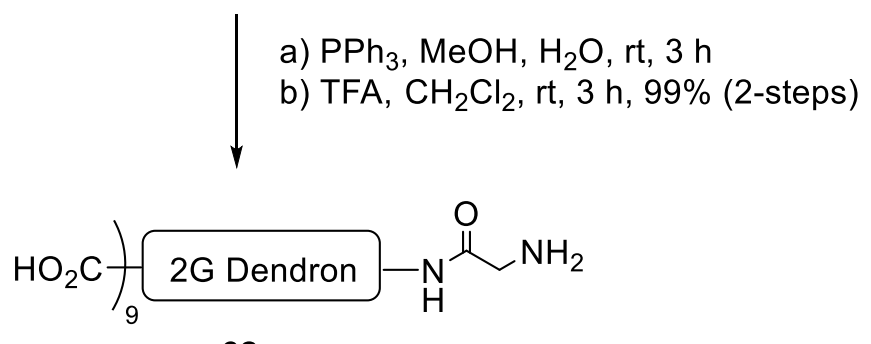

62

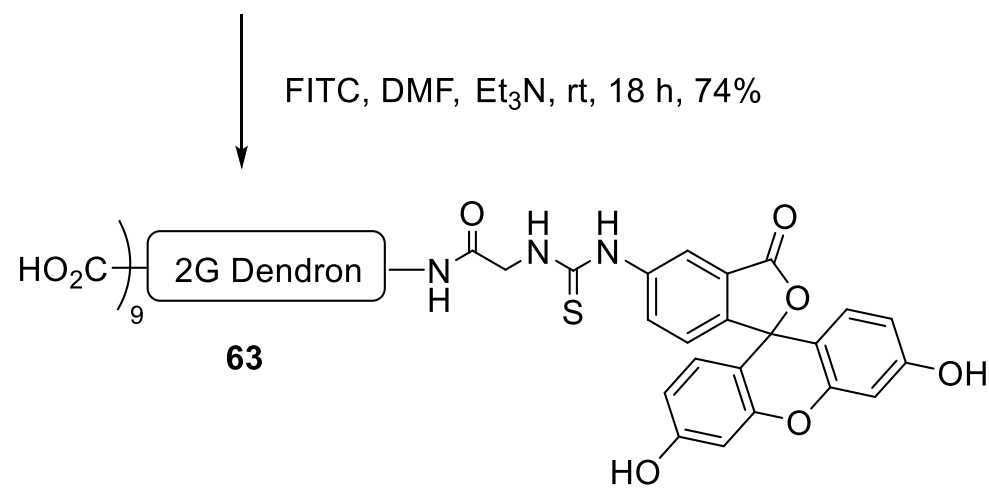

Scheme 16. Synthesis of fluorescent dendron 63. 


\subsection{Conclusion}

In summary, the design and synthesis of a novel glycodendron has been presented. Even though the field for dendrimer chemistry has rapidly expanded in the last decades, with many dendron corestructures now being commercially available, the presented strategy allows for the synthesis of a novel dendron core structure that can be functionalised as required. In the presented synthetic route, a first generation dendron was synthesised from the TRIS core moiety in 2-steps. Here, optimisation of the amine protection, as well as the alkylation reaction, resulted in the formation of the first generation dendron 47 on a multi-gram scale. Next, the double exponential growth approach was used, which allows for the rapid 3-step assembly of a second generation dendron $\mathbf{5 0}$ in $81 \%$ yield on a gram scale. Here, the combination of silica gel column chromatography as well as reverse phase $\left(\mathrm{C}_{18}\right)$ column chromatography proved to be very successful in the purification of these macromolecules.

With the bi-functional second generation dendron in hand, rapid functionalisation allows for use of these dendrons in various applications. Accordingly, a core-biotin derivatised dendron was then synthesised in two steps, and after ester deprotection, the dendron was subjected to glycosylation through the use of the oxyamine amine functionalised $N$-acetylglucosamine $\mathbf{5 3}$ to give the biotinylated glycodendron 54.

In addition, a multivalent fluorescent glycodendron was synthesised for use in microscopy and flow cytometry. Here, it was required to globally deprotect the second generation dendron $\mathbf{5 0}$ to the amino acid before coupling with FITC to give the desired fluorescent dendron $\mathbf{6 3}$ in a good yield. This fluorescent dendron was later utilised for conjugation with the Lewis ${ }^{\mathrm{X}}$ glycan, as will be discussed in Chapter 5.

Taken as a whole, the novel and efficient synthesis of second generation dendrons allows for the rapid functionalisation of the dendron with the substrate of choice. Here, the conjugation with D-biotin or a fluorescent moiety allows for the use of these dendrons in various biological assays, including microscopy, ELISA and flow cytometry. Moreover, it was demonstrated that the effective use of the oxyamine linker strategy for glycan conjugation allows for the efficient assembly of complex multivalent bio-molecules. 


\subsection{Experimental}

General procedure. Prior to use, THF (Pancreac) was distilled from sodium and benzophenone, $\mathrm{CH}_{2} \mathrm{Cl}_{2}$ (Fisher) was distilled from $\mathrm{P}_{2} \mathrm{O}_{5}, \mathrm{DMF}$ was distilled from $\mathrm{BaO}, \mathrm{Et}_{3} \mathrm{~N}$ (Sigma) was distilled from $\mathrm{KOH}$, and $\mathrm{H}_{2} \mathrm{O}$ was distilled. Bromoacetic acid (Sigma), $\mathrm{NaN}_{3}(\mathrm{BDH})$, conc $\mathrm{HCl}$ (Panreac), $N$-hydroxysuccinamide (Aldrich), $\quad N, N^{\prime}$-Dicyclohexylcarbodiimide $\quad$ (Aldrich), TRIS(hydroxymethyl)aminomethane (Sigma), NaH (Aldrich, 60\% dispersed in mineral oil), tertbutyl bromoacetate (Apollo Scientific), trifluoroacetic acid (Sigma), Raney-Nickel (Sigma-Aldrich), HBTU (Apollo Scientific), D-biotin (Acros), $\mathrm{Ph}_{3} \mathrm{P}$ (Acros), fluorescein isothiocyanate isomer I (Sigma), anhydrous $\mathrm{Et}_{2} \mathrm{O}$ (Pancreac), EtOAc (Pancreac), petroleum ether (Pure Science), toluene (Panreac), $\mathrm{MeOH}$ (Pure Science), $\mathrm{CHCl}_{3}$ (Pancreac), EtOH (absolute, Pure Science), $\mathrm{NaHCO}_{3}$ (Pure Science), $\mathrm{Na}_{2} \mathrm{HPO}_{4} .7 \mathrm{H}_{2} \mathrm{O}$ (J.T.Baker), $\mathrm{NaOH}$ (Pure Science), $\mathrm{MgSO}_{4}$ (Pure Science) and $\mathrm{NaCl}$ (Pancreac) were used as received.

All solvents were removed by evaporation under reduced pressure. Reactions were monitored by TLC-analysis on Macherey-Nagel silica gel coated plastic sheets $(0.20 \mathrm{~mm}$, with fluorescent indicator $\mathrm{UV}_{254}$ ) with detection by UV-absorption (short wave UV - $254 \mathrm{~nm}$; long wave UV - $366 \mathrm{~nm}$ ), by dipping in $10 \% \mathrm{H}_{2} \mathrm{SO}_{4}$ in EtOH followed by charring at $\sim 150{ }^{\circ} \mathrm{C}$, by dipping in $\mathrm{I}_{2}$ in silica, or by dipping into a solution of ninhydrin in $\mathrm{EtOH}$ followed by charring at $\sim 150{ }^{\circ} \mathrm{C}$. Column chromatography was performed on Pure Science silica gel (40 - 63 micron). AccuBOND II ODS-C 18 (Agilent) was used for reverse phase chromatography. Infrared spectra were recorded as thin films using a Bruker Tensor 27 FTIR spectrometer equipped with an Attenuated Total Reflectance (ATR) sampling accessory and are reported in wave numbers $\left(\mathrm{cm}^{-1}\right)$. Nuclear magnetic resonance spectra were recorded at $20{ }^{\circ} \mathrm{C}$ in $\mathrm{D}_{2} \mathrm{O}, \mathrm{CD}_{3} \mathrm{OD}, \mathrm{CDCl}_{3}$, or pyridine- $\mathrm{d}_{5}$ using either a Varian INOVA operating at $500 \mathrm{MHz}$ or Varian VNMRS operating at $600 \mathrm{MHz}$. Chemical shifts are given in ppm

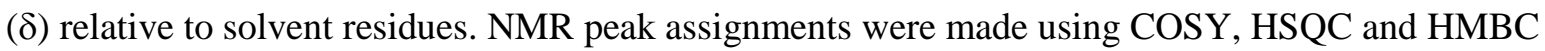
$2 \mathrm{D}$ experiments. 


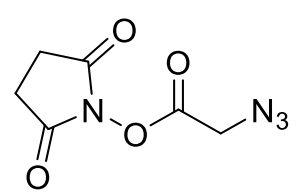

$N$-Hydroxysuccinimidyl azidoacetate (45). To a solution of bromoacetic acid $43(15.1 \mathrm{~g}, 0.11 \mathrm{~mol})$ in water $(30 \mathrm{~mL}), \mathrm{NaN}_{3}(30.0 \mathrm{~g}, 0.43 \mathrm{mmol})$ was added and the reaction mixture was stirred at r.t. for $3 \mathrm{~h}$. The reaction mixture was carefully diluted with $6 \mathrm{M}$ aq. $\mathrm{HCl}(50 \mathrm{~mL})$ (CAUTION: formation of hydrazoic acid $)$ and extracted with $\mathrm{Et}_{2} \mathrm{O}(2 \times 100 \mathrm{~mL})$. The organic layers were dried with $\mathrm{MgSO}_{4}$, filtered and concentrated in vacuo, to give azide 44 as a colourless oil $(10.7 \mathrm{~g}, 0.11 \mathrm{mmol}, 99 \%)$. The crude reaction product was used in the esterification reaction without further purification. To a solution of crude 2-azido acetic acid $44(9.70 \mathrm{~g}, 96.0 \mathrm{mmol})$ in dry $\mathrm{CH}_{2} \mathrm{Cl}_{2}(100 \mathrm{~mL}), \mathrm{N}$-hydroxysuccinamide $(13.2 \mathrm{~g}, 115 \mathrm{mmol})$ and $N, N^{\prime}$-dicyclohexylcarbodiimide $(17.8 \mathrm{~mL}, 115 \mathrm{mmol})$ were added at $0{ }^{\circ} \mathrm{C}$ and the reaction mixture was stirred at r.t. for $18 \mathrm{~h}$. The reaction mixture was filtered and concentrated in vacuo. To the crude residue, EtOAc $(150 \mathrm{~mL})$ was added and the solids were removed by filtration and the mixture was concentrated in vacuo. The residue was purified by silica gel column chromatography (PE/EtOAc, 75/25 $\rightarrow 25 / 75$, v/v) and crystallised from $\mathrm{CH}_{2} \mathrm{Cl}_{2} / \mathrm{PE}$ to yield title compound 45 as a white solid (17.9 g, 90.2 mmol, 94\%). $\mathrm{R}_{f}=0.25$ (PE/EtOAc, 1/1, v/v); Mp 110$115{ }^{\circ} \mathrm{C}$; IR (film) 2995, 2935, 2110, 1818, 1781, 1727, 1428, 1416, 1369, 1278, 1199, $1087 \mathrm{~cm}^{-1} ;{ }^{1} \mathrm{H}$ NMR $\left(500 \mathrm{MHz}, \mathrm{CDCl}_{3}\right) \delta 4.24\left(\mathrm{~s}, 2 \mathrm{H}, \mathrm{CH}_{2}-\mathrm{N}_{3}\right), 2.88\left(\mathrm{~s}, 4 \mathrm{H}, 2 \times \mathrm{CH}_{2} \mathrm{C}=\mathrm{O}\right) ;{ }^{13} \mathrm{C} \mathrm{NMR}(125 \mathrm{MHz}$, $\left.\mathrm{CDCl}_{3}\right) \delta 168.6(2 \times N-\mathrm{C}=\mathrm{O}), 164.3(O-\mathrm{C}=\mathrm{O}), 48.1\left(\mathrm{CH}_{2}-\mathrm{N}_{3}\right), 25.7\left(2 \times \alpha \mathrm{CH}_{2}\right)$.

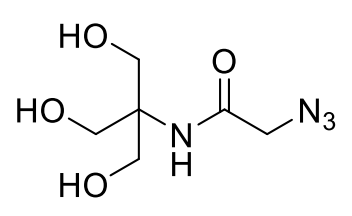

$N$-Azidoacetyl-1,1,1-tris(hydroxymethyl)aminomethane (46). To a solution of succinimidyl ester 45 (8.83 g, $44.6 \mathrm{mmol})$ in freshly distilled DMF (100 mL), TRIS(hydroxymethyl)aminomethane 1 (26.9 g, $222 \mathrm{mmol}$ ) and $\mathrm{Et}_{3} \mathrm{~N}(0.6 \mathrm{~mL}, 4.5 \mathrm{mmol})$ were added and the reaction mixture was stirred at r.t. for $18 \mathrm{~h}$. The reaction mixture was concentrated in vacuo, then co-evaporated with $\mathrm{H}_{2} \mathrm{O}$ to remove traces of DMF. The residue was purified by silica gel column chromatography (dry-loading, PE:EtOAc, 50/50 $\rightarrow$ 0/100, v/v) yielded the title compound $\mathbf{4 6}$ as a white foam (7.84 g, $38.4 \mathrm{mmol}$, $86 \%) . \mathrm{R}_{f}=0.50\left(\mathrm{CH}_{2} \mathrm{Cl}_{2} / \mathrm{EtOH} / \mathrm{MeOH} / \mathrm{NH}_{3}\right.$ (aq. 33\%), 5/2/2/1, v/v/v/v); IR (film) 3359, 2979, 2946, 2890, 2113, 1739, 1650, 1540, 1454, 1367, 1282, 1229, 1217, $1053 \mathrm{~cm}^{-1} ;{ }^{1} \mathrm{H}$ NMR (500 MHz, D $\left.\mathrm{O}\right)$ $\delta 4.00\left(\mathrm{~s}, 2 \mathrm{H}, \mathrm{CH}_{2}-\mathrm{N}_{3}\right), 3.76\left(\mathrm{~s}, 6 \mathrm{H}, 3 \times \mathrm{CH}_{2}-\mathrm{O}\right) ;{ }^{13} \mathrm{C} \mathrm{NMR}\left(125 \mathrm{MHz}, \mathrm{D}_{2} \mathrm{O}\right) \delta 170.4(\mathrm{C}=\mathrm{O}$ Amide $)$, 62.1 (Cq tris), $60.1\left(3 \times \mathrm{CH}_{2}-O\right), 51.9\left(\mathrm{CH}_{2}-\mathrm{N}_{3}\right) ; \mathrm{HRMS}(\mathrm{ESI}) \mathrm{m} / z$ calcd. for $\left[\mathrm{C}_{6} \mathrm{H}_{13} \mathrm{~N}_{4} \mathrm{O}_{4}\right]^{+}: 205.0931$, obsd.: 205.0930 . 
<smiles>CC(C)(C)OC(=O)COCC(COCC(=O)OC(C)(C)C)(COCC(=O)OC(C)(C)C)NC(=O)CN</smiles>

$N$-Azidoacetyl-1,1,1-tris(tert-butyloxycarbonylmethyloxymethyl)aminomethane (47). To a solution of triol 46 (366 mg, $1.79 \mathrm{mmol})$ in DMF $(9 \mathrm{~mL})$ and toluene $(9 \mathrm{~mL})$, tert-butyl bromoacetate $(1.06 \mathrm{~mL}, 7.16 \mathrm{mmol})$ and $\mathrm{NaH}(286 \mathrm{mg}, 7.16$ mmol) were added and the reaction mixture was stirred at r.t. for 4 $h$. The reaction mixture was quenched by the addition of ice water $(20 \mathrm{~mL})$ and was then extracted with $\mathrm{Et}_{2} \mathrm{O}(2 \times 100 \mathrm{~mL})$. The organic layers were dried with $\mathrm{MgSO}_{4}$, filtered and concentrated in vacuo. Purification by silica gel column chromatography (PE:EtOAc, $85 / 15 \rightarrow 75 / 25$, v/v) and reverse phase column chromatography $\left(\mathrm{C}_{18}, \mathrm{H}_{2} \mathrm{O} / \mathrm{MeOH}, 50 / 50 \rightarrow 20 / 80, \mathrm{v} / \mathrm{v}\right)$ yielded tri-alkylated 47 as a white foam (464 mg, $0.85 \mathrm{mmol}$, 47\%). $\mathrm{R}_{f}=0.44(\mathrm{PE} / \mathrm{EtOAc}, 70 / 30$, v/v); IR (film) 3316, 2979, 2935, 2107, 1745, 1684, 1536, 1473, 1428, 1393, 1368, 1229, 1158, 1119, 1037, 971, 915, 845, 731, $647 \mathrm{~cm}^{-1} ;{ }^{1} \mathrm{H}$ NMR (500 MHz, CDCl $)$ $\delta 7.86(\mathrm{~s}, 1 \mathrm{H}, \mathrm{NH}), 3.95\left(\mathrm{~s}, 6 \mathrm{H}, 3 \times \mathrm{CH}_{2} \mathrm{C}=\mathrm{O}\right), 3.92\left(\mathrm{~s}, 6 \mathrm{H}, 3 \times \mathrm{CH}_{2}-\mathrm{O}\right), 3.85\left(\mathrm{~s}, 2 \mathrm{H}, \mathrm{CH}_{2}-\mathrm{N}_{3}\right), 1.45$ $\left(\mathrm{s}, 27 \mathrm{H}, \mathrm{CH}_{3} t \mathrm{Bu}\right) ;{ }^{13} \mathrm{C} \mathrm{NMR}\left(125 \mathrm{MHz}, \mathrm{CDCl}_{3}\right) \delta 170.2(3 \times \mathrm{C}=\mathrm{O}$ ester $), 167.5(1 \times \mathrm{C}=\mathrm{O}$ amide $)$, $82.0(3 \times \mathrm{Cq} t \mathrm{Bu}), 70.1\left(3 \times \mathrm{CH}_{2}-\mathrm{O}\right), 68.9\left(3 \times \mathrm{CH}_{2} \mathrm{C}=\mathrm{O}\right), 60.1(\mathrm{Cq}$ tris $), 52.9\left(\mathrm{CH}_{2}-\mathrm{N}_{3}\right), 28.2(9 \times$ $\mathrm{CH}_{3} t \mathrm{Bu}$ ); HRMS(ESI) $m / z$ calcd. for $\left[\mathrm{C}_{24} \mathrm{H}_{43} \mathrm{~N}_{4} \mathrm{O}_{10}\right]^{+}:$547.2974, obsd.: 547.2981.

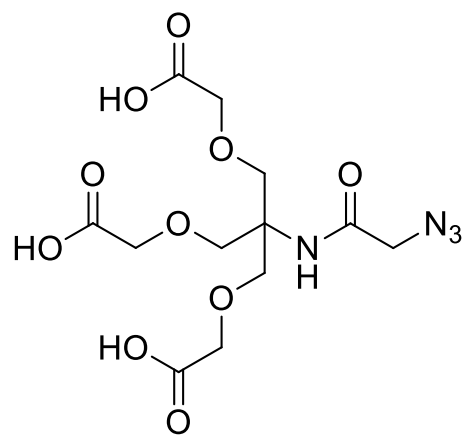

$N$-Azidoacetyl-1,1,1-tris(carboxymethyloxymethyl)aminomethane (48). To a solution of dendron 47 (508 $\mathrm{mg}, 0.93 \mathrm{mmol})$ in $\mathrm{CH}_{2} \mathrm{Cl}_{2}(5 \mathrm{~mL})$, freshly distilled trifluoroacetic acid $(5 \mathrm{~mL})$ was added and the reaction mixture was stirred at r.t. for $2 \mathrm{~h}$. The reaction mixture was then concentrated in vacuo and coevaporated with $\mathrm{CH}_{2} \mathrm{Cl}_{2}(10 \mathrm{~mL})$. The residue was purified by reverse phase column chromatography $\left(\mathrm{C}_{18}, \mathrm{H}_{2} \mathrm{O} / \mathrm{MeOH}, 100 / 0 \rightarrow 70 / 30\right.$, v/v) yielded acid 48 as a colourless oil. $\mathrm{R}_{f}=0.20\left(\mathrm{CH}_{2} \mathrm{Cl}_{2} / \mathrm{EtOH} / \mathrm{MeOH} / \mathrm{NH}_{3}\right.$ (aq. 33\%), 5/2/2/1, v/v/v/v); IR (film) 3418, 2930, 2114, 1727, 1661, 1547, 1427, 1249, $1121 \mathrm{~cm}^{-1} ;{ }^{1} \mathrm{H}$ NMR $\left(500 \mathrm{MHz}, \mathrm{D}_{2} \mathrm{O}\right) \delta 4.20(\mathrm{~s}$, $\left.6 \mathrm{H}, 3 \times \mathrm{CH}_{2} \mathrm{C}=\mathrm{O}\right), 3.98\left(\mathrm{~s}, 6 \mathrm{H}, 3 \times \mathrm{CH}_{2}-\mathrm{O}\right), 3.90\left(\mathrm{~s}, 2 \mathrm{H}, \mathrm{CH}_{2}-\mathrm{N}_{3}\right) ;{ }^{13} \mathrm{C} \mathrm{NMR}\left(125 \mathrm{MHz}, \mathrm{CDCl}_{3}\right) \delta$ $174.3(3 \times \mathrm{C}=\mathrm{O}), 170.2(1 \times \mathrm{C}=\mathrm{O}$ amide $), 69.6\left(3 \times \mathrm{CH}_{2}-\mathrm{O}\right), 68.1\left(3 \times \mathrm{CH}_{2} \mathrm{C}=\mathrm{O}\right), 59.9(\mathrm{Cq}$ tris $), 52.0$ $\left(\mathrm{CH}_{2}-\mathrm{N}_{3}\right)$; HRMS(ESI) $\mathrm{m} / z$ calcd. for $\left[\mathrm{C}_{12} \mathrm{H}_{19} \mathrm{~N}_{4} \mathrm{O}_{10} \mathrm{Na}\right]^{+}: 401.0915$, obsd.: 401.0920. 
<smiles>CC(C)(C)OC(=O)COCC(COCC(=O)OC(C)(C)C)(COCC(=O)OC(C)(C)C)NC(=O)CN</smiles>

N-Glycyl-1,1,1-tris(tert-butyloxycarbonylmethyloxymethyl)aminomethane (49). To a solution of dendron 47 (220 mg, 0.40 mmol) in ethanol $(1 \mathrm{~mL})$ and THF $(1 \mathrm{~mL})$, activated RaneyNickel $(120 \mathrm{mg})$ was added and hydrogen gas was then bubbled through the reaction mixture at r.t. for $18 \mathrm{~h}$. The reaction mixture was filtered, washed with sat. $\mathrm{NaCl}$ (aq.) $(50 \mathrm{~mL})$ containing $1 \mathrm{M}$ $\mathrm{NaOH}(1 \mathrm{~mL})$, extracted with $\mathrm{CH}_{2} \mathrm{Cl}_{2}(2 \times 50 \mathrm{~mL})$ and the combined organic layers were dried, filtered and concentrated in vacuo. The residue was purified by silica gel column chromatography (EtOAc/MeOH, 100/0 $\rightarrow$ 85/15, v/v) yielded amine 49 as a colourless oil (207 mg, $0.40 \mathrm{mmol}, 99 \%) . \mathrm{R}_{f}=0.09$ (EtOAc/MeOH, 90/10, v/v); IR (film) 3336, 2978, 3933, 1745, 1674, 1520, 1368, 1300, 1229, 1159, 1121, 1044, 847 $\mathrm{cm}^{-1} ;{ }^{1} \mathrm{H}$ NMR $\left(500 \mathrm{MHz}, \mathrm{CDCl}_{3}\right) \delta 7.82(\mathrm{~s}, 1 \mathrm{H}, \mathrm{NH}), 3.92\left(\mathrm{~s}, 6 \mathrm{H}, 3 \times \mathrm{CH}_{2} \mathrm{C}=\mathrm{O}\right), 3.88(\mathrm{~s}, 6 \mathrm{H}, 3 \times$ $\mathrm{CH}_{2}-\mathrm{O}$ ), 3.30 (s, $2 \mathrm{H}, \mathrm{CH}_{2}-\mathrm{NH}_{2}$ ), 2.07 (bs, $\left.2 \mathrm{H}, \mathrm{NH}_{2}\right) 1.42$ (s, 27H, $\left.9 \times \mathrm{CH}_{3} t \mathrm{Bu}\right) ;{ }^{13} \mathrm{C} \mathrm{NMR}(125 \mathrm{MHz}$, $\left.\mathrm{CDCl}_{3}\right) \delta 173.2(1 \times \mathrm{C}=\mathrm{O}$ amide $), 170.3(3 \times \mathrm{C}=\mathrm{O}$ ester $), 81.8(3 \times \mathrm{Cq} t \mathrm{Bu}), 70.2\left(3 \times \mathrm{CH}_{2}-\mathrm{O}\right), 69.0$ $\left(3 \times \mathrm{CH}_{2} \mathrm{C}=\mathrm{O}\right), 59.5(\mathrm{Cq}$ tris $), 45.6\left(\mathrm{CH}_{2}-\mathrm{NH}_{2}\right), 28.2\left(9 \times \mathrm{CH}_{3} t \mathrm{Bu}\right) ; \mathrm{HRMS}(\mathrm{ESI}) \mathrm{m} / z$ calcd. for $\left[\mathrm{C}_{24} \mathrm{H}_{45} \mathrm{~N}_{2} \mathrm{O}_{10} \mathrm{Na}\right]^{+}:$543.2888, obsd.: 543.2891.

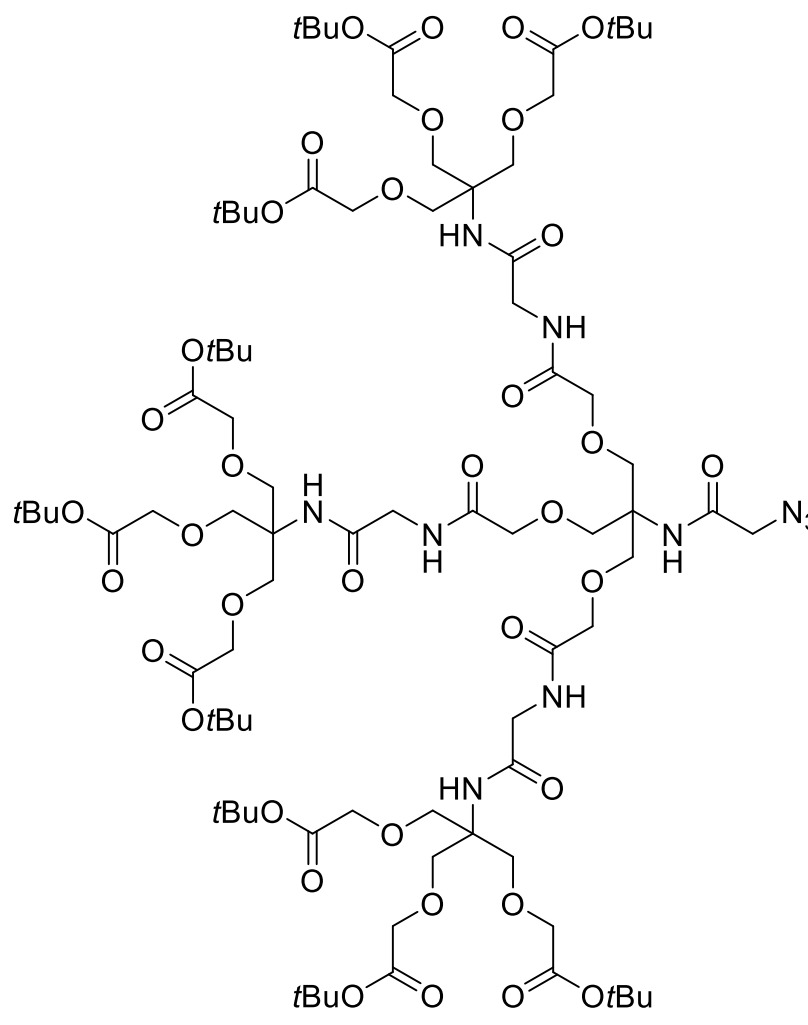

$N$-Azidoacetyl-1,1,1-tris(1,1,1-tris[tertmethyl)aminomethane (50). First generation tri-acid $48(118 \mathrm{mg}, 0.31 \mathrm{mmol})$ and primary amine 49 (728 mg, $1.40 \mathrm{mmol})$ were co-evaporated with DMF $(2 \times 5 \mathrm{~mL})$. To the mixture DMF $(3.1 \mathrm{~mL})$, 
HBTU (590 mg, $1.56 \mathrm{mmol})$ and $\mathrm{NEt}_{3}(0.65 \mathrm{~mL}, 4.67 \mathrm{mmol})$ were added and the reaction mixture was stirred at r.t. for $14 \mathrm{~h}$. The reaction mixture was diluted with $\mathrm{Et}_{2} \mathrm{O}(50 \mathrm{~mL})$ and washed with $0.1 \mathrm{M}$ $\mathrm{HCl}(\mathrm{aq})(2 \times 50 \mathrm{~mL})$. The combined water layers were extracted with $\mathrm{Et}_{2} \mathrm{O}(2 \times 50 \mathrm{~mL})$ and the combined organic layers were dried with $\mathrm{MgSO}_{4}$, filtered and concentrated in vacuo. The residue was purified by silica gel column chromatography (PE/EtOAc, 75/25 $\rightarrow$ 0/100, v/v; followed by EtOAc/MeOH $100 / 0 \rightarrow 95 / 5, v / v)$ and reverse phase column chromatography $\left(\mathrm{C}_{18}, \mathrm{H}_{2} \mathrm{O} / \mathrm{MeOH}\right.$, $50 / 50 \rightarrow 0 / 100, \mathrm{v} / \mathrm{v}$ ) yielded second generation dendron $\mathbf{5 0}$ as a colourless oil (475 $\mathrm{mg}, 0.25 \mathrm{mmol}$, $81 \%) . \mathrm{R}_{f}=0.48($ EtOAc/MeOH, 95/5, v/v); IR (film) 3316, 3970, 2934, 2107, 1745, 1670, 1526, 1368, 1231, 1159, 1120, 917, 846, $731 \mathrm{~cm}^{-1}$; ${ }^{1} \mathrm{H}$ NMR (500 MHz, $\left.\mathrm{D}_{2} \mathrm{O}\right) \delta 7.62$ (s, 3H, $\left.3 \times \mathrm{NH}-\mathrm{TRIS}\right)$, $7.44(\mathrm{~s}, 1 \mathrm{H}, 1 \times \mathrm{NH}-\mathrm{TRIS}), 7.24\left(\mathrm{t}, 3 \mathrm{H}, J_{N H, C H 2}=5.0 \mathrm{~Hz}, 3 \times \mathrm{NH}\right.$ gly), $4.06\left(\mathrm{~s}, 6 \mathrm{H}, 3 \times \mathrm{CH}_{2} \mathrm{C}=\mathrm{O}\right)$, $4.01\left(\mathrm{~d}, 6 \mathrm{H}, J_{C H 2, N H}=4.5 \mathrm{~Hz}, 3 \times \mathrm{CH}_{2}-\mathrm{N}\right), 3.98\left(\mathrm{~s}, 2 \mathrm{H}, \mathrm{CH}_{2}-\mathrm{N}_{3}\right), 3.95\left(\mathrm{~s}, 18 \mathrm{H}, 9 \times \mathrm{CH}_{2} \mathrm{C}=\mathrm{O}\right), 3.93(\mathrm{~s}$, $\left.6 \mathrm{H}, 3 \times \mathrm{CH}_{2}-\mathrm{O}\right), 3.89\left(\mathrm{~s}, 18 \mathrm{H}, 9 \times \mathrm{CH}_{2}-\mathrm{O}\right), 1.45\left(\mathrm{~s}, 54 \mathrm{H}, 18 \times \mathrm{CH}_{3} t-\mathrm{Bu}\right) ;{ }^{13} \mathrm{C} \mathrm{NMR}\left(125 \mathrm{MHz}, \mathrm{CDCl}_{3}\right)$ $\delta 170.3(9 \times \mathrm{C}=\mathrm{O}$ ester $), 169.6(3 \times \mathrm{C}=\mathrm{O}$ amide $), 168.5(3 \times \mathrm{C}=\mathrm{O}$ amide $), 168.2(1 \times \mathrm{C}=\mathrm{O}$ amide $)$, $82.0(\mathrm{Cq} t \mathrm{Bu}), 70.7\left(3 \times \mathrm{CH}_{2}-\mathrm{O}\right), 70.6\left(3 \times \mathrm{CH}_{2} \mathrm{C}=\mathrm{O}\right), 70.2\left(9 \times \mathrm{CH}_{2}-\mathrm{O}\right), 68.9\left(9 \times \mathrm{CH}_{2} \mathrm{C}=\mathrm{O}\right), 60.0$ $\left(3 \times\right.$ Cq tris), $59.8(1 \times \mathrm{Cq}$ tris $) 52.5\left(\mathrm{CH}_{2}-\mathrm{N}_{3}\right), 42.2\left(3 \times \mathrm{CH}_{2}-\mathrm{NH}\right), 28.3\left(27 \times \mathrm{CH}_{3} t \mathrm{Bu}\right) ; \mathrm{HRMS}(\mathrm{ESI})$ $m / z$ calcd. for $\left[\mathrm{C}_{84} \mathrm{H}_{146} \mathrm{~N}_{10} \mathrm{O}_{37}\right]^{2+}:$ 943.4920, obsd.: 943.4929 .

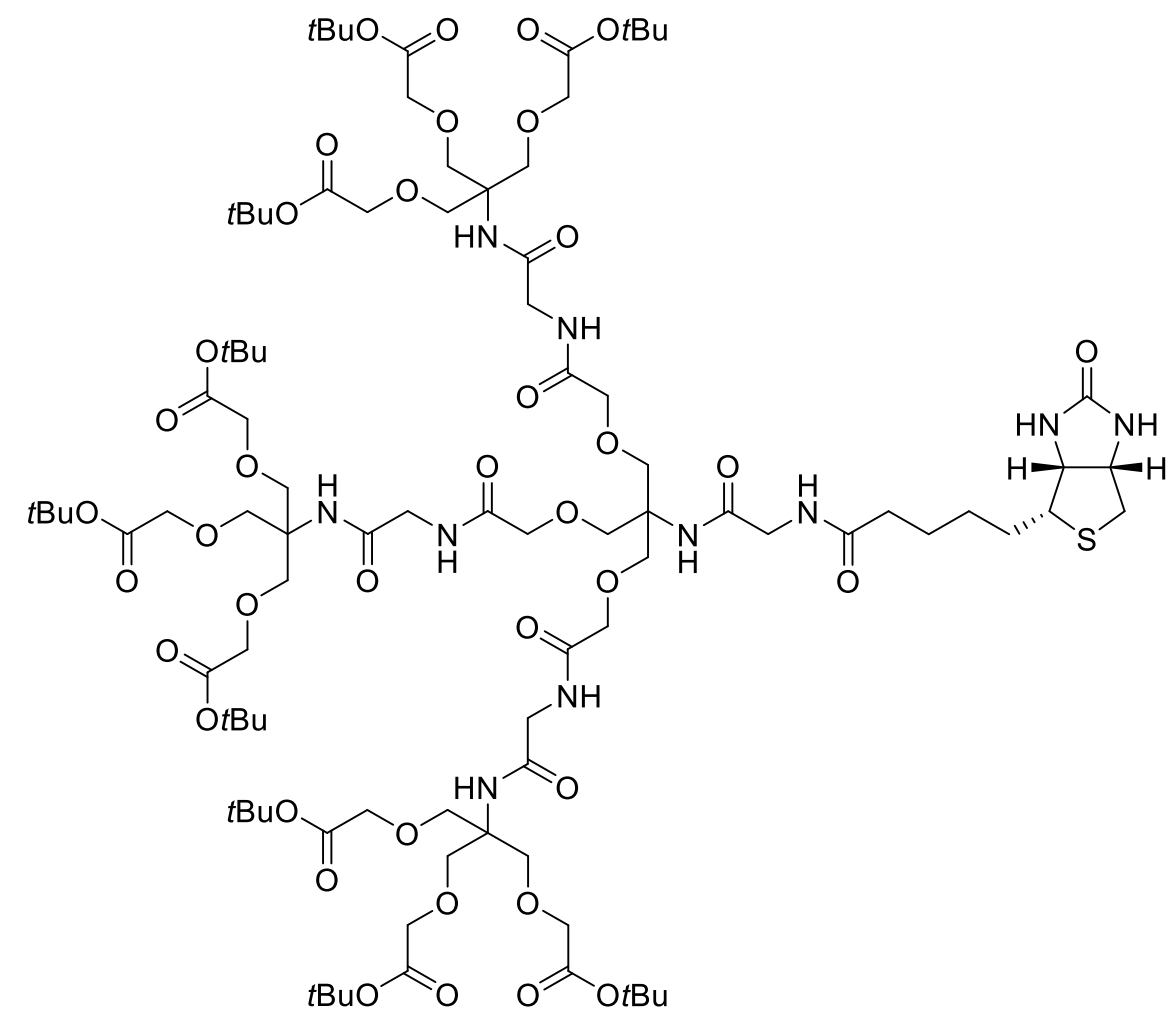

$N$-(2-D-Biotinylamidoacetyl)-1,1,1-tris(1,1,1-tris[tert-butyl-oxycarbonylmethyloxymethyl]methylamidocarbonylmethylamidocarbonylmethyloxymethyl)aminomethane (51). To a solution of second generation dendron 50 (139 mg, $73.7 \mu \mathrm{mol})$ in ethanol $(1 \mathrm{~mL})$ and THF $(1 \mathrm{~mL})$, activated Raney-Nickel (100 
mg) was added and hydrogen gas was bubbled through the reaction mixture at r.t. for $20 \mathrm{~h}$. The reaction mixture was filtered, washed with sat. aq. $\mathrm{NaCl}(50 \mathrm{~mL})$ containing $1 \mathrm{M} \mathrm{NaOH}(1 \mathrm{~mL})$, extracted with $\mathrm{CH}_{2} \mathrm{Cl}_{2}(2 \times 50 \mathrm{~mL})$ and concentrated in vacuo. The resulting oil was then passed through a silica gel plug $(\mathrm{EtOAc} / \mathrm{MeOH}, 100 / 0 \rightarrow 85 / 15$, v/v) to yield crude amine as a colourless oil, which was then used without further purification. HRMS(ESI) $m / z$ calcd. for $\left[\mathrm{C}_{84} \mathrm{H}_{148} \mathrm{~N}_{8} \mathrm{O}_{37}\right]^{2+}$ : 930.4967, obsd.: 930.4960.

To crude second generation dendron amine in DMF (0.73 mL), D-biotin $(27.0 \mathrm{mg}, 110 \mu \mathrm{mol}), \mathrm{HBTU}$ (55.9 $\mathrm{mg}, 147 \mu \mathrm{mol})$ and $\mathrm{NEt}_{3}(25 \mu \mathrm{L})$ were added and the reaction mixture was stirred at r.t. for 18 h. The reaction was diluted with $\mathrm{Et}_{2} \mathrm{O}(50 \mathrm{~mL})$ and washed with sat. aq. $\mathrm{NaCl}(2 \times 50 \mathrm{~mL})$. The combined water layers were extracted with $\mathrm{Et}_{2} \mathrm{O}(2 \times 50 \mathrm{~mL})$ and the combined organic layers were dried with $\mathrm{MgSO}_{4}$, filtered and concentrated in vacuo. Purification by column chromatography $\left(\mathrm{CH}_{2} \mathrm{Cl}_{2} / \mathrm{MeOH}, 100 / 0 \rightarrow 90 / 10\right.$, v/v) and reverse phase column chromatography $\left(\mathrm{C}_{18}, \mathrm{H}_{2} \mathrm{O} / \mathrm{MeOH}\right.$, $50 / 50 \rightarrow 0 / 100, \mathrm{v} / \mathrm{v})$ yielded $2^{\text {nd }}$ generation dendron $\mathbf{5 1}$ as a colourless oil (146 mg, $70.1 \mu \mathrm{mol}, 95 \%$ ). $\mathrm{R}_{f}=0.50\left(\mathrm{CH}_{2} \mathrm{Cl}_{2} / \mathrm{MeOH}, 90 / 10, \mathrm{v} / \mathrm{v}\right)$; IR (film) 3309, 3064, 2979, 2933, 1744, 1668, 1527, 1368, 1230, 1158, 1118, 1035, 846, $733 \mathrm{~cm}^{-1} ;{ }^{1} \mathrm{H}$ NMR (500 MHz, $\left.\mathrm{CDCl}_{3}\right) \delta 7.66$ (s, 3H, $\left.3 \times \mathrm{NH}-\mathrm{TRIS}\right)$, $7.64\left(\mathrm{t}, 1 \mathrm{H}, J_{C H 2, N H}=5.7 \mathrm{~Hz}, \mathrm{NH}-\mathrm{gly}\right), 7.57(\mathrm{~s}, 1 \mathrm{H}, \mathrm{NH}-\mathrm{TRIS}), 7.54\left(\mathrm{t}, 3 \mathrm{H}, J_{C H 2, N H}=5.1 \mathrm{~Hz}, 3 \times \mathrm{NH}-\right.$ gly), 6.30 (s, 1H, NH biotin), 5.65 (s, 1H, NH biotin), 4.45 (dd, $1 \mathrm{H}, J_{7,8 a}=5.3 \mathrm{~Hz}, J_{6,7}=7.0 \mathrm{~Hz}, \mathrm{H} 7-$ biotin), $4.27\left(\mathrm{dd}, 1 \mathrm{H}, J_{5,6}=5.1 \mathrm{~Hz}, J_{6,7}=7.0 \mathrm{~Hz}, \mathrm{H} 6\right.$-biotin), $4.00\left(\mathrm{~s}, 6 \mathrm{H}, 3 \times \mathrm{CH}_{2} \mathrm{O}\right), 3.97(\mathrm{t}, 6 \mathrm{H}$, $\left.J_{C H 2, N H}=4.3 \mathrm{~Hz}, 3 \times \mathrm{CH}_{2} \mathrm{~N}\right), 3.92\left(\mathrm{~s}, 18 \mathrm{H}, 9 \times \mathrm{CH}_{2} \mathrm{O}\right), 3.88-3.80\left(\mathrm{~m}, 26 \mathrm{H}, 9 \times \mathrm{CH}_{2} \mathrm{C}=\mathrm{O}, 3 \times\right.$ $\mathrm{CH}_{2} \mathrm{C}=\mathrm{O}, \mathrm{CH}_{2} \mathrm{~N}$ ), $3.08\left(\mathrm{dt}, 1 \mathrm{H}, J_{5,6}=4.8 \mathrm{~Hz}, J_{4,5}=7.0 \mathrm{~Hz}, \mathrm{H} 5\right.$-biotin), $2.85\left(\mathrm{dd}, 1 \mathrm{H}, J_{7,8 a}=5.3 \mathrm{~Hz}\right.$, $J_{8 a, 8 b}=13.0 \mathrm{~Hz}, \mathrm{H} 8 \mathrm{a}$-biotin), 2.69 (d, 1H, $J_{8 a, 8 b}=12.8 \mathrm{~Hz}$, H8b-biotin), $2.28-2.16$ (m, 2H, $\mathrm{CH}_{2}-1$ biotin), $1.71-1.50$ (m, 6H, $\mathrm{CH}_{2}-2, \mathrm{CH}_{2}-3, \mathrm{CH}_{2}$-4-biotin), 1.41 (s, 81H, $\left.27 \times \mathrm{CH}_{3} t \mathrm{Bu}\right) ;{ }^{13} \mathrm{C} \mathrm{NMR}$ $\left(125 \mathrm{MHz}, \mathrm{CDCl}_{3}\right) \delta 174.4(\mathrm{C}=\mathrm{O}$ biotin $), 170.6(\mathrm{C}=\mathrm{O}$ dendron $), 170.1(9 \times \mathrm{C}=\mathrm{O}), 170.0(3 \times \mathrm{C}=\mathrm{O})$, $169.0(3 \times \mathrm{C}=\mathrm{O}), 163.9(\mathrm{C}=\mathrm{O}$ carbamide biotin $), 81.9(9 \times \mathrm{Cq} t \mathrm{Bu}), 70.6\left(3 \times \mathrm{CH}_{2} \mathrm{C}=\mathrm{O}\right), 70.4(3 \times$ $\left.\mathrm{CH}_{2}-\mathrm{O}\right), 70.0\left(9 \times \mathrm{CH}_{2}-\mathrm{O}\right), 68.8\left(9 \times \mathrm{CH}_{2} \mathrm{C}=\mathrm{O}\right), 61.8$ (C6-biotin), 60.1 (C7-biotin), $59.9(3 \times \mathrm{Cq}$ tris), $59.5\left(1 \times\right.$ Cq tris), $55.5\left(\right.$ C5-biotin), $43.7\left(1 \times \mathrm{CH}_{2}-\mathrm{N}\right), 42.2\left(3 \times \mathrm{CH}_{2}-\mathrm{N}\right), 40.3$ (C8-biotin), $35.2(\mathrm{C} 1$ biotin), 28.2 (C2-biotin), 28.0 (27 $\times \mathrm{CH}_{3}$ ), 27.9 (C4-biotin), 25.3 (C3-biotin); HRMS(ESI) $\mathrm{m} / z$ calcd. for $\left[\mathrm{C}_{94} \mathrm{H}_{162} \mathrm{~N}_{10} \mathrm{O}_{39} \mathrm{~S}\right]^{2+}:$ 1043.5355, obsd.: 1043.5351 . 


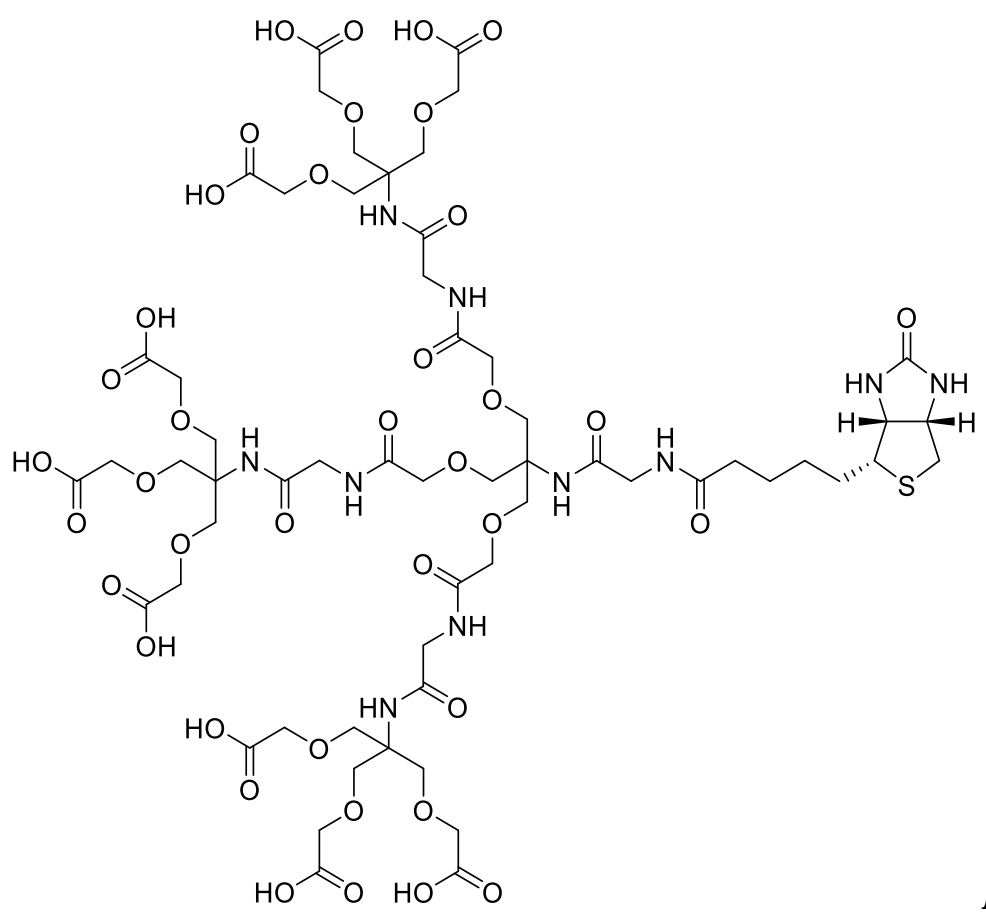

$N$-(2-D-Biotinylamidoacetyl)-

\section{1,1,1-tris(1,1,1-tris[carboxymethyloxymethyl]methylamidocarbonylmethylamidocarbonyl-}

methyloxymethyl)aminomethane (52). To a solution of second generation dendron $\mathbf{5 1}$ (12.0 mg, $5.8 \mu \mathrm{mol})$ in $\mathrm{CH}_{2} \mathrm{Cl}_{2}(1 \mathrm{~mL})$, freshly distilled trifluoroacetic acid $(1 \mathrm{~mL})$ was added and the reaction mixture was stirred at r.t. for $3 \mathrm{~h}$. The crude reaction mixture was concentrated in vacuo and coevaporated with water $(10 \times 1 \mathrm{~mL})$. Purification by reverse phase column chromatography $\left(\mathrm{C}_{18}\right.$, $\mathrm{H}_{2} \mathrm{O} / \mathrm{MeOH}, 100 / 0 \rightarrow 70 / 30$, v/v) yielded nona-acid $52(8.8 \mathrm{mg}, 5.6 \mathrm{mmol}, 97 \%) . \mathrm{R}_{f}=0.1$ (EtOAc/MeOH, 80/20, v/v); IR (film) 3360, 3005, 2990, 2978, 2948, 1721, 1656, 1552, 1472, 1431, $1343,1245,1200,1123,1032,1018,979 \mathrm{~cm}^{-1} ;{ }^{1} \mathrm{H}$ NMR $\left(500 \mathrm{MHz}, \mathrm{D}_{2} \mathrm{O}\right) \delta 4.57\left(\mathrm{dd}, 1 \mathrm{H}, J_{7,8 a}=5.1\right.$ $\mathrm{Hz}, J_{6,7}=7.5 \mathrm{~Hz}, \mathrm{H} 7$-biotin), 4.39 (dd, 1H, $J_{5,6}=4.6, J_{6,7}=7.5 \mathrm{~Hz}, \mathrm{H6}$-biotin), 4.18 (s, $18 \mathrm{H}, 9 \times$ $\mathrm{CH}_{2} \mathrm{CO}$ ), 4.12 (s, $6 \mathrm{H}, 3 \times \mathrm{CH}_{2} \mathrm{CO}$ ), 3.95 (s, $\left.6 \mathrm{H}, 3 \times \mathrm{CH}_{2} \mathrm{NH}\right), 3.88\left(\mathrm{~s}, 18 \mathrm{H}, 9 \times \mathrm{CH}_{2} \mathrm{O}\right), 3.86(\mathrm{~s}, 6 \mathrm{H}$, $3 \times \mathrm{CH} 2 \mathrm{O}), 3.28\left(\mathrm{dt}, 1 \mathrm{H}, J_{5,6}=5.3 \mathrm{~Hz}, J_{4,5}=8.0 \mathrm{~Hz}, \mathrm{H} 5\right.$-biotin $), 2.96\left(\mathrm{dd}, 1 \mathrm{H}, J_{7,8 a}=5.0 \mathrm{~Hz}, J_{8 a, 8 b}=\right.$ $13.0 \mathrm{~Hz}, \mathrm{H} 8 \mathrm{a}$-biotin), 2.74 (d, $1 \mathrm{H}, J_{8 a, 8 b}=13.0 \mathrm{~Hz}, \mathrm{H} 8 \mathrm{~b}$-biotin), 2.30 (t, $2 \mathrm{H}, J_{l, 2}=7.2 \mathrm{~Hz}, \mathrm{CH}_{2}-1-$ biotin), $1.74-1.50$ (m, 4H, $\mathrm{CH}_{2}$-4-biotin, $\mathrm{CH}_{2}$-2-biotin), 1.39 (p, $2 \mathrm{H}, J_{2,3}=J_{3,4}=7.4 \mathrm{~Hz}, \mathrm{CH}_{2}-3$ biotin); ${ }^{13} \mathrm{C}$ NMR (125 MHz, $\left.\mathrm{CDCl}_{3}\right) \delta 177.1(\mathrm{C}=\mathrm{O}$ biotin), $174.2(9 \times \mathrm{C}=\mathrm{O}), 172.8(3 \times \mathrm{C}=\mathrm{O}), 171.3$ $(1 \times \mathrm{C}=\mathrm{O}), 170.7(3 \times \mathrm{C}=\mathrm{O}), 165.2(\mathrm{C}=\mathrm{O}$ carbamide biotin $), 70.0\left(3 \times \mathrm{CH}_{2} \mathrm{C}=\mathrm{O}\right), 69.8\left(3 \times \mathrm{CH}_{2}-\mathrm{O}\right)$, $69.7\left(9 \times \mathrm{CH}_{2}-\mathrm{O}\right), 68.0\left(9 \times \mathrm{CH}_{2} \mathrm{C}=\mathrm{O}\right), 62.0$ (C6-biotin), 60.2 (C7-biotin), 59.8 (3 $\times$ Cq tris), $59.6(1$ $\times$ Cq tris), 55.2 (C5-biotin), $43.0\left(1 \times \mathrm{CH}_{2}-\mathrm{N}\right), 42.4\left(3 \times \mathrm{CH}_{2}-\mathrm{N}\right), 39.6(\mathrm{C} 8$-biotin), 35.1 (C1-biotin), 27.9 (C2-biotin), 27.6 (C4-biotin), 25.0 (C3-biotin); HRMS(ESI) $m / z$ calcd. for $\left[\mathrm{C}_{58} \mathrm{H}_{90} \mathrm{~N}_{10} \mathrm{O}_{39} \mathrm{~S}^{2+}\right.$ : 791.2538, obsd.: 791.2536 . 


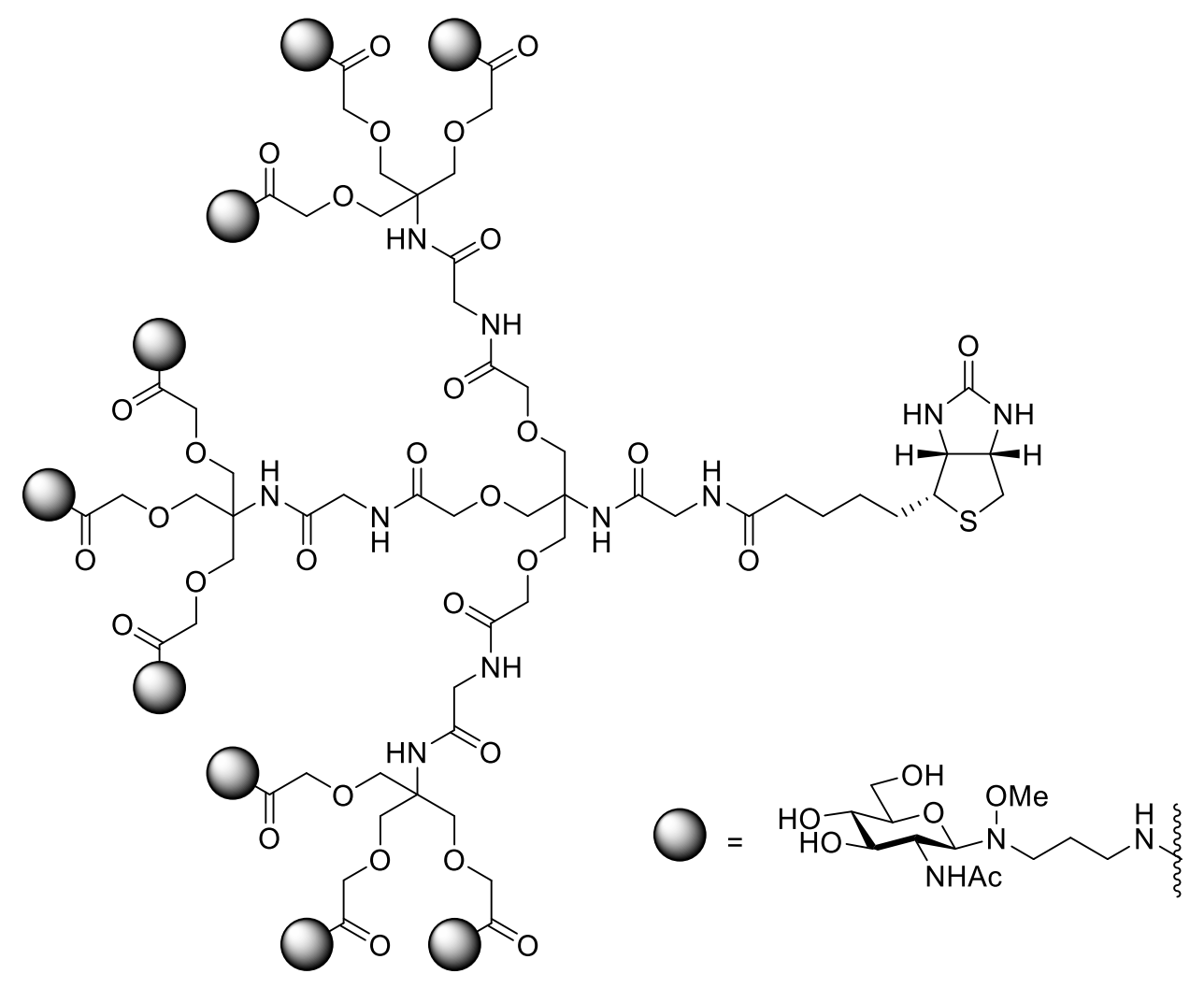

$N$-(2-D-Biotinylamido-acetyl)-1,1,1-tris(1,1,1-tris[3-( $N$-(2-acetamido-2-deoxy- $\beta$-Dglucopyranosyl)- $O$-methyl-hydroxylamine)propylamidocarbonylmethyloxymethyl]methylamidocarbonylmethylamidocarbonylmethyloxy-methyl)aminomethane (54). Second generation dendron nona-acid $52(1.5 \mathrm{mg}, 0.95 \mu \mathrm{mol})$ and GlcNAc-amine $53(5.3 \mathrm{mg}, 17.1 \mu \mathrm{mol})$ were coevaporated with freshly distilled DMF $(2 \times 2 \mathrm{~mL})$. To the mixture DMF $(0.2 \mathrm{~mL})$ was added and the reaction mixture was half concentrated under reduced pressure to remove traces of $\mathrm{Et}_{2} \mathrm{NH}$. To the reaction mixture, HBTU $(13.7 \mathrm{mg}, 36 \mu \mathrm{mol})$ and distilled $\mathrm{NEt}_{3}(25 \mu \mathrm{L})$ were added and the reaction mixture was stirred at r.t. for 14 h. The reaction mixture was diluted with water $(2 \mathrm{~mL})$ and purified using a dialysis in a $\mathrm{Na}_{2} \mathrm{HPO}_{4}$ solution $(1 \mathrm{~g} / \mathrm{L})$, to remove the conjugation byproducts. The water was replaced twice a day for 4 days. The dendron was then purified using reverse phase column chromatography $\left(\mathrm{C}_{8}, \mathrm{H}_{2} \mathrm{O} / \mathrm{MeOH}, 100 / 0 \rightarrow 70 / 30\right.$, v/v) to give glycodendron $\mathbf{5 4}$ as a colourless oil $(2.8 \mathrm{mg}, 0.68 \mu \mathrm{mol}, 72 \%) .{ }^{1} \mathrm{H}$ NMR $\left(600 \mathrm{MHz}, \mathrm{D}_{2} \mathrm{O}\right) \delta 4.58\left(\mathrm{dd}, 1 \mathrm{H}, J_{7,8 a}=5.1 \mathrm{~Hz}, J_{6,7}=7.7 \mathrm{~Hz}\right.$, H7-biotin), 4.40 (dd, $1 \mathrm{H}, J_{5,6}=4.7 \mathrm{~Hz}, J_{6,7}=7.7 \mathrm{~Hz}, \mathrm{H6}$-biotin), $4.32\left(\mathrm{~d}, 9 \mathrm{H}, J_{1,2}=9.7 \mathrm{~Hz}, 9 \times \mathrm{H}-1\right.$ GlcNAc), 4.12 (s, 6H, $3 \times \mathrm{CH}_{2} \mathrm{C}=\mathrm{O}$ dendron), 4.07 (s, $18 \mathrm{H}, 9 \times \mathrm{CH}_{2} \mathrm{C}=\mathrm{O}$ dendron), 3.96 (s, $6 \mathrm{H}, 3 \times$ $\mathrm{CH}_{2}$-N dendron), $3.92-3.83$ (m, 37H, $1 \times \mathrm{CH}_{2} \mathrm{~N}$ dendron, $9 \times \mathrm{H}-2$ GlcNAc, $9 \times \mathrm{H}-6 \mathrm{a}$ GlcNAc, $9 \times$ $\mathrm{CH}_{2}$-dendron), $3.72\left(\mathrm{dd}, 9 \mathrm{H}, J_{5,6 a}=5.5 \mathrm{~Hz}, J_{6 a, 6 b}=12.4 \mathrm{~Hz}, 9 \times \mathrm{H}-6 \mathrm{~b}\right.$ GlcNAc), 3.51 (dd, 9H, $J_{2,3}=$ $\left.8.8 \mathrm{~Hz}, J_{3,4}=9.8 \mathrm{~Hz}, 9 \times \mathrm{H}-3 \mathrm{GlcNAc}\right), 3.48\left(\mathrm{~s}, 27 \mathrm{H}, 9 \times \mathrm{NOCH}_{3}\right), 3.43-3.28\left(\mathrm{~m}, 28 \mathrm{H}, 9 \times \mathrm{CH}_{2} \mathrm{a}-3\right.$ linker, $9 \times$ H-4 GlcNAc, $9 \times$ H-5 GlcNAc, H5-biotin), 3.24 (dt, 9H, $J_{2,3}=7.2 \mathrm{~Hz}, J_{3 a, 3 b}=13.4 \mathrm{~Hz}, 9 \times$ $\mathrm{CH}_{2}$ b-3 linker), $3.01-2.87$ (m, $19 \mathrm{H}, 9 \times \mathrm{CH}_{2}-1$ linker, H8a-biotin), $2.75\left(\mathrm{~d}, 1 \mathrm{H}, J_{8 a, 8 b}=12.9 \mathrm{~Hz}\right.$, 
H8b-biotin), 2.30 (t, 2H, $J_{1,2}=7.3 \mathrm{~Hz}, \mathrm{CH}_{2}$-1-biotin), 2.03 (s, 27H, $9 \times N$-Ac), $1.88-1.78$ (m, $1 \mathrm{H}$, H4a-biotin), 1.77 - 1.69 (m, 18H, $9 \times \mathrm{CH} 2-2$ linker), 1.69 - 1.58 (m, 1H, H4a-biotin) 1.58 - 1.50 (m, 2H, $\mathrm{CH}_{2}$-2-biotin), $1.44-1.36\left(\mathrm{~m}, 2 \mathrm{H}, \mathrm{CH}_{2}\right.$-3-biotin); HRMS(ESI) $\mathrm{m} / \mathrm{z}$ calcd. for $\left[\mathrm{C}_{166} \mathrm{H}_{298} \mathrm{~N}_{37} \mathrm{O}_{84} \mathrm{~S}\right]^{3+}:$ 1395.6641, obsd.: 1395.6648 .<smiles>NCC(=O)NC(COCC(=O)NCC(=O)NC(COCC(=O)O)(COCC(=O)O)COCC(=O)O)(COCC(=O)NCC(=O)NC(COCC(=O)O)(COCC(=O)O)COCC(=O)O)COCC(=O)NC(COCC(=O)O)(COCC(=O)O)COCC(=O)O</smiles>

$N$-Glycyl-1,1,1-tris(1,1,1-tris[carboxymethyloxymethyl]methylamidocarbonylmethylamidocarbonylmethyloxymethyl)amino-

methane trifluoroacetic acid (62). To a solution dendron $\mathbf{5 0}(60.5 \mathrm{mg}, 31.8 \mu \mathrm{mol})$ in $\mathrm{MeOH} / \mathrm{H}_{2} \mathrm{O} / \mathrm{CH}_{2} \mathrm{Cl}_{2}(3 \mathrm{~mL}, 3 / 1 / 1$, v/v/v), triphenylphosphine (25.0 mg, $95.4 \mu \mathrm{mol})$ was added and the reaction mixture was stirred at $\mathrm{rt}$ for $18 \mathrm{~h}$. The crude reaction mixture was concentrated in vacuo and co-evaporated with $\mathrm{CH}_{2} \mathrm{Cl}_{2}(2 \times 10 \mathrm{~mL})$. The residue was dissolved in $\mathrm{CH}_{2} \mathrm{Cl}_{2}(3 \mathrm{~mL})$, TFA (3 $\mathrm{mL}$ ) was added and the reaction mixture was stirred at $\mathrm{rt}$ for $3 \mathrm{~h}$. The solvent was evaporated by an argon stream, $\mathrm{H}_{2} \mathrm{O}(10 \mathrm{~mL})$ was added, and the phosphine salts were removed via filtration, and the resulting residue was concentrated in vacuo to give crude amino acid 62. (45.9 mg, $31.6 \mu \mathrm{mol}, 99 \%$ ). $\mathrm{R}_{f}=0.05\left(t \mathrm{BuOH} / \mathrm{AcOH} / \mathrm{H}_{2} \mathrm{O}, 4 / 1 / 1, \mathrm{v} / \mathrm{v} / \mathrm{v}\right)$; IR (film) 3344, 3003, 2981, 2950, 2937, 2930, 1718, 1651, 1543, 1473, 1458, 1434, 1241, 1199, 1120, 1032, $952 \mathrm{~cm}^{-1} ;{ }^{1} \mathrm{H}$ NMR $\left(500 \mathrm{MHz}, \mathrm{D}_{2} \mathrm{O}\right) \delta 4.06$ $\left(\mathrm{s}, 18 \mathrm{H}, 9 \times \mathrm{CH}_{2} \mathrm{C}=\mathrm{O}\right), 4.00\left(\mathrm{~s}, 6 \mathrm{H}, 3 \times \mathrm{CH}_{2} \mathrm{C}=\mathrm{O}\right), 3.83\left(\mathrm{~s}, 6 \mathrm{H}, 3 \times \mathrm{CH}_{2} \mathrm{~N}\right), 3.77\left(\mathrm{~s}, 6 \mathrm{H}, 3 \times \mathrm{CH}_{2} \mathrm{O}\right)$, $3.75\left(\mathrm{~s}, 18 \mathrm{H}, 9 \times \mathrm{CH}_{2} \mathrm{O}\right), 3.71\left(\mathrm{~s}, 2 \mathrm{H}, 1 \times \mathrm{CH}_{2} \mathrm{NH}_{2}\right) ;{ }^{13} \mathrm{C} \mathrm{NMR}\left(125 \mathrm{MHz}, \mathrm{D}_{2} \mathrm{O}\right) \delta 174.1(9 \times \mathrm{C}=\mathrm{O}$ $\left.\mathrm{CO}_{2} \mathrm{H}\right), 172.6(3 \times \mathrm{C}=\mathrm{ONH}), 170.6(3 \times \mathrm{C}=\mathrm{O}), 166.7(1 \times \mathrm{C}=\mathrm{O}), 162.8,162.5,162.2,161.9(\mathrm{C}=\mathrm{O}$ 
TFA), 119.4, 117.1, 114.8, 112.5 ( $\mathrm{CF}_{3}$ TFA $), 69.83,69.77\left(3 \times \mathrm{CH}_{2} \mathrm{O}, 3 \times \mathrm{CH}_{2} \mathrm{C}=\mathrm{O}\right), 69.6(9 \times$ $\left.\mathrm{CH}_{2} \mathrm{O}\right), 67.9\left(9 \times \mathrm{CH}_{2} \mathrm{C}=\mathrm{O}\right), 59.9(1 \times \mathrm{Cq}$ tris $), 59.7(3 \times \mathrm{Cq}$ tris $), 42.3\left(3 \times \mathrm{CH}_{2} \mathrm{NH}\right), 40.6(1 \times$ $\mathrm{CH}_{2} \mathrm{NH}_{2}$ ); HRMS(ESI) $\mathrm{m} / z$ calcd. for $\left[\mathrm{C}_{48} \mathrm{H}_{76} \mathrm{~N}_{8} \mathrm{O}_{37}\right]^{2+}$ : 678.2150, obsd.: 678.2136.<smiles>O=C(O)CNC(=S)NC(COCC(=O)NCC(=O)NCC(=O)Nc1ccc2c(c1)C(=O)OC21c2ccc(O)cc2Oc2cc(O)ccc21)(COCC(=O)NCC(=O)NC(COCC(=O)O)(COCC(=O)O)COCC(=O)O)COCC(=O)NCC(=O)NC(COCC(=O)O)(COCC(=O)O)COCC(=O)O</smiles>

$N$-[N-([(3',6'-Dihydroxy-3-oxospiro[isobenzofuran-1(3H),9'-(9H)xanthen]-5-yl)amino]-

thioxomethyl)glycyl]-1,1,1-tris(1,1,1-tris[carboxymethyloxymethyl]methylamido-

carbonylmethylamidocarbonylmethyloxymethyl)aminomethane (63). To a solution of dendron $62(25.1 \mathrm{mg}, 17.3 \mu \mathrm{mol})$ in DMF (3 mL), fluorescein isothiocyanate isomer I (7.4 mg, $19.0 \mu \mathrm{mol})$ and $\mathrm{Et}_{3} \mathrm{~N}(1 \mathrm{~mL})$ were added and the reaction mixture was stirred at $\mathrm{rt}$ for $18 \mathrm{~h}$. The crude reaction mixture was concentrated in vacuo. The residue was purified with size exclusion chromatography (LH-20, $\mathrm{CH}_{2} \mathrm{Cl}_{2} / \mathrm{MeOH}, 50 / 50, \mathrm{v} / \mathrm{v}$ ) and concentrated in vacuo to give the trimethylamine salt of dendron 63. The dendron was co-evaporated with $\mathrm{H}_{2} \mathrm{O}$ and purified using ion exchange chromatography (Dowex $\mathrm{H}^{+}$, elute with $\left.\mathrm{H}_{2} \mathrm{O}\right)$ to give dendron $\mathbf{6 3}(22.3 \mathrm{mg}, 12.8 \mathrm{mmol}, 74 \%)$ as a yellow oil. $\mathrm{R}_{f}=0.05\left(t \mathrm{BuOH} / \mathrm{AcOH} / \mathrm{H}_{2} \mathrm{O}, 4 / 1 / 1, \mathrm{v} / \mathrm{v} / \mathrm{v}\right)$; IR (film) 3463, 3366, 3074, 3015, 2970, 2946, 1738, 1656, 1548, 1436, 1366, 1229, 1216, 1206, 1119, 1051, 1032, $897 \mathrm{~cm}^{-1}$; ${ }^{1} \mathrm{H}$ NMR (500 $\left.\mathrm{MHz}, \mathrm{CD}_{3} \mathrm{OD}\right) \delta 8.22(\mathrm{~s}, 1 \mathrm{H}, \mathrm{H}-3), 8.13-7.98(\mathrm{~m}, \mathrm{NH}), 7.94(\mathrm{~s}, 3 \times \mathrm{NH}), 7.88(\mathrm{~s}, 1 \times \mathrm{NH}), 7.84(\mathrm{~d}$, $\left.1 \mathrm{H}, J_{5,6}=8.8 \mathrm{~Hz}, \mathrm{H}-5\right), 7.17\left(\mathrm{~d}, 1 \mathrm{H}, J_{5,6}=8.8 \mathrm{~Hz}, \mathrm{H}-6\right), 6.78-6.63(\mathrm{~m}, 4 \mathrm{H}, 2 \times \mathrm{H}-13,2 \times \mathrm{H}-10)$, 
$6.58\left(\mathrm{~d}, 2 \mathrm{H}, J_{9,10}=8.2 \mathrm{~Hz}, 2 \times \mathrm{H}-11\right), 4.35\left(\mathrm{~s}, 2 \mathrm{H}, 1 \times \mathrm{CH}_{2} \mathrm{NH}\right), 4.20-4.02\left(\mathrm{bs}, 24 \mathrm{H}, 3 \times \mathrm{CH}_{2} \mathrm{C}=\mathrm{O}\right.$, $\left.9 \times \mathrm{CH}_{2} \mathrm{C}=\mathrm{O}\right), 4.02-3.92\left(\mathrm{~m}, 12 \mathrm{H}, 3 \times \mathrm{CH}_{2} \mathrm{O}, 3 \times \mathrm{CH}_{2} \mathrm{NH}\right), 3.92-3.86\left(\mathrm{~m}, 18 \mathrm{H}, 9 \times \mathrm{CH}_{2} \mathrm{O}\right) ;{ }^{13} \mathrm{C}$ NMR (125 MHz, CD $\left.{ }_{3} \mathrm{OD}\right) \delta 183.5(\mathrm{C}=\mathrm{S}), 174.5(9 \times \mathrm{C}=\mathrm{O}), 173.0(3 \times \mathrm{C}=\mathrm{O}), 172.0(1 \times \mathrm{C}=\mathrm{O}$ glyc $)$, 171.4 (3 × C=O glyc), 171.2 (C=O C-1), 161.5, 154.2 (C-12, C14), 149.7 (C-7), 142.2 (C-2/4/8), 132.1 (C-5), 130.4 (C-13), 130.3 (C-2/4/8), 128.9 (C-2/4/8), 125.8 (C-6), 120.3 (C-3), 113.8 (C-11), $111.5(\mathrm{C}-9), 103.6(\mathrm{C}-10), 71.4\left(3 \times \mathrm{CH}_{2}-\mathrm{O}\right), 71.0\left(9 \times \mathrm{CH}_{2} \mathrm{O}\right), 69.3\left(3 \times \mathrm{CH}_{2} \mathrm{C}=\mathrm{O}\right), 69.2(9 \times$ $\left.\mathrm{CH}_{2} \mathrm{C}=\mathrm{O}\right), 61.3\left(3 \times \mathrm{C}_{\mathrm{q}}\right.$ tris $), 61.2\left(1 \times \mathrm{C}_{\mathrm{q}}\right.$ tris $), 48.9\left(1 \times \mathrm{CH}_{2} \mathrm{NH}\right), 43.5\left(3 \times \mathrm{CH}_{2} \mathrm{NH}\right) ; \mathrm{HRMS}(\mathrm{ESI})$ $m / z$ calcd. for $\left[\mathrm{C}_{69} \mathrm{H}_{87} \mathrm{~N}_{9} \mathrm{O}_{42} \mathrm{~S}\right]^{2+}: 872.7329$, obsd.: 872.7335 . 


\subsection{References}

(1) Astruc, D.; Boisselier, E.; Ornelas, C. 'Dendrimers Designed for Functions: From Physical, Photophysical, and Supramolecular Properties to Applications in Sensing, Catalysis, Molecular Electronics, Photonics, and Nanomedicine' Chem. Rev. 2010, 110, 1857-1959.

(2) Nantalaksakul, A.; Reddy, D. R.; Bardeen, C. J.; Thayumanavan, S. 'Light Harvesting Dendrimers'. Photosynthesis Research, 2006, 87, 133-150.

(3) Caminade, A. M.; Hameau, A.; Majorai, J. P. 'Multicharged And/or Water-Soluble Fluorescent Dendrimers: Properties and Uses' Chem. Eur. J. 2009, 15, 9270-9285.

(4) De Jesús, E.; Flores, J. C. 'Dendrimers: Solutions for Catalyst Separation and Recycling-a Review'. Industrial and Engineering Chemistry Research, 2008, 47, 7968-7981.

(5) Gajbhiye, V.; Palanirajan, V. K.; Tekade, R. K.; Jain, N. K. 'Dendrimers as Therapeutic Agents: A Systematic Review.' J. Pharm. Pharmacol. 2009, 61, 989-1003.

(6) Fulton, D. A.; Elemento, E. M.; Aime, S.; Chaabane, L.; Botta, M.; Parker, D. 'Glycoconjugates of Gadolinium Complexes for MRI Applications.' Chem. Comm. 2006, 1064-1066.

(7) Aaron, A. J.; Bumb, A.; Brechbiel, M. W. 'Macromolecules, Dendrimers, and Nanomaterials in Magnetic Resonance Imaging: The Interplay between Size, Function, and Pharmacokinetics' Chem. Rev. 2010, 110, 2921-2959.

(8) Heegaard, P. M. H.; Boas, U.; Sorensen, N. S. 'Dendrimers for Vaccine and Immunostimulatory Uses. A Review' Bioconjug. Chem. 2010, 21, 405-418.

(9) Wang, S.-K.; Liang, P.-H.; Astronomo, R. D.; Hsu, T.-L.; Hsieh, S.-L.; Burton, D. R.; Wong, C.-H. 'Targeting the Carbohydrates on HIV-1: Interaction of Oligomannose Dendrons with Human Monoclonal Antibody 2G12 and DC-SIGN.' Proc. Natl. Acad. Sci. U. S. A. 2008, 105, 3690-3695.

(10) Gupta, U.; Agashe, H. B.; Asthana, A.; Jain, N. K. 'A Review of in Vitro-in Vivo Investigations on Dendrimers: The Novel Nanoscopic Drug Carriers'. Nanomedicine: Nanotechnology, Biology, and Medicine, 2006, 2, 66-73.

(11) Bernardes, G. J. L.; Kikkeri, R.; Maglinao, M.; Laurino, P.; Collot, M.; Hong, S. Y.; Lepenies, B.; Seeberger, P. H. 'Design, Synthesis and Biological Evaluation of CarbohydrateFunctionalized Cyclodextrins and Liposomes for Hepatocyte-Specific Targeting.' Org. Biomol. Chem. 2010, 8, 4987-4996.

(12) Sampathkumar, S.; Yarema, K. 'Nanotechnologies Life ...'; 2007; Vol. 7.

(13) Yiyun, C.; Tongwen, X. 'Dendrimers as Potential Drug Carriers. Part I. Solubilization of NonSteroidal Anti-Inflammatory Drugs in the Presence of Polyamidoamine Dendrimers' Eur. J. Med. Chem. 2005, 40, 1188-1192.

(14) Mammen, M.; Choi, S.-K.; Whitesides, G. M. 'Polyvalent Interactions in Biological Systems: Implications for Design and Use of Multivalent Ligands and Inhibitors' Angew. Chem. Int. Ed. Engl. 1998, 37, 2754-2794.

(15) Walter, M. V; Malkoch, M. 'Simplifying the Synthesis of Dendrimers: Accelerated Approaches'. Chem. Soc. Rev., 2012, 41, 4593.

(16) Newkome, G. R.; Lin, X. 'Symmetrical, Four-Directional, Poly(ether-Amide) Cascade Polymers' Macromolecules 1991, 24, 1443-1444.

(17) Chabre, Y. M.; Contino-Pépin, C.; Placide, V.; Shiao, T. C.; Roy, R. 'Expeditive Synthesis of Glycodendrimer Scaffolds Based on Versatile TRIS and Mannoside Derivatives.' J. Org. Chem. 2008, 73, 5602-5605.

(18) Kaplánek, R.; Bříza, T.; Havlík, M.; Martásek, P.; Král, V. 'Three-Fold Polyfluoroalkylated Amines and Isocyanates Based on Tris(hydroxymethyl)aminomethane (TRIS)' J. Fluor. Chem. 2007, 128, 179-183. 
(19) Ashton, P. R.; Boyd, S. E.; Brown, C. L.; Jayaraman, N.; Nepogodiev, S. A.; Stoddart, J. F. 'A Convergent Synthesis of Carbohydrate-Containing Dendrimers' Chem. Eur. J. 1996, 2, $1115-1128$.

(20) Newkome, G. R.; Lin, X.; Young, J. K. 'Syntheses of Amine Building Blocks for Dendritic Macromolecule Construction' Synlett 1992, 1992, 53-54.

(21) Sliedregt, L. A. J. M.; Rensen, P. C.; Rump, E. T.; van Santbrink, P. J.; Bijsterbosch, M. K.; Valentijn, A. R. P. M.; van der Marel, G. A.; van Boom, J. H.; van Berkel, T. J.; Biessen, E. A. L. 'Design and Synthesis of Novel Amphiphilic Dendritic Galactosides for Selective Targeting of Liposomes to the Hepatic Asialoglycoprotein Receptor.' J. Med. Chem. 1999, 42, 609-618.

(22) Rensen, P. C. N.; van Leeuwen, S. H.; Sliedregt, L. A. J. M.; van Berkel, T. J. C.; Biessen, E. A. L. 'Design and Synthesis of Novel N-Acetylgalactosamine-Terminated Glycolipids for Targeting of Lipoproteins to the Hepatic Asialoglycoprotein Receptor.' J. Med. Chem. 2004, 47, 5798-5808.

(23) Schlick, K. H.; Morgan, J. R.; Weiel, J. J.; Kelsey, M. S.; Cloninger, M. J. 'Clusters of Ligands on Dendrimer Surfaces.' Bioorg. Med. Chem. Lett. 2011, 21, 5078-5083.

(24) Chabre, Y. M.; Giguère, D.; Blanchard, B.; Rodrigue, J.; Rocheleau, S.; Neault, M.; Rauthu, S.; Papadopoulos, A.; Arnold, A. A.; Imberty, A.; Roy, R. 'Combining Glycomimetic and Multivalent Strategies toward Designing Potent Bacterial Lectin Inhibitors.' Chemistry 2011, 17, 6545-6562.

(25) Ribeiro-Viana, R.; García-Vallejo, J. J.; Collado, D.; Pérez-Inestrosa, E.; Bloem, K.; van Kooyk, Y.; Rojo, J. 'BODIPY-Labeled DC-SIGN-Targeting Glycodendrons Efficiently Internalize and Route to Lysosomes in Human Dendritic Cells.' Biomacromolecules 2012, 13, 3209-3219.

(26) Ornelas, C.; Broichhagen, J.; Weck, M. 'Strain-Promoted Alkyne Azide Cycloaddition for the Functionalization of Poly(amide)-Based Dendrons and Dendrimers.' J. Am. Chem. Soc. 2010, 132, 3923-3931.

(27) Gonzaga, F.; Sadowski, L. P.; Rambarran, T.; Grande, J.; Adronov, A.; Brook, M. A. 'Highly Efficient Divergent Synthesis of Dendrimers via Metal-Free "click" Chemistry' J. Polym. Sci.

Part A Polym. Chem. 2013, 51, 1272-1277.

(28) Astruc, D.; Liang, L.; Rapakousiou, A.; Ruiz, J. 'Click Dendrimers and Triazole-Related Aspects: Catalysts, Mechanism, Synthesis, and Functions. A Bridge between Dendritic Architectures and Nanomaterials.' Accounts Chem. Res. 2012, 45, 630-640.

(29) Lallana, E.; Fernandez-Trillo, F.; Sousa-Herves, A.; Riguera, R.; Fernandez-Megia, E. 'Click Chemistry with Polymers, Dendrimers, and Hydrogels for Drug Delivery.' Pharm. Res. 2012, 29, 902-921.

(30) Ribeiro-Viana, R.; Sánchez-Navarro, M.; Luczkowiak, J.; Koeppe, J. R.; Delgado, R.; Rojo, J.; Davis, B. G. 'Virus-like Glycodendrinanoparticles Displaying Quasi-Equivalent Nested Polyvalency upon Glycoprotein Platforms Potently Block Viral Infection.' Nat. Commun. 2012, 3, 1303.

(31) Lowe, A. B. 'Thiol-Ene "click" Reactions and Recent Applications in Polymer and Materials Synthesis' Polym. Chem. 2010, $1,17$.

(32) Gingras, M.; Chabre, Y. M.; Roy, M.; Roy, R. 'How Do Multivalent Glycodendrimers Benefit from Sulfur Chemistry?' Chem. Soc. Rev. 2013, 42, 4823-4841.

(33) Gómez-García, M.; Benito, J. M.; Butera, A. P.; Ortiz Mellet, C.; García Fernández, J. M.; Jiménez Blanco, J. L. 'Probing Carbohydrate-Lectin Recognition in Heterogeneous Environments with Monodisperse Cyclodextrin-Based Glycoclusters.' J. Org. Chem. 2012, 77, 1273-1288.

(34) Dupuy, C.; Viguier, R.; Dupraz, A. 'Polyalkylation of 2,2-Bis(hydroxymethyl)-1-Alkanols with Tert-Butyl Bromoacetate' Synth. Commun. 2001, 31, 1307-1313.

(35) Martinez, A. J.; Pendri, A.; Greenwald, R. B.; Choe, Y. H.; Roberts \& Mercanti, L. L. P. 'Terminally-Branched Polymeric Linkers and Polymeric Conjugates Containing the Same', 1999, $1,1-33$. 
(36) Ashton, P. R.; Hounsell, E. F.; Jayaraman, N.; Nilsen, T. M.; Spencer, N.; Stoddart, J. F.; Young, M. 'Synthesis and Biological Evaluation of R - D -Mannopyranoside-Containing Dendrimers $\dagger^{\prime}$ 1998, 3263, 3429-3437.

(37) Furneaux, R. H.; Pakulski, Z.; Tyler, P. C. 'New Mannotriosides and Trimannosides as Potential Ligands for Mannose-Specific Binding Proteins' Can. J. Chem. 2002, 80, 964-972.

(38) Edwards, A. S.; Wybrow, R. A. J.; Johnstone, C.; Adams, H.; Harrity, J. P. A. 'A New Approach to Functionalised Spiropiperidines through Tandem RCM and Nitrogen-Directed Reactions' Chem. Comm. 2002, 1542-1543.

(39) Agami, C.; Couty, F. 'The Reactivity of the N-Boc Protecting Group: An Underrated Feature' Tetrahedron 2002, 58, 2701-2724.

(40) Edman, P.; Högfeldt, E.; Sillén, L. G.; Kinell, P.-O. 'Method for Determination of the Amino Acid Sequence in Peptides.' Acta Chem. Scand. 1950, 4, 283-293.

(41) Jullian, M.; Hernandez, A.; Maurras, A.; Puget, K.; Amblard, M.; Martinez, J.; Subra, G. 'NTerminus FITC Labeling of Peptides on Solid Support: The Truth behind the Spacer' Tetrahedron Lett. 2009, 50, 260-263.

(42) Yang, P. Y.; Wu, H.; Lee, M. Y.; Xu, A.; Srinivasan, R.; Yao, S. Q. 'Solid-Phase Synthesis of Azidomethylene Inhibitors Targeting Cysteine Proteases' Org. Lett. 2008, 10, 1881-1884. 


\section{Chapter 4.}

\section{Highly efficient synthesis of Lewis ${ }^{\mathrm{X}}$ via a versatile late-stage crystalline trisaccharide intermediate.}

\subsection{Introduction}

The Lewis antigens are a related set of glycans and are part of glycoconjugates (i.e.glycolipids and/or glycoproteins) found in nature. They are divided into two subgroups, the Type-1 and Type-2 Lewis antigens, which are characterised by the presence of the Fuc- $\alpha-(1 \rightarrow 4)-$ GlcNAc or Fuc- $\alpha-(1 \rightarrow 3)-$ GlcNAc residues, respectively. ${ }^{1}$ In addition, several of the Lewis glycans may also be sialyated and/or sulfated (e.g. Sialyl Lewis ${ }^{\mathrm{x}}$ ). These antigens are synthesised in the tissue and subsequently distributed throughout the body via excretion of glycolipids into the plasma. ${ }^{2}$ The Type-1 antigens, also known as the Lewis blood group antigens (e.g. Lewis ${ }^{\mathrm{a}}$ and Lewis ${ }^{\mathrm{b}}$, Figure 1 ), are incorporated into erythrocytes and various tissues. ${ }^{2,3}$ The other sub-class, Type-2 antigens (i.e. Lewis ${ }^{\mathrm{X}}$, Lewis ${ }^{\mathrm{Y}}$, Sialyl Lewis ${ }^{\mathrm{X}}$, 3'-Sulfo-Lewis ${ }^{\mathrm{X}}$, 6-Sulfo-Sialyl Lewis ${ }^{\mathrm{X}}$, 6'-Sulfo-Sialyl Lewis $^{\mathrm{X}}$ and 6,6'-Sialyl Lewis ${ }^{\mathrm{X}}$ ) are serological characters widely distributed in human and animal organisms. ${ }^{2,3}$ These Lewis glycans have important roles in biology and members of both subsets of Lewis antigens have been implicated in favourable and unfavourable selectin-dependent leukocyte and cell adhesion processes ${ }^{2,3}$ with, for example, Sialyl Lewis ${ }^{\mathrm{A}}$ and Sialyl Lewis ${ }^{\mathrm{X}}$ being used as serological markers for cancer prognosis and progression, ${ }^{4-6}$ while Sialyl Lewis ${ }^{\mathrm{X}}$ also has an essential role in human fertilisation. ${ }^{7}$ Targeting the dendritic cell (DC) C-type lectin, DC-SIGN, which binds Lewis glycans (i.e. Lewis ${ }^{\mathrm{X}}$, Lewis ${ }^{\mathrm{Y}}$, Lewis ${ }^{\mathrm{b}}$ and Lewis ${ }^{\mathrm{a}}$ ), has been shown to increase antigen cross-presentation and enhance vaccine efficacy for cancer and intracellular pathogens. ${ }^{8-12}$ Accordingly, there has been much interest in targeting this lectin using antigenconjugated antibodies or antigen-conjugated Lewis glycans (e.g. Lewis ${ }^{\mathrm{X}}$ ), whereby the latter approach is thought to be advantageous due to reduced side-effects and the ease of synthesis. ${ }^{13}$ In 
this chapter the synthesis of the Lewis ${ }^{\mathrm{X}}$ ligand will be discussed, with the aim to synthesise a versatile trisaccharide intermediate, which can be used towards the synthesis of other Lewis glycans.

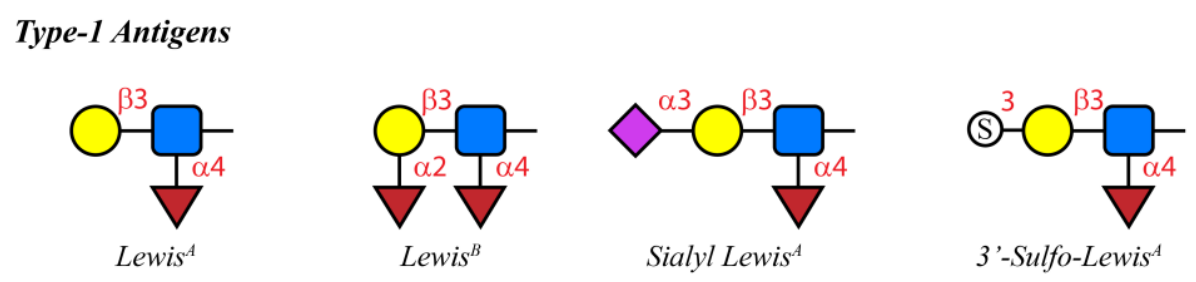

Type-2 Antigens
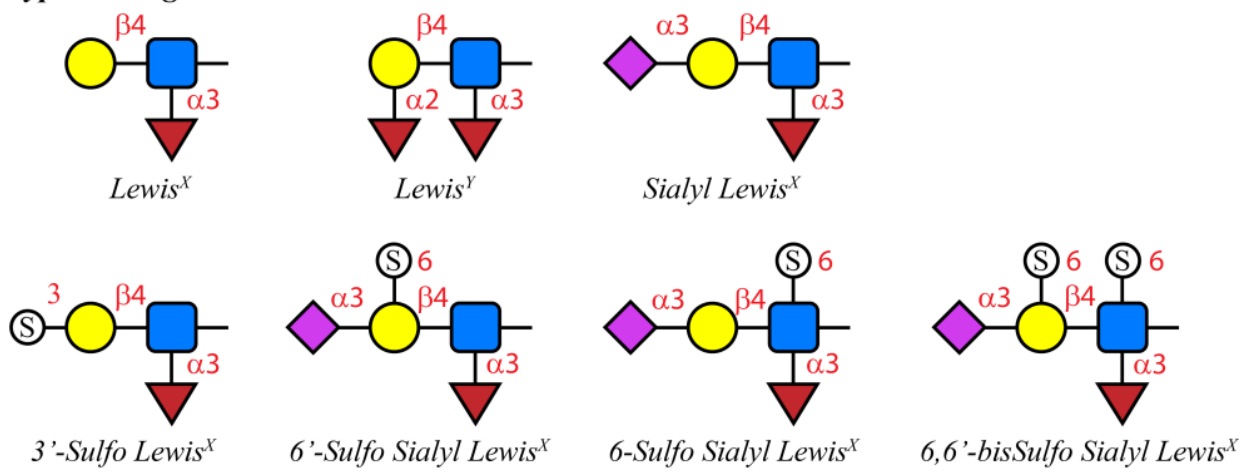

Figure 1. Schematical presentation of Type-1 and Type-2 Lewis antigens; $\bigcirc=$ Galactose; $\square=N$-Acetylglucosamine; $\nabla=$ Fucose $; \diamond=$ Sialic acid $;$ (S) sulfate .

\subsubsection{Lewis ${ }^{\mathrm{X}}$ Synthesis}

Although many of the Lewis structures are commercially available, they are rather expensive, with for example, $10 \mathrm{mg}$ of Lewis ${ }^{\mathrm{X}}$ costing $\sim 1000$ NZD. ${ }^{14}$ The availability of Lewis ${ }^{\mathrm{X}}$ at a lower cost would allow for further investigation into the biological functions of Lewis ${ }^{\mathrm{x}}$, particularly as more complex Lewis ${ }^{\mathrm{X}}$ constructs (e.g. fluorescent derivatives) can be more readily prepared. Moreover, the efficient synthesis of Lewis ${ }^{\mathrm{x}}$ would not only provide greater amounts of material, but if the synthetic route is developed with an appropriate protecting group strategy other more complex glycan ligands, such as Sialyl Lewis ${ }^{\mathrm{X}}$, Lewis ${ }^{\mathrm{Y}}$ and sulfated derivatives can also be synthesised.

The goal of this section of research is to develop an efficient and scalable route towards the synthesis of Lewis ${ }^{\mathrm{X}}$. Here, several aspects come to play, including using building blocks that can be readily prepared (i.e. preferably in a low number of synthetic steps and in a highly convergent route). To begin, however, an overview of the key strategies for the synthesis of Lewis ${ }^{\mathrm{X}}$ will be described. 


\subsubsection{Chemoenzymatic synthesis}

Lewis ${ }^{\mathrm{X}}$ has been previously synthesised chemoenzymatically using two glycosyltransferases: $\beta$-1,4-galaclosyltransferase and $\alpha$-1,3-fucosyltansferase (Scheme 1). ${ }^{15}$ Using these transferases, Lewis ${ }^{\mathrm{X}}$ glycans can be synthesised in two steps and good yield (ca 80\%). In addition, the glycan could be synthesised or elongated on the surface of the desired complex, for example, on glycodendrons, ${ }^{16}$ glyco-nanoparticles ${ }^{17}$ or glycolipids. ${ }^{15}$ Other enzymes, such as $\alpha$-2,3-sialyl transferases, can also be used to prepare more complex glycans from the Lewis ${ }^{X}$ core. On the other hand, chemoenzymatic synthesis can be costly. The required building blocks, UDP-galactose and GDP-fucose, as well as the glycosyltransferases themselves, are rather expensive and only available in small quantities. In addition, glycan complexity is restricted to the enzymes available thus limiting the diversity of the enzymatically produced antigens. Increasing the scale of the enzymatic reactions may also be problematic as enzymes can be affected by temperature, pressure, chemical environment and the concentration of substrate. Even though the use of chemoenzymatic routes is becoming more popular, the above mentioned limitations allow for more efficient chemical syntheses of complex glycans.
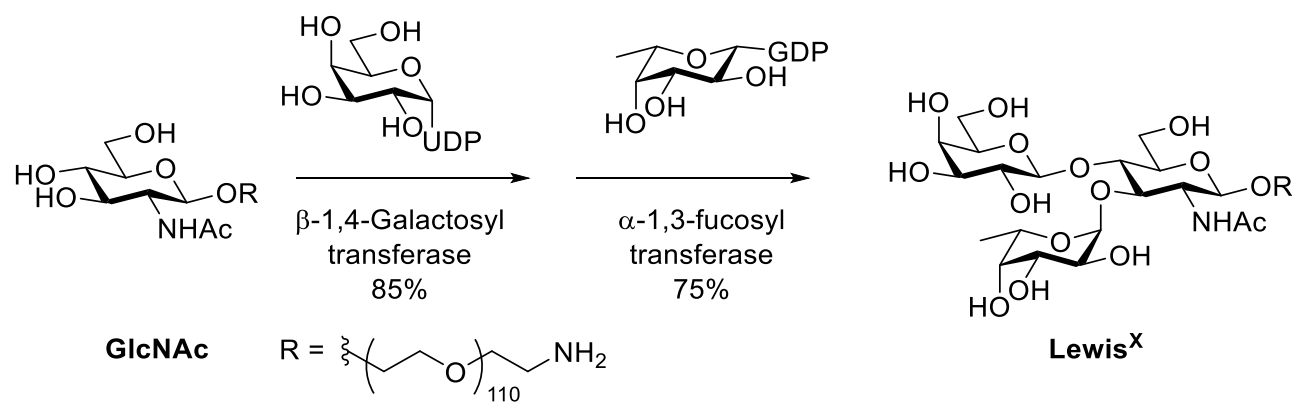

Scheme 1. The use of glycosyltransferases to synthesise Lewis ${ }^{X}$ derivative. ${ }^{18}$

\subsubsection{Chemical synthesis}

Retrosynthetically, there are three main strategies that have been used for the synthesis of Lewis ${ }^{\mathrm{X}}$ I (Scheme 2). The key differences in the routes are whether the Fuc- $\alpha-(1 \rightarrow 3)-G l c N$ II or Gal- $\beta$ $(1 \rightarrow 4)$-GlcN III backbone is synthesised first, and whether backbone II can be prepared via a regioselective glycosylation. In the first approach, "Route A", the first glycosylation reaction is between a glucosamine acceptor and a fucosyl donor to obtain the Fuc $\alpha(1 \rightarrow 3) \mathrm{GlcN}$ backbone, which upon further modification can be used as an acceptor for the glycosylation with a galactose donor to obtain the desired Lewis ${ }^{\mathrm{X}}$ structure. In all previously reported "Route A" syntheses, benzylidene protected glucosamine acceptors were used for the selective 3-O-glycosylation with a variety of fucosyl donors. The benzylidene acetal was then converted to the 6-O-benzyl protecting group, followed by glycosylation of the glucosamine 4-OH with a galactose donor. In the "Route B" strategy, the galactose donor is coupled to the 4-OH of a glucosamine acceptor, followed by protecting group manipulation and glycosylation with the fucose donor. Key in this 
approach is to temporarily protect the glucosamine 3-OH with a specific protecting group, which after galactosylation allows for selective deprotection. This route can be improved when a regioselective glycosylation is used thereby allowing for two consecutive couplings in "Route $C$ ". This section will give a brief overview on each of these different approaches towards the synthesis of Lewis ${ }^{\mathrm{X}}$.
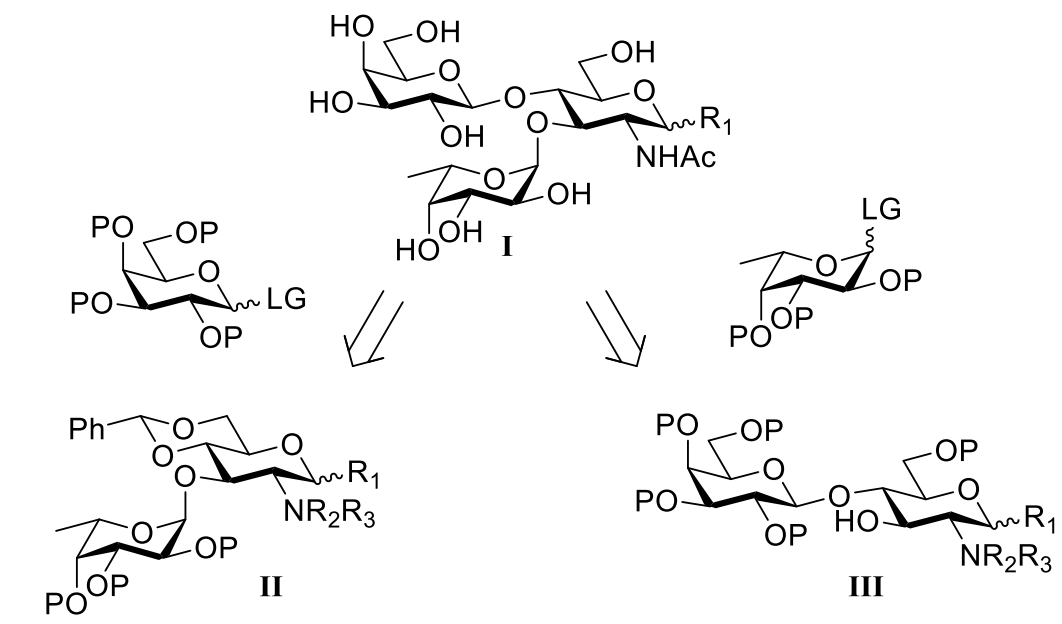

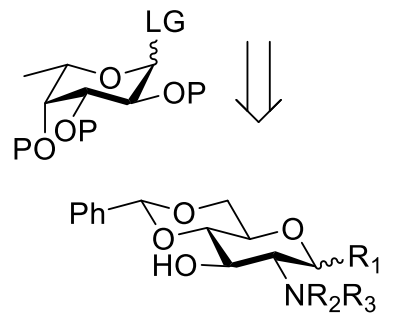

"Route A"
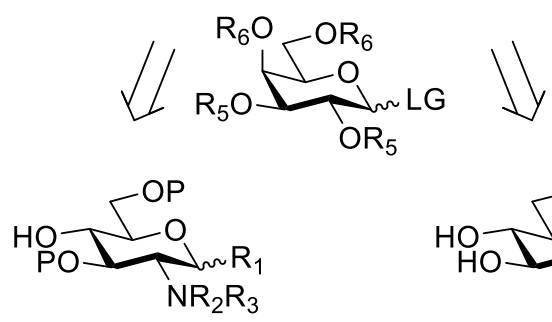

"Route B"

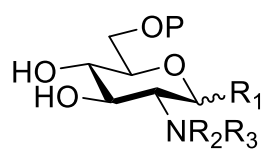

"Route C"

Scheme 2. Retrosynthetic approaches towards the synthesis of Lewis ${ }^{X}$.

\section{Route A. Lewis X synthesis via the Fuca-1,3-GlcNAc disaccharide}

In the "Route $A$ " approach, the glucosamine acceptor is coupled with a fucoside donor before glycosylation with the galactose donor en route to the synthesis Lewis ${ }^{\mathrm{x}}$. This route was first employed by Hans Lönn in 1984, whereby acceptor 1 was reacted with glycosyl bromide 2 to give disaccharide 3 in $81 \%$ with the $\alpha$-fucoside linkage (Scheme 3). ${ }^{19,20}$ Reductive ring-opening of the benzylidene acetal with trimethylamine-borane complex and aluminum trichloride then gave acceptor 4 in 66\% yield. Glycosylation of the acceptor proved sluggish and, according to the authors, could only be achieved with galactosyl bromide 5 to give protected Lewis ${ }^{\mathrm{x}} \mathbf{6}$ in $53 \%$ yield. Here, the participating $2-O$-acetate gives rise to the $\beta$-linked galactose moiety. The combined yield over these three steps was $28 \%$ from glucosamine acceptor $1 .{ }^{20}$ 


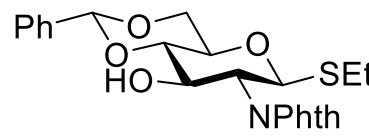

1

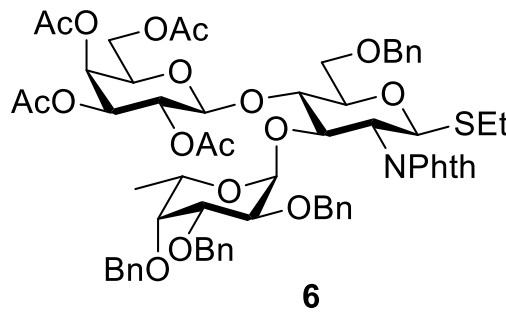

(28\% from 1)
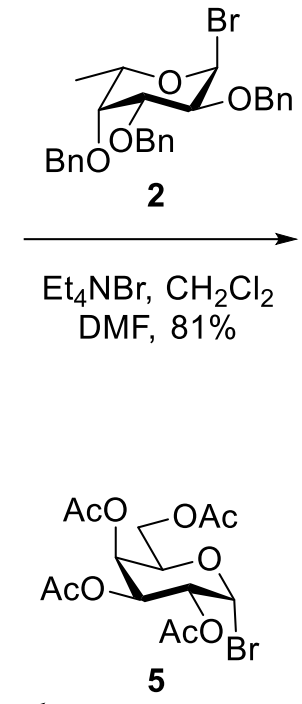

AgOTf, sym-collidine

$\mathrm{CH}_{2} \mathrm{Cl}_{2}$, Toluene $-20{ }^{\circ} \mathrm{C}, 53 \%$
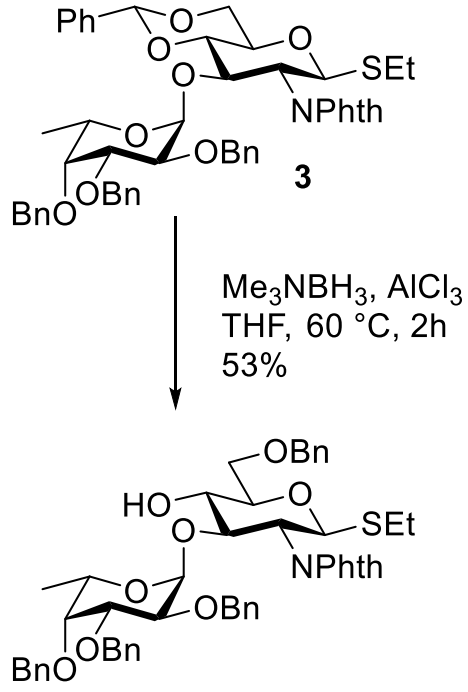

4

Scheme 3. First synthesis of Lewis ${ }^{X}$ derivative using the "Route A" approach. ${ }^{19}$

Following the seminal work of Lönn, several other groups have also used the "Route A" approach for the synthesis of Lewis ${ }^{X}$ and related glycans. In 1995, Stahl et al. successfully reacted acetamide acceptor $\mathbf{7}$ with fucosyl donor $\mathbf{8}$ using mild activation conditions to give disaccharide 9 in a good yield and $\alpha$-selectivity (Scheme 4). A subsequent regioselective reductive ring-opening of the benzylidene acetal with $\mathrm{NaCNBH}_{3} / \mathrm{HCl}$ then gave acceptor 10 in $90 \%$. Next, the acceptor was used in a glycosylation reaction with galactose donor $\mathbf{1 1}$ to give trisaccharide 12 in $75 \%$ yield. ${ }^{21}$ Here, it is interesting to note that a relatively large amount of donor (1.7 equivalents) was required to achieve sufficient coupling yields. This is probably due to the unreactive nature of the 4-OH acceptor. Nevertheless, the overall yield of 54\% over these three steps is the highest reported yield to date for the assembly of the Lewis ${ }^{\mathrm{X}}$ backbone, with the use of an $\mathrm{N}$-acetylglucosamine moiety. The use of the $\mathrm{N}$-acetyl glucosamine moiety throughout the synthesis has the obvious advantage in that it does not require late stage deprotection or conversion steps, when compared to N-protected glycans. 


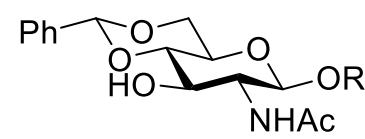

7

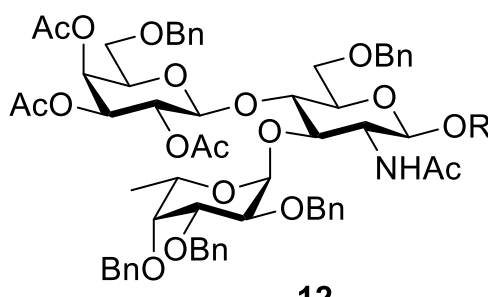

12

(54\% from 7$)$

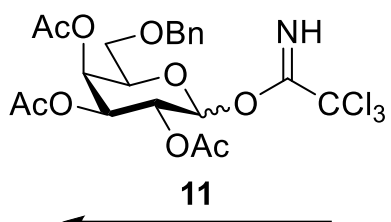

$\mathrm{BF}_{3} . \mathrm{OEt}_{2}, \mathrm{CH}_{2} \mathrm{Cl}_{2}$ $\mathrm{rt}, 4 \mathrm{~h}, 75 \%$

$\mathrm{R}=-\left(\mathrm{CH}_{2}\right)_{6} \mathrm{NHCbz}$

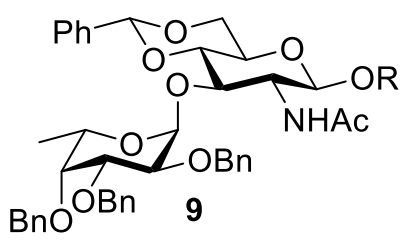

$\mathrm{NaCNBH}_{3}$ $\mathrm{HCl}$ in $\mathrm{Et}_{2} \mathrm{O}$, THF $\mathrm{rt}, 90 \%$

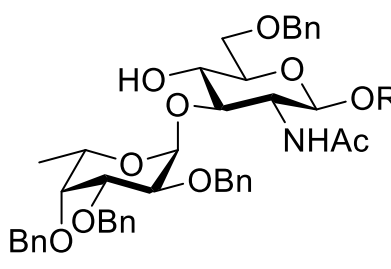

10

Scheme 4. The use of $N$-acetyl glucosamine acceptor 7. This synthesis of Lewis ${ }^{X} 12$ has the advantage that it does not require a deprotection or conversion step in the global deprotection sequence. $^{21}$

The difficulty of 4-OH glycosylation with 3-O-fucosylated glucosamine acceptors, such as $\mathbf{1 0}$ above, has also been reported by others in the synthesis of Lewis ${ }^{\mathrm{X}}$ and related structures. ${ }^{20,22-25}$ This lack of reactivity has been attributed to the steric hindrance around the glucosamine 4-OH. ${ }^{23}$ For example, while reacting highly benzylated acceptor 13 with donor 14, Hendel et al. observed that the resulting trisaccharide $\mathbf{1 5}$ could undergo degradation under acidic conditions, due to the acid lability of the armed fucosyl group, to give fucose 16 and LacNAc 17, thus resulting in low yielding glycosylation reactions (Scheme 5). ${ }^{23}$ These results have also been supported by Wang et al. who found similar degradation products in their glycosylation reactions. ${ }^{25}$ 


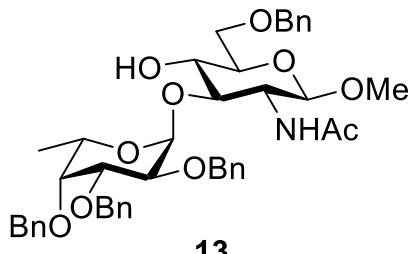

13
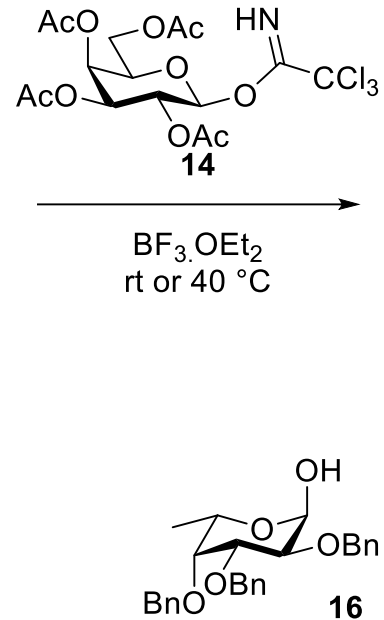

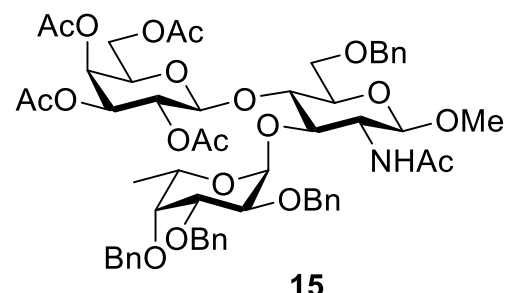

15

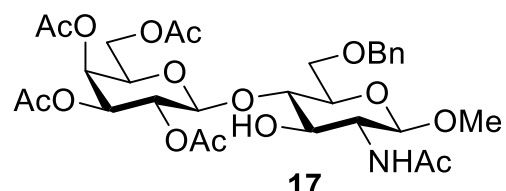

Scheme 5. Poor reactivity of disaccharide acceptor 13 and degradation of trisaccharide $\mathbf{1 5}$ results in low yielding glycosylation reactions. ${ }^{23}$

In order to solve this problem, Schmidt and co-workers reacted the less bulky and more stable, 2$O$-benzyl-3,4-di- $O$-acetyl fucose protected acceptor 18 with donor 19 to obtain Lewis ${ }^{\mathrm{X}}$ derivative 20 in a good yield of $77 \%$ (Scheme 6). ${ }^{26,27}$ This, however, requires more steps for the fucose building block synthesis and results in a less versatile trisaccharide $\mathbf{2 0}$, which complicates the synthesis of larger oligosaccharides. Global deprotection, however, resulted in the formation of methyl glycoside $\mathbf{2 1}$ in good yield.

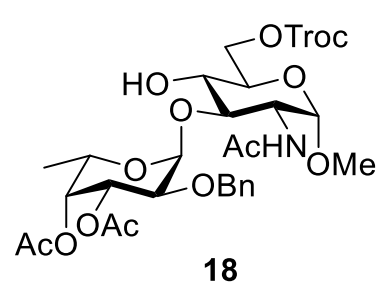

1) 19, TMSOTf, $\mathrm{CH}_{2} \mathrm{Cl}_{2}$ 2) $\mathrm{Zn}, \mathrm{THF}, \mathrm{AcOH}, 77 \%$

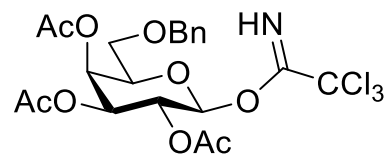

19

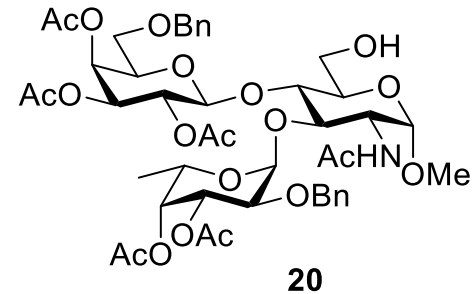

1) $\mathrm{Pd} / \mathrm{C}, \mathrm{H}_{2}, \mathrm{MeOH}, \mathrm{AcOH}$

2) $\mathrm{MeNH}_{2}, 82 \%$

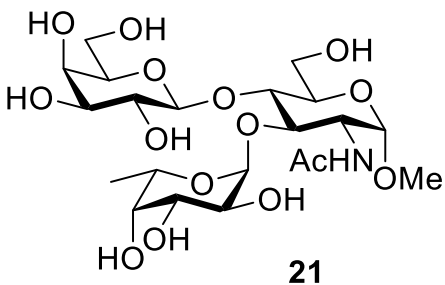

(63\% from 18$)$

Scheme 6. Utilisation of the more electron withdrawing 2-O-benzyl-3,4-di-O-acetyl protected fucose residue in acceptor 18 results in higher glycosylation yields. ${ }^{26}$ 
Finally, it should be noted that $N$-protection increases the reactivity of the glucosamine acceptor. In 2001, it was revealed by Crich and Dudkin that 4-OH of $N$-acetyl glucosamine acceptors are less reactive, compared to azide and $N$-phthalimide protected amines. ${ }^{28}$ Thus, in order to increase the reactivity of the acceptor, different nitrogen protection group strategies have been employed. For example, $N$-Troc ${ }^{29-32}$ as well as $N$-Phth ${ }^{20,24,33,34}$ protecting groups have been successfully used to slightly increase glycosylation yields en route towards glycans containing the core Lewis ${ }^{\mathrm{X}}$ motif. These protecting groups, however, are introduced at an early stage of the synthesis and require an additional deprotection step in the late stage synthesis of oligosaccharides, which can sometimes be problematic.

Interestingly, the combination of the $\mathrm{N}$-protection and the 2-O-benzyl-3,4-di- $O$-acetyl fucose protecting group strategy was used by Boons et al. in a remarkable one-pot synthesis of a variety of oligosaccharides, including a protected Lewis ${ }^{\mathrm{X}} .{ }^{35}$ Here, the glycosylation reaction of acceptor 22 and donor $\mathbf{2 3}$ was combined with the reductive opening of the benzylidene acetal and the glycosylation with galactose donor $\mathbf{2 4}$ to form protected Lewis ${ }^{\mathrm{x}} \mathbf{2 5}$ in $67 \%$ yield from acceptor 22. Indeed, this is the highest yield to date for Lewis ${ }^{\mathrm{X}}$ compounds, synthesised via "Route A".

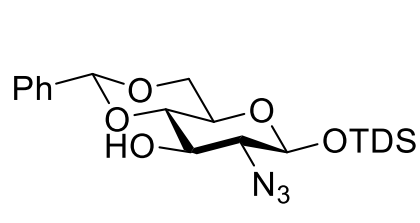

22

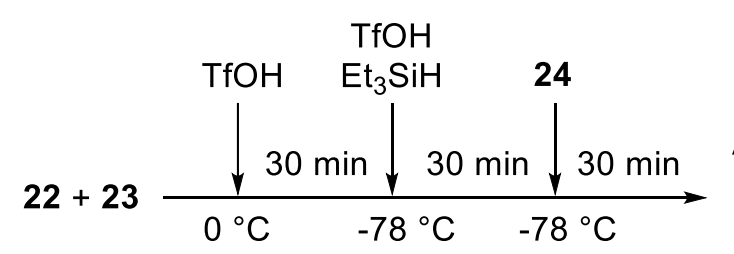

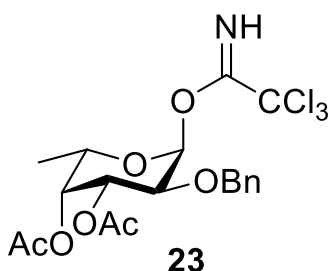

23
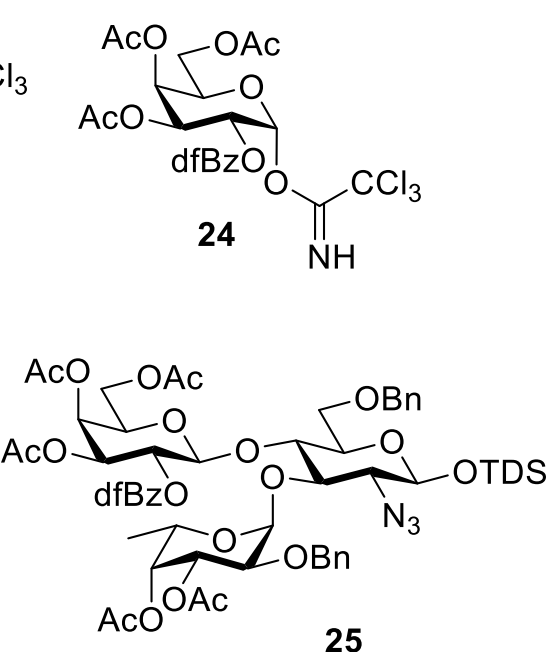

(67\% from 22)

Scheme 7. One-pot synthesis of Lewis ${ }^{X}$ oligosaccharide 25 , combining the reductive benzylidene opening with two glycosylation reactions. ${ }^{35}$

In summary, several groups have reported the synthesis of Lewis ${ }^{\mathrm{x}}$ derivatives via "Route A". Here, the use of benzylidene protected glucosamine derivatives is used to introduce the fucose moiety on the $3-\mathrm{OH}$. Next, selective ring-opening results in the disaccharide acceptor for glycosylation with galactose acceptors, while different protection strategies result in different reactivities of the acceptor and/or donor. A major challenge of this synthetic route, however, is the poor reactivity of the Fuc- $\alpha-(1 \rightarrow 3)-G l c N$ acceptor and the potential lability of the fucosyl group during the glycosylation reaction. On the contrary, other synthetic strategies such as in 
"Route $B$ " and "Route $C$ " avoid this problem by synthesising the Gal- $\beta-(1 \rightarrow 4)$-GlcNAc disaccharide first.

\section{Route B. Lewis ${ }^{X}$ synthesis via the Gal-ß-1,4-GlcNAc disaccharide}

The "Route B" approach towards the synthesis of Lewis ${ }^{\mathrm{X}}$ was first employed by Nicolaou and co-workers, who glycosylated a 3-O-allyl protected acceptor $\mathbf{2 6}$ with galactosyl fluoride donor 27, which is compatible with the thioglycoside acceptor, to obtain disaccharide $\mathbf{2 8}$ in $\mathbf{7 2 \%}$ (Scheme 8$).{ }^{36}$ Selective removal of the allyl protecting group with a ruthenium hydride catalyst then gave acceptor 29. Again, a fluoride donor $\mathbf{3 0}$ was required to obtain the protected Lewis ${ }^{\mathrm{X}}$ derivative 31, which was then used in the synthesis of an impressive trimeric $\mathrm{Le}^{\mathrm{X}}$ glycolipid. Here, the $N$-phthalamide protecting group was converted into the acetamide at a late stage in the synthesis. To avoid this late stage conversion, Nicolaou reported a slightly improved method, in which a 3-O-allyl protected $\mathrm{N}$-acetylglucosamine acceptor was used for the synthesis of Sialyl Lewis $^{\mathrm{X}} \cdot 37$

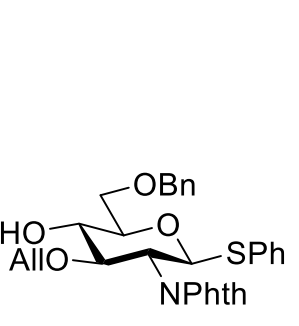

26

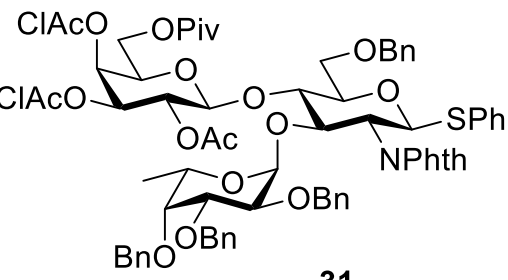

31

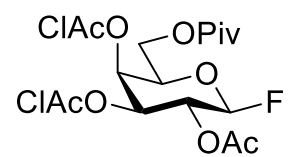

27

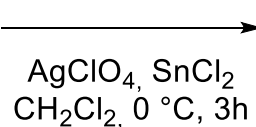

$72 \%$

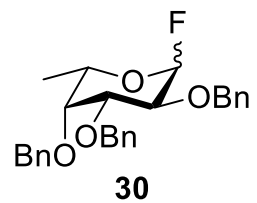

$\mathrm{AgClO}_{4}, \mathrm{SnCl}_{2}$

$\mathrm{Et}_{2} \mathrm{O},-30{ }^{\circ} \mathrm{C}, 3 \mathrm{~h}$ $87 \%$

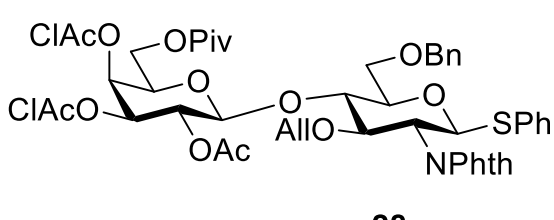

28

$\mathrm{H}_{2} \mathrm{Ru}\left(\mathrm{PPh}_{3}\right)_{4}$ EtOH, $95^{\circ} \mathrm{C}, 1.5 \mathrm{~h}$ then $\mathrm{TsOH}, \mathrm{MeOH}$ $\mathrm{rt}, 2.5 \mathrm{~h}, 86 \%$

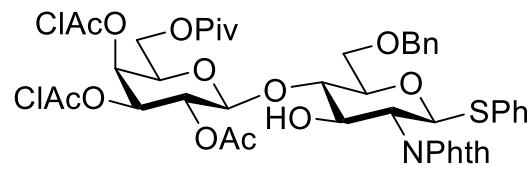

29

(54\% from 26$)$

Scheme 8. First Lewis ${ }^{X}$ derivative synthesis employing the "Route B" strategy. ${ }^{36}$

Following Nicolaou's publications, several groups have employed the "Route B" strategy using different protecting groups for the 3-hydroxyl of glucosamine, with a number of groups using a 3-O-chloroacetyl. ${ }^{38-43}$ Alternatively, carbonates ${ }^{44}$ and para-methoxybenzy ${ }^{45,46}$ protecting groups have been used as temporary protecting groups. In addition, some syntheses of Lewis ${ }^{\mathrm{X}}$ derivatives 
via "Route $B$ " involve the use of the $N$-acylated amine, while others have employed $N$-TCA ${ }^{47,48}$ or $N$-phthalimido protecting groups. ${ }^{36}$

Via these approaches, a number of different Lewis ${ }^{\mathrm{x}}$ derivatives have been prepared with overall yields ranging from 16 to $70 \%$ for the assembly of the core glycan structure (i.e. excluding the preparation of building blocks).

To date, however, the highest yield of $78 \%$ for the synthesis of Sialyl Lewis ${ }^{\mathrm{x}}$, which contains the Lewis ${ }^{\mathrm{X}}$ motif, over three steps, was reported by Seeberger and co-workers (Scheme 9). Here, trisaccharide acceptor 32, containing the 3-O-levulinoyl protecting group was coupled with donor 33 to give the pentasaccharide 34. Subsequent selective removal of the 3-O-levulinoyl protecting group with hydrazine gave acceptor $\mathbf{3 5}$, which was glycosylated with imidate donor $\mathbf{3 6}$ to give the desired hexasaccharide $\mathbf{3 7} \cdot .^{47,48}$

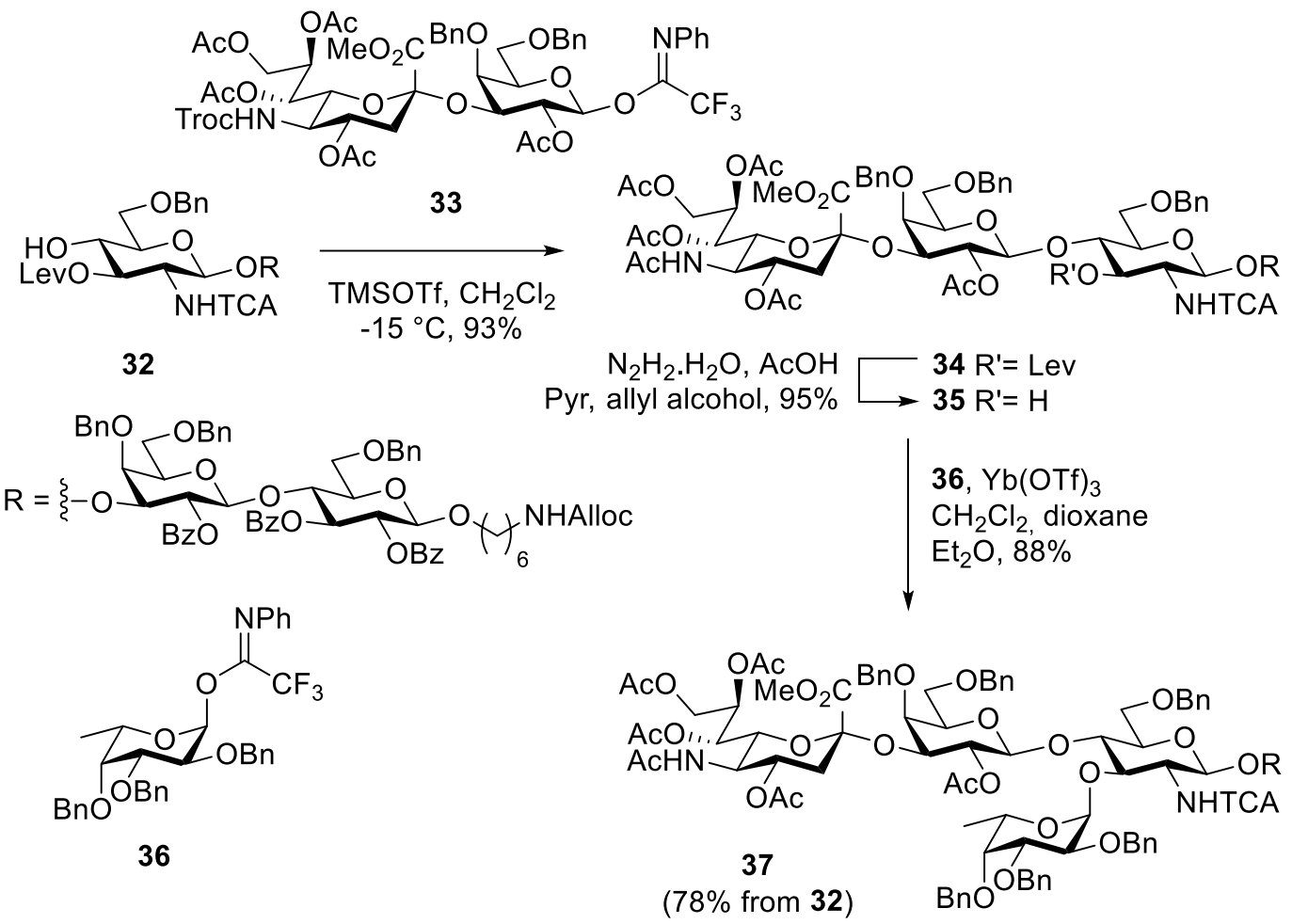

Scheme 9. Most efficient synthesis of a Sialyl Lewis ${ }^{X}$ derivative by Seeberger and co-workers, employing the "Route B" approach with a 3-O-Lev protecting group. ${ }^{47}$

In contrast to "Route A", this synthetic approach allows for the synthesis of the linear saccharides, followed by fucosylation to obtain Lewis antigens. In comparison "Route $B$ " has been shown to be slightly higher yielding, which could be explaned by the use of less reactive disaccharide acceptors employed in "Route A". Although both routes have successfully been employed in Lewis ${ }^{\mathrm{x}}$ syntheses, "Route $C$ " has particular merit since it allows for more straightforward glucosamine building block syntheses. In addition, two consecutive glycosylation reactions are used to obtain Lewis ${ }^{\mathrm{X}}$ derivatives, which thus allows for a rapid assembly of the Lewis ${ }^{\mathrm{X}}$ backbone. 


\section{Route C. Regioselective glycosylation towards LacNAc derivatives.}

The first regioselective glycosylation en route to Lewis ${ }^{\mathrm{X}}$ was reported in 1996, by Roy and co-workers (Scheme 10). ${ }^{49} \mathrm{Here}$, the 6-O-(tert-butyl diphenylsilyl) protected azido-GlcNAc acceptor $\mathbf{3 8}$ was successfully used in glycosylation with thioglycoside donor $\mathbf{3 9}$ to give the target disaccharide in $82 \%$ yield. It was suggested that the bulky $6-O$-silane protecting group blocked the top side of the GlcNAc, thereby favouring the regioselective glycosylation of the 4-hydroxyl. The resulting disaccharide $\mathbf{4 0}$ was then used in a glycosylation reaction with fucosyl donor $\mathbf{8}$ to give the protected Lewis ${ }^{\mathrm{X}}$ trisaccharide $\mathbf{4 1}$ in $64 \%$ yield over two steps. To date, this is the highest reported yield over the two glycosylations, while using an $\mathrm{N}$-acetylglucosamine acceptor.

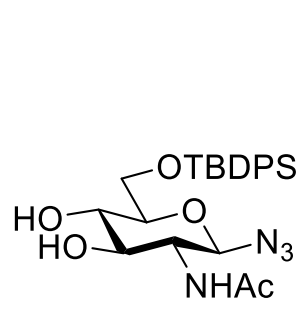

38

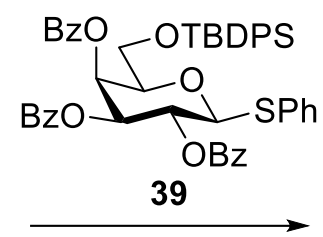

$\mathrm{NIS}, \mathrm{TfOH}$ $\mathrm{CH}_{2} \mathrm{Cl}_{2}, \mathrm{MeCN}$ $-45{ }^{\circ} \mathrm{C}, 82 \%$
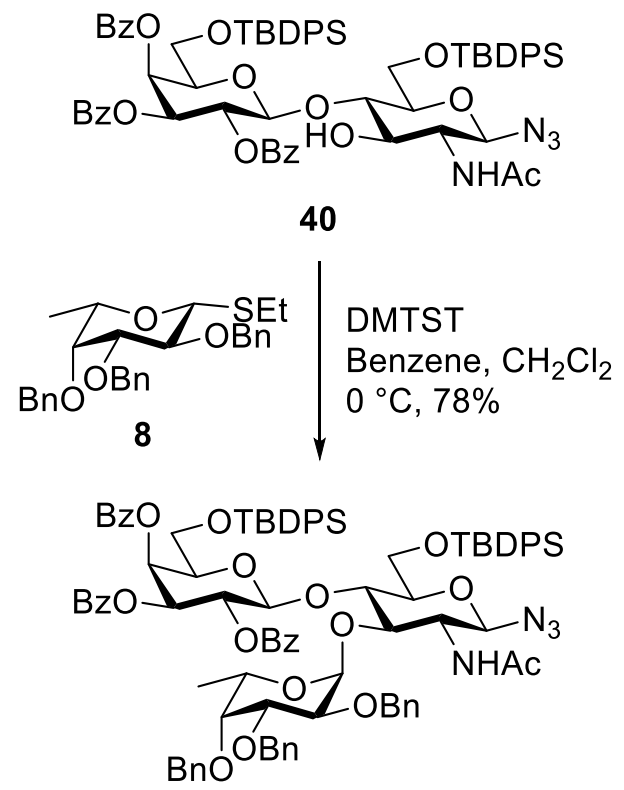

41

(64\% from 38)

Scheme 10. Regioselective glycosylation of 3,4-dihydroxyl glucosamine acceptor 38 with glycosyl donor 39. The resulting disaccharide acceptor $\mathbf{4 0}$ is then reacted with fucose donor 8 to obtain Lewis ${ }^{X}$ derivative $41 .^{49}$

Since then, "Route $C$ " has been used by numerous research groups who made slight modifications to either the donor or the acceptor en route to the synthesis of Lewis ${ }^{\mathrm{X}}$ and related structures. ${ }^{49-65}$ For example, the use of the $\mathrm{N}$-phthalimide protecting group has been used to increase the yields in glycosylation reactions. Here, $\mathrm{N}$-phthalimide acceptor $\mathbf{4 2}$ was glycosylated with fluoride donor 43 to give disaccharide 44 , which in turn was glycosylated with fucoside donor 8 to give protected Lewis ${ }^{\mathrm{X}} \mathbf{4 5}$ in $68 \%$ yield over the two steps (Scheme 11). ${ }^{50}$ Other examples include the use of 
"Route $C$ " for the automated one-pot synthesis of a Lewis ${ }^{\mathrm{x}}$ thioglycoside by Huang and coworkers in $68-71 \%$ yield. ${ }^{51}$ This work builds on earlier work by Tanaka et al. for the synthesis of dimeric and trimeric Lewis ${ }^{\mathrm{X}}{ }^{52,53}$ Although the use of automated one-pot strategies allows for rapid assembly of the Lewis ${ }^{\mathrm{X}}$ derivatives, relatively large amounts (5 equivalents) of donors are required to obtain high yields. In addition, the building blocks used require extensive protecting group manipulation.
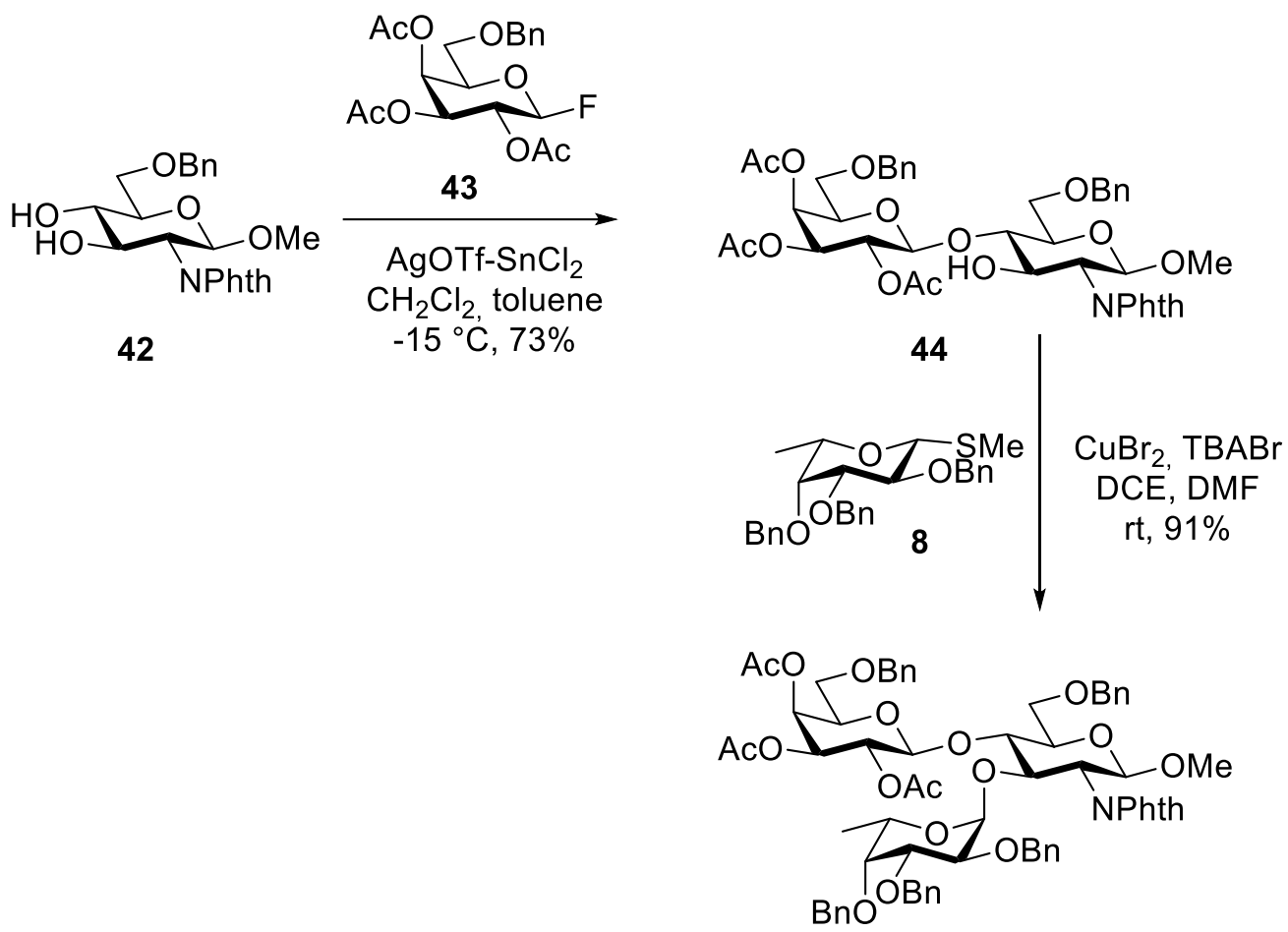

45

(68\% from 42)

Scheme 11. The use of the N-phthalimide protecting group in the synthesis of Lewis ${ }^{X}$ derivative 45. ${ }^{50}$

To date, the highest yield (80\%) in the regioselective glycosylation reactions, was reported by Cao et al. for the assembly of Sialyl Lewis ${ }^{\mathrm{X}} 46$ (Scheme 12). ${ }^{54}$ Here, diol acceptor 47 was glycosylated with Sia- $\alpha(2 \rightarrow 3)$ Gal donor 48 to give trisaccharide $\mathbf{4 9}$ in $87 \%$, which in turn was reacted with thiofucoside $\mathbf{5 0}$ to form Sialyl Lewis ${ }^{\mathrm{X}} \mathbf{4 6}$ in $92 \%$ yield. Even though this route allows for the construction of protected Lewis ${ }^{\mathrm{X}}$ derivatives in high yields, a late stage conversion of the $N$-phthalimide into the acetamide is required, which ultimately increases the number of steps in the final stages of the synthesis of these complex antigens. Moreover, this route also does not allow for the synthesis of other Lewis glycans. 


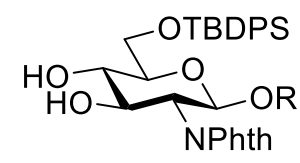

47

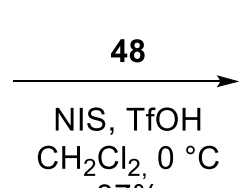

$87 \%$
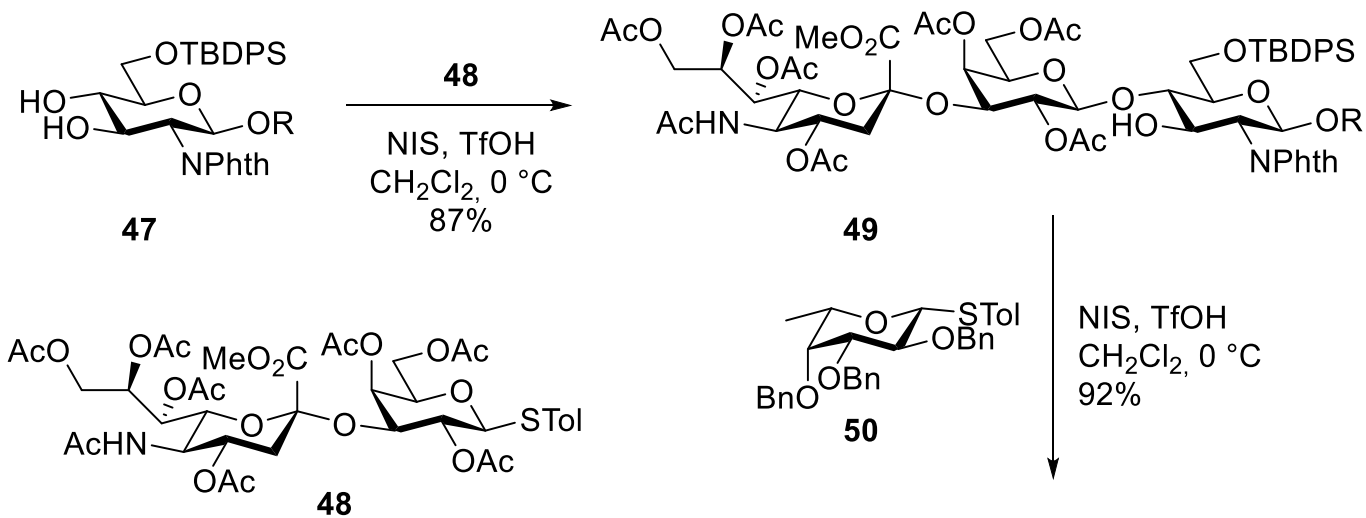

49

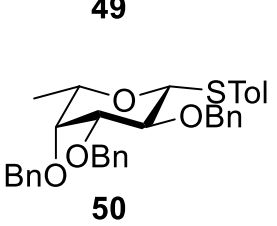

NIS, TfOH

$\mathrm{CH}_{2} \mathrm{Cl}_{2}, 0^{\circ} \mathrm{C}$ $92 \%$<smiles>[R][Y](=O)CCCCN</smiles>

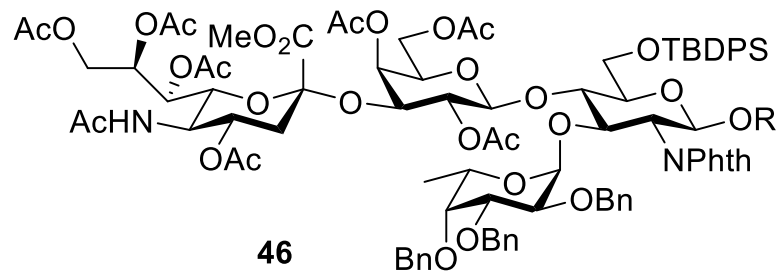

$(80 \%$ from 47$)$

Scheme 12. Highest yielding glycosylation sequence for the synthesis of Sialyl Lewis ${ }^{X} \mathbf{6}$ by Cao et al. ${ }^{54}$

\subsubsection{Building block syntheses and glycosylation strategies}

As described above, many different glycosyl donors and acceptors can be used for the synthesis of Lewis ${ }^{X}$ and related structures. Here, the protecting group strategy of the glucosamine acceptor dictates the route by which Lewis ${ }^{\mathrm{X}}$ can be synthesised. For example, when $N$-acetylglucosamine is protected at the anomeric position, and a 4,6-O-benzylidene acetal is installed, the acceptor is suitable for the "Route $A$ " approach. Instead of a glycosylation reaction, a 3-O-protecting group could be installed, followed by the reductive opening of the benzylidene acetal to give an acceptor suitable for "Route B". The acceptor for "Route C", however, can be obtained more efficiently, by protecting the anomeric position, as well as the primary 6-OH of $\mathrm{N}$-acetylglucosamine in two steps. The glycosyl donors, however, do not differ between the different synthetic strategies, and thus similar donors were used throughout all routes.

In order to obtain the $\alpha$-fucoside, a non-participating protecting group at the 2-hydroxyl is required, and thus per-benzylated donors are often used. However, these armed fucosides can be more prone to hydrolysis, and thus other protecting groups (e.g. acetates) for the 3- and 4hydroxyls of the carbohydrate have been used. ${ }^{35,55,56}$ Alternatively, a fucose donor with TBDMS protecting groups at the 2-, 3- and 4-position was used en route to protected Lewis ${ }^{\mathrm{X}}$ glycans, however, these bulky protecting groups resulted in a low-yielding (24\%) glycosylation reaction. ${ }^{57}$ Alternatively, the less-bulky triethylsilane (TES) protected fucose donor was employed by Kopitzki et al., however, a 1:1 $\alpha$ : $\beta$-ratio was observed in the glycosylation reaction. ${ }^{58}$ The activation method of the fucoside donor depends on the type of glycoside, for example thioethyl fucoside donors can be activated with DMTST, ${ }^{49}$ NIS/TfOH, ${ }^{59}$ or more mildly with 
$\mathrm{Cu}(\mathrm{Br})_{2} / \mathrm{TPABr}^{66}$ Alternatively, imidate donors, ${ }^{47,55}$ or bromide/fluoride donors ${ }^{60}$ can be activated in the presence of a thioglycoside acceptor, thereby allowing for the use of glucosamine donors. ${ }^{20,34,36,51,61}$ Here, however, it is interesting to note that the halide and imidate donors are often obtained from a fucosyl thioglycoside donor, which increases the number of steps to synthesise the donor, and thus lowers the overall efficiency of the route.

To introduce the $\beta$-galactose moiety in Lewis ${ }^{\mathrm{x}}$, a participating 2-O-protecting group on the galactose donor is required. Accordingly, benzoyl and acetate protecting groups at this position have been employed. The protecting group strategy for the 3-, 4- and 6-OH differs between the Lewis ${ }^{\mathrm{X}}$ glycan syntheses and depends on the desired functionality. For example, per-acetylated thioglycoside donors can be obtained in 2 steps from galactose, and thus allow for efficient donor synthesis. Alternatively, 4,6-O-benzylidene acetal donors have been used to obtain an orthogonally protected product that more readily allows for the synthesis of other Lewis ${ }^{\mathrm{x}}$ glycans. In addition, $\operatorname{Sia} \alpha(2 \rightarrow 3) \mathrm{Gal}$ disaccharide donors have been used to obtain Sialyl Lewis ${ }^{\mathrm{X}}$ structures, ${ }^{27,29,42,43,45,47,54,56,62}$ though again, this restricts the type of Lewis glycan that can be prepared.

\subsubsection{Deprotection strategies}

Many synthetic routes appear to have focussed on the assembly of the backbone of desired Lewis ${ }^{\mathrm{X}}$ glycans and have omitted their global deprotection strategies, even though these late stage reactions can be tedious and difficult to optimise. Although each protected oligosaccharide requires a unique deprotection sequence, depending on the protecting groups used, a few relevant examples are given.

In 2008, Cao et al. reported the global deprotection of Sialyl Lewis ${ }^{\mathrm{x}} \mathbf{5 1}$ where the silyl protecting group was removed with HF.pyridine, followed by the conversion of the $N$-phthalimide to the acetamide (Scheme 13, A).$^{54}$ Here, the phthalimide was reacted with hydrazine.hydrate in methanol under reflux conditions to give the deacetylated glycosylamine, which in turn was peracetylated. Next, azide reduction with 1,3-propanedithiol, deacetylation under Zemplén conditions and a $\mathrm{Pd}(\mathrm{OH})_{2}$ mediated hydrogenation gave tetrasaccharide $\mathbf{5 2}$ in 35\% over 6 steps. More recently, this deprotection sequence was improved by Lu et al. who reported a two-step deacetylation and phthalimide conversion in $88 \%$ yield, followed by the quantitative conversion of trisaccharide 53 into Lewis ${ }^{\mathrm{X}} \mathbf{5 4}$ (Scheme 13, B). ${ }^{63}$ This deprotection sequence features a hydrazine deacetylation/phthalimide removal, followed by selective $N$-acetylation and a hydrogenation with $\mathrm{Pd} / \mathrm{C}$ in THF: $\mathrm{H}_{2} \mathrm{O}: \mathrm{AcOH}$ under 1 atm. hydrogen. 
A)

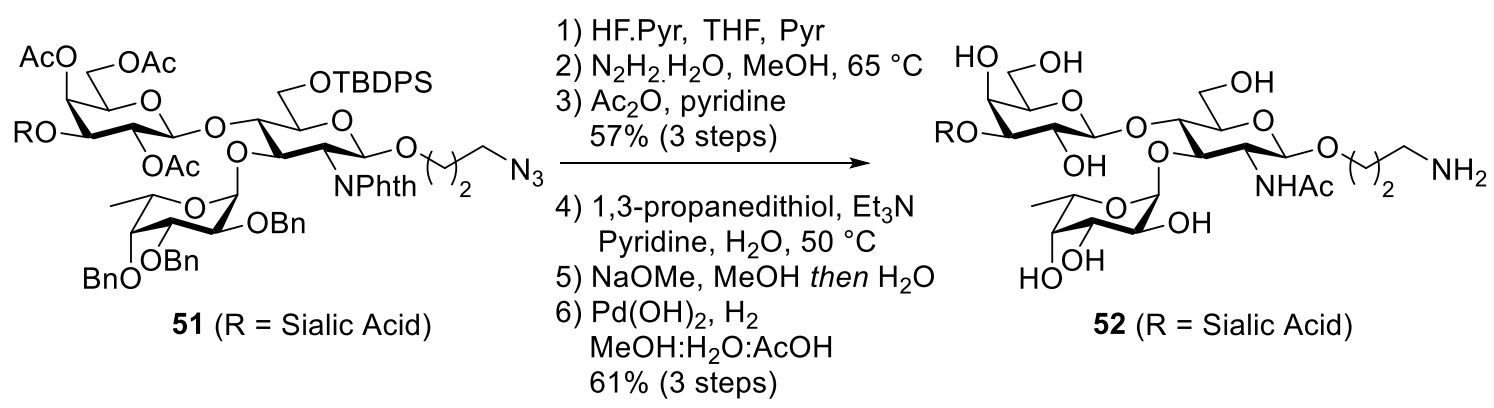

B)

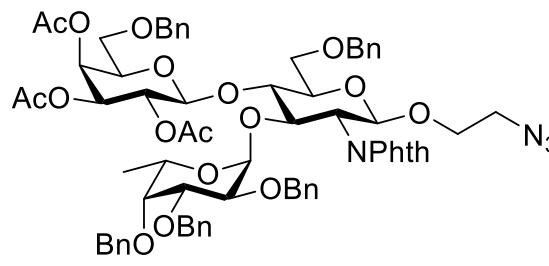

53

C) $\mathrm{Ph}$

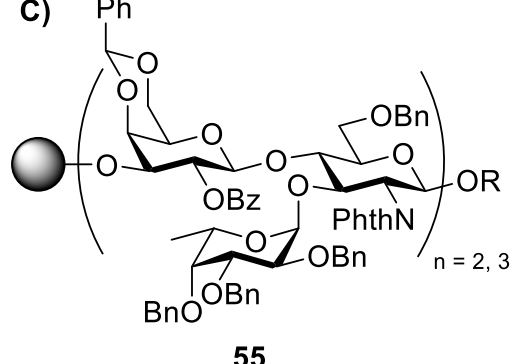

55
1) $\mathrm{N}_{2} \mathrm{H}_{2}$, $\mathrm{EtOH}, \mathrm{H}_{2} \mathrm{O}$

2) $\mathrm{Ac}_{2} \mathrm{O}, \mathrm{MeOH}, \mathrm{CH}_{2} \mathrm{Cl}_{2}$ $88 \%$ (2 steps)
3) $\mathrm{Pd} / \mathrm{C}, \mathrm{H}_{2}$ THF: $\mathrm{H}_{2} \mathrm{O}: \mathrm{AcOH} 4: 2: 1$ quant

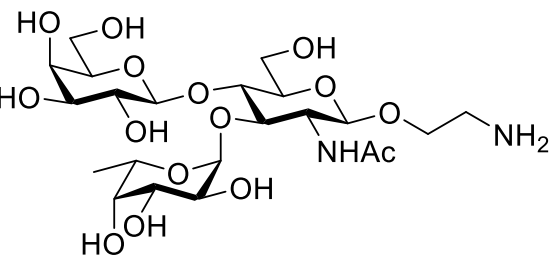

54

= Solid Phase Resin

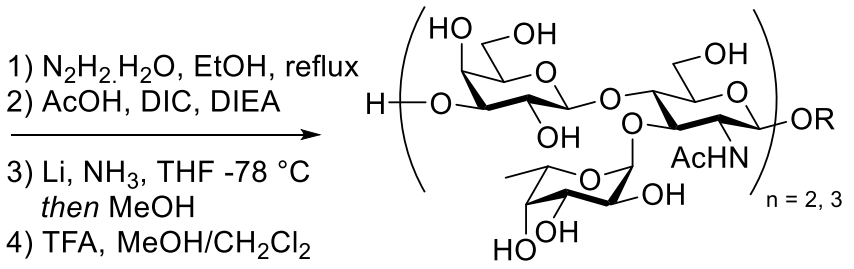

56

Scheme 13. Global deprotection strategies to obtain Lewis ${ }^{X}$ derivatives. ${ }^{53,54,63}$

Alternatively, a Birch reaction can be employed for the global deprotection of oligosaccharides. For example, dimeric and trimeric Lewis ${ }^{\mathrm{X}}$ structures $\mathbf{5 5}$ were fully deprotected in a 4-step sequence (Scheme 13, C). ${ }^{53}$ First, the phthalimide was converted to the acetamide in two steps. Next, a Birch reaction removed all benzyl and benzylidene acetal protecting groups. When the Birch reaction was quenched with methanol, the formation of $\mathrm{NaOMe}$ resulted in benzoate deprotection. Finally, cleavage of the resin with TFA, $\mathrm{MeOH} / \mathrm{CH}_{2} \mathrm{Cl}_{2}$ resulted in the formation of dimeric and trimeric Lewis ${ }^{\mathrm{x}} \mathbf{5 6}$. 


\subsubsection{Retrosynthesis of Lewis ${ }^{X}$}

The main goal in this chapter is to develop an efficient synthesis for Lewis ${ }^{\mathrm{X}}$ and other Lewis antigens. As described above, three general synthetic routes have been reported to synthesise the Lewis ${ }^{\mathrm{x}}$ antigen. Here, "Route $C$ " is the most efficient strategy, employing a regioselective glycosylation reaction, followed by fucosylation to obtain Lewis ${ }^{\mathrm{x}}$ derivatives. This route allows for more efficient acceptor synthesis compared to other routes. In addition, the two consecutive glycosylations are high yielding and do not require an extra deprotection step. Thus, it was envisioned that the Lewis ${ }^{\mathrm{X}}$ trisaccharide $\mathbf{6 7}$ could be synthesised via global deprotection of the protected trisaccharide 68 (Scheme 14). Trisaccharide 68 can in turn be obtained via an initial glycosylation of $\mathrm{N}$-acetylglucosamine diol acceptor 69 with galactosyl donor 70, followed by the coupling with fucose donor 71. Here, the thioglycoside donors were chosen as they are more stable (i.e. increase shelf life) and easily prepared, compared to glycosyl imidates or halides. All three monosaccharide building blocks were previously synthesised from the corresponding monosaccharides $N$-acetyl-D-glucosamine, ${ }^{67}$ D-galactose ${ }^{68,69}$ and L-fucose. ${ }^{70}$

Herein, it should be noted that the protected Lewis ${ }^{\mathrm{X}} \mathbf{6 8}$ is a versatile building block for the synthesis of the other Lewis glycans. For example, removal of the benzoates, followed by a regioselective glycosylation with a sialic acid building block, allows for rapid access to a protected Sialyl Lewis ${ }^{\mathrm{x}}{ }^{37,71}$ Alternatively, sulfonation of the 3 '-hydroxyl results in the $3^{\text {' } \text {-sulfo Lewis }}{ }^{\mathrm{X}}$ derivative. ${ }^{21}$ On the other hand, removal of the benzylidene acetal, followed by sulfonation gives 6 '-sulfo Lewis ${ }^{\mathrm{X}}$ motifs. ${ }^{72}$ Protecting group manipulation to obtain the galactose 2-OH acceptor allows for the synthesis of Lewis ${ }^{\mathrm{Y}}$ glycans. ${ }^{34}$ Derivatisation of the glucosamine 6position, can be achieved after removal of the silane protecting group, and allows for the assembly of 6-sulfo Lewis structures. ${ }^{72}$ Thus, all seven type-2 Lewis structures can be obtained using trisaccharide 68.

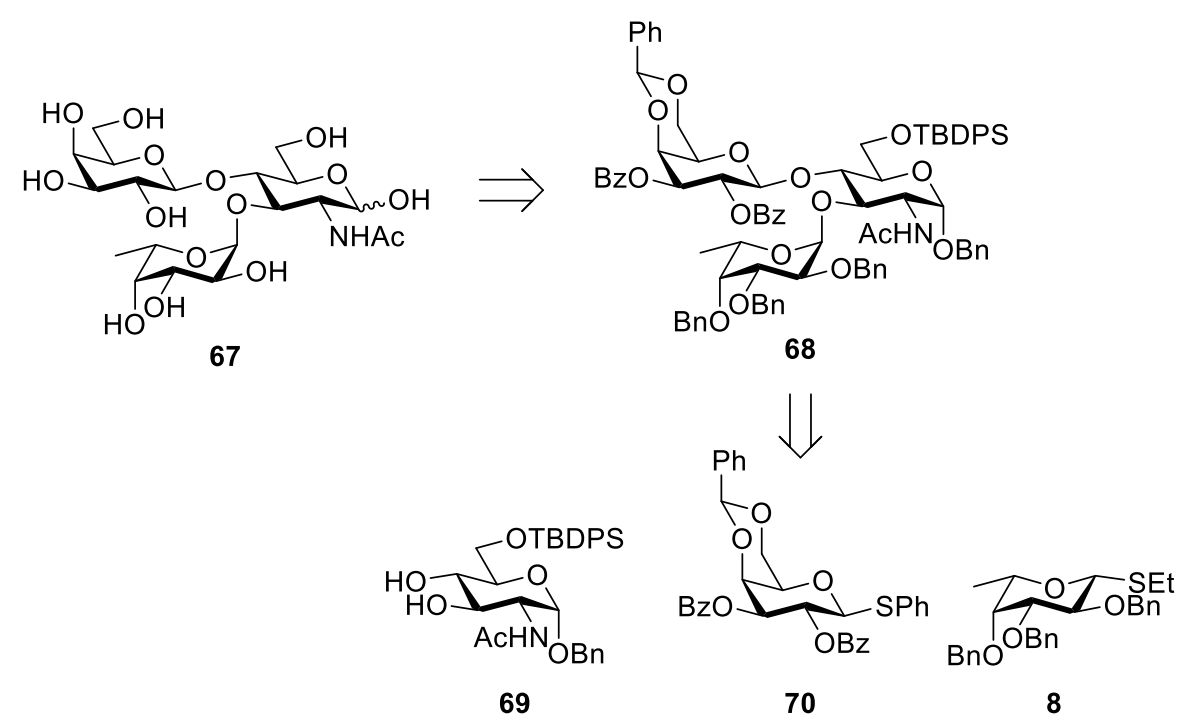

Scheme 14. Retrosynthesis of Lewis ${ }^{X} 67$. 


\subsection{Results and discussion}

\subsubsection{Synthesis of Lewis ${ }^{\mathrm{X}}$ building blocks}

The glucosamine acceptor 69 was synthesised in two steps, starting with a Fischer glycosylation of benzyl alcohol with $N$-acetyl glucosamine (71) (Scheme 15). This reaction was performed on a 6.5 gram scale to obtain the $\alpha$-benzyl glycoside $\mathbf{7 2}$, which was isolated in $74 \%$ yield. ${ }^{73,74}$ The benzyl glycoside was then used in the selective silylation reaction with TBDPS-Cl and DMAP in pyridine to obtain acceptor $69 .{ }^{67}$ In our hands, the reaction was sluggish at room temperature due to the insolubility of the sugar moiety, but by increasing the temperature of the reaction to $40{ }^{\circ} \mathrm{C}$, a smooth conversion of the triol into diol-acceptor 69 was achieved.

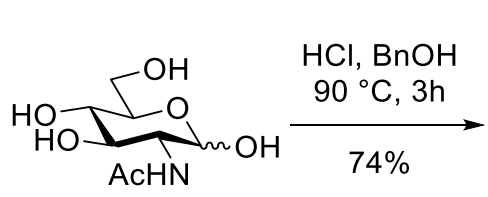

71

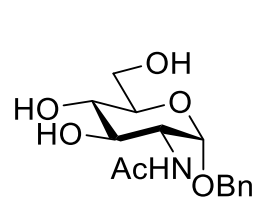

72

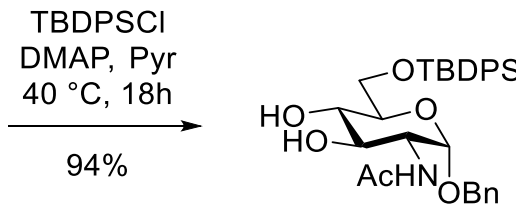

69

Scheme 15. Two step synthesis of N-acetyl glucosamine acceptor 69.

The galactose donor $\mathbf{7 0}$ was synthesised in 5 steps, on a multi-gram scale, starting from D-galactose (73). ${ }^{68,75}$ Here, peracetylation of galactose gave pentaacetate $\mathbf{7 4}$ that was used without purification. Activation of the anomeric acetate in $\mathbf{7 4}$ with $\mathrm{SnCl}_{4}$ in the presence of thiophenol gave thioglycoside 75, which was treated with $\mathrm{NaOMe}$ to give the tetraol $\mathbf{7 6} .^{68} \mathrm{Next}$, the $4,6-O$ benzylidene protecting group was installed using benzaldehyde dimethyl acetal and pTsOH in acetonitrile at $40{ }^{\circ} \mathrm{C}$ to give diol 77. ${ }^{68}$ The diol was then treated with benzoyl chloride in pyridine to give the desired crystalline donor $\mathbf{7 0}$ in good yield. ${ }^{69}$ Here, the ability of the benzoyl protecting group to participate is required for $\beta$-selective glycosylation with acceptor 69.

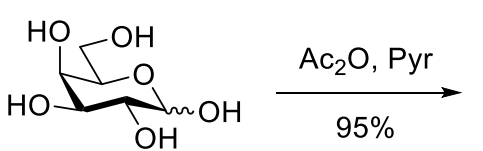

73

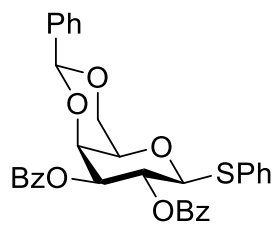

70

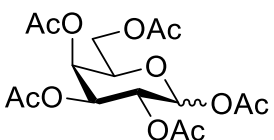

74

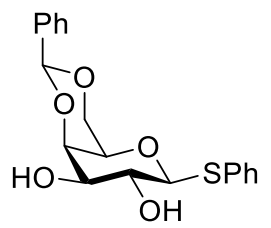

77

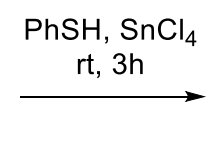

$\mathrm{PhCH}(\mathrm{OMe})_{2}$

pTsOH, MeCN

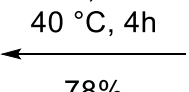

$78 \%$

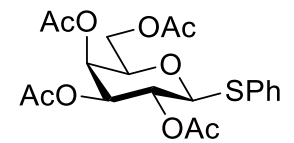

75

$\left.\begin{array}{r}\mathrm{NaOMe}, \mathrm{MeOH} \\ \mathrm{rt}, 4 \mathrm{~h}\end{array}\right\rfloor 82 \%($ from 74)

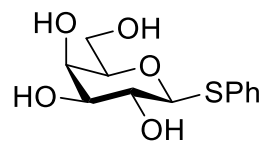

76

Scheme 16. Synthesis of galactose building block 70. 
The third building block, fucose donor $\mathbf{8}$, was synthesised in five steps from L-fucose (78) (Scheme 17). Here, acetylation gave fucoside 79, which was reacted with $\mathrm{HBr}$ to give bromide 80. Next, the bromide was treated with ethanethiol and sodium hydride to give thioglycoside $\mathbf{8 1}$, which was deacetylated to give triol 82 in $68 \%$ over four steps. In order to obtain the $\alpha$-fucopyranoside during glycosylation, a non-participating protecting group was required at the 2-position. To this end, triol $\mathbf{8 2}$ was perbenzylated using benzyl bromide, TBAI and sodium hydride to give thioglycoside 8 in $82 \%$ yield. ${ }^{76}$ This allowed for a highly efficient synthesis of the fucose donor, although it should be noted that others have found such highly armed fucose donors to be labile during glycosylation reaction, as previously described. ${ }^{23}$

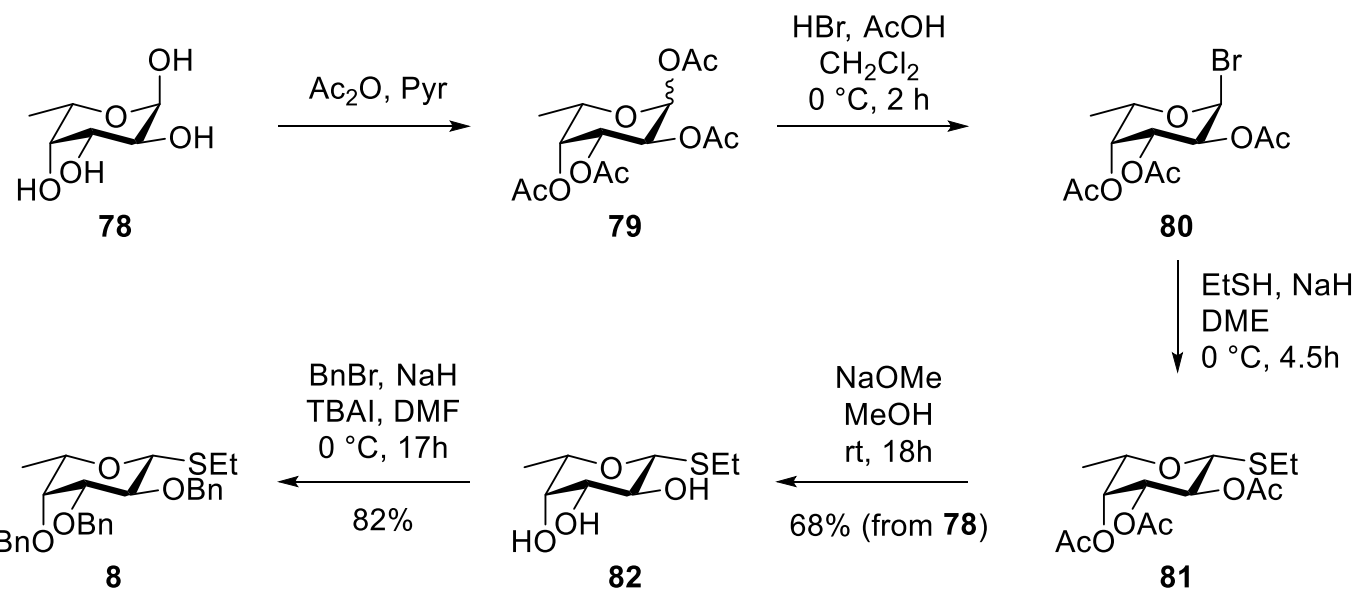

Scheme 17. Building block synthesis fucose donor 8 .

\subsubsection{Glycosylation reactions}

After the synthesis of the necessary building blocks, the assembly of the Lewis ${ }^{\mathrm{X}}$ trisaccharide backbone started with the regioselective glycosylation of glucosamine acceptor 69 with thioglycoside donor 70 to give disaccharide 83 (Scheme 18). In trial reactions on small scale, it was found that slow addition of the donor (1.2 equivalents) to a mixture of the acceptor, NIS and $\mathrm{TfOH}$ at $-50{ }^{\circ} \mathrm{C}$ resulted in a high yielding $(74 \%)$ regioselective glycosylation. It was also observed that when the reaction was performed at higher temperatures (or allowed to warm to 0 ${ }^{\circ} \mathrm{C}$ ) small amounts of the over-glycosylated trimer were formed. Given that the acceptor is synthesised in fewer steps than the donor, in subsequent gram scale couplings, the acceptor was used in slight excess. 


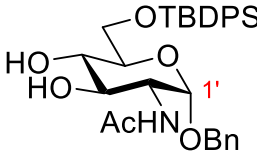

69

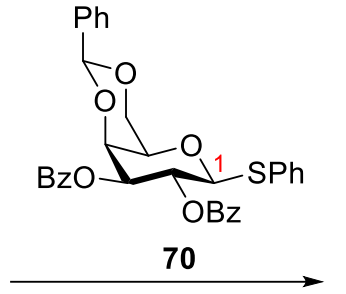

1.1 eq TfOH, NIS, $\mathrm{CH}_{2} \mathrm{Cl}_{2}$ $-50{ }^{\circ} \mathrm{C}, 15 \mathrm{~min}$ then, $70, \mathrm{CH}_{2} \mathrm{Cl}_{2}$ $-50{ }^{\circ} \mathrm{C}, 1 \mathrm{~h}, 78 \%$

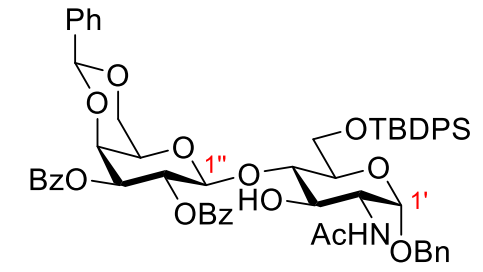

83
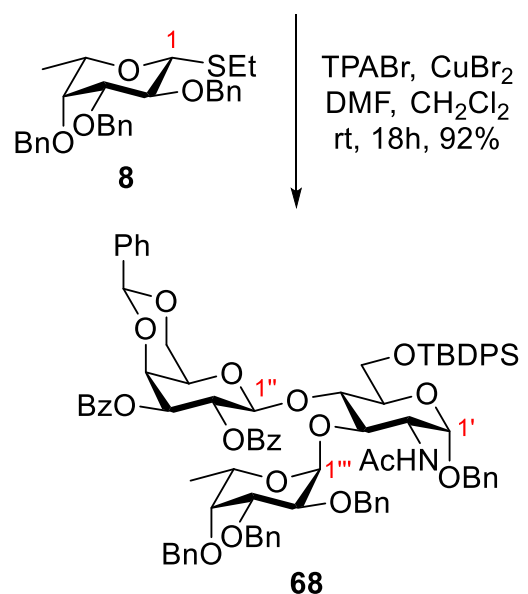

Scheme 18. Glycosylation reactions to obtain protected Lewis ${ }^{X} \mathbf{6 8}$.

The best yield (78\%) on a gram scale was obtained when TfOH was added to a mixture of NIS and a slight excess of GlcNAc acceptor 69 (1.1 equiv.) at $-50{ }^{\circ} \mathrm{C}$, prior to the addition of galactosyl donor 70 (1 equiv.). The resulting disaccharide 83 was purified by silica gel flash column chromatography and was obtained as the desired $\beta$-pyranoside, whereas the $\alpha$-anomer was not observed.

The configuration at the anomeric centre was confirmed by NMR analysis, which revealed a $J_{1^{\prime \prime}, 2^{\prime \prime}}$ coupling of $8.2 \mathrm{~Hz}$ while an $\mathrm{HMBC}$ between $\mathrm{H}-1$ " and $\mathrm{C}-5$ " confirmed the pyranose configuration. A combination of 1D- and 2D-NMR analysis was used to assign all proton and carbon resonances, and for example, the regioselectivity of this method was confirmed using HMBC NMR analysis, in which correlations between $\mathrm{H}-1^{\prime \prime}$ and C-4', as well between $\mathrm{H}-4^{\prime}$ and C-1" were observed (Figure 2). 


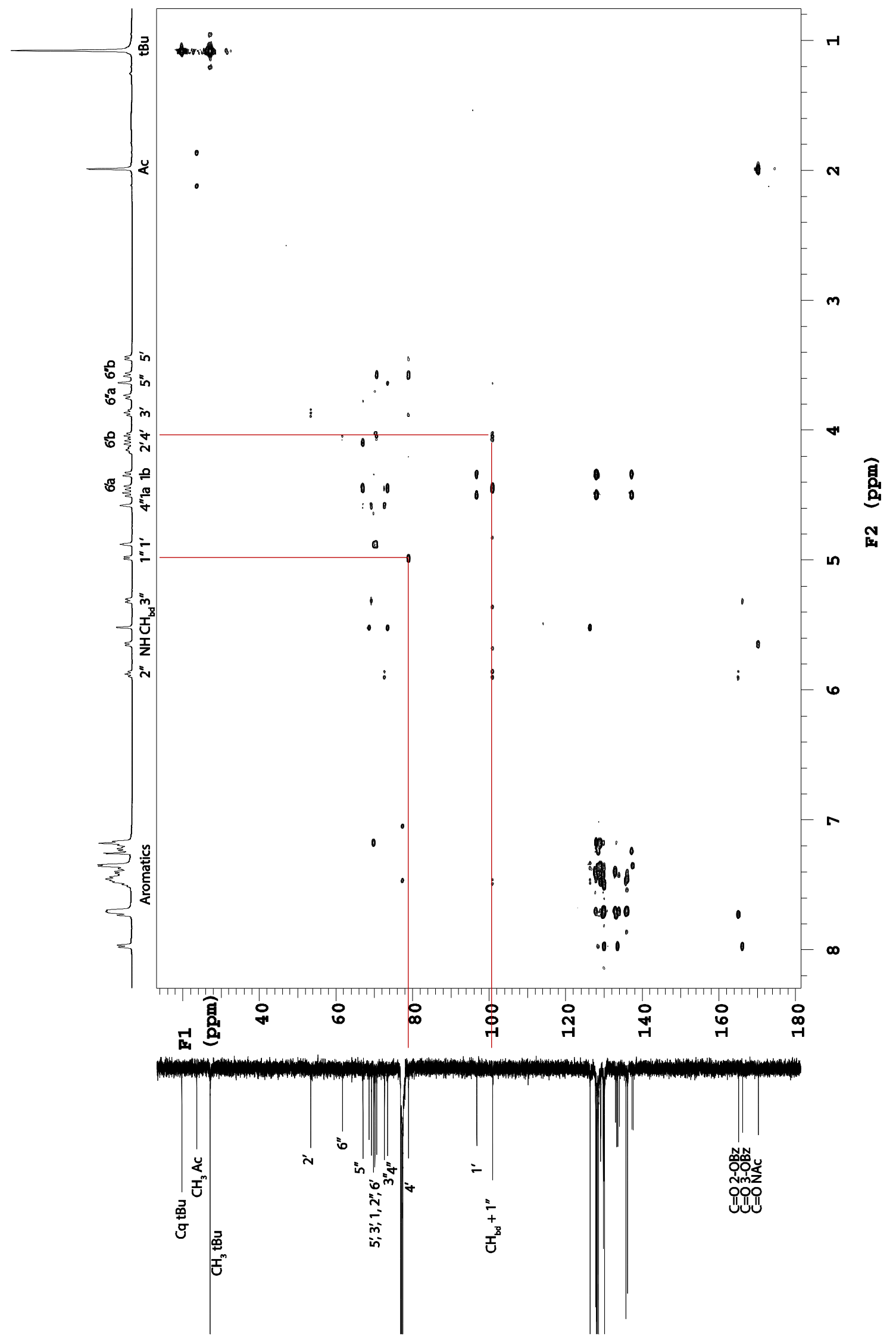

Figure 2. HMBC analysis of disaccharide $\mathbf{8 3}$ confirms the regioselectivity of the glycosylation reaction. 
With the disaccharide acceptor in hand, the glycosylation between disaccharide $\mathbf{8 3}$ and L-fucose donor $\mathbf{8}$ was attempted. Here, the thioethyl donor $\mathbf{8}$ was activated with copper(II)bromide and tetrapropylammonium bromide in DMF and $\mathrm{CH}_{2} \mathrm{Cl}_{2}$ at room temperature, which resulted in the smooth formation of the desired trisaccharide 68. Although the $J_{l^{\prime \prime}, 2}{ }^{\prime \prime}$ coupling constant could not be observed, the ${ }^{1} J_{H-1}{ }^{\prime \prime}, C-1$ "' $c$ coupling constant of $170 \mathrm{~Hz}$ confirmed the formation of the $\alpha$-anomer, whereas the $\beta$-anomer was not observed. ${ }^{77}$ To explain the $\alpha$-selectivity, the reaction mechanism needs to be considered (Scheme 19). ${ }^{78}$ Here, the $S_{\mathrm{N}} 2$-like activation of the thioglycoside 8 results in the formation of the relatively stable $\alpha$-bromide 84, which in turn anomerises 'in situ' to the more reactive $\beta$-bromoglycoside $\mathbf{8 5}$ in the presence of the ' $\mathrm{Br}^{\text {' }}$ ' nucleophile, that originates from tetrapropylammonium bromide (TPABr). The relative reaction rate between the $\alpha$ - and $\beta$-bromides, whereby the $\alpha$-bromide reacts slowly while the $\beta$-bromide reacts more quickly, then results in the selective formation of the $\alpha$-glycoside $\mathbf{6 8}$ via an $S_{\mathrm{N}} 2$ like substitution with the acceptor, whereas $\beta$-glycoside $\mathbf{8 6}$ was not observed.

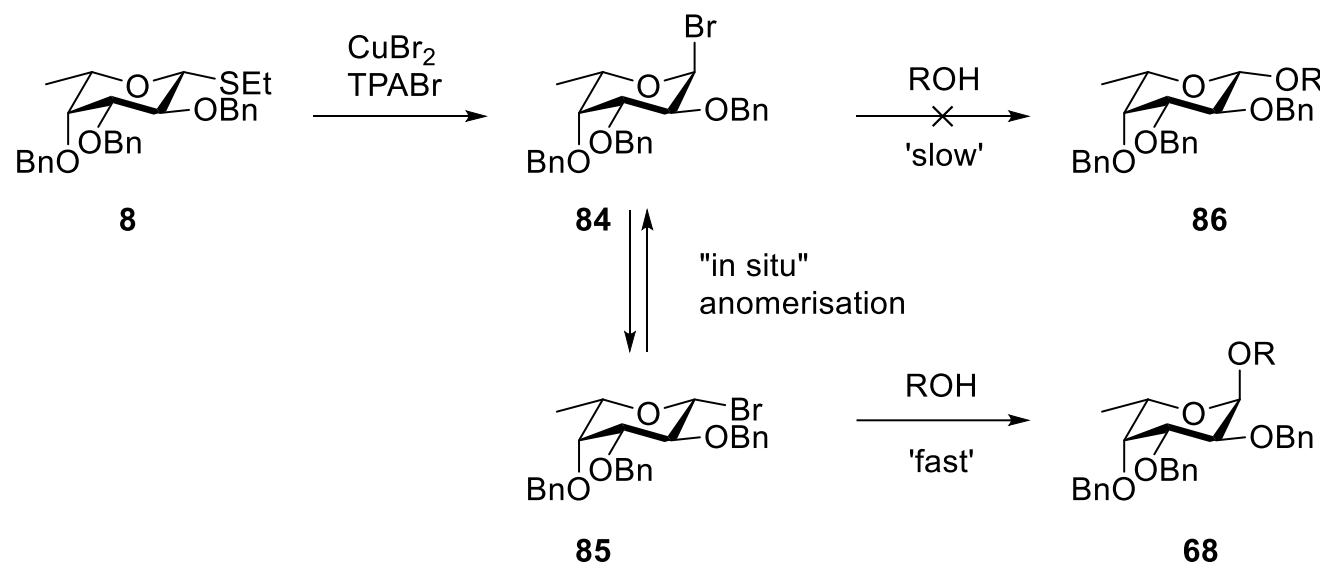

Scheme 19. In situ anomerisation with $T P A B r$ results in the selective formation of the $\alpha-L$ fucopyranoside 68.

Finally, the trisaccharide $\mathbf{6 8}$ was purified using silica gel column chromatography $\left(\mathrm{R}_{f}=0.23,2 / 1\right.$ $\mathrm{PE} / \mathrm{EtOAc}, \mathrm{v} / \mathrm{v}$ ), however, the trisaccharide can also be crystallised from methanol. This allows for crystallisation of the trisaccharide from the crude reaction mixture, thus significantly simplifying the purification process, especially during large-scale synthesis. Recrystallisation of the initial crystalline product, resulted in large $(1-2 \mathrm{~mm})$ crystals that were suitable for X-ray crystallography (Figure 3). The structure of trisaccharide $\mathbf{6 8}$ was confirmed and, in particular, the confirmation of the glycosidic linkages were clearly as required. 


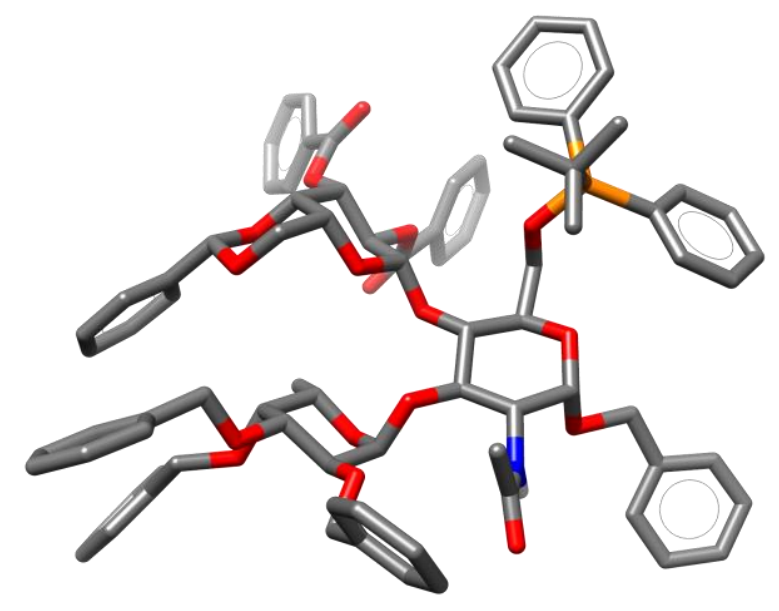

Figure 3. XRD Crystal structure of the protected Lewis ${ }^{X}$ trisaccharide 68.

Interestingly, only one protected Lewis ${ }^{\mathrm{X}}$ derivative has previously been reported as a crystalline compound, however no crystal structure of this material was obtained. ${ }^{79}$ Here, Lewis ${ }^{\mathrm{x}} \mathbf{8 7}$ was synthesised in 8 steps from trifluoroacetyl glucosamine 88 (Scheme 20). The 3-OH of glucosamine $\mathbf{8 8}$ was temporarily protected with a carbonate to give acceptor $\mathbf{8 9}$, which underwent glycosylation with imidate $\mathbf{9 0}$, followed by acetylation to yield disaccharide $\mathbf{9 1}$. Removal of the carbonate via hydrogenation of the $O$-alloc intermediate, followed by fucosylation gave trisaccharide 87. Unfortunately, the authors did not report the full global deprotection, and instead only removed the benzyl and acetate protecting groups. In contrast to their extensive synthetic route, the synthetic route employed in this thesis only requires four steps to obtain Lewis ${ }^{\mathrm{X}} \mathbf{6 8}$ in good yields from $\mathrm{N}$-acetyl glucosamine. In addition, trisaccharide $\mathbf{6 8}$ contains a benzylidene acetal protecting group, which allows for further functionalisation of Lewis ${ }^{\mathrm{X}}$ into more complex ligands, such as Sialyl Lewis ${ }^{\mathrm{X}}$ derivatives and/or sulfated glycans.

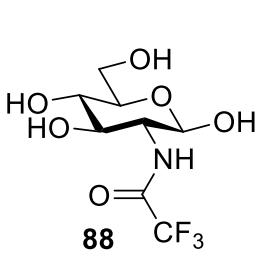

1) TBDPSCI, Imidazole DMF, $89 \%$ 2) $\mathrm{LiOH}, \mathrm{H}_{2} \mathrm{O}$, THF

3) $\mathrm{p}-\mathrm{O}_{2} \mathrm{~N}\left(\mathrm{C}_{6} \mathrm{H}_{4}\right) \mathrm{OCOCl}$ $\mathrm{NaHCO}_{3}, \mathrm{MeCN}, \mathrm{H}_{2} \mathrm{O}$ $85 \%$ (2 steps)
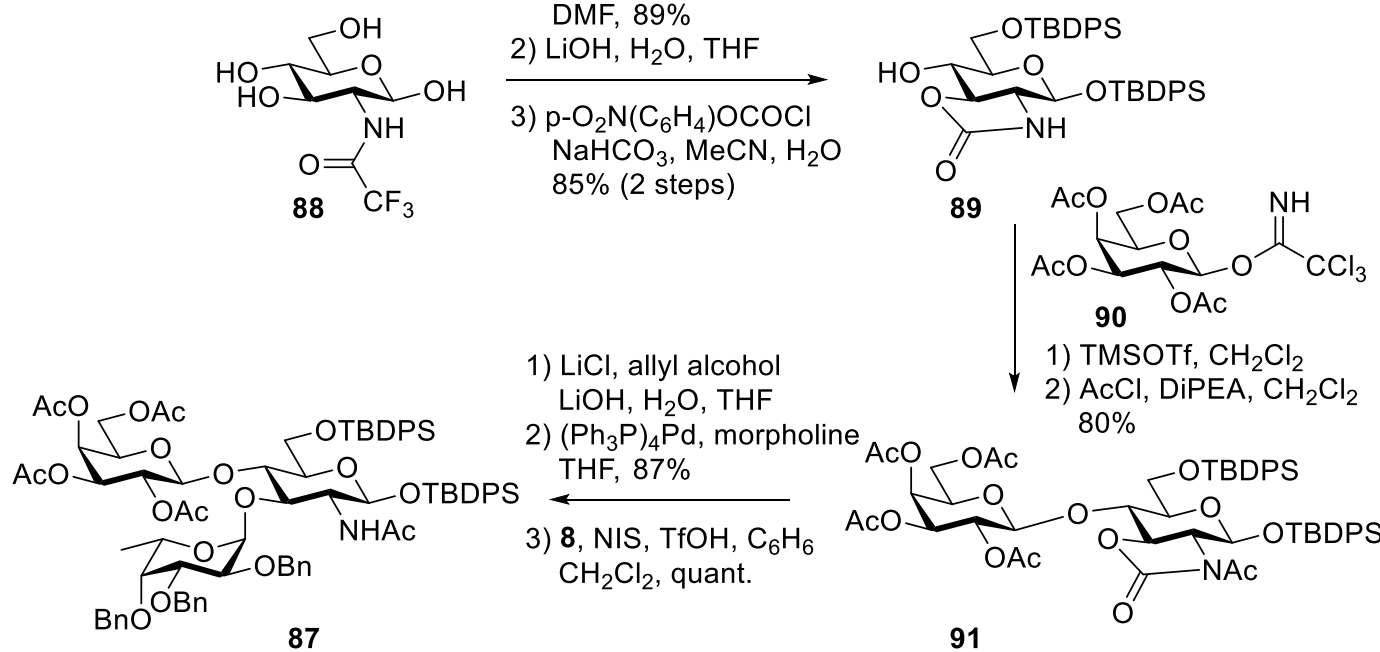

1) $\mathrm{LiCl}$, allyl alcohol $\mathrm{LiOH}, \mathrm{H}_{2} \mathrm{O}$, THF
$\left(\mathrm{Ph}_{3} \mathrm{P}\right)_{4} \mathrm{Pd}$, morpholine

2) $\left(\mathrm{Ph}_{3} \mathrm{P}\right)_{4} \mathrm{Pd}$, morpholine $\mathrm{AcO}$ _OAc 2) $\mathrm{AcCl}, \mathrm{DiPEA}, \mathrm{CH}_{2} \mathrm{Cl}_{2}$ $80 \%$

3) $8, \mathrm{NIS}, \mathrm{TfOH}, \mathrm{C}_{6} \mathrm{H}_{6}$ $\mathrm{CH}_{2} \mathrm{Cl}_{2}$, quant.

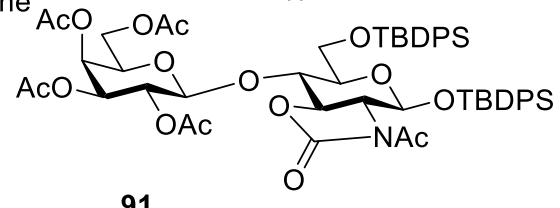

Scheme 20. Synthesis by Nagai et al. towards crystalline Lewis ${ }^{X} 91 .{ }^{79}$ 
Another advantage of the isolation of a late stage crystalline compounds is that it could allow for shorter and thus cheaper Good Manufacturing Practices (GMP)-controlled syntheses of Lewis ${ }^{\mathrm{X}}$ containing pharmaceutical products. GMP-synthesis is an expensive process due to many regulations, guidelines, extensive documentation such as good laboratory and clinical practices, hygiene and safety regulations. A late stage crystalline material is therefore advantageous as it reduces the number of steps required in GMP-synthesis, as crystalline material can conveniently be used as feedstock, without needing to analyse prior intermediates using GMP-guidelines.

Taken as a whole, the new synthetic strategy reported in this thesis has resulted in the highest yield of a protected Lewis ${ }^{\mathrm{X}}$ derivative (72\% from 69) utilising an $N$-acetylglucosamine acceptor. While other groups have reported higher yielding syntheses using $N$-phtalimido protected glucosamines, ${ }^{52,54,59}$ two extra steps are required at a late-stage synthesis to obtain the desired $\mathrm{N}$ acetyl derivative. In addition, the versatile protecting group strategy of crystalline trisaccharide $\mathbf{6 8}$ allows for further derivatisation of Lewis ${ }^{\mathrm{X}}$, for example in sulfated Lewis ${ }^{\mathrm{X}}$ derivatives, as well as Sialyl Lewis ${ }^{\mathrm{X}}$. To complete the synthesis of Lewis ${ }^{\mathrm{X}}$, global deprotection was required.

\subsubsection{Deprotection strategy}

Deprotection of trisaccharide $\mathbf{6 8}$ started with debenzoylation under Zemplén conditions in $\mathrm{MeOH}: \mathrm{CH}_{2} \mathrm{Cl}_{2}(1 / 1, \mathrm{v} / \mathrm{v})$ (Scheme 21). The reaction was quenched with Dowex $\mathrm{H}^{+}$and concentrated in vacuo and the crude mixture was then submitted to HF.pyridine in pyridine to give triol 92 in an excellent (99\%) yield after purification by silica column chromatography. With the triol in hand, several hydrogenolysis methods were examined in order to obtain the Lewis ${ }^{\mathrm{X}}$ trisaccharide (67). 

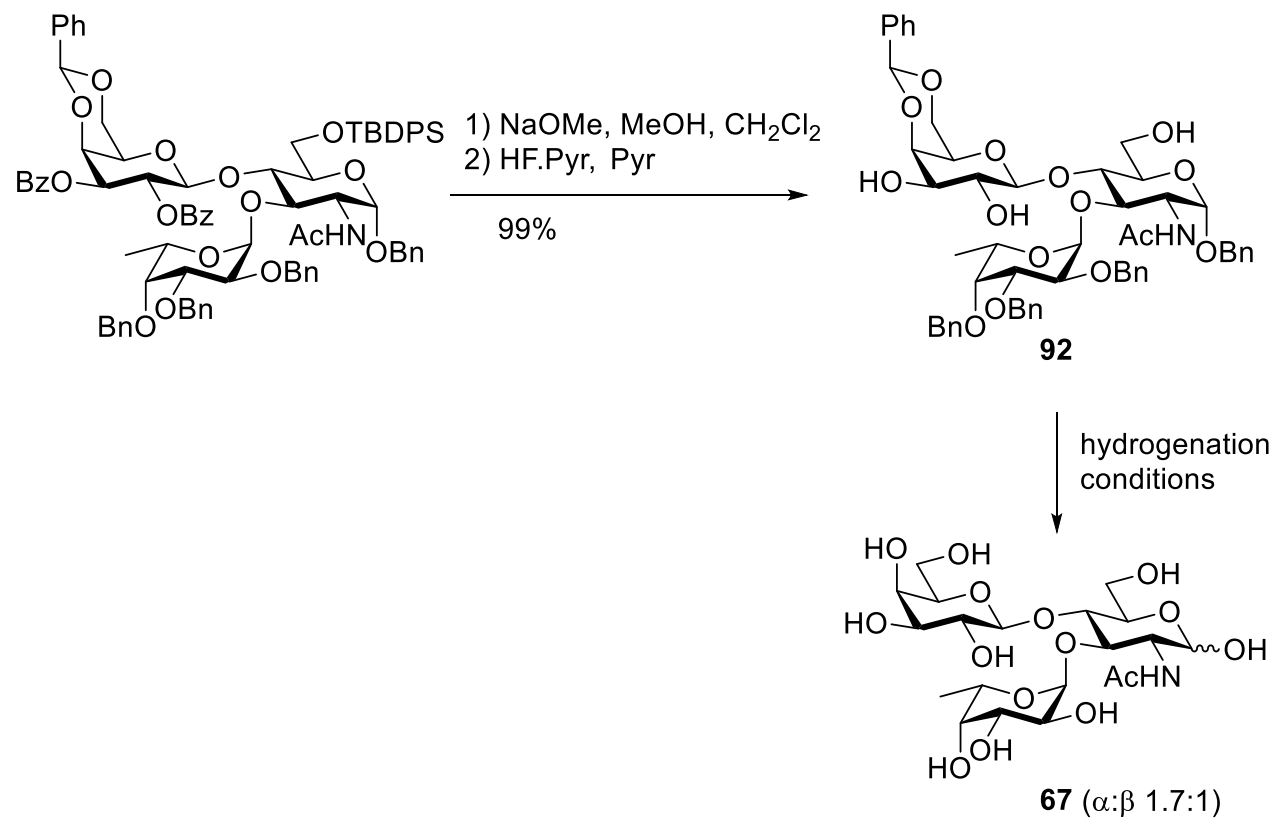

Scheme 21. Three step deprotection sequence to synthesise Lewis ${ }^{X}$.

Although a few groups have reported high yielding global deprotection strategies for the synthesis of Lewis ${ }^{\mathrm{x}}$ and related glycans, in our hands, it took much effort to optimise the final deprotection step (Table 1). When methanol was used as a solvent, the reaction was slow and after 5 days, debenzylation was complete, yet a significant amount of the defucosylated disaccharide $\mathbf{9 3}$ (ca $80 \%$ ) was also formed (entry 1). Here, it should be noted that a large excess of palladium, or filtration followed by resubmission of the reaction did not increase the yield of the desired trisaccharide. By using 3.5 bar of hydrogen gas in a Fisher Porter bottle, the hydrogenation occurred faster, however, again the fucose moiety was cleaved (entry 2). When $\mathrm{Pd} / \mathrm{C}$, rather than $\mathrm{Pd}(\mathrm{OH})_{2}$, was used similar results were observed (entry 3 ). To speed up the reaction, a mixture of $\mathrm{CHCl}_{3}$ : $\mathrm{MeOH}$ was used, which is known to be slightly acidic and thus favor the hydrogenolysis reaction (entry 4). Although these conditions resulted in a rapid reaction, they also favoured cleavage of the fucose moiety. With the addition of formic acid, the reaction was complete overnight, but again the disaccharide was the only product observed (entry 5). Thus, given the known acid sensitivity of the fucosyl linkage, it was envisioned to keep the reaction mixture basic.

To this end, the addition of $\mathrm{NH}_{4} \mathrm{HCO}_{2}$, which forms $\mathrm{NH}_{3}, \mathrm{H}_{2}$ and $\mathrm{CO}_{2}$ during the reaction, ${ }^{80}$ resulted in a sluggish low yielding reaction accompanied by undesired disaccharide $\mathbf{9 3}$ formation (entry 6). When the hydrogenolysis reaction was performed in the presence of pyridine (residue from the HF.pyridine silane cleavage), a smooth but slow (5 days) conversion to Lewis ${ }^{\mathrm{X}}$ (67) was observed (entry 7). Unfortunately, pyridine was hydrogenated to form piperidine, which proved difficult to remove with either reverse phase or size exclusion chromatography. Notwithstanding, this result suggested that the addition of a base might aid in the synthesis of trisaccharide (67) and 
thus other bases were therefore investigated. The use of ammonia in methanol resulted in disaccharide formation as well as the formation of what was thought to be a methylated amine, as evidenced by mass spectrometry (entry 8 ). This by-product may have been formed by a reductive amination of the acetamide, with traces of formaldehyde present in methanol. The use of triethylamine resulted in the isolation of the product in moderate yield, however, again disaccharide formation was observed (entry 9). Here it should also be noted that a large excess of $\mathrm{Et}_{3} \mathrm{~N}$ most likely poisoned the catalyst and resulted in the long reaction time. Nevertheless, after 5 days, $34 \%$ of the desired product was obtained on a small scale reaction.

During the course of these studies, the hydrogenation reactions were monitored using HRMS, and it was observed that the benzyl protecting groups were removed within 24 hours, whereas the benzylidene acetal removal proved sluggish. And upon further literature research, it was found that the use of $\mathrm{AcOH}$ greatly increases the reaction speed and, in particular, seems essential for the deprotection of benzylidene acetals. Accordingly, triol 92 was submitted to the hydrogenation conditions reported by Lu et al. ${ }^{63}$ and gratifyingly it was found that the hydrogenation with $\mathrm{Pd} / \mathrm{C}$ in $\mathrm{THF} / \mathrm{H}_{2} \mathrm{O} / \mathrm{AcOH}(4 / 2 / 1, \mathrm{v} / \mathrm{v} / \mathrm{v})$ and atmospheric hydrogen gas was completed within three hours, with clean conversion to the desired Lewis ${ }^{\mathrm{X}}$ antigen $\mathbf{6 7}$, which was isolated in a high (98\%) yield as an $\alpha / \beta$-mixture. 
Table 1. Hydrogenation conditions for the formation of Lewis ${ }^{X}$.
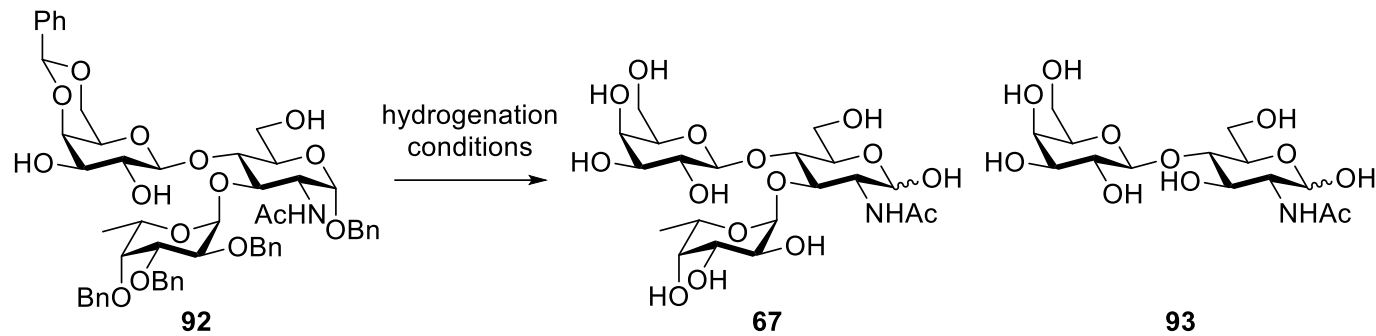

\begin{tabular}{|c|c|c|c|c|c|c|}
\hline Entry & Cat. & Solvent & Additives & Time & $\begin{array}{l}\text { Isolated } \\
\text { Yield } 67\end{array}$ & Observed \\
\hline $1^{\mathrm{a}}$ & $\mathrm{Pd}(\mathrm{OH})_{2}$ & $\mathrm{MeOH}$ & - & $5 \mathrm{~d}$ & $\sim 10 \%$ & $67+93$ \\
\hline $2^{b}$ & $\mathrm{Pd}(\mathrm{OH})_{2}$ & $\mathrm{MeOH}$ & - & $5 \mathrm{~d}$ & $\sim 10 \%$ & $67+93$ \\
\hline $3^{\mathrm{b}}$ & $\mathrm{Pd} / \mathrm{C}$ & $\mathrm{MeOH}$ & - & $5 \mathrm{~d}$ & $\sim 10 \%$ & $67+93$ \\
\hline $4^{\mathrm{a}}$ & $\mathrm{Pd}(\mathrm{OH})_{2}$ & $\mathrm{MeOH}: \mathrm{CHCl}_{3}$ & - & $18 \mathrm{~h}$ & - & 93 \\
\hline $5^{\mathrm{a}}$ & $\mathrm{Pd}(\mathrm{OH})_{2}$ & $\mathrm{MeOH}$ & $\mathrm{HCO}_{2} \mathrm{H}$ & $18 \mathrm{~h}$ & - & 93 \\
\hline $6^{\mathrm{a}, \mathrm{b}}$ & $\mathrm{Pd}(\mathrm{OH})_{2}$ & $\mathrm{MeOH}$ & $\mathrm{NH}_{4} \mathrm{HCO}_{2}$ & $5 \mathrm{~d}$ & $15 \%$ & $67+93$ \\
\hline $7^{\mathrm{a}}$ & $\mathrm{Pd}(\mathrm{OH})_{2}$ & $\mathrm{MeOH}$ & Pyridine & $5 \mathrm{~d}$ & $\sim 40 \%$ & $67^{\mathrm{c}}$ \\
\hline $8^{\mathrm{a}}$ & $\mathrm{Pd}(\mathrm{OH})_{2}$ & $\mathrm{MeOH}$ & $\mathrm{NH}_{3}(\mathrm{MeOH})$ & $1 \mathrm{~d}$ & - & $\mathbf{9 3}^{\mathrm{d}}$ \\
\hline $10^{\mathrm{a}}$ & $\mathrm{Pd}(\mathrm{OH})_{2}$ & $\mathrm{EtOH}$ & $\mathrm{Et}_{3} \mathrm{~N}$ & $5 \mathrm{~d}$ & $34 \%$ & $67^{\mathrm{e}}$ \\
\hline $11^{\mathrm{a}}$ & $\mathrm{Pd} / \mathrm{C}$ & $\begin{array}{l}\text { 4:2:1 THF: } \\
\mathrm{H}_{2} \mathrm{O}: \mathrm{AcOH}\end{array}$ & & $3 \mathrm{~h}$ & $98 \%$ & 67 \\
\hline a) & \multicolumn{6}{|c|}{ Reaction performed at $1.0 \mathrm{bar}$} \\
\hline b) & \multicolumn{6}{|c|}{ Reaction performed at 3.5 bar } \\
\hline c) & \multicolumn{6}{|c|}{ Contaminated with piperidine } \\
\hline d) & \multicolumn{6}{|c|}{ Methylated byproduct formed } \\
\hline e) & ca. $5 \%$ of di & ner 93 was observ & & & & \\
\hline
\end{tabular}




\subsection{Conclusion}

In summary, a number of different strategies can be used for the synthesis of Lewis ${ }^{\mathrm{X}}$ derivatives. In the "Route A" approach, a benzylidene acetal glucosamine acceptor is used in the glycosylation with fucosyl donors. Next, reductive opening of the benzylidene acetal gives the disaccharide acceptor for glycosylations with galactose donors. Even though several groups reported the successful assembly of Lewis ${ }^{\mathrm{X}}$ using this route, others noted the unreactivity of this type of acceptors. To avoid this problem, "Route B" can be used which involves the glycosylation of a 3-O-protected glucosamine acceptor with a galactosyl donor to give a lactosamine derivative. Selective deprotection of the 3-hydroxy protecting group then gave the disaccharide acceptor, which in turn was fucosylated. This route was further improved by employing a regioselective glycosylation of a diol acceptor with a galactose donor, followed by the consecutive glycosylation with a fucosyl donor ( "Route C"). This route allows for a more efficient acceptor synthesis, and more importantly, results in the high yielding assembly of Lewis ${ }^{\mathrm{x}}$ derivatives.

Thus, in this thesis, the "Route $C$ " strategy was employed to synthesise Lewis ${ }^{\mathrm{x}}$. First, the required monosaccharide building blocks were synthesised in good yields (2-5 step, 54-70\% overall yield). The regioselective glycosylation of the glucosamine acceptor with the galactose donor resulted in the required disaccharide acceptor in good yield. Next, the $\alpha$-selective fucosylation was performed with the fucose donor, and the resulting trisaccharide was obtained as a crystalline material. Compared to previous reported synthetic routes, Lewis ${ }^{\mathrm{x}}$ was obtained in an excellent overall yield of $72 \%$ from the glucosamine acceptor. With the versatile trisaccharide in hand, global deprotection afforded DC-SIGN antigen Lewis ${ }^{\mathrm{X}}$. Although the final hydrogenation step proved troublesome, an overall deprotection yield of $97 \%$ could be achieved if the benzoyl and TBDPS groups were removed first, and the hydrogenolysis was performed using the conditions previously reported by Lu et al. It is envisioned that this highly efficient route (36\% over 10-steps) will find further application, not only in the synthesis of Lewis ${ }^{\mathrm{X}}$ probes for studying the function of DC-SIGN (see Chapter 5), but also for the synthesis of other members of the Type-2 family of Lewis antigens. 


\subsection{Experimental}

General procedure. Unless otherwise stated all reactions were performed under argon. Prior to use, THF (Pancreac) was distilled from sodium and benzophenone, pyridine was distilled and

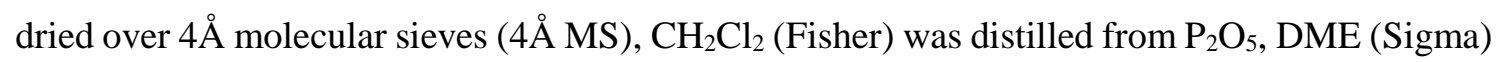
was distilled from $\mathrm{LiAlH}_{4}, \mathrm{MeCN}$ (Panreac) was distilled from CaH, DMF was distilled from $\mathrm{BaO}$, and $\mathrm{H}_{2} \mathrm{O}$ was distilled. N-Acetylglucosamine (Fluka), D-galactose (J. T. Baker), L-fucose (Pfanstiehl Inc.), BnOH (Ajax Chemicals), conc HCl (Panreac), TBDPS-Cl (Aldrich), DMAP (Pierce), $\mathrm{SnCl}_{4}$ (Aldrich), $\mathrm{PhSH}$ (Koch-Light Laboratories), KF (Riedel-de Haën), NaOMe (freshly prepared from sodium [Aldrich] and $\mathrm{MeOH}$ ), benzaldehyde dimethyl acetal (Aldrich), pTsOH (Aldrich), $\mathrm{BzCl}$ (Aldrich), $\mathrm{Ac}_{2} \mathrm{O}$ (Peking Reagent), $\mathrm{HBr}$ (33\% in $\left.\mathrm{AcOH}, \mathrm{Acros}\right), \mathrm{LiAlH}_{4}$ (Aldrich), EtSH (Sigma-Aldrich), BnBr (Fluka), TBAI (Riedel-de Haën), NaH (Aldrich, 60\% dispersed in mineral oil), $\mathrm{N}$-iodosuccinimide (Aldrich), TfOH (Aldrich), TPABr (Aldrich), $\mathrm{CuBr}_{2}$ (Chempur), HF.Pyridine (Aldrich), CaOAc (Sigma), AcOH (Sigma-Aldrich), Pd/C (Aldrich), Et3N (Sigma), Dowex $\mathrm{H}^{+}$(Sigma-Aldrich 50WX8), 4A molecular sieves (ROTH), Toluene (Panreac), anhydrous $\mathrm{Et}_{2} \mathrm{O}$ (Pancreac), EtOAc (Pancreac), petroleum ether (Pure Science), $\mathrm{MeOH}$ (Pure Science), $\mathrm{CHCl}_{3}$ (Pancreac), EtOH (absolute, Pure Science), $\mathrm{NaHCO}_{3}$ (Pure Science), $\mathrm{Na}_{2} \mathrm{~S}_{2} \mathrm{O}_{3}$ (Merck) and $\mathrm{NaCl}$ (Pancreac) were used as received. All solvents were removed by evaporation under reduced pressure. Reactions were monitored by TLC-analysis on MachereyNagel silica gel coated plastic sheets $\left(0.20 \mathrm{~mm}\right.$, with fluorescent indicator $\left.\mathrm{UV}_{254}\right)$ with detection by UV-absorption (short wave UV - $254 \mathrm{~nm}$; long wave UV - $366 \mathrm{~nm}$ ), by dipping in $10 \% \mathrm{H}_{2} \mathrm{SO}_{4}$ in EtOH followed by charring at $\sim 150{ }^{\circ} \mathrm{C}$, by dipping in $\mathrm{I}_{2}$ in silica, or by dipping into a solution of ninhydrin in $\mathrm{EtOH}$ followed by charring at $\sim 150^{\circ} \mathrm{C}$. Column chromatography was performed on Pure Science silica gel (40-63 micron). AccuBOND II ODS-C $\mathrm{C}_{18}$ (Agilent) was used for reverse phase chromatography. Infrared spectra were recorded as thin films using a Bruker Tensor 27 FTIR spectrometer equipped with an Attenuated Total Reflectance (ATR) sampling accessory and are reported in wave numbers $\left(\mathrm{cm}^{-1}\right)$. Nuclear magnetic resonance spectra were recorded at $20{ }^{\circ} \mathrm{C}$ in $\mathrm{D}_{2} \mathrm{O}, \mathrm{CD}_{3} \mathrm{OD}, \mathrm{CDCl}_{3}$, or pyridine-d $\mathrm{d}_{5}$ using either a Varian INOVA operating at $500 \mathrm{MHz}$ or Varian VNMRS operating at $600 \mathrm{MHz}$. Chemical shifts are given in ppm $(\delta)$ relative to solvent residues. NMR peak assignments were made using COSY, HSQC and HMBC $2 \mathrm{D}$ experiments.

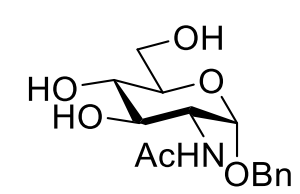

Benzyl 2-acetamido-2-deoxy-a-D-glucopyranoside (2). ${ }^{73,74}$ To a solution of $N$-acetylglucosamine $(6.40 \mathrm{~g}, 28.9 \mathrm{mmol})$ in benzyl alcohol $(50 \mathrm{~mL}, 480$ $\mathrm{mmol})$, conc. aq. $\mathrm{HCl}(3.0 \mathrm{~mL})$ was added and the reaction mixture was stirred at $90{ }^{\circ} \mathrm{C}$ for $3 \mathrm{~h}$. The crude mixture was cooled down to rt, poured into $\mathrm{Et}_{2} \mathrm{O}(500 \mathrm{~mL})$ and 
left to crystallise at $-4{ }^{\circ} \mathrm{C}$ for $18 \mathrm{~h}$. The crystalline product was filtered and washed with petroleum ether. Purification by silica gel column chromatography $\left(\mathrm{CH}_{2} \mathrm{Cl}_{2} / \mathrm{MeOH}, 95 / 5 \rightarrow 88 / 12\right.$, v/v) gave benzyl glycoside 2 (6.67 gr, $21.4 \mathrm{mmol}, 74 \%)$ as a white foam. $\mathrm{R}_{f}=0.25\left(\mathrm{CH}_{2} \mathrm{Cl}_{2} / \mathrm{MeOH}, 90 / 10\right.$, $\mathrm{v} / \mathrm{v}) ; \mathrm{Mp} 182-184^{\circ} \mathrm{C} ; \alpha_{\mathrm{D}}{ }^{17.8}=+222(\mathrm{c}=0.1, \mathrm{MeOH})$; IR (film) 3298, 3092, 2938, 2901, 2844, $1648,1552,1497,1455,1375,1309,1230,1156,1093,1047,778,732,695 \mathrm{~cm}^{-1}$; ${ }^{1} \mathrm{H}$ NMR (500 $\left.\mathrm{MHz}, \mathrm{CD}_{3} \mathrm{OD}\right) \delta 7.40-7.25\left(\mathrm{~m}, 5 \mathrm{H}, \mathrm{CH}_{\mathrm{arom}}\right), 4.85\left(\mathrm{~d}, 1 \mathrm{H}, J_{l^{\prime}, 2^{\prime}}=3.6 \mathrm{~Hz}, \mathrm{H}-1^{\prime}\right), 4.74\left(\mathrm{~d}, 1 \mathrm{H}, J_{1 a, 1 b}\right.$ $=12.0 \mathrm{~Hz}, \mathrm{H}-1 \mathrm{a}), 4.49$ (d, $\left.1 \mathrm{H}, J_{l a, l b}=12.0 \mathrm{~Hz}, \mathrm{H}-1 \mathrm{~b}\right), 3.89\left(\mathrm{dd}, 1 \mathrm{H}, J_{l^{\prime}, 2^{\prime}}=3.6 \mathrm{~Hz}, J_{2^{\prime}, 3^{\prime}}=10.8 \mathrm{~Hz}\right.$ H-2'), $3.83\left(\mathrm{dd}, 1 \mathrm{H}, J_{5^{\prime}, 6 a^{\prime}}=1.6 \mathrm{~Hz}, J_{6 a^{\prime}, 6 b^{\prime}}=11.4 \mathrm{~Hz}, \mathrm{H}-6 \mathrm{a}^{\prime}\right), 3.73-3.64$ (m, 3H, H-6b', H-3’, H$\left.4^{\prime}\right), 3.36\left(\mathrm{dd}, 1 \mathrm{H}, J_{4^{\prime}, 5^{\prime}}=9.6 \mathrm{~Hz}, \mathrm{H}-5^{\prime}\right), 1.95\left(\mathrm{~s}, 3 \mathrm{H}, \mathrm{CH}_{3} \mathrm{Ac}\right) ;{ }^{13} \mathrm{C} \mathrm{NMR}\left(125 \mathrm{MHz}, \mathrm{CD}_{3} \mathrm{OD}\right) \delta$ $173.6\left(\mathrm{C}=\mathrm{O}\right.$ Ac), 139.0 (Cqarom), $129.4\left(\mathrm{C}-o_{\text {arom }}\right), 129.3$ (C- $\left.m_{\text {arom }}\right), 128.8$ (C- $\left.p_{\text {arom }}\right), 97.5\left(\mathrm{C}-1^{\prime}\right)$, 74.1 (C-4'), 72.7 (C-3’), 72.5 (C-5'), 70.1 (C-1), 62.7 (C-6'), 55.4 (C-2'), $22.5\left(\mathrm{CH}_{3} \mathrm{Ac}\right.$ ); HRMS(ESI) $m / z$ calcd. for $\left[\mathrm{C}_{15} \mathrm{H}_{22} \mathrm{NO}_{6}\right]^{+}: 312.1442$, obsd.: 312.1446 .

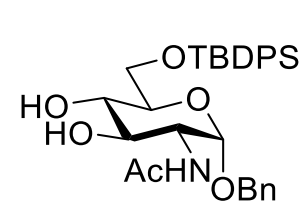

Benzyl 2-acetamido-6-O-tert-butyldiphenylsilyl-2-deoxy- $\alpha$-Dglucopyranoside (3). To a solution of benzyl glycoside 2 (837 $\mathrm{mg}, 8.74$ $\mathrm{mmol})$ in pyridine $(10 \mathrm{~mL})$, TBDPS-Cl $(2.75 \mathrm{~mL}, 33.9 \mathrm{mmol})$ and DMAP (200 mg, $1.64 \mathrm{mmol}$ ) were added and the reaction mixture was stirred at 40 ${ }^{\circ} \mathrm{C}$ for $18 \mathrm{~h}$. The crude mixture was concentrated in vacuo and purified by silica gel column chromatography $\left(\mathrm{CH}_{2} \mathrm{Cl}_{2} / \mathrm{MeOH}, 100 / 0 \rightarrow 95 / 5\right.$, v/v) to give diol $\mathbf{3}(1.39$ gram, $94 \%)$ as a white solid. $\mathrm{R}_{f}=0.50\left(\mathrm{CH}_{2} \mathrm{Cl}_{2} / \mathrm{MeOH}, 95 / 5, \mathrm{v} / \mathrm{v}\right) ; \alpha_{\mathrm{D}}{ }^{17.8}=+58.4$ (c = 0.5, $\left.\mathrm{CHCl}_{3}\right) ; \mathrm{IR}$ (film) 3324, 3071, 2930, 2857, 1739, 1655, 1544, 1472, 1456, 1428, 1376, 1217, 1112, 1048, 1025, 823, 775, 739, $701 \mathrm{~cm}^{-1} ;{ }^{1} \mathrm{H}$ NMR $\left(500 \mathrm{MHz}, \mathrm{CDCl}_{3}\right) \delta 7.73-7.68\left(\mathrm{~m}, 4 \mathrm{H}, \mathrm{CH}_{\text {arom }}\right), 7.46-7.27(\mathrm{~m}, 11 \mathrm{H}$, $\left.\mathrm{CH}_{\text {arom }}\right), 5.84\left(\mathrm{~d}, 1 \mathrm{H}, J_{2^{\prime}, N H}=8.5 \mathrm{~Hz}, \mathrm{NH}\right), 4.88\left(\mathrm{~d}, 1 \mathrm{H}, J_{l^{\prime}, 2^{\prime}}=3.0 \mathrm{~Hz}, \mathrm{H}-1^{\prime}\right), 4.72\left(\mathrm{~d}, 1 \mathrm{H}, J_{1 a, l b}=\right.$ $11.8 \mathrm{~Hz}, \mathrm{H}-1 \mathrm{a}), 4.44$ (d, 1H, $\left.J_{l a, 1 b}=11.8 \mathrm{~Hz}, \mathrm{H}-1 \mathrm{~b}\right), 4.14-4.06$ (m, 1H, H-2'), $3.94-3.85$ (m,

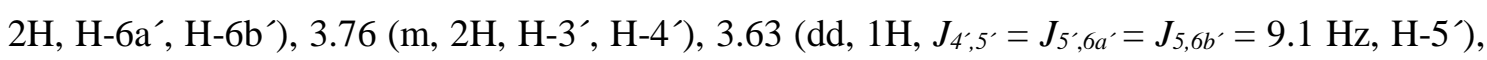
3.24 (bs, 1H, OH), 2.86 (bs, 1H, OH), 2.00 (s, 3H, $\mathrm{CH}_{3} \mathrm{Ac}$ ), 1.07 (s, 9H, $\left.\mathrm{CH}_{3} t \mathrm{Bu}\right) ;{ }^{13} \mathrm{C} \mathrm{NMR}$ $\left(125 \mathrm{MHz}, \mathrm{CDCl}_{3}\right) \delta 172.0(\mathrm{C}=\mathrm{O}), 137.1,135.81,135.78,133.3,133.2,130.0,128.8,128.3$, 128.2, 127.9 ( $\mathrm{C}_{\text {arom }}$ ), 96.5 (C-1'), 74.5 (C-3’), 72.9 (C-5’), 71.5 (C-4’), 69.4 (C-1), 64.3 (C-6’), $53.8\left(\mathrm{C}-2^{\prime}\right), 27.0\left(\mathrm{CH}_{3} t \mathrm{Bu}\right), 23.4\left(\mathrm{CH}_{3} \mathrm{Ac}\right), 19.4(\mathrm{Cq} t \mathrm{Bu})$; HRMS(ESI) $\mathrm{m} / z$ calcd. for $\left[\mathrm{C}_{31} \mathrm{H}_{40} \mathrm{NO}_{6} \mathrm{Si}\right]^{+}:$550.2619, obsd.: 550.2620.

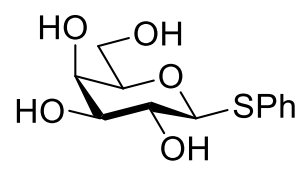

Phenyl 1-thio- $\beta$-D-galactopyranoside (76). 1,2,3,4,6-penta- $O$-acetyl- $\beta$-Dgalactopyranose 74 (20.0 g, $51.2 \mathrm{mmol})$ was co-evaporated with toluene (2x), dissolved in $\mathrm{CH}_{2} \mathrm{Cl}_{2}(150 \mathrm{~mL})$ and cooled to $0{ }^{\circ} \mathrm{C}$. Thiophenol $(6.3$ $\mathrm{mL}, 61.4 \mathrm{mmol})$ and $\mathrm{SnCl}_{4}(0.6 \mathrm{~mL}, 5.12 \mathrm{mmol})$ were added and the reaction mixture and stirred at $0{ }^{\circ} \mathrm{C}$ for $3 \mathrm{~h}$. under argon. The reaction was quenched with a $3 \mathrm{M}$ solution of potassium fluoride 
$(100 \mathrm{~mL})$ and the organic layer was washed with water, saturated sodium bicarbonate solution, brine and dried over $\mathrm{MgSO}_{4}$, filtered and concentrated in vacuo. The residue was dissolved in EtOAc and passed through a plug of silica gel to remove residual tin by-products, to give the crude thioglycoside $\mathbf{7 5}$ as a yellow oil (23.9 g). The data obtained for this compound matched literature values. ${ }^{68}$

To a suspension of the crude mixture $(23.9 \mathrm{~g})$ in methanol $(200 \mathrm{~mL})$, sodium methoxide was slowly added until the solution reached $\mathrm{pH}$ 13. The reaction mixture was stirred for $4 \mathrm{~h}$ and neutralised with Dowex $-\mathrm{H}^{+}$. The resin was removed by filtration and the methanolic solution was concentrated in vacuo to give crude tetraol $\mathbf{7 6}$ as a white solid, which was used without further purification. The data obtained for this compound matched literature values. ${ }^{6}{ }^{6}$

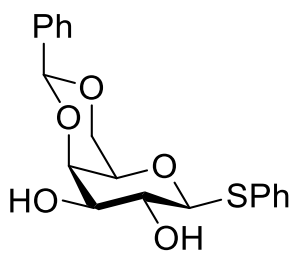

Phenyl 4,6- $O$-benzylidene-1-thio- $\beta$-D-galactopyranoside (77). To a solution of phenyl 1-thio- $\beta$-D-galactopyranoside 76 (4.80 g, $17.6 \mathrm{mmol})$ in acetonitrile $(100 \mathrm{~mL})$, benzaldehyde dimethyl acetal $(2.9 \mathrm{~mL}, 19.4 \mathrm{mmol})$ and $\mathrm{pTsOH}(200 \mathrm{mg}, 1.64 \mathrm{mmol})$ were added and the reaction mixture was stirred at $40{ }^{\circ} \mathrm{C}$ for $4 \mathrm{~h}$. The reaction mixture was neutralised with triethylamine $(0.4 \mathrm{~mL}, 3 \mathrm{mmol})$, concentrated in vacuo and purified by silica gel column chromatography $(\mathrm{CH} 2 \mathrm{Cl} 2 / \mathrm{MeOH}, 100 / 0$ $\rightarrow$ 95/5, v/v). Crystallisation from PE/EtOAc (2/1, v/v) afforded diol 77 (4.96 gram, 13.7 mmol, $78 \%$ ) as white crystals. The data obtained for this compound matched literature values. ${ }^{68}$

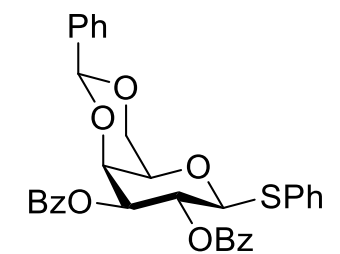

Phenyl 2,3-di- $O$-benzoyl-4,6- $O$-benzylidene-1-thio- $\beta$-D-galactopyranoside (70). To a solution of diol 77 (4.90 g, $13.6 \mathrm{mmol})$ in pyridine (68 $\mathrm{mL})$, benzoyl chloride ( $4.7 \mathrm{~mL}, 40.8 \mathrm{mmol}$ ) was added dropwise and the reaction mixture was stirred at r.t. for $16 \mathrm{~h}$. The crude mixture was diluted with $\mathrm{CH}_{2} \mathrm{Cl}_{2}(100 \mathrm{~mL})$ and washed with aq. $1 \mathrm{M} \mathrm{HCl}(100 \mathrm{~mL})$, and the water layer was extracted with $\mathrm{CH}_{2} \mathrm{Cl}_{2}(50 \mathrm{~mL})$. The combined organics were washed with sat. $\mathrm{NaHCO}_{3}(100 \mathrm{~mL})$ and brine $(100 \mathrm{~mL})$, dried with $\mathrm{MgSO}_{4}$, filtered and concentrated in vacuo. The product was crystallised from $\mathrm{CH}_{2} \mathrm{Cl}_{2} / \mathrm{PE}$ to give donor $70(6.65 \mathrm{~g}, 86 \%)$ as white crystalls. $\mathrm{R}_{f}=0.55$ $(\mathrm{PE} / \mathrm{EtOAc}, 1 / 1, \mathrm{v} / \mathrm{v}) ; \mathrm{Mp} 210{ }^{\circ} \mathrm{C} ; \alpha_{\mathrm{D}}{ }^{17.8}=+52\left(\mathrm{c}=0.1, \mathrm{CHCl}_{3}\right)$; IR (film) 3062, 2937, 2855, 1726, 1601, 1584, 1480, 1451, 1403, 1368, 1316, 1275, 1219, 1176, 1130, 1093, 1070, 1027, 944, 817, 772, $709 \mathrm{~cm}^{-1} ;{ }^{1} \mathrm{H}$ NMR (300 MHz, $\left.\mathrm{CDCl}_{3}\right) \delta 8.02$ - 7.91 (m, 4H, $\left.\mathrm{CH}_{\text {arom }}\right), 7.65-7.60$ (m, $2 \mathrm{H}, \mathrm{CH}_{\text {arom }}$ ), $7.56-7.22\left(\mathrm{~m}, 14 \mathrm{H}, \mathrm{CH}_{\text {arom }}\right), 5.82\left(\mathrm{t}, 1 \mathrm{H}, J_{2,3}=10.0 \mathrm{~Hz}, \mathrm{H}-2\right), 5.52(\mathrm{~s}, 1 \mathrm{H}, \mathrm{CHPh})$, $5.37\left(\mathrm{dd}, 1 \mathrm{H}, J_{2,3}=10.0 \mathrm{~Hz}, J_{3,4}=3.5 \mathrm{~Hz}, \mathrm{H}-3\right), 4.97\left(\mathrm{~d}, 1 \mathrm{H}, J_{1,2}=9.8 \mathrm{~Hz}, \mathrm{H}-1\right), 4.61\left(\mathrm{dd}, 1 \mathrm{H}, J_{3,4}\right.$ $\left.=3.3 \mathrm{~Hz}, J_{4,5}=0.6 \mathrm{~Hz}, \mathrm{H}-4\right), 4.46\left(\mathrm{dd}, 1 \mathrm{H}, J_{5,6 \mathrm{a}}=1.6 \mathrm{~Hz}, \mathrm{~J}_{6 \mathrm{a}, 6 b}=12.5 \mathrm{~Hz}, \mathrm{H}-6 \mathrm{a}\right), 4.10\left(\mathrm{~d}, 1 \mathrm{H}, J_{5,6 b}\right.$ $\left.=1.6 \mathrm{~Hz}, J_{6 a, 6 b}=12.4 \mathrm{~Hz}, \mathrm{H}-6 \mathrm{~b}\right), 3.77$ (bs, $\left.1 \mathrm{H}, \mathrm{H}-5\right) ;{ }^{13} \mathrm{C} \mathrm{NMR}\left(125 \mathrm{MHz}, \mathrm{CDCl}_{3}\right) \delta 166.3(\mathrm{C}=\mathrm{O}$ 
Bz), 165.1 (C=O Bz), 137.7, 134.1, 133.5, 133.3, 131.2, 130.1, 129.9, 129.8, 129.2, 129.2, 128.9, 128.5, 128.4, 128.3, 126.6 ( $\mathrm{C}_{\text {arom }}$ ), 101.1 (CHPh), 85.5 (C-1), 74.2 (C-3), 73.8 (C-4), 70.1 (C-5), 69.3 (C-6), 67.2 (C-2); HRMS(ESI) $\mathrm{m} / z$ calcd. for $\left[\mathrm{C}_{33} \mathrm{H}_{32} \mathrm{NO}_{7} \mathrm{~S}\right]^{+}: 586.1894$, obsd.: 586.1920 .

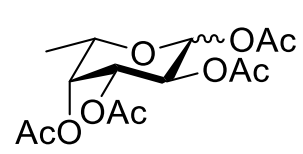

1,2,3,4-Tetra- $\boldsymbol{O}$-acetyl- $\boldsymbol{\alpha} / \boldsymbol{\beta}$-L-fucopyranose (79). L-Fucose (78) $(4.00 \mathrm{~g}$, $24.4 \mathrm{mmol})$ was dissolved in acetic anhydride $(25 \mathrm{ml})$ and pyridine $(50 \mathrm{~mL})$ and the reaction mixture was stirred at r.t. for $18 \mathrm{~h}$. The reaction mixture was concentrated in vacuo, co-evaporated three times with toluene, redissolved in EtOAc, washed with water and brine, dried with $\mathrm{MgSO}_{4}$, filtered and concentrated in vacuo. The crude peracetylated product $79(8.4 \mathrm{~g})$ was used without further purification. The data obtained for this compound matched literature values. ${ }^{70}$

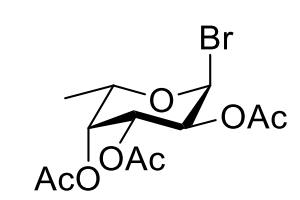

2,3,4-Tri- $O$-acetyl- $\alpha$-L-fucopyranosyl bromide (80). To a solution of crude $79(8.4 \mathrm{~g}, 24.4 \mathrm{mmol})$ in $\mathrm{CH}_{2} \mathrm{Cl}_{2}(16 \mathrm{~mL})$ at $0{ }^{\circ} \mathrm{C}, \mathrm{HBr}(16 \mathrm{~mL}, 33 \%$ in $\mathrm{AcOH})$ was slowly added and the reaction mixture was stirred for $2.5 \mathrm{~h}$, where it was allowed to warm to r.t. The reaction mixture was co-evaporated with toluene $(4 \times 25 \mathrm{~mL})$ and the resulting crude bromide $\mathbf{8 0}$ (9.05 g) was used without further purification. The data obtained for this compound matched literature values. ${ }^{70}$

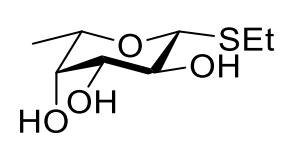

Ethyl $\boldsymbol{\alpha}$-L-thiofucopyranoside (82). To a solution of sodium hydride (2.40 $\mathrm{g}, 60.0 \mathrm{mmol})$ in DME $\left(53 \mathrm{~mL}\right.$, distilled from $\left.\mathrm{LiAlH}_{4}\right)$ at $0^{\circ} \mathrm{C}$, ethanethiol $(5.40 \mathrm{~mL}, 101 \mathrm{mmol})$ was slowly added while vigorously stirring the reaction mixture. After $30 \mathrm{~min}$, a solution of crude bromide 80 (9.01 g, $24.4 \mathrm{mmol})$ in DME (27 mL, distilled from $\mathrm{LiAlH}_{4}$ ) was added and the reaction mixture stirred at r.t. for $3.5 \mathrm{~h}$. After TLCanalysis (PE/EtOAc, 50/50, v/v) showed complete conversion of the starting material, $\mathrm{MeOH}(80$ $\mathrm{mL}$ ) and $\mathrm{NaOMe}$ were added until the reaction mixture reached $\mathrm{pH} 10$, and the reaction mixture was stirred at r.t. for $18 \mathrm{~h}$. The reaction mixture was neutralised with Dowex $\mathrm{H}^{+}$, filtered, washed with $\mathrm{MeOH}$, and concentrated in vacuo. Purification by column chromatography $\left(\mathrm{CH}_{2} \mathrm{Cl}_{2} / \mathrm{MeOH}\right.$, $100 / 0 \rightarrow 90 / 10, \mathrm{v} / \mathrm{v})$ yielded 82 as a colourless oil (3.43 g, 68\% over four steps) which was crystallised from $\mathrm{Et}_{2} \mathrm{O}$ to yield white crystals. The data obtained for this compound matched literature values. ${ }^{70}$

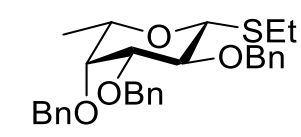

Ethyl 2,3,4-tri- $\boldsymbol{O}$-benzyl- $\boldsymbol{\beta}$ - L-fucopyranoside (8). Thiofucoside 82 (1.13 g, $5.44 \mathrm{mmol})$ was co-evaporated with DMF $(3 \times 50 \mathrm{~mL})$, and for the final coevaporation the solution was partially concentrated leaving ca. $27 \mathrm{~mL}$ DMF. 
The reaction was cooled to $0{ }^{\circ} \mathrm{C}$ under inert atmosphere, benzyl bromide $(2.33 \mathrm{~mL}, 19.6 \mathrm{mmol})$, sodium hydride $(0.849 \mathrm{~g}, 21.2 \mathrm{mmol})$ and TBAI $(100 \mathrm{mg})$ were added at $0{ }^{\circ} \mathrm{C}$ and the reaction mixture was stirred for $17 \mathrm{~h}$. and allowed to warm to r.t. The reaction mixture was quenched with methanol $(100 \mathrm{~mL})$, concentrated in vacuo, dissolved in EtOAc, washed with water and brine, dried $\left(\mathrm{MgSO}_{4}\right)$, filtered and concentrated in vacuo. The residue was purified by silica gel column chromatography (PE/EtOAc, 98/2 $\rightarrow 90 / 10$, v/v) yielded $\mathbf{8}$ as a colourless oil which crystallised upon refrigeration $(2.14 \mathrm{~g}, 82 \%) . \mathrm{R}_{f}=0.36(\mathrm{PE} / \mathrm{EtOAc}, 5 / 1, \mathrm{v} / \mathrm{v}) ; \mathrm{Mp} 51.0-52.1{ }^{\circ} \mathrm{C} ;[\alpha]_{\mathrm{D}}{ }^{24}=$ +6.0 (c= 1.0, $\mathrm{CHCl}_{3}$ ); IR (film) 3088, 3063, 3030, 2977, 2929, 2868, 1497, 1454, 1357, 1208, 1165, 1125, 1089, 1067, 1048, 912, 876, 697, $667 \mathrm{~cm}^{-1} ;{ }^{1} \mathrm{H}$ NMR (500 MHz, $\left.\mathrm{CDCl}_{3}\right) \delta 7.42-$ $7.28\left(\mathrm{~m}, 15 \mathrm{H}, \mathrm{CH}_{\text {arom }}\right), 5.00\left(\mathrm{~d}, 1 \mathrm{H}, J_{C H 2 a, C H 2 b}=11.7 \mathrm{~Hz}, \mathrm{CH}_{2} \mathrm{a} 4-O-\mathrm{Bn}\right), 4.91\left(\mathrm{~d}, 1 \mathrm{H}, J_{C H 2 a, C H 2 b}=\right.$ $\left.10.0 \mathrm{~Hz}, \mathrm{CH}_{2} \mathrm{a} 2-O-\mathrm{Bn}\right), 4.81\left(\mathrm{~d}, 1 \mathrm{H}, J_{\text {CH2a, СН2b }}=10.3 \mathrm{~Hz}, \mathrm{CH}_{2} \mathrm{~b} 2-O-\mathrm{Bn}\right), 4.78$ (d, $1 \mathrm{H}, J_{\text {CH2a,СH2b }}$ $\left.=11.7 \mathrm{~Hz}, \mathrm{CH}_{2} \mathrm{a} 3-O-\mathrm{Bn}\right), 4.75\left(\mathrm{~d}, 1 \mathrm{H}, J_{C H 2 a, \text { СH2b }}=12.0 \mathrm{~Hz}, \mathrm{CH}_{2} \mathrm{~b} 3-O-\mathrm{Bn}\right), 4.71\left(\mathrm{~d}, 1 \mathrm{H}, J_{C H 2 a, \text { СH2b }}\right.$ $\left.=11.7 \mathrm{~Hz}, \mathrm{CH}_{2} \mathrm{~b} 4-O-\mathrm{Bn}\right), 4.40\left(\mathrm{~d}, 1 \mathrm{H}, J_{l, 2}=9.8 \mathrm{~Hz}, \mathrm{H}-1\right), 3.83\left(\mathrm{t}, 1 \mathrm{H}, J_{l, 2}=J_{2,3}=9.5 \mathrm{~Hz}, \mathrm{H}-2\right)$, $3.62\left(\mathrm{~d}, 1 \mathrm{H}, J_{3,4}=2.9 \mathrm{~Hz}, \mathrm{H}-4\right), 3.57\left(\mathrm{dd}, 1 \mathrm{H}, J_{2,3}=9.3 \mathrm{~Hz}, J_{3,4}=2.7 \mathrm{~Hz}, \mathrm{H}-3\right), 3.49\left(\mathrm{q}, 1 \mathrm{H}, J_{5,6}=\right.$ $6.3 \mathrm{~Hz}, \mathrm{H}-5), 2.79$ (dq, 1H, $J_{\text {СH2a, СН } 2 b}=12.5 \mathrm{~Hz}, J_{C H 2 a, C H 3}=7.6 \mathrm{~Hz}, \mathrm{CH}_{2} \mathrm{a} \mathrm{SEt}$ ), 2.72 (dq, $1 \mathrm{H}$, $\left.J_{C H 2 a, C H 2 b}=12.5 \mathrm{~Hz}, J_{C H 2 b, \mathrm{CH} 3}=7.6 \mathrm{~Hz}, \mathrm{CH}_{2} \mathrm{~b} \mathrm{SEt}\right), 1.31\left(\mathrm{t}, 3 \mathrm{H}, J_{\mathrm{CH} 2, \mathrm{CH} 3}=7.6 \mathrm{~Hz}, \mathrm{CH}_{3} \mathrm{SEt}\right), 1.21$ $\left(\mathrm{d}, 3 \mathrm{H}, J_{5,6}=6.3 \mathrm{~Hz}, \mathrm{CH}_{3} \mathrm{H}-6\right) ;{ }^{13} \mathrm{C} \mathrm{NMR}\left(125 \mathrm{MHz}, \mathrm{CDCl}_{3}\right) \delta 138.8,138.6,138.5,128.6,128.4$, 128.3, 128.3, 127.8, 127.8, 127.7, 127.6 ( $\mathrm{C}_{\text {arom }}$ ), 85.1 (C-1), 84.6 (C-3), 78.5 (C-2), 76.6 (C-4), $75.8\left(\mathrm{CH}_{2} 2-O-\mathrm{Bn}\right), 74.7$ (C-5), $74.6\left(\mathrm{CH}_{2} 4-O-\mathrm{Bn}\right), 73.0\left(\mathrm{CH}_{2} 3-\mathrm{O}-\mathrm{Bn}\right), 24.8\left(\mathrm{CH}_{2} \mathrm{SEt}\right), 17.4(\mathrm{C}-$ 6), $15.1\left(\mathrm{CH}_{3} \mathrm{SEt}\right)$; HRMS(ESI) $\mathrm{m} / z$ calcld. for $\left[\mathrm{C}_{29} \mathrm{H}_{34} \mathrm{O}_{4} \mathrm{SNa}\right]^{+}:$501.2076, obsd.: 501.2079.

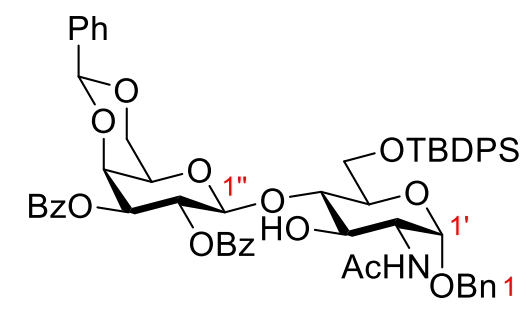

Benzyl 2-acetamido-4- $O$-(2,3-di- $O$-benzoyl-4,6- $O$ benzylidene- $\beta$-D-galactopyranosyl)-2-deoxy-6-O-tertbutyldiphenylsilyl- $\alpha$-D-glucopyranoside (10). Glycosyl acceptor 3 (680 mg, $1.24 \mathrm{mmol}$ ) was co-evaporated with toluene $(3 \times 3 \mathrm{~mL})$ and dissolved in dry $\mathrm{CH}_{2} \mathrm{Cl}_{2}(5 \mathrm{~mL})$. In a separate flask, glycosyl donor $\mathbf{6}(798 \mathrm{mg}, 1.40 \mathrm{mmol})$ was co-evaporated with toluene $(3 \times 3 \mathrm{~mL})$ and dissolved in dry $\mathrm{CH}_{2} \mathrm{Cl}_{2}(10 \mathrm{~mL})$. Activated molsieves (4 $\left.\mathrm{A}\right)$ were added to both the donor and the acceptor solutions, and both solutions were stirred at $\mathrm{rt}$ for $30 \mathrm{~min}$. $\mathrm{N}$-iodosuccinamide $(631 \mathrm{mg}, 2.81 \mathrm{mmol}$ ) was added to the mixture with acceptor 3 and the reaction mixture was cooled to $-50{ }^{\circ} \mathrm{C}$. Freshly distilled $\mathrm{TfOH}(124 \mu \mathrm{L})$ was added at $-50{ }^{\circ} \mathrm{C}$ and the reaction mixture was stirred for $15 \mathrm{~min}$ before adding the solution of donor $\mathbf{6}$. The crude reaction mixture was stirred for $1 \mathrm{~h}$ at $-50^{\circ} \mathrm{C}$ and when TLC analysis showed full conversion of the glycosyl donor, the reaction mixture was quenched by the addition of $\mathrm{NaHCO}_{3}(830 \mathrm{mg})$. The crude reaction mixture was diluted with $\mathrm{CH}_{2} \mathrm{Cl}_{2}(50 \mathrm{~mL})$, washed with sat. aq. $\mathrm{Na}_{2} \mathrm{~S}_{2} \mathrm{O}_{3}(30 \mathrm{~mL})$ and the water layer was extracted with $\mathrm{CH}_{2} \mathrm{Cl}_{2}(3 \times 10 \mathrm{~mL})$. The combined organic layers were dried with $\mathrm{MgSO}_{4}$, filtered and concentrated in vacuo. Purification by silica gel flash column chromatography (PE/EtOAc 
$75 / 25 \rightarrow 100 / 0, \mathrm{v} / \mathrm{v})$ gave disaccharide $10(909 \mathrm{mg}, 73 \%)$ as a white foam. $\mathrm{R}_{f}=0.56$ (EtOAc); $\alpha_{\mathrm{D}}^{17.8}=+91.1$ (c = 1, $\mathrm{CHCl}_{3}$ ); IR (film) 3422, 3382, 3069, 2931, 2889, 2857, 1722, 1668, 1602, 1531, 1452, 1428, 1369, 1315, 1273, 1219, 1177, 1112, 1070, 1040, 1027, 821, 773, 741, $708 \mathrm{~cm}^{-}$ ${ }^{1} ;{ }^{1} \mathrm{H}$ NMR $\left(300 \mathrm{MHz}, \mathrm{CDCl}_{3}\right) \delta 7.97\left(\mathrm{~d}, 2 \mathrm{H}, J_{o-m}=7.3 \mathrm{~Hz}, \mathrm{CH}-o \mathrm{Bz}\right), 7.76-7.66$ (m, 6H, $\mathrm{CH}_{\text {arom}}$ ), $7.55-7.30$ (m, $16 \mathrm{H}, \mathrm{CH}_{\text {arom }}$ ), $7.28-7.14$ (m, $8 \mathrm{H}, \mathrm{CH}_{\text {arom }}$ ), 5.88 (t, $1 \mathrm{H}, J_{1^{\prime \prime}, 2^{\prime \prime}}=J_{2^{\prime \prime}, 3^{\prime \prime}}=J_{2^{\prime \prime}, N H}=8.8$ $\left.\mathrm{Hz}, \mathrm{H}-2^{\prime \prime}\right), 5.64\left(\mathrm{~d}, 1 \mathrm{H}, J_{2^{\prime}, N H}=8.8 \mathrm{~Hz}, \mathrm{NH}\right) 5.52(\mathrm{~s}, 1 \mathrm{H}, \mathrm{CHPh}), 5.31\left(\mathrm{dd}, 1 \mathrm{H}, J_{3^{\prime \prime}, 4^{\prime \prime}}=3.0 \mathrm{~Hz}\right.$, $\left.J_{2^{\prime \prime}, 3^{\prime \prime}}=10.7 \mathrm{~Hz}, \mathrm{H}-3^{\prime \prime}\right), 4.98\left(\mathrm{~d}, 1 \mathrm{H}, J_{I^{\prime \prime}, 2^{\prime \prime}}=8.2 \mathrm{~Hz}, \mathrm{H}-1^{\prime \prime}\right), 4.88\left(\mathrm{~d}, 1 \mathrm{H}, J_{I^{\prime}, 2^{\prime}}=3.2 \mathrm{~Hz}, \mathrm{H}-1^{\prime}\right)$, $4.58\left(\mathrm{~d}, 1 \mathrm{H}, J_{3^{\prime \prime}, 4^{\prime \prime}}=J_{4^{\prime \prime}, 5^{\prime \prime}}=3.0 \mathrm{~Hz}, \mathrm{H}-4^{\prime \prime}\right), 4.50\left(\mathrm{~d}, 1 \mathrm{H}, J_{1 a, 1 b}=12.0 \mathrm{~Hz}, \mathrm{H}-1 \mathrm{a}\right), 4.45\left(\mathrm{~d}, 1 \mathrm{H}, J_{6 a^{\prime}, 6 b^{\prime}}\right.$ $\left.=12.0 \mathrm{~Hz}, \mathrm{H}-6 \mathrm{a}^{\prime}\right), 4.34$ (d, 1H, $\left.J_{1 a, 1 b}=12.0 \mathrm{~Hz}, \mathrm{H}-1 \mathrm{~b}\right), 4.20$ - 4.00 (m, 3H, H-2', H-6b', H-4'), $3.87\left(\mathrm{t}, 1 \mathrm{H}, J_{2^{\prime}, 3^{\prime}}=J_{3^{\prime}, 4^{\prime}}=9.8 \mathrm{~Hz}, \mathrm{H}-3^{\prime}\right), 3.75\left(\mathrm{~d}, 1 \mathrm{H}, J_{6 a^{\prime \prime}, 6 b^{\prime \prime}}=11.6 \mathrm{~Hz}, \mathrm{H}-6 \mathrm{a}^{\prime \prime}\right), 3.64$ (bs, $1 \mathrm{H}, \mathrm{H}-$ $\left.5^{\prime \prime}\right), 3.57\left(\mathrm{~d}, 1 \mathrm{H}, J_{6 a^{\prime \prime}, 6 b^{\prime \prime}}=11.6 \mathrm{~Hz}, \mathrm{H}-6 \mathrm{~b}^{\prime \prime}\right), 3.45$ (d, 1H, $\left.J_{4^{\prime}, 5^{\prime}}=9.9 \mathrm{~Hz}, \mathrm{H}-5^{\prime}\right), 1.99$ (s, 3H, $\mathrm{CH}_{3}$ Ac), $1.08\left(\mathrm{~s}, 9 \mathrm{H}, \mathrm{CH}_{3} t \mathrm{Bu}\right) ;{ }^{13} \mathrm{C} \mathrm{NMR}\left(125 \mathrm{MHz}, \mathrm{D}_{2} \mathrm{O}\right) \delta 170.3(\mathrm{C}=\mathrm{O} \mathrm{Ac}), 166.2(\mathrm{C}=\mathrm{O} 3-O-\mathrm{Bz})$, $165.1(\mathrm{C}=\mathrm{O} 2-O-\mathrm{Bz}), 137.6,137.3,136.1,135.7,134.0,133.6,133.3,133.0,130.1,130.0,129.8$, $129.2,129.1,128.6,128.5,128.3,128.1,128.0,127.8,126.4\left(\mathrm{C}_{\text {arom }}\right), 100.93(\mathrm{CPh}), 100.91(\mathrm{C}-$ $\left.1^{\prime \prime}\right), 96.8$ (C-1'), 79.0 (C-4'), 73.5 (C-4') 72.7 (C-3') 70.7 (C-5'), 70.2 (C-3'), 69.8 (C-1), 69.3 $\left(\mathrm{C}-2^{\prime \prime}\right), 68.6\left(\mathrm{C}-6^{\prime}\right), 67.0\left(\mathrm{C}-5^{\prime \prime}\right), 61.7\left({\left.\mathrm{C}-6^{\prime \prime}\right)}^{\prime}, 53.4\left(\mathrm{C}-2^{\prime}\right), 27.1\left(\mathrm{CH}_{3} t \mathrm{Bu}\right), 23.6\left(\mathrm{CH}_{3} \mathrm{Ac}\right), 19.7\right.$ $(\mathrm{Cq} t \mathrm{Bu}) ; \mathrm{HRMS}(\mathrm{ESI}) \mathrm{m} / z$ calcd. for $\left[\mathrm{C}_{58} \mathrm{H}_{62} \mathrm{NO}_{13} \mathrm{Si}\right]^{+}: 1008.3985$, obsd.: 1008.4009 .

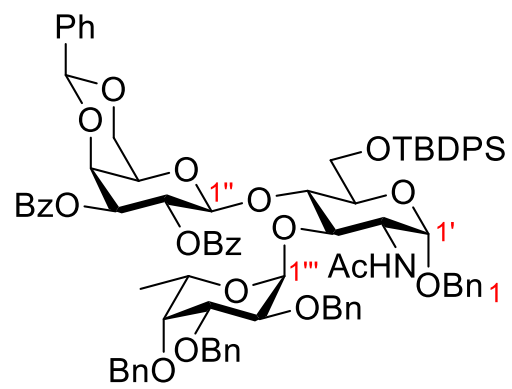

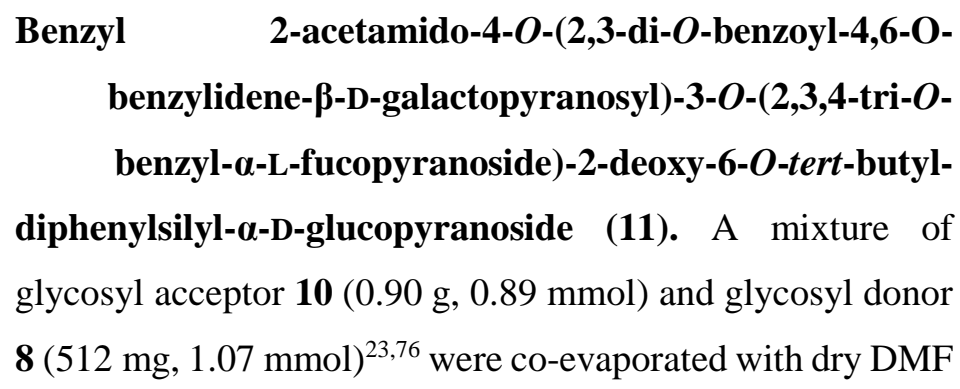
$(3 \times 3 \mathrm{~mL})$ and dissolved in dry DMF $(5 \mathrm{~mL})$ and dry $\mathrm{CH}_{2} \mathrm{Cl}_{2}(10 \mathrm{~mL})$. Activated molsieves $(4 \AA)$ were added and the reaction mixture was stirred at $\mathrm{rt}$ for $30 \mathrm{~min}$. TPABr (570 $\mathrm{mg}, 2.14 \mathrm{mmol})$ and $\mathrm{CuBr}_{2}$ (480 mg, $2.14 \mathrm{mmol}$ ) were added and the reaction mixture was stirred ar rt for $15 \mathrm{~h}$. When TLC analysis showed full conversion of the glycosyl acceptor, the crude reaction mixture was quenched by the addition of sat. aq. $\mathrm{Na}_{2} \mathrm{~S}_{2} \mathrm{O}_{3}(30 \mathrm{~mL})$. The water layer was extracted with $\mathrm{CH}_{2} \mathrm{Cl}_{2}(3 \times 10 \mathrm{~mL})$, and the combined organic layers were washed with brine, dried with $\mathrm{MgSO}_{4}$, filtered and concentrated in vacuo. The residue was purified by silica gel flash column chromatography (PE/EtOAc, 9/1 $\rightarrow$ 2/1, v/v) and crystallised from methanol to afford trisaccharide 11 (1.05 g, $0.74 \mathrm{mmol}, 92 \%)$ as white crystals. $\mathrm{R}_{f}=0.23(\mathrm{PE} / \mathrm{EtOAc}, 2 / 1, \mathrm{v} / \mathrm{v}) ; \alpha_{\mathrm{D}}^{24.4}$ $=+110\left(\mathrm{c}=0.5, \mathrm{CHCl}_{3}\right)$; IR (film) 3088, 3064, 3032, 3007, 2932, 2894, 2859, 1735, 1671, 1497, 1453, 1428, 1367, 1315, 1273, 1250, 1250, 1219, 1165, 1143, 1098, 1061, 1046, 1027, 1002, 772 , 708, $699 \mathrm{~cm}^{-1} ;{ }^{1} \mathrm{H}$ NMR (500 MHz, $\left.\mathrm{CDCl}_{3}\right) \delta 8.05$ (d, 2H, $J_{o, m}=7.7 \mathrm{~Hz}, \mathrm{CH}-o$ Bz), $7.87-7.81$ (m, 4H, $\left.\mathrm{CH}_{\text {arom }}\right), 7.64-7.07\left(\mathrm{~m}, 39 \mathrm{H}, \mathrm{CH}_{\text {arom }}\right), 5.88\left(\mathrm{t}, 1 \mathrm{H}, J_{2^{\prime \prime}, 3^{\prime \prime}}=9.0 \mathrm{~Hz}, \mathrm{C}-2^{\prime \prime}\right), 5.60(\mathrm{~s}, 1 \mathrm{H}$, 
$\mathrm{CHPh}$ ), 5.49 (d, 1H, $\left.J_{2^{\prime}, N H}=9.9 \mathrm{~Hz}, \mathrm{NH}\right), 5.28-5.20$ (m, 3H, H-1"”, H-1"', H-3”'), 4.95 (q, 1H, $\left.J_{5}{ }^{\prime \prime}, 6^{\prime \prime \prime}=6.7 \mathrm{~Hz}, \mathrm{H}-5^{\prime \prime \prime}\right), 4.84-4.77$ (m, 2H, H-1', $\left.\mathrm{CH}_{2}-\mathrm{a} 2^{\prime \prime \prime}-O-\mathrm{Bn}\right), 4.73\left(\mathrm{~d}, 1 \mathrm{H}, J_{a, b}=11.7 \mathrm{~Hz}\right.$, $\mathrm{CH}_{2}$-a 3"'-O-Bn), 4.68 (d, 1H, Ja,b $=11.7 \mathrm{~Hz}, \mathrm{CH}_{2}$-b 3"'-O-Bn), $4.58-4.48$ (m, 4H, H-4"', $\mathrm{CH}_{2}$ b 3'"'-O-Bn, H-2', H-6a'), $4.41-4.31$ (m, 3H, H-1a, H-1b, H-4'), 4.20 (d, 1H, Ja,b $=11.4$ Hz, $\mathrm{CH}_{2}$-a $\left.4^{\prime \prime \prime}-O-\mathrm{Bn}\right), 4.12\left(\mathrm{~d}, 1 \mathrm{H}, J_{6 a^{\prime}, 6 b^{\prime}}=12.1 \mathrm{~Hz}, \mathrm{H}-6 \mathrm{~b}^{\prime}\right), 4.06-4.01\left(\mathrm{~m}, 1 \mathrm{H}, \mathrm{H}-3^{\prime \prime \prime}\right), 3.99$ (t, 1H,

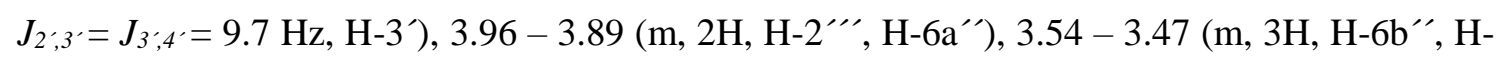
5", $\mathrm{CH}_{2}$-b 4"'-O-Bn), 3.29 (d, 1H, J4,5" $\left.=9.8 \mathrm{~Hz}, \mathrm{C}-5^{\prime}\right), 3.25$ (bs, $\left.1 \mathrm{H}, \mathrm{H}-4^{\prime \prime \prime}\right), 1.81$ (s, $3 \mathrm{H}, \mathrm{CH}_{3}$ $\mathrm{Ac}), 1.33\left(\mathrm{~d}, 3 \mathrm{H}, J_{5}{ }^{\prime \prime}, 6^{\prime \prime}=6.2 \mathrm{~Hz} \mathrm{C}-6 "{ }^{\prime \prime}\right), 1.14\left(\mathrm{~s}, 9 \mathrm{H}, \mathrm{CH}_{3} t \mathrm{Bu}\right) ;{ }^{13} \mathrm{C} \mathrm{NMR}\left(125 \mathrm{MHz}, \mathrm{CDCl}_{3}\right) \delta$ $169.7(\mathrm{C}=\mathrm{O} \mathrm{Ac}), 166.0(\mathrm{C}=\mathrm{O} 3-O \mathrm{Bz}), 164.8(\mathrm{C}=\mathrm{O} 2-O \mathrm{Bz}), 139.9,139.8,138.6,137.8,137.1$, 136.2, 135.6, 134.0, 133.6, 133.2, 132.4, 130.3, 130.1, 130.0, 129.7, 129.2, 129.1, 129.0, 128.7, $128.64,128.55,128.52,128.48,128.45,128.35,128.3,128.23,128.20,128.1,128.0,127.9$, 127.7, 127.6, 127.5, 127.4, 127.1, 126.9, 125.9 (C $\mathrm{C}_{\text {arom }}$ ), 99.9 (C-1"'), 99.8 (CHPh), 97.9 (C-1“"), 96.8 (C-1'), 79.4 (C-4"”), 78.8 (C-3"“), 75.3 (C-2”'), $75.0\left(\mathrm{CH}_{2} 4^{\prime \prime \prime}-\mathrm{O}-\mathrm{Bn}\right), 73.9$ (C-4'), 73.6 (C-4"'), 72.9 (C-3"', $\left.\mathrm{CH}_{2} 2^{\prime \prime \prime}-\mathrm{O}-\mathrm{Bn}\right), 72.7$ (C-3'), $72.0\left(\mathrm{CH}_{2} 3^{\prime \prime \prime}-\mathrm{O}-\mathrm{Bn}\right), 71.3(\mathrm{C}-5), 70.1\left(\mathrm{CH}_{2}\right.$

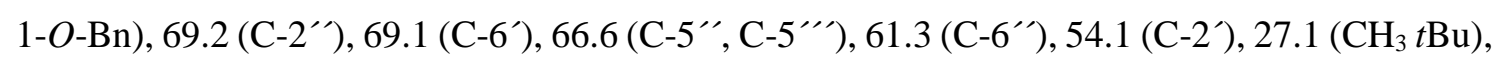
$23.7\left(\mathrm{CH}_{3} \mathrm{Ac}\right), 19.7(\mathrm{Cq} t \mathrm{Bu}), 16.4\left(\mathrm{C}^{-6}{ }^{\prime \prime \prime}\right)$; HRMS(ESI) $\mathrm{m} / z$ calcd. for $\left[\mathrm{C}_{85} \mathrm{H}_{89} \mathrm{NO}_{17} \mathrm{SiNa}\right]^{+}$: 1446.5792, obsd.: 1446.5797 .

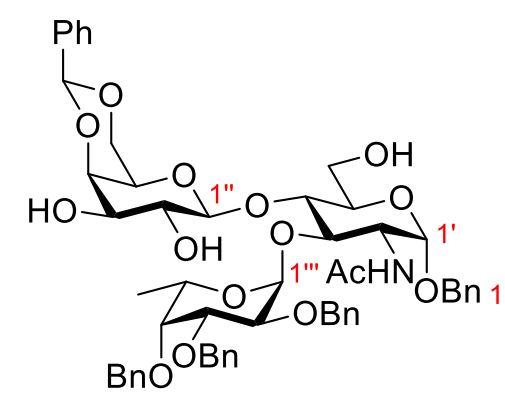

\section{Benzyl 2-acetamido-4- $O$-(4,6-O-benzylidene- $\beta$-D-galacto-} pyranosyl)-3- $O$-(2,3,4-tri- $O$-benzyl- $\alpha$-L-fucopyranosyl)-2deoxy- $\alpha$-D-glucopyranoside (12). To a solution of trisaccharide $11(201 \mathrm{mg}, 141 \mu \mathrm{mol})$ in methanol $(3.0 \mathrm{~mL})$ and $\mathrm{CH}_{2} \mathrm{Cl}_{2}(3.0 \mathrm{~mL}), 1 \mathrm{M}$ methanolic $\mathrm{NaOMe}(2.0 \mathrm{~mL})$ was added and the reaction mixture was stirred at $\mathrm{rt}$ for $4 \mathrm{~h}$. The reaction mixture was quenched by the addition of Dowex $\mathrm{H}^{+}$and the reaction mixture was filtered, washed with $\mathrm{MeOH}$ and concentrated in vacuo. The crude product was used in the next step without further purification. $\quad \mathrm{R}_{f}=0.57$ (PE/EtOAc, 1/1, v/v); HRMS(ESI) $\mathrm{m} / \mathrm{z}$ calcd. for $\left[\mathrm{C}_{71} \mathrm{H}_{81} \mathrm{NO}_{15} \mathrm{SiNa}\right]^{+}:$1238.5268, obsd.: 1238.5276.; The crude trisaccharide was dissolved in pyridine $(5 \mathrm{~mL})$, HF.pyridine $(0.5 \mathrm{~mL})$ was added at $0{ }^{\circ} \mathrm{C}$ and the reaction mixture was stirred ar $\mathrm{rt}$ for $18 \mathrm{~h}$. After TLC analysis showed complete conversion, the mixture was diluted with $\mathrm{CH}_{2} \mathrm{Cl}_{2}$ $(25 \mathrm{~mL})$ and washed with $1 \mathrm{M}$ aq. $\mathrm{Ca}(\mathrm{OAc})_{2}(50 \mathrm{~mL})$. The aqeaous layer was extracted with $\mathrm{CH}_{2} \mathrm{Cl}_{2}(2 \times 25 \mathrm{~mL})$ and the combined organic extracts were washed with brine, dried with $\mathrm{MgSO}_{4}$, filtered and concentrated in vacuo. The residue was co-evaporated with toluene $(3 \times 5$ $\mathrm{mL}$ ) in order to remove traces of pyridine, and purified by silica gel flash column chromatography $($ EtOAc/MeOH, 100/0 $\rightarrow$ 95/5, v/v) to afford triol $12(137 \mathrm{mg}, 140 \mu \mathrm{mol}, 99 \%)$ as a white foam. $\mathrm{R}_{f}=0.45(\mathrm{EtOAc} / \mathrm{MeOH}, 8 / 1, \mathrm{v} / \mathrm{v}), \alpha_{\mathrm{D}}^{24.6}=-24.2\left(\mathrm{c}=0.5, \mathrm{CHCl}_{3}\right) ; \mathrm{IR}($ film) 3428, 3334, 3063, 3031, 2974, 2929, 2906, 2872, 1658, 1543, 1497, 1454, 1397, 1363, 1338, 1246, 1213, 1165 , 
1136, 1093, 1047, 966, 909, 858, 734, $697 \mathrm{~cm}^{-1} ;{ }^{1} \mathrm{H}$ NMR (500 MHz, $\left.\mathrm{CDCl}_{3}\right) \delta 7.48-7.42(\mathrm{~m}$, $\left.2 \mathrm{H}, \mathrm{CH}_{\text {arom }}\right), 7.31-7.14\left(\mathrm{~m}, 22 \mathrm{H}, \mathrm{CH}_{\text {arom }}\right), 7.11$ (t, $\left.1 \mathrm{H}, J=7.6 \mathrm{~Hz}, \mathrm{CH}_{\text {arom }}\right), 7.00$ (t, 2H, J= 7.6 $\left.\mathrm{Hz}, \mathrm{CH}_{\text {arom }}\right), 6.79\left(\mathrm{~d}, 1 \mathrm{H}, J_{N H, 2^{\prime}}=7.2 \mathrm{~Hz}, \mathrm{NH}\right), 5.47(\mathrm{~s}, 1 \mathrm{H}, \mathrm{CHPh}), 5.38\left(\mathrm{~d}, 1 \mathrm{H}, J_{1^{\prime \prime}, 2^{\prime \prime}}=3.2 \mathrm{~Hz}\right.$,

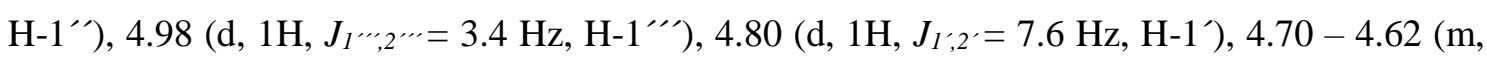
$\left.3 \mathrm{H}, \mathrm{CH}_{2} \mathrm{a} 2-O-\mathrm{Bn}, \mathrm{CH}_{2} \mathrm{a} 3-O-\mathrm{Bn}, \mathrm{CH}_{2} \mathrm{a} 4-O-\mathrm{Bn}\right), 4.60\left(\mathrm{~d}, 1 \mathrm{H}, J_{C H 2 a, C H} b=10.4 \mathrm{~Hz}, \mathrm{CH}_{2} \mathrm{~b} 2-O-\right.$ $\mathrm{Bn}), 4.55\left(\mathrm{~d}, 1 \mathrm{H}, J_{1 a, 1 b}=12.0 \mathrm{~Hz}, \mathrm{CH}_{2} \mathrm{a}-1-O-\mathrm{Bn}\right), 4.52\left(\mathrm{~d}, 1 \mathrm{H}, J_{C H 2 a, C H 2 b}=\mathrm{CH}_{2} \mathrm{~b} 3-O-\mathrm{Bn}\right), 4.35$ $\left(\mathrm{d}, 1 \mathrm{H}, J_{l a, 1 b}=12.0 \mathrm{~Hz}, \mathrm{CH}_{2} \mathrm{~b}-1-O-\mathrm{Bn}\right), 4.31-4.20$ (m, 2H, H-6a"' $\left.\mathrm{H}-5^{\prime \prime \prime}\right), 4.16$ (dd, $J_{2,3}^{\prime}=9.1$ $\left.\mathrm{Hz}, J_{3^{\prime}, 4^{\prime}}=10.2 \mathrm{~Hz}, \mathrm{H}-3^{\prime}\right), 4.10-4.03$ (m, 2H, H-4', H-4'”), 4.00 (bd, $\left.J_{6 a^{\prime}, 6 b^{\prime}}=12.2 \mathrm{~Hz}, \mathrm{H}-6 \mathrm{a}^{\prime}\right)$,

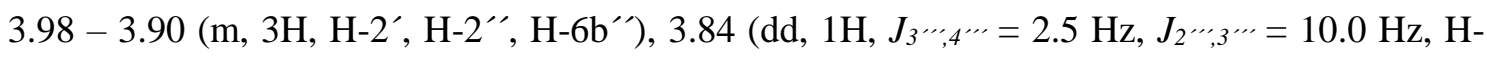
$3^{\prime \prime \prime}$ ), $3.74-3.64$ (m, 3H, H-2”, H-5', H-6b'), 3.59 - 3.51 (m, 2H, H-4"', H-5'”), 3.51 - 3.44 (m, 2H, H-3", OH), 2.85 (bs, 1H, OH), 1.70 (bs, 2H, $2 \times \mathrm{OH}), 1.45$ (s, 3H, $\mathrm{CH}_{3} \mathrm{Ac}$ ), 1.04 (d, 3H, $\left.J_{5}{ }^{\prime \prime}, 6^{\prime \prime}=6.6 \mathrm{~Hz}, \mathrm{H}-6^{\prime \prime \prime}\right) ;{ }^{13} \mathrm{C} \mathrm{NMR}\left(125 \mathrm{MHz}, \mathrm{CDCl}_{3}\right) \delta 170.3(\mathrm{C}=\mathrm{O}, \mathrm{Ac}), 138.9,137.8,137.5$, 137.5 ( $\left.\mathrm{CH}_{\text {arom }}\right), 129.2,128.8,128.6,128.51,128.50,128.33,128.28,128.10,128.06,128.0,127.7$, 127.3, 126.5 ( $\left.\mathrm{CH}_{\text {arom }}\right), 102.3$ (C-1”), 101.1 (CHPh), 98.6 (C-1“”), 96.3 (C-1'), 79.4 (C-3””), 77.7 (C-4"'), 77.5 (C-2”'), 76.7 (C-3'), 75.6 (C-4'), 75.3 (C-4”), $75.1\left(\mathrm{CH}_{2} 4-\mathrm{OBn}\right), 74.7\left(\mathrm{CH}_{2} 2-\right.$

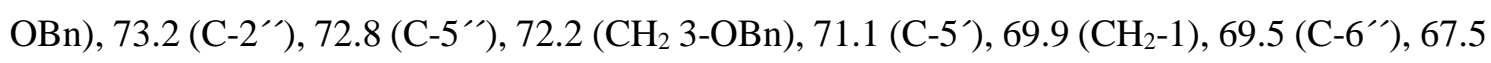
(C-5”'), 67.0 (C-3”), 61.0 (C-6'), 54.1 (C-2'), 22.8 (CH3 Ac), 17.1 (C-6"'); HRMS(ESI) m/z calcd. for $\left[\mathrm{C}_{55} \mathrm{H}_{64} \mathrm{NO}_{15}\right]^{+}$: 978.4270 , obsd.: 978.4274 .

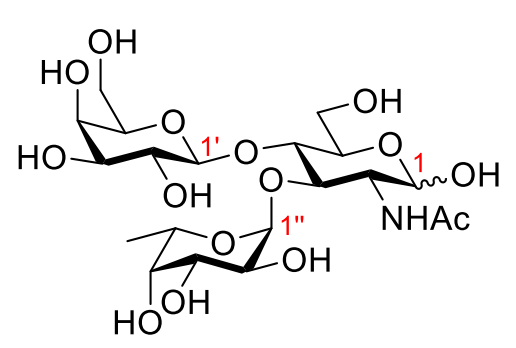

2-Acetamido-2-deoxy-3-O-( $\alpha$-L-fucopyranosyl)-4-O-( $\beta$-Dgalactopyranosyl))- $\alpha / \beta$-D-glucopyranoside $\quad\left(\operatorname{Le}^{\mathrm{x}}\right)$. To trisaccharide $12(137 \mathrm{mg}, 140 \mu \mathrm{mol})$ in distilled THF $(4 \mathrm{~mL})$, $\mathrm{H}_{2} \mathrm{O}(2 \mathrm{~mL}), \mathrm{AcOH}(1.0 \mathrm{~mL})$ and $\mathrm{Pd} / \mathrm{C}(100 \mathrm{mg})$ were added and hydrogen gas was bubbled though the reaction mixture at $\mathrm{rt}$ for $3 \mathrm{~h}$. The crude mixture was diluted with water $(20 \mathrm{~mL})$, filtered over paper (Whatman 42) and lyophilised. Purification by size exclusion chromatography (BioGel P-2, $1200 \times 10 \mathrm{~mm}$ ) gave $\mathrm{Le}^{\mathrm{X}}(72.5 \mathrm{mg} 137 \mu \mathrm{mol}, 98 \%)$ as a white foam. $\mathrm{R}_{f}=0.12$ (n-butanol/AcOH/ $\mathrm{H}_{2} \mathrm{O}, 4 / 1 / 1$, v/v/v); IR (film) 3313, 2971, 2938, 1640, 1555, 1427, 1378, 1162 , $1118,1071,1034,1021,968,811 \mathrm{~cm}^{-1}$; ${ }^{1} \mathrm{H}$ NMR $\left(300 \mathrm{MHz}, \mathrm{D}_{2} \mathrm{O}\right) \underline{\alpha \text {-anomer }}: \delta 5.11-5.08(\mathrm{~m}$, $\left.2 \mathrm{H}, \mathrm{H}-1^{\prime}, \mathrm{H}-1\right), 4.84$ (q, $\left.1 \mathrm{H}, J_{5^{\prime \prime}}, 6^{\prime \prime}=6.7 \mathrm{~Hz}, \mathrm{H}-5^{\prime \prime}\right), 4.46$ (d, $\left.1 \mathrm{H}, J_{I^{\prime}, 2^{\prime}}=7.8 \mathrm{~Hz}, \mathrm{H}-1^{\prime} \alpha\right), 4.15$ (dd, $\left.1 \mathrm{H}, J_{1,2}=3.5 \mathrm{~Hz}, J_{2,3}=10.4 \mathrm{~Hz}, \mathrm{H}-2 \alpha\right), 4.03-3.92$ (m, 4H, H-3, H-4, H-5, H-6a), $3.92-3.81$ (m, 8H, H-6b, H-3", H-4'), 3.79 (d, 1H, $\left.J_{3^{\prime \prime}, 4^{\prime \prime}}=J_{4^{\prime \prime}} 5^{\prime \prime}=3.0 \mathrm{~Hz}, \mathrm{H}-4^{\prime \prime}\right), 3.77-3.62$ (m, 4H, H6’a, H-6’b, H-2”', H-3'), 3.62 - 3.57 (m, 1H, H-5'), 3.53 - 3.46 (m, 1H, H-2'), 2.03 (s, 3H, CH Ac), $1.19-1.15$ (m, 3H, H-6"); ㅅ-anomer: $\delta 5.11-5.08\left(\mathrm{~m}, 1 \mathrm{H}, \mathrm{H}-1^{\prime}\right), 4.84\left(\mathrm{q}, 1 \mathrm{H}, J_{5^{\prime \prime}}, 6^{\prime \prime}=6.7\right.$ Hz, H-5' ), 4.72 (d, 1H, $\left.J_{1,2}=8.1 \mathrm{~Hz}, \mathrm{H}-1 \beta\right), 4.45$ (d, 1H, $\left.J_{1^{\prime}, 2^{\prime}}=7.8 \mathrm{~Hz}, \mathrm{H}-1^{\prime} \beta\right), 4.03-3.92$ (m, 
2H, H-4, H-6a), 3.92 - 3.81 (m, 5H, H-6b, H-3"', H-4', H-2, H-3), 3.79 (d, 1H, $J_{3^{\prime \prime}} 4^{\prime \prime}=J_{4^{\prime \prime}} 5^{\prime \prime}=$ 3.0 Hz, H-4”'), 3.77 - 3.62 (m, 4H, H-6’a, H-6’b, H-2”, H-3’), 3.62 - 3.57 (m, 2H, H-5, H-5'), $3.53-3.46$ (m, 1H, H-2'), 2.03 (s, 6H, $\left.\mathrm{CH}_{3} \mathrm{Ac}\right), 1.19-1.15$ (m, 6H, H-6"); ${ }^{13} \mathrm{C}$ NMR (125 $\left.\mathrm{MHz}, \mathrm{D}_{2} \mathrm{O}\right) \delta 174.4(\mathrm{C}=\mathrm{O} \beta), 174.2(\mathrm{C}=\mathrm{O} \alpha), 101.76\left(\mathrm{C}-1^{\prime} \beta\right), 101.74\left(\mathrm{C}-1^{\prime} \alpha\right), 98.6\left(\mathrm{C}-1^{\prime \prime} \beta\right)$, $98.5\left(\mathrm{C}-1^{\prime \prime} \alpha\right), 94.7$ (C-1 $\left.\beta\right), 91.0(\mathrm{C}-1 \alpha), 75.4(\mathrm{C}-5 \beta), 74.91(\mathrm{C}-3 \beta), 74.85\left(\mathrm{C}-5^{\prime} \alpha+\beta\right), 73.2$ $(\mathrm{C}-4 \alpha+\beta), 72.8(\mathrm{C}-3 \alpha), 72.4\left(\mathrm{C}-3^{\prime} \alpha+\beta\right), 71.8\left(\mathrm{C}-4^{\prime \prime} \alpha+\beta\right), 71.3(\mathrm{C}-5 \alpha), 71.0\left(\mathrm{C}-2^{\prime} \alpha+\beta\right), 69.20$ $\left(\mathrm{C}-3^{\prime \prime} \alpha\right), 69.15\left(\mathrm{C}-3^{\prime \prime} \beta\right), 68.3\left(\mathrm{C}-4^{\prime} \alpha+\beta\right), 67.6\left(\mathrm{C}-2^{\prime \prime} \alpha+\beta\right), 66.6\left(\mathrm{C}-5^{\prime \prime} \alpha+\beta\right), 61.5\left(\mathrm{C}-6^{\prime} \alpha+\beta\right)$, $59.7(\mathrm{C}-6 \beta), 59.6(\mathrm{C}-6 \alpha), 56.9(\mathrm{C}-2 \beta), 54.0(\mathrm{C}-2 \alpha), 22.2\left(\mathrm{CH}_{3} \mathrm{Ac} \beta\right), 21.9\left(\mathrm{CH}_{3} \mathrm{Ac} \alpha\right), 15.2(\mathrm{C}-$ $6^{\prime \prime} \alpha+\beta$ ); HRMS(ESI) $m / z$ calcd. for $\left[\mathrm{C}_{20} \mathrm{H}_{36} \mathrm{NO}_{15}\right]^{+}: 530.2079$, obsd.: 530.2093. 


\subsection{References}

(1) Soejima, M.; Koda, Y. 'Molecular Mechanisms of Lewis Antigen Expression'. Legal Medicine, 2005, 7, 266-269.

(2) Stanley, P.; Cummings, R. D. Structures Common to Different Glycans. In Essentials of Glycobiology. 2nd edition.; 2009.

(3) Schenkel-Brunner, H. 'Blood Group Antigens' Comprehensive Glycoscience; From Chemistry to Systems Biology 2007, 343-372.

(4) Rho, J. H.; Mead, J. R.; Wright, W. S.; Brenner, D. E.; Stave, J. W.; Gildersleeve, J. C.; Lampe, P. D. 'Discovery of Sialyl Lewis A and Lewis X Modified Protein Cancer Biomarkers Using High Density Antibody Arrays' J. Proteomics 2014, 96, 291-299.

(5) Saldova, R.; Wormald, M. R.; Dwek, R. a; Rudd, P. M. 'Glycosylation Changes on Serum Glycoproteins in Ovarian Cancer May Contribute to Disease Pathogenesis.' Dis. Markers 2008, 25, 219-232.

(6) Shiozaki, K.; Yamaguchi, K.; Takahashi, K.; Moriya, S.; Miyagi, T. 'Regulation of Sialyl Lewis Antigen Expression in Colon Cancer Cells by Sialidase NEU4' J. Biol. Chem. 2011, 286, 21052-21061.

(7) Clark, G. F. 'A Role for Carbohydrate Recognition in Mammalian Sperm-Egg Binding' Biochem. Biophys. Res. Commun. 2014, 450, 1195-1203.

(8) Van Kooyk, Y.; Unger, W. W. J.; Fehres, C. M.; Kalay, H.; García-Vallejo, J. J. 'GlycanBased DC-SIGN Targeting to Enhance Antigen Cross-Presentation in Anticancer Vaccines'. OncoImmunology, 2013, 2, e23040.

(9) Appelmelk, B. J.; van Die, I.; van Vliet, S. J.; Vandenbroucke-Grauls, C. M. J. E.; Geijtenbeek, T. B. H.; van Kooyk, Y. 'Cutting Edge: Carbohydrate Profiling Identifies New Pathogens That Interact with Dendritic Cell-Specific ICAM-3-Grabbing Nonintegrin on Dendritic Cells' J. Immunol. 2003, 170, 1635-1639.

(10) Hesse, C.; Ginter, W.; Förg, T.; Mayer, C. T.; Baru, A. M.; Arnold-Schrauf, C.; Unger, W. W. J.; Kalay, H.; van Kooyk, Y.; Berod, L.; Sparwasser, T. 'In Vivo Targeting of Human DC-SIGN Drastically Enhances CD8+ T-Cell-Mediated Protective Immunity' Eur. J. Immunol. 2013, 43, 2543-2553.

(11) Wang, J.; Zhang, Y.; Wei, J.; Zhang, X.; Zhang, B.; Zhu, Z.; Zou, W.; Wang, Y.; Mou, Z.; Ni, B.; Wu, Y. 'Lewis X Oligosaccharides Targeting to DC-SIGN Enhanced AntigenSpecific Immune Response' Immunology 2007, 121, 174-182.

(12) Tacken, P. J.; Ginter, W.; Berod, L.; Cruz, L. J.; Joosten, B.; Sparwasser, T.; Figdor, C. G.; Cambi, A. 'Targeting DC-SIGN via Its Neck Region Leads to Prolonged Antigen Residence in Early Endosomes, Delayed Lysosomal Degradation, and Cross-Presentation' Blood 2011, 118, 4111-4119.

(13) Van Kooyk, Y.; Unger, W. W. J.; Fehres, C. M.; Kalay, H.; García-Vallejo, J. J. 'GlycanBased DC-SIGN Targeting Vaccines to Enhance Antigen Cross-Presentation'. Molecular Immunology, 2013, 55, 143-145.

(14) www.carbosynth.com.

(15) Flitsch, S. L.; Goodridge, D. M.; Guilbert, B.; Revers, L.; Webberley, M. C.; Wilson, I. B. H. 'The Chemoenzymatic Synthesis of Neoglycolipids and Lipid-Linked Oligosaccharides Using Glycosyltransferases' Bioorg. Med. Chem. 1994, 2, 1243-1250.

(16) Matsushita, T.; Nagashima, I.; Fumoto, M.; Ohta, T.; Yamada, K.; Shimizu, H.; Hinou, H.; Naruchi, K.; Ito, T.; Kondo, H.; Nishimura, S.-I. 'Artificial Golgi Apparatus: Globular Protein-like Dendrimer Facilitates Fully Automated Enzymatic Glycan Synthesis.' J. Am. Chem. Soc. 2010, 132, 16651-16656.

(17) Van Kasteren, S. I.; Campbell, S. J.; Serres, S.; Anthony, D. C.; Sibson, N. R.; Davis, B. G. 'Glyconanoparticles Allow Pre-Symptomatic in Vivo Imaging of Brain Disease.' Proc. Natl. Acad. Sci. U. S. A. 2009, 106, 18-23.

(18) Etxebarria, J.; Serna, S.; Beloqui, A.; Martin-Lomas, M.; Reichardt, N. C. 'ThreeDimensional Arrays Using GlycoPEG Tags: Glycan Synthesis, Purification and Immobilisation' Chem. Eur. J. 2013, 19, 4776-4785. 
(19) Lönn, H. 'Synthesis of a Tri- and a Hepta-Saccharide Which Contain A-L-Fucopyranosyl Groups and Are Part of the Complex Type of Carbohydrate Moiety of Glycoproteins' Carbohydr. Res. 1985, 139, 105-113.

(20) Lönn, H. 'Synthesis of a Tetra- and a Nona-Saccharide Which Contain Alpha-LFucopyranosyl Groups and Are Part of the Complex Type of Carbohydrate Moiety of Glycoproteins.' Carbohydr. Res. 1985, 139, 115-121.

(21) Stahl, W.; Sprengard, U.; Kretzschmar, G.; Schmidt, D. W.; Kunz, H. 'Synthesis of Sulfated Le ${ }^{\mathrm{x}}$-Trisaccharides' J. für Prakt. Chemie/Chemiker-Zeitung 1995, 337, 441-445.

(22) Kiyoi, T.; Nakai, Y.; Kondo, H.; Ishida, H.; Kiso, M.; Hasegawa, A. 'A Highly Practical Synthesis of the Sialyl Lewis X Pentasaccharide and an Investigation of Binding to E-, P, and L-Selectins' Bioorg. Med. Chem. 1996, 4, 1167-1176.

(23) Hendel, J. L.; Wang, J.-W.; Jackson, T. a; Hardmeier, K.; De los Santos, R.; Auzanneau, F.-I. 'How the Substituent at O-3 of N-Acetylglucosamine Impacts Glycosylation at O-4: A Comparative Study.' J. Org. Chem. 2009, 74, 8321-8331.

(24) Shimizu, H.; Yoshimura, Y.; Hinou, H.; Nishimura, S.-I. 'A New Glycosylation Method Part 3: Study of Microwave Effects at Low Temperatures to Control Reaction Pathways and Reduce Byproducts' Tetrahedron 2008, 64, 10091-10096.

(25) Wang, J. W.; Asnani, A.; Auzanneau, F. I. 'Synthesis of a BSA-Le ${ }^{\mathrm{x}}$ Glycoconjugate and Recognition of $\mathrm{Le}^{\mathrm{x}}$ Analogues by the Anti-Le ${ }^{\mathrm{x}}$ Monoclonal Antibody SH1: The Identification of a Non-Cross Reactive Analogue' Bioorgan. Med. Chem. 2010, 18, 7174 7185.

(26) Gege, C.; Geyer, A.; Schmidt, R. R. 'Carbohydrate-Carbohydrate Recognition Between Lewis X Blood Group Antigens, Mediated by Calcium Ions' Eur. J. Org. Chem. 2002, 2002, 2475.

(27) Gege, C.; Geyer, A.; Schmidt, R. R. 'Synthesis and Molecular Tumbling Properties of Sialyl Lewis X and Derived Neoglycolipids' Chem. Eur. J. 2002, 8, 2454-2463.

(28) Crich, D.; Dudkin, V. 'Why Are the Hydroxy Groups of Partially Protected N Acetylglucosamine Derivatives Such Poor Glycosyl Acceptors, and What Can Be Done about It? A Comparative Study of the Reactivity of N -Acetyl-, N -Phthalimido-, and 2Azido-2-Deoxy-Glucosamine Derivativ' J. Am. Chem. Soc. 2001, 123, 6819-6825.

(29) Gege, C.; Oscarson, S.; Schmidt, R. 'Synthesis of Fluorescence Labeled Sialyl Lewis ${ }^{\mathrm{X}}$ Glycosphingolipids' Tetrahedron Lett. 2001, 14, 5105-5110.

(30) Mukhopadhyay, B.; Maurer, S. V; Rudolph, N.; van Well, R. M.; Russell, D. A.; Field, R. A. 'From Solution Phase to "on-Column" Chemistry: Trichloroacetimidate-Based Glycosylation Promoted by Perchloric Acid-Silica.' J. Org. Chem. 2005, 70, 9059-9062.

(31) Baumann, K.; Kowalczyk, D.; Kunz, H. 'Total Synthesis of the Glycopeptide Recognition Domain of the P-Selectin Glycoprotein Ligand 1.' Angew. Chem. Int. Ed. Engl. 2008, 47, 3445-3449.

(32) Vohra, Y.; Buskas, T.; Boons, G.-J. 'Rapid Assembly of Oligosaccharides: A Highly Convergent Strategy for the Assembly of a Glycosylated Amino Acid Derived from PSGL-1.' J. Org. Chem. 2009, 74, 6064-6071.

(33) De la Fuente, J. M.; Penadés, S. 'Synthesis of Lex-Neoglycoconjugate to Study Carbohydrate-carbohydrate Associations and Its Intramolecular Interaction' Tetrahedron Assymetr. 2002, 13, 1879-1888.

(34) De Paz, J. L.; Ojeda, R.; Barrientos, Á. G.; Penadés, S.; Martín-Lomas, M. 'Synthesis of a Le $^{\mathrm{y}}$ Neoglycoconjugate and Le ${ }^{\mathrm{y}}$ - Functionalized Gold Glyconanoparticles' Tetrahedron Assymetr. 2005, 16, 149-158.

(35) Vohra, Y.; Vasan, M.; Venot, A.; Boons, G.-J. 'One-Pot Synthesis of Oligosaccharides by Combining Reductive Openings of Benzylidene Acetals and Glycosylations.' Org. Lett. 2008, 10, 3247-3250.

(36) Nicolaou, K. C.; Caulfield, T. J.; Kataoka, H.; Stylianides, N. A. 'Total Synthesis of the Tumor-Associated Le ${ }^{\mathrm{X}}$ Family of Glycosphingolipids' J. Am. Chem. Soc. 1990, 112, 3693-3695.

(37) Nicolaou, K. C.; Hummel, C. W.; Bockovich, N. J.; Wong, C.-H. 'Stereocontrolled Synthesis of Sialyl Lex, the Oligosaccharide Binding Ligand to ELAM-1 (sialyl =NAcetylneuramin)'. J. Chem. Soc. Chem. Comm., 1991, 870. 
(38) Wang, A.; Auzanneau, F.-I. 'Synthesis of Le ${ }^{a} e^{x}$ Oligosaccharide Fragments and Efficient One-Step Deprotection.' Carbohydr. Res. 2010, 345, 1216-1221.

(39) Guillemineau, M.; Auzanneau, F. 'Challenging Deprotection Steps during the Synthesis of Tetra- and Pentasaccharide Fragments of the Le ${ }^{\mathrm{a}} \mathrm{Le}^{\mathrm{x}}$ Tumor-Associated Hexasaccharide Antigen.' J. Org. Chem. 2012, 77, 8864-8878.

(40) Wang, A.; Hendel, J.; Auzanneau, F.-I. 'Convergent Syntheses of Le Analogues.' Beilstein J. Org. Chem. 2010, 6, 17.

(41) Hendel, J. L.; Auzanneau, F.-I. 'Convergent Preparation of DimLe ${ }^{\mathrm{x}}$ Hexasaccharide Analogues' Eur. J. Org. Chem. 2011, 2011, 6864-6876.

(42) Titz, A.; Marra, A.; Cutting, B.; Smieško, M.; Papandreou, G.; Dondoni, A.; Ernst, B. 'Conformational Constraints: Nature Does It Best with Sialyl Lewis X' Eur. J. Org. Chem. 2012, 2012, 5534-5539.

(43) Pazynina, G. V.; Sablina, M. A.; Tuzikov, A. B.; Chinarev, A. A.; Bovin, N. V. 'Synthesis of Complex a2-3 Sialooligosaccharides, Including Sulfated and Fucosylated Ones, Using Neu5Aca2-3Gal as a Building Block' Mendeleev Commun. 2003, 13, 245-248.

(44) Herzner, H.; Kunz, H. 'Spacer-Separated Sialyl LewisX Cyclopeptide Conjugates as Potential E-Selectin Ligands.' Carbohydr. Res. 2007, 342, 541-557.

(45) Yamaguchi, M.; Ishida, H.; Galustian, C.; Feizi, T.; Kiso, M. 'Synthesis and SelectinBinding Activity of N-Deacetylsialyl Lewis X Ganglioside.' Carbohydr. Res. 2002, 337, 2111-2117.

(46) Yamaguchi, M.; Ishida, H.; Kanamori, A.; Kannagi, R.; Kiso, M. 'Studies on the Endogenous L-Selectin Ligands: Systematic and Highly Efficient Total Synthetic Routes to Lactamized-Sialyl 6-O-Sulfo Lewis X and Other Novel Gangliosides Containing Lactamized Neuraminic Acid.' Carbohydr. Res. 2003, 338, 2793-2812.

(47) Hanashima, S.; Castagner, B.; Esposito, D.; Nokami, T.; Seeberger, P. H. 'Synthesis of a Sialic Acid alpha(2-3) Galactose Building Block and Its Use in a Linear Synthesis of Sialyl Lewis X.' Org. Lett. 2007, 9, 1777-1779.

(48) Schlegel, M. K.; Hütter, J.; Eriksson, M.; Lepenies, B.; Seeberger, P. H. 'Defined Presentation of Carbohydrates on a Duplex DNA Scaffold.' Chembiochem 2011, 12, 2791-2800.

(49) Gan, Z.; Cao, S.; Wu, Q.; Roy, R. 'Regiospecific Syntheses of N -Acetyllactosamine Derivatives and Application Toward a Highly Practical Synthesis of Lewis X Trisaccharide' J. Carbohydr. Chem. 1999, 18, 755-773.

(50) Xia, J.; Srikrishnan, T.; Alderfer, J. L.; Jain, R. K.; Piskorz, C. F.; Matta, K. L. 'Chemical Synthesis of Sulfated Oligosaccharides with a Beta-D-Gal-(1-->3).' Carbohydr. Res. 2000, 329, 561-577.

(51) Miermont, A.; Zeng, Y.; Jing, Y.; Ye, X. S.; Huang, X. 'Syntheses of Lewis ${ }^{\mathrm{X}}$ and Dimeric Lewis $\mathrm{X}$ : Construction of Branched Oligosaccharides by a Combination of Preactivation and Reactivity Based Chemoselective One-Pot Glycosylations' J. Org. Chem. 2007, 72, 8958-8961.

(52) Tanaka, H.; Matoba, N.; Tsukamoto, H.; Takimoto, H.; Yamada, H.; Takahashi, T. 'Automated Parallel Synthesis of a Protected Oligosaccharride Library Based upon the Structure of Dimeric Lewis X by One-Pot Sequential Glycosylation' Synlett 2005, 824828.

(53) Tanaka, H.; Ishida, T.; Matoba, N.; Tsukamoto, H.; Yamada, H.; Takahashi, T. 'Efficient Polymer-Assisted Strategy for the Deprotection of Protected Oligosaccharides.' Angew. Chem. Int. Ed. Engl. 2006, 45, 6349-6352.

(54) Cao, H.; Huang, S.; Cheng, J.; Li, Y.; Muthana, S.; Son, B.; Chen, X. 'Chemical Preparation of Sialyl Lewis X Using an Enzymatically Synthesized Sialoside Building Block.' Carbohydr. Res. 2008, 343, 2863-2869.

(55) Hudak, J. E.; Yu, H. H.; Bertozzi, C. R. 'Protein Glycoengineering Enabled by the Versatile Synthesis of Aminooxy Glycans and the Genetically Encoded Aldehyde Tag.' $J$. Am. Chem. Soc. 2011, 133, 16127-16135.

(56) Xia, J.; Alderfer, J. L.; Locke, R. D.; Piskorz, C. F.; Matta, K. L. 'Complex Oligosaccharide Investigations: Synthesis of an Octasaccharide Incorporating the Dimeric Le $^{x}$ Structure of PSGL-1.' J. Org. Chem. 2003, 68, 2752-2759. 
(57) Daly, R.; McCabe, T.; Scanlan, E. M. 'Development of Fully and Partially Protected Fucosyl Donors for Oligosaccharide Synthesis.' J. Org. Chem. 2013, 78, 1080-1090.

(58) Kopitzki, S.; Dilmaghani, K. A.; Thiem, J. 'Synthesis of Benzaldehyde-Functionalized LewisX Trisaccharide Analogs for Glyco-SAM Formation' Tetrahedron 2013, 69, 1062110636.

(59) Mandal, P. K.; Turnbull, W. B. 'Studies on the Synthesis of Lewis-Y Oligosaccharides.' Carbohydr. Res. 2011, 346, 2113-2120.

(60) Zhang, Y.; Dong, D.; Qu, H.; Sollogoub, M.; Zhang, Y. 'Regio- and Stereocontrolled Synthesis of 2d-Deoxy Lewisx Pentasaccharide' Eur. J. Org. Chem. 2011, 2011, 71337139.

(61) Yang, B.; Jing, Y.; Huang, X. 'Fluorous-Assisted One-Pot Oligosaccharide Synthesis.' Eur. J. Org. Chem. 2010, 2010, 1290-1298.

(62) Xia, J.; Alderfer, J. L.; Piskorz, C. F.; Matta, K. L. 'The 2-Naphthylmethyl (NAP) Group in Carbohydrate Synthesis: First Total Synthesis of the GlyCAM-1 Oligosaccharide Structures.' Chemistry 2001, 7, 356-367.

(63) Lu, D.; Hu, Y.; He, X.; Sollogoub, M.; Zhang, Y. 'Total Synthesis of a Sialyl Lewis ${ }^{\mathrm{x}}$ Derivative for the Diagnosis of Cancer.' Carbohydr. Res. 2014, 383, 89-96.

(64) Nishida, Y.; Tsurumi, T.; Sasaki, K.; Watanabe, K.; Dohi, H.; Kobayashi, K. 'Design and Synthesis of C3-Symmetric Lewis ${ }^{\mathrm{X}}$ Antigen' Org. Lett. 2003, 5, 3775-3778.

(65) Mukherjee, D.; Sarkar, S. K.; Chattopadhyay, P.; Shankar Chowdhury, U. 'Synthesis of Two Lewis $\mathrm{X}$ Trisaccharides Using Regiospecific Glycosylation Reactions' J. Carbohydr. Chem. 2005, 24, 251-259.

(66) Guillemineau, M.; Auzanneau, F.-I. 'Matched and Mismatched Acceptor/donor Pairs in the Glycosylation of a Trisaccharide Diol Free at O-3 of Two N-Acylated Glucosamine Residues.' Carbohydr. Res. 2012, 357, 132-138.

(67) Gallo-Rodriguez, C.; Gil-Libarona, M. A.; Mendoza, V. M.; de Lederkremer, R. M. 'Synthesis of B-D-Galp- $(1 \rightarrow 3)-B-D-G a l p-(1 \rightarrow 6)-[\beta-D-G a l f-(1 \rightarrow 4)]-D-G l c N A c, \quad$ a Tetrasaccharide Component of Mucins of Trypanosoma Cruzi' Tetrahedron 2002, 58, 9373-9380.

(68) Dangerfield, E. M.; Cheng, J. M. H.; Knight, D. A.; Weinkove, R.; Dunbar, P. R.; Hermans, I. F.; Timmer, M. S. M.; Stocker, B. L. 'Species-Specific Activity of Glycolipid Ligands for Invariant NKT Cells' Chembiochem 2012, 13, 1349-1356.

(69) Bera, S.; Linhardt, R. J. 'Design and Synthesis of Unnatural Heparosan and Chondroitin Building Blocks' J. Org. Chem. 2011, 76, 3181-3193.

(70) Ruttens, B.; Kováč, P. 'A Facile Synthesis of Armed and Disarmed Colitose Thioglycosides' Synthesis (Stuttg). 2004, 2505-2508.

(71) Pudelko, M.; Kowalczyk, D.; Kunz, H. 'Regioselective Deacetylation and Glycosylation in the Synthesis of the Sialyl lewis0 X Tetrasaccharide, a Key Component of the Recognition Site of PSGL-1' Synlett 2010, 2010, 3023-3026.

(72) Misra, A. K.; Ding, Y.; Lowe, J. B.; Hindsgaul, O. 'A Concise Synthesis of the 6-O- and 6'-O-Sulfated Analogues of the Sialyl Lewis X Tetrasaccharide' Bioorg. Med. Chem. Lett. 2000, 10, 1505-1509.

(73) Kuhn, R.; Baer, H. H.; Seeliger, A. 'Zur Methylierung Von N-AcetylglucosaminDerivaten' Justus Liebigs Ann. Chem. 1958, 611, 236-241.

(74) Wasonga, G.; Tatara, Y.; Kakizaki, I.; Huang, X. 'Synthesis of N-Acetyl Glucosamine Analogs as Inhibitors for Hyaluronan Biosynthesis' J. Carbohydr. Chem. 2013, 32, 392409.

(75) Tiwari, P.; Misra, A. K. 'Acylation of Carbohydrates over $\mathrm{Al}_{2} \mathrm{O}_{3}$ : Preparation of Partially and Fully Acylated Carbohydrate Derivatives and Acetylated Glycosyl Chlorides' Carbohydr. Res. 2006, 341, 339-350.

(76) Calosso, M.; Charpentier, D.; Vaillancourt, M.; Bencheqroun, M.; St-Pierre, G.; Wilkes, B. C.; Guindon, Y. 'A New Approach to Explore the Binding Space of PolysaccharideBased Ligands: Selectin Antagonists' ACS Med. Chem. Lett. 2012, 3, 1045-1049.

(77) Bubb, W. A. 'NMR Spectroscopy in the Study of Carbohydrates: Characterizing the Structural Complexity' Concepts Magn. Reson. Part A Bridg. Educ. Res. 2003, 19, 1-19. 
(78) Lemieux, R. U.; Hendriks, K. B.; Stick, R. V; James, K. 'Halide Ion Catalyzed Glycosidation Reactions. Syntheses of .alpha.-Linked Disaccharides' J. Am. Chem. Soc. 1975, 97, 4056-4062.

(79) Nagai, Y.; Ito, N.; Sultana, I.; Sugai, T. 'Regio- and Chemoselective Manipulation under Mild Conditions on Glucosamine Derivatives for Oligosaccharide Synthesis and Its Application toward N-Acetyl-D-Lactosamine and Lewis X Trisaccharide' Tetrahedron 2008, 64, 9599-9606.

(80) Anwer, M.; Sherman, D. 'Applications of Ammonium Formate Catalytic Transfer Hydrogenation. 6. Analysis of Catalyst, Donor Quantity, and Solvent Effects upon the Efficacy of Dechlorination' J. Org. Chem. 1989, 1289, 1284-1289. 


\section{Chapter 5.}

\section{The synthesis and biological evaluation of fluorescent Lewis $^{\mathrm{x}}$ glycodendrons to target DC-SIGN lectins on macrophages.}

\subsection{Introduction}

When monocytes migrate into the tissue in response to pathogenic infection or inflammation, they differentiate into macrophages (M $\phi s$ ) and dendritic cells (DCs). Here, the upregulation of pathogen recognising receptors (PRRs), such as Toll-like receptors (TLRs) and C-type Lectin receptor (CLRs), is essential in modulating the immune responses. ${ }^{1,2}$ In particular, signaling through the C-type lectin Dendritic Cell-Specific Intercellular adhesion molecule-3-Grabbing Non-integrin (DC-SIGN) on DCs and $\mathrm{M} \phi \mathrm{s}$, can result in the internalisation of the pathogen via endocytosis and antigen processing, which can lead to immune activation against the pathogen through major histocompatibility complex class II (MHC-II)-mediated antigen presentation to CD4 ${ }^{+}$T-cells. ${ }^{3}$ DC-SIGN binds several carbohydrate ligands, including high mannose and Lewis glycans such as the trisaccharide Lewis ${ }^{\mathrm{X}}{ }^{4}$

Viruses, such as human immunodeficiency virus (HIV), Ebola and Hepatitis C, however, can also use the DC-SIGN-mediated internalisation to invade and infect the host. In this context, the virus can use the glycosylation mechanism of the host cell to glycosylate their viral proteins in order to prevent immune detection. The highly glycosylated proteins form a multivalent display of native ligands which can then efficiently bind to host cells, and allow for the subsequent internalisation of the virus by the host. ${ }^{5}$ 
In a similar fashion, glycan-based multivalent substances can be utilised to target lectins on specific cells. These agents have the potential to be developed into novel therapeutics and may be employed in vaccination strategies. Accordingly, the objective of this research is to selectively target DCs and M $\phi s$ through DC-SIGN by using highly glycosylated Lewis antigen glycodendrons. In particular, the use of fluorescent glycodendrons would allow for the biological evaluation of DC-SIGN ${ }^{+}$cells as a flow cytometry marker and demonstrate the potential of the Lewis ${ }^{\mathrm{X}}$ glycodendron for other biological applications.

\subsubsection{DC-SIGN expression on subsets of macrophages}

When monocytes migrate from the blood into the tissue, they differentiate into either DCs or M $\phi \mathrm{s}$ depending on the presence or absence of specific cytokines. ${ }^{6} \mathrm{M} \phi$ s can differentiate into either 'M1-like' or 'M2-like' macrophages, whereby these descriptors represent the two general subsets of $M \phi s^{7,8}$ Herein, it should also be noted that $M \phi s$ exhibit great plasticity and can change phenotype depending on their local environment.

'M1-like' macrophages are characterised as pro-inflammatory macrophages as they produce antimicrobial cytotoxic cytokines, and can activate T-cells in a TH1 mediated pro-inflammatory response. ${ }^{9}$ Polarisation into 'M1-like' M $\phi$ s is induced by lipopolysaccharide (LPS), interferon- $\gamma$ (IFN- $\gamma$ ), tumour necrosis factor- $\alpha$ (TNF- $\alpha$ ) and granulocyte macrophage-colony stimulating factor (GM-CSF). ${ }^{7,10}$ On the other hand, 'M2-like' M $\phi$ s are immunosuppressive cells and are distinguished by the abundant expression of scavenger receptors, such as the mannose receptor. ${ }^{7,9}$ 'M2-like' M $\phi$ s can participate in tissue repair, and promote a TH2 immune response. ${ }^{9} \mathrm{M} \phi \mathrm{s}$ can be polarised into 'M2-like' M $\phi$ s by stimulation with Interleukin-4 (IL-4), IL-10, IL-13, IL-33, transforming growth factor- $\beta$ (TGF- $\beta$ ) and macrophage-colony stimulating factor (M-CSF). ${ }^{11}$

DC-SIGN expression is highly upregulated upon stimulation with M-CSF, and as a result, the receptor can be found in high concentrations on 'M2-like' macrophages, whereas the expression on the 'M1-like' phenotype is minimal. ${ }^{12}$ Moreover, DC-SIGN is expressed on tumour associated macrophages (TAM), which contributes to tumour growth, survival and metastasis, as well as immune suppression against the tumour. ${ }^{12}$ In tumour environments, M-CSF is highly upregulated which causes 'M2-like' polarisation and $\mathrm{M} \phi$ recruitment that stimulates pro-tumourigenic activities such as blood vessel preparation. This makes DC-SIGN an attractive target for anti-tumour therapeutics. 


\subsubsection{THP-1 derived DCs and Macrophages}

Puig-Kröger et al. reported on the DC-SIGN expression of the human THP-1 leukemic cells, monocytes and M $\phi s .{ }^{13}$ These THP-1 leukemic cells are widely used in monocyte-macrophage differentiation and serve as a model to study macrophages. Stimulation of these THP-1 cells with phorbol-12-myristate-13-acetate (PMA) results in the differentiation of these monocytes into M $\phi s$. Stimulation with PMA+IL-4 results in 'M2-like' M $\phi s$, whereas PMA+IFN- $\gamma+L P S$ stimulation gives rise to 'M1-like' M $\phi$ s. Thus, by changing the stimuli that THP-1 cells are exposed to in solution, different $\mathrm{M} \phi$-phenotypes can be generated.

Puig-Kröger et al. observed that THP-1 cells, as well as PMA-stimulated cells, have low expression levels of DC-SIGN, however, when THP-1 cells were differentiated with PMA and IL-4, these leukocytes produced high levels of DC-SIGN, comparable to the DC-SIGN transfected K562-cells. In these studies, the pro-inflammatory 'M1-like' M $\phi$ s were not examined for DC-SIGN expression.

\subsubsection{Development of DC-SIGN-mediated therapeutics}

As was discussed in section 1.6.5, several glycoconjugates have been synthesised to target the DC-SIGN lectin on DCs and $\mathrm{M} \phi \mathrm{s}$, including glycodendrons and glycoproteins. For example, Wong and co-workers synthesised high-mannose glycodendrons in the development of a novel DC-SIGN-mediated vaccine. ${ }^{14}$ They showed that these glycodendrons could bind DC-SIGN and inhibit the interaction between gp120 on HIV and DC-SIGN on DCs, and thus possibly prevent HIV infection. Moreover, Davies and co-workers synthesised a glycodendrinanoparticle containing 1620 mannose antigens to prevent HIV infection by blocking the DC-SIGN receptor and demonstrated that these nanoparticles could completely prevent HIV infection. ${ }^{15}$

Van Kooyk and co-workers have also reported the synthesis of several PAMAM-based glycodendrons through the use of reductive amination methodology. ${ }^{16}$ In particular, glycodendrons bearing the Lewis ${ }^{\mathrm{X}}$ antigens have been prepared and shown to have high affinity for DC-SIGN. ${ }^{16}$ These glycodendrons were used to target DC-SIGN selectively and, in addition, can be used to prevent HIV infection through DC-SIGN. Moreover, DC-SIGN could be targeted using glycodendrons bearing immunogenic peptide antigens for the development of novel vaccines. ${ }^{17}$ Taken as a whole, Lewis antigen glycodendrons show great promise in the development of novel therapeutics, and accordingly there is a need for the rapid assembly of these glycoconjugates. 


\subsubsection{Assembly of Lewis ${ }^{\mathrm{X}}$-glycodendrons}

As described above, the DC-SIGN lectin is an interesting target in the development of novel therapeutics. Several synthetic strategies have been reported for the synthesis of DC-SIGN binding glycodendrons, however, further development of these strategies can result in better substrates that target DC-SIGN. Besides the development of DC-SIGN mediated vaccines, the selective targeting of DC-SIGN on tumour associated macrophages has great potential in the treatment of many types of cancers. ${ }^{18}$ With Lewis glycans being excellent substrates for the DCSIGN lectin, and by increasing binding affinity for these glycans through the use of multivalent glycodendron scaffolds, it was envisioned that multivalent Lewis ${ }^{\mathrm{X}}$ glycodendrons could bind DCSIGN $^{+}$cells with high selectivity. As discussed in Chapter 3, the oxyamine ligation strategy was utilised to conjugate $\mathrm{N}$-acetylglucosamine to multivalent substrates such as a biotinylated dendron scaffold, to obtain highly glycosylated dendrons. Accordingly, it was proposed to synthesise a fluorescent Lewis ${ }^{\mathrm{x}}$ glycodendron $\mathbf{1}$ by conjugating the Lewis ${ }^{\mathrm{x}}$ glycan $\mathbf{2}$ to a fluorescent second generation dendron 3 via the use of a bi-functional oxyamine linker 4 (Scheme 1). The synthesis of these three individual components was discussed in Chapters 2, 3 and 4.
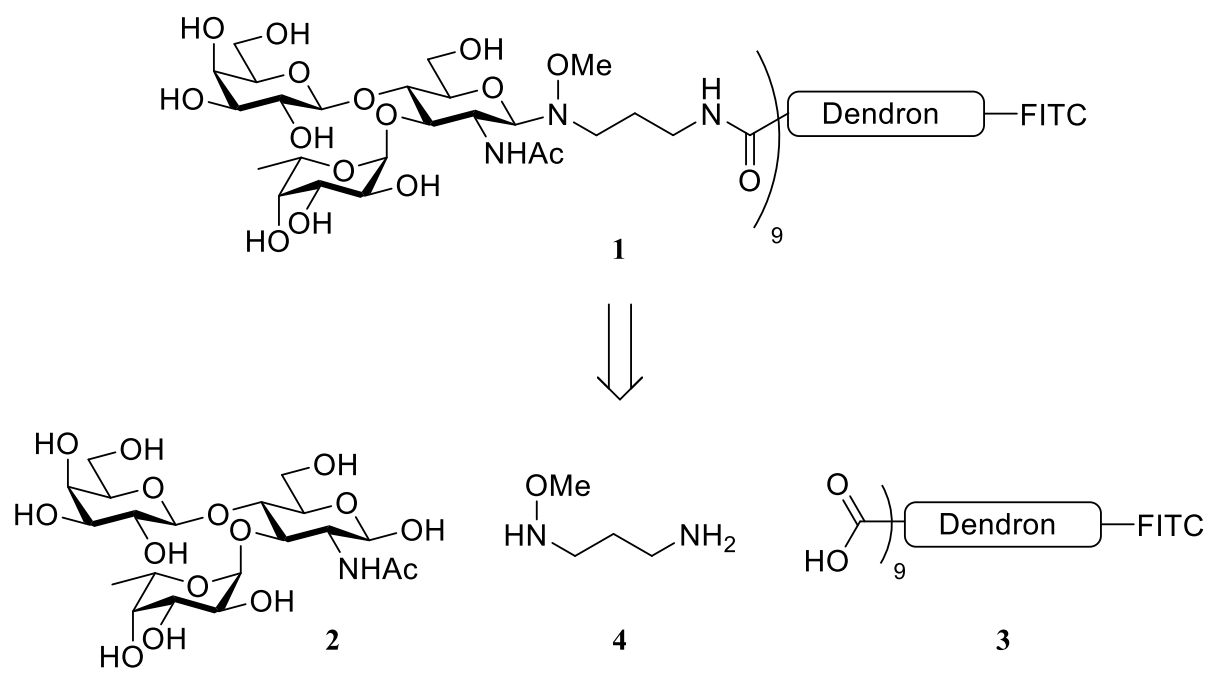

Scheme 1. Retrosynthesis of fluorescent multivalent Lewis ${ }^{X}$ glycodendron 1.

The fluorescent glycodendron 1 can then be used in the detection of DC-SIGN ${ }^{+}$cells via flow cytometry assays, while using the anti-DC-SIGN antibody as a positive control and unglycosylated dendron $\mathbf{3}$ as a negative control. First, the glycodendron will be tested against various types of human THP-1 derived M $\phi$ s ('M0-like', 'M1-like' and 'M2-like'), where the 'M2like' M $\phi$ s should show high DC-SIGN expression, and the other subsets have lower DC-SIGN levels. The specificity for DC-SIGN-mediated binding will be examined via the co-staining with the fluorescent anti-DC-SIGN-antibody. These results will indicate if these fluorescent 
glycodendrons are suitable as flow cytometry reagents for DC-SIGN ${ }^{+}$cells. Moreover, these fluorescent glycodendrons can be utilised to study DC-SIGN-mediated internalisation, which may give a better insight into the biological functions of this lectin, but could also lead to the further development of DC-SIGN-mediated therapeutics, such as vaccines and anti-tumourigenic agents. To achieve this, alteration of the Lewis ${ }^{\mathrm{X}}$ glycodendron with antigenic peptides instead of fluorophores may result in the DC-SIGN-mediated internalisation by DCs and M $\phi$ s followed by the antigen presentation to $\mathrm{CD} 4^{+} \mathrm{T}$-cells, a desired effect in vaccination strategies. In a similar fashion, the synthesis of glycodendrons bearing toxins may find its use in the depletion of tumour associated M $\phi \mathrm{s}$. 


\subsection{Results and discussion}

\subsubsection{Glycodendron synthesis}

As described in Chapter 2, the conjugation of natural glycan antigens with the substrate of choice can be achieved using bi-functional oxyamine linkers. These reactions can be performed under buffered conditions without the use of protecting groups and, depending on the type of linker used, allow for the rapid assembly of a variety of glycoconjugates. For the assembly of the target glycodendron, Lewis ${ }^{\mathrm{X}}$ (2) was condensed with amine-functionalised bi-functional linker 4 (Scheme 2). Initially, the reaction was performed at room temperature, however, only a small amount of glycoconjugate was observed. When the reaction was heated to $40{ }^{\circ} \mathrm{C}$ for 36 hours, Lewis ${ }^{\mathrm{X}}$ was converted into the desired trisaccharide and direct purification by size exclusion chromatography (BioGel P2) then allowed for the isolation of the desired trisaccharide as the ammonium formate salt. The ammonium formate salt, however, could not be used in peptide ligation strategies due to the conjugation of formic acid to form the formamide derivative. Accordingly, ion exchange chromatography with Dowex $\mathrm{OH}^{-}$resulted in the formation of the free amine 5 in a good yield (88\%) and the resulting trisaccharide was suitable for conjugation with the fluorescent dendron.

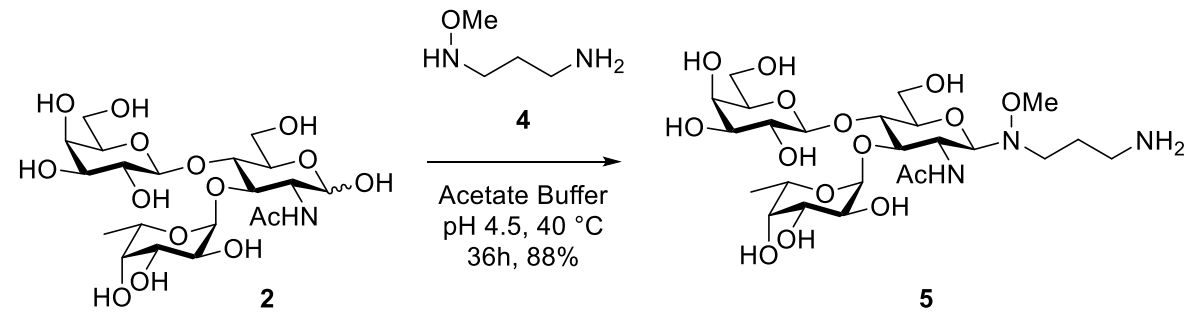

$$
\prod_{O}^{O} \overbrace{3}^{-N}
$$

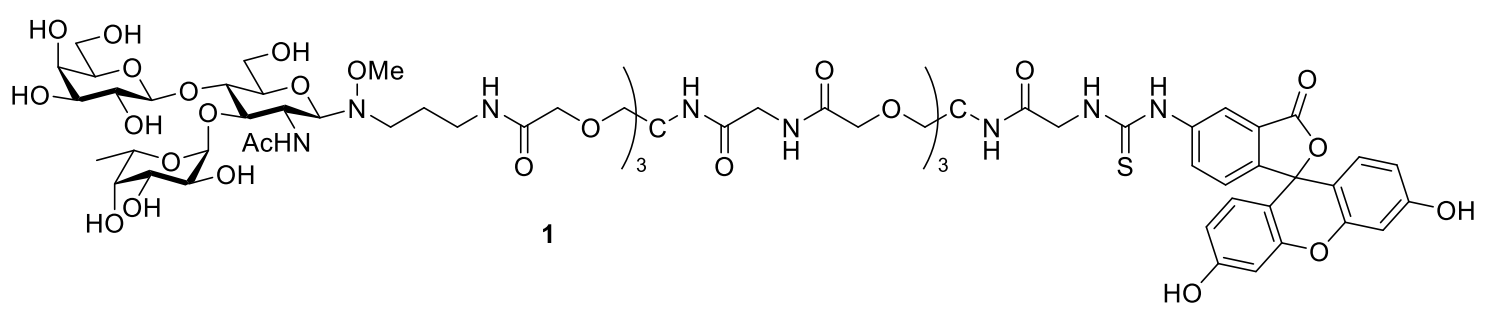

Scheme 2. Synthesis of glycodendron 1 by conjugating Lewis ${ }^{X} 2$ to the fluorescein derived second generation dendron 3 by utilising the bi-functional methoxyamine linker 4. 
Next, conjugation with the fluorescein isothiocyanate (FITC)-labelled second generation dendron 3 was performed using the HBTU-mediated peptide ligation methodology. Here, the fluorescent dendron 3 (1 equiv.) was dissolved in freshly distilled DMF and the volume of the solvent was then reduced by half, so as to remove traces of dimethylamine which could react with the activated ester. This solution was then added to the Lewis ${ }^{\mathrm{X}}$ glycan $\mathbf{5}$ (18 equiv.), and reacted by the addition of HBTU (27 equiv.) and $\mathrm{Et}_{3} \mathrm{~N}$ (36 equiv.). Here, the order of addition is important as the Lewis ${ }^{\mathrm{X}}$ glycan 5 has poor solubility in DMF, however, completely dissolves after the addition of HBTU and $\mathrm{Et}_{3} \mathrm{~N}$. After one hour, the reaction mixture was analysed using HRMS and it was observed that a mixture of the hepta-, octa- and nona-valent glycodendron was formed $(\mathrm{m} / \mathrm{z}$ for $\left[\mathrm{C}_{285} \mathrm{H}_{476} \mathrm{~N}_{36} \mathrm{O}_{168} \mathrm{~S}\right]^{4+}$ calcd.: 1781.4903 , obsd.: 1781.4906). When the reaction mixture was stirred overnight, the MS showed a similar product ratio, and thus the glycodendron was purified using size exclusion chromatography (Sephadex $\mathrm{CM} \mathrm{C}-25,0.1 \mathrm{M}$ aq. $\mathrm{NH}_{4} \mathrm{HCO}_{2}$ ) to obtain the fluorescent glycodendron product. The glycodendron was lyophilised twice and dissolved in $\mathrm{H}_{2} \mathrm{O}$ $(1 \mathrm{~mL})$. HRMS analysis of the glycodendrons, showed the presence of the hexa-, hepta-, octaand nona-glycosylated dendrons (Figure 1). To determine the final concentration, the solution was analysed using a colorimetric assay (UV-VIS) by diluting 50, 100, 150 and $200 \mu \mathrm{L}$ with 1500 $\mu \mathrm{L}$ water (Figure 2). Here the amount of stock solution is related to the absorbance (Figure $2 \mathbf{A}$ ), and this can then be correlated to the amount of glycodendron present in the stock solution (Figure 2 B) based on an average molecular weight of $6.5 \mathrm{kDa}$ and the extinction coefficient of 85000 . The concentration of the stock solution was thus determined to be $0.66 \mathrm{mg} / \mathrm{mL}(0.1 \mu \mathrm{M})$. The stock solution was used in the biological evaluation of the C-type lectin DC-SIGN.

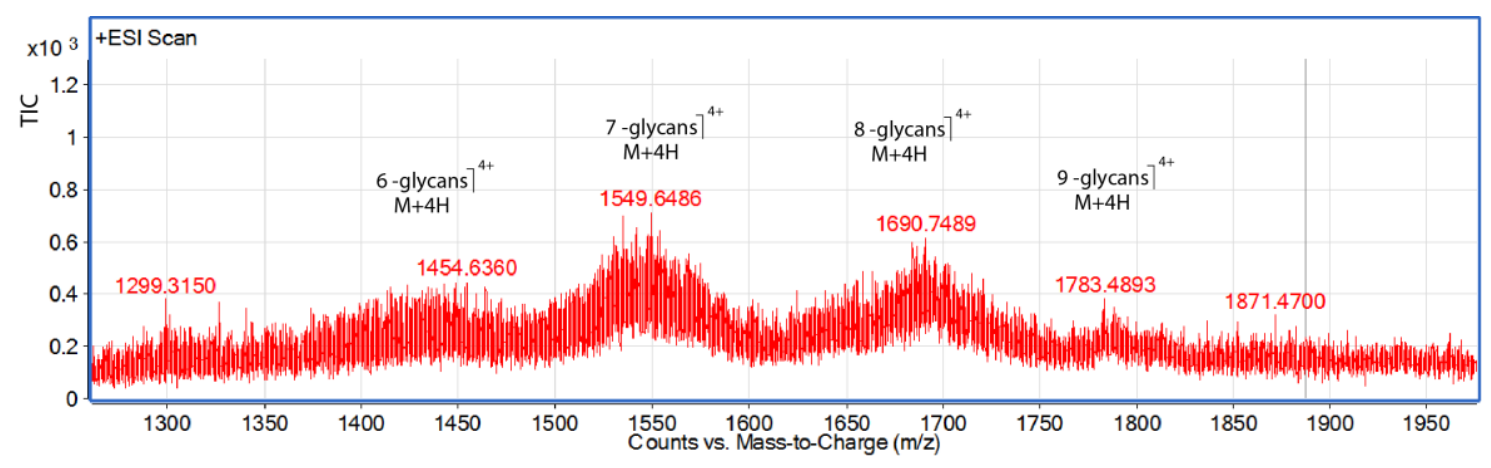

Figure 1. HRMS of the fluorescent Lewis ${ }^{X}$ glycodendron stock solution. 

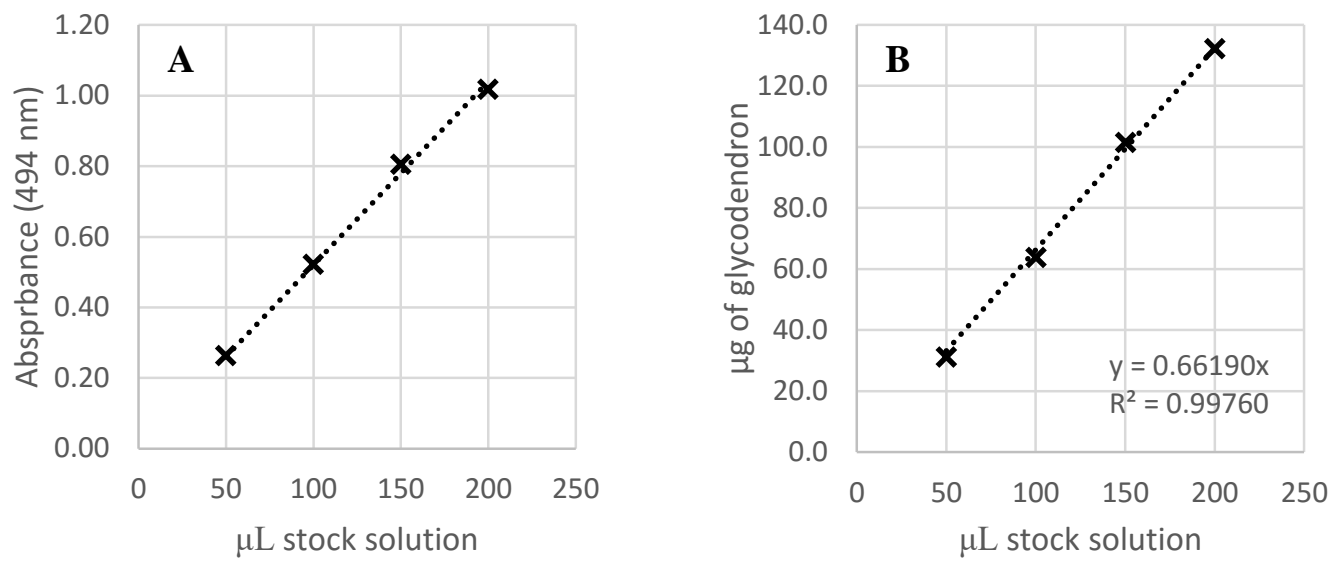

Figure 2. Colorimetric assay showed a concentration of $0.66 \mathrm{mg} / \mathrm{mL}(100 \mu \mathrm{M})$ of glycodendron 1 stock solution.

\subsubsection{Biological evaluation of fluorescent glycodendron}

The THP-1 acute monocytic lymphoma cell line was cultured in Fetal Calf Serum (FCS), Glutamax and Penstrep to obtain enough cells for differentiation and subsequent flow cytometry analysis. Next, the cells were differentiated into 'M0-like' M $\phi s$ (PMA), 'M1-like' M $\phi s$ (PMA + IFN- $\gamma+$ LPS), or 'M2-like' M $\phi$ s (PMA + IL-4). For the flow cytometry assays, the different types of cells were incubated for 1 hour at $4{ }^{\circ} \mathrm{C}$ with the various substrates (FITC glycodendron, positive and negative control), washed twice, and analysed accordingly.

\subsubsection{Preliminary Flow cytometry results}

First, the THP-1 cells as well as the different types of M $\phi$ s were compared by CD14, CD86 and DC-SIGN expression (Figure 3). Interestingly, it was observed that undifferentiated THP-1 cells express high levels of DC-SIGN which is in contrast with previously reported experiments. ${ }^{13}$ When these THP-1 cells were stimulated with PMA, the expression of CD14 and DC-SIGN was reduced. Stimulation of these THP-1+PMA derived M $\phi$ s with LPS and IFN- $\gamma$ upregulates CD14, CD86 as well as DC-SIGN. Stimulation with IL-4, however, did not result in the upregulation of DC-SIGN and, in addition, did not affect CD14 and CD86 expression. 

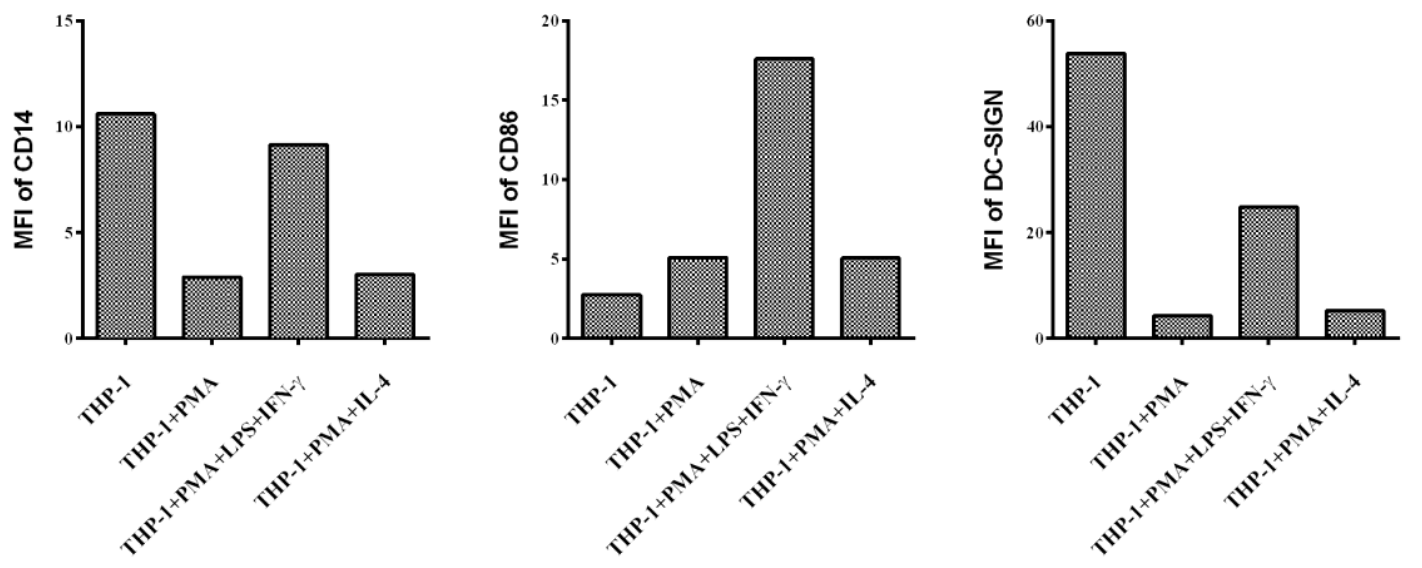

Figure 3. Cellular expression of CD14, CD86 and DC-SIGN on different subsets of cells; MFI (median fluorescent intensity).

The THP-1 cells stimulated with PMA and IL-4 were used to study the DC-SIGN binding of the anti-DC-SIGN antibody (positive control), the unglycosylated dendron $\mathbf{3}$ (negative control) and the Lewis ${ }^{\mathrm{X}}$ glycodendron 1 (Figure 4). Here, it was observed that the anti-DC-SIGN antibody did bind to the $M \phi s$ in a concentration-dependent manner. The negative control did not show binding to the $\mathrm{M} \phi \mathrm{s}$ in low $\mu \mathrm{M}$ concentration, whereas the highest concentration tested $(100 \mu \mathrm{M})$ gave a small increase in mean fluorescence. The Lewis ${ }^{\mathrm{x}}$ glycodendron also bound to the cells in a concentration-dependent manner, with observed binding above $0.1 \mu \mathrm{M}$ concentrations, whereas binding was not observed in the low nanomolar concentration range.

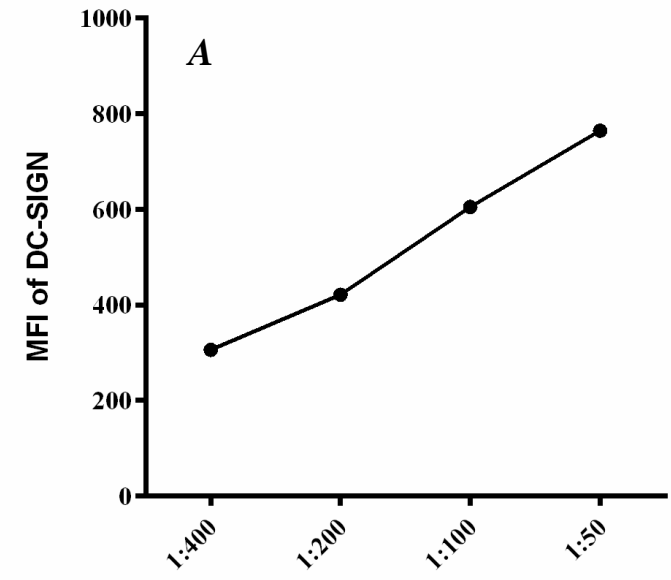

Anti-DC-SIGN antibody dilution

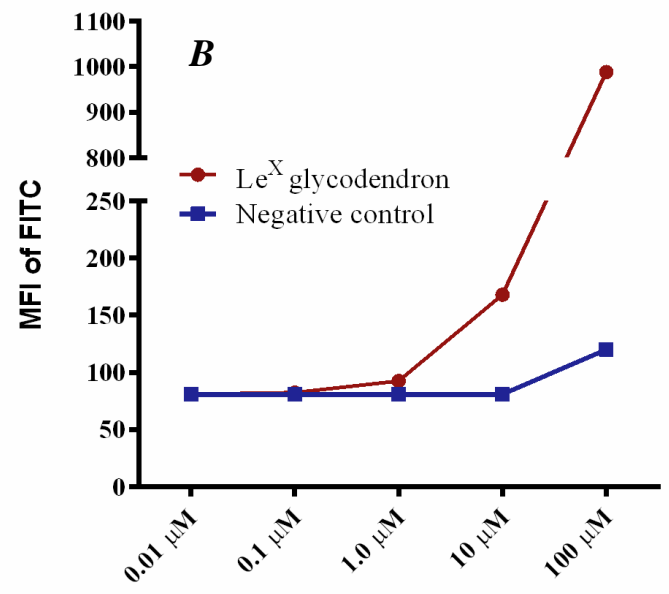

Concentration of dendrons

Figure 4. The expression of DC-SIGN on THP-1+PMA+IL-4 cells measured with A) anti-DCSIGN antibody and B) unglycosylated dendron 3 (negative control) and the fluorescent Lewis ${ }^{X}$ glycodendron 1. 
To investigate the DC-SIGN mediated binding of Lewis ${ }^{\mathrm{X}}$ glycodendrons, co-staining of the glycodendron with the anti-DC-SIGN antibody was then conducted on the 'M2-like' M $\phi$ s (Figure 5). Here, it is important to note that the co-staining with the antibody did not interfere with the glycan-mediated binding, as the co-stain resulted in similar binding of the fluorescent glycodendron when compared to the glycodendron single staining (Figure 5A).

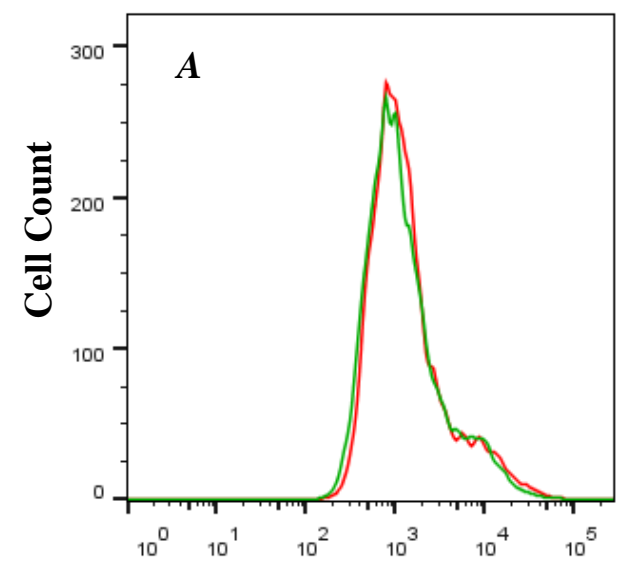

FI of FITC

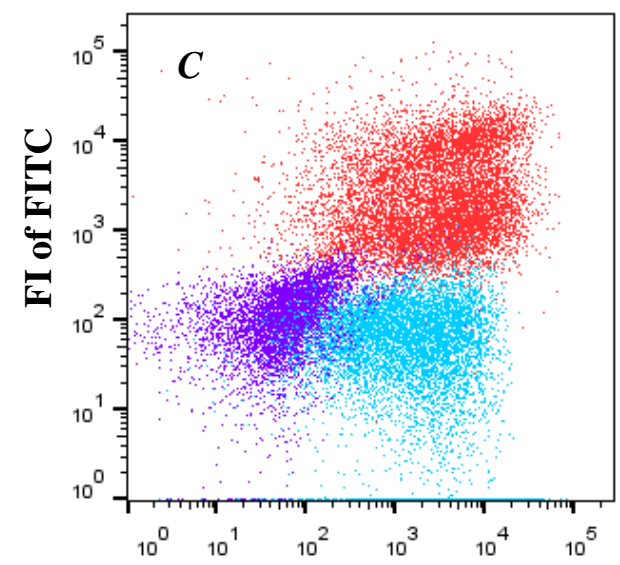

FI of anti-DC-SIGN-AF647

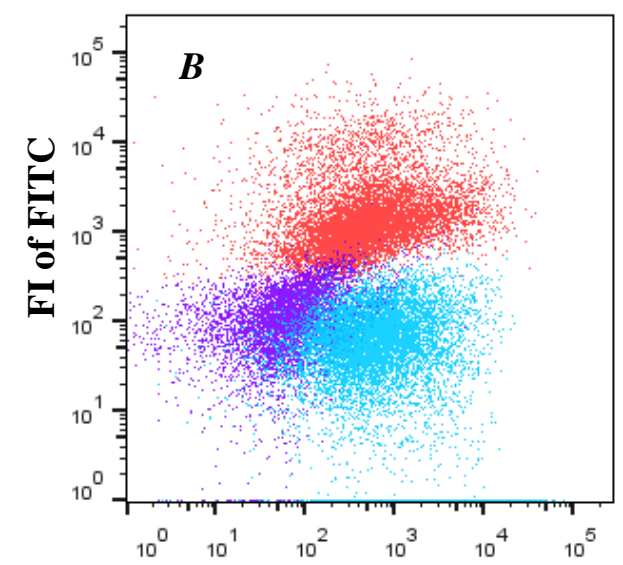

FI of anti-DC-SIGN-AF647

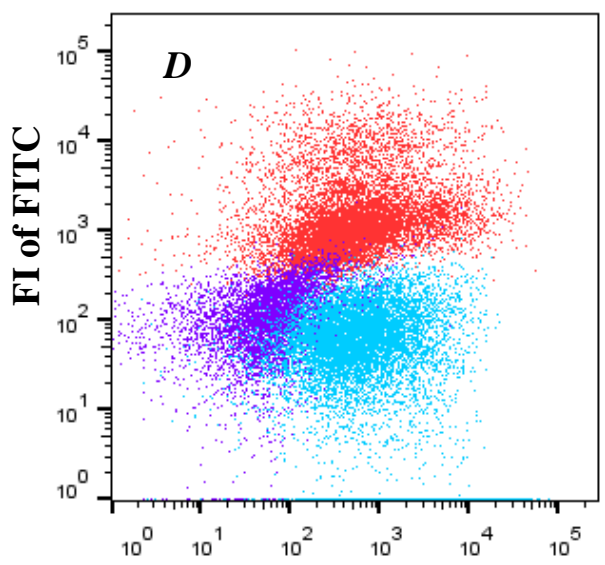

FI of anti-DC-SIGN-AF647

Figure 5. (A) The costaining with the antibody does not affect glycodendron binding to $T H P-1+P M A+I L-4$ cells, single staining with $100 \mu M$ glycodendron (green), co-staining of the anti-DC-SIGN antibody with 100 $\mu M$ glycodendron (red); (B-D) The expression of DC-SIGN measured with the glycodendron and the anti-DC-SIGN antibody on B) THP-1+PMA cells; $(C)$ $T H P-1+P M A+L P S+I F N-\gamma$ cells; (D) THP-1+PMA+IL-4 cells, using single staining with the anti-DC-SIGN antibody (blue), co-staining of the antibody with $100 \mu \mathrm{M}$ glycodendron (red) and unstained cells (purple).

Next, the co-staining of the different type of M $\phi$ s was conducted using the anti-DC-SIGN antibody and the glycodendron 1 (100 $\mu \mathrm{M}$ concentration) (Figure 5B, 5C and 5D). Here it was observed that all DC-SIGN ${ }^{+}$cells are $\mathrm{FITC}^{+}$. Interestingly, in the 'M1-like' M $\phi$ s there are two FITC $^{+}$populations which are both DC-SIGN ${ }^{+}$, but show different glycodendron binding, which 
might either indicate non-specific binding or binding to other lectins. However, as the negative control (non-glycosylated dendron 3) showed low levels of unspecific binding, it appears that the glycan-probe is binding to receptors other than DC-SIGN. Lewis ${ }^{\mathrm{X}}$, however, is only known to bind to Langerin (on Langerhans cells) and MGL-1 (on mouse M $\phi s$ ), and accordingly, this requires further investigation.

Taken as a whole, these data demonstrate that the Lewis ${ }^{\mathrm{x}}$ glycodendron can be used to target $\mathrm{M} \phi \mathrm{s}$. This binding appears to occur predominantly via the DC-SIGN lectin, however, there also seems to be a small sub-population of $\mathrm{M} \phi \mathrm{s}$ which bind the glycodendron independent of DC-SIGN. This will require further investigation using additional antibodies which are known to bind other lectins (i.e. Langerin or E-, P- and L-selectins), or biotinylated constructs for the purification of these lectins via streptavidin-columns. Moreover, to explore whether the glycodendron can be used to distinguish between 'M1-like' $\mathrm{M} \phi \mathrm{s}$ and 'M2-like' $\mathrm{M} \phi \mathrm{s}$, different types of macrophages need to be produced, as the THP-1 cells generated in these assays contain low levels of DC-SIGN, regardless of their differentiation conditions. 


\subsection{Conclusion}

In summary, the synthesis of a complex fluorescent glycodendron has been achieved by using a bi-functional oxyamine linker to conjugate the Lewis ${ }^{\mathrm{x}}$ antigen to a multivalent dendron scaffold. Here, condensation of Lewis ${ }^{\mathrm{X}}$ with the bi-functional oxyamine linker gave the amine-functionalised glycoconjugate in a good $88 \%$ yield and the amide ligation with acid-functionalised dendrons also proved to be extremely efficient with eight to nine conjugations per dendron scaffold. The glycodendron was purified by size exclusion chromatography and, to determine the glycodendron concentration of the stock solution, a UV-VIS colorimetric assay was performed.

As proof-of-concept, the fluorescent glycodendron was used as a fluorescent marker in flow cytometry for the C-type lectin DC-SIGN on human THP-1 derived M $\phi$ s. It was observed that the glycodendron binds to THP-1 derived M $\phi s$ in a concentration-dependent manner, with binding being observed at micromolar concentrations. Although preliminary results indicate that these fluorescent glycodendrons are suitable as flow cytometry markers, further investigations are required.

Overall, the combination of glycan antigens, bi-functional oxyamine linkers and multivalent dendron scaffolds has proven to be an effective strategy towards the rapid assembly of complex glycodendrons which then allows for the biological evaluation of these macromolecules. Ideally, future work will demonstrate that these and related Lewis antigen glycodendrons have great potential as DC-SIGN-mediated therapeutics. 


\subsection{Experimental}

General procedure. Prior to use, DMF was distilled from $\mathrm{BaO}, \mathrm{Et}_{3} \mathrm{~N}$ (Sigma) was distilled from $\mathrm{KOH}$, and $\mathrm{H}_{2} \mathrm{O}$ was purified by millipore. $\mathrm{AcOH}$ (Ajax Finechem), $\mathrm{NH}_{4} \mathrm{OAc}$ (Sigma Aldrich), $\mathrm{NH}_{4} \mathrm{HCO}_{2}$ (Sigma Aldrich) and HBTU (Apollo Scientific) were used as received. Reactions were monitored by TLC-analysis on Macherey-Nagel silica gel coated plastic sheets $(0.20 \mathrm{~mm}$, with fluorescent indicator $\mathrm{UV}_{254}$ ) with detection by UV-absorption (short wave UV - $254 \mathrm{~nm}$; long wave $\mathrm{UV}-366 \mathrm{~nm}$ ), by dipping in $10 \% \mathrm{H}_{2} \mathrm{SO}_{4}$ in $\mathrm{EtOH}$ followed by charring at $\sim 150{ }^{\circ} \mathrm{C}$, or by dipping into a solution of ninhydrin in $\mathrm{EtOH}$ followed by charring at $\sim 150{ }^{\circ} \mathrm{C}$. Infrared spectra were recorded as thin films using a Bruker Tensor 27 FTIR spectrometer equipped with an Attenuated Total Reflectance (ATR) sampling accessory and are reported in wave numbers ( $\mathrm{cm}^{-}$ ${ }^{1}$ ). Nuclear magnetic resonance spectra were recorded at $20{ }^{\circ} \mathrm{C}$ in $\mathrm{D}_{2} \mathrm{O}$ using either a Varian INOVA operating at $500 \mathrm{MHz}$ or Varian VNMRS operating at $600 \mathrm{MHz}$. Chemical shifts are given in ppm $(\delta)$ relative to solvent residues. NMR peak assignments were made using COSY, HSQC and HMBC 2D experiments.
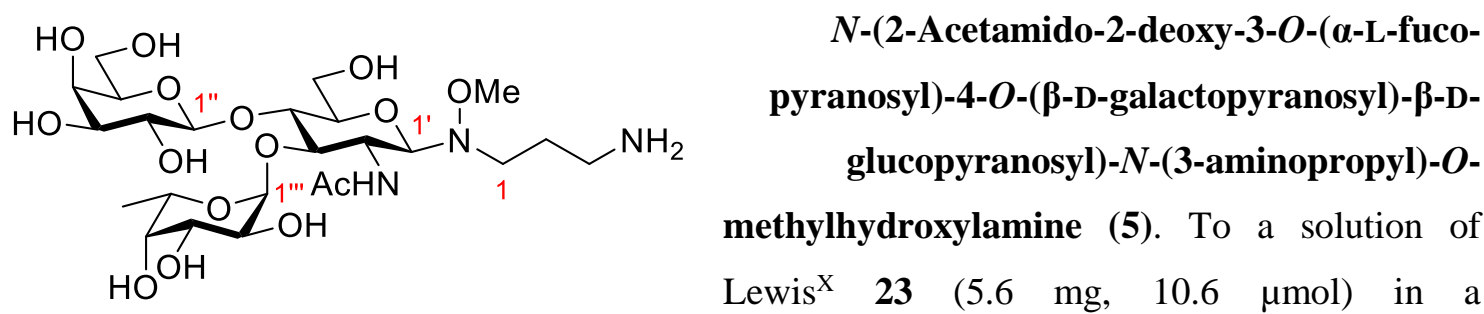

$\mathrm{AcOH} / \mathrm{NH}_{4} \mathrm{OAc}$ buffer $(0.5 \mathrm{~mL}, 2 \mathrm{M}$, freshly prepared, $\mathrm{pH} 4.5)$, 3-(methoxyamino)propanylamine hydrochloride $4(15.9 \mathrm{mg}, 113.2 \mu \mathrm{mol})$ was added and the reaction mixture was stirred at $40{ }^{\circ} \mathrm{C}$ for $35 \mathrm{~h}$. The crude mixture was directly loaded onto a size exclusion column (Bio-Gel P$2,1200 \times 18 \mathrm{~mm}$ ) and eluted with $0.1 \mathrm{M}$ aq. $\mathrm{NH}_{4} \mathrm{HCO}_{2}$. Lyophilisation of the product fractions afforded neoglycoside 24 (5.7 mg, 88\%). $\mathrm{R}_{f}=0.10\left(\mathrm{CH}_{2} \mathrm{Cl}_{2} / \mathrm{EtOH} / \mathrm{MeOH} / \mathrm{NH}_{3}\right.$ (aq. 35\%), 5/2/2/1, $\mathrm{v} / \mathrm{v} / \mathrm{v} / \mathrm{v}) ; \alpha_{\mathrm{D}}^{19.5}=-9.3(\mathrm{c}=0.2, \mathrm{MeOH}) ; \mathrm{IR}($ film) $3341,2925,2852,17117,1647,1586,1466$, 1451, 1415, 1380, 1350, 1302, 1233, 1193, 1085, 1026, 968, $917 \mathrm{~cm}^{-1} ;{ }^{1} \mathrm{H}$ NMR (500 MHz, $\left.\mathrm{D}_{2} \mathrm{O}\right)$ $\delta 5.12\left(\mathrm{~d}, 1 \mathrm{H}, J_{1^{\prime \prime \prime}, 2^{\prime \prime \prime}}=3.9 \mathrm{~Hz}, \mathrm{H}-1^{\prime \prime \prime}\right), 4.84\left(\mathrm{q}, 1 \mathrm{H}, J_{5^{\prime \prime \prime}, 6^{\prime \prime \prime}}=6.7 \mathrm{~Hz}, \mathrm{H}-5^{\prime \prime \prime}\right), 4.49-4.42(\mathrm{~m}, 2 \mathrm{H}$, H-1', H-1"), 4.05 (m, 2H, H-2', H-6a'), 3.93 - 3.81 (m, 5H, H-3'"', H-4"', H-4', H-3', H-6b'), 3.80 $\left(\mathrm{d}, 1 \mathrm{H}, J_{3^{\prime \prime \prime}, 4^{\prime \prime \prime}}=J_{4^{\prime \prime \prime}, 5^{\prime \prime \prime}}=2.9 \mathrm{~Hz}, \mathrm{H}-4^{\prime \prime \prime}\right), 3.80-3.68$ (m, 3H, H-6a", H-6b", H-2"'), 3.64 (dd, 1H, $\left.J_{3^{\prime \prime}, 4^{\prime \prime}}=3.2 \mathrm{~Hz}, J_{2^{\prime \prime}, 3^{\prime \prime}}=9.9 \mathrm{~Hz}, \mathrm{H}-3^{\prime \prime}\right), 3.60-3.55$ (m, 1H, H-5"), $3.54-3.46$ (m, 1H, H-5"), 3.54 - 3.46 (m, 5H, $\left.\mathrm{OCH}_{3}, \mathrm{H}-5^{\prime}, \mathrm{H}-2^{\prime \prime}\right), 3.11-2.92$ (m, 4H, $\mathrm{CH}_{2}-1, \mathrm{CH}_{2}-3$ ), 2.02 (s, 3H, $\mathrm{CH}_{3} \mathrm{Ac}$ ), 1.95 (m, 2H, $\left.\mathrm{CH}_{2}-2\right), 1.17$ (d, 3H, $\left.J_{5^{\prime \prime \prime}, 6^{\prime \prime \prime}}=6.7 \mathrm{~Hz}, \mathrm{H}-6{ }^{\prime \prime \prime}\right) ;{ }^{13} \mathrm{C}$ NMR $\left(125 \mathrm{MHz}, \mathrm{D}_{2} \mathrm{O}\right) 170.5(\mathrm{C}=\mathrm{O})$, 101.8 (C-1"'), 98.7 (C-1'"'), 90.5 (C-1'), 76.9 (C-5'), 76.1 (C-3'), 74.8 (C-5"), 73.2 (C-4'), 72.4 (C3"), 71.8 (C-4"'), 70.9 (C-2"), 69.1 (C-3"'), 68.3 (C-4"), 67.6 (C-2"'), 66.7 (C-5'"'), 61.4 (C-6"), 
$60.9\left(\mathrm{OCH}_{3}\right), 59.7\left(\mathrm{C}-6^{\prime}\right), 52.5\left(\mathrm{C}-2^{\prime}\right), 47.2(\mathrm{C}-1), 37.5(\mathrm{C}-3), 24.5(\mathrm{C}-2), 22.1\left(\mathrm{CH}_{3} \mathrm{Ac}\right), 15.2(\mathrm{C}-$ 6"'); HRMS(ESI) $m / z$ calcd. for $\left[\mathrm{C}_{24} \mathrm{H}_{46} \mathrm{~N}_{3} \mathrm{O}_{15}\right]^{+}:$616.2923, obsd.: 616.2938 .

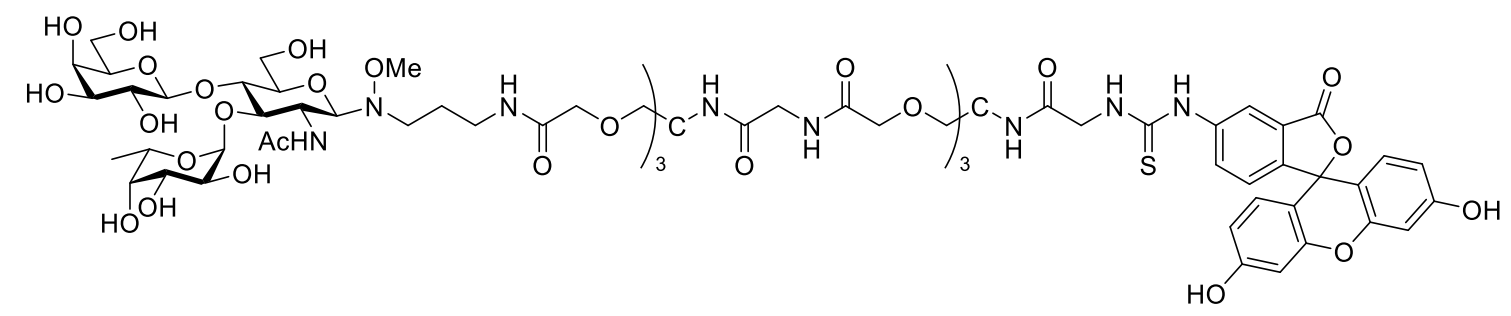

$N$-[N-([(3',6'-Dihydroxy-3-oxospiro[isobenzofuran-1 $(3 H), 9^{\prime}$-(9H)xanthen]-5-yl)amino]thioxomethyl)glycyl]-1,1,1-tris(1,1,1-tris[3-( $N$-(2-acetamido-2-deoxy-3- $O$-( $\alpha$-L-fucopyranosyl)-4- $O$-( $\beta$-D-galactopyranosyl)- $\beta$-D-gluco-pyranosyl)- $N$-(3-aminopropyl)- $O$-)- $O$ methyl-hydroxylamine)propylamidocarbonylmethyloxymethyl]methylamidocarbonylmethylamidocarbonylmethyloxy-methyl)aminomethane (1). To Lewis ${ }^{\mathrm{X}}$ trisaccharide 5 (6 mg, $9.8 \mu \mathrm{mol})$, a solution of FITC labeled second generation dendron $3(0.94 \mathrm{mg}, 0.54 \mu \mathrm{mol})$ in freshly distilled DMF $(250 \mu \mathrm{L})$ was added followed by the addition of HBTU $(5.6 \mathrm{mg}, 15 \mu \mathrm{mol})$ and $\mathrm{Et}_{3} \mathrm{~N}(10 \mu \mathrm{L}, 72 \mu \mathrm{mol})$ and the reaction mixture was stirred at $\mathrm{rt}$ for $18 \mathrm{~h}$. The crude mixture was diluted with $\mathrm{H}_{2} \mathrm{O}(1 \mathrm{~mL})$ and purified by size exclusion chromatography (Sephadex CM C25, $0.1 \mathrm{M}$ aq. $\mathrm{NH}_{4} \mathrm{HCO}_{2}$ ). Lyophilisation of the product afforded fluorescent glycodendron $\mathbf{1}$ as a yellow foam. HRMS(ESI) $\mathrm{m} / z$ calcd. for $\left[\mathrm{C}_{285} \mathrm{H}_{476} \mathrm{~N}_{36} \mathrm{O}_{168} \mathrm{~S}\right]^{4+}: 1781.4903$, obsd.: 1781.4906 .

THP-1 derived Mфs differentiation. The following experiments were performed by Kristel Kodar. The THP-1 acute monocytic lymphoma cell line were cultured in RPMI 1640 medium supplemented with 10\% Fetal Calf Serum (FCS), 1\% Glutamax and 1\% Penstrep, and seeded $2.5 \times 10^{5}$ cells $/ \mathrm{mL}$. Differentiation of the THP- 1 cells was induced via the treatment with PMA (50 ng/mL, 48 h.) to obtain 'M0-like' M $\phi$ s, followed by addition of IL-4 (20 ng/mL, $24 \mathrm{~h}$.) for 'M2-like' M $\phi$ s, or IFN- $\gamma(10 \mathrm{ng} / \mathrm{mL})$ and LPS (10 ng/mL, 24h) for 'M1-like' M $\phi s$. For subsequent analysis, the differentiated cells were detached from the tissue culture plates by incubating the cells in PBS on ice.

Flow cytometry. The various types of $\mathrm{M} \phi$ s were stained by incubating for 1 hour at $4{ }^{\circ} \mathrm{C}$ with either the FITC-labelled glycodendron 1, negative control $\mathbf{3}$ or the anti-DC-SIGN antibody, washed twice with PBS, and DC-SIGN expression was measured by flow cytometry. The fluorescent Lewis ${ }^{\mathrm{X}}$ glycodendron and the negative control (non-glycosylated dendron $\mathbf{3}$ ) were used in various concentrations $\left(10^{-1}, 10^{1}, 10^{2}, 10^{3}, 10^{4}\right.$ and $10^{5} \mathrm{nM}$, diluted in $10 \%$ FCS $)$, while the human anti-DC-SIGN antibody (Phycoerythrin-labelled) positive control was diluted by a factor of $2(1 / 50 \rightarrow 1 / 1600, v / v)$. 


\subsection{References}

(1) Medzhitov, R. 'Recognition of Microorganisms and Activation of the Immune Response.' Nature 2007, 449, 819-826.

(2) Gordon, S. 'Pattern Recognition Receptors: Doubling up for the Innate Immune Response'. Cell, 2002, 111, 927-930.

(3) Fehres, C. M.; Unger, W. W. J.; Garcia-Vallejo, J. J.; van Kooyk, Y. 'Understanding the Biology of Antigen Cross-Presentation for the Design of Vaccines against Cancer' Front. Immunol. 2014, 5, 1-10.

(4) Guo, Y.; Feinberg, H.; Conroy, E.; Mitchell, D. a; Alvarez, R.; Blixt, O.; Taylor, M. E.; Weis, W. I.; Drickamer, K. 'Structural Basis for Distinct Ligand-Binding and Targeting Properties of the Receptors DC-SIGN and DC-SIGNR.' Nat. Struct. Mol. Biol. 2004, 11, 591-598.

(5) Geijtenbeek, T. B.; Kwon, D. S.; Torensma, R.; van Vliet, S. J.; van Duijnhoven, G. C.; Middel, J.; Cornelissen, I. L.; Nottet, H. S.; KewalRamani, V. N.; Littman, D. R.; Figdor, C. G.; van Kooyk, Y. 'DC-SIGN, a Dendritic Cell-Specific HIV-1-Binding Protein That Enhances Trans-Infection of T Cells.' Cell 2000, 100, 587-597.

(6) Yang, J.; Zhang, L.; Yu, C.; Yang, X.-F.; Wang, H. 'Monocyte and Macrophage Differentiation: Circulation Inflammatory Monocyte as Biomarker for Inflammatory Diseases' Biomark. Res. 2014, 2, 1.

(7) Brown, M. B.; von Chamier, M.; Allam, A. B.; Reyes, L. 'M1/M2 Macrophage Polarity in Normal and Complicated Pregnancy' Front. Immunol. 2014, 5, 1-10.

(8) Martinez, F. O.; Gordon, S. 'The M1 and M2 Paradigm of Macrophage Activation: Time for Reassessment.' F1000Prime Rep. 2014, 6, 13.

(9) Mantovani, A.; Sica, A.; Sozzani, S.; Allavena, P.; Vecchi, A.; Locati, M. 'The Chemokine System in Diverse Forms of Macrophage Activation and Polarization'. Trends in Immunology, 2004, 25, 677-686.

(10) Mills, C. D.; Kincaid, K.; Alt, J. M.; Heilman, M. J.; Hill, A. M. 'M-1/M-2 Macrophages and the Th1/Th2 Paradigm.' J. Immunol. 2000, 164, 6166-6173.

(11) Mosser, D. M.; Edwards, J. P. 'Exploring the Full Spectrum of Macrophage Activation.' Nat. Rev. Immunol. 2008, 8, 958-969.

(12) Domínguez-Soto, A.; Sierra-Filardi, E.; Puig-Kröger, A.; Pérez-Maceda, B.; GómezAguado, F.; Corcuera, M. T.; Sánchez-Mateos, P.; Corbí, A. L. 'Dendritic Cell-Specific ICAM-3-Grabbing Nonintegrin Expression on M2-Polarized and Tumor-Associated Macrophages Is Macrophage-CSF Dependent and Enhanced by Tumor-Derived IL-6 and IL-10.' J. Immunol. 2011, 186, 2192-2200.

(13) Puig-Kröger, A.; Serrano-Gómez, D.; Caparrós, E.; Domínguez-Soto, A.; Relloso, M.; Colmenares, M.; Martínez-Muñoz, L.; Longo, N.; Sánchez-Sánchez, N.; Rincon, M.; Rivas, L.; Sánchez-Mateos, P.; Fernández-Ruiz, E.; Corbí, A. L. 'Regulated Expression of the Pathogen Receptor Dendritic Cell-Specific Intercellular Adhesion Molecule 3 (ICAM-3)-Grabbing Nonintegrin in THP-1 Human Leukemic Cells, Monocytes, and Macrophages' J. Biol. Chem. 2004, 279, 25680-25688.

(14) Wang, S.-K.; Liang, P.-H.; Astronomo, R. D.; Hsu, T.-L.; Hsieh, S.-L.; Burton, D. R.; Wong, C.-H. 'Targeting the Carbohydrates on HIV-1: Interaction of Oligomannose Dendrons with Human Monoclonal Antibody 2G12 and DC-SIGN.' Proc. Natl. Acad. Sci. U. S. A. 2008, 105, 3690-3695.

(15) Ribeiro-Viana, R.; Sánchez-Navarro, M.; Luczkowiak, J.; Koeppe, J. R.; Delgado, R.; Rojo, J.; Davis, B. G. 'Virus-like Glycodendrinanoparticles Displaying Quasi-Equivalent Nested Polyvalency upon Glycoprotein Platforms Potently Block Viral Infection.' Nat. Commun. 2012, 3, 1303.

(16) Garcia-Vallejo, J. J.; Koning, N.; Ambrosini, M.; Kalay, H.; Vuist, I.; SarramiForooshani, R.; Geijtenbeek, T. B. H.; van Kooyk, Y. 'Glycodendrimers Prevent HIV Transmission via DC-SIGN on Dendritic Cells.' Int. Immunol. 2013, 25, 221-233. 
(17) García-Vallejo, J. J.; Ambrosini, M.; Overbeek, A.; van Riel, W. E.; Bloem, K.; Unger, W. W. J.; Chiodo, F.; Bolscher, J. G.; Nazmi, K.; Kalay, H.; van Kooyk, Y. 'Multivalent Glycopeptide Dendrimers for the Targeted Delivery of Antigens to Dendritic Cells' Mol. Immunol. 2013, 53, 387-397.

(18) Allavena, P.; Sica, A.; Solinas, G.; Porta, C.; Mantovani, A. 'The Inflammatory MicroEnvironment in Tumor Progression: The Role of Tumor-Associated Macrophages' Crit. Rev. Oncol. Hematol. 2008, 66, 1-9. 


\section{Chapter 6.}

\section{Conclusion and future prospects.}

\subsection{Conclusion}

The overall objective of this dissertation was to develop new and efficient strategies for the synthesis of multivalent glycoconjugates. The research was divided into three separate projects: the oxyamine glycan conjugation methodology, the synthesis of functionalised multivalent dendrons and the total synthesis of a Lewis antigen. The elements of these research projects were then combined to complete the synthesis of fluorescent Lewis ${ }^{\mathrm{X}}$ glycodendrons for the detection of the C-type lectin DC-SIGN on macrophages.

In Chapter 2, novel methodology was presented for the synthesis of a variety of bi-functional oxyamine linkers in high yield and few (3-4) steps. The oxyamine linkers were employed for the conjugation of representative glycan antigens to the substrate of choice, without the need for protecting groups. Here the oxyamine was used in the glycan conjugation, whereas the second functional group on the linker allowed for a variety of ligation reactions, including peptide ligation, thiol-maleimide Michael addition and sulfonylation. The substrates conjugated to the oxyamine-functionalised glycans via these ligation reactions included molecular probes, such as biotin and fluorescent molecules, as well as multivalent substrates, such as proteins and dendrons. Moreover, the hydrolytic stability of the oxyamine linkage was investigated, and it was determined that these glycoconjugates have excellent half-lives under physiological conditions.

Multivalent ligation is required to induce strong biological responses, and accordingly, the synthesis of multivalent dendrons was the focus of Chapter 3. Here, an efficient double exponential growth strategy allowed for the rapid assembly of a novel second generation dendron core. Functionalisation of this dendron scaffold with biotin, followed by glycan conjugation, allowed for the synthesis of biotinylated glycodendrons which can be used in a number of 
applications. In addition, the synthesis of fluorescently labelled glycodendrons allowed for the use of these macromolecules in fluorescent microscopy as well as in flow cytometry, as described in Chapter 5.

En route to the formation of complex glycoconjugates, the synthesis of a novel trisaccharide crystalline intermediate which can be used in the synthesis of most Type-2 Lewis antigens was described in Chapter 4. In the synthetic route, all monosaccharide building blocks were readily synthesised on a large scale, in few steps, and in good yields. The regioselective glycosylation between the galactose donor and the 3,4-di-hydroxyl GlcNAc acceptor yielded the Gal- $\beta$ - $(1,4)$ GlcNAc disaccharide, which was then 3-O-fucosylated to give the Lewis ${ }^{\mathrm{X}}$ trisaccharide intermediate as a crystalline product. In summary, the trisaccharide was obtained in good yields and in few steps (38\% over 7 steps, longest linear route). For the purpose of this thesis, the trisaccharide was globally deprotected to obtain the Lewis ${ }^{\mathrm{X}}$ antigen in excellent yield.

Finally, Chapter 5 concerned the synthesis of the complex Lewis ${ }^{\mathrm{x}}$ glycodendrons, by conjugating the glycan antigen and multivalent dendron scaffold through the use of the bi-functional oxyamine linker. As a proof-of-concept, a fluorescent Lewis ${ }^{\mathrm{X}}$ glycodendron was synthesised and tested as a flow cytometry marker for the C-type lectin DC-SIGN on human THP-1 derived macrophages. Preliminary results indicated that these Lewis ${ }^{\mathrm{X}}$ glycodendrons can be used in the selective targeting of DC-SIGN ${ }^{+}$cells, however, further investigations are required to confirm these studies, and will be reported in due course. 


\subsection{Future prospects}

The work in this doctoral thesis discussed the rapid assembly of complex glycoconjugates via the use of novel bi-functional linker methodology. In particular, the synthesis of a "Type B" oxyamine is discussed, however, in order to investigate the relative reactivity of both "Type A" and "Type B" oxyamines, future work requires the direct comparison between these two types of linkers. To this end, both types of glycoconjugates could be directly compared in various hydrolysis studies whereby the effect of glycan concentration, buffer strength and $\mathrm{pH}$ on hydrolytic stability could be compared.

Further insight into the formation and hydrolysis of oxyamine-linked glycoconjugates may also result in the development of more stable glycoconjugates, and may result in higher glycan conversions and thus higher yielding glycoconjugate syntheses. Moreover, the oxyaminemediated conjugation of other glycan epitopes, such as tumour associated glycan antigens, to multivalent substrates such as dendrons, proteins and microarray slides, would allow for the development of further glycoconjugates that can be studied for their biological function, and in addition, may allow for the development of novel therapeutics such as anti-cancer vaccines.

As described in Chapter 3, dendritic structures are required for the multivalent presentation of glycan antigens. It was shown by Van Kooyk and co-workers that the larger size dendrimers have lower $\mathrm{IC}_{50}$ values, compared to more compact glycodendrimers. With this in mind, bi-functional linkers of various lengths could be synthesised in order to investigate the effect of dendron size on biological activity. Alternatively, the second generation dendron scaffold could be elongated with polyethylene glycol linkers to increase the size of the glycodendrons.

In Chapter 4, the synthesis of Lewis ${ }^{\mathrm{x}}$, a glycan antigen that plays an important role in targetting of the C-type lectin DC-SIGN, was described. Although Chapter 4 concerns the synthesis of Lewis $^{\mathrm{X}}$, other Type-2 Lewis antigens (i.e. Lewis ${ }^{\mathrm{Y}}$, Sialyl Lewis ${ }^{\mathrm{X}}$ and sulfated derivatives) could be synthesised from the orthogonally protected trisaccharide intermediate. Moreover, the common intermediate may find use in the synthesis of tumour associated glycan antigens such as dimeric Lewis ${ }^{\mathrm{X}}$, which may aid in the development of anti-tumour therapeutics.

As described above, bi-functional oxyamine linkers are powerful tools in the synthesis of glycodendrons. Accordingly, Lewis ${ }^{\mathrm{x}}$ glycodendrons were synthesised for the targeting of DC-SIGN. Further experiments are required to demonstrate that the Lewis ${ }^{\mathrm{X}}$ glycodendrons can be used to selectively target DC-SIGN, which will be undertaken in due course. If, however, these macromolecules can be used to selectively target this lectin, leading to endocytosis by dendritic cells and macrophages, these glycodendrons can be utilised in vaccines. Derivatisation of the glycodendron with antigenic peptides for the DC-SIGN-mediated peptide delivery to antigen 
presenting cell may lead to immune activation against the peptide antigen through $\mathrm{CD}-4^{+} \mathrm{T}$-cells (Figure 1). Moreover, toxic molecules could be added to the dendron so as to facilitate cell-selective depletion of DC-SIGN ${ }^{+}$cells. This concept could be employed as anti-cancer therapeutics, whereby specific lectins on cancer cells are targeted for toxin delivery. Other glycodendrons could also be prepared for the use in other diseases. For example, the synthesis of 6'-O-sulfo-sialyl Lewis ${ }^{\mathrm{X}}$ would allow for the selective targeting of Siglec-8, which is expressed on inflammatory cells such as eosinophils and mast cells. Upon ligation of the siglec, eosinophil apoptosis is induced. Accordingly, 6'-O-sulfo-sialyl Lewis ${ }^{\mathrm{X}}$ glycodendrons could be used to decrease eosinophil levels in eosinophilia patients and, in addition, the glycodendron might find use for the relief of symptoms in patients with asthma.
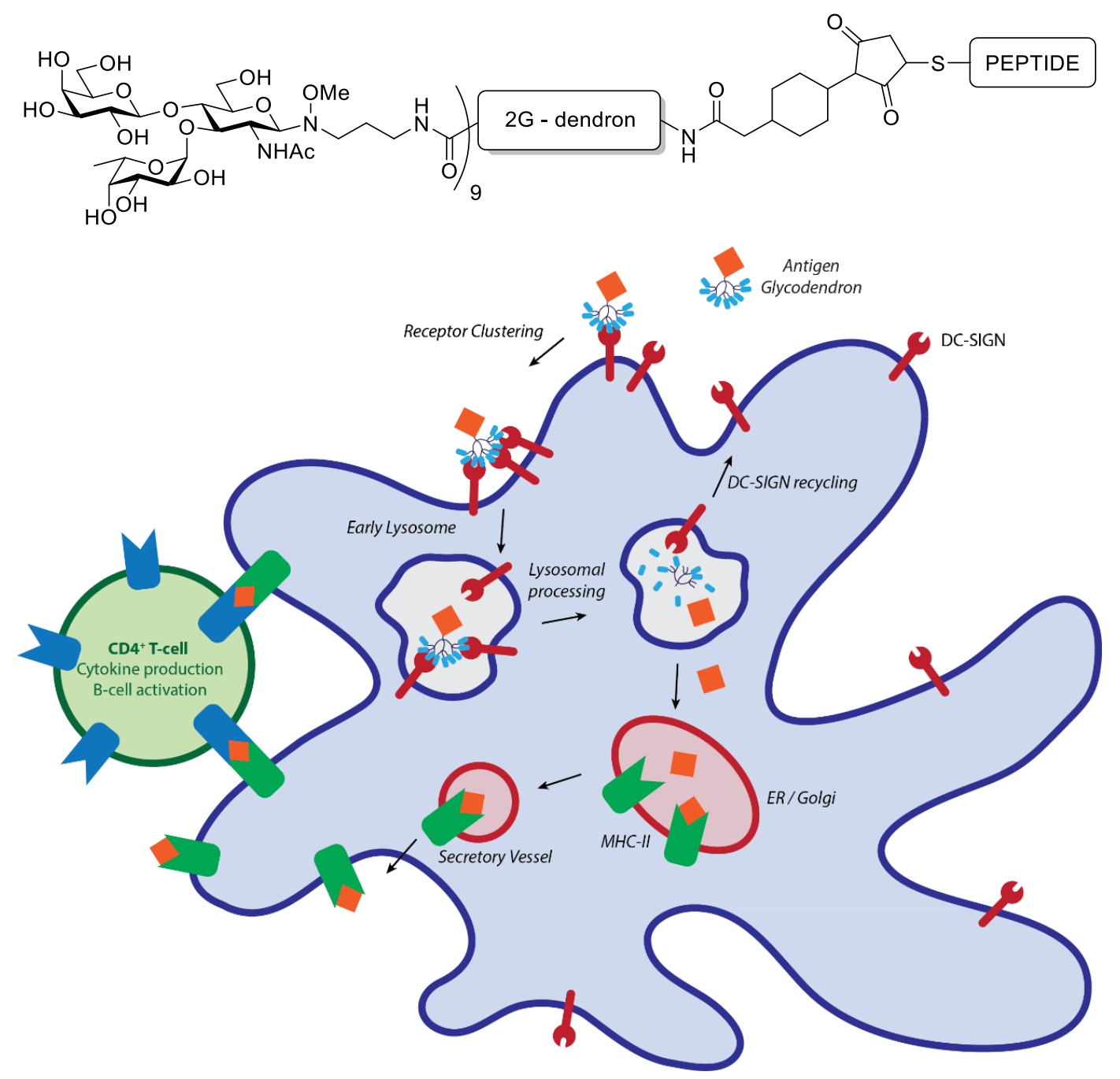

Figure 1. Antigen-functionalised Lewis ${ }^{X}$ glycodendrons can selectively target DCs and macrophages through the lectin DC-SIGN. Multivalent binding with Lewis antigens results in internalisation in lysosomes, where the antigens get processed, and DC-SIGN recycles to the cellsurface. The antigen then gets transferred to the endoplasmic reticulum (ER) and Golgi apparatus where it is bound to MHC-II, and the complex is secreted to the cell surface. This allows for activation of $\mathrm{CD}^{+}$T-cells against specific antigens, through the MHC-II mediated antigen presentation to $T$-cell receptors. 


\section{Appendix Chapter 2.}

\section{The Rapid and Facile Synthesis of Oxyamine Linkers for the Preparation of Novel Glycoconjugates.}

\section{Table of contents}

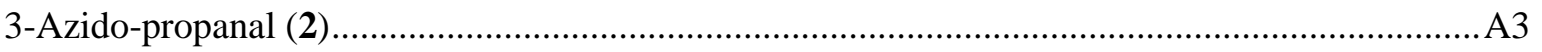

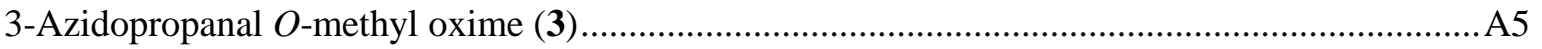

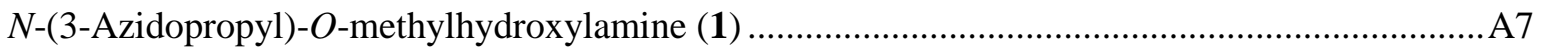

3-(Methoxyamino)propan-1 -amine hydrochloride (4) .......................................................... 9

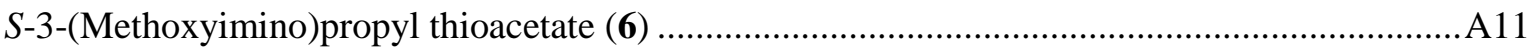

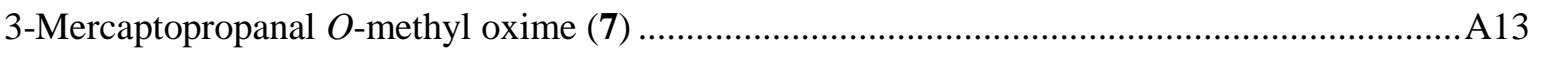

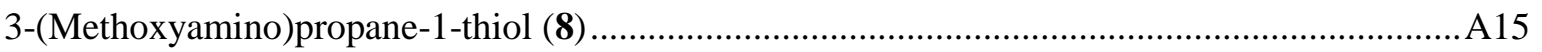

2-(3-(Methoxyamino)propylthio)ethanamine (9) ..................................................................... 17

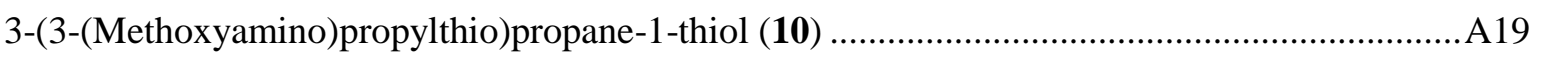

2-(3-(Methoxyamino)propylthio)acetic acid (11) ................................................................... 21

$S$-(3-(Methoxyamino)propyl)- $N$-Boc-L-cysteine (12) ............................................................. 23

$\mathrm{N}$-(2-Acetamido-2-deoxy- $\beta$-D-glucopyranosyl)- $N$-(3-azidopropyl)- $O$ -

methylhydroxylamine (14).

$N$-(2-Acetamido-2-deoxy- $\beta$-D-glucopyranosyl)- $N$-(3-aminopropyl)-O-

methylhydroxylamine (15)

A30 
2-(3-(Methoxy(2-acetamido-2-deoxy- $\beta$-D-glucopyranosyl)amino)propylthio)-

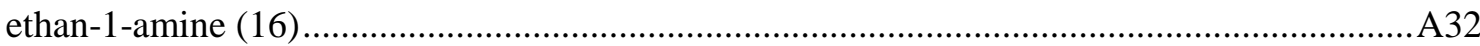

D-gluco-2-(1-Acetamido-2,3,4,5-tetrahydroxy-pentyl)-3-methoxy-1,3-thiazinane (18) ..............A34

D-gluco-2-(1-Acetamido-2,3,4,5-tetra-acetoxy-pentyl)-3-methoxy-1,3-thiazinane (19) ..............A36

1,2-Bis(3-[3-(methoxy[2-acetamido-2-deoxy- $\beta$-D-glucopyranosyl]amino)propylthio]propyl)-

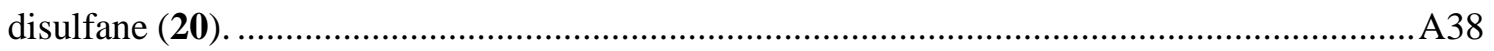

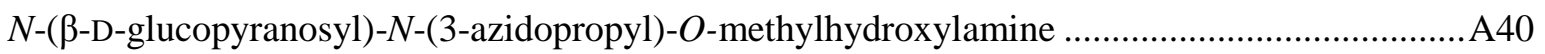

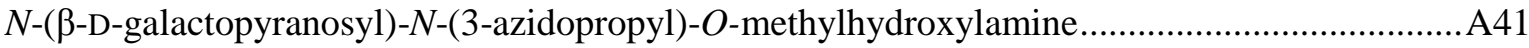

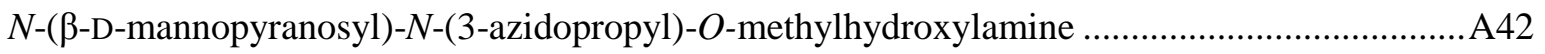

$N$-(4-O-( $\alpha$-D-glucopyranosyl)- $\beta$-D-glucopyranosyl)- $N$-(3-azidopropyl)- $O$ methylhydroxylamine (22)

$N$-(2-Acetamido-2-deoxy-3- $O$-( $\alpha$-L-fucopyranosyl)-4- $O$-( $\beta$-D-galactopyranosyl)- $\beta$-Dglucopyranosyl)- $N$-(3-aminopropyl)- $O$-methylhydroxylamine (24)

(1-(3-(Methoxy(2-acetamido-2-deoxy-b-D-glucosyl)amino)propyl)-1H-1,2,3-triazol-4-yl)methanol (25)

N-(3-(methoxy[2-acetamido-2-deoxy- $\beta$-D-glucopyranosyl]amino)propyl)-D-

biotinamide (26) A52

5-(dimethylamino)- $N$-(2-((3-(methoxy(2-acetamido-2-deoxy- $\beta$-D-glucopyranosyl)amino)propyl)thio)ethyl)naphthalene-1-sulfonamide (28) A54

1-(2-D-biotinamidoethyl)-1H-pyrrole-2,5-dione (29) ............................................................. 56

1-(2-D-biotinamidoethyl)-3-((3-((3-(methoxy[2-acetamido-2-deoxy-b-Dglucopyranosyl]amino)propyl)thio)propyl)thio)pyrrolidine-2,5-dione (34) A58

Glycosylated BSA (37) Maldi-TOF A60 


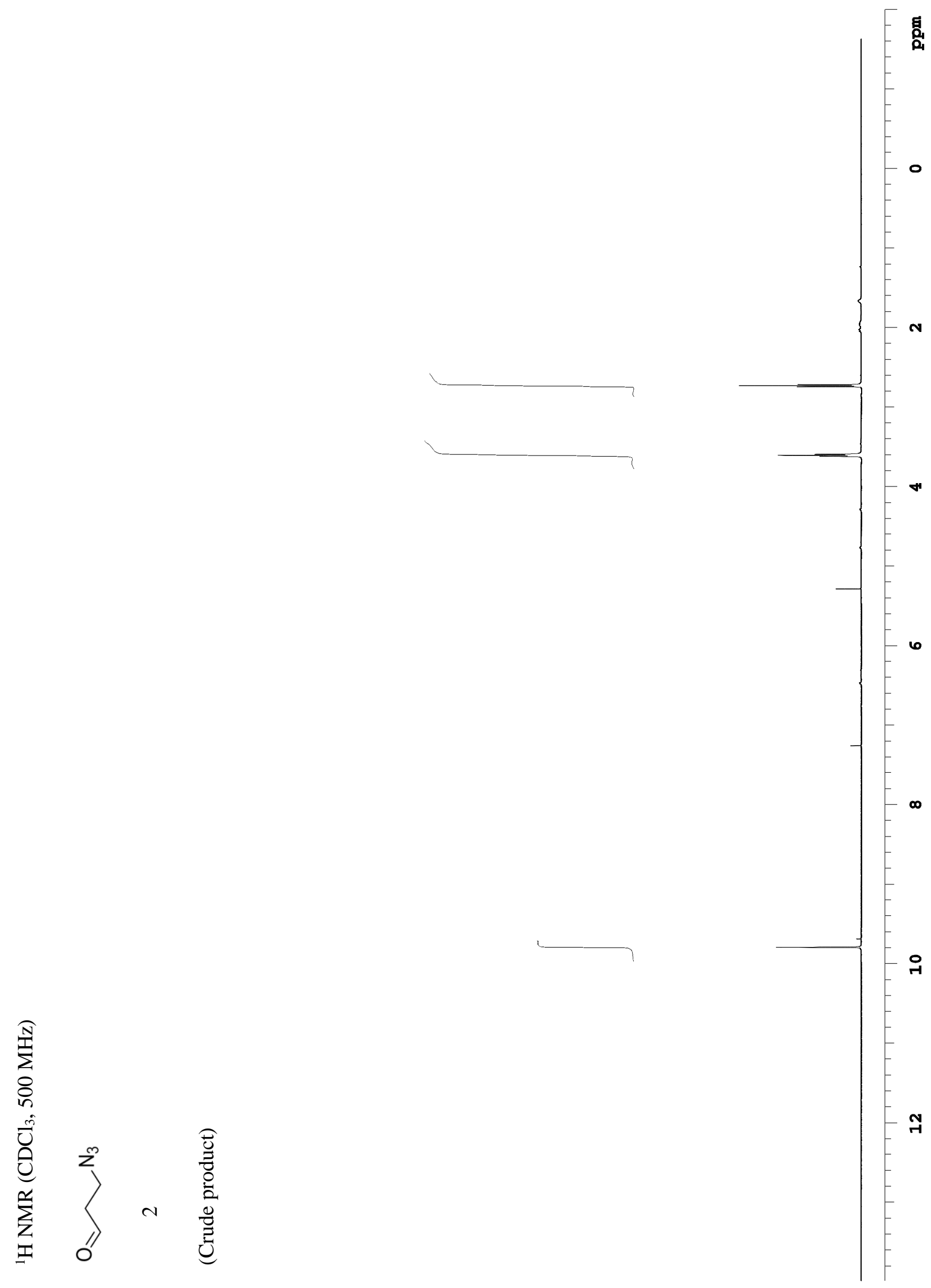

Appendix - 3 

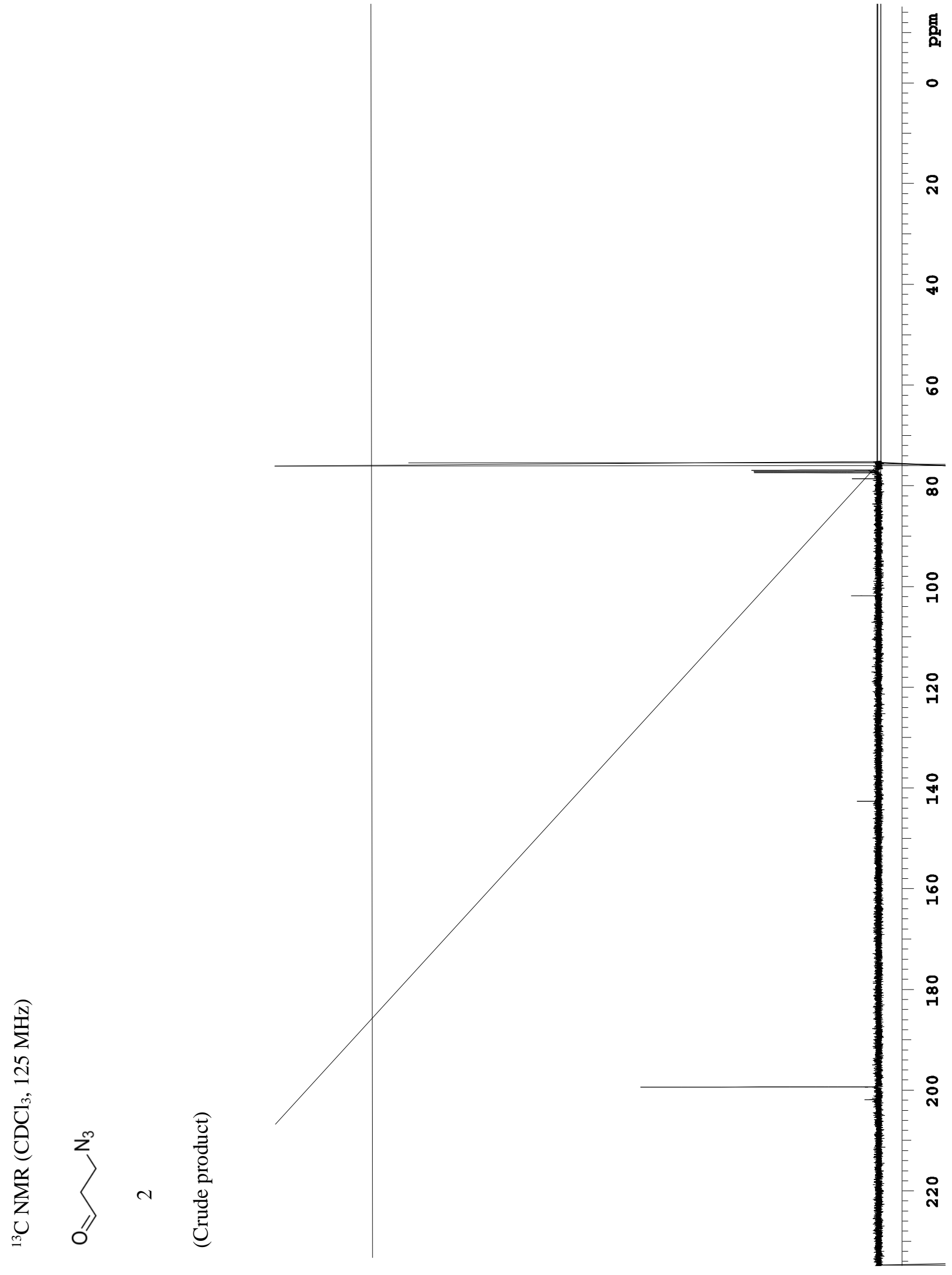

Appendix - 4 


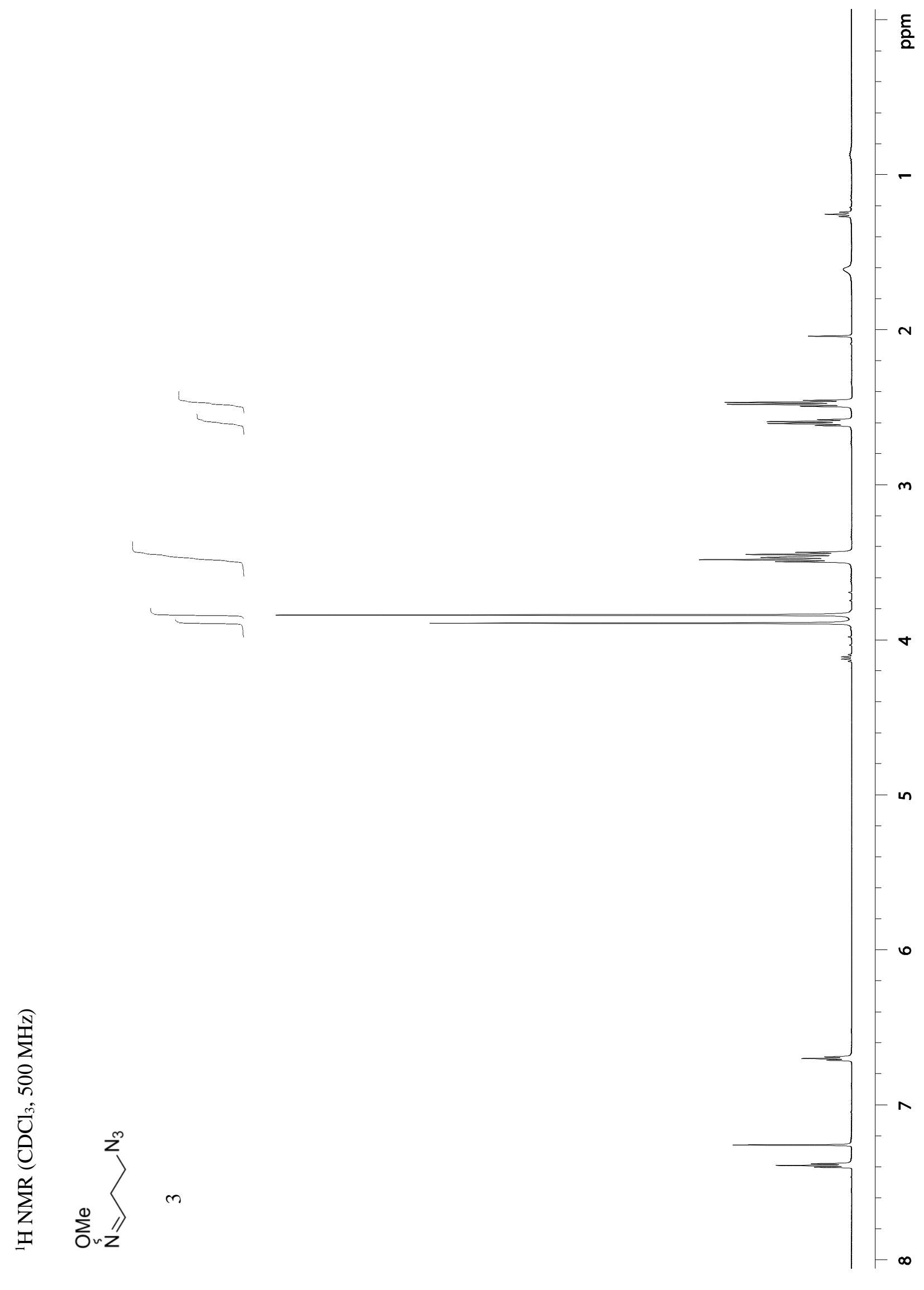

Appendix - 5 


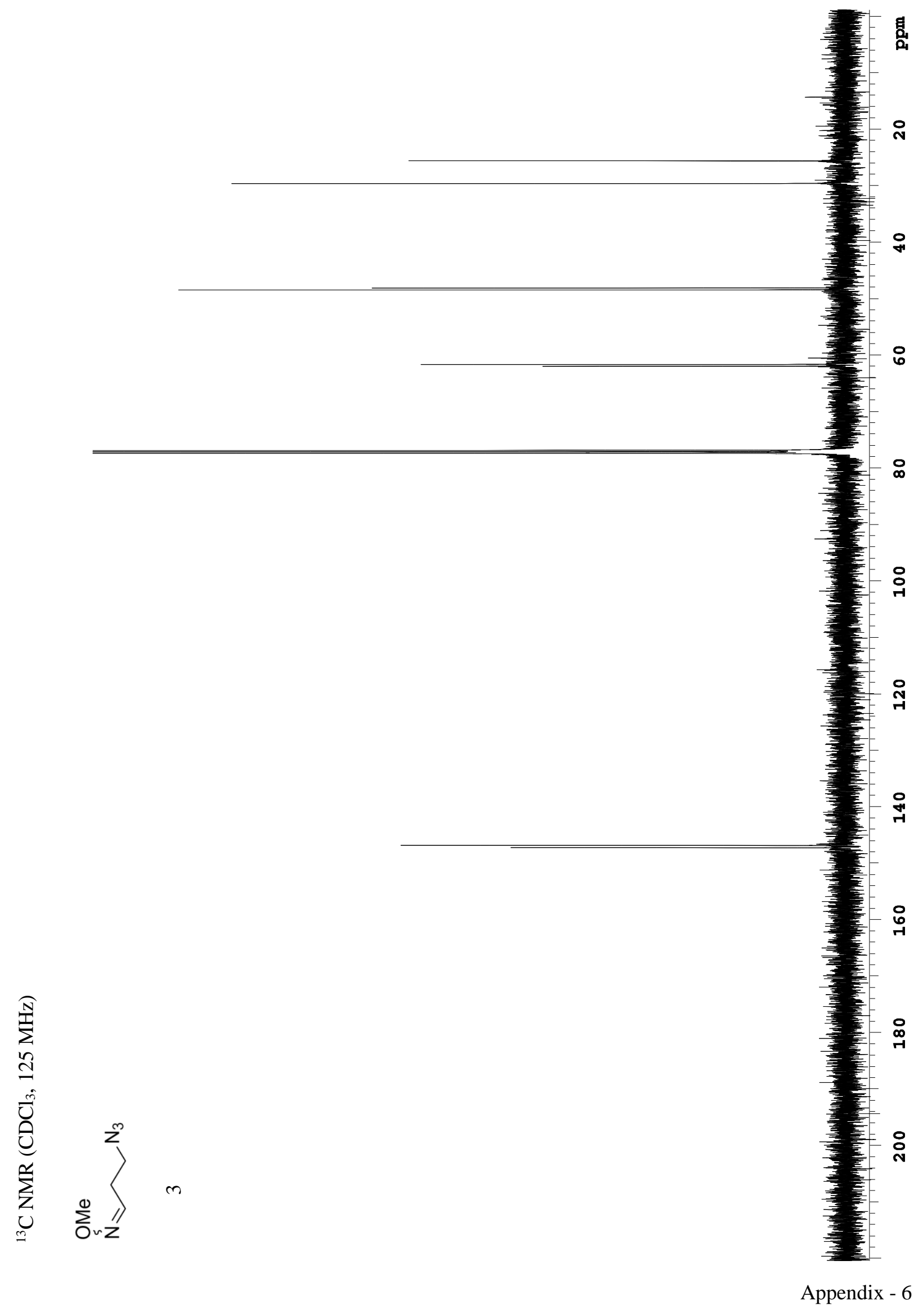




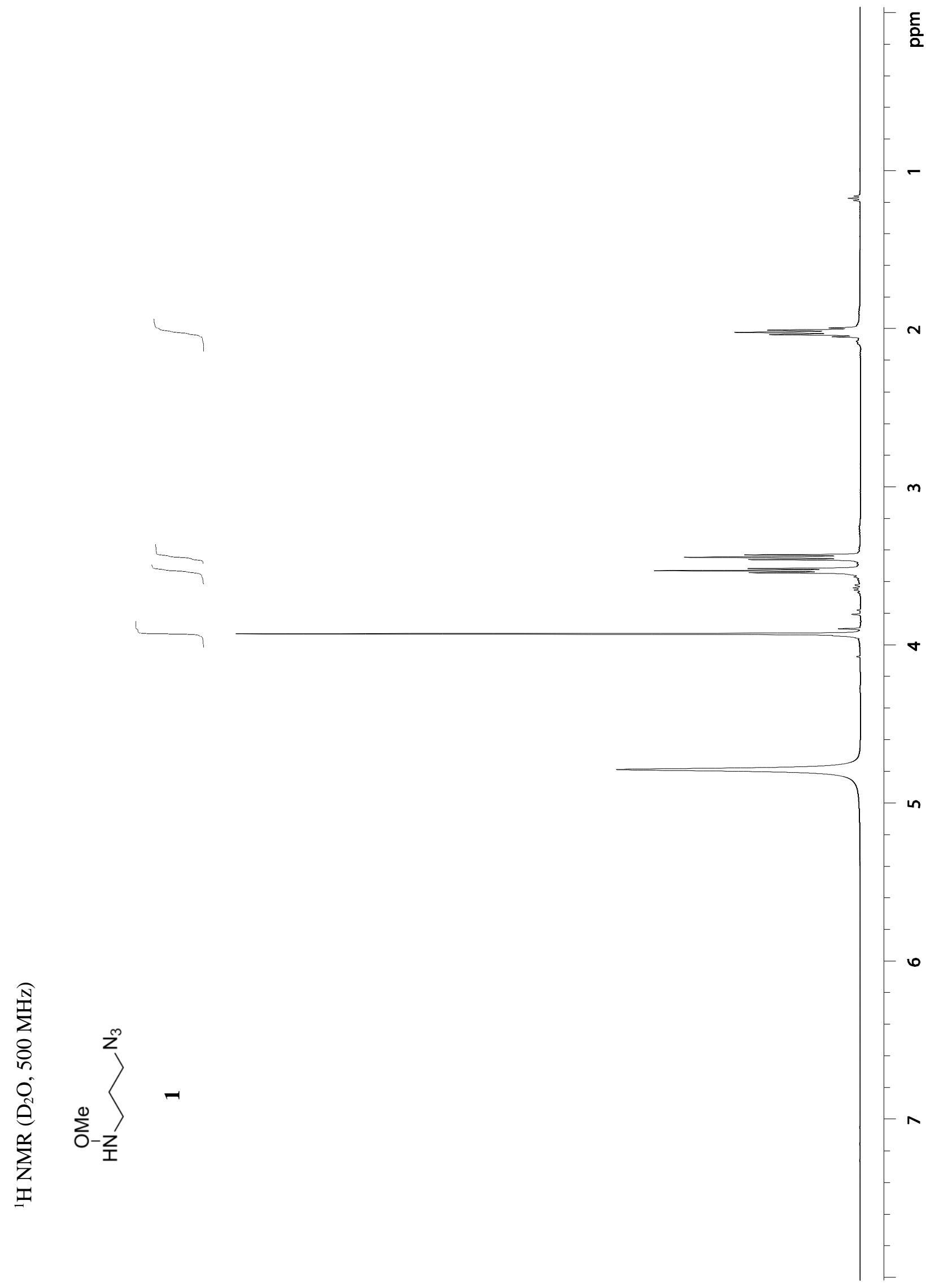

Appendix - 7 


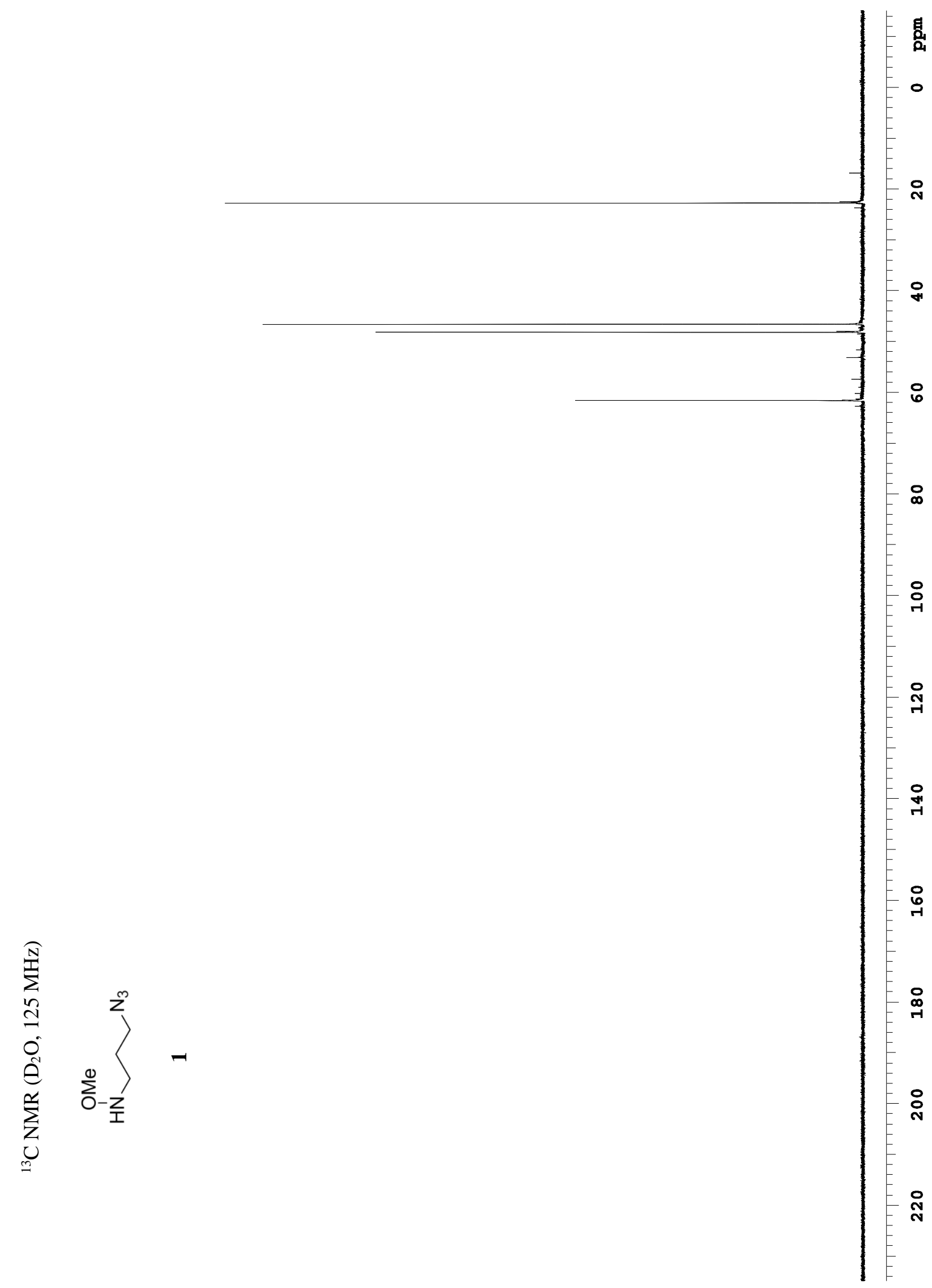

Appendix - 8 


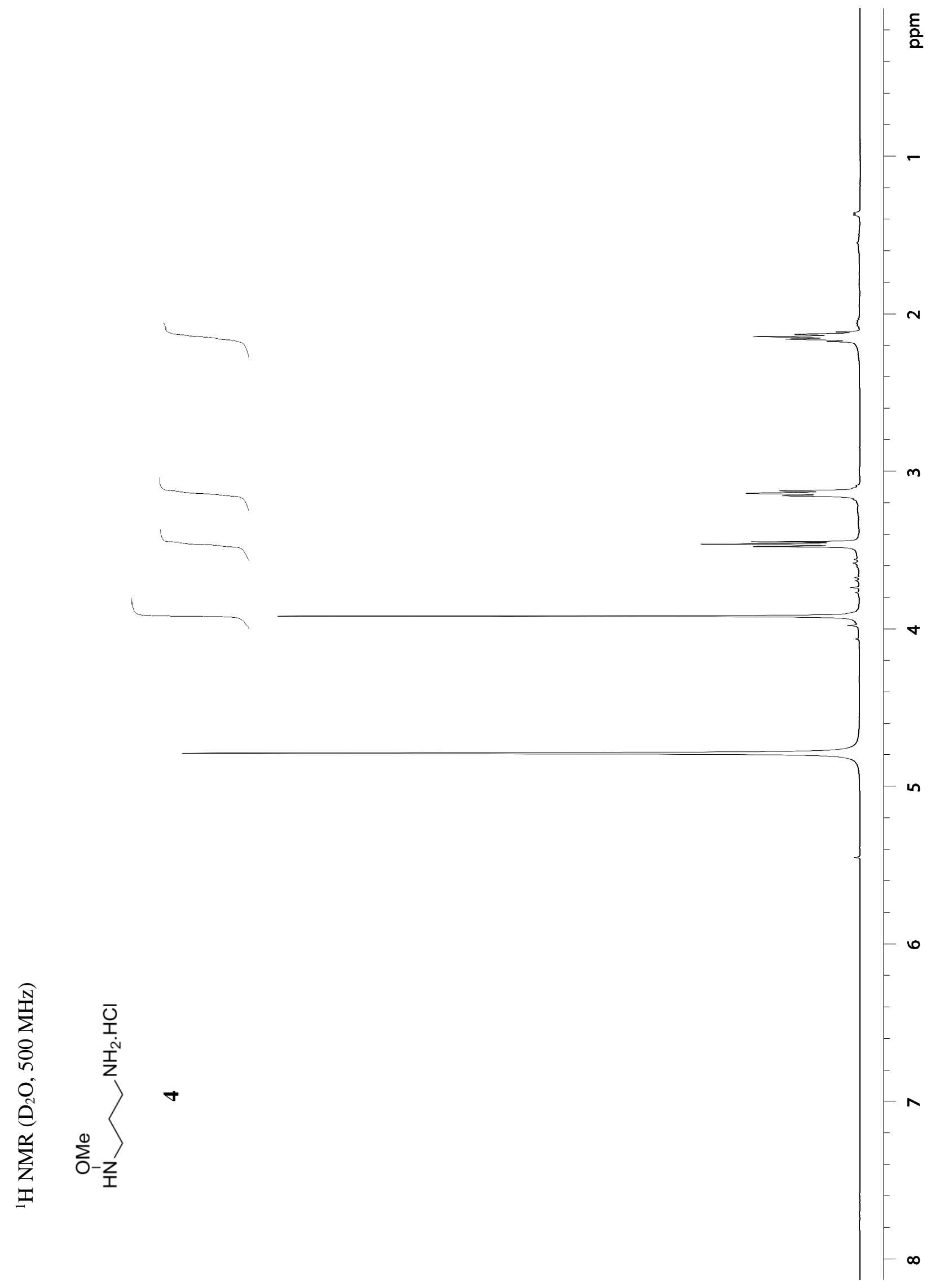

Appendix - 9 


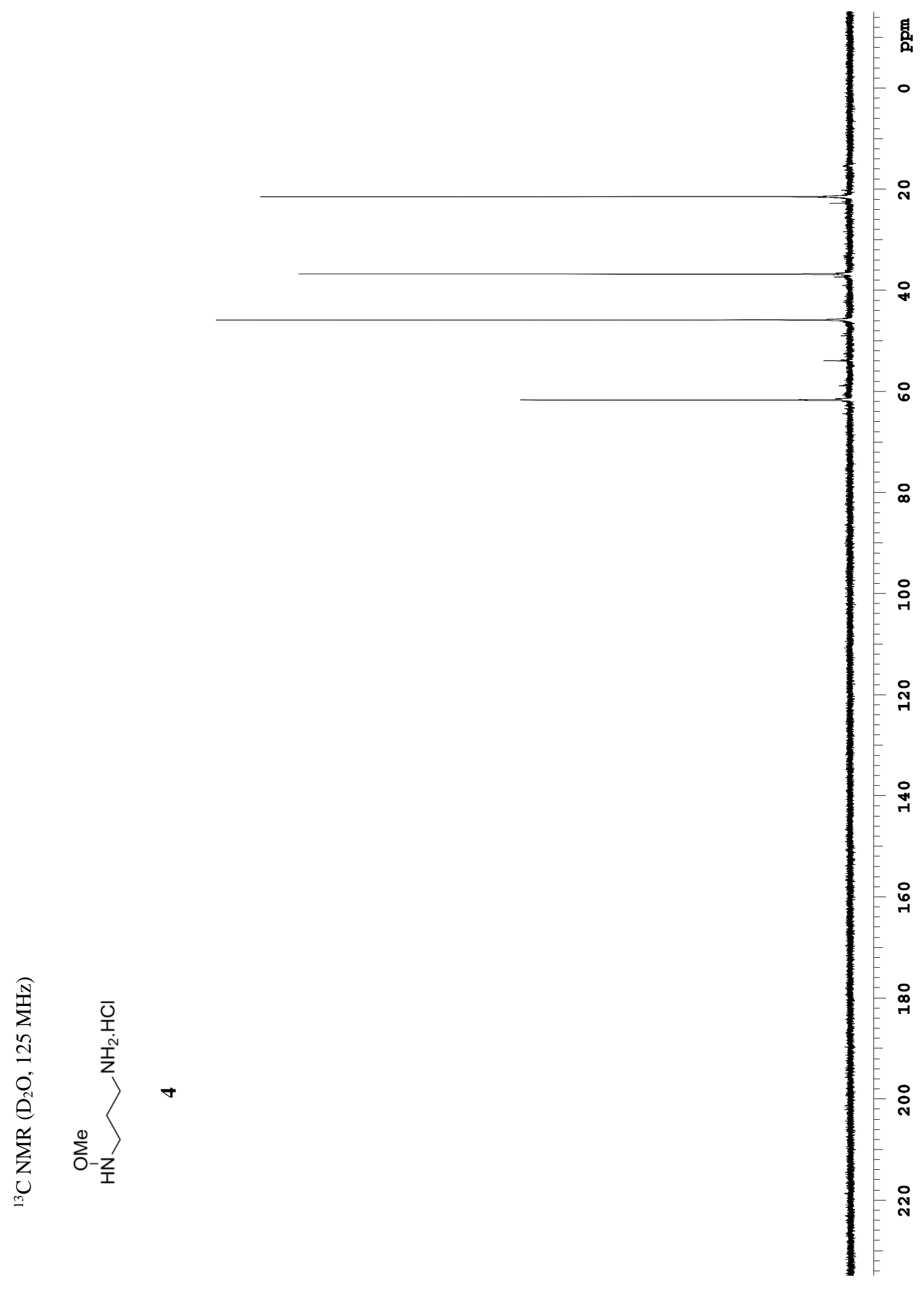

Appendix - 10 


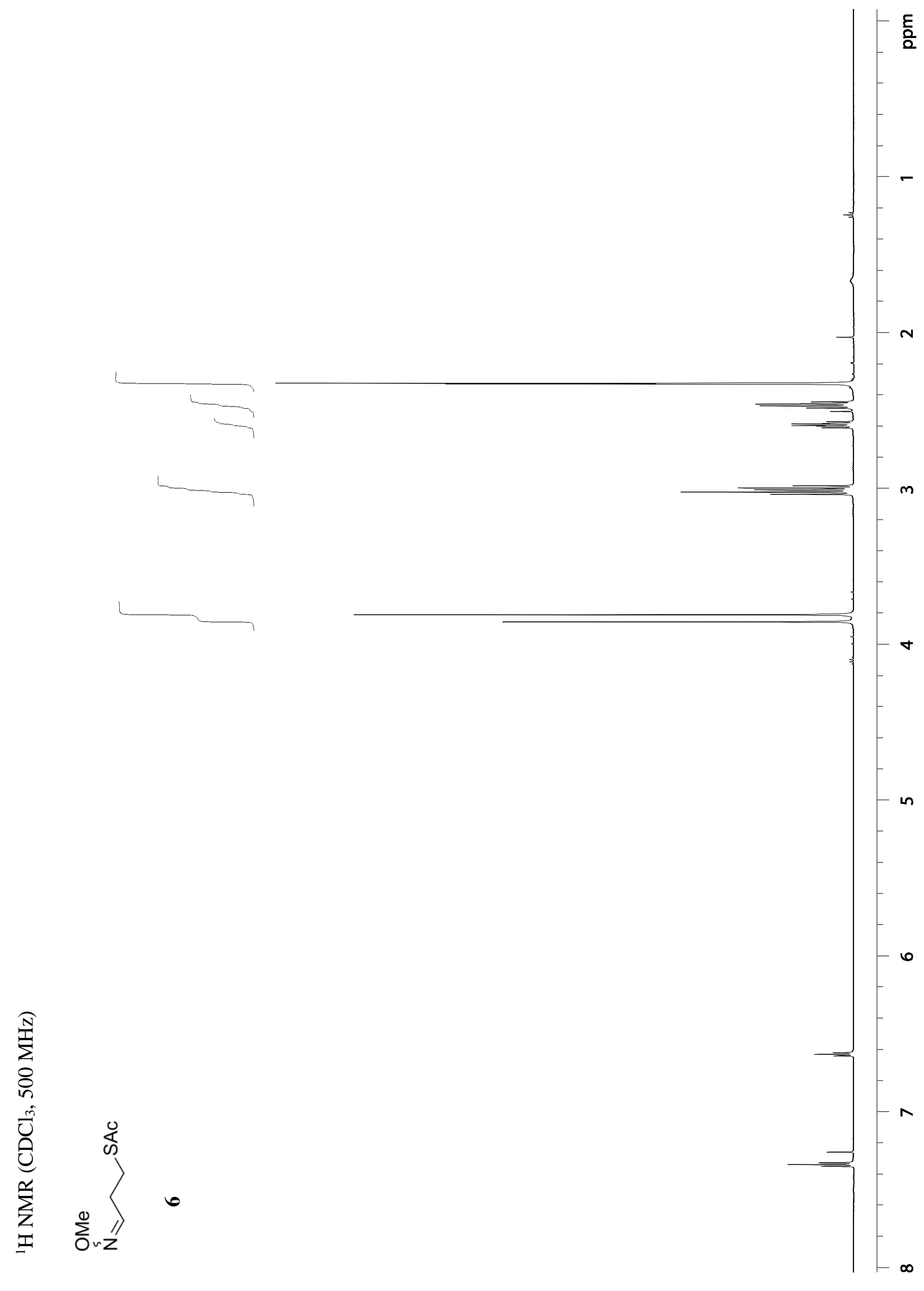

Appendix - 11 


$$
1
$$




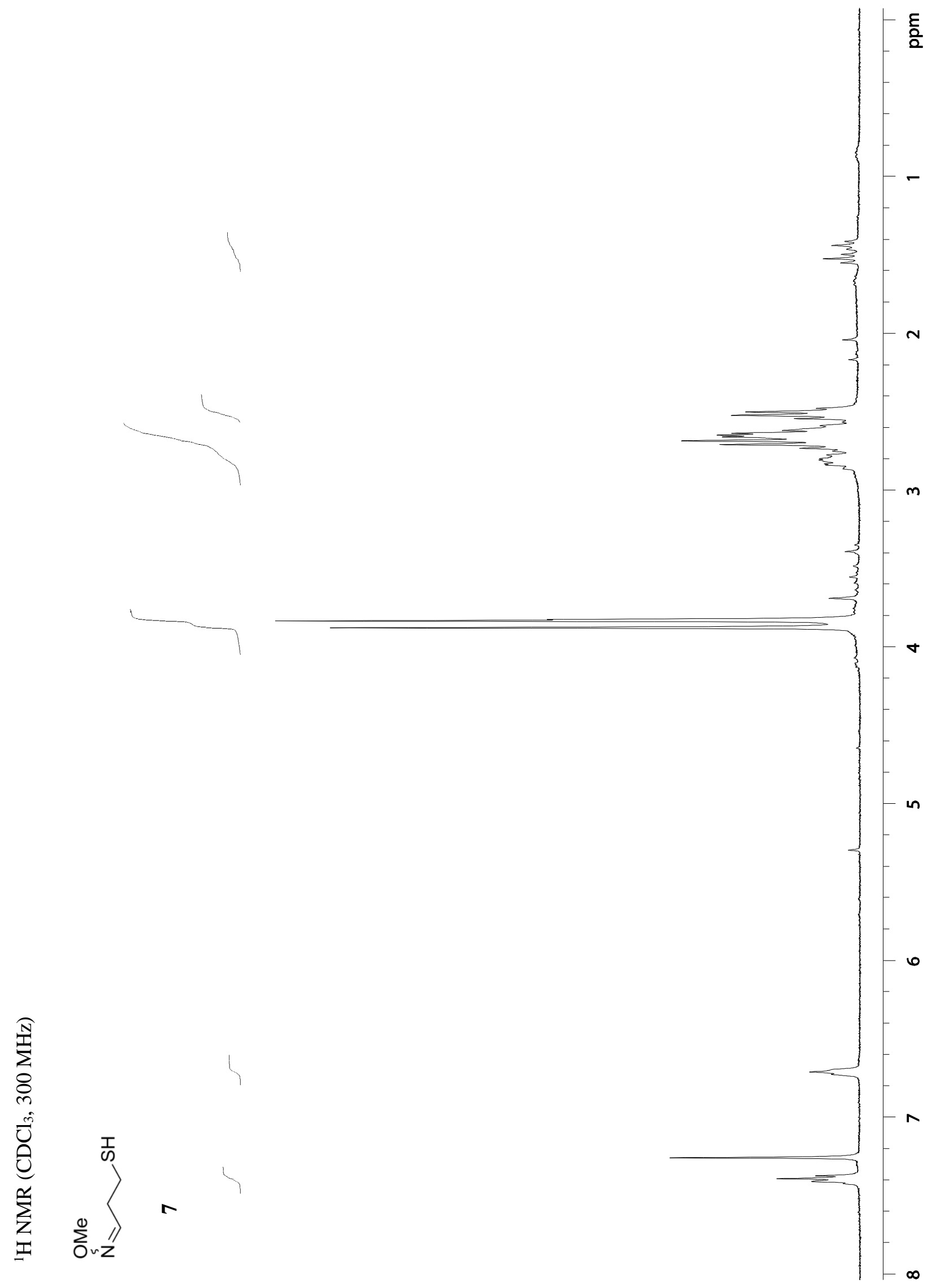

Appendix - 13 


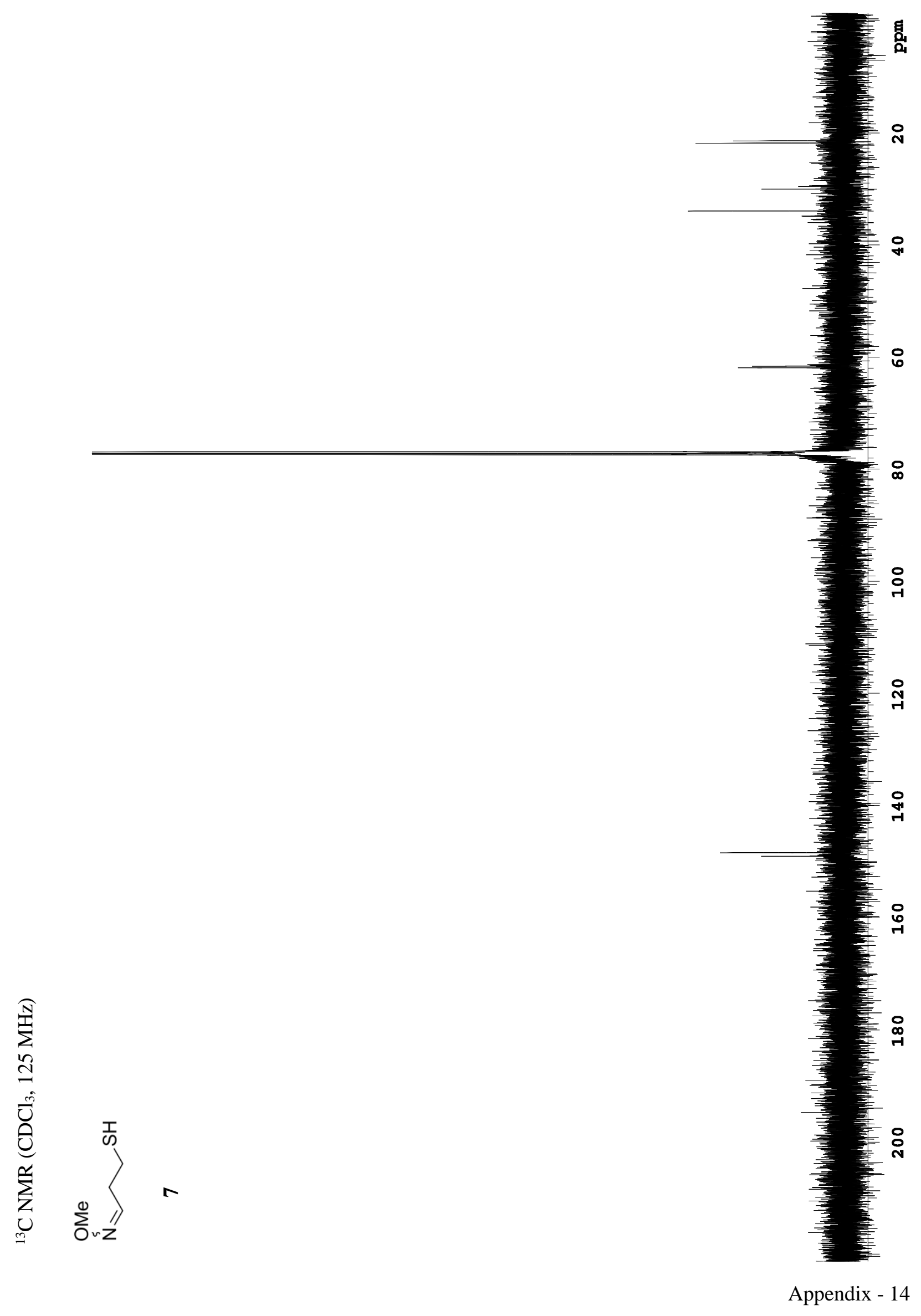




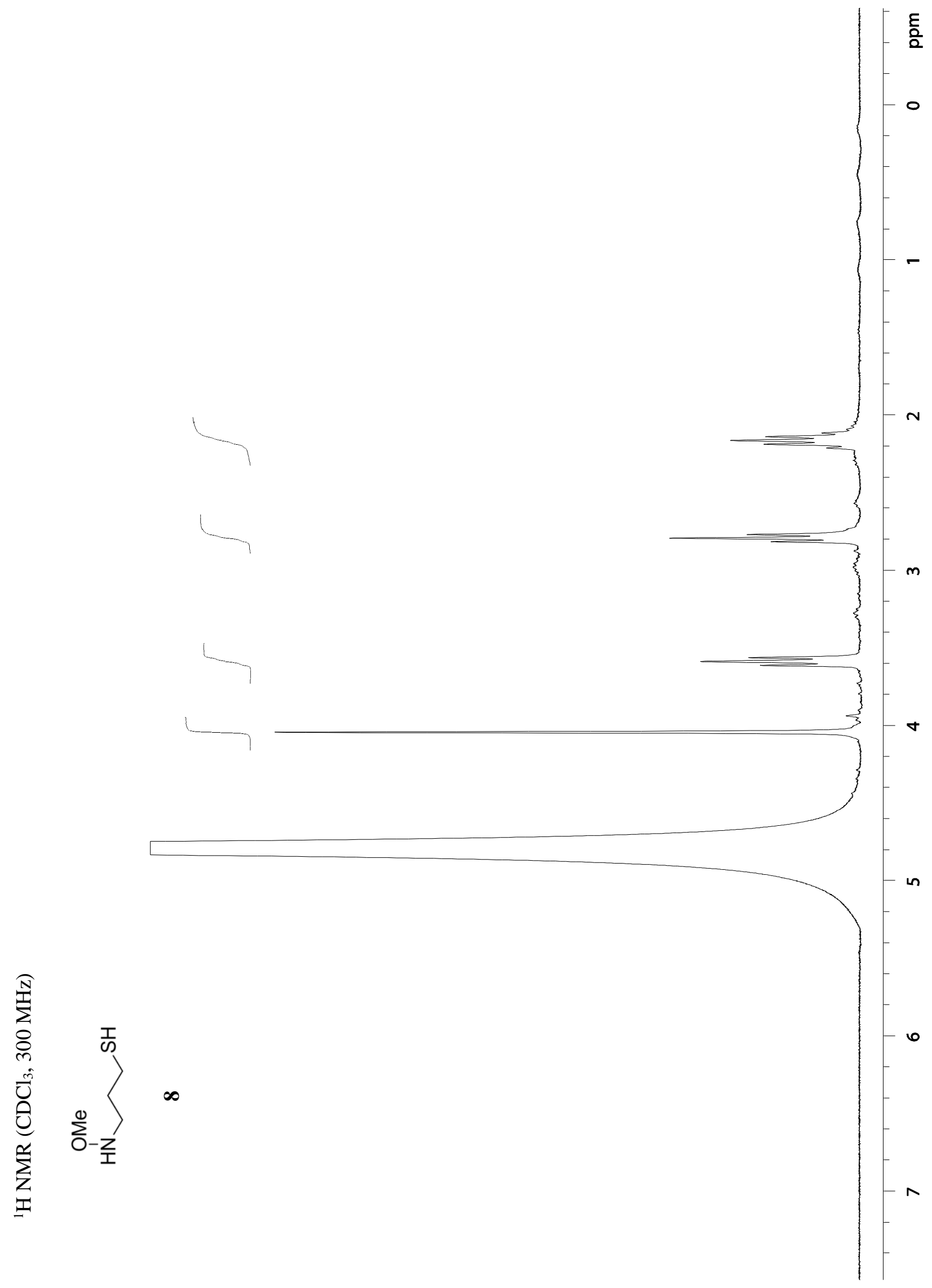

Appendix - 15 



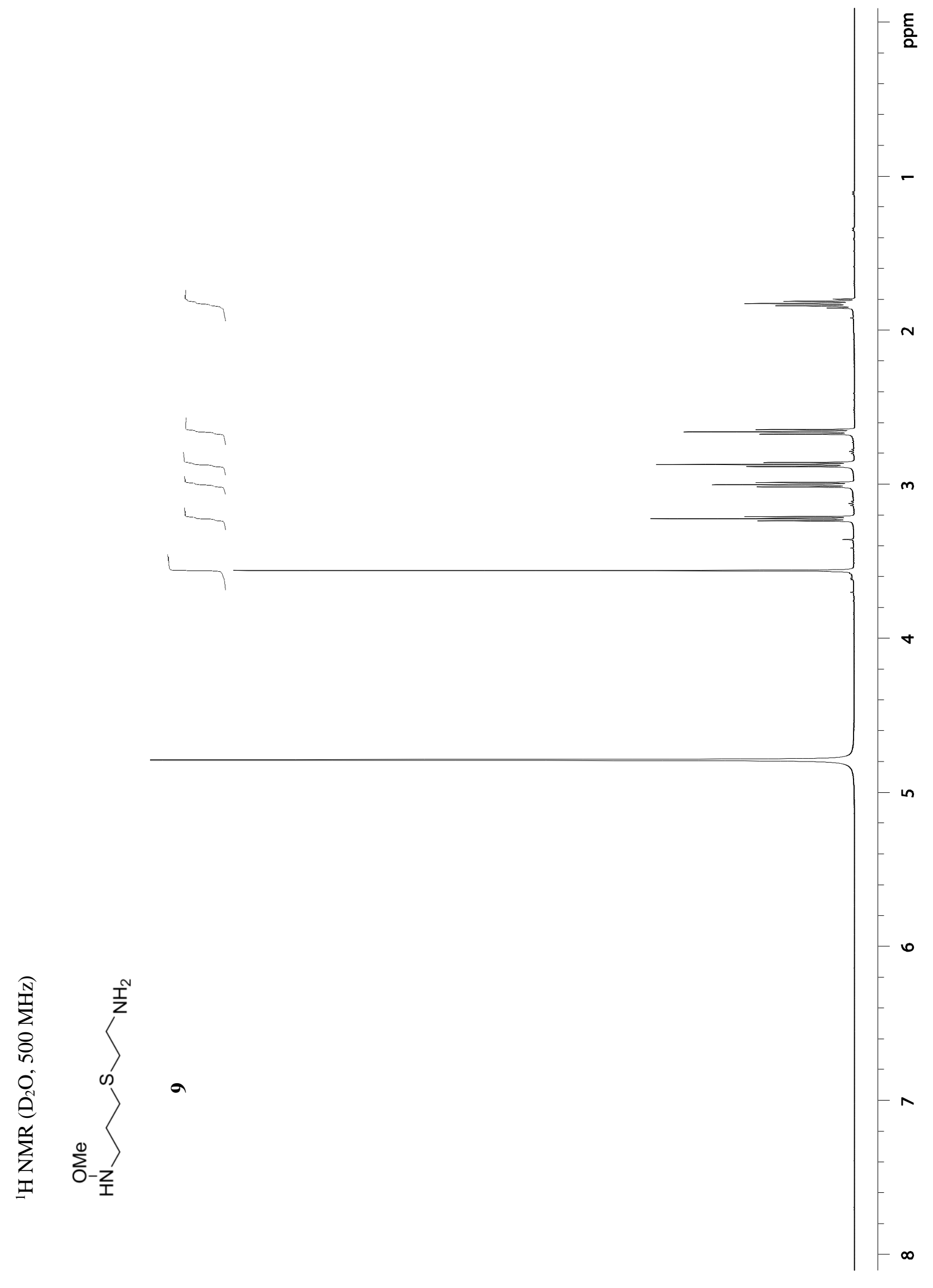

Appendix - 17 


$$
\text { W }
$$




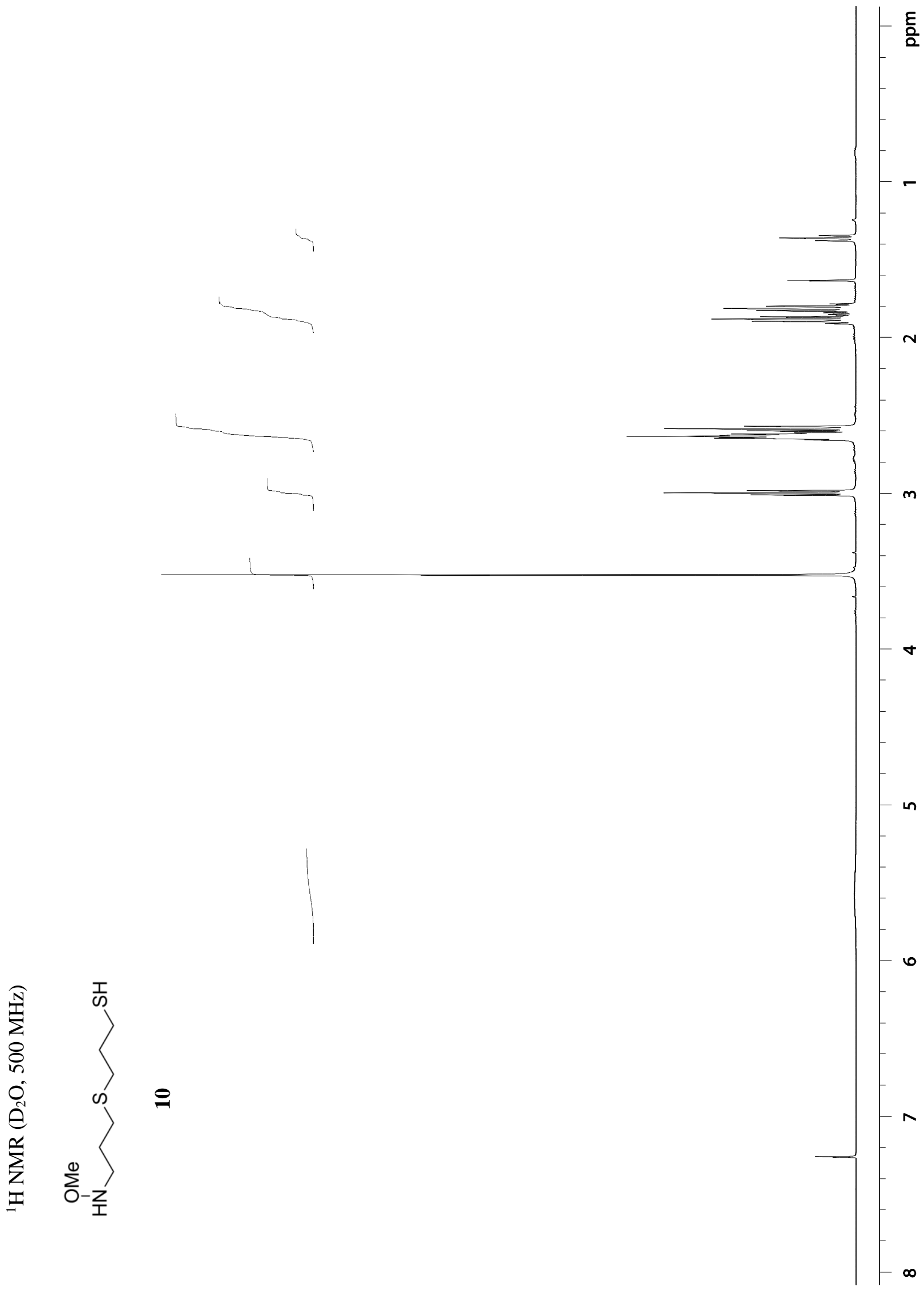

Appendix - 19 


$$
1
$$




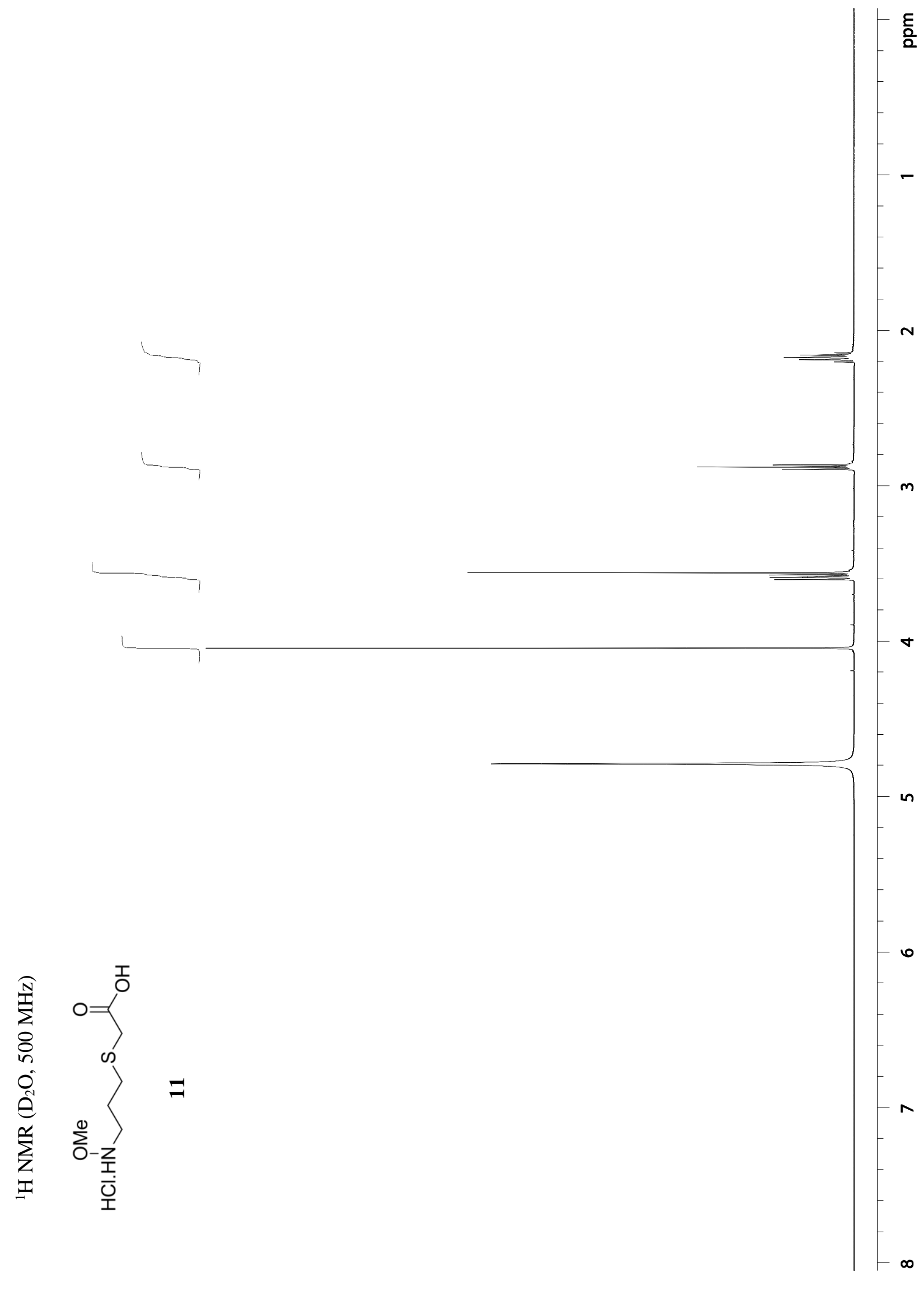

Appendix - 21 


$$
\mid
$$




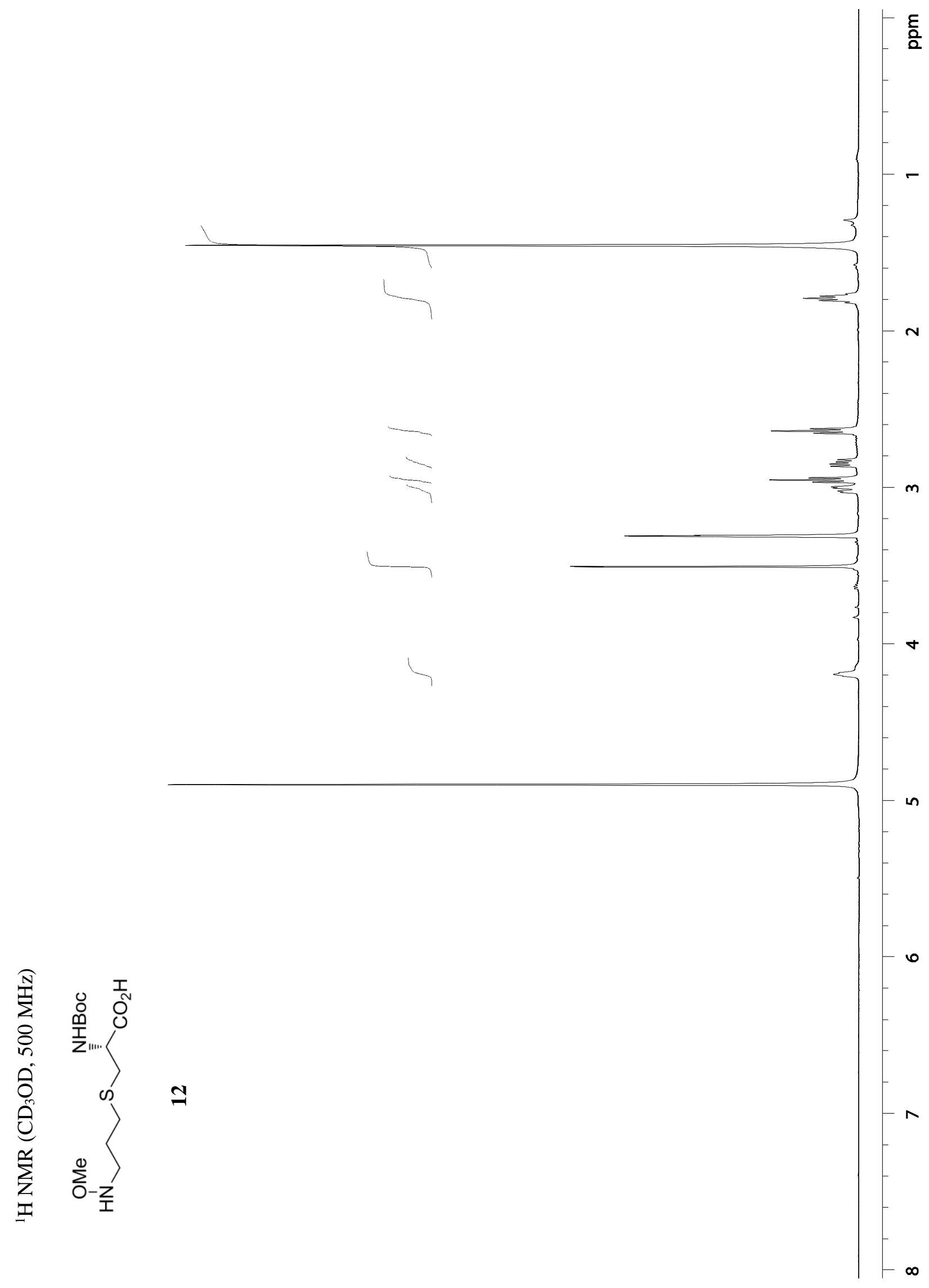

Appendix - 23 


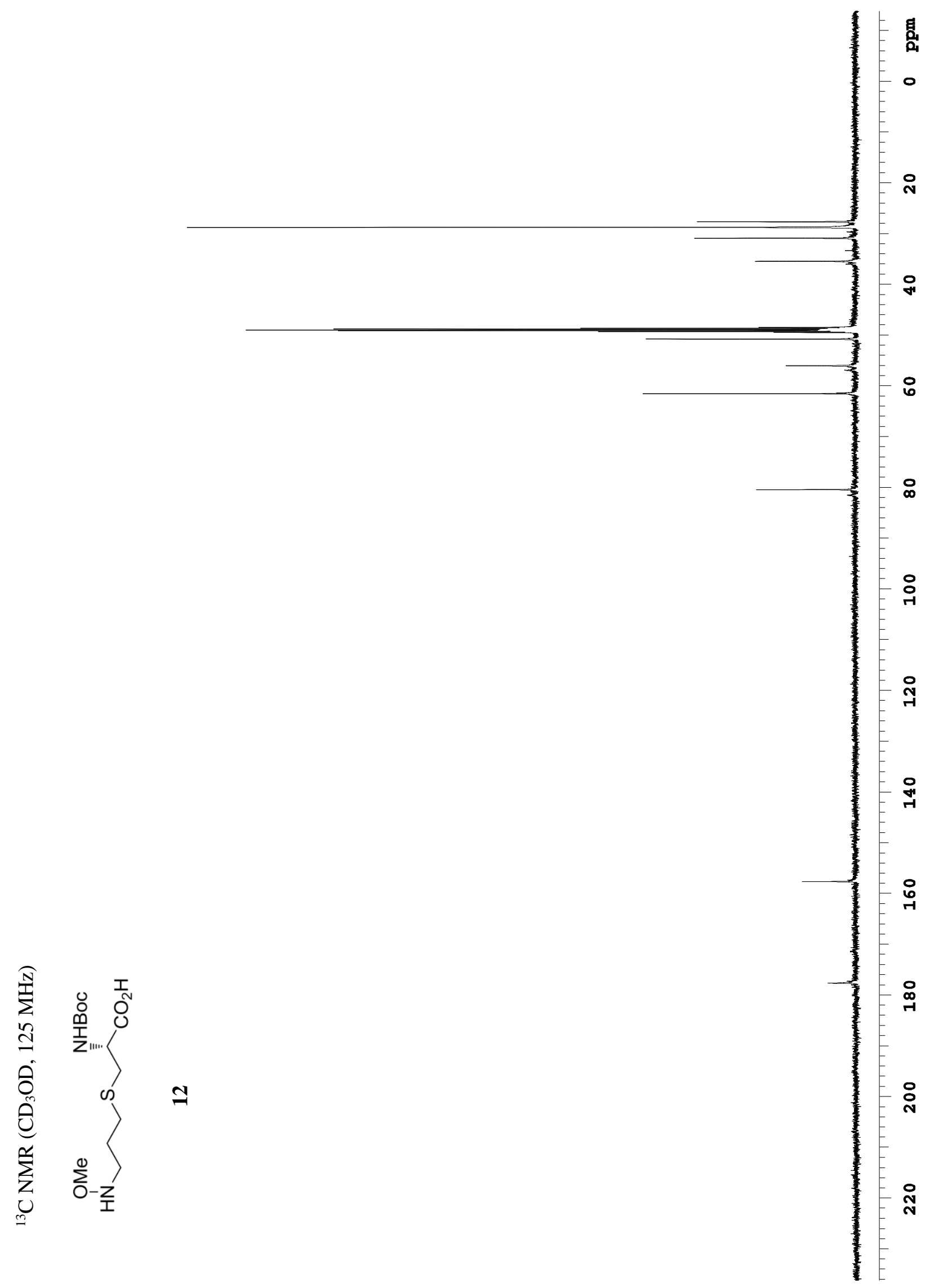

Appendix - 24 


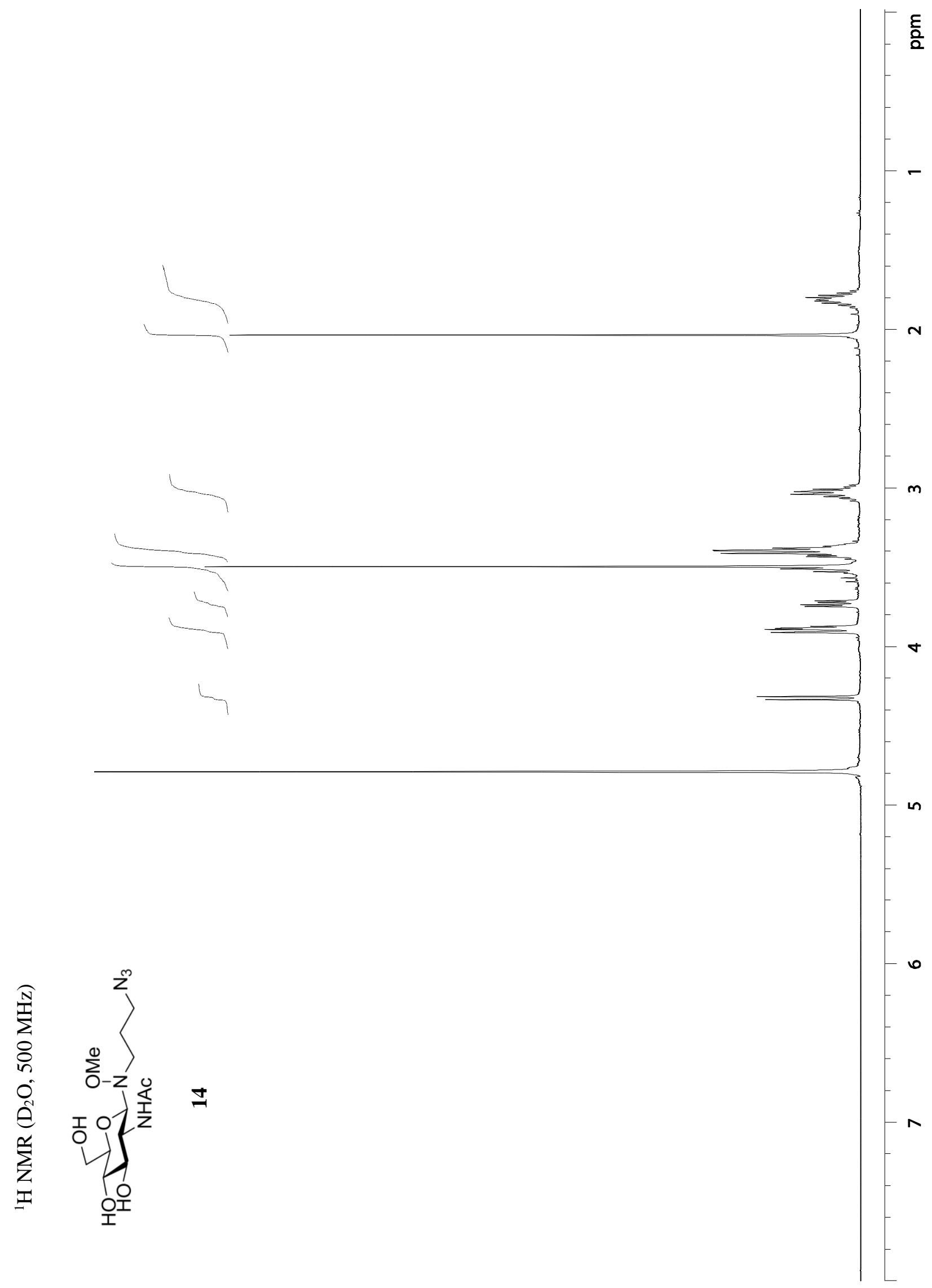

Appendix - 25 


$$
1
$$




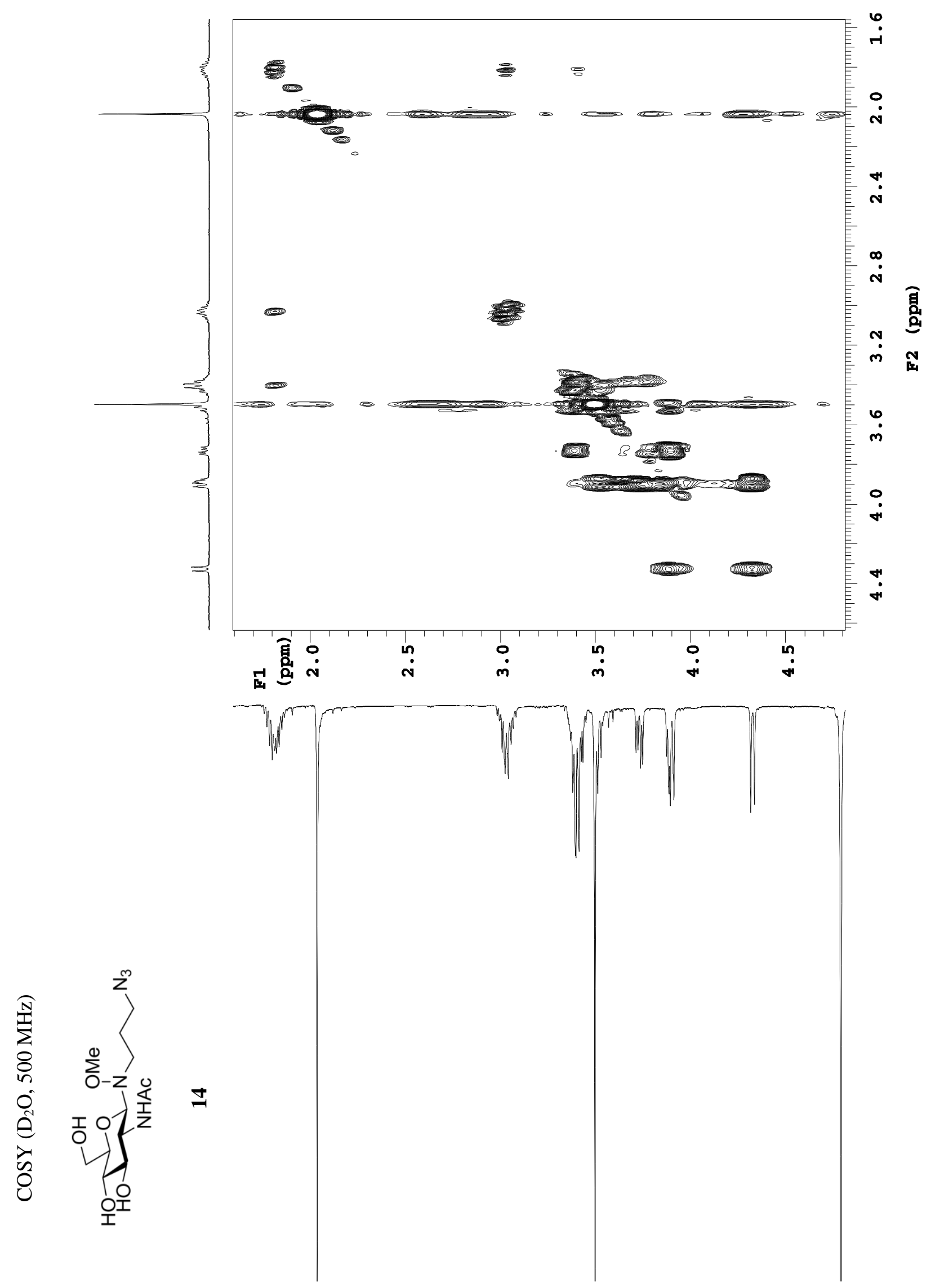

Appendix - 27 


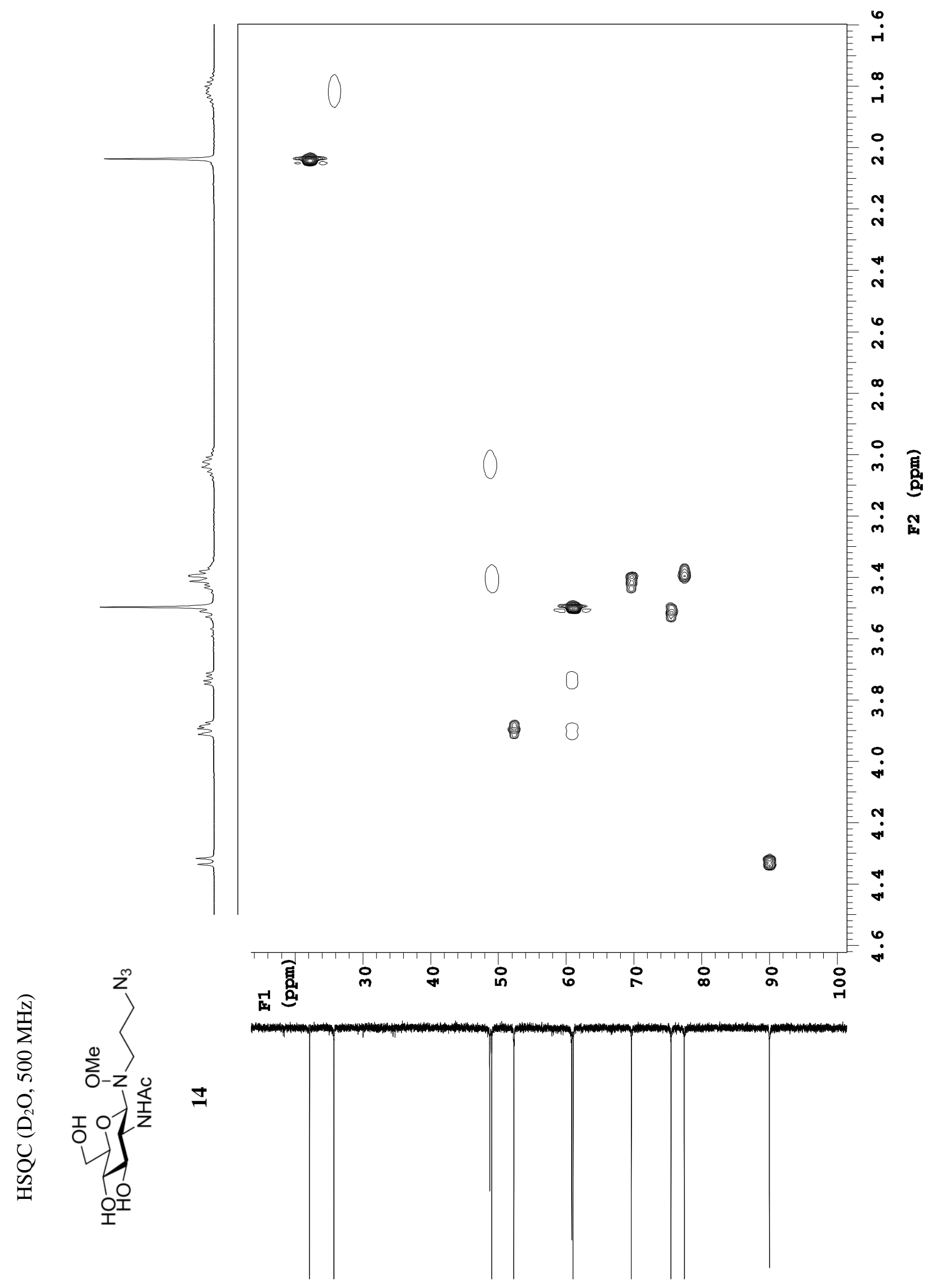

Appendix - 28 


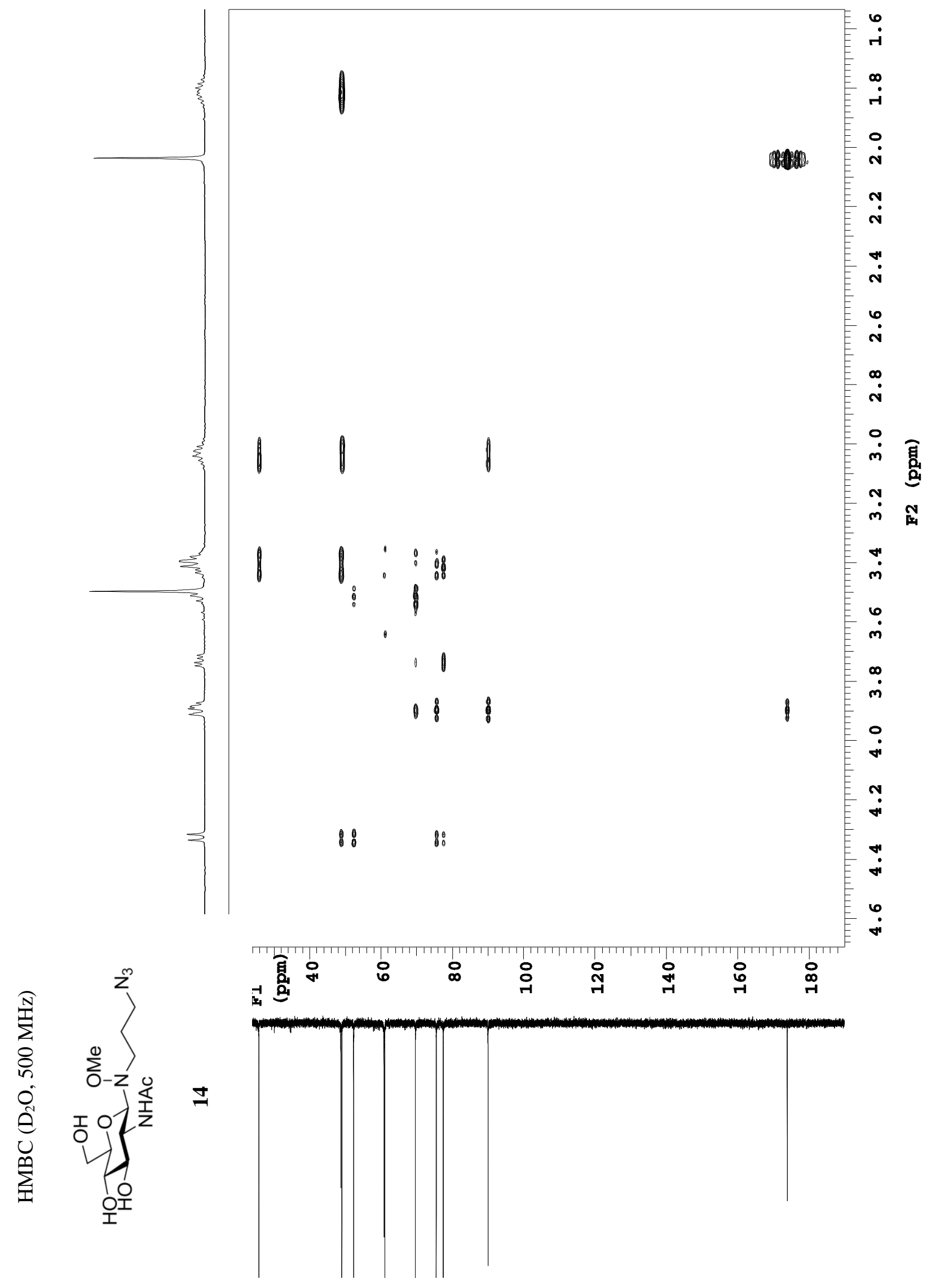

Appendix - 29 


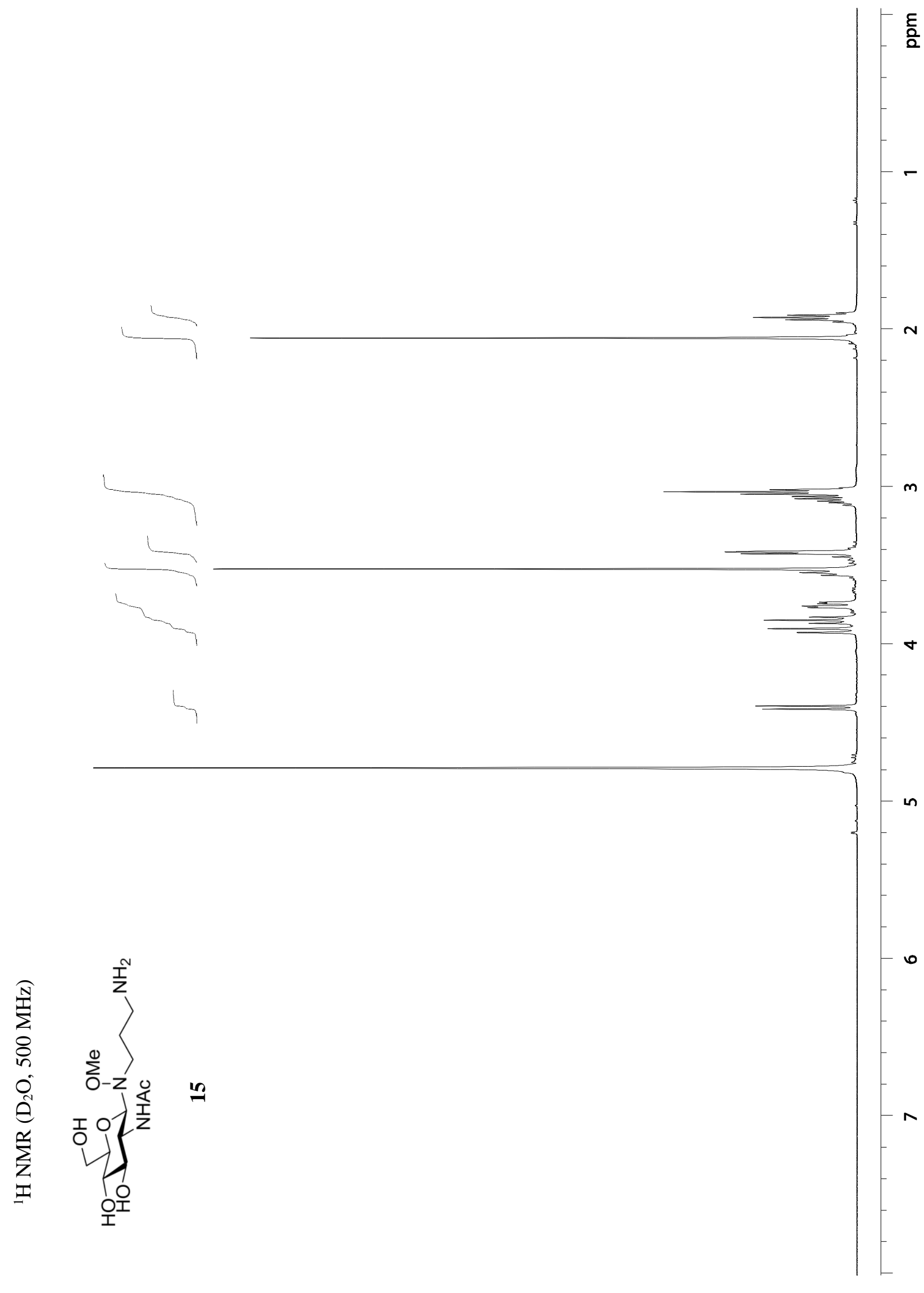

Appendix - 30 


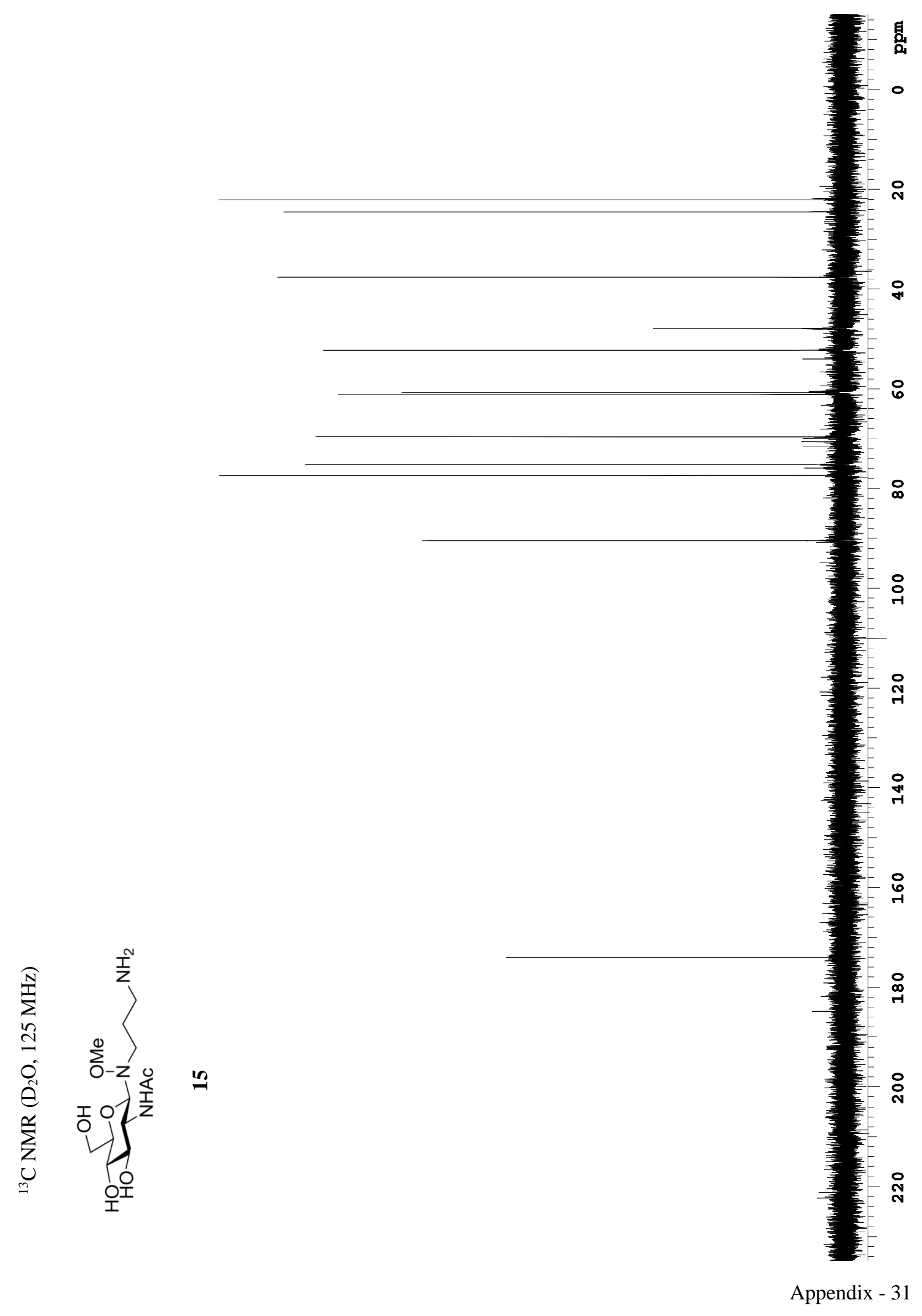




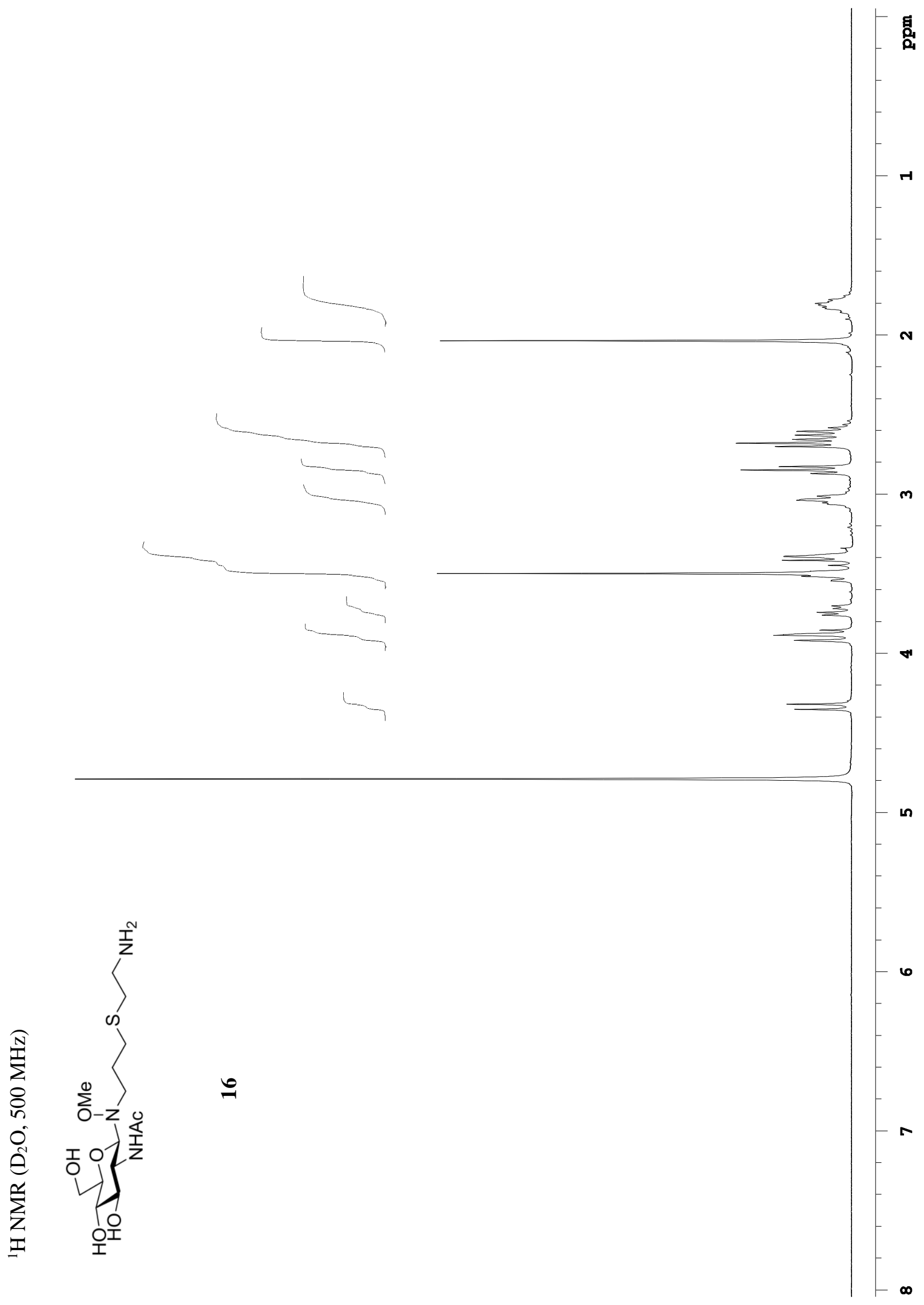

总

$-1$

N

เก

Appendix - 32 


$$
1
$$




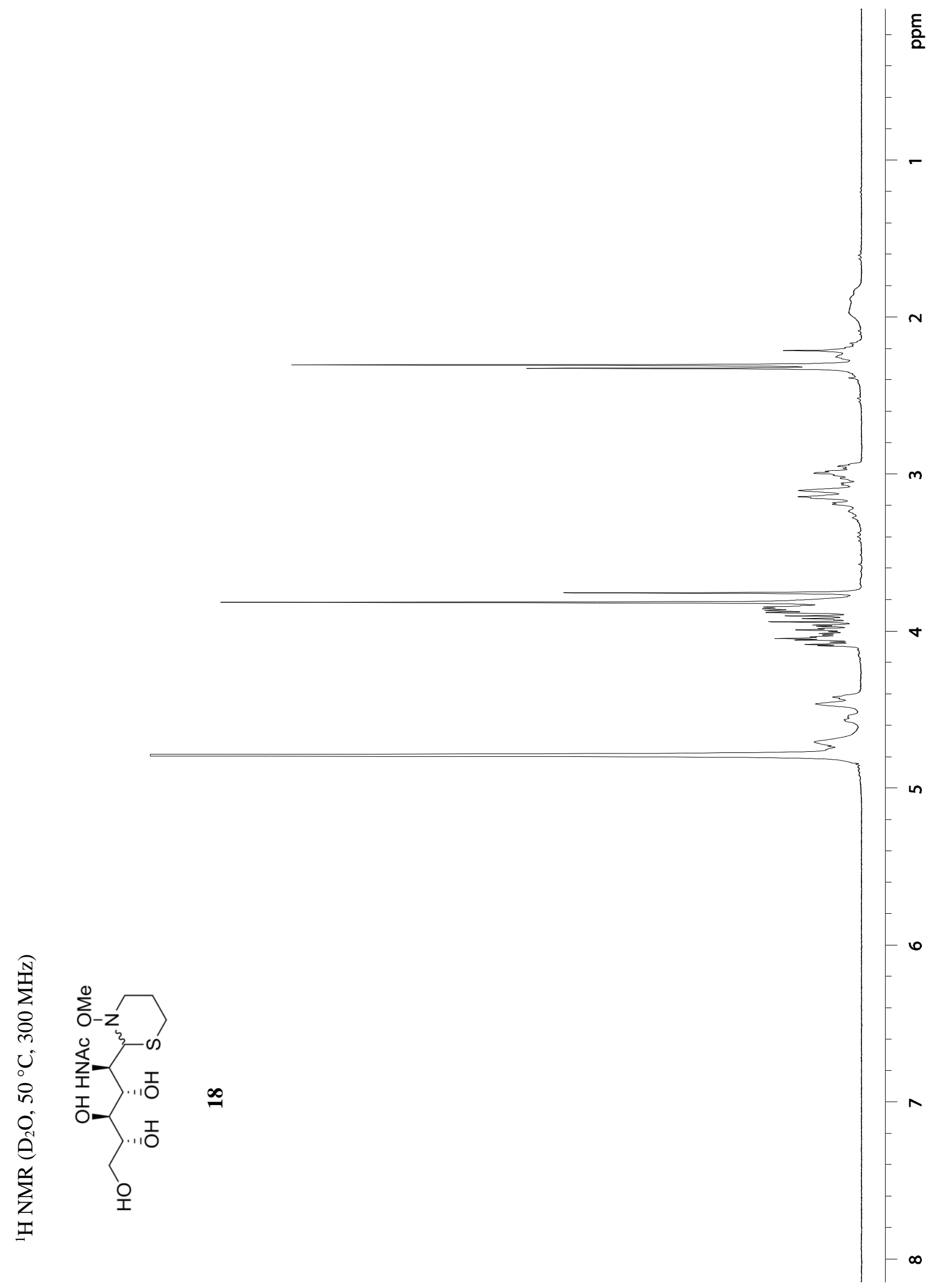

Appendix - 34 


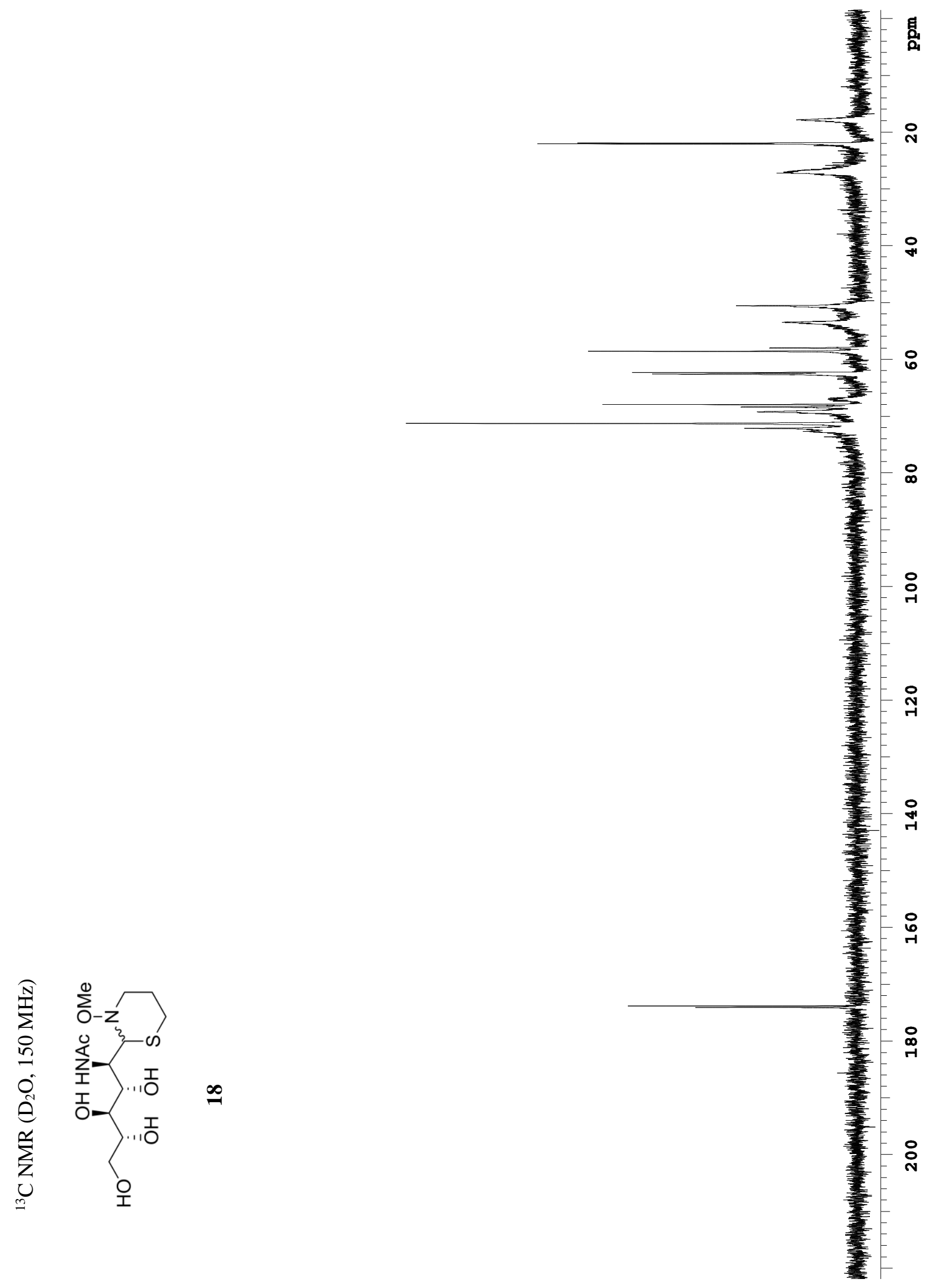

Appendix - 35 


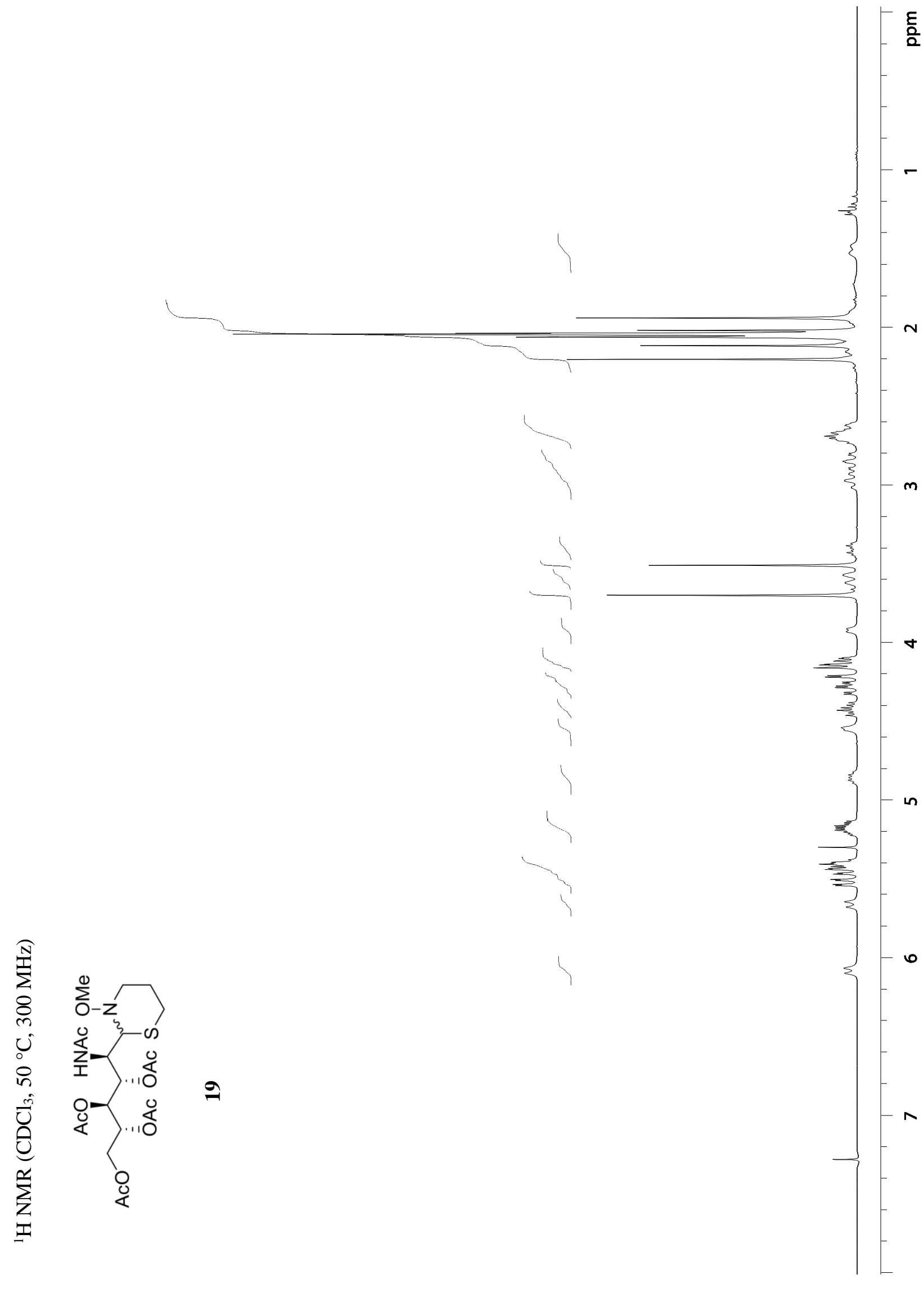

Appendix - 36 


$$
1
$$




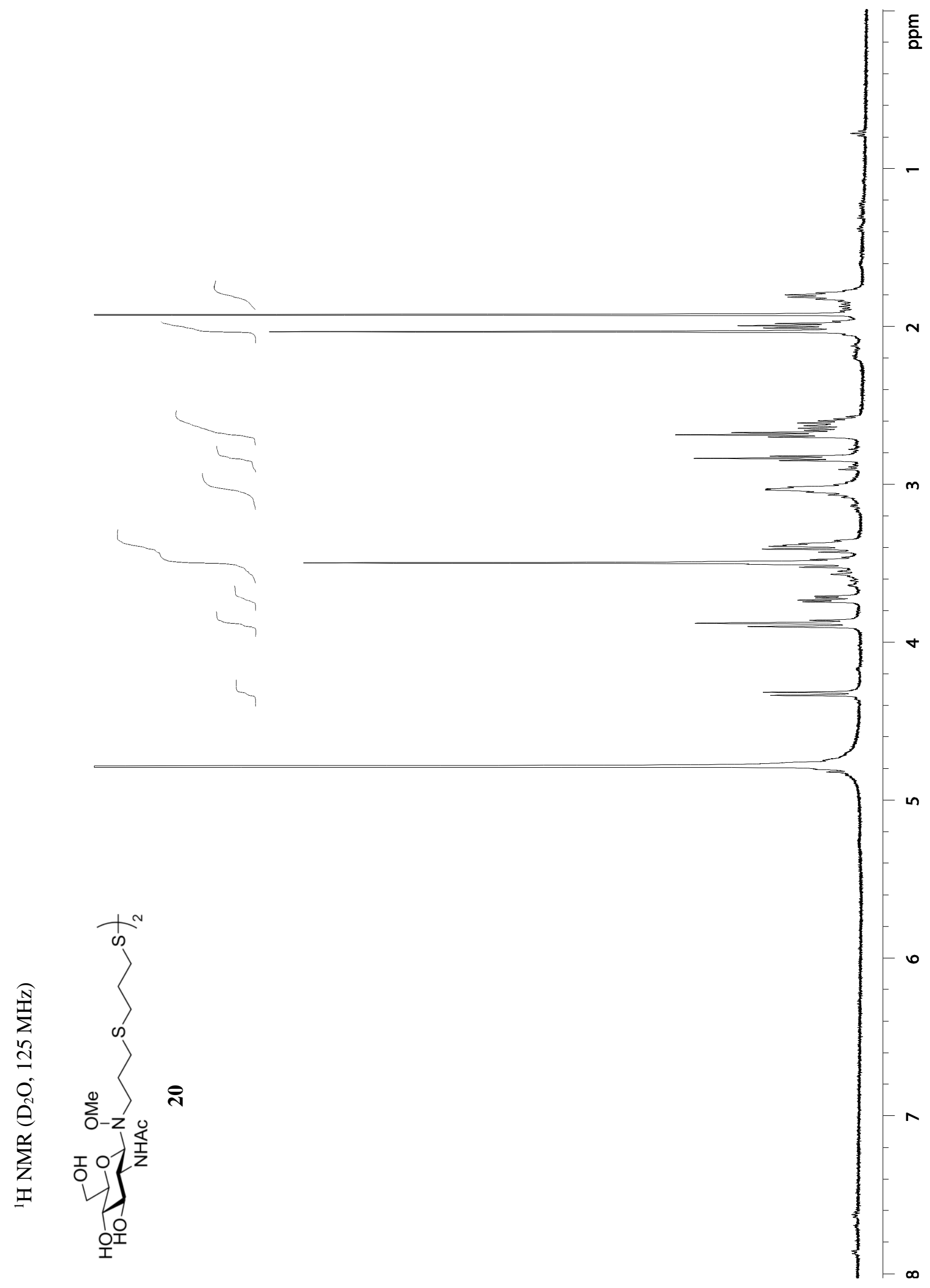

Appendix - 38 


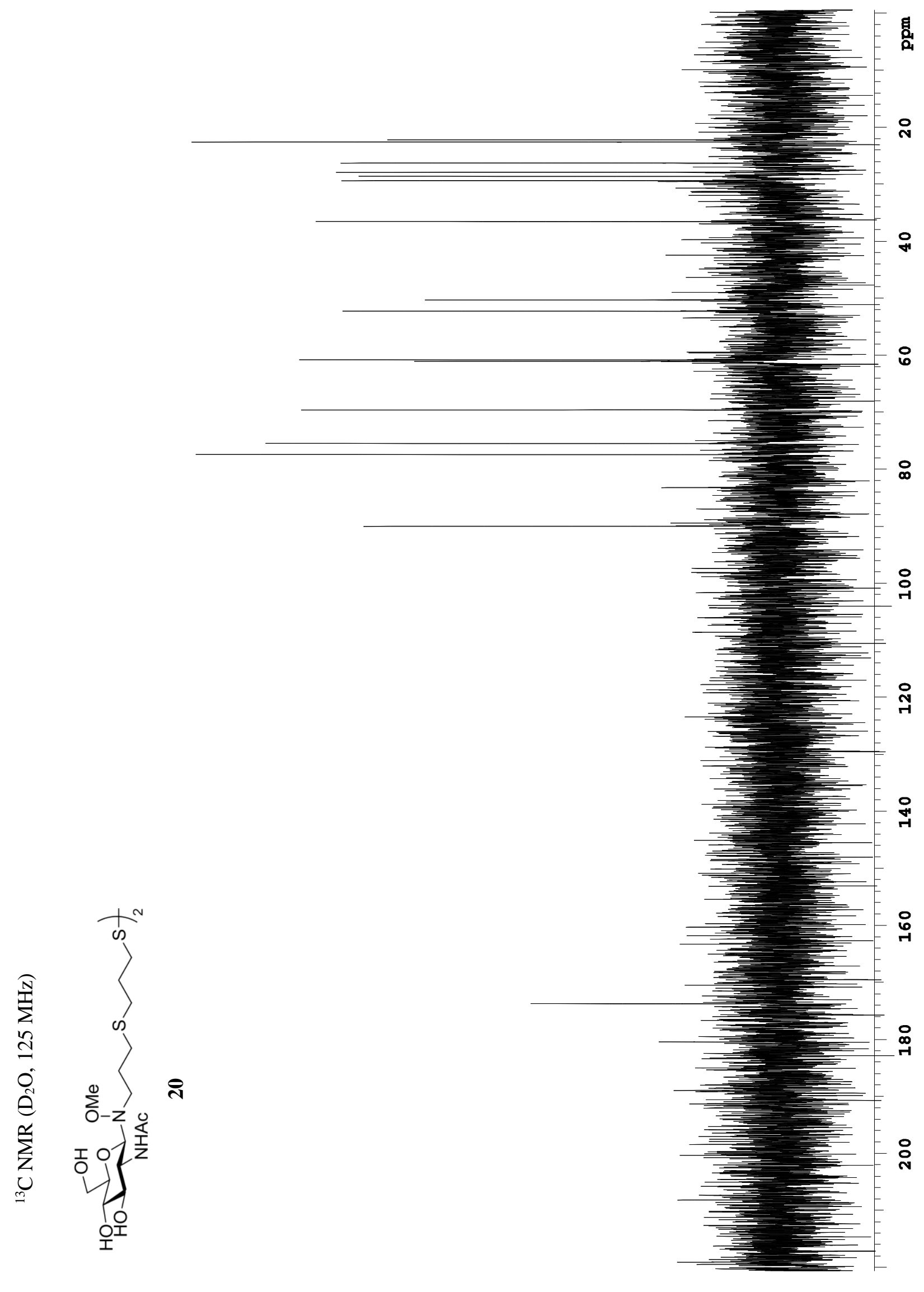

Appendix - 39 

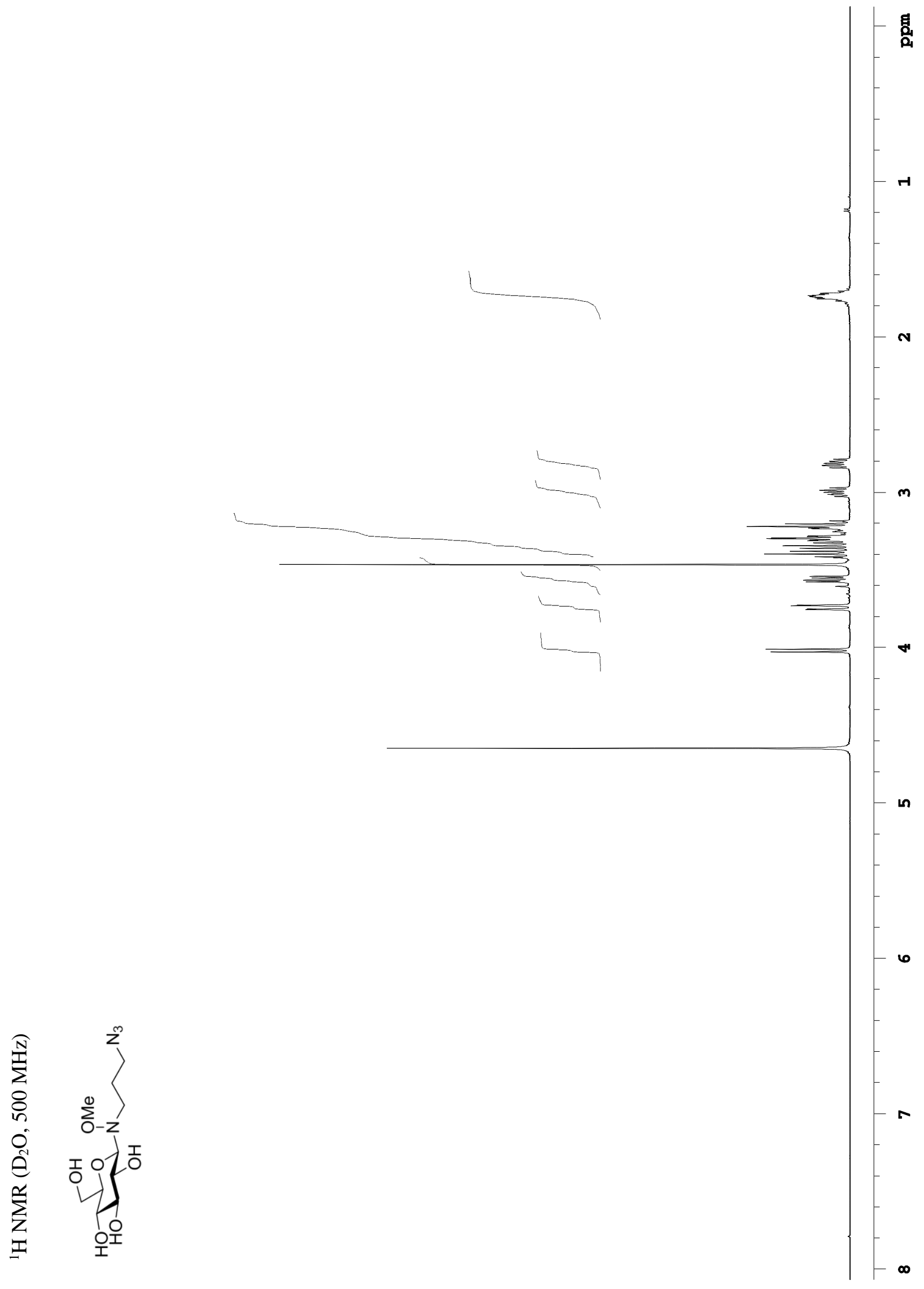

Appendix - 40 


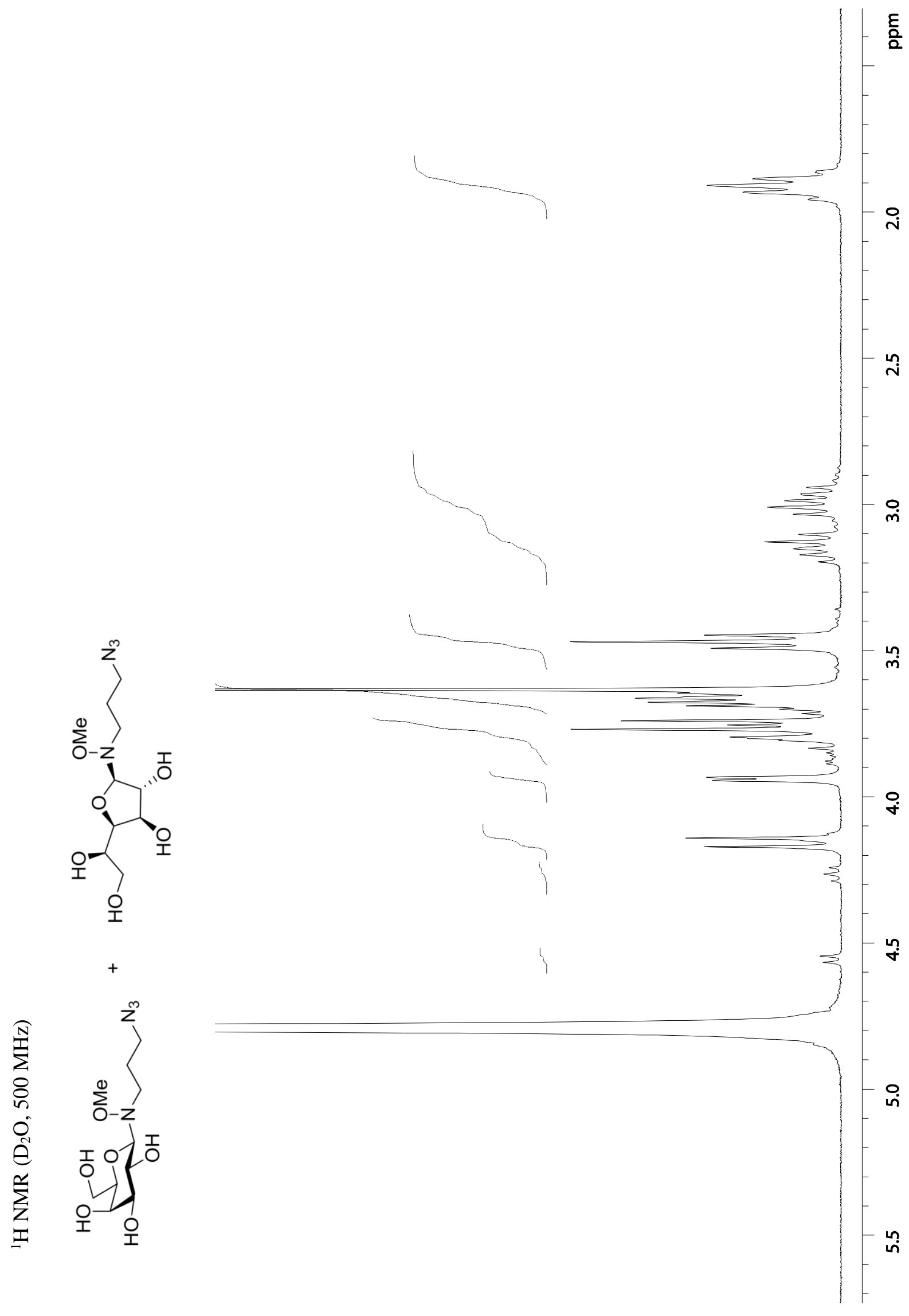

Appendix - 41 


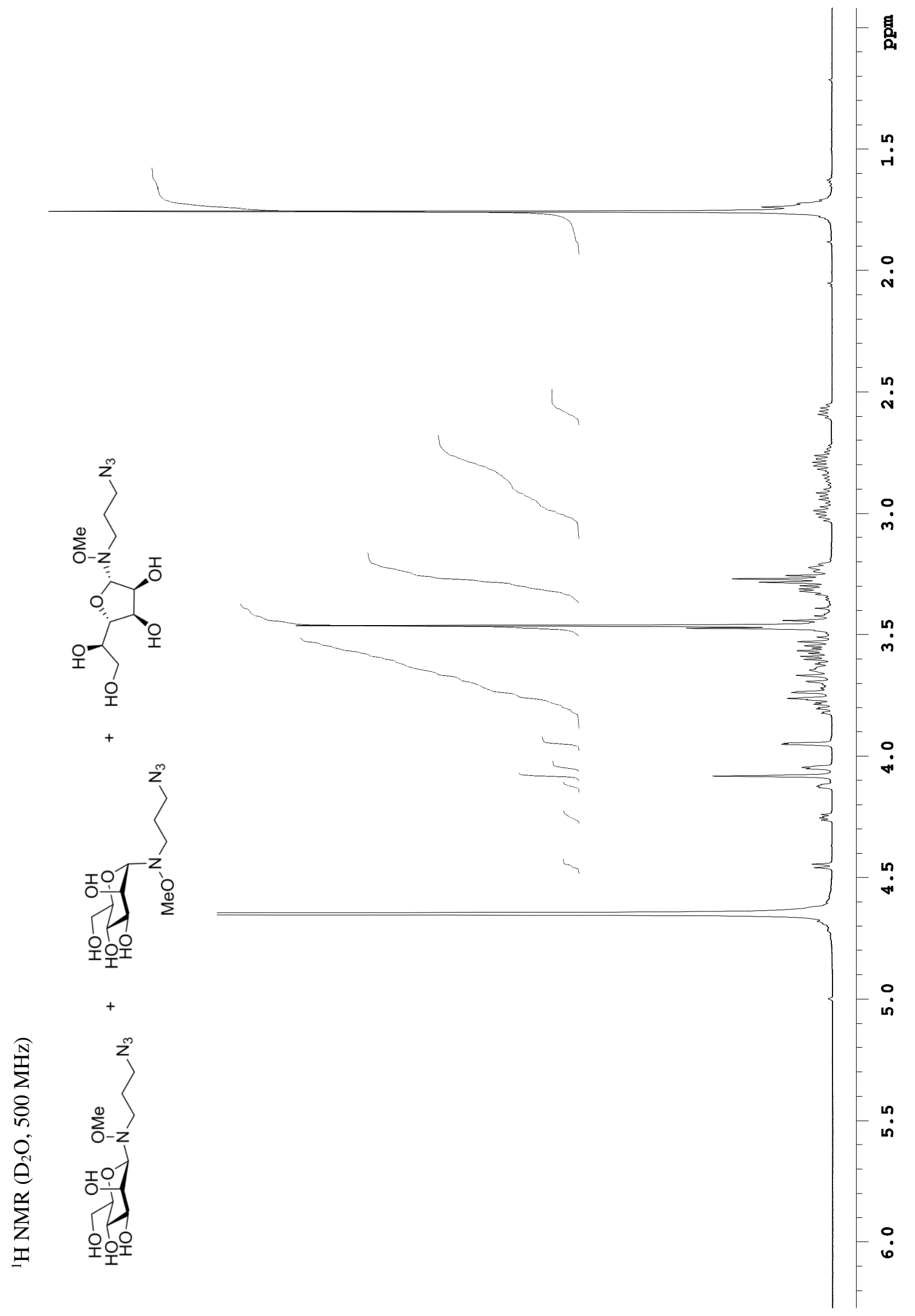

Appendix - 42 


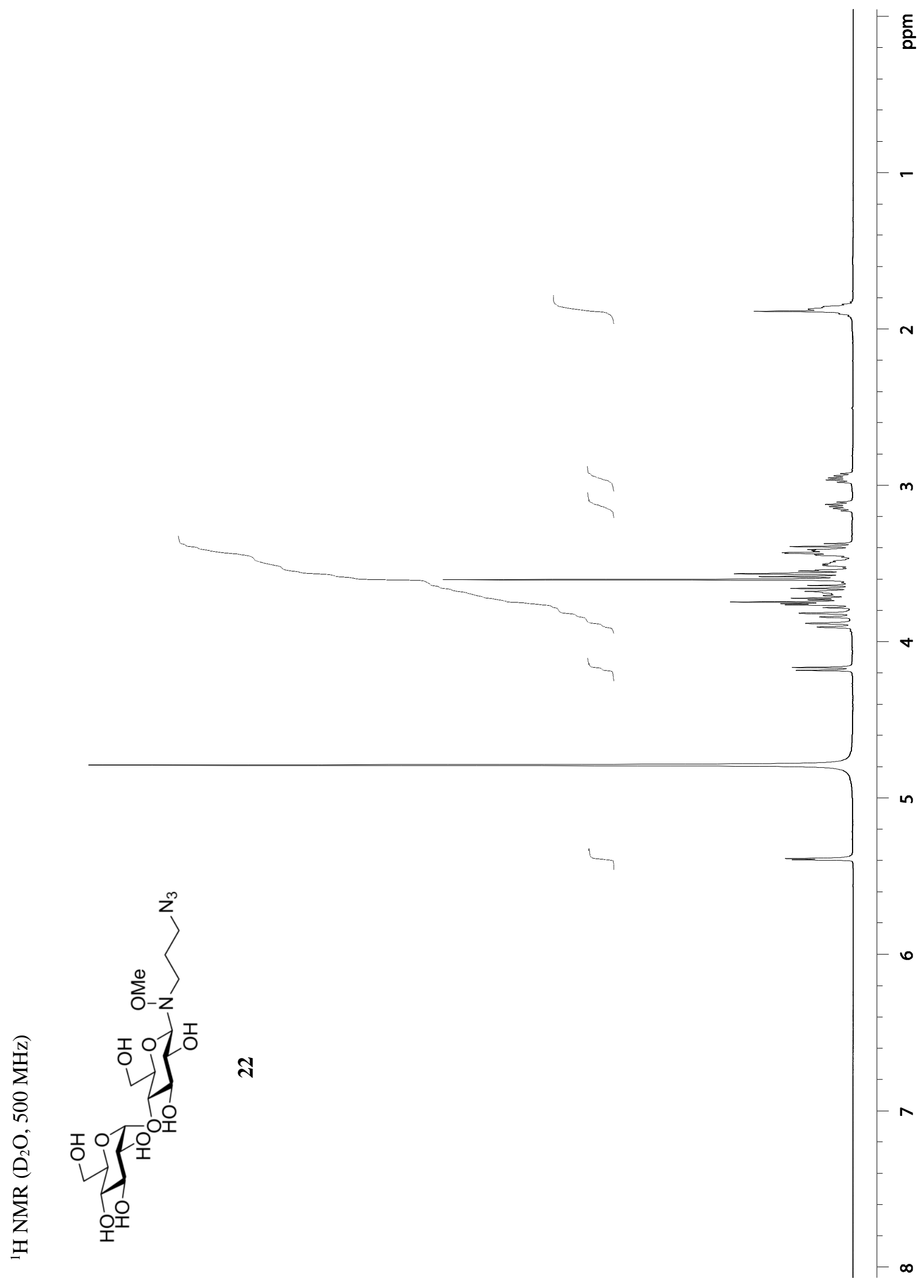

Appendix - 43 

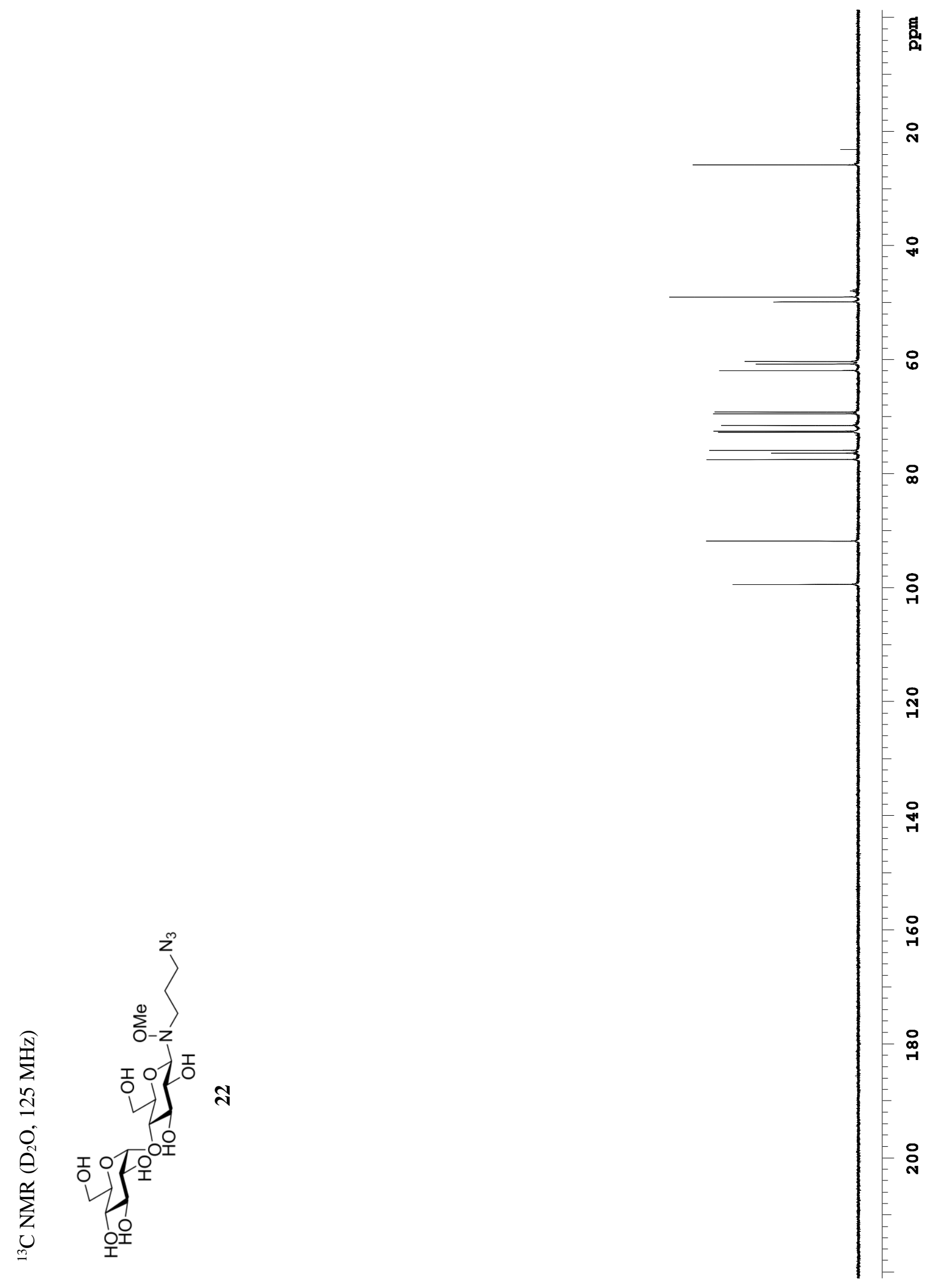

Appendix - 44 


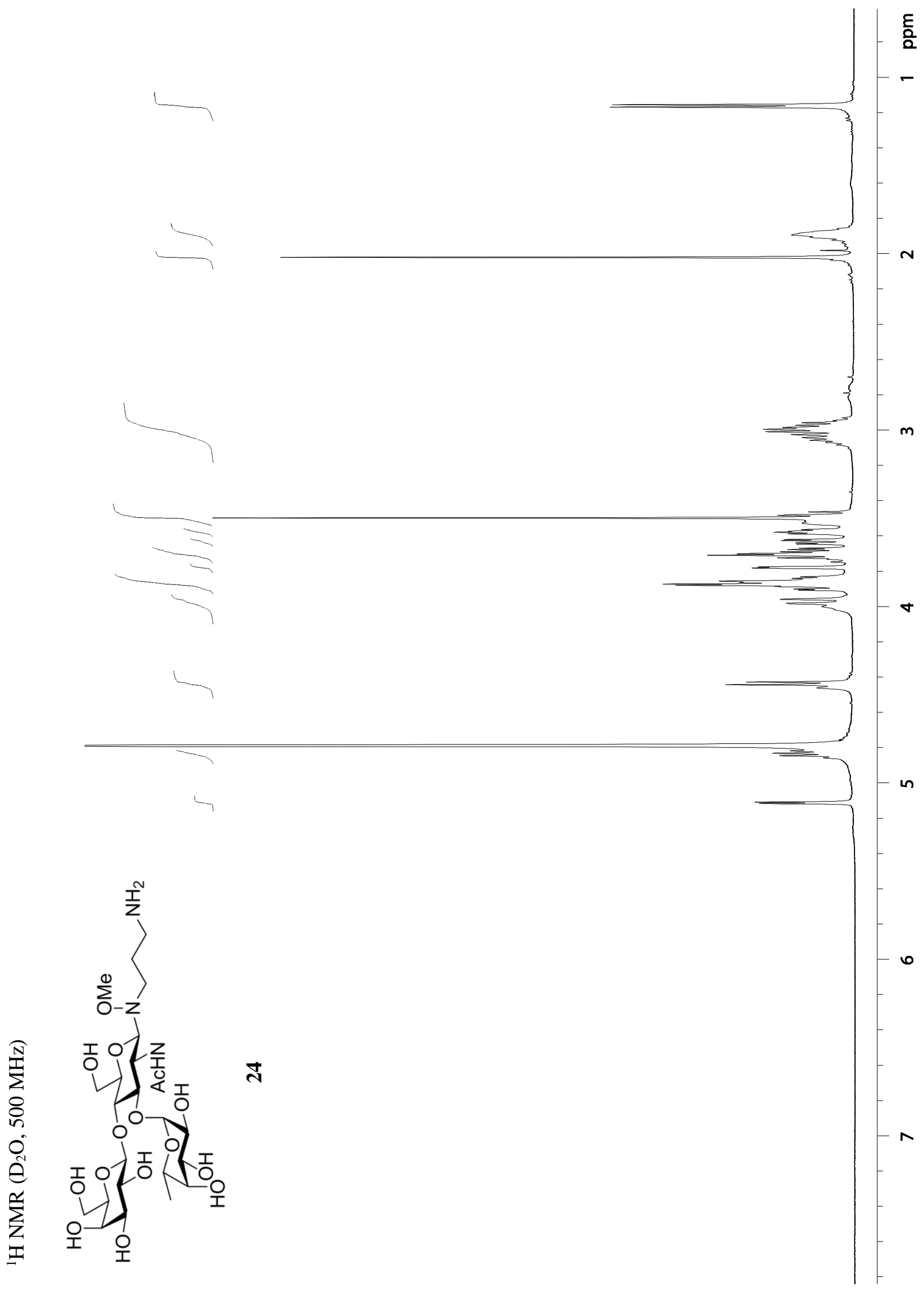

Appendix - 45 

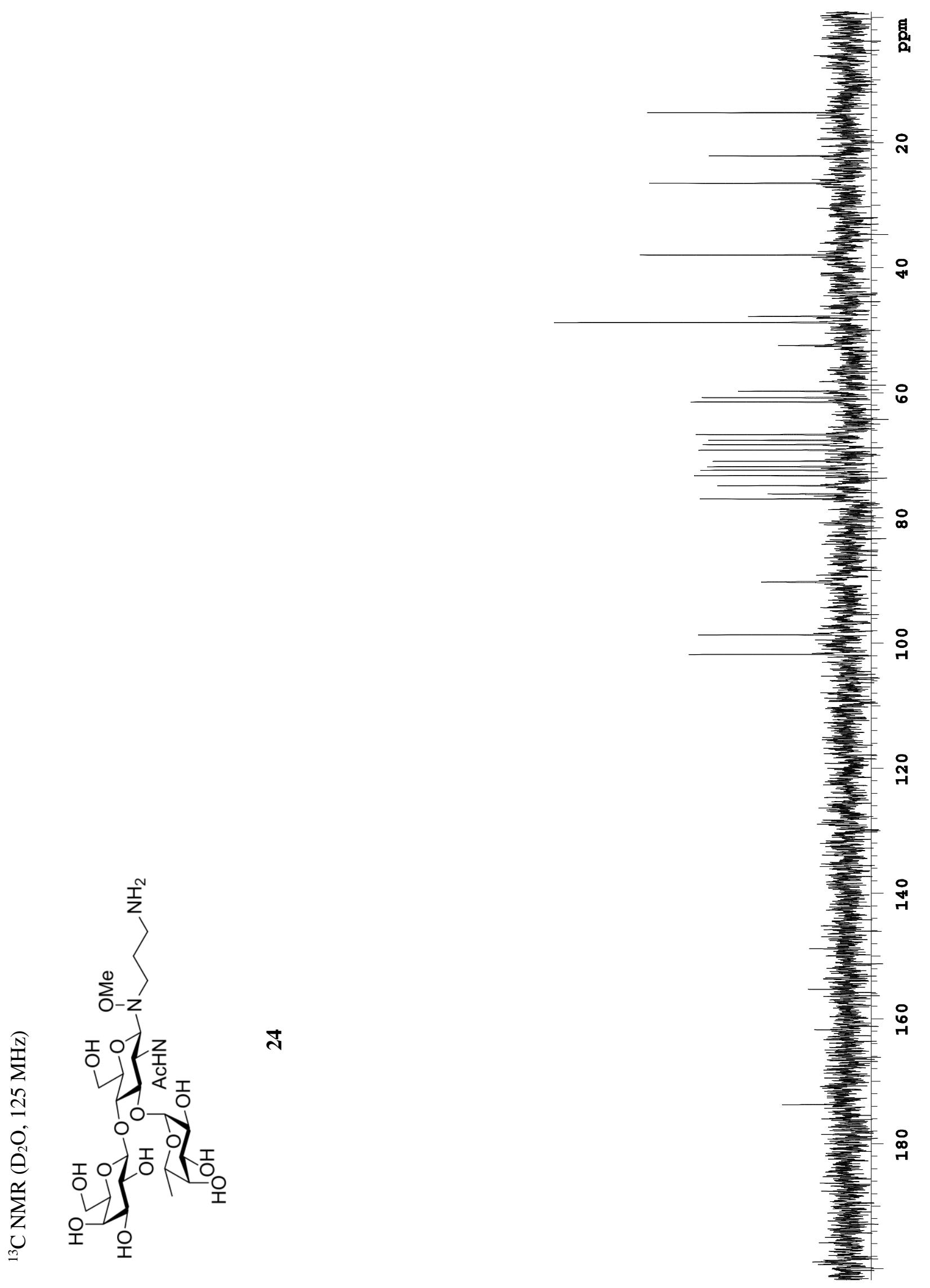

Appendix - 46 


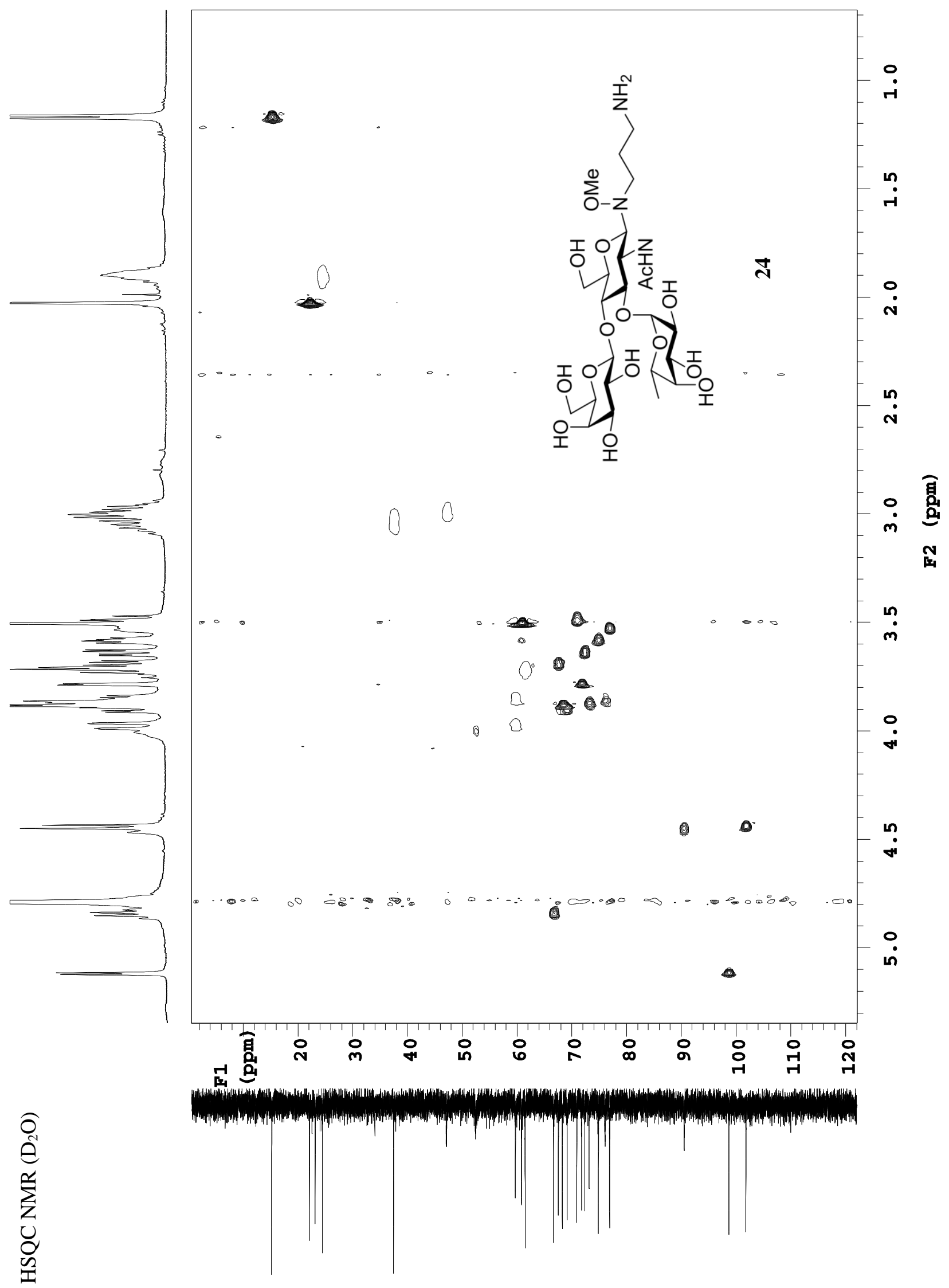

Appendix - 47 


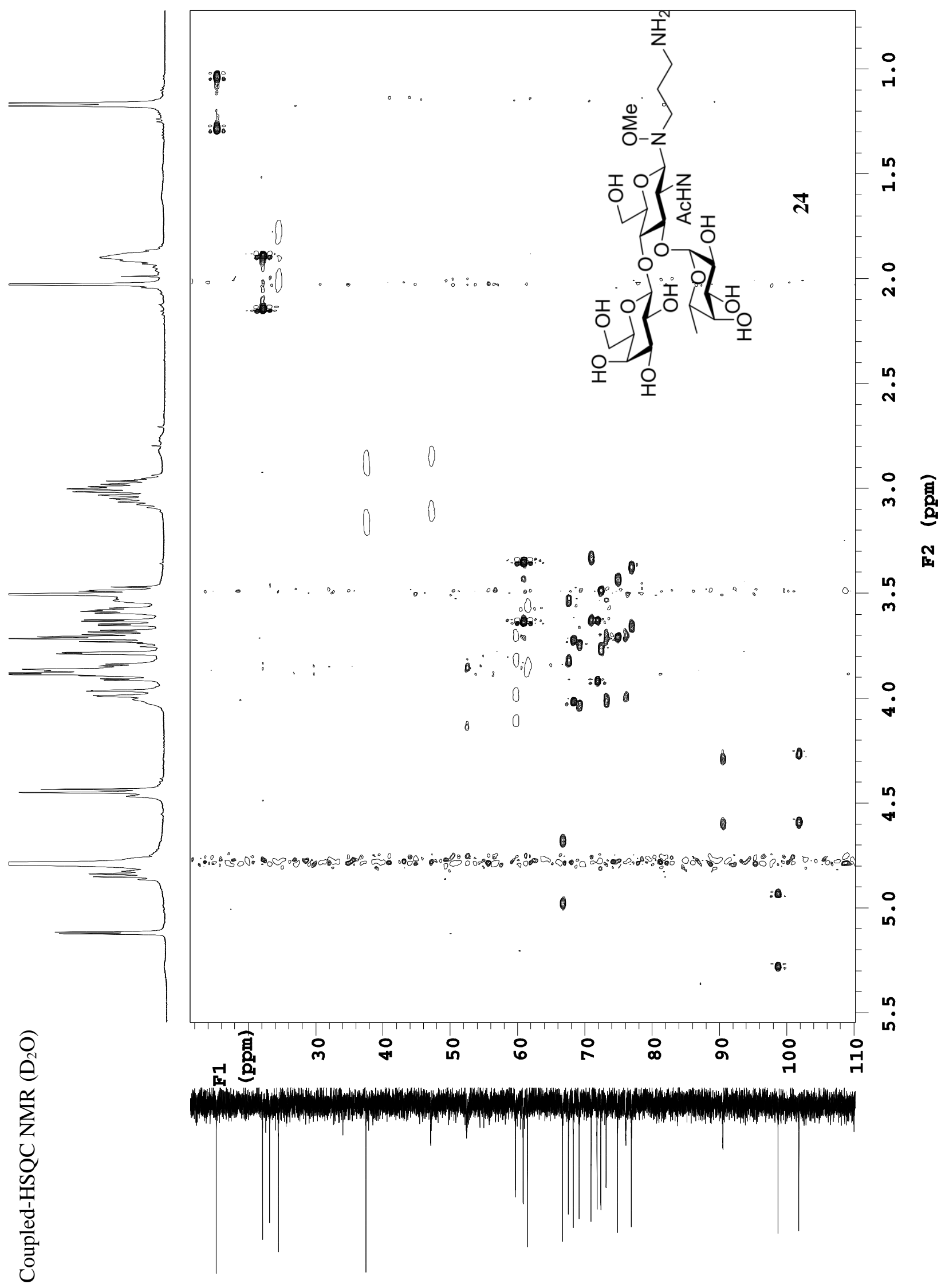

Appendix - 48 


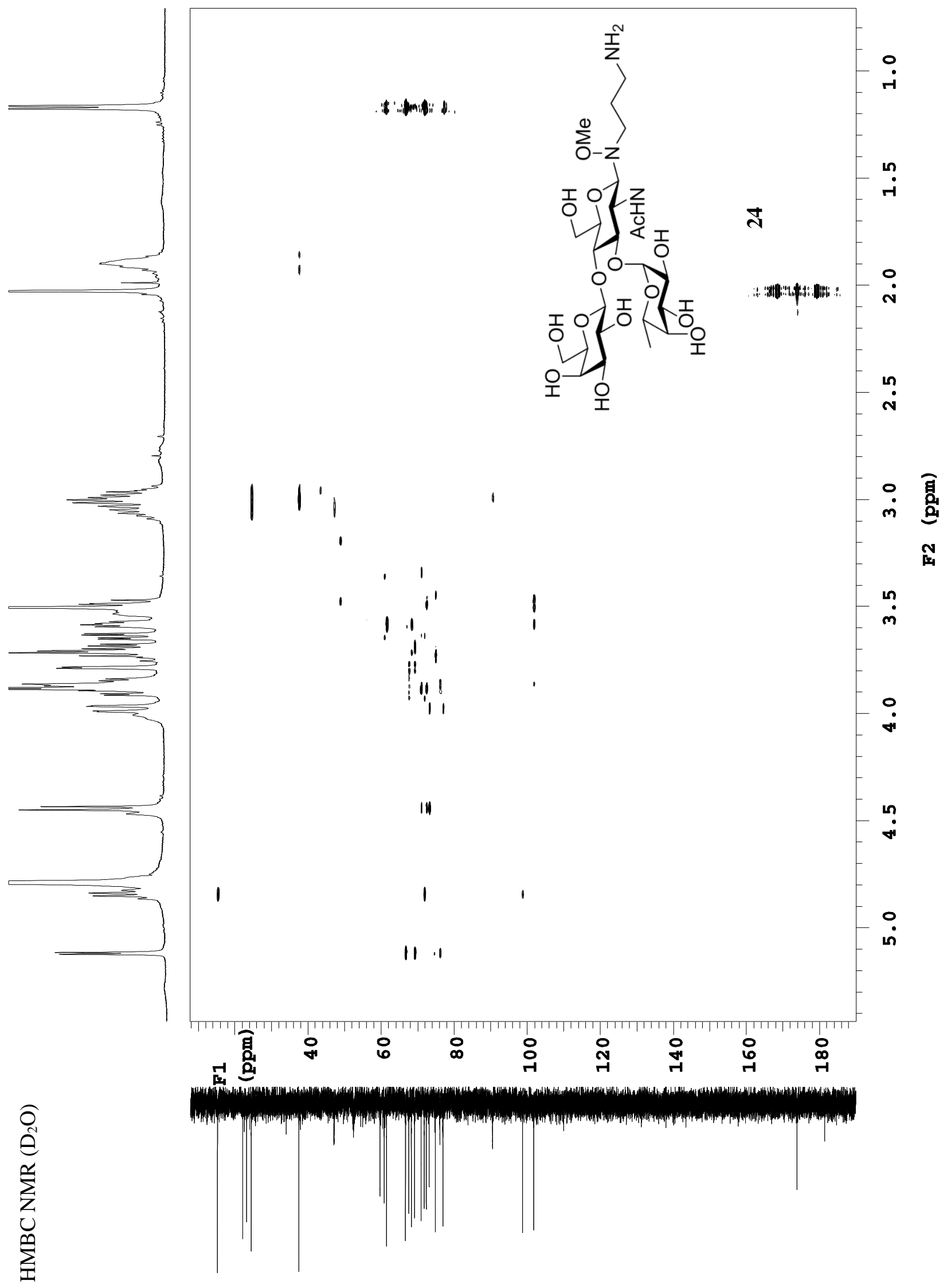

Appendix - 49 


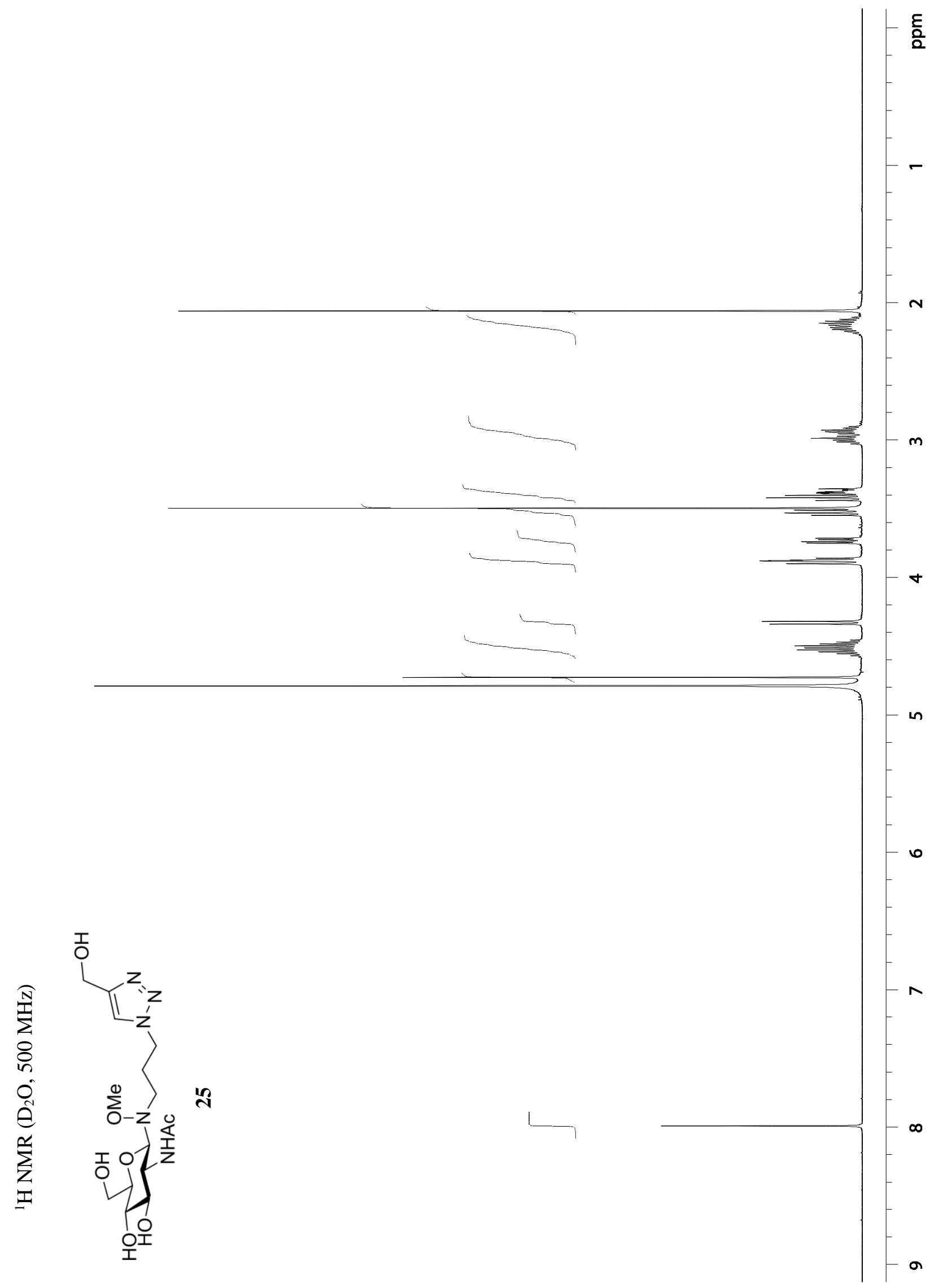

Appendix - 50 


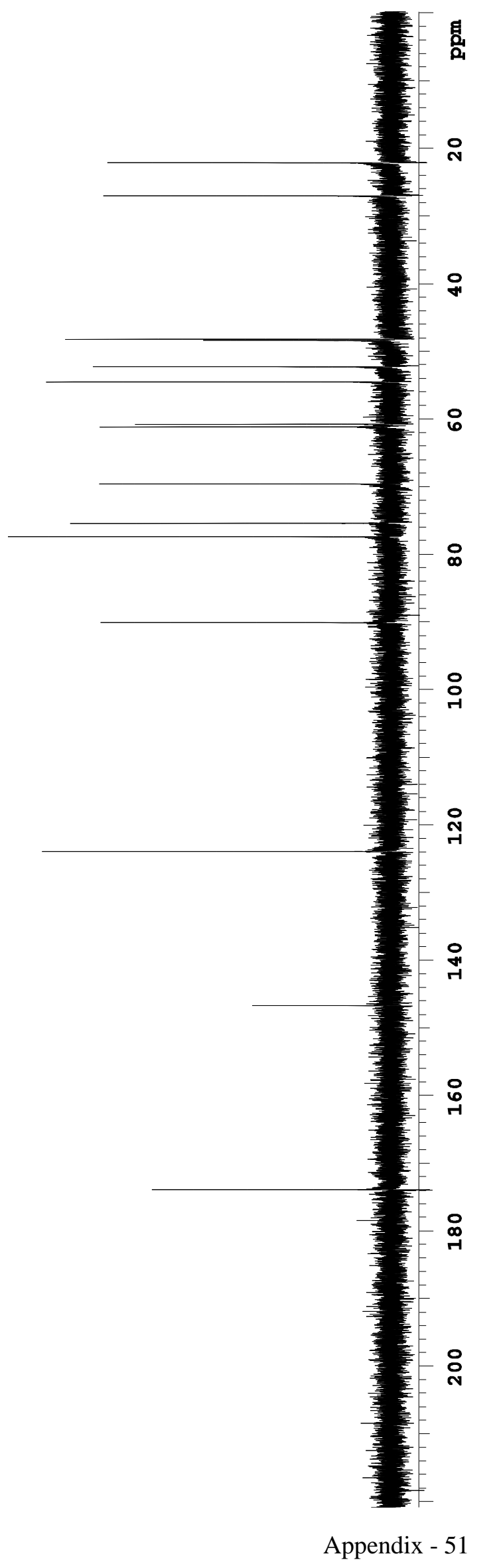




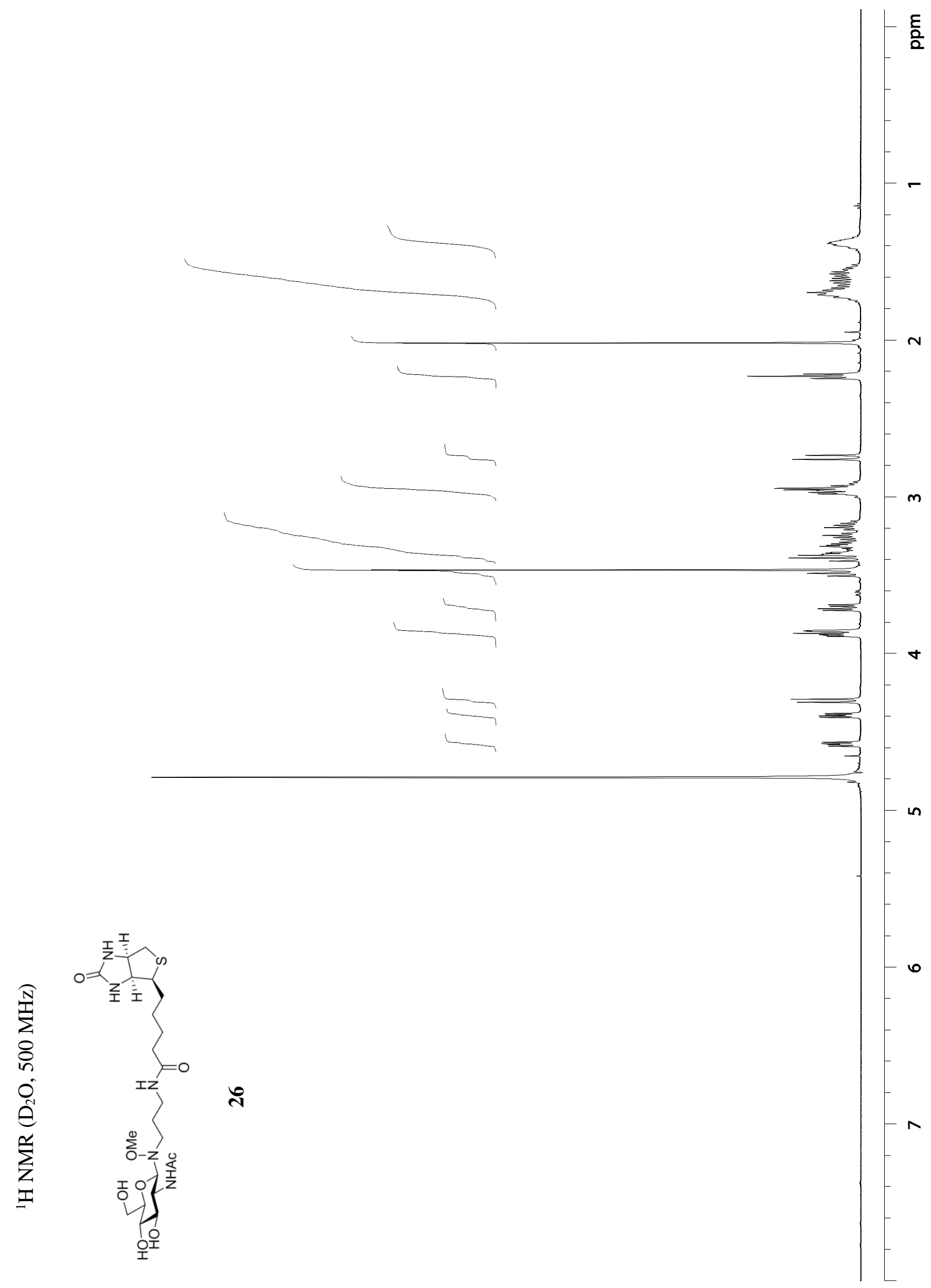

Appendix - 52 


$$
1
$$




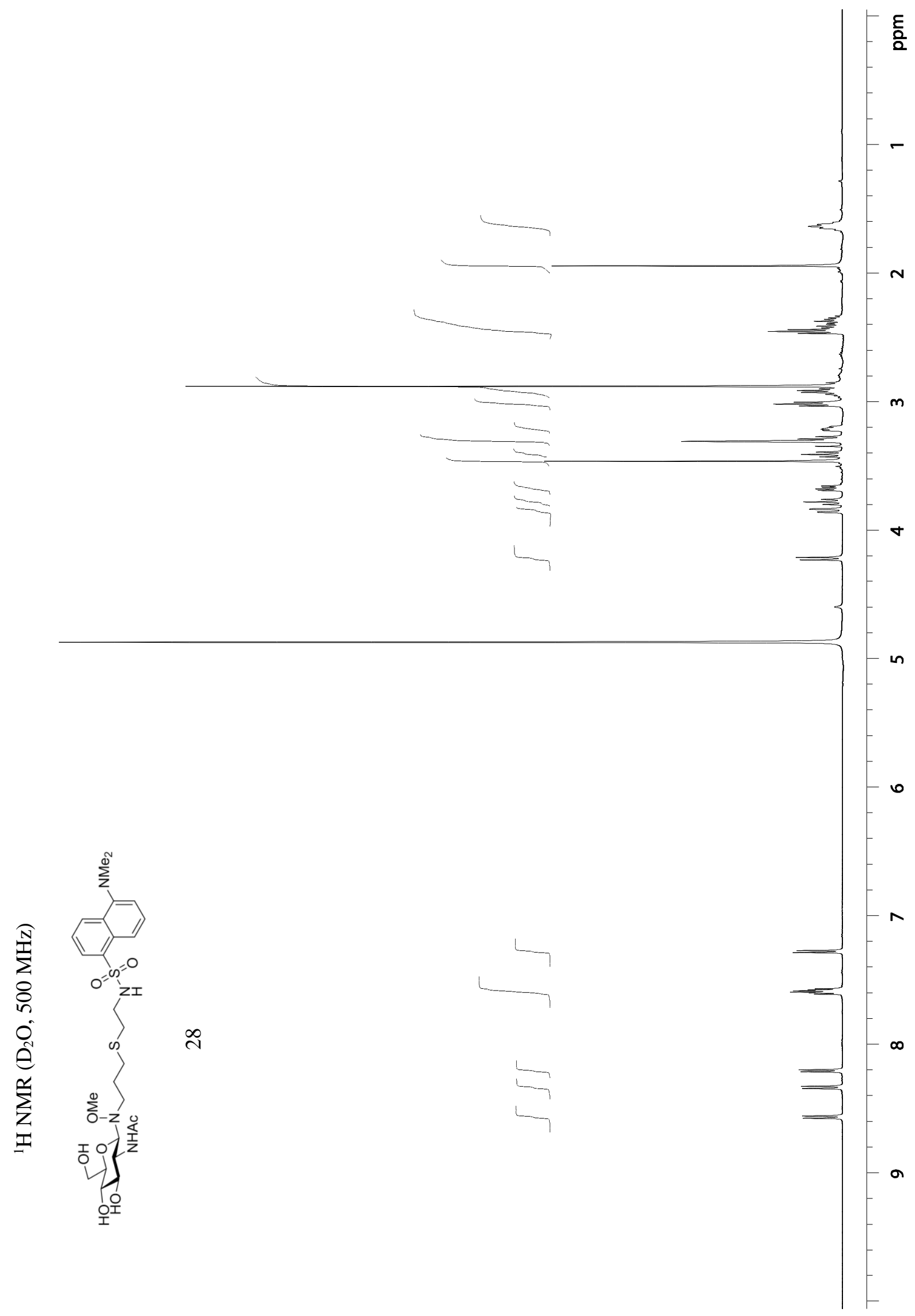

Appendix - 54 


$$
4
$$




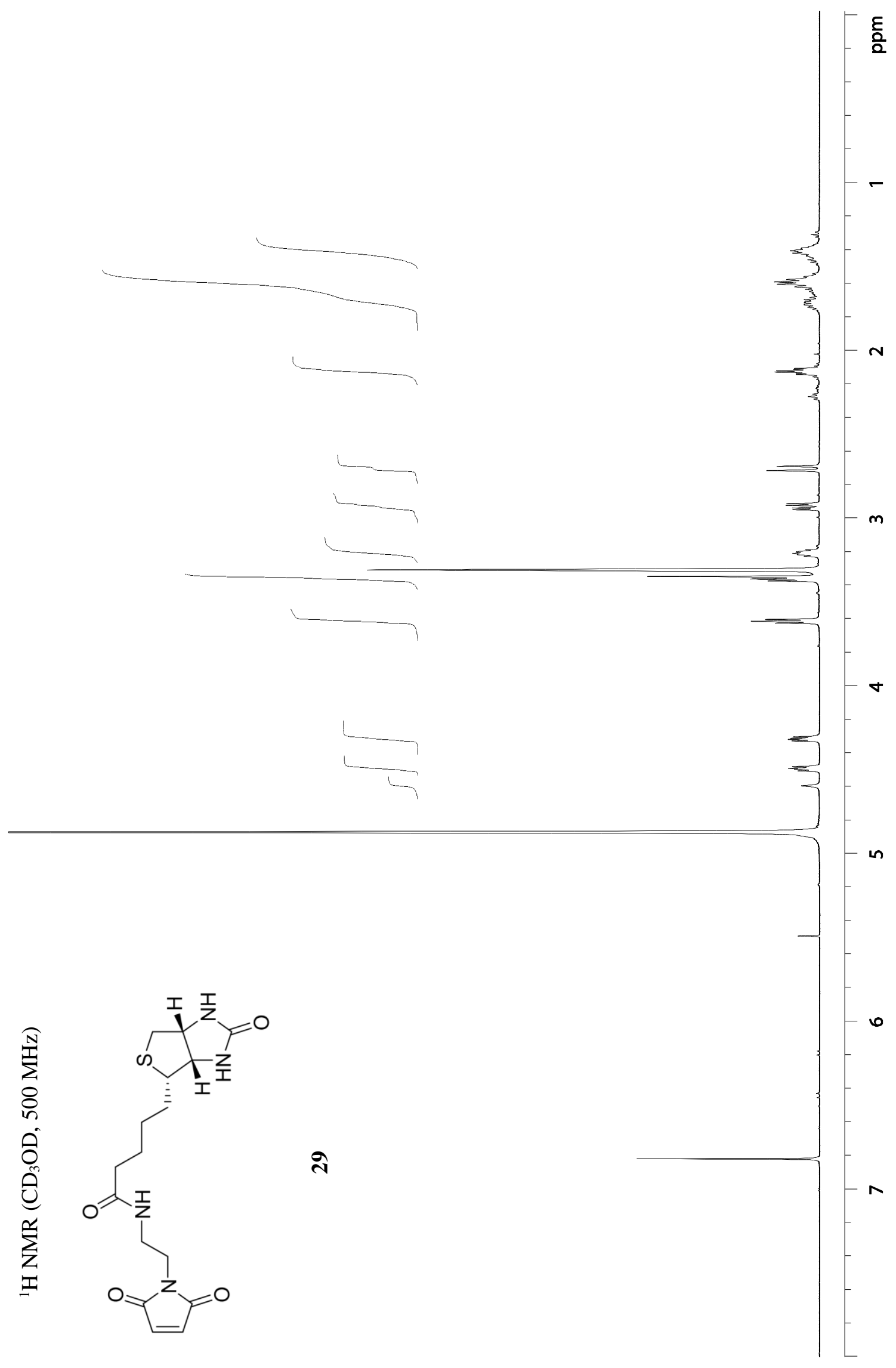

Appendix - 56 


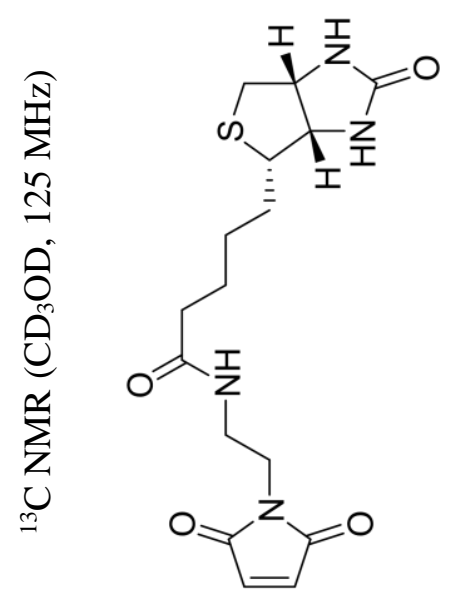

สิ

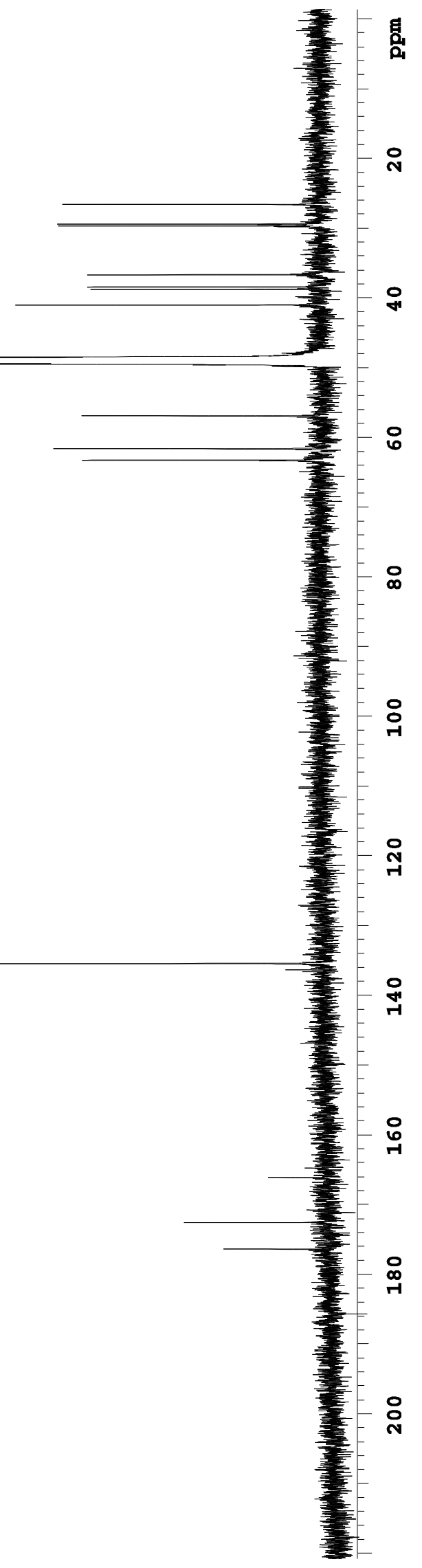

Appendix - 57 


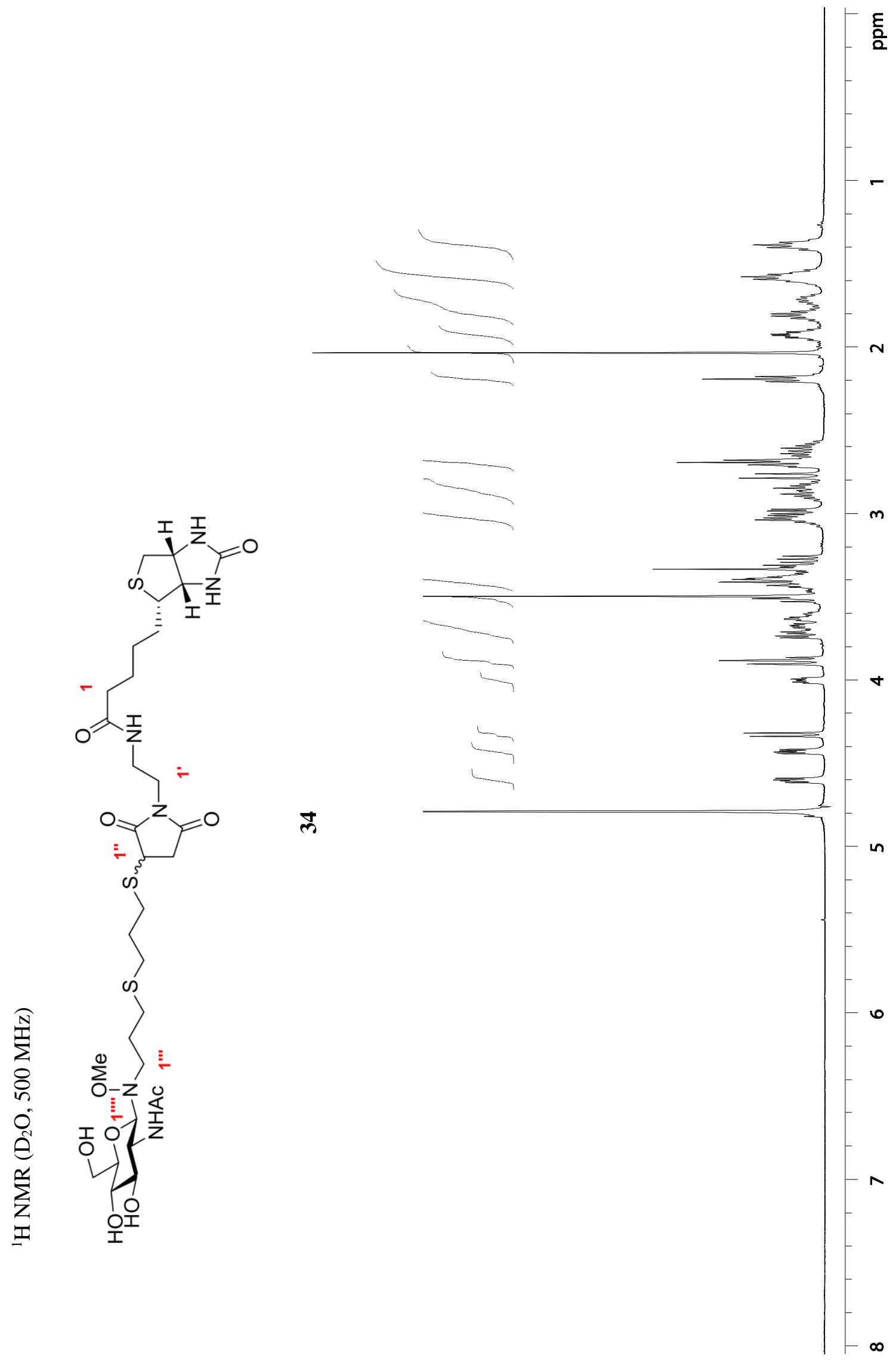

Appendix - 58 


$$
11
$$



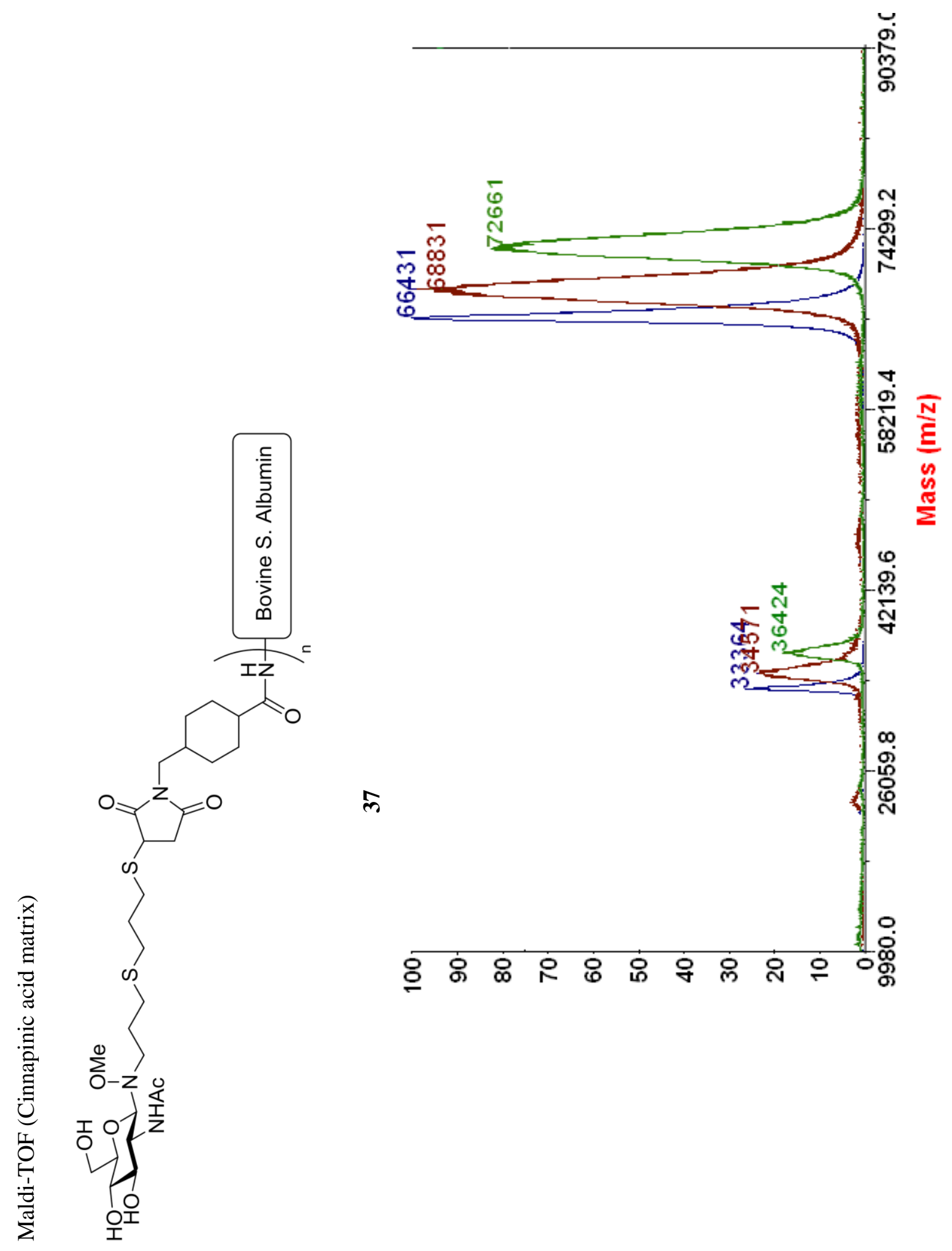

Appendix - 60 


\section{Supporting Information Chapter 3.}

\section{Design and synthesis of novel fluorescent and biotinylated multivalent glycodendrons.}

\section{Table of content}

$N$-Azidoacetyl-1,1,1-tris(hydroxymethyl)aminomethane (46). A62

$N$-Azidoacetyl-1,1,1-tris(tert-butyloxycarbonylmethyloxymethyl)aminomethane (47) A64

$N$-Azidoacetyl-1,1,1-tris(carboxymethyloxymethyl)aminomethane (48) .................. A66

N-Glycyl-1,1,1-tris(tert-butyloxycarbonylmethyloxymethyl)aminomethane (49)....... A68

$N$-Azidoacetyl-1,1,1-tris(1,1,1-tris[tert-butyloxycarbonylmethyloxymethyl]methylamidocarbonylmethylamidocarbonylmethyloxymethyl)aminomethane (50)

$N$-(2-D-Biotinylamido-acetyl)-1,1,1-tris(1,1,1-tris[tert-butyloxycarbonylmethyloxymethyl]methylamidocarbonylmethylamidocarbonylmethyloxymethyl)aminomethane (51)

$\mathrm{N}$-(2-D-Biotinylamidoacetyl)-1,1,1-tris(1,1,1-tris[carboxymethyloxymethyl]methylamidocarbonylmethylamidocarbonylmethyloxymethyl)aminomethane (52)

$N$-(2-D-Biotinylamido-acetyl)-1,1,1-tris(1,1,1-tris[3-( $N$-(2-acetamido-2-deoxy- $\beta$-Dglucopyranosyl)- $O$-methylhydroxylamine)propylamidocarbonylmethyloxymethyl]methylamidocarbonylmethylamidocarbonylmethyloxy-methyl)aminomethane (54)

$N$-Glycyl-1,1,1-tris(1,1,1-tris[carboxymethyloxymethyl]methylamidocarbonylmethylamidocarbonylmethyloxymethyl)amino-methane trifluoroacetic acid (62)

$N$-[N-([(3',6'-Dihydroxy-3-oxospiro[isobenzofuran-1(3H),9'-(9H)xanthen]-5-yl)amino]thioxomethyl)glycyl]-1,1,1-tris(1,1,1-tris[carboxymethyloxymethyl]methylamidocarbonylmethylamidocarbonylmethyloxymethyl)aminomethane (63) 

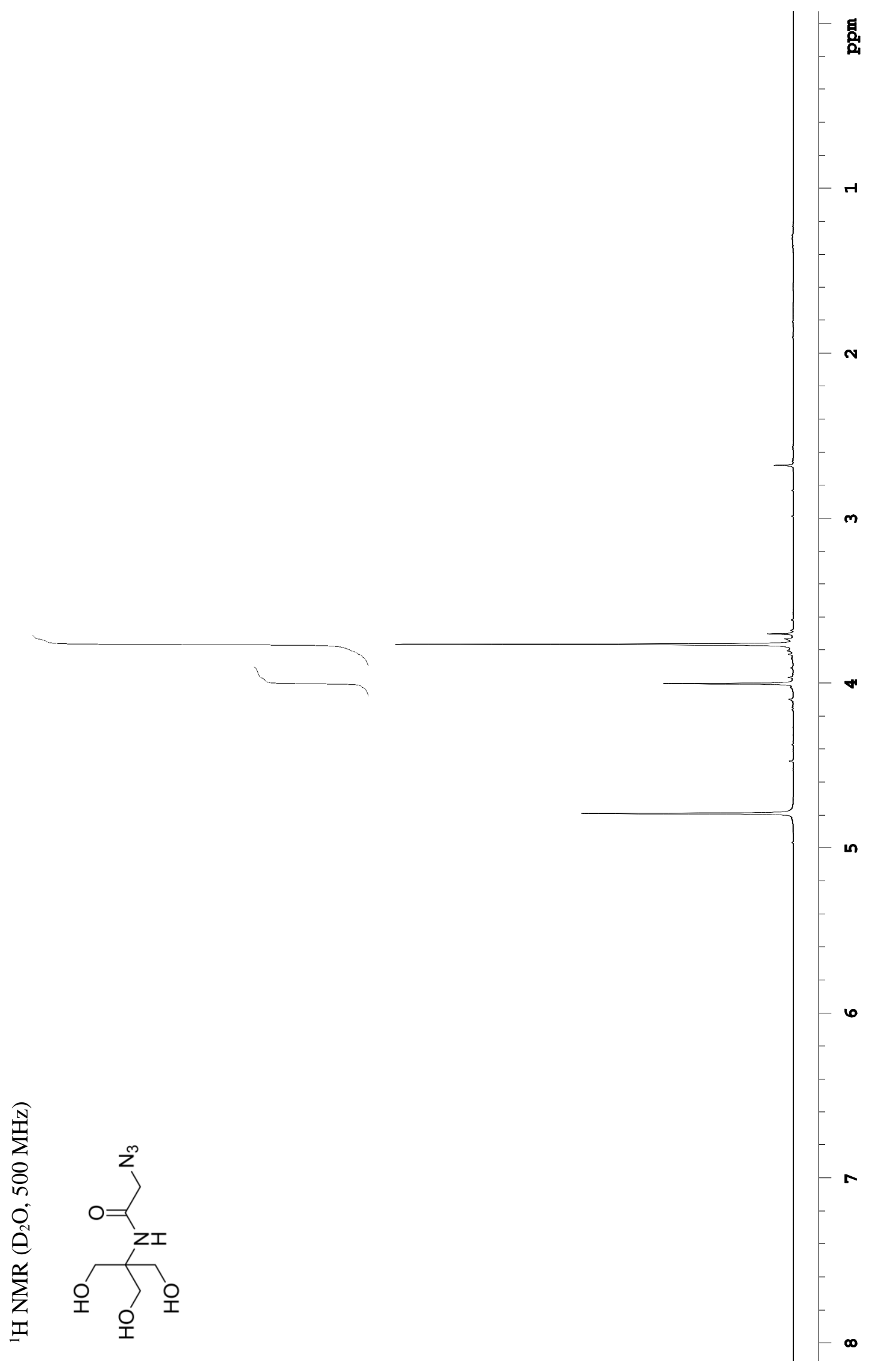

Appendix - 62 

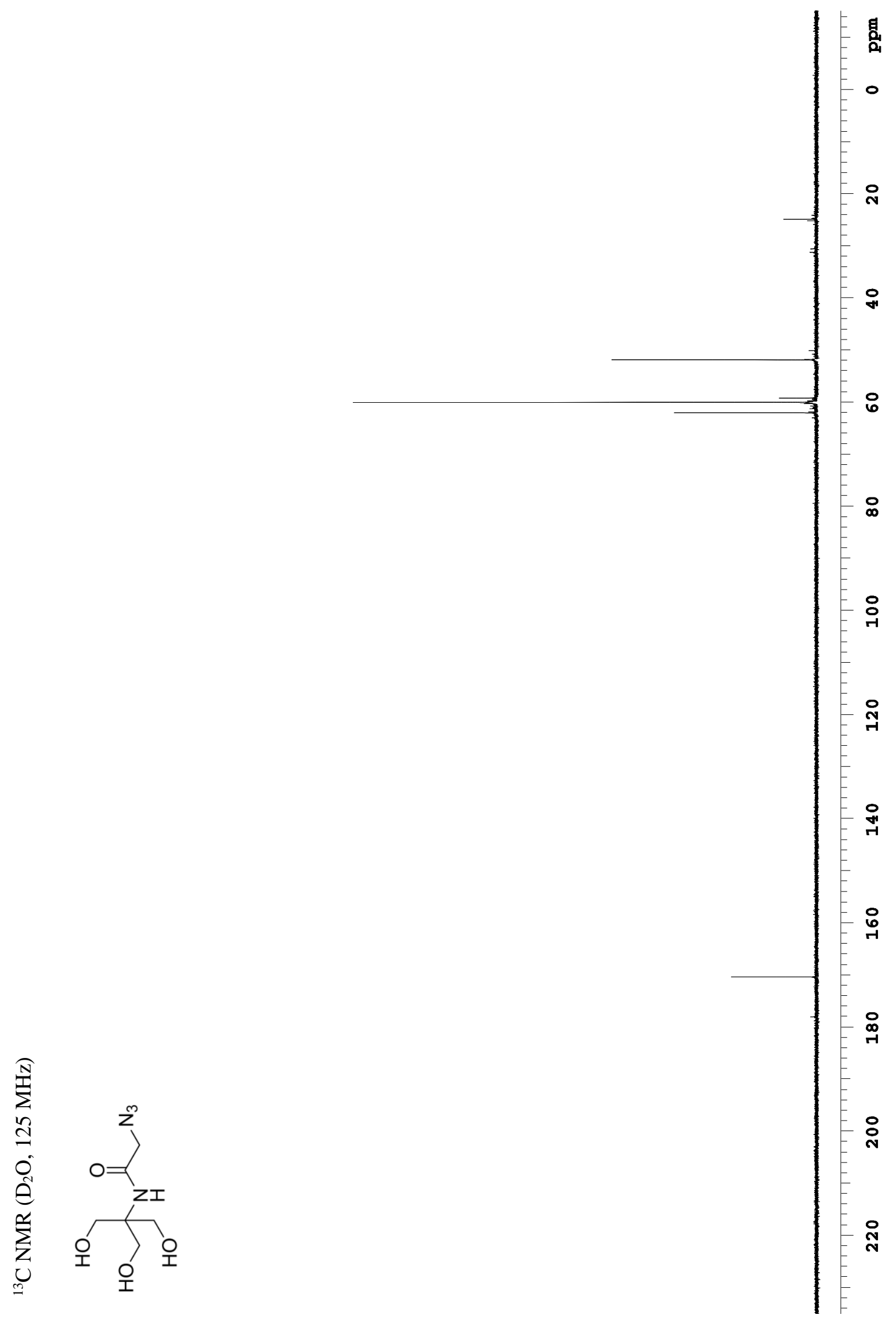

Appendix - 63 


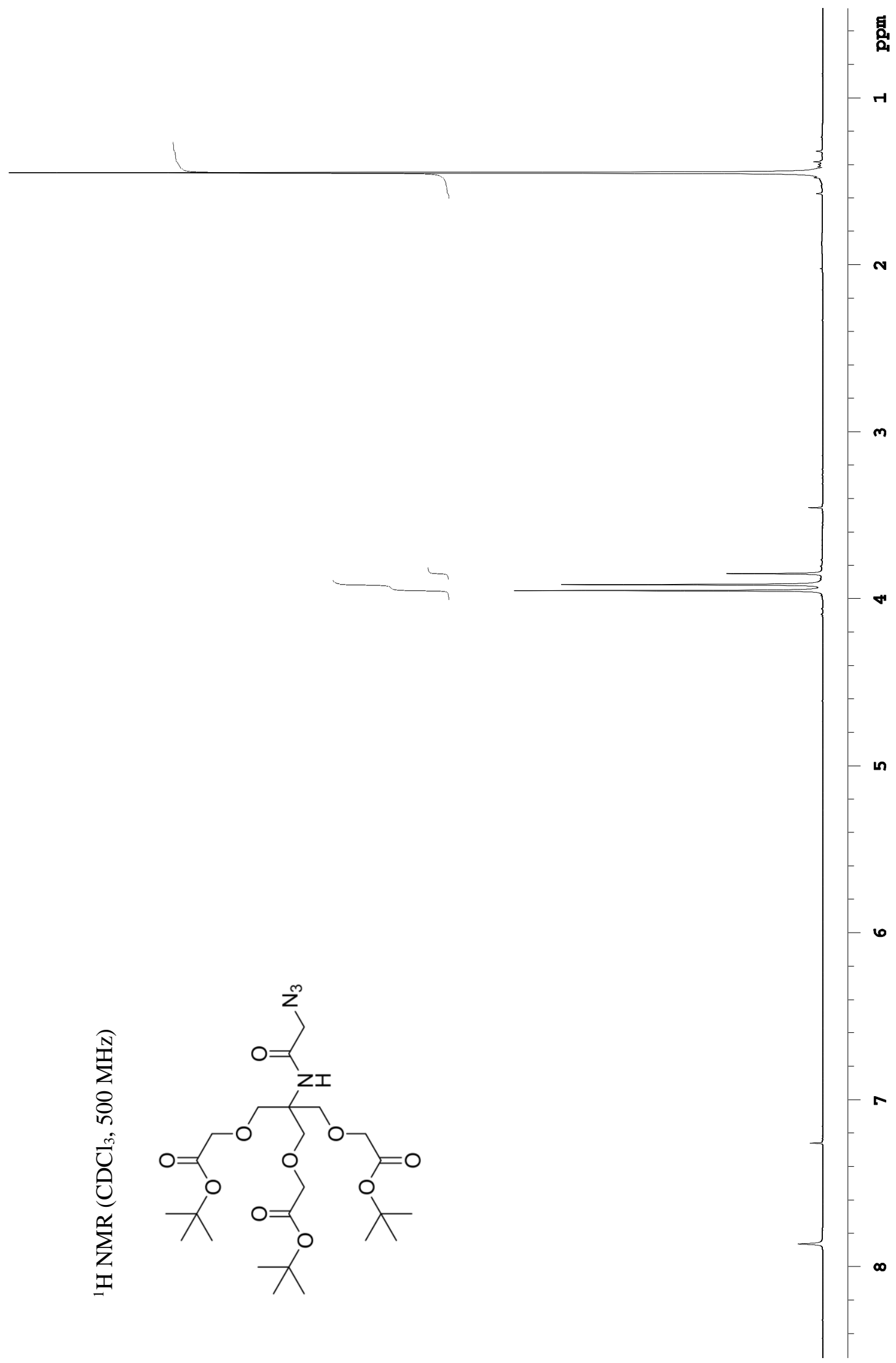

Appendix - 64 


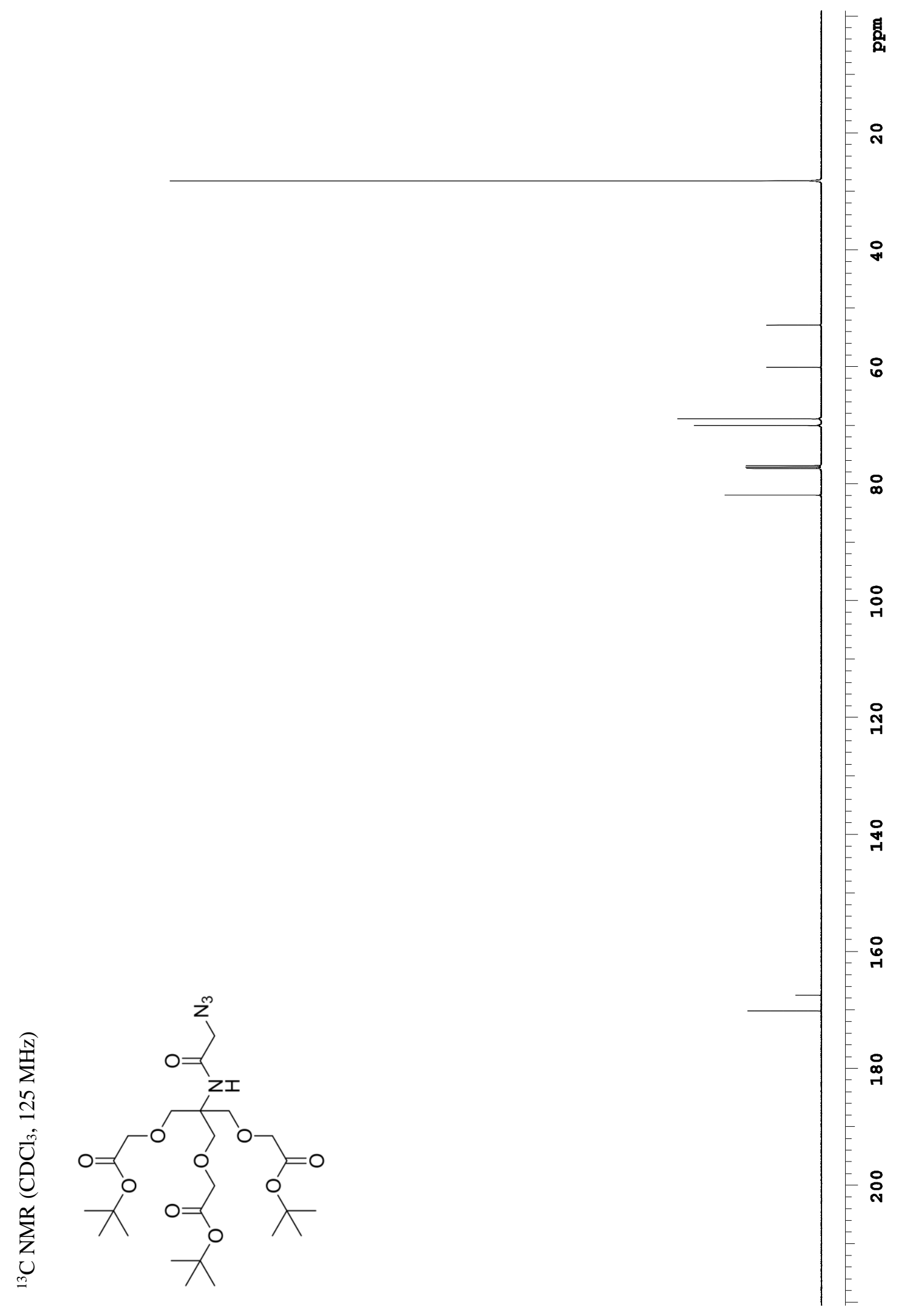

Appendix - 65 

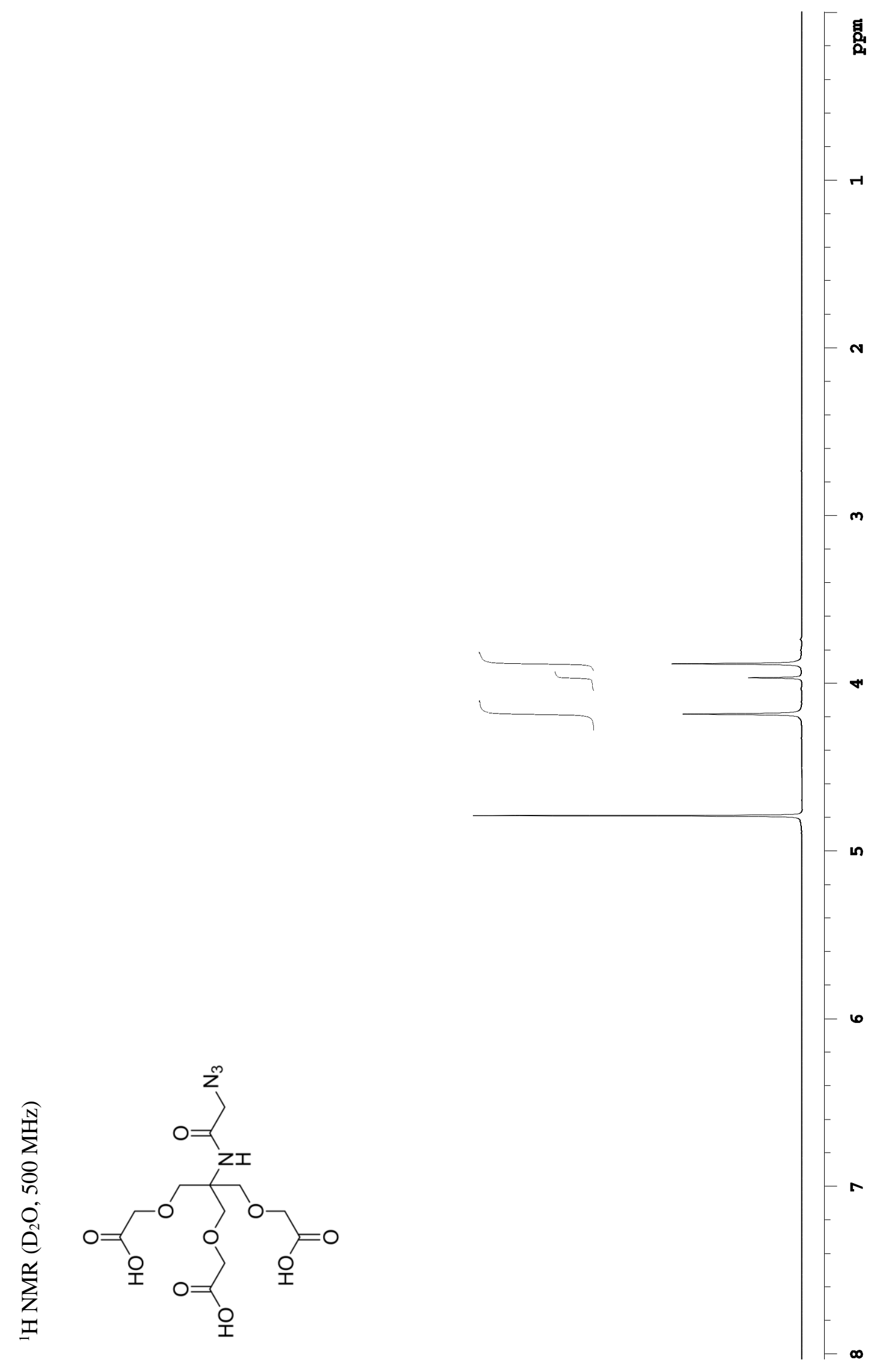

Appendix - 66 

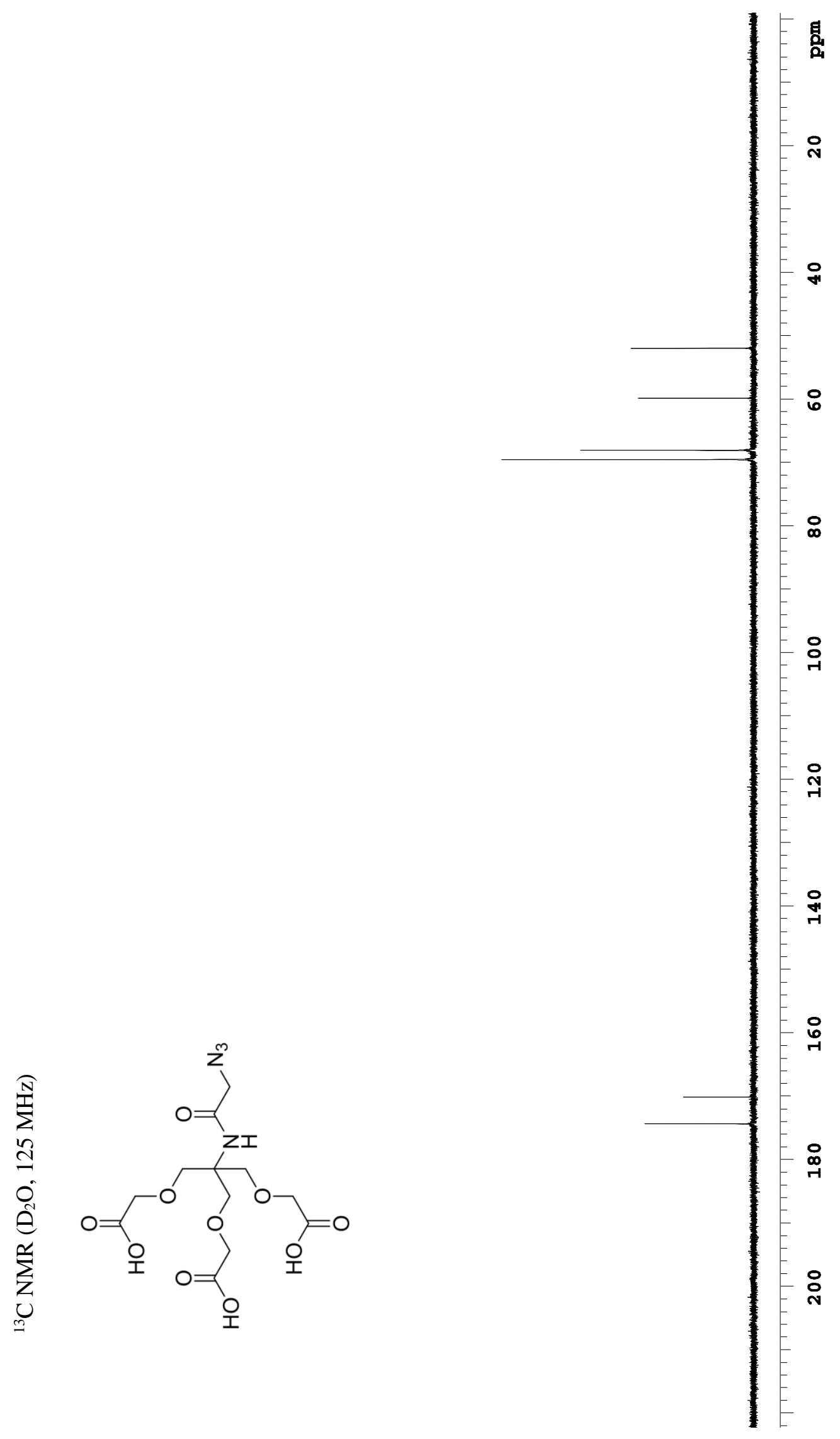

Appendix - 67 


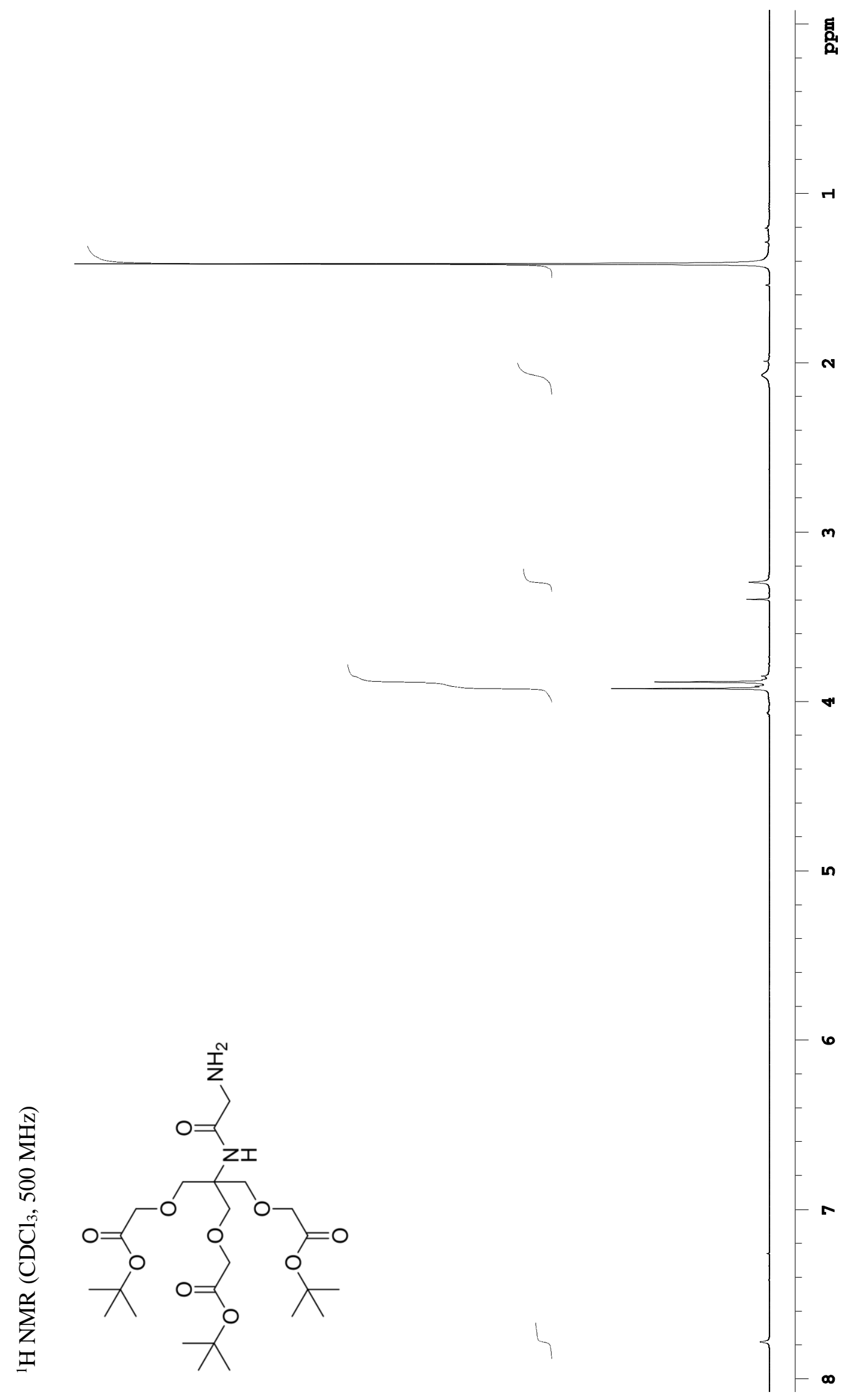

Appendix - 68 


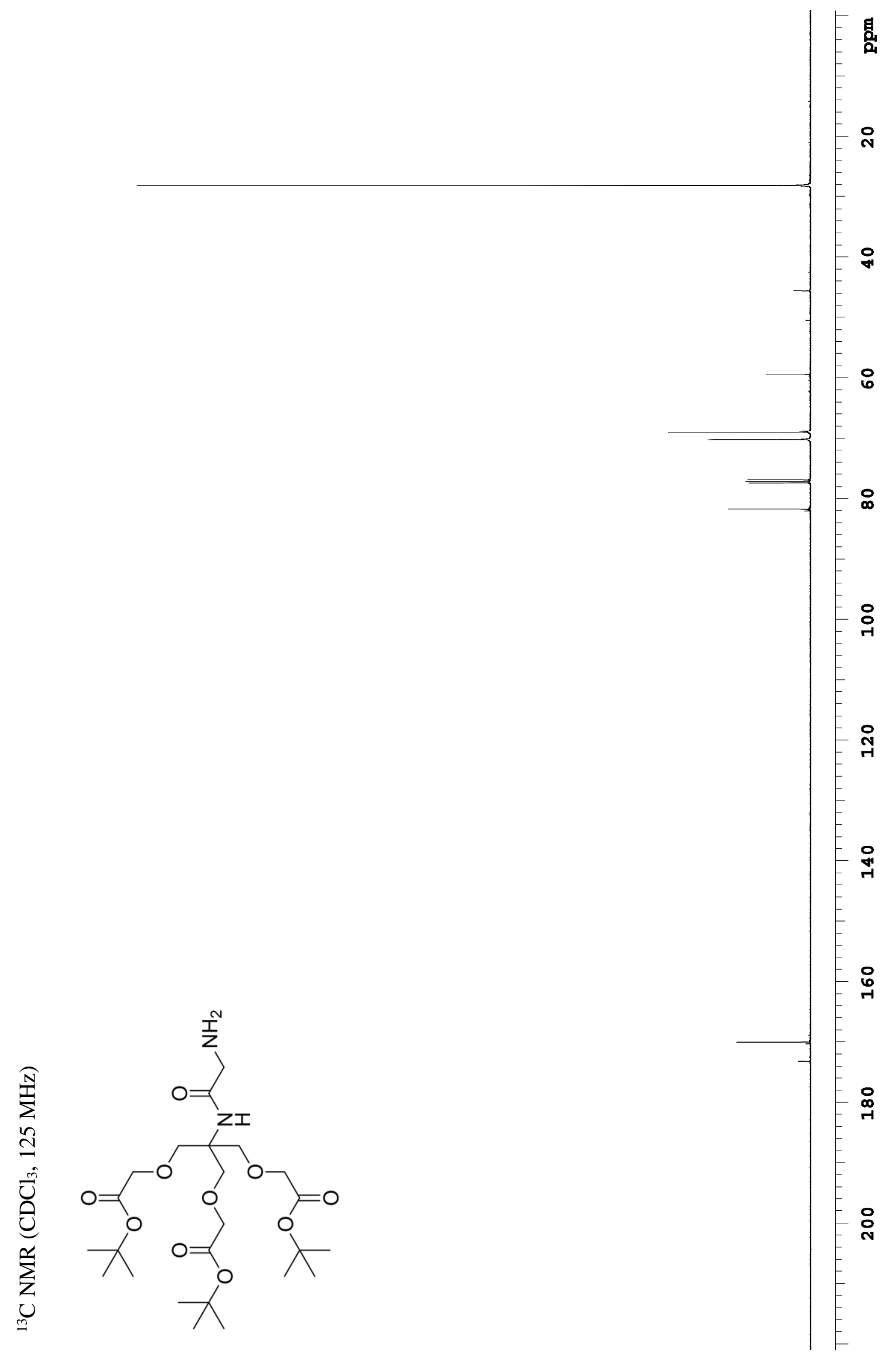

Appendix - 69 


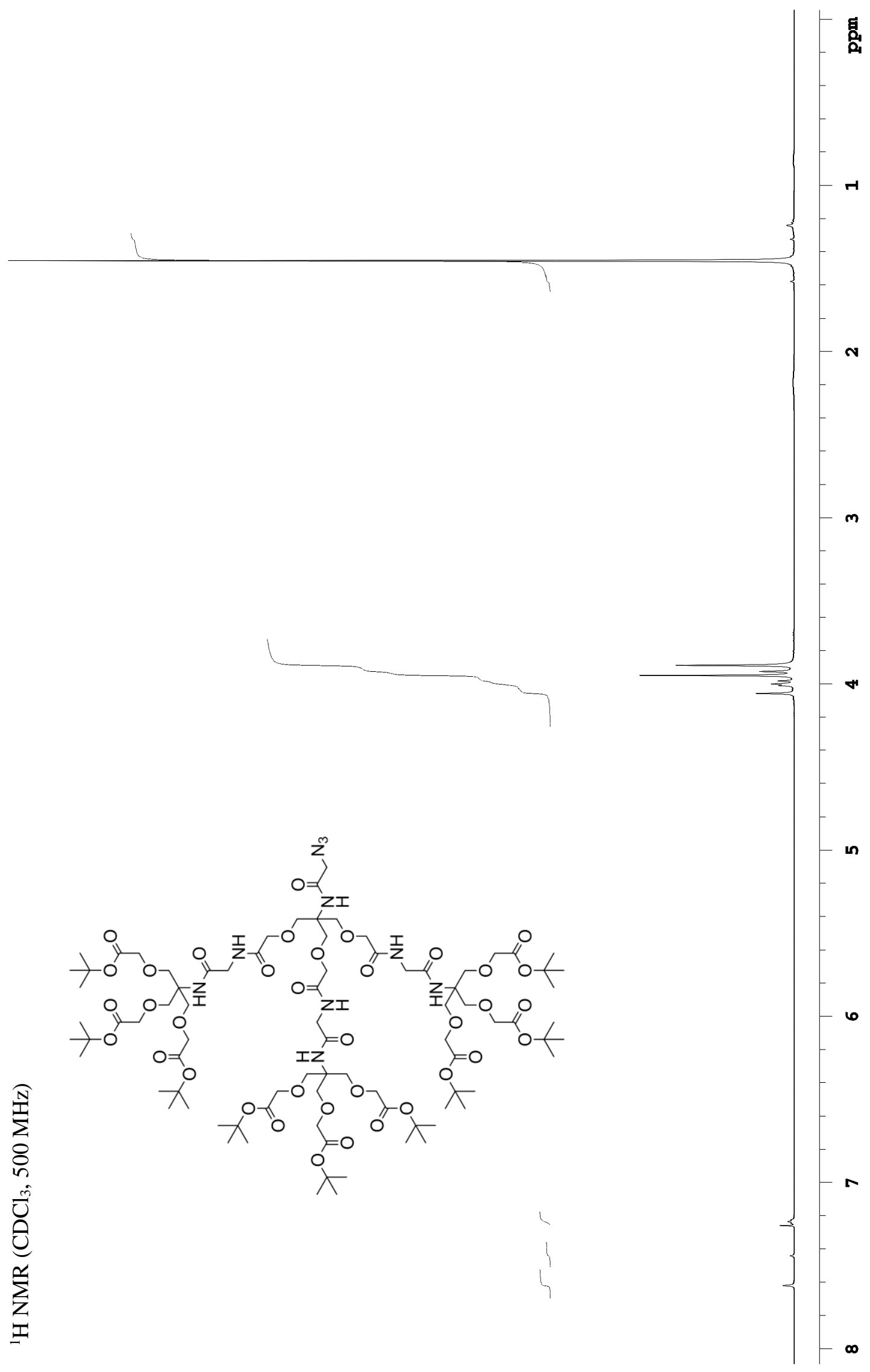

Appendix - 70 


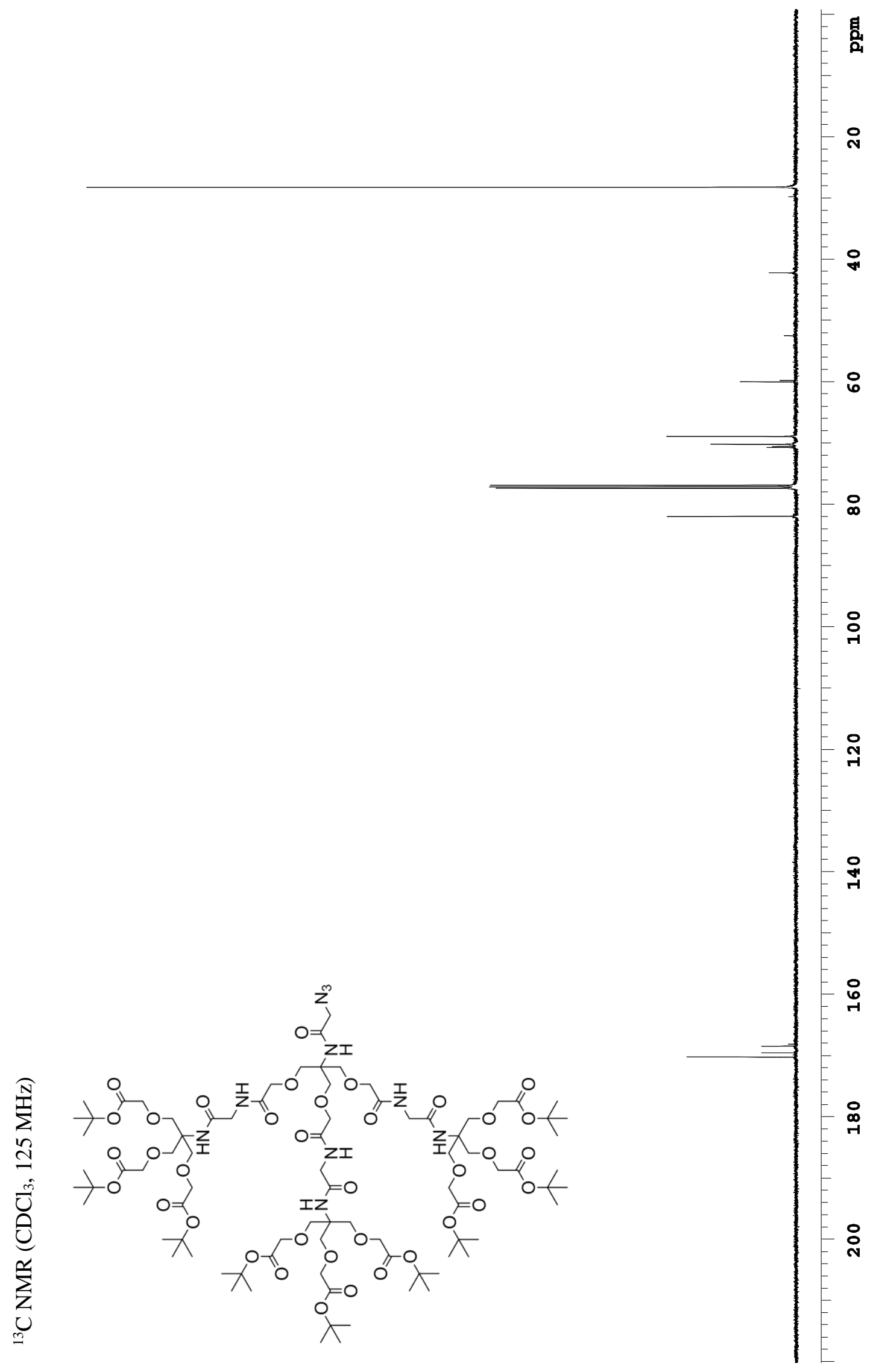

Appendix - 71 


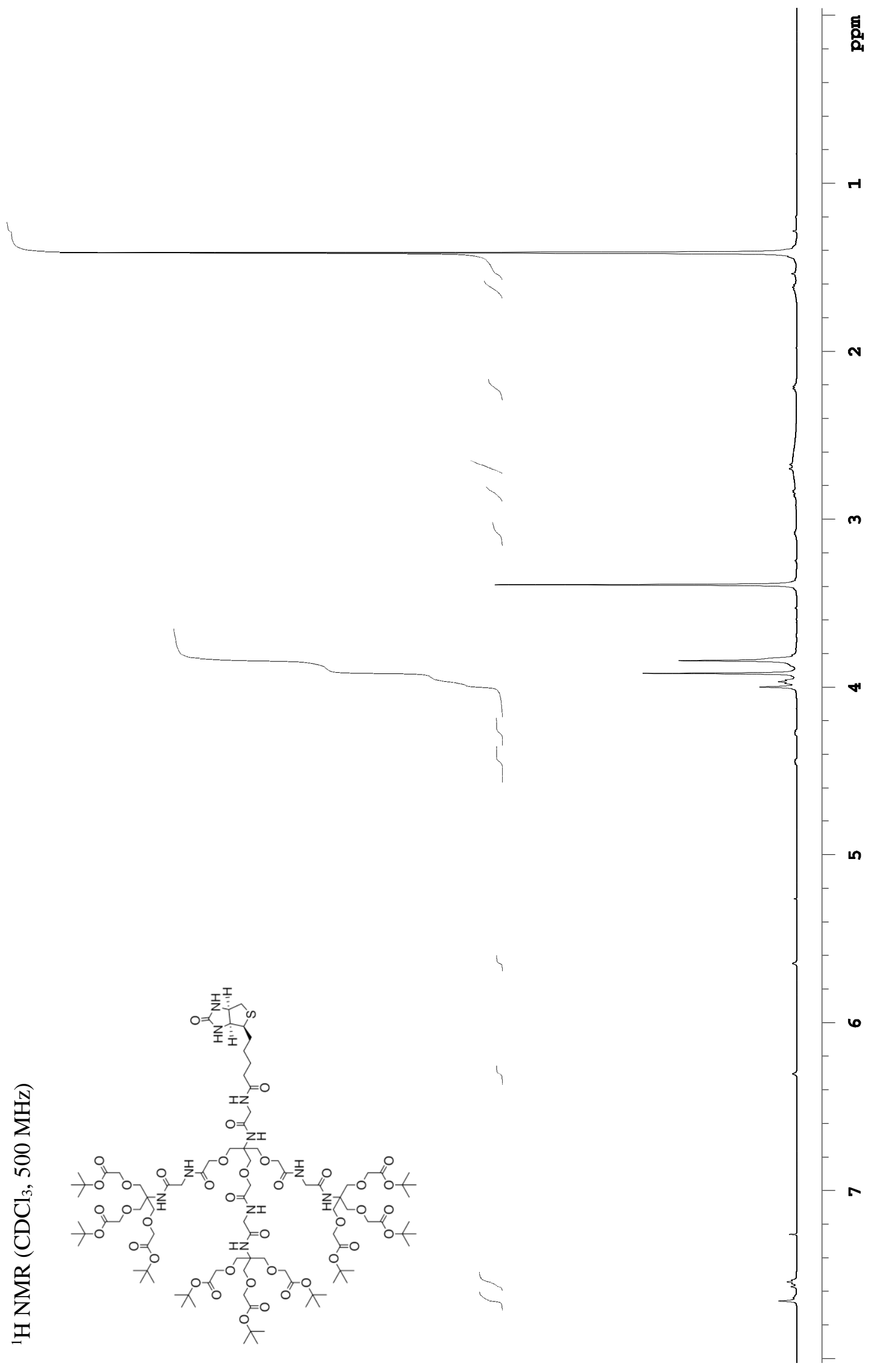

Appendix - 72 


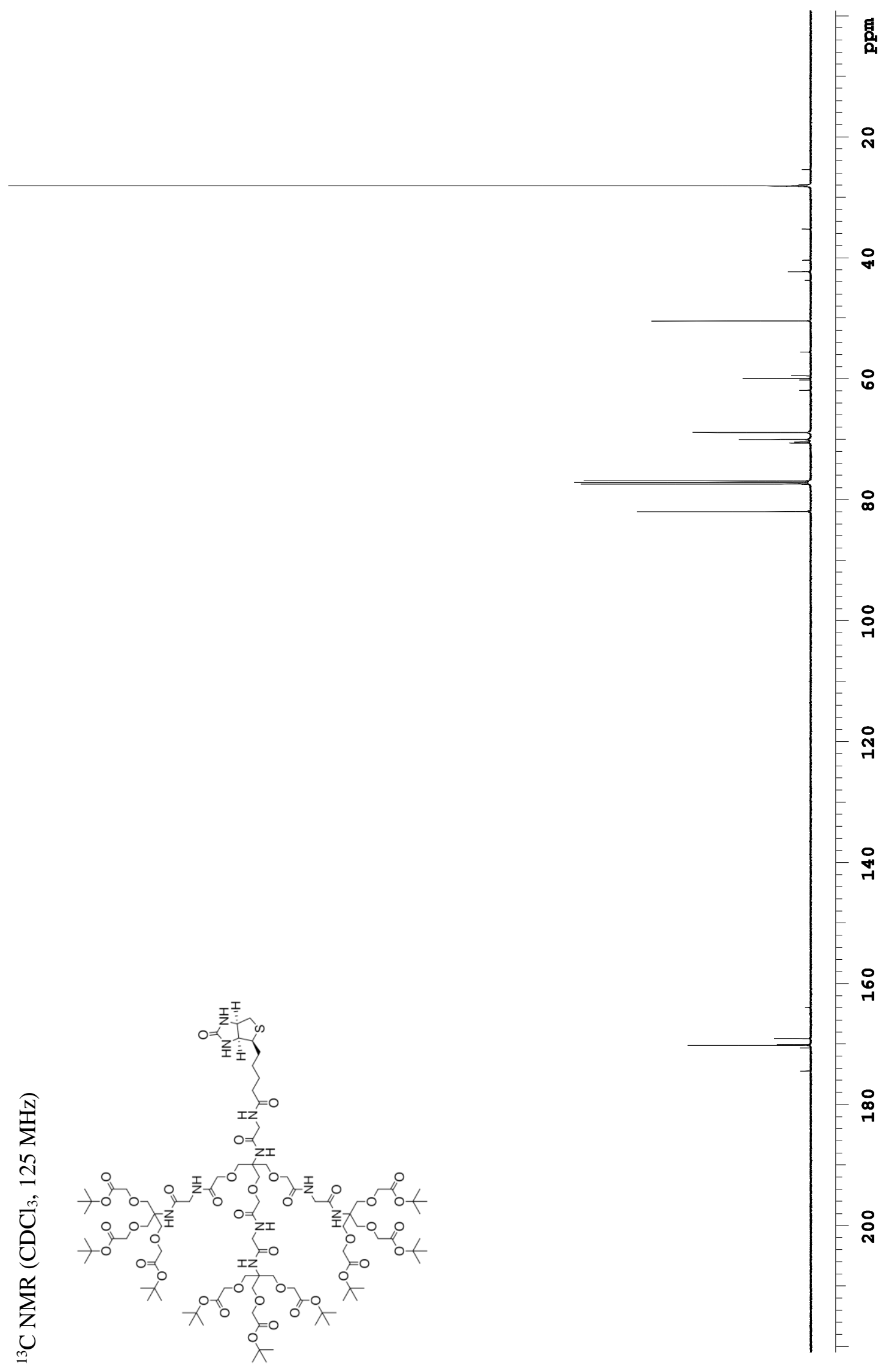

Appendix - 73 


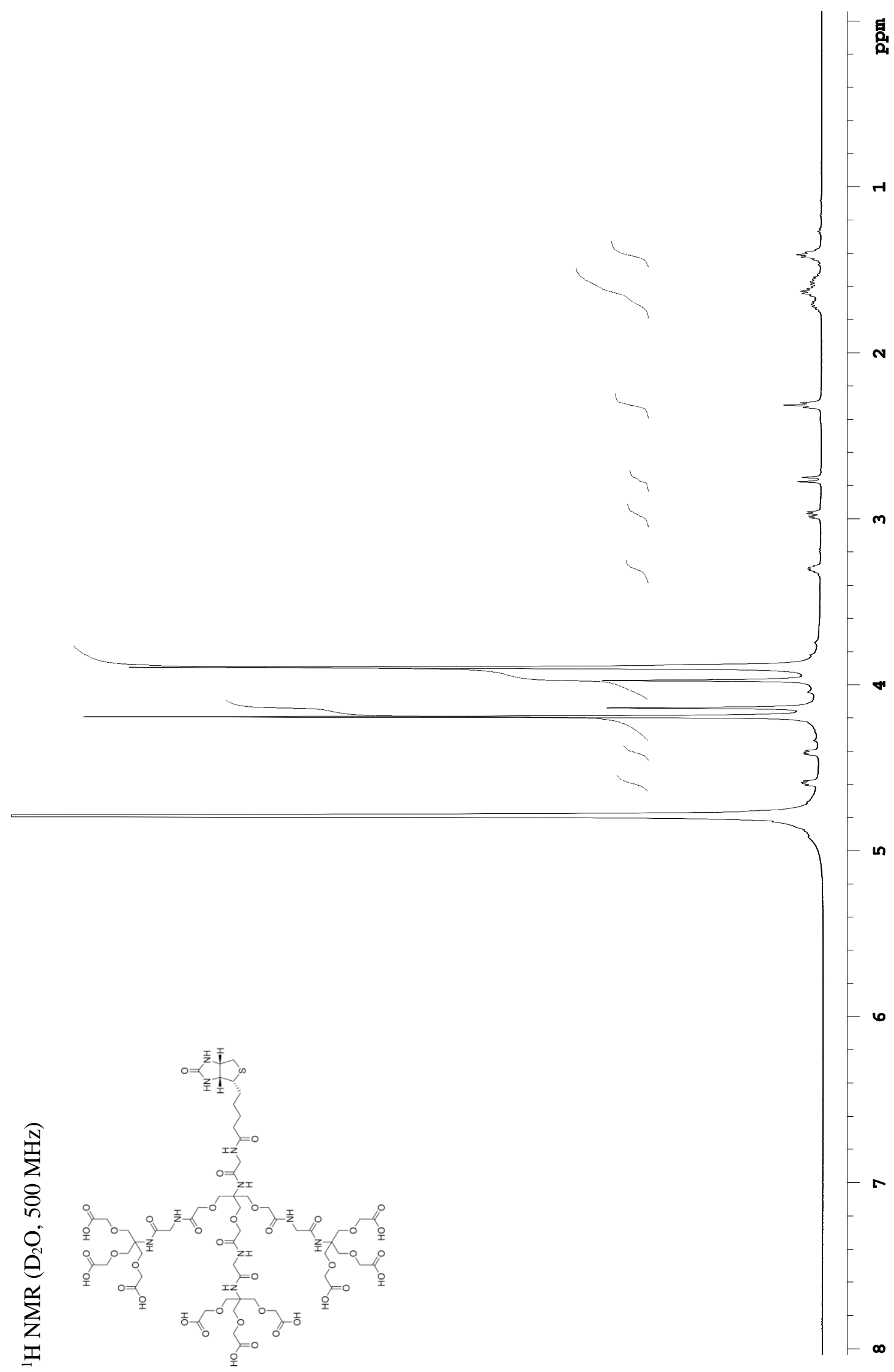

Appendix - 74 


$$
1
$$




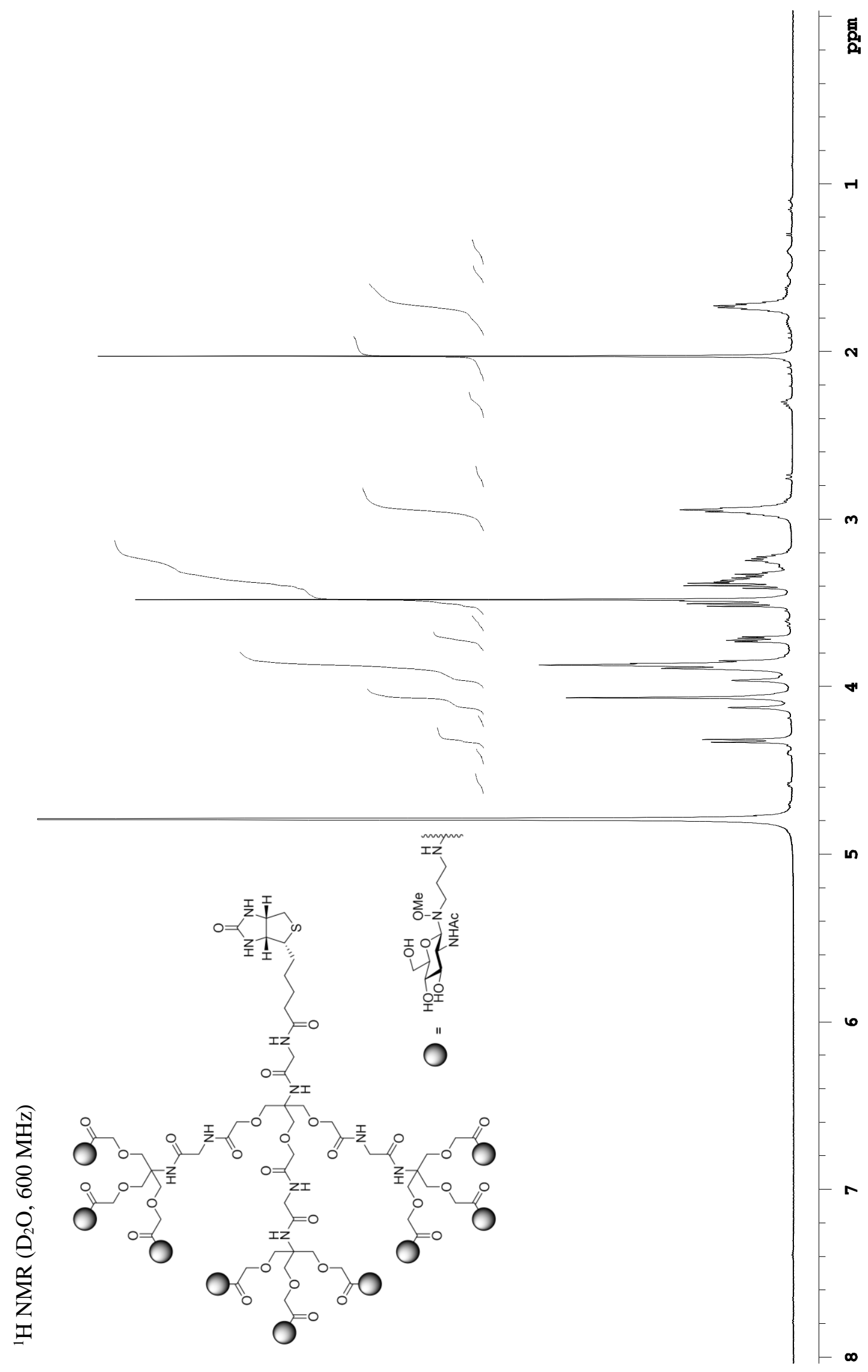

Appendix - 76 


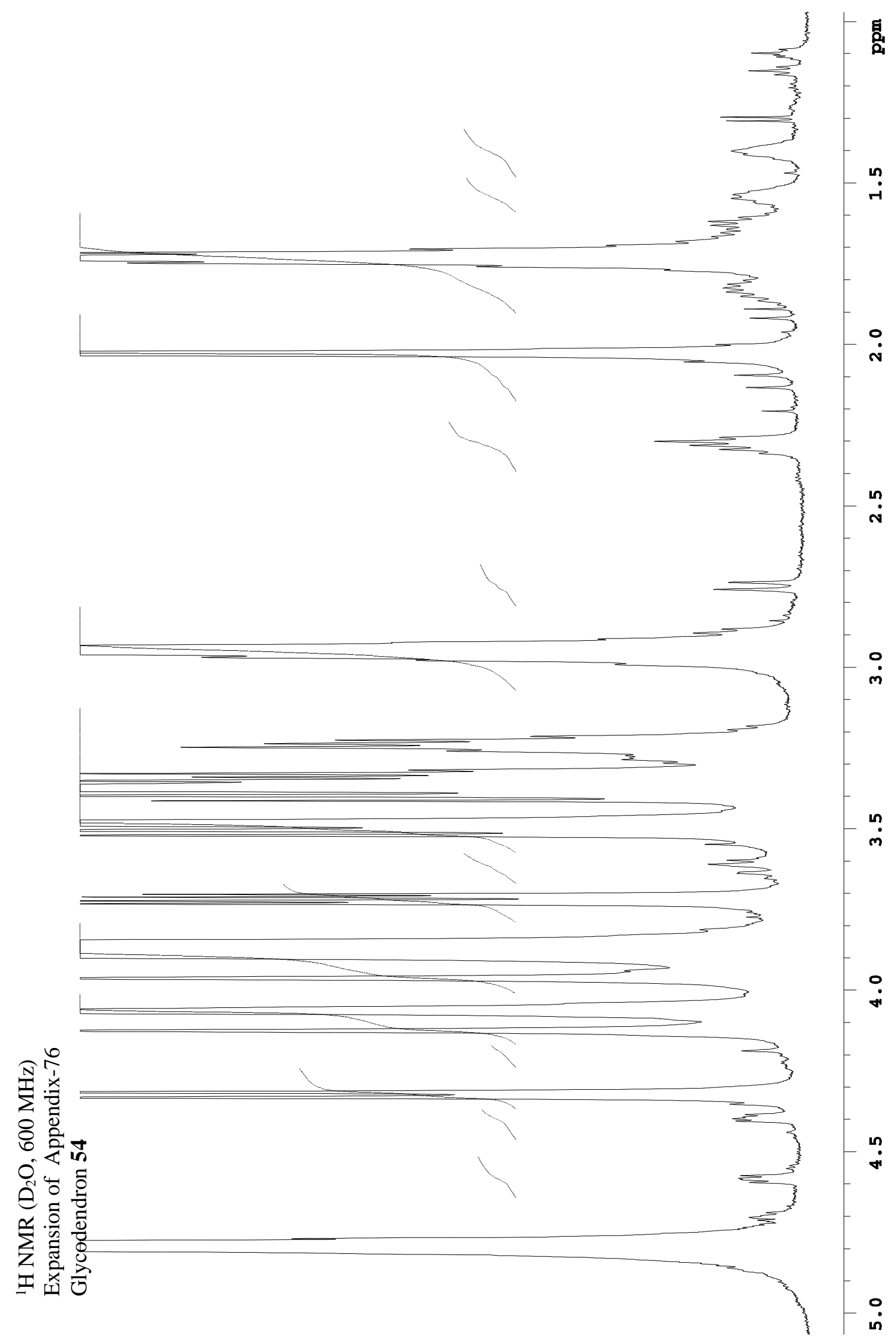

Appendix - 77 


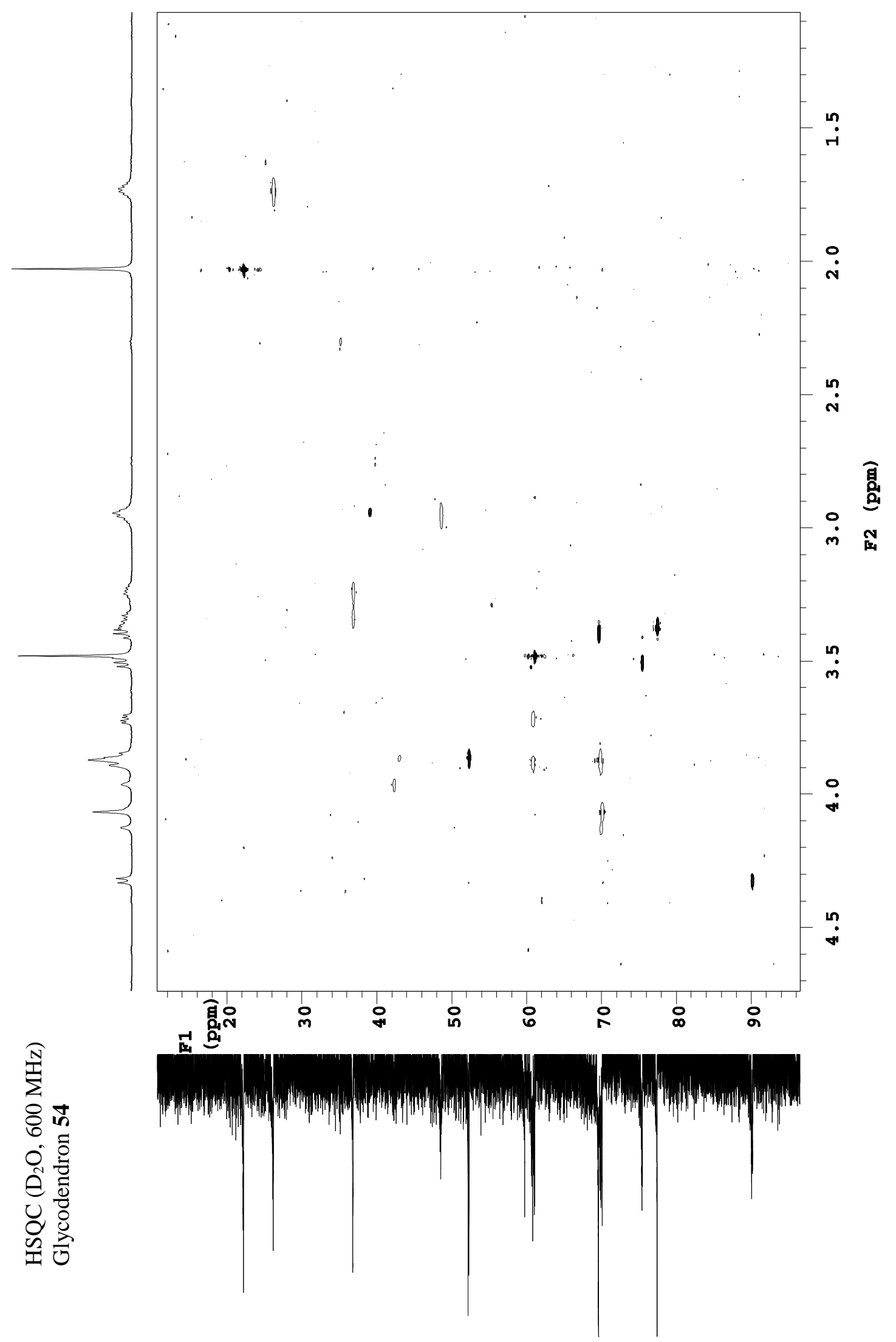

Appendix - 78 


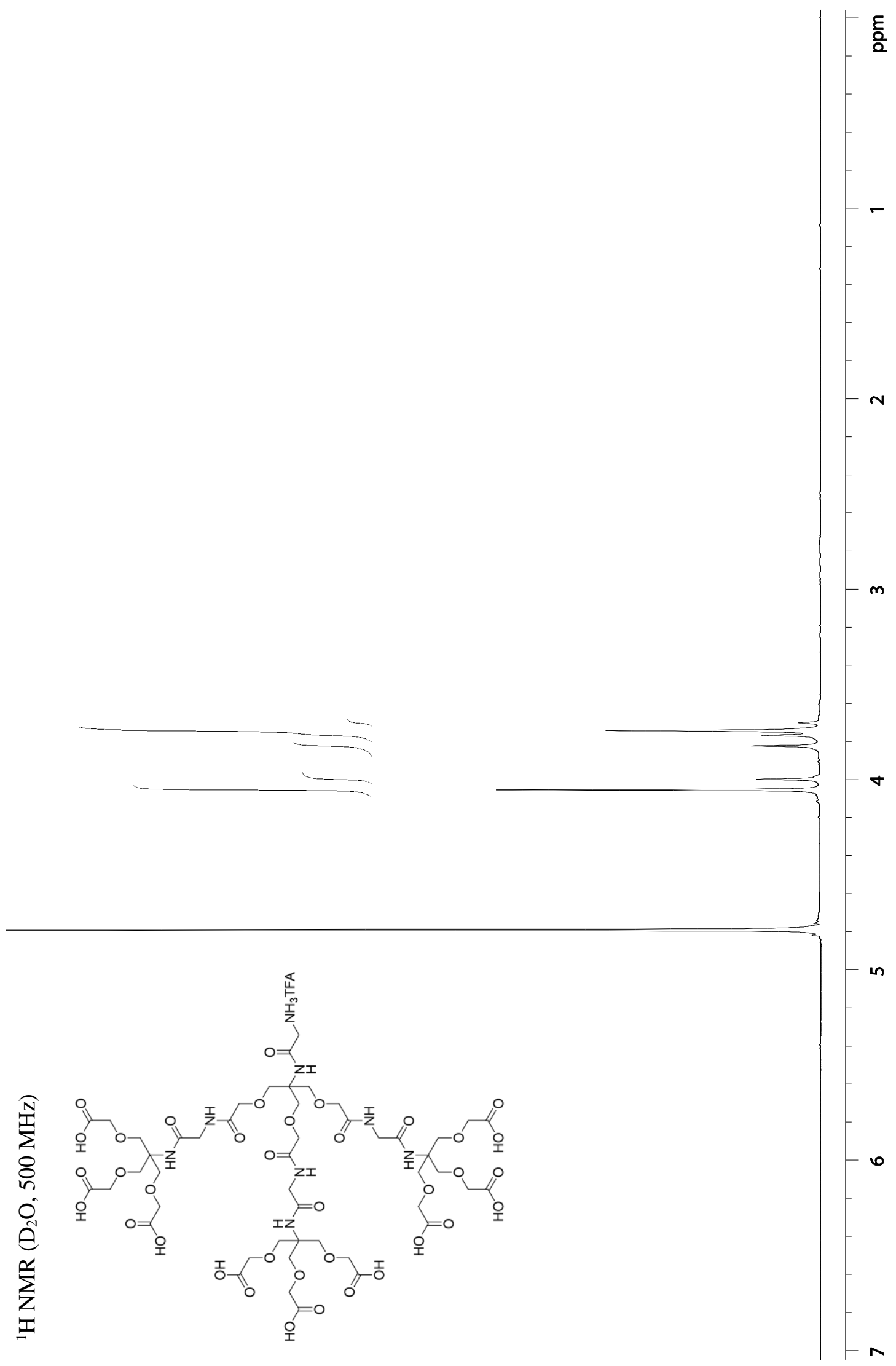

Appendix - 79 


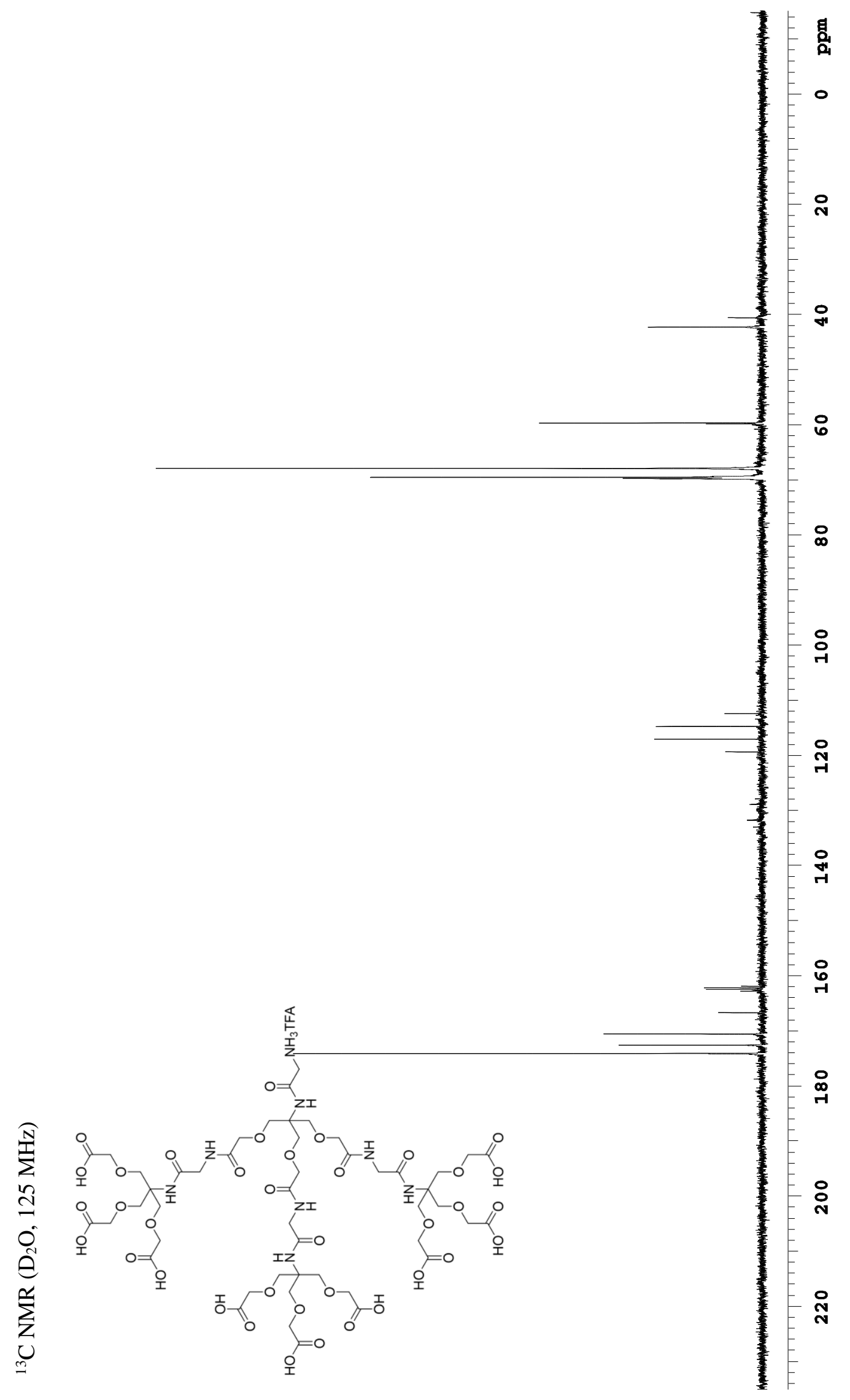

Appendix - 80 


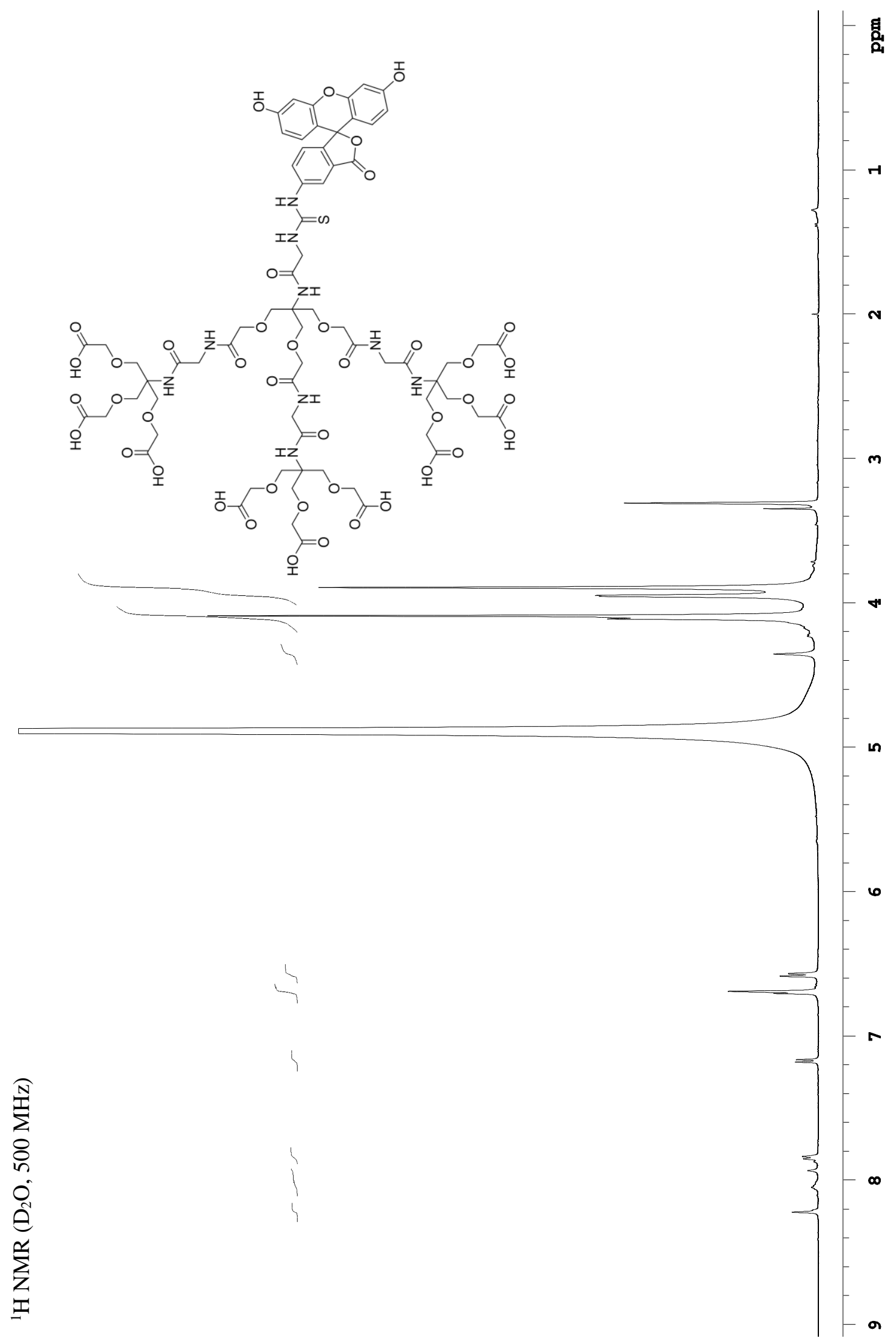

Appendix - 81 


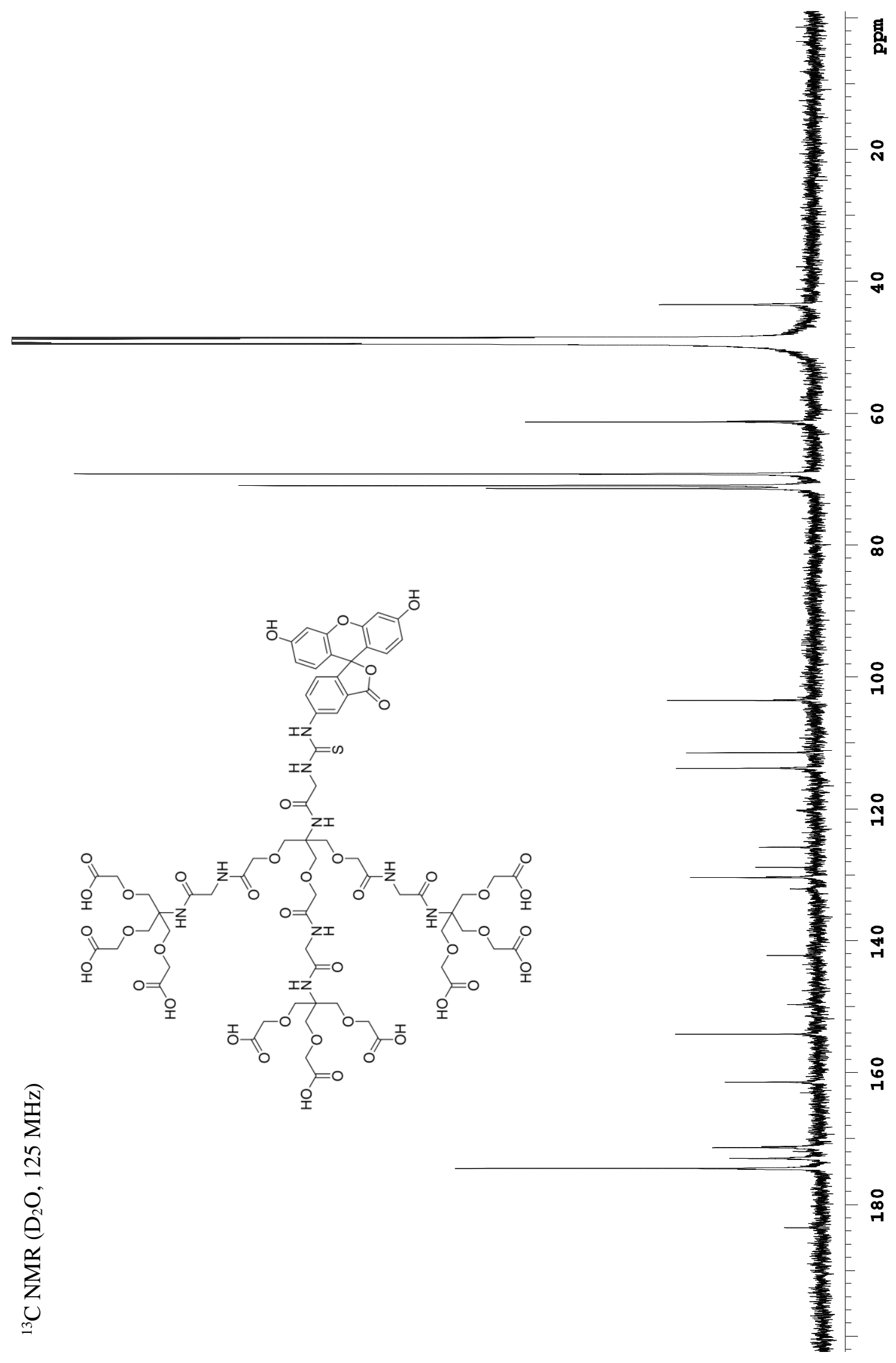

Appendix - 82 


\section{Appendix Chapter 4.}

\section{The Highly Efficient Synthesis of a crystalline trisaccharide for the construction of Lewis ${ }^{x}$ and related antigens.}

\section{Table of content}

Benzyl 2-acetamido-2-deoxy- $\alpha$-D-glucopyranoside (2).............................................A84

Benzyl 2-acetamido-6-O-tert-butyldiphenylsilyl-2-deoxy- $\alpha$-Dglucopyranoside (3).

Phenyl 2,3-di-O-benzoyl-4,6-O-benzylidene-1-thio- $\beta$-Dgalactopyranoside (70)

Ethyl 2,3,4-tri- $O$-benzyl- $\beta$ - L-fucopyranoside (8).

Benzyl 2-acetamido-4- $O$-(2,3-di- $O$-benzoyl-4,6- $O$-benzylidene- $\beta$-D-galactopyranoside)-2-deoxy-6-O-tert-butyldiphenylsilyl- $\alpha$-D-glucopyranoside (10)

Benzyl 2-acetamido-3- $O$-(2,3,4-tri- $O$-benzyl- $\alpha$-L-fucopyranoside)-4- $O$-(2,3di-O-benzoyl-4,6-O-benzylidene- $\beta$-D-galactopyranoside)-2-deoxy-6-Otert-butyldiphenylsilyl- $\alpha$-D-glucopyranoside (11) .

Benzyl 2-acetamido-3-O-(2,3,4-tri- $O$-benzyl- $\alpha$-L-fucopyranoside)-4- $O$ -

(4,6-O-benzylidene- $\beta$-D-galactopyranoside)-2-deoxy- $\alpha$-Dglucopyranoside (12).

2 -acetamido-2-deoxy-3-( $\alpha$-L-fucopyranoside)-4-( $\alpha / \beta$-D-galactopyranoside $))$ $\alpha$-D-glucopyranoside (Lewis ${ }^{\mathbf{x}}$ ) 


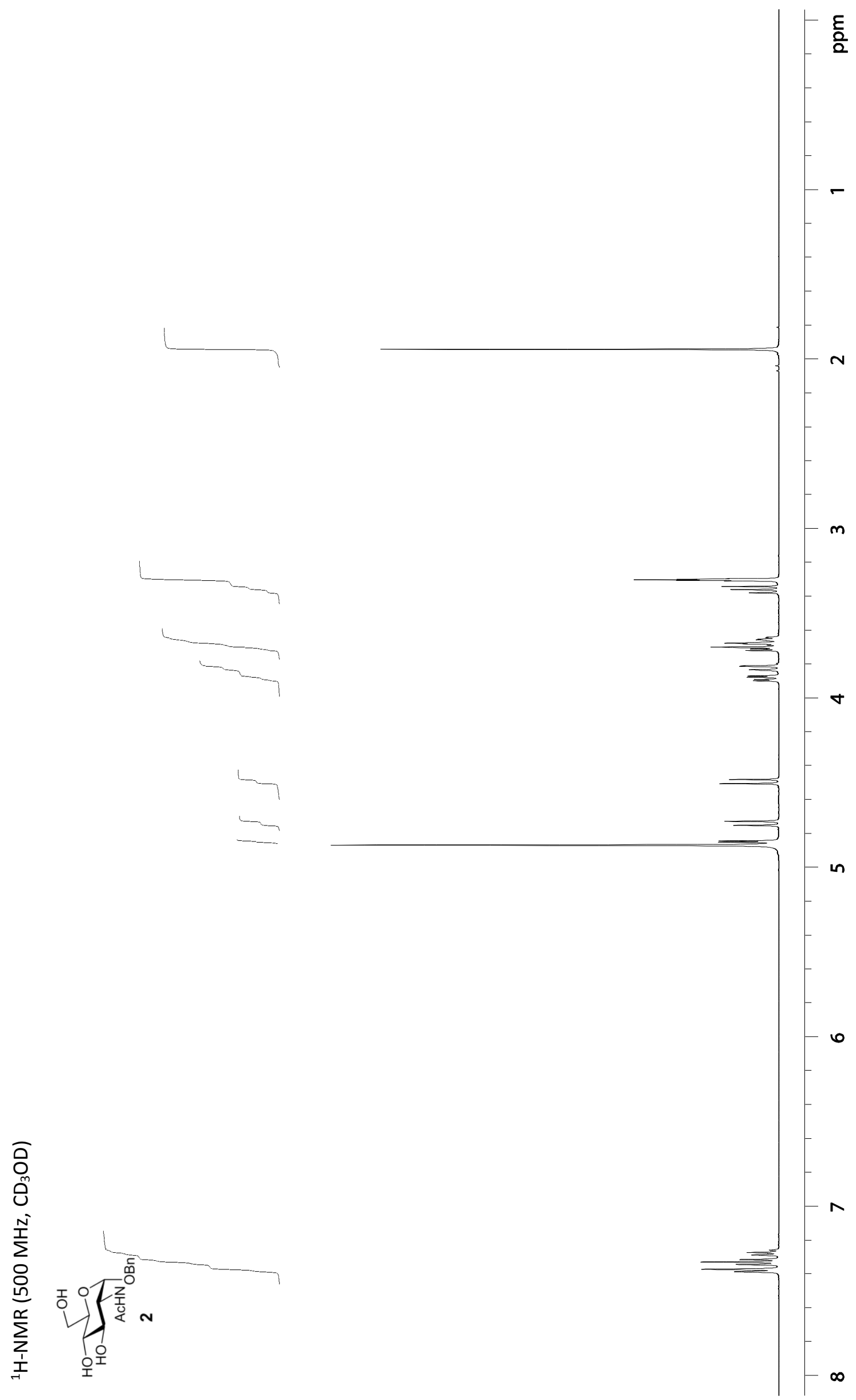

Appendix - 84 


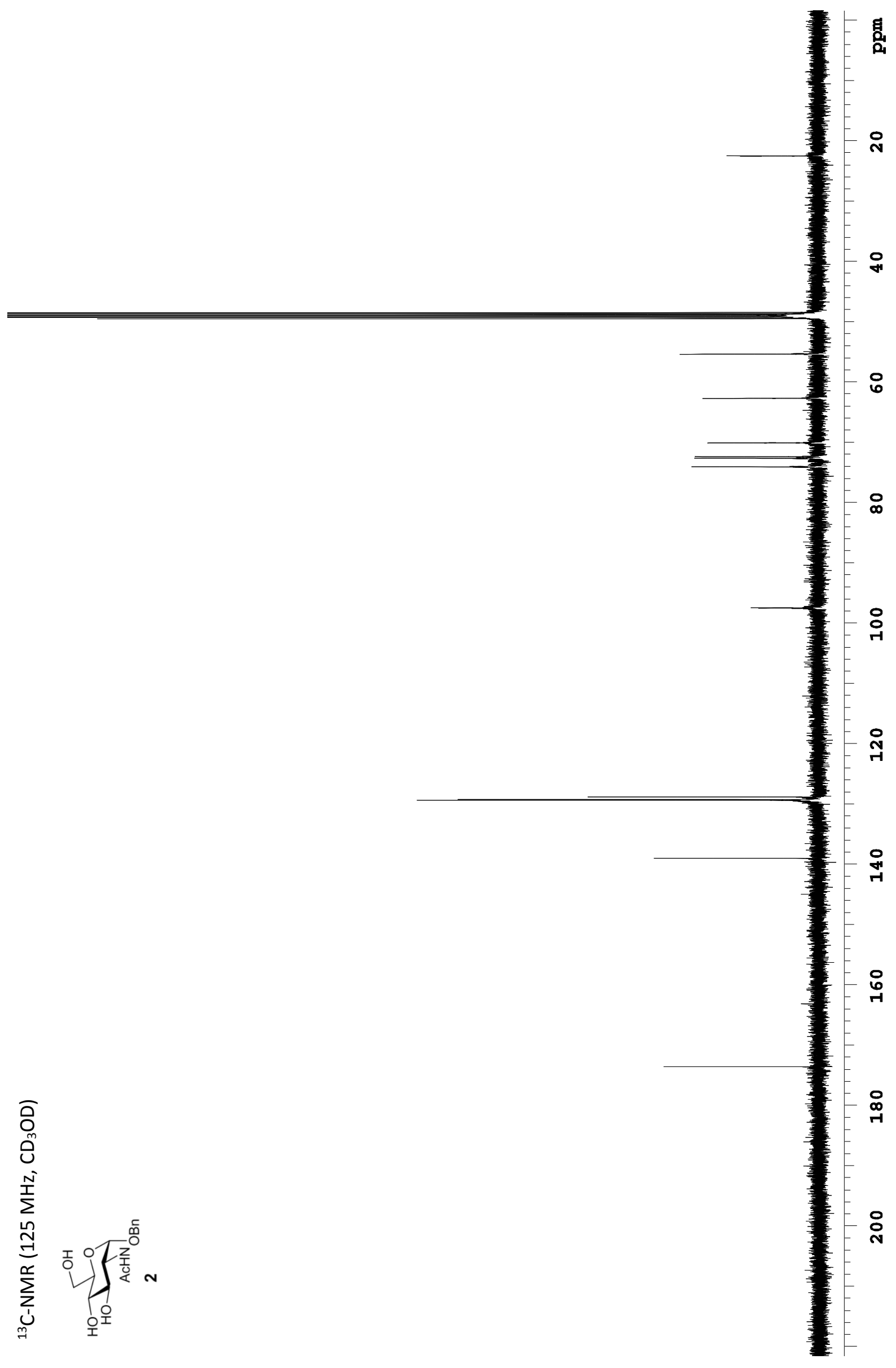

Appendix - 85 


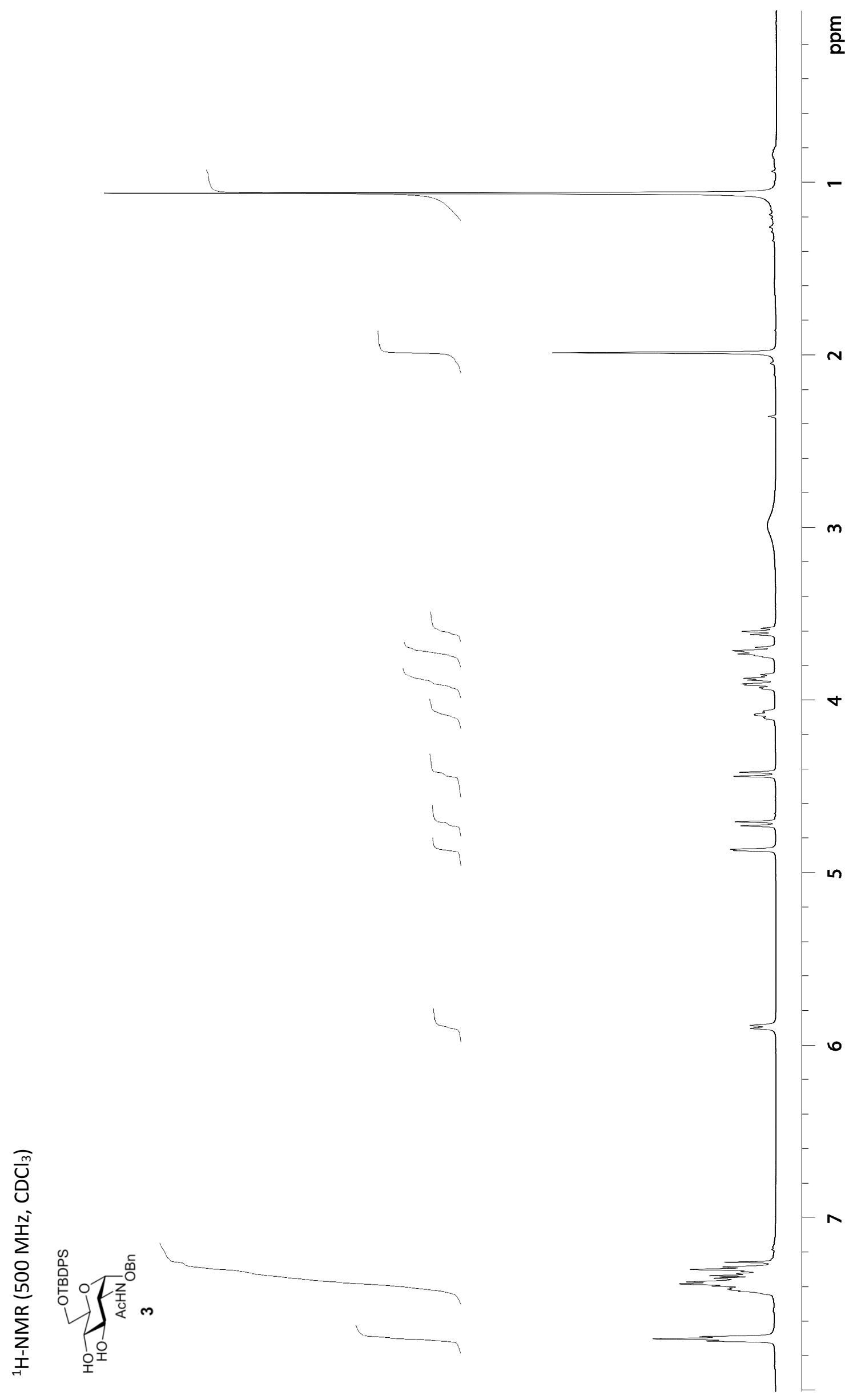

Appendix - 86 


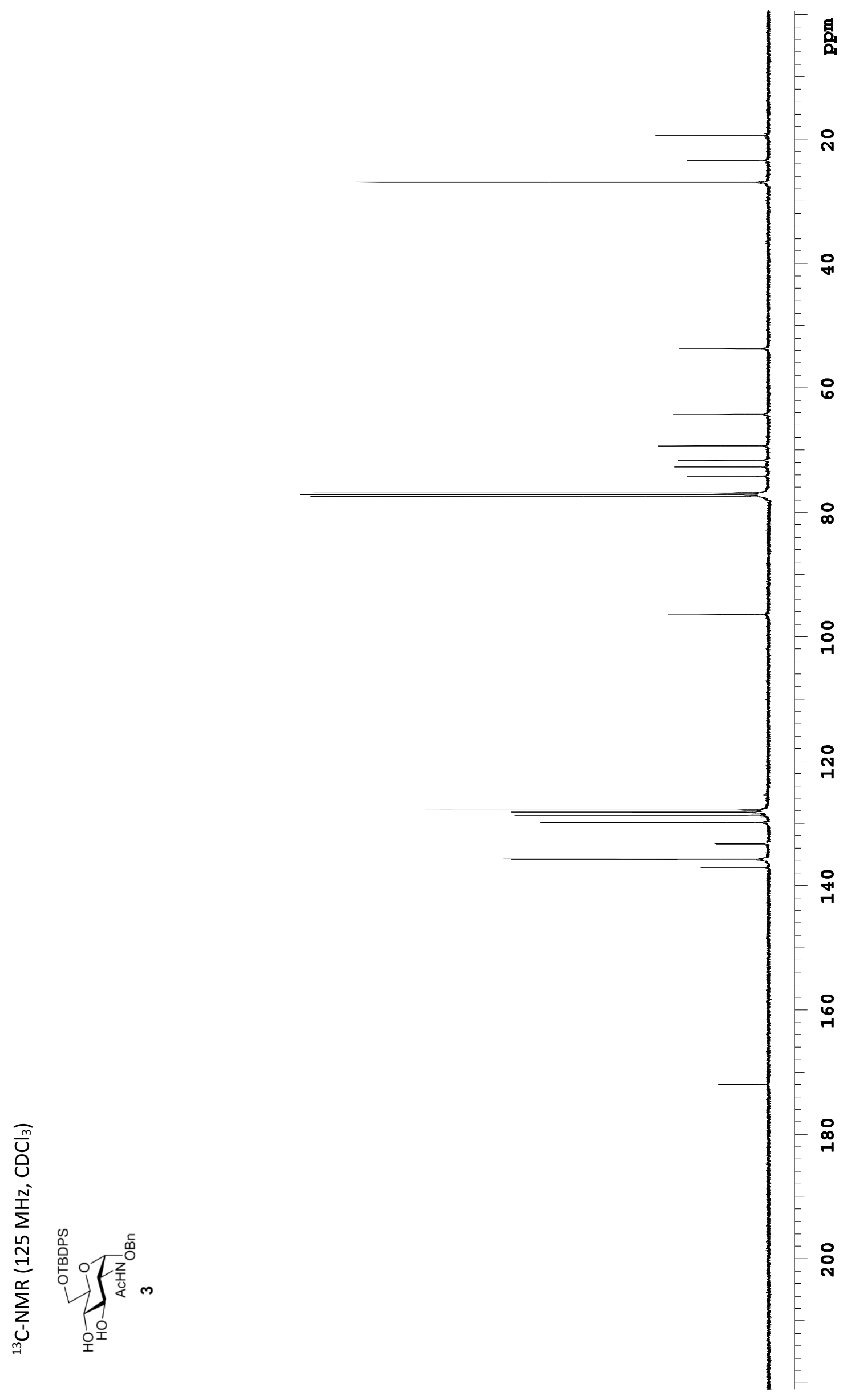



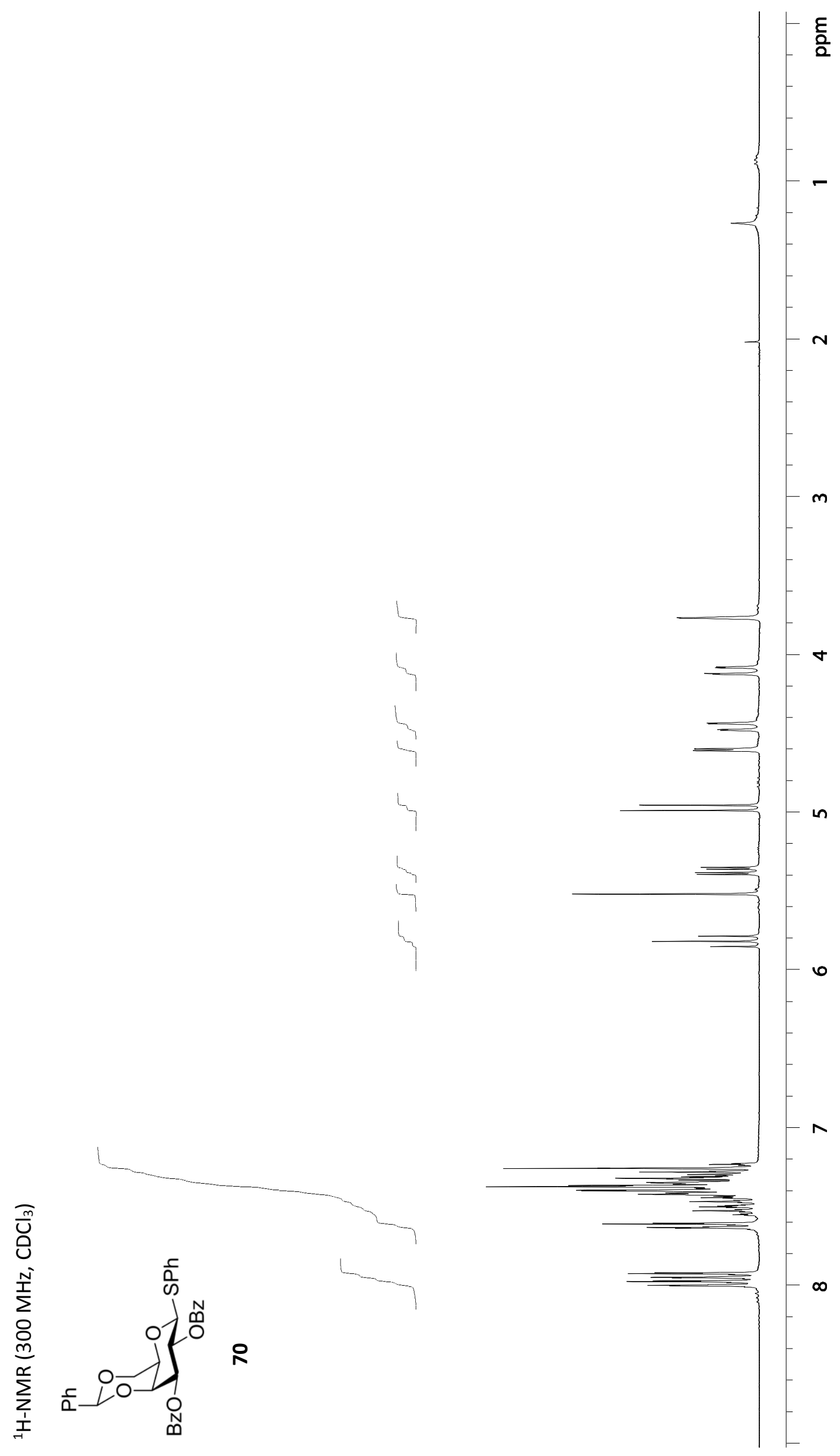

Appendix - 88 


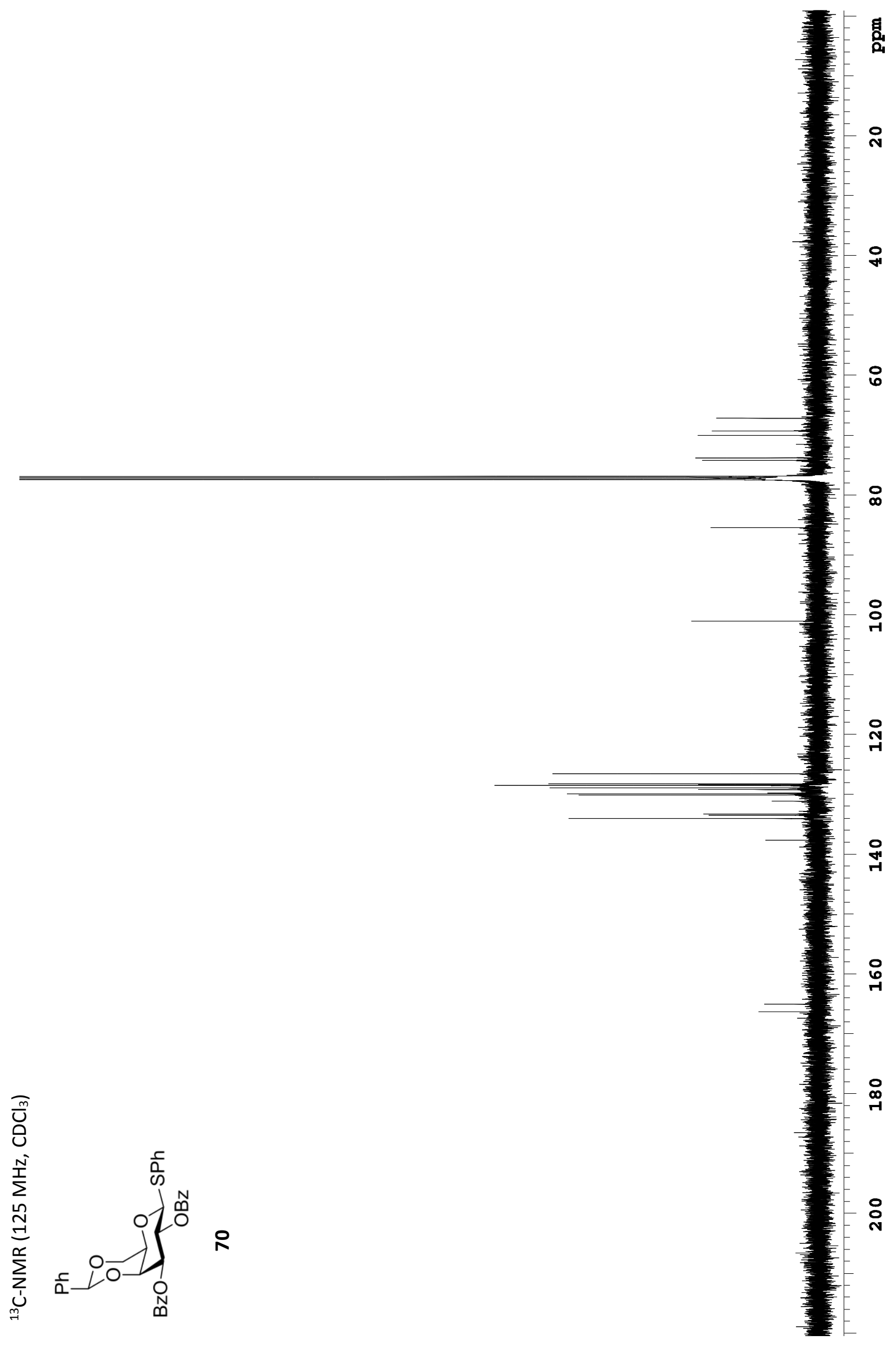

Appendix - 89 


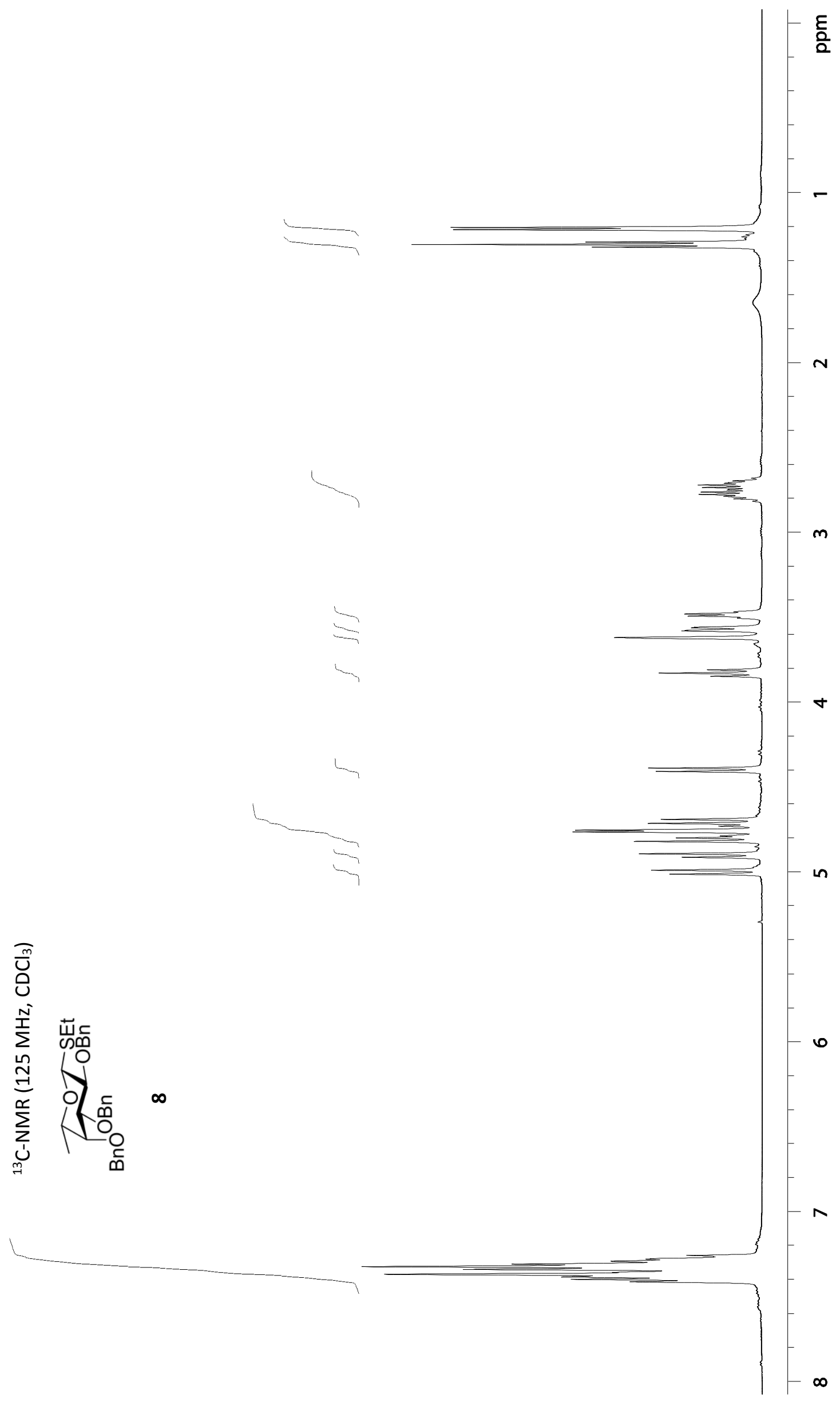

Appendix - 90 


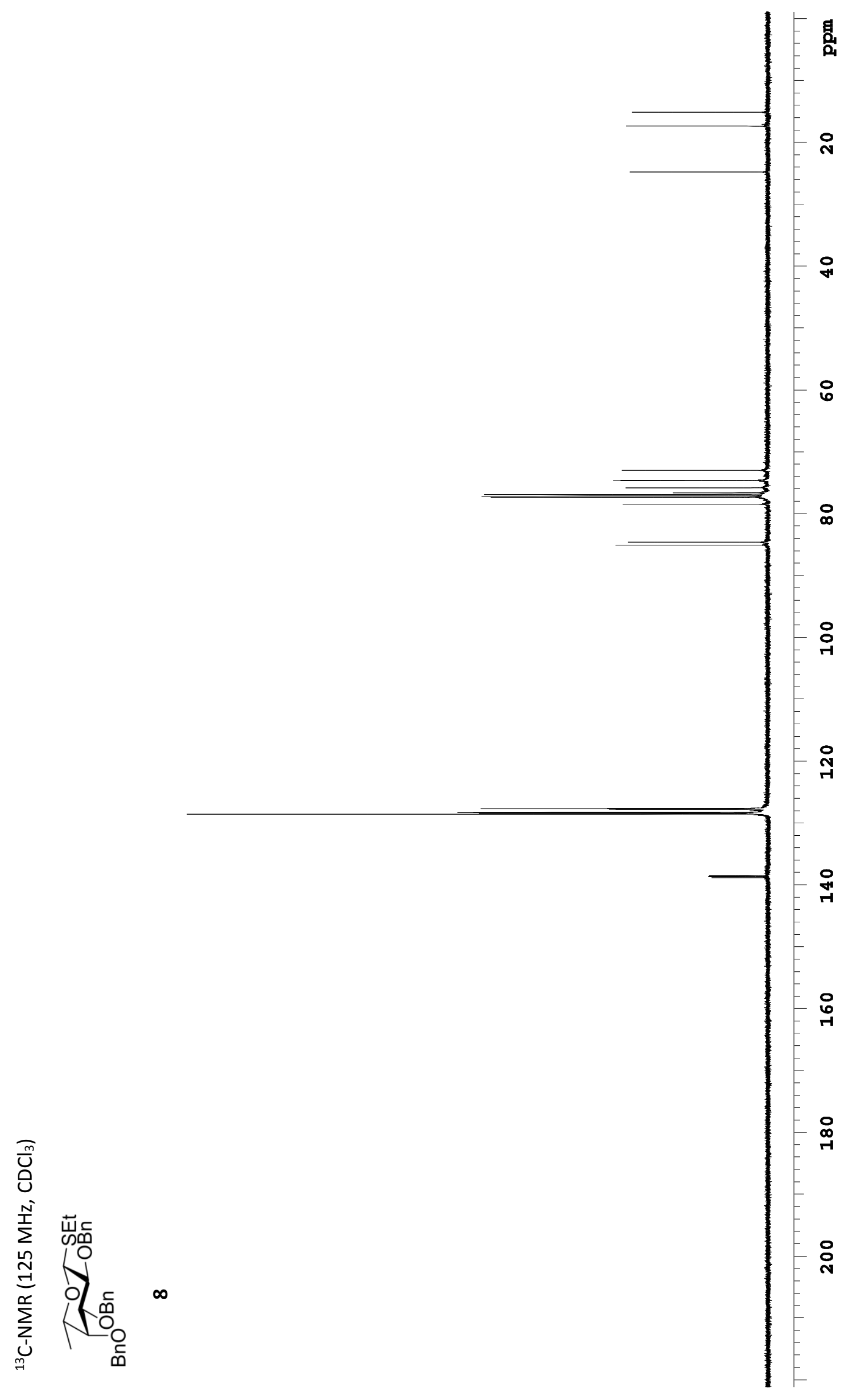




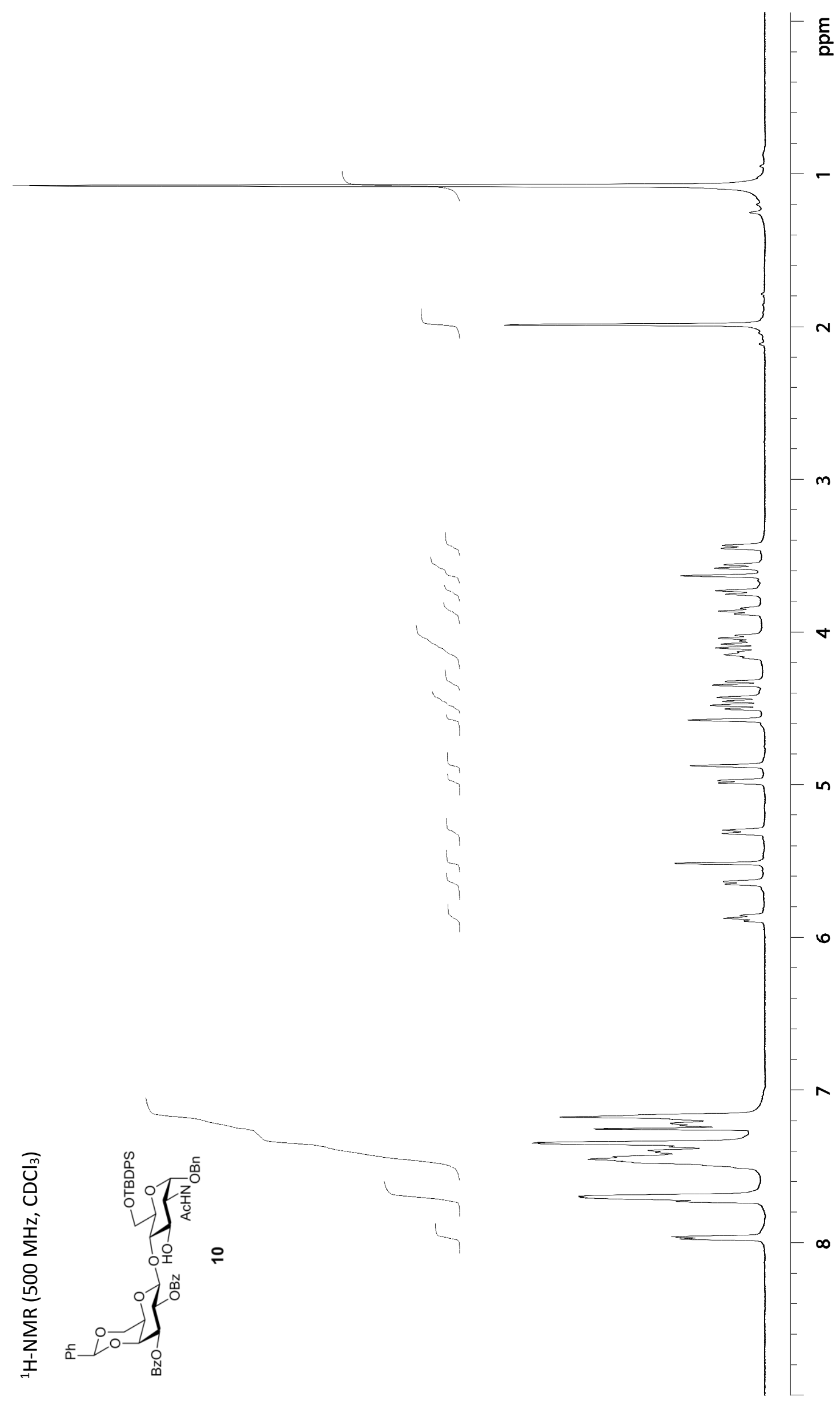

Appendix - 92 


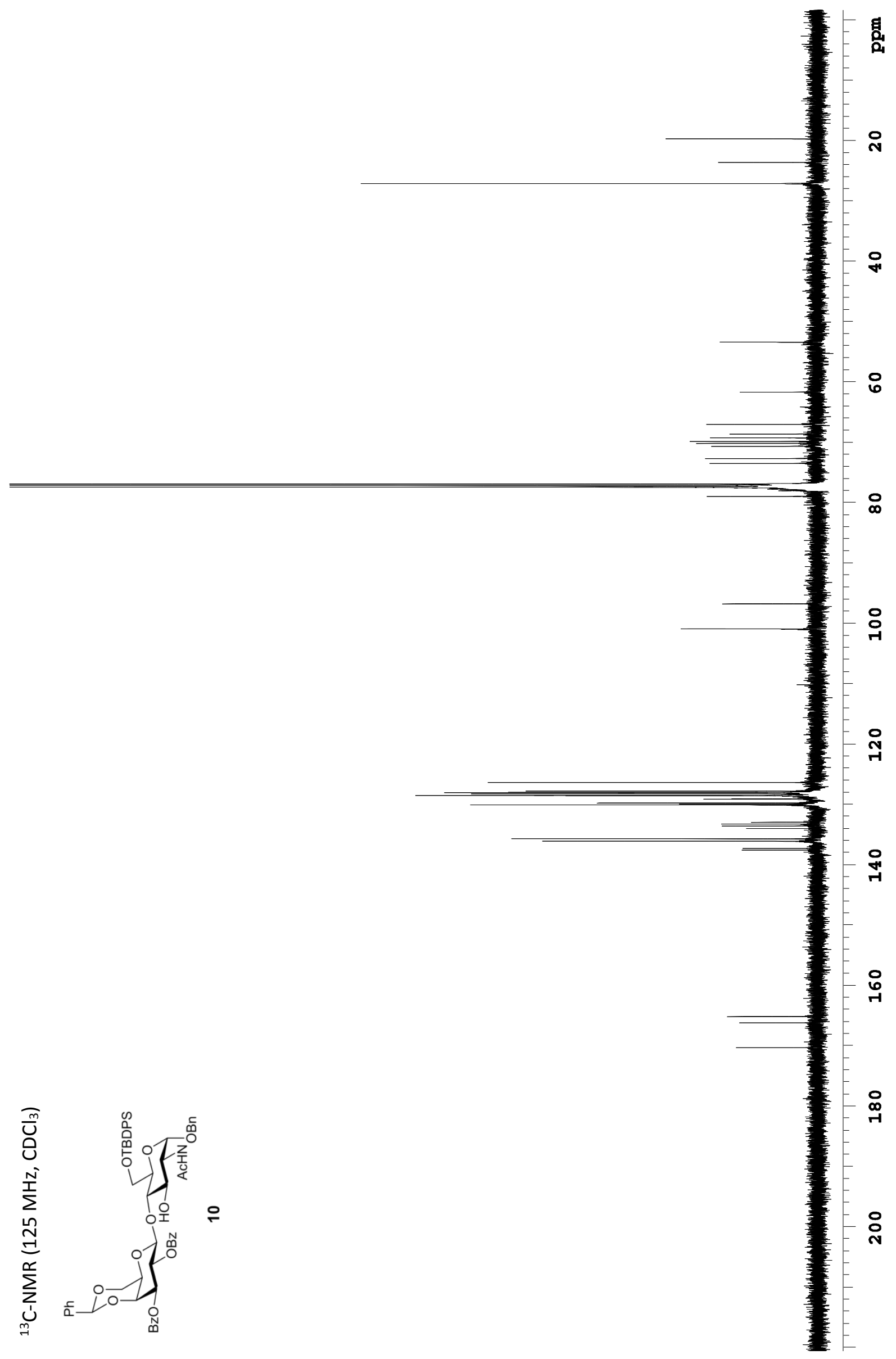

Appendix - 93 


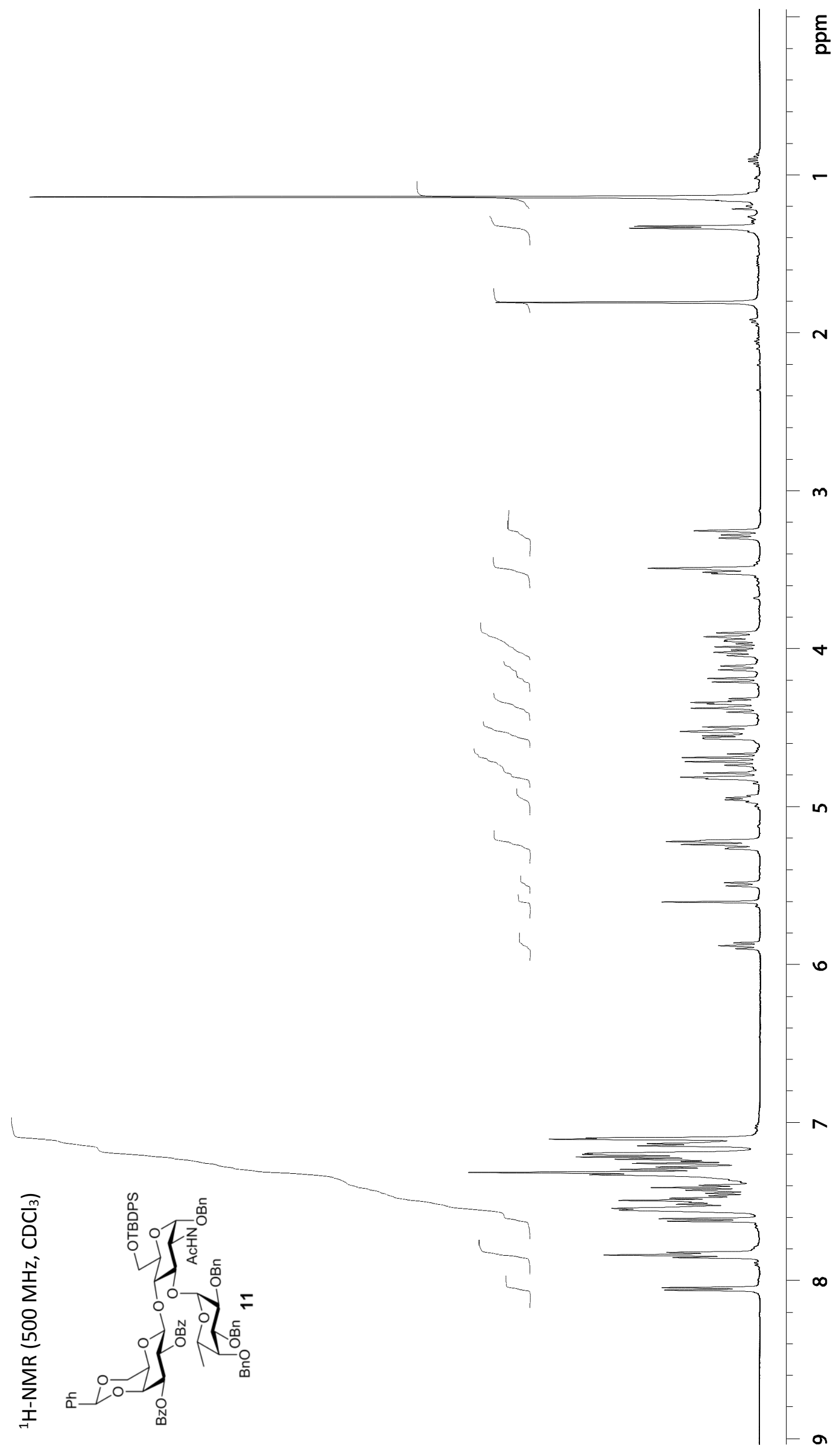

Appendix - 94 


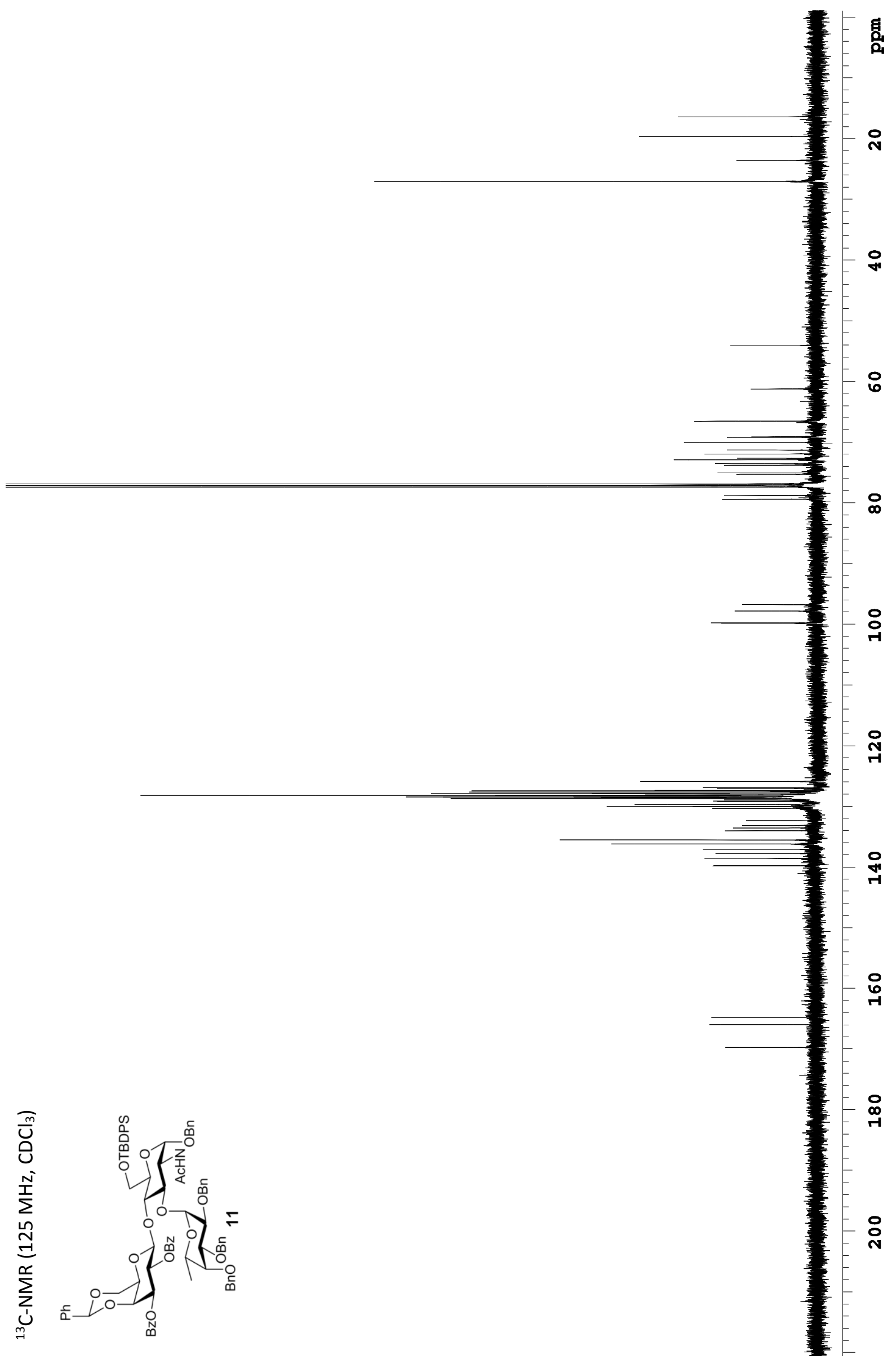

Appendix - 95 


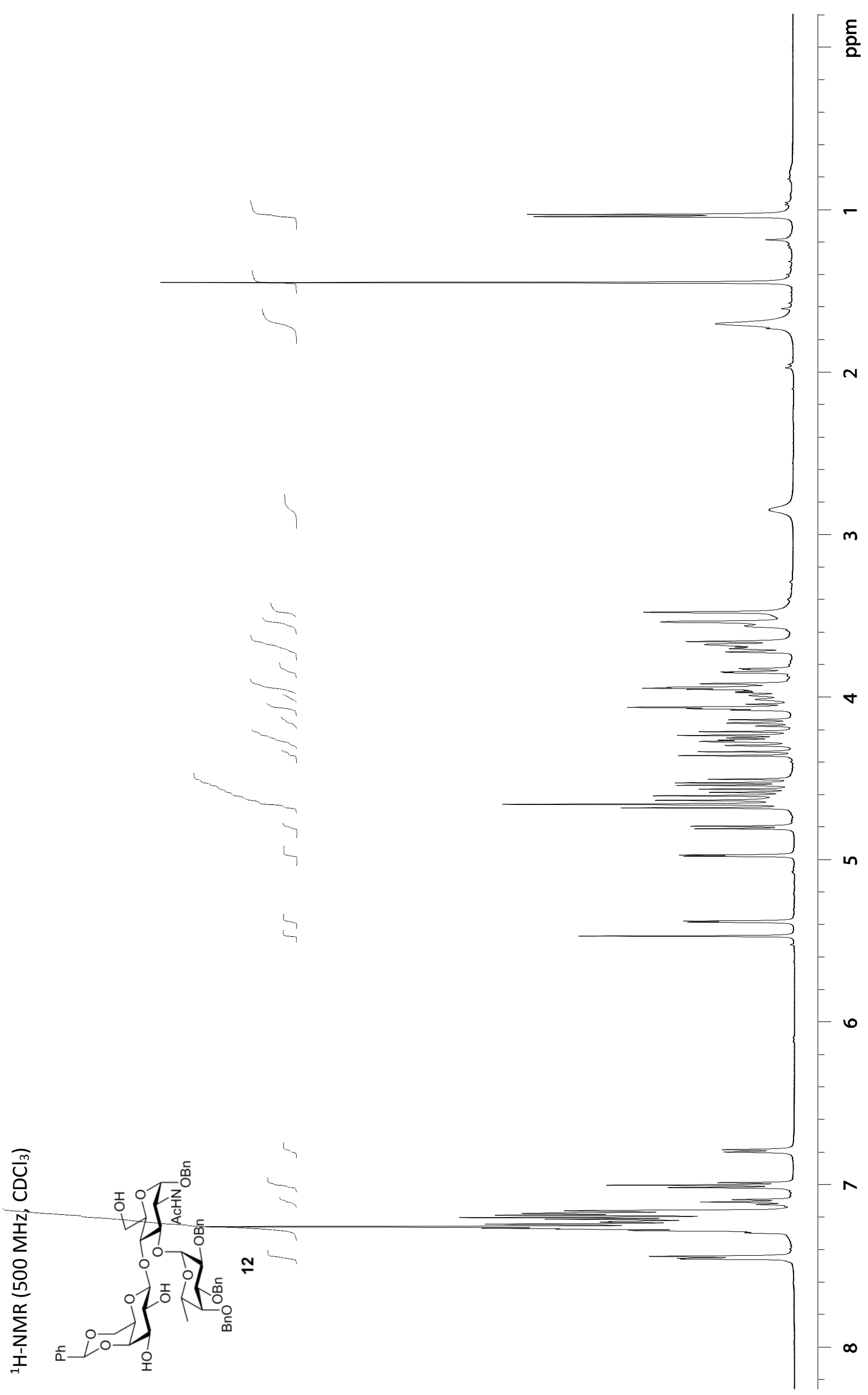

Appendix - 96 


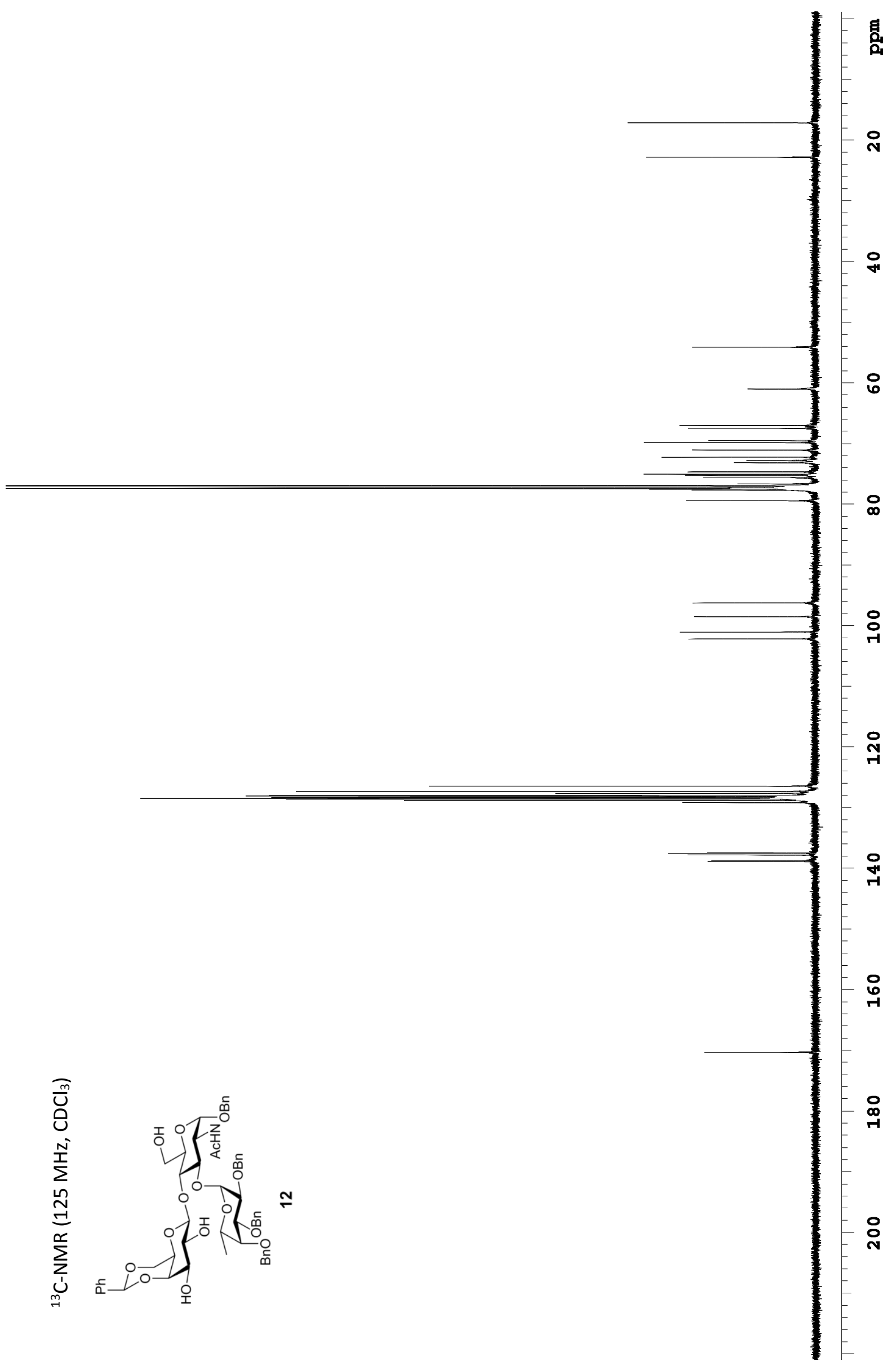

Appendix - 97 


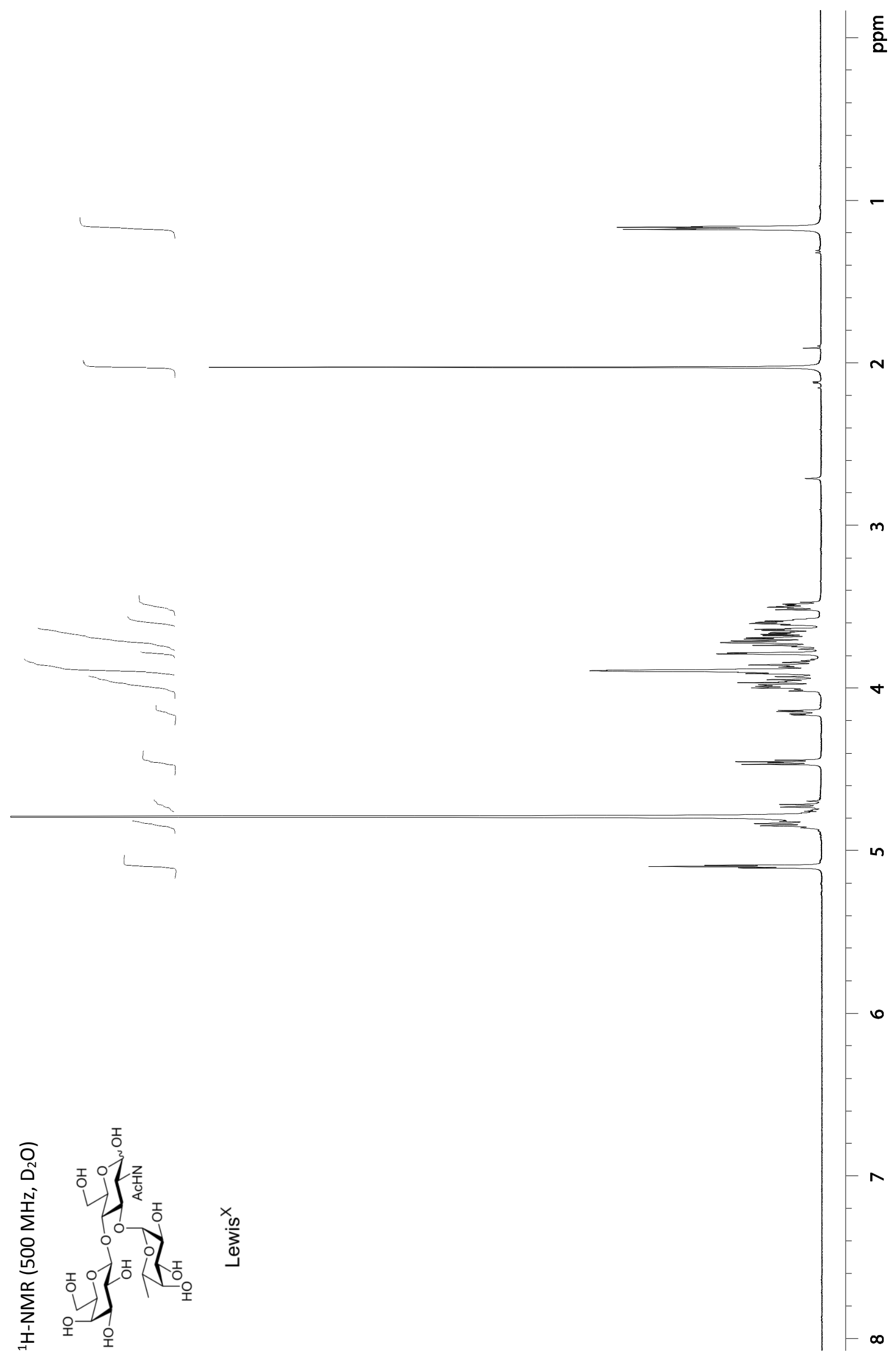




$$
1
$$




\section{Appendix Chapter 5.}

\section{The synthesis and biological evaluation of fluorescent Lewis ${ }^{\mathrm{X}}$ glycodendrons to target DC-SIGN lectins on Macrophages and Dendritic cells.}

\section{Table of content}

\section{$\underline{\text { NMR spectra }}$}

$N$-(2-Acetamido-2-deoxy-3- $O$-( $\alpha$-L-fucopyranosyl)-4- $O$-( $\beta$-D-galactopyranosyl)- $\beta$-Dglucopyranosyl)- $N$-(3-aminopropyl)- $O$-methylhydroxylamine (24)

\section{$\underline{U V-V I S \text { spectra }}$}

$N$-[N-([(3',6'-Dihydroxy-3-oxospiro[isobenzofuran-1(3H),9'-(9H)xanthen]-5-yl)amino]thioxomethyl)glycyl]-1,1,1-tris(1,1,1-tris[3-( $N$-(2-acetamido-2-deoxy-3-O-( $\alpha$-L-fucopyranosyl)-4- $O$-( $\beta$-D-galactopyranosyl)- $\beta$-D-gluco-pyranosyl)- $N$-(3-aminopropyl)- $O-)-$ $O$-methyl-hydroxylamine)propylamidocarbonylmethyloxymethyl]methylamidocarbonylmethylamidocarbonylmethyloxy-methyl)aminomethane (1). 


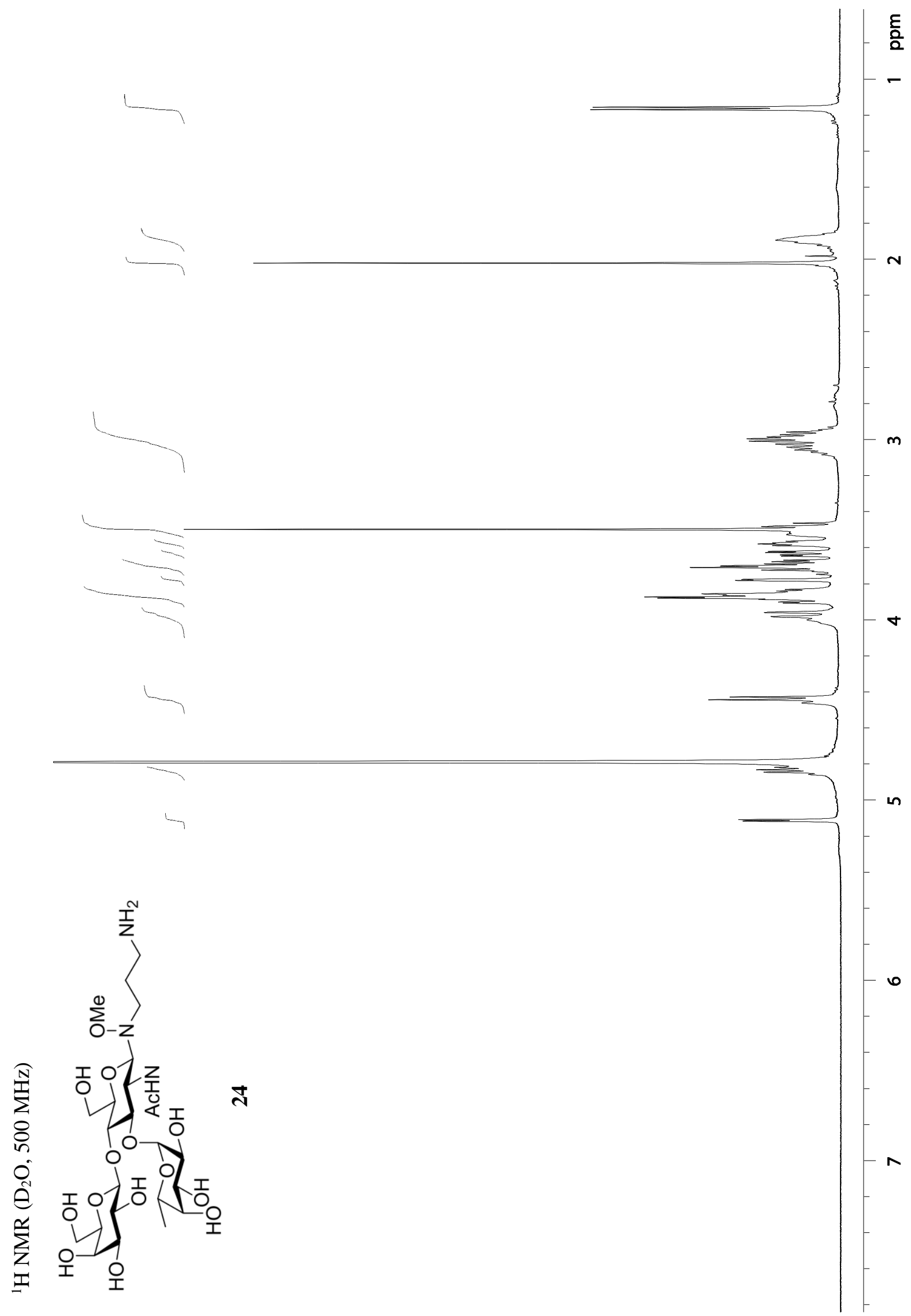



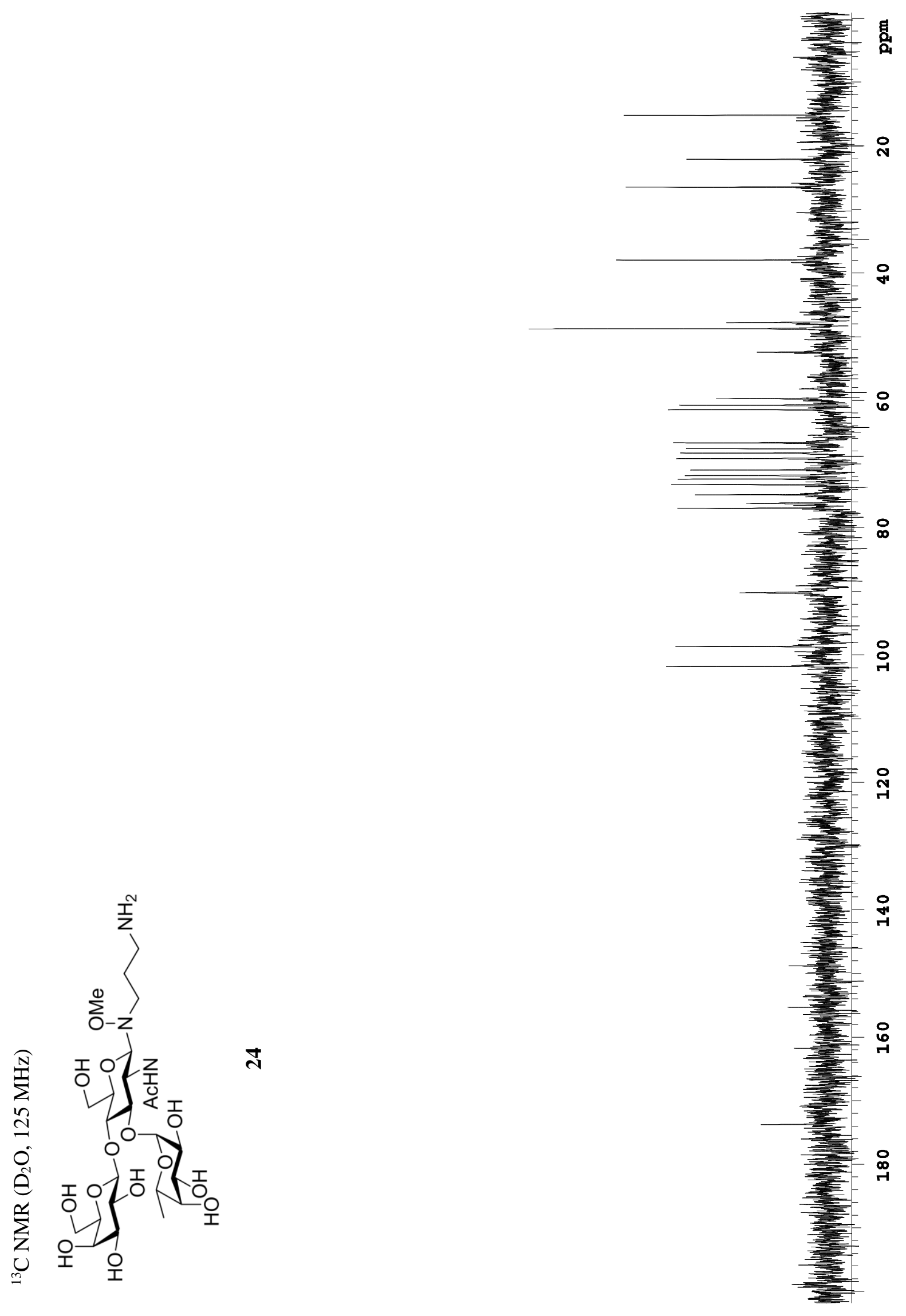
UV-VIS spectra of fluorescent Lewis ${ }^{X}$ glycodendron

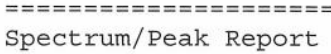

Date 5/26/2015 Time 18:00:44 Page 1 of 1

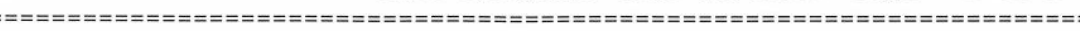

Method file:

<untitled>

Information : Default Method

Data File

D: \Ste fanM \LEWISX-DENDRON-10 0UL-160 0UL .SD

$16: 44: 15$

Created : 5/6/15

Overlaid spectra:

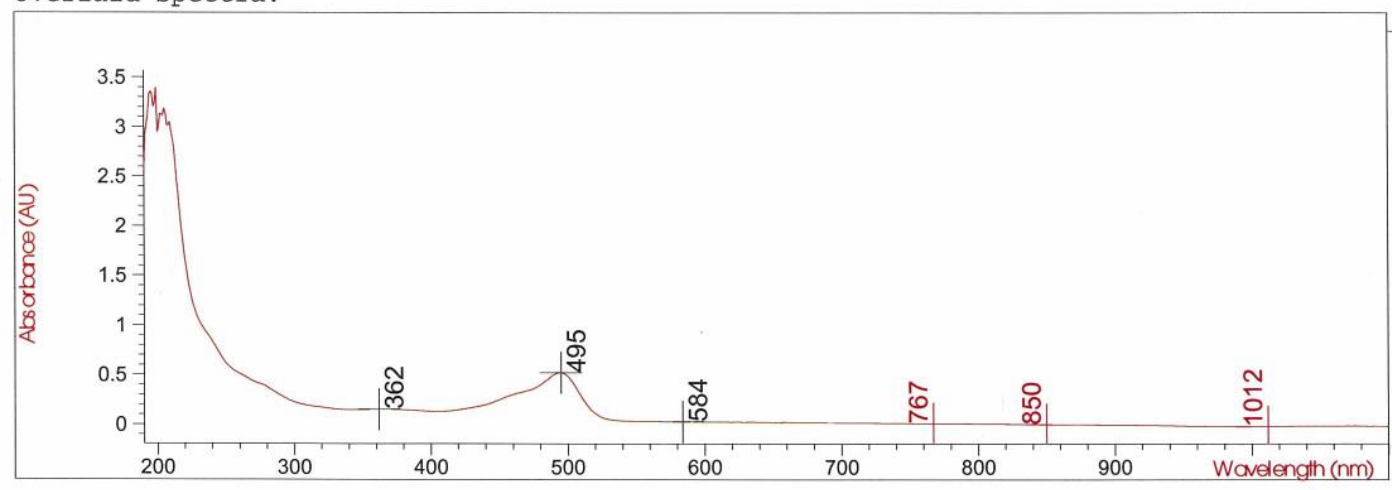

\begin{tabular}{lrrrr}
$\#$ Name & Peaks $(\mathrm{nm})$ & $\mathrm{Abs}(\mathrm{AU})$ & $\mathrm{Valleys}(\mathrm{nm})$ & $\mathrm{Abs}(\mathrm{AU})$ \\
\hline 1 & 495.0 & 0.52230 & 1012.0 & $-2.0363 \mathrm{E}-2$ \\
1 & 362.0 & 0.15248 & 850.0 & $1.0519 \mathrm{E}-3$ \\
1 & 584.0 & $2.7593 \mathrm{E}-2$ & 767.0 & $8.9579 \mathrm{E}-3$ \\
2 & 495.0 & 0.52230 & 1012.0 & $-2.0363 \mathrm{E}-2$ \\
2 & 362.0 & 0.15248 & 850.0 & $1.0519 \mathrm{E}-3$ \\
2 & 584.0 & $2.7593 \mathrm{E}-2$ & 767.0 & $8.9579 \mathrm{E}-3$
\end{tabular}

Report generated by : Service

Signature:

*** End Spectrum/Peak Report *** 